\title{
Proteomic analysis of glutathione transferases from \\ Lucilia cuprina
}

\author{
By \\ Ramavati Pal
}

\begin{abstract}
A thesis
Submitted to the Victoria University of Wellington

in fulfilment of the requirements for the degree of Doctor of Philosophy in Cell and Molecular Bioscience
\end{abstract}

Victoria University of Wellington 2010 


\section{Acknowledgements}

I would like to thank all people who have helped and inspired me during my $\mathrm{PhD}$.

First and foremost, my utmost gratitude to my supervisor, Dr. Alan Clark, whose sincerity and encouragement I will never forget. Dr. Alan has been my inspiration as I hurdle all the obstacles in the completion of this research work.

I was delighted to interact with Dr. Paul Teesdale-Spittle during our monthly meetings and have him as my co-supervisor. He has shared valuable insights in the relevance of the study and critically reviewed this manuscript.

I would like to thank Dr. Bill Jordon for allowing me to use the Proteomics facilities at the lab.

Many thanks go to Dr. Lifeng Peng for running my samples using LC-MS and providing me the results in time.

I am heartily thankful to my lab-mate Xue Ding, for teaching me all the experimental proteomic techniques from the start of this project. I would also like to thank other members of the lab for their support and valuable input especially Jonathan Dunne and Graeme Lindsay.

Thanks to Chris and Laurent for supplying flies throughout the study.

Many thanks due to Mary, Sandra and Patricia for administrative help.

Thanks to VUW PhD scholarship and the Wellington Medical Research Foundation for supporting me financially during my research.

Many thanks to my husband, Uppender Raghav; without his support, it would have been so hard to complete this project in time! Thanks chaku, for helping me during my tough time and supporting me wholeheartedly!!

Last but not the least, thanks to my family especially my mother and father, whose decision to allow me to come so far from India to study in New Zealand. Thanks to my brothers Mukesh and Santosh for their encouragement over phone during the stumbling phase of my research.

Thank God!! I am done!!! 


\section{Abstract}

The glutathione transferases are a family of multifunctional enzymes involved in detoxification of xenobiotic and endogenous electrophilic compounds. Interest in insect GSTs has primarily focused on their role in insecticide resistance. The sheep blowfly, Lucilia cuprina is a major economic problem for the sheep meat and wool industries in Australasia and hence this thesis has attempted the study of the Lucilia cuprina GST family, using proteomics, with a view to eventually determining their role in insecticide resistance.

Combinations of different affinity matrices (glutathione-Sepharose matrix a followed by dinitrophenyl-glutathione-Sepharose matrix (DNP-GSH)) and twodimensional electrophoresis has successfully isolated members from major four insect GST classes: Sigma, Delta, Epsilon and Omega. Drosophila melanogaster has been used as a model insect throughout as a basis for comparison. To characterise Lucilia GSTs, the whole metazoan fragmentation database was used for sequence alignment with Lucilia peptides. This approach is broad and speculative but predicts a possible classification of the GSTs based on $\%$ similarity and $\%$ identity. This method of characterisation yielded match scores that provided a basis for classification, which must at present be regarded as tentative and in need of confirmation.

In D. melanogaster and L. cuprina, GSH affinity-purified extracts showed the presence of only Sigma and Delta GSTs. In D. melanogaster, the DNP-GSH affinity-purified GSTs showed mostly the presence of Epsilon and Omega GSTs whereas in L. cuprina no Omega GSTs were detected. In both species, the migration pattern of Delta GST on 2D PAGE gel indicated possible post-translational 
modification. The results from analysis of LC-MS/MS data by the software PEAKS suggested deamidation at asparagine and glutamine residues in a limited number of the matched peptides of Delta GST.

GST activity was present in all developmental stages of $L$. cuprina. The number of isoenzymes and their extent of expression vary as the insect develops. Delta GSTs were present in all developmental stages. The Sigma GST started expressing from the larval stage and was abundantly present in adult stage. The DNP-GSH affinity matrix purified GSTs which have been tentatively classified as Mu-like GSTs were present in egg, larvae and pupae but totally absent in adult stage.

The GST families were characterised by proteomics in the main body sections of L. cuprina. Higher GST activity towards 1-chloro-2, 4-dinitrobenzene (CDNB) was found in the thorax $(65.2 \%)$ followed by the abdomen $(19.6 \%)$ and the head $(15.2 \%)$.

The cytosolic GSTs of a resistant strain (PY81) of L. cuprina had significantly higher (2.26- and 2.6- fold) activity than the susceptible strains (NSW and CSIRO) towards CDNB and 2, 3-dichloro, 4-nitrobenzene (DCNB) respectively. The proteomic analysis of DNP-GSH purified extract from susceptible and resistant strains showed quantitatively higher expression of GSTs on 2D PAGE gel of the PY81 strain. The in vitro interaction of purified GSTs and model insecticides studied by high performance liquid chromatography revealed that Delta and DNP-GSH affinity-purified GSTs catalyse the conjugation of the insecticides to reduced glutathione but Sigma GST had almost no activity. 


\section{Table of contents}

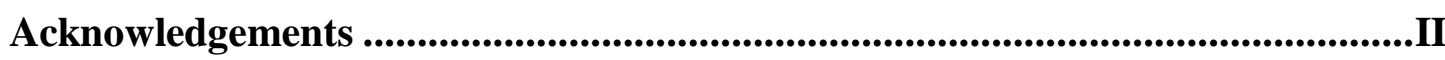

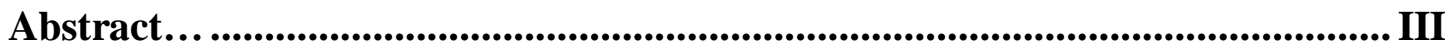

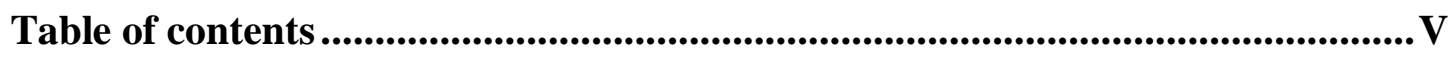

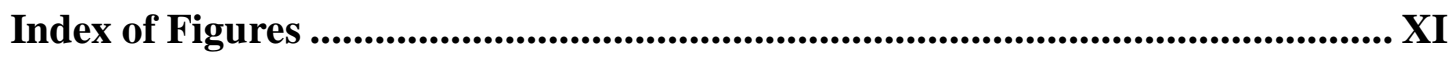

Index of Tables.................................................................................................

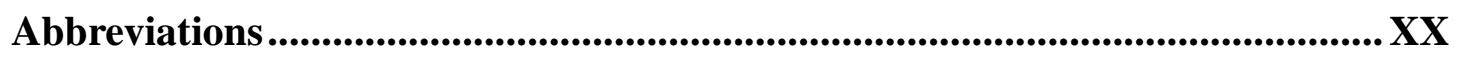

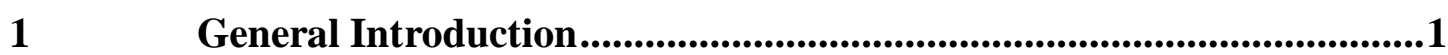

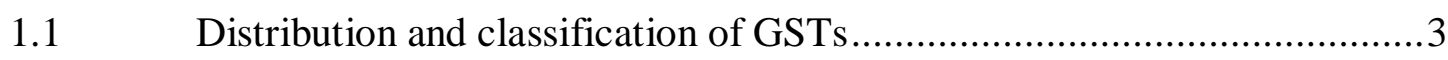

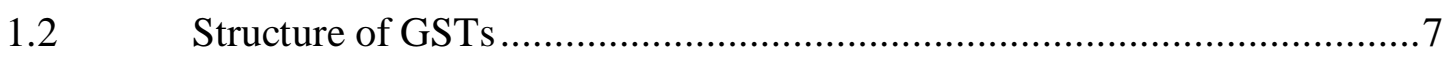

1.3 Mechanism of action of GSTs....................................................... 10

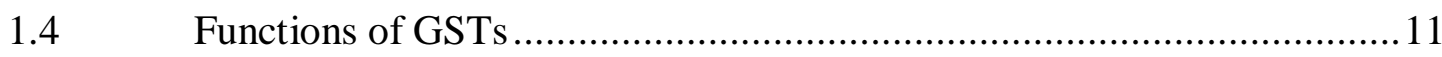

1.4.1 Glutathione conjugation and detoxification ....................................... 11

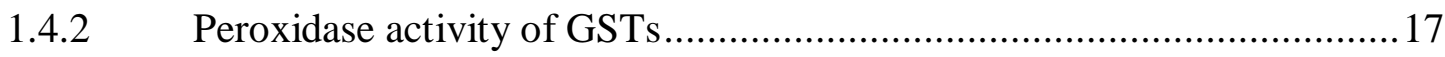

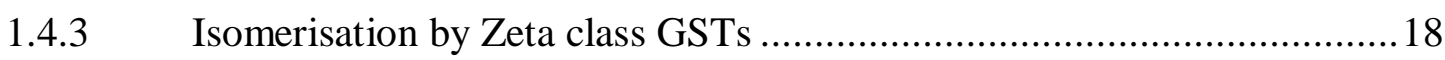

1.4.4 GSTs and the processing of odorant signals ...................................... 19

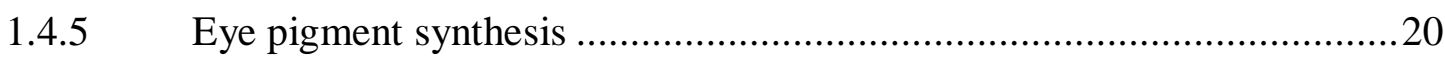

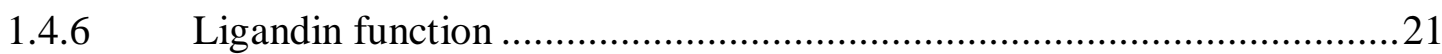

1.4.7 GSTs as regulators of the MAP kinase pathway ..................................22

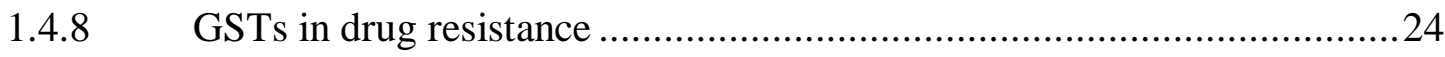

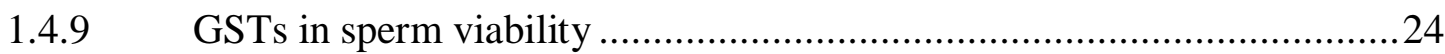

1.4.10 GSTs involved in haematin binding and in wound healing ...................25

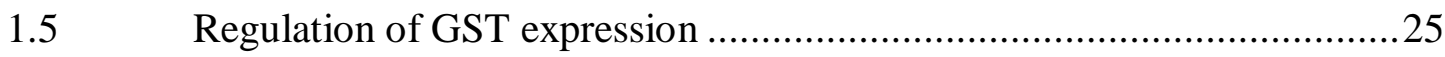

Insecticide resistance and role of GSTs ............................................26

$1.7 \quad$ Lucilia cuprina - an economically significant fly ................................30

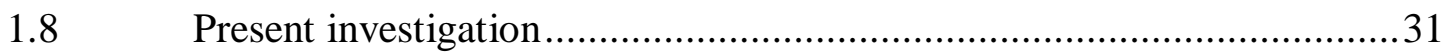

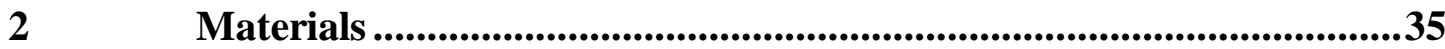




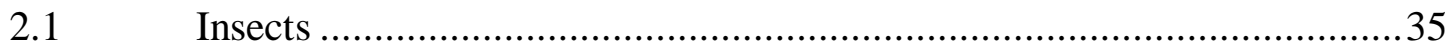

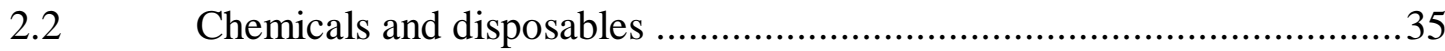

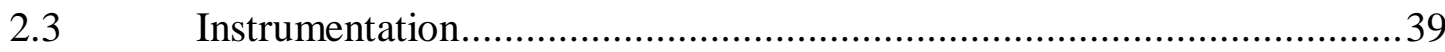

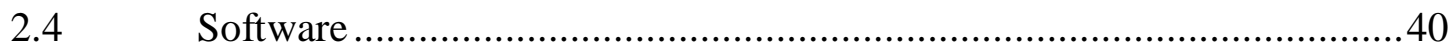

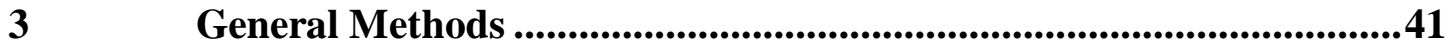

3.1 Introduction to Methods: basic principles underlying experimental

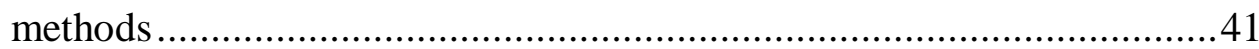

3.1.1 Chromatographic techniques..................................................... 41

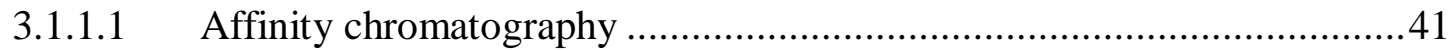

3.1.1.2 Ion exchange chromatography ................................................... 41

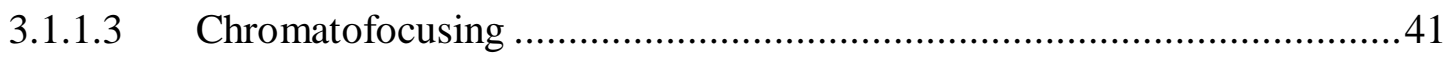

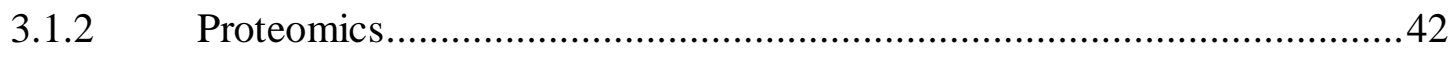

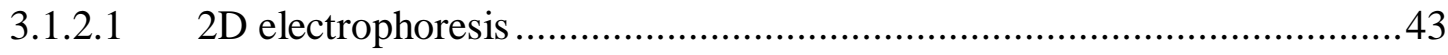

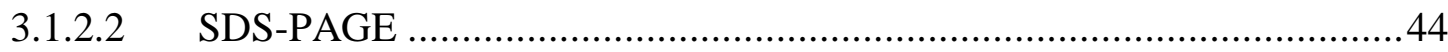

3.1.2.3 Matrix Assisted Laser Desorption/Ionisation -Time Of Flight (MALDI-

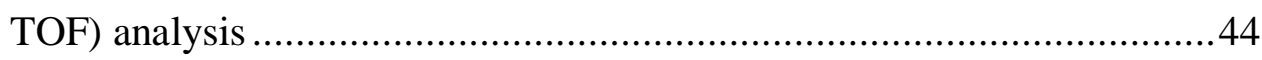

3.1.3 Reverse-phase high performance liquid chromatography .....................47

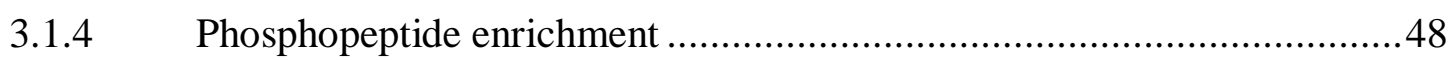

3.1.5 PEAKS: a tandem mass spectrometry software package ......................48

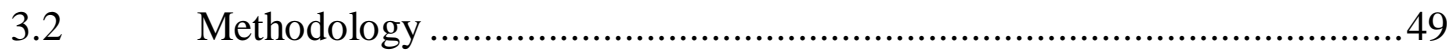

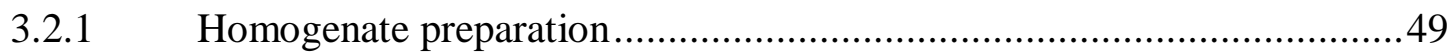

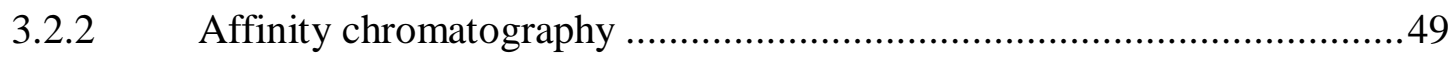

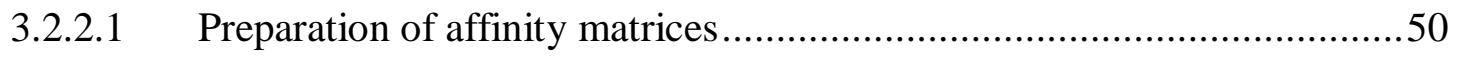

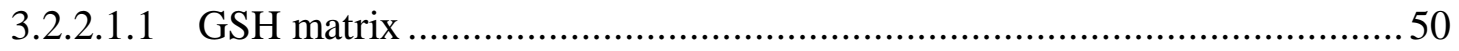

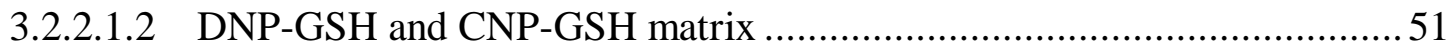

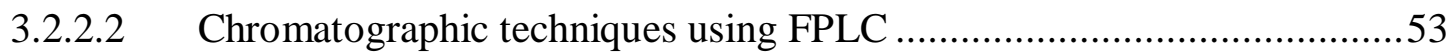

3.2.2.2.1 Desalting / de-pigmenting method protocol .........................................53

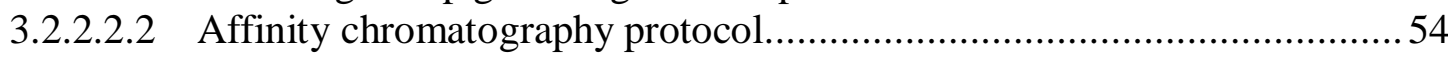

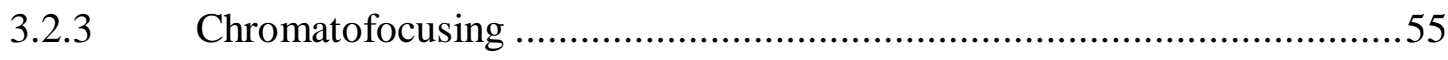

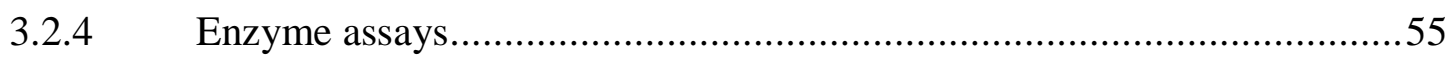

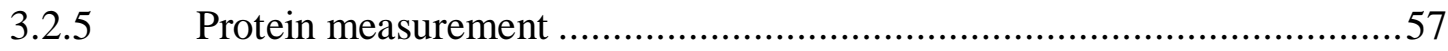

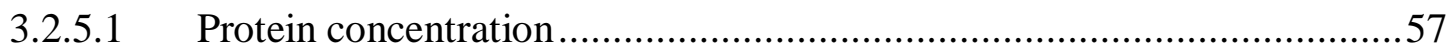




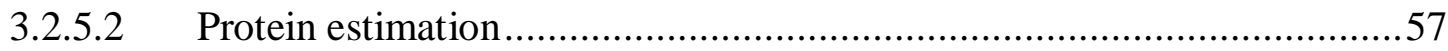

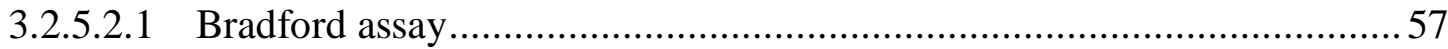

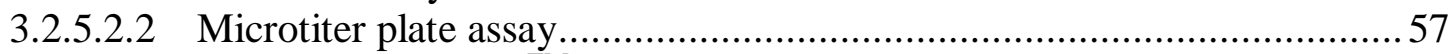

3.2.5.2.3 2D Quant kit (Ettan ${ }^{\mathrm{TM}}$ sample preparation kit and reagents) ................... 58

3.2.6 Protein precipitation of samples for electrophoresis ............................59

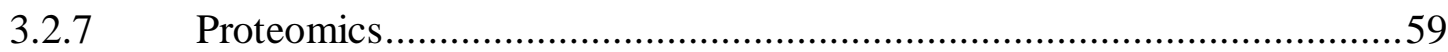

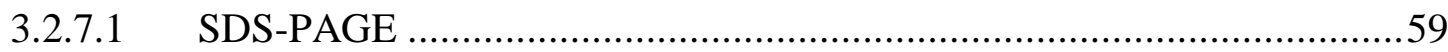

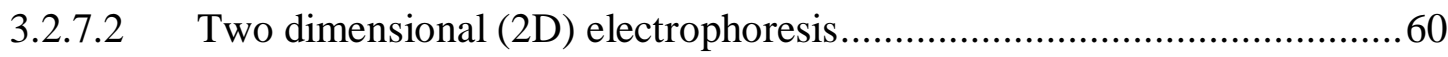

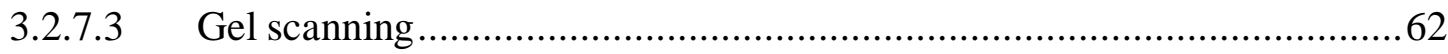

3.2.7.4 Destaining of the gel spots and in-gel digestion .................................63

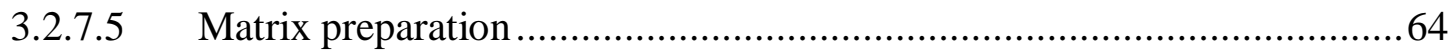

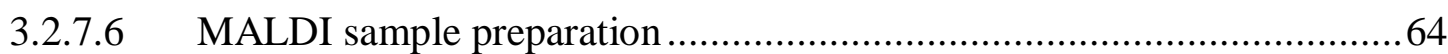

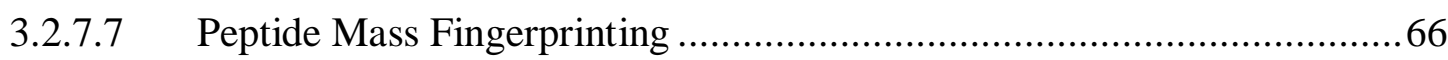

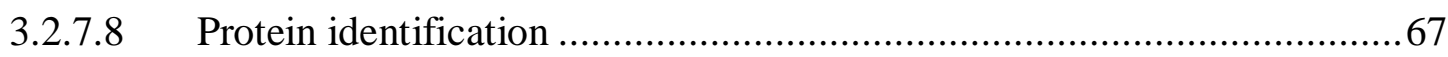

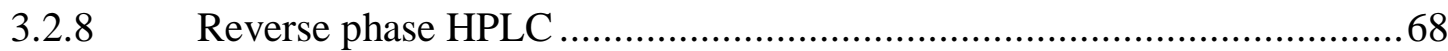

3.2.9 Techniques to determine post-translational modifications ......................70

3.2.9.1 Pro- $\mathrm{Q}^{\circledR}$ Diamond phosphoprotein gel stain .......................................... 70

3.2.9.2 SYPRO ${ }^{\circledR}$ Ruby protein gel stain ....................................................... 71

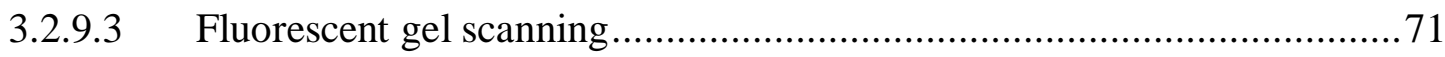

3.2.10 Enrichment strategies for phosphopeptide analysis ............................72

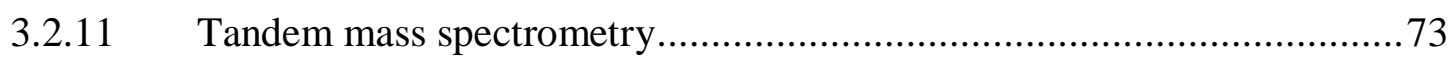

3.2.11.1 Sample preparation for LC-MS/MS analysis ................................... 73

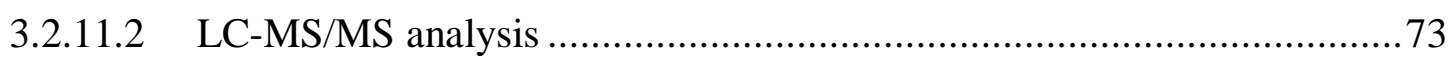

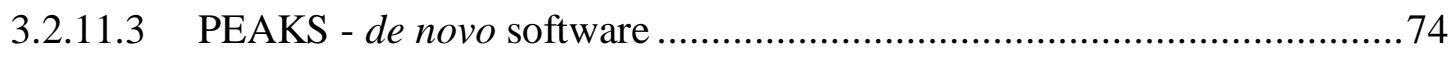

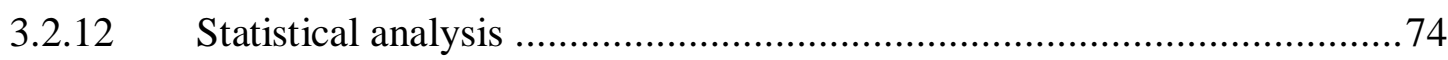

4 Purification and identification of GSTs from Drosophila melanogaster and Lucilia cuprina ........................................................75

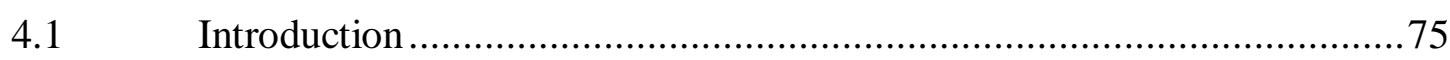

4.1.1 Drosophila melanogaster as a model insect .........................................79

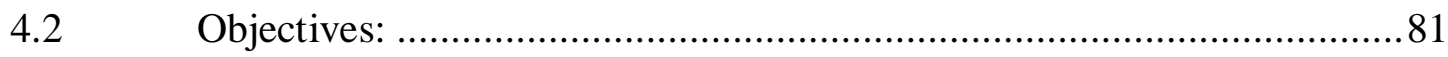

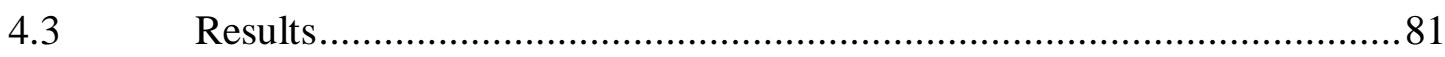

4.3.1 Similarity of L. cuprina Delta GST with D. melanogaster GSTs.............81 


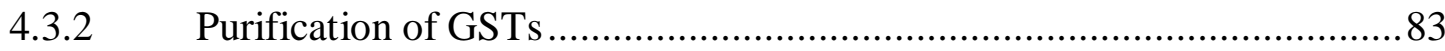

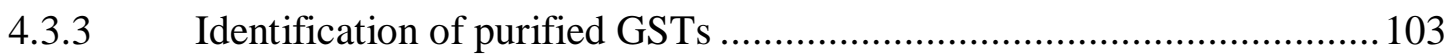

4.3.3.1 Development of a consensus sequence-based approach for tentative characterisation of L. cuprina GSTs............................................... 104

4.3.4 Separation of Sigma and Delta class GSTs...................................... 126

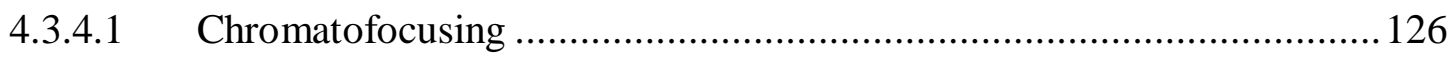

4.3.4.2 Ion exchange chromatography …................................................... 131

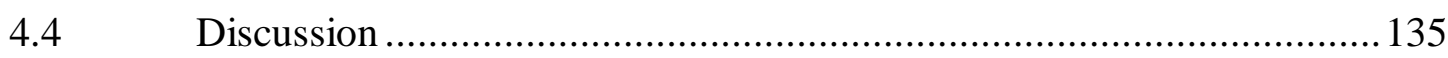

$5 \quad$ Examination of qualitative and quantitative variation of GSTs during the life span of Lucilia cuprina and in main body parts of the adult fly ..........................................................................................145

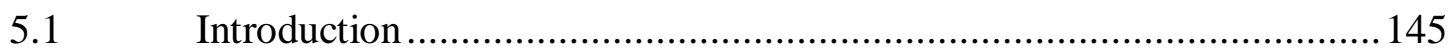

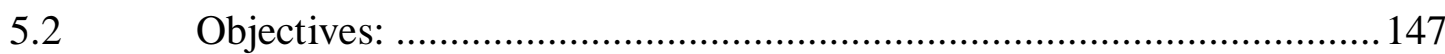

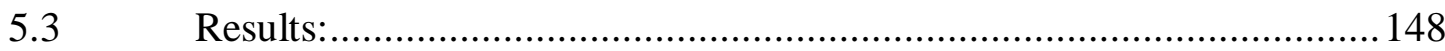

5.3.1 Purification of GSTs from eggs, larvae, pupae and adult ..................... 148

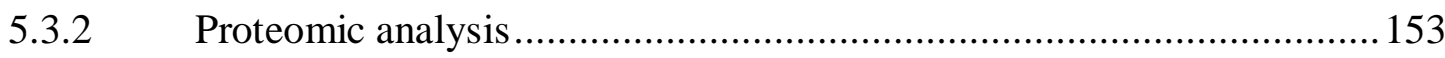

Insect body distribution of GSTs............................................... 168

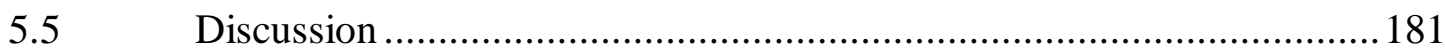

6 Involvement of glutathione S-transferases in the development of insecticide resistance in Lucilia cuprina ...............................................188

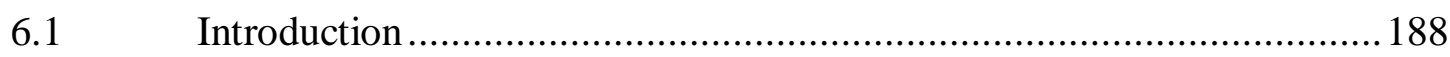

6.1.1 The Lucilia cuprina enzyme system and insecticide resistance.............. 193

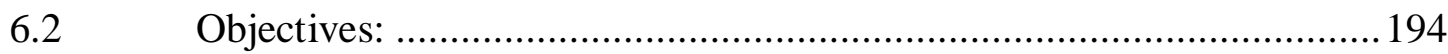

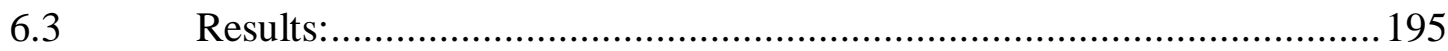

6.3.1 Purification of GSTs from susceptible and resistant strains of

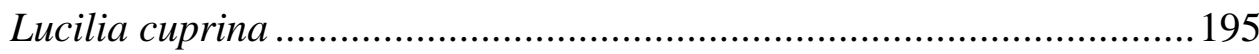

6.3.2 Proteomic analysis of GSTs from susceptible and resistant strains ........201

6.4 In vitro conjugation of insecticides with GSTs determined by reverse

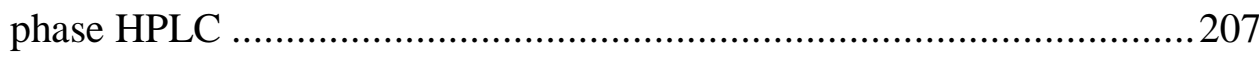

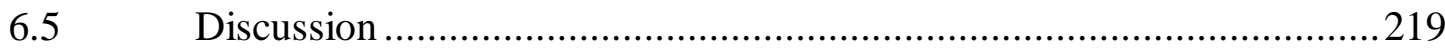




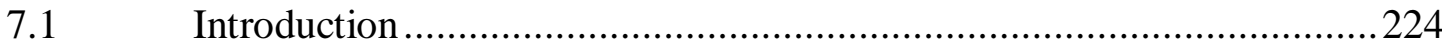

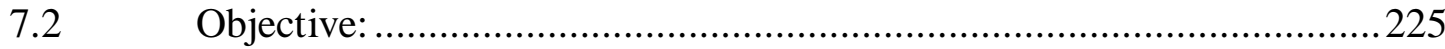

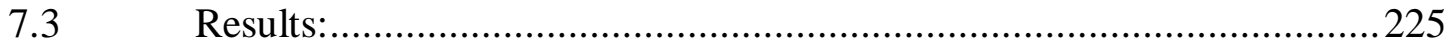

7.3.1 Phosphorylation as a possible PTM of Delta GSTs ............................2225

7.3.2 Use of PTMFinder in PEAKS software.............................................2234

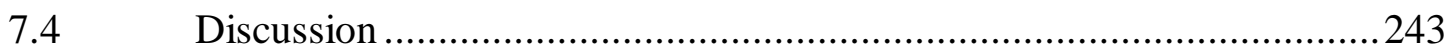

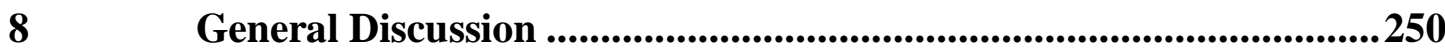

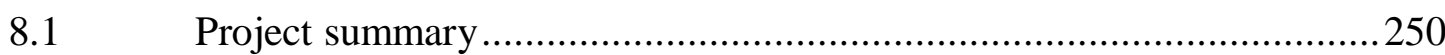

8.1.1 How reliable is the consensus sequence-based approach for the characterisation of L. cuprina GSTs? ...............................................252

8.1.2 Do insects possess Mu-like GSTs?.............................................256

8.1.3 A comparison of the GST proteomes of D. melanogaster and $L$. cuprina .259

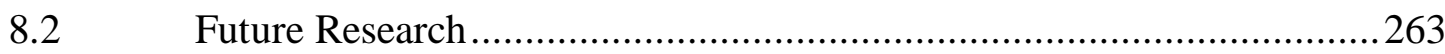

8.2.1 GST study at molecular genetics level ............................................263

8.2.2 Use of further GST purification techniques ......................................264

8.2.3 Study of interaction of GSTs with insecticides ..................................265

8.2.4 Comparative developmental study of D. melanogaster .......................266

8.2.5 Post-translational modification of Delta GSTs .................................266

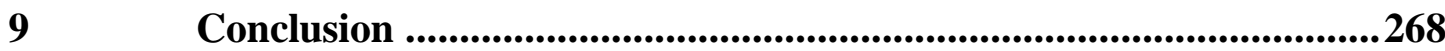

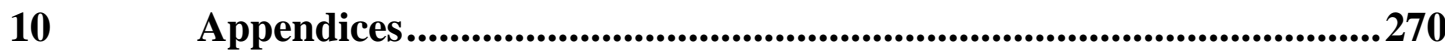

10.1 Reagent preparation ............................................................... 270

10.2 Enzyme assay purification parameters and calculation .......................22

10.3 Mass spectra and identification of spots using PROFOUND .................2273

10.3.1 Spectra of spots labelled in Figure 4-17 ........................................2274

10.3.2 Identification of spots labelled in Figure 4-17 using PROFOUND ........276

10.3.3 Spectra of spots labelled in Figure 4-18 …......................................2279

10.3.4 Identification of spots labelled in Figure 4-18 using PROFOUND ........281 
10.3.5 Spectra of spots labelled in Figure 4-20 (A) ......................................224

10.3.6 Identification of spots labelled in Figure 4-20 (A) using PROFOUND..288

10.3.7 Spectra of spots labelled in Figure 4-20 (B) .....................................294

10.3.8 Identification of spots labelled in Figure 4-20 (B) using PROFOUND ..296

10.3.9 Spectra of spots labelled in Figure 4-21 ............................................300

10.3.10 Identification of spots labelled in Figure 4-21 using PROFOUND ........304

10.3.11 Spectra of spots labelled in Figure 5-8 E ............................................311

10.3.12 Identification of spots labelled in Figure 5-8 E using PROFOUND.......312

10.4 Consensus sequences for GST classes............................................. 313

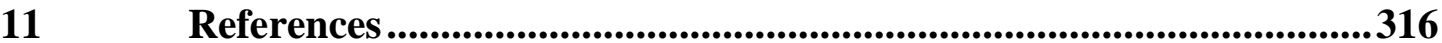




\section{Index of Figures}

Figure 1-1: Chemical structure of glutathione (Merck Index, $7^{\text {th }}$ edition). 1

Figure 1-2: Glutathione conjugation to a generic electrophilic xenobiotic (RX) by GST....... 2

Figure 1-3: Phylogenetic tree of insect GST classes. 6

Figure 1-4: The 3-D structures of GSTs obtained from the RCSB Protein Data Bank 8

Figure 1-5: The 3-D structures of GSTs obtained from the RCSB Protein Data Bank.

Figure 1-6: Model substrates used in the study of GST (Hayes and Pulford, 1995).

Figure 1-7: Model substrates used in the study of GSTs (Ayyadevara et al., 2007; Hayes and Pulford, 1995; Kim et al., 2006; Singh et al., 2001; Yang et al., 2003).

Figure 1-8: Detoxification of Herbicides and Insecticides (Hayes and Pulford, 1995; Oppenoorth et al., 1979; Wilson and Clark, 1996). 15

Figure 1-9: Isomerization of $\Delta^{5}$-androstene-3,17-dione and maleylacetoacetic acid catalysed by GST (Hayes and Pulford, 1995).

Figure 1-10: Interaction of GST Mu with ASK1 .23

Figure 1-11: GST-mediated detoxification of organophosphate insecticides (Che-Mendoza et al., 2009)

Figure 3-1: Proposed structure of A) DNP-GSH conjugate and B) CNP-GSH conjugate covalently linked to epichlorhydrin activated Sepharose $6 \mathrm{~B}$. .52

Figure 3-2: MALDI-plate spot pattern (Invitrogen Protocols)...............................65

Figure 3-3: HPLC mobile phase gradient 69

Figure 4-1: Phylogenetic relation of L. cuprina Delta GST with D. melanogaster GSTs. 82

Figure 4-2: The BLAST result of alignment of GST sequence of $D$. melanogaster and L. cuprina. 
Figure 4-3: SDS PAGE gels of different GSH affinity-purified GSTs from $L$.

cuprina.

Figure 4-4: SDS PAGE gel of GSH $(10 \mu \mathrm{mol} / \mathrm{ml})$ affinity-purified GSTs from $D$. melanogaster and L. cuprina. .86

Figure 4-5: SDS PAGE gel of GSH affinity-purified GSTs from different batches and strains of $L$. cuprina and D. melanogaster.

Figure 4-6: SDS PAGE gel of GSH affinity-purified GSTs from different sugarfed strains of L. cuprina.

Figure 4-7: Schematic workflow of strategies for the purification and identification of GSTs used in this study....

Figure 4-8: Typical chromatography elution profiles for partial purification of D. melanogaster and L. cuprina GSTs by manual sequential affinity chromatography. .92

Figure 4-9: Typical elution chromatograms for the purification method of GSTs using FPLC.

Figure 4-10: SDS PAGE gels of DNP-GSH and CNP-GSH affinity-purified GSTs from D. melanogaster (A) and L. cuprina PY81 strain (B).

Figure 4-11: Comparison of substrate-specific total activities of unbound and bound fractions of D. melanogaster from different affinity matrices. ....98

Figure 4-12: Comparison of substrate-specific total activities of unbound and bound fractions of L. cuprina from different affinity matrices. 100

Figure 4-13: Comparison of specific activities of D. melanogaster and $L$. cuprina purified GSTs from different affinity matrices.

Figure 4-14: BLAST result of D. melanogaster Sigma GSTS1 against the Arthropoda fragmentation database 106

Figure 4-15: ClustalW2 alignment result of Sigma GST sequences from the different insects.

Figure 4-16: Alignment result in Jalview. 108

Figure 4-17: Two dimensional gel electrophoresis of GSH affinity-purified $D$. melanogaster GSTs. 
Figure 4-18: Two dimensional gel electrophoresis of GSH affinity-purified $L$. cuprina GSTs.

Figure 4-19: Alignment of peptide sequences of L. cuprina spot 1(Figure 4-18) matched against $M$. domestica (A) and D. melanogaster (B) (Table 4-10) using ClustalW2.

Figure 4-20: Two dimensional gel electrophoresis of DNP-GSH and CNP-GSH affinity-purified D. melanogaster GSTs after purification by GSH matrix.

Figure 4-21: Two dimensional gel electrophoresis of DNP-GSH affinity-purified

L. cuprina GSTs after purification by GSH matrix.

Figure 4-22: Typical FPLC chromatogram, for the separation of $D$. melanogaster Delta GSTs by Mono P HPLC chromatofocusing at $\mathrm{pH} 7-4$.

Figure 4-23: Magnified image of peak II of Figure 4-22 showing separation of D. melanogaster Delta GSTs by Mono P HPLC chromatofocusing at $\mathrm{pH}$ 7-4.

Figure 4-24: Characterization of $D$. melanogaster GST subunits by SDS PAGE.... 130

Figure 4-25: Separation of Sigma and Delta GSTs by ion exchange chromatography.

Figure 4-26: Resolution of Sigma (peak I) and Delta GSTs (peak II) from $D$. melanogaster by $2 \mathrm{D}$ electrophoresis.

Figure 4-27: Alignment of L. cuprina Sigma GST sequence with other known insect Sigma GST sequences from Musca domestica and Drosophila melanogaster using ClustalW2.

Figure 5-1: Substrate-specific total activity of crude and affinity-purified GSTs from different developmental stage of $L$. cuprina towards the model substrates.

Figure 5-2: Substrate-specific specific activity of crude and affinity-purified GSTs from different developmental stage of $L$. cuprina towards the model substrates. 
Figure 5-3: A typical SDS PAGE of eluents from fractions that bound to GSH and DNP-GSH affinity matrices from different developmental stages of L. cuprina.

Figure 5-4: Two dimensional electrophoresis gels of GSH affinity-purified fractions from egg, larvae, pupae and adult stages of $L$. cuprina.

Figure 5-5: Quantification of the GSH affinity-purified protein spots expressed in different developmental stages of L. cuprina. 156

Figure 5-6: Two dimensional electrophoresis gels of DNP-GSH affinity-purified fractions from egg, larvae, pupae and adult stages of $L$. cuprina.

Figure 5-7: Changes in expression of DNP-GSH affinity-purified proteins in different developmental stages of $L$. cuprina.

Figure 5-8: Changes in expression of DNP-GSH affinity-purified proteins in different developmental stages of $L$. cuprina.

Figure 5-9: A tentative approach to classify the spot $2 *$ GST by matching to consensus sequences of GST classes from other species.

Figure 5-10: A tentative approach to classify the spot $3^{*}$ GST by matching to consensus sequences of GST classes from other species.

Figure 5-11: Quantitation of differentially expressed DNP-GSH purified GSTs in developmental stages of L. cuprina.

Figure 5-12: Quantitation of differentially expressed DNP-GSH purified GSTs in developmental stages of L. cuprina.

Figure 5-13: Comparative study of GST production and activity towards CDNB from different stages of purification of head, thorax and abdomen. ..... 170

Figure 5-14: Substrate-specific total activity of crude and affinity-purified GSTs from head, thorax and abdomen towards the model substrates.

Figure 5-15: Substrate-specific specific activity of crude and affinity-purified GSTs from head, thorax and abdomen towards the model substrates... 172

Figure 5-16: SDS PAGE of affinity-purified fractions of head, thorax and abdomen of L. cuprina

Figure 5-17: Two dimensional electrophoresis gels of GSH affinity-purified fractions from head, thorax and abdomen of $L$. cuprina. 
Figure 5-18: Differential expression of Sigma and Delta GSTs on 2D gel of GSH affinity-purified extracts from head, thorax and abdomen.

Figure 5-19: Quantification of the GSH affinity-purified protein spots expressed in head, thorax and abdomen of L. cuprina

Figure 5-20: Two dimensional electrophoresis gels of DNP-GSH affinitypurified fractions of head, thorax and abdomen of L. cuprina.

Figure 5-21: Quantification of the DNP-GSH affinity-purified protein spots expressed in head, thorax and abdomen of L. cuprina.

Figure 6-1: OP insecticides being metabolised by GSTs via dealkylation and dearylation reactions.

Figure 6-2: Amount of protein recovered from the GSH and DNP-GSH affinity matrices.

Figure 6-3: Substrate-specific total activity of crude GSTs and affinity-purified GSTs with different model substrates relative to CSIRO strain

Figure 6-4: Substrate-specific specific activities of crude GSTs and affinitypurified GSTs with different model substrates relative to CSIRO strain.

Figure 6-5: SDS PAGE on bound GSH and bound DNP-GSH affinity-purified fractions of NSW, PY81 and CSIRO strains.

Figure 6-6: Two dimensional electrophoresis gels of the GSH affinity-purified fractions from susceptible and resistant strains of $L$. cuprina...... .202

Figure 6-7: Quantitative analysis of GSH affinity-purified GSTs in different $L$. cuprina strains. .203

Figure 6-8: Two dimensional electrophoresis gels of the DNP-GSH affinitypurified fractions from susceptible and resistant strains of $L$. cuprina. .204

Figure 6-9: Quantitative analysis of DNP-GSH affinity-purified GSTs from different $L$. cuprina strains. .206

Figure 6-10: Calibration curve for methyl parathion. .208

Figure 6-11: Typical HPLC chromatograms under the given gradient condition at $254 \mathrm{~nm}$. .209 
Figure 6-12: Typical HPLC chromatograms of methyl parathion with and without GSH and affinity-purified enzymes from L. cuprina (PY81 strain) at $280 \mathrm{~nm}$.

Figure 6-13: Typical HPLC chromatograms of conjugation of methyl parathion by de-pigmented enzyme from L. cuprina (PY81 strain) at $280 \mathrm{~nm}$ with and without GSH.

Figure 6-14: Metabolism of methyl parathion by affinity-purified GSTs. 215

Figure 6-15: Metabolism of methyl parathion by GST isoforms and partially purified DNP-GSH eluents.

Figure 6-16: GST activity in metabolising OPs from different strains of $L$. cuprina

Figure 7-1: NetPhos 2.0 Server prediction results .227

Figure 7-2: Pro-Q Diamond and SYPRO Ruby gel staining. .228

Figure 7-3: Analysis of a SDS-PAGE gel using ImageQuant version 5.2 software. .229

Figure 7-4: D. melanogaster Delta GST separation by 2D gel electrophoresis. 231

Figure 7-5: Comparison of MALDI-TOF spectrum of native GSTD1 and phosphopeptide enriched GST D1 (spot 3) as shown in Figure 7-4. ....233

Figure 7-6: PTMFinder result with identification of protein spot 3 in Figure 7-4...236

Figure 7-7: PTM Finder result with identification of protein spot 5 in Figure 7-4...237

Figure 7-8: PTM Finder result with identification of protein spot 6 in Figure 7-4...238

Figure 7-9: Alignment of matched deamidated peptides with DmGSTD1 sequence.

Figure 7-10: Comparison of the mass spectra of the peptide AVGVELNKK and modified peptide AVGVELN[3]KK suggested by PTMFinder in PEAKS

Figure 7-11: Alignment of D. melanogaster DmGSTD1 sequence with $L$. cuprina and Musca domestica D1 sequences using ClustalW2.

Figure 8-1: Alignment of L. cuprina peptide sequences matched against "Other Metazoa" with Delta GST consensus sequence .253 
Figure 8-2: Alignment of L. cuprina peptide sequences matched against "Other Metazoa" with Epsilon GST consensus sequence.

Figure 8-3: Alignment of L. cuprina peptide sequences matched against "Other Metazoa" with consensus sequences of different GST class .255

Figure 8-4: Dendrogram showing the relationship between D. melanogaster and human GSTs (Ranson et al., 2001).

Figure 8-5: Alignment of various peptide sequences with Mu GST consensus sequence

Figure 10-1: A MALDI-TOF spectrum of the external standard CalMix 2 .273

Figure 10-2: MALDI-TOF spectra of the GSH affinity-purified D. melanogaster spots on 2D gel labelled in Figure 4-17

Figure 10-3: MALDI-TOF spectra of the GSH affinity-purified L. cuprina spots on 2D gel labelled in Figure 4-18 280

Figure 10-4: MALDI-TOF spectra of the DNP-GSH affinity-purified $D$. melanogaster spots on 2D gel labelled in Figure 4-20 (A) .287

Figure 10-5: MALDI-TOF spectra of the CNP-GSH affinity-purified $D$. melanogaster spots on 2D gel labelled in Figure 4-20 (B) .295

Figure 10-6: MALDI-TOF spectra of the DNP-GSH affinity-purified L. cuprina spots on 2D gel labelled in Figure 4-21

Figure 10-7: MALDI-TOF spectra of the DNP-GSH affinity-purified Lucilia cuprina egg spots on 2D gel labelled in Figure 5-8 E

Figure 10-8: Comparison of MALDI-TOF spectra of DmGSTD1 spots from Figure 7-4 


\section{Index of Tables}

Table 3-1: Proteases and their cleavage specificities.............................................46

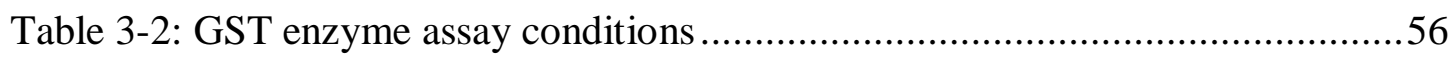

Table 4-1: Purification of GSTs from L. cuprina by three different GSH matrices.

Table 4-2: Comparative DCNB activity with D. melanogaster and L. cuprina unbound and bound GSH affinity eluents. 90

Table 4-3: Glutathione S-transferase activity of $D$. melanogaster...........................94

Table 4-4: Glutathione S-transferase activity of $L$. cuprina ...................................95

Table 4-5: Substrate-specific total activities of GSTs from D. melanogaster. ............99

Table 4-6: Substrate-specific specific activities of GSTs from D. melanogaster.......99

Table 4-7: Substrate-specific total activity of GSTs from $L$. cuprina ..................... 101

Table 4-8: Substrate-specific specific activity of GSTs from L. cuprina ................. 101

Table 4-9: Identification of D. melanogaster GSTs purified by GSH affinity matrix

Table 4-10: Identification of $L$. cuprina GSTs purified by GSH affinity matrix......112

Table 4-11: Assignment of class of GST using the ClustalW2 programme. 115

Table 4-12: Identification of $D$. melanogaster GSTs purified by sequential DNPGSH affinity matrix.

Table 4-13: Identification of L. cuprina GSTs purified by sequential DNP-GSH affinity matrix

Table 4-14: Assignment of class of GST using the ClustalW2 programme.

Table 4-15: Purification of GSTs from D. melanogaster.

Table 4-16: Purification of D. melanogaster GSTs by ion exchange chromatography.

Table 4-17: Substrate specificities of Sigma and Delta GSTs from $D$. melanogaster and L. cuprina. Four g of flies were used. The specific activity is from three individual experiments with triplicate measurements in each and values represent mean \pm S. D. 
Table 5-1: Purification of GSTs from developmental stages of L. cuprina. 149

Table 5-2: Identification of spots present in Zone E in Figure 5-8 ....................... 161

Table 5-3: Assignment of class of GST using the ClustalW2 programme............... 162

Table 5-4: Distribution of the total GST activity in adults of L. cuprina towards CDNB.

Table 6-1: Purification of GSTs from susceptible (NSW and CSIRO) and resistant strain (PY81) of L. cuprina.

Table 6-2: Total activities of affinity-purified GSTs and crude enzyme from $L$. cuprina (PY81 strain) towards insecticides determined by HPLC.

Table 6-3: Specific activities of affinity-purified GSTs and crude enzyme from L. cuprina (PY81 strain) towards insecticides determined by HPLC. ..213 Table 6-4: Metabolism of methyl parathion and diazinon by NSW, PY81 and

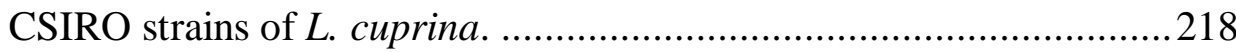

Table 7-1: Setting parameters for each application in PEAKS..............................2235

Table 7-2: List of modified peptides suggested by PTMFinder............................2239

Table 10-1: Identification of D. melanogaster GSTs purified by CNP-GSH affinity matrix 


\section{Abbreviations}

\begin{tabular}{|c|c|}
\hline GSH & Glutathione \\
\hline CDNB & 1-chloro, 2,4- dinitrobenzene \\
\hline DCNB & 1,2-dichloro, 4-nitrobenzene \\
\hline $\mathrm{PBO}$ & trans- 4-phenyl-3-buten-2-one \\
\hline EA & Ethacrynic Acid \\
\hline EPNP & 1,2-epoxy-3-nitrophenoxy propane \\
\hline BSP & Bromosulphophthalein \\
\hline NPA & 4-Nitrophenyl acetate \\
\hline TNE & trans-2-nonenal \\
\hline DHA & Dehydroascorbic acid \\
\hline BSP-GSH & Bromosulphophthalein-glutathione conjugate \\
\hline $\mathrm{CF}$ & Chromatofocusing \\
\hline SDS-PAGE & Sodium Dodyl Sulphate- Polyacrylamide Gel Electrophoreisis \\
\hline $2 \mathrm{DE}$ & 2-Dimensional Electrophoresis \\
\hline DDT & 1,1,1-trichloro-2,2-di(4-chlorophenyl)ethane \\
\hline DDE & 1,1-bis-(4-chlorophenyl)-2,2-dichloroethene \\
\hline PMF & Peptide Mass Fingerprinting \\
\hline PTM & Post-Translational Modification \\
\hline DNA & Deoxyribonucleic acid \\
\hline RNA & Ribonucleic Acid \\
\hline IEF & Iso Electric Focusing \\
\hline HPLC & High Pressure Liquid Chromatography \\
\hline TLC & Thin Layer Chromatography \\
\hline PMF & Peptide Mass Fingerprinting \\
\hline
\end{tabular}




$\begin{array}{ll}\text { DNP-GSH } & \text { S- 2,4-(Dinitrophenyl)-glutathione } \\ \text { CNP-GSH } & \text { S-2,4-(Chloronitrophenyl)-glutathione } \\ \text { TFA } & \text { Trifluroacetic acid } \\ \text { ACN } & \text { Acetonitrile } \\ \text { RT } & \text { Room Temperature } \\ \text { GR } & \text { Glutathione Reductase } \\ \text { GSSG } & \text { Glutathione disulfide } \\ \text { TEMED } & \text { N,N, } N^{\prime}, N^{\prime} \text {-tetramethyl-ethane-1,2-diamine } \\ \text { M.W. } & \text { Molecular Weight } \\ \text { PCR } & \text { Polymerase Chain Reaction } \\ \text { MALDI-TOF } & \text { Matrix-Assisted Laser Desorption/Ionizatin-Time of Flight Mass } \\ \text { MS } & \text { Spectrometry } \\ \text { LC-MS/MS } & \text { Liquid Chromatography- Tandem Mass Spectrometry }\end{array}$




\section{General Introduction}

Glutathione, a tri-peptide (gamma-L-glutamyl-L-cysteinyl-glycine, Figure 1-1), was first reported in 1888 as a "Philothion" by a French scientist de Rey Pailhade, who found evidence that yeast cells produce hydrogen sulphide when crushed with elemental sulphur (de Rey-Pailhade, 1888). In 1929, Hopkins isolated philothion from the yeast extract and renamed it as "glutathione" (Hopkins, 1929). Glutathione $(\mathrm{GSH})$ is found in the intracellular space of plants, animals and microorganisms and is believed to have two major general functions: to remove toxic metabolites from the cell and to maintain cellular sulfhydryl groups in their reduced form (Liebman and Greenberg, 1988). The high number of hydrophilic functional groups in glutathione combined with its low molecular weight leads to high water solubility (Kosower, 1976).

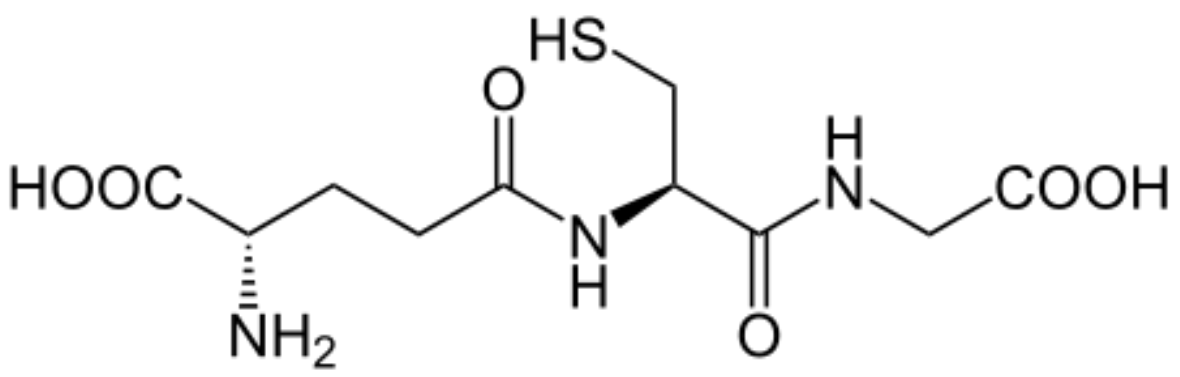

Figure 1-1: Chemical structure of glutathione (Merck Index, $7^{\text {th }}$ edition).

There are a number of glutathione-dependent enzymes which play important roles in cellular protection against endogenous and xenobiotic toxic compounds. Among these, glutathione reductase catalyzes the reduction of oxidised GSH (GSSG) using NADPH and hence maintains the high cellular reductive potential (KrohneEhrich et al., 1977). Selenium-dependent glutathione peroxidases are other GSHlinked enzymes that catalyze the reduction of peroxides using GSH as the reducing 
agent (Arthur, 2000; Margis et al., 2008). Another important GSH-dependent system comprises glyoxalase I and II which are responsible for catalysing the conversion of methylglyoxal (a by-product in glycolysis) to lactic acid (Allen et al., 1993a; Allen et al., 1993b). Glyoxalase I also catalyses the isomerization of the hemithioacetal, formed spontaneously from alpha-oxoaldehyde and GSH, to $S$-2hydroxyacylglutathione derivatives $[\mathrm{RCOCH}(\mathrm{OH})-\mathrm{SG}->\mathrm{RCH}(\mathrm{OH}) \mathrm{CO}-\mathrm{SG}]$, and in so-doing decreases the steady-state concentrations of physiological alphaoxoaldehydes and associated glycation reactions (Thornalley, 2003). The active site residue Tyr-175 in human glyoxalase II contributes to the binding of glutathione derivatives (Ridderstrom et al., 2000). Finally, glutathione transferases (GSTs) are also GSH-dependent enzymes with a number of functions amongst which catalysis of the GSH conjugation to various electrophilic compounds is one of the most investigated.

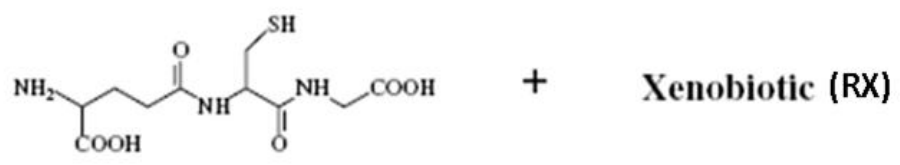

Glutathione

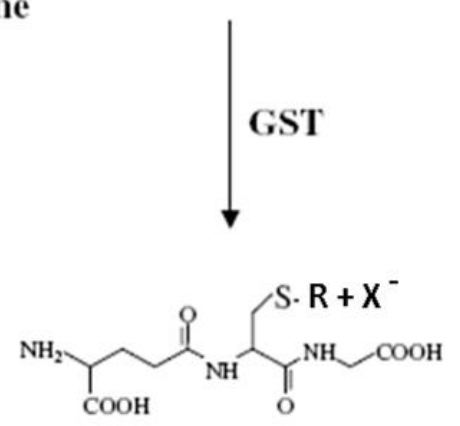

Glutathione S-conjugate

Figure 1-2: Glutathione conjugation to a generic electrophilic xenobiotic (RX) by GST.

GSTs were first discovered as enzymes in 1961 (Booth et al., 1961). Today, as a result of many years of research the picture of what exactly the proteins of this 
superfamily do is more complex than ever. GSTs are mostly known as the cellular catalysts of conjugation of the nucleophilic sulfhydryl group of glutathione to various electrophilic toxic compounds as depicted in Figure 1-2. However, today the catalytic function of GSTs is known not to be restricted to detoxication. Some GST isoenzymes have a metabolic role in catalyzing synthesis of steroids and prostaglandins (Beuckmann et al., 2000b; Hayes et al., 2005; Johansson and Mannervik, 2001; Pettersson and Mannervik, 2000; Pettersson and Mannervik, 2001). Lately, another function, namely the regulatory role of GSTs in the situations of stress-induced signaling, drug resistance, and transport has been reported (Adler $e t$ al., 1999; Awasthi et al., 2008; Chekhun et al., 2009; Jang et al., 2001; Johansson et al., 2007) which has added a new dimension to the importance of this large family of proteins. The detailed functions of GSTs are described later in this chapter.

\subsection{Distribution and classification of GSTs}

GSTs are widely distributed in nature, being found in bacteria (Laroche and Leisinger, 1990; Orser et al., 1993), yeast (Tamaki et al., 1990; Tamaki et al., 1991), fungi (Sheehan and Casey, 1993), molluscs (Tomarev and Zinovieva, 1988; Tomarev et al., 1993), crustaceans (Stenersen et al., 1987), insects (Clark, 1989; Toung et al., 1990), plants (Grove et al., 1988), fish (Dominey et al., 1991), birds (Liu and Tam, 1991) and mammals (Mannervik et al., 1985; Meyer and Thomas, 1995). They are widely found in most aerobic eukaryotes and prokaryotes (Sheehan et al., 2001).

Enzymes of the GST family include membrane-bound, cytosolic and mitochondrial proteins. The human membrane-bound proteins include microsomal GSTs and leukotriene $\mathrm{C}_{4}$ synthase, members of the superfamily MAPEG (membrane associated proteins in eicosanoid and glutathione metabolism) (Jakobsson et al., 1996; Morgenstern et al., 1982). Microsomal GST is involved in the detoxification of 
xenobiotic compounds while leukotriene $\mathrm{C}_{4}$ synthase conjugates leukotriene $\mathrm{A}_{4}$ with GSH. Neither of these GSTs share sequence homology with the cytosolic GST enzymes (Dejong et al., 1988; Lam et al., 1994). The human cytosolic or soluble GSTs are not only in the cytoplasm but may also be localized in the mitochondria and peroxisomes or the nucleus (Johansson and Mannervik, 2001; Morel et al., 2004; Pearson, 2005; Pemble et al., 1996). The mammalian cytosolic GSTs are so far divided into eight classes based on their amino acid sequences similarities. These are Alpha, Kappa, Mu, Omega, Pi, Sigma, Theta and Zeta (Board, 1998; Board et al., 2000; Mannervik et al., 1985; Meyer et al., 1991; Meyer and Thomas, 1995; Pemble et al., 1996).

In insects, very little is known about the microsomal GSTs. A single microsomal GST gene is present in the genome of fruitfly Drosophila melanogaster (D. melanogaster) whereas the mosquito Anopheles gambiae has three microsomal GST genes (Ranson et al., 2002; Toba and Aigaki, 2000). Although they are very different in size, structure and origin, they have conjugation activities similar to those of the cytosolic GSTs (Pearson, 2005) and this does not exclude them having a role in detoxification (Frova, 2006; Hayes et al., 2005; Oakley, 2005). However, so far only the cytosolic GSTs have been known to be implicated in insecticide resistance (Enayati et al., 2005; Hemingway et al., 2004; Ranson and Hemingway, 2005).

Insect cytosolic GSTs were initially assigned to a particular class based on their amino acid sequence homology and immunological properties (Beall et al., 1992; Fournier et al., 1992; Toung et al., 1990). The current criteria for inclusion of a GST in a class include to have an identity of over $40 \%$ of the amino acid sequence and other properties such as immunological character, tertiary structure, their ability to form heterodimers and chromosomal location (Ding et al., 2003; Hemingway et 
al., 2004; Ranson and Hemingway, 2005). At least eight classes of cytosolic GSTs have been identified in dipteran and other insect species, designated Delta, Epsilon, Omega, Sigma, Theta and Zeta (Chelvanayagam et al., 2001; Ding et al., 2003; Ranson and Hemingway, 2005; Tu and Akgul, 2005) and Xi and Iota (Lumjuan et al., 2007) with the possibility of the existence of novel GST classes.

Insect Delta-class GSTs have been characterized in Musca domestica, Drosophila melanogaster, Anopheles gambiae, Anopheles dirus and Lucilia cuprina (Prapanthadara et al., 1998; Ranson et al., 1997; Snyder and Maddison, 1997; Wilce et al., 1995). Insect Sigma-class GSTs have been found in Manduca sexta, Blattella germanica, Drosophila melanogaster and Apis mellifera macedonica (Arruda et al., 1997; Beall et al., 1992; Papadopoulos et al., 2004b; Snyder et al., 1995). In addition, an insect Epsilon-class GST has been identified from Drosophila melanogaster (Sawicki et al., 2003) and eight members of Epsilon class GSTs have been identified in A. gambiae (Ranson et al., 2002). The Omega GSTs, in most of the species including A. gambiae, seem to be encoded by a single gene, however five putative Omega GSTs have been identified in D. melanogaster (Ding et al., 2003). Omega GST has also been identified in the Silk Moth, Bombyx mori (Yamamoto et al., 2009a). The Theta GSTs are found in a diverse range of organisms and were originally postulated to be the progenitor of all GST classes. However, as more GSTs were identified, it became apparent that many GSTs, including the insect Delta class, were inappropriately assigned to this class, and these GSTs were subsequently renamed. Two Theta GST genes have been identified in A. gambiae (Ding et al., 2003) and five putative Theta GSTs have been identified in A. aegypti (Lumjuan et $a l ., 2007)$. The Zeta GST has been identified in Silk Moth, Bombyx mori (Yamamoto et al., 2009b) and a single Zeta GST gene is found in A. gambiae (Ding et al., 2003). 
The $\mathrm{Xi}$ and Iota GSTs have so far been found uniquely in mosquitoes - A. aegypti and clear orthologs of these GSTs were found in A. gambiae (Lumjuan et al., 2007). The evolutionary relationship between the insect GSTs has been shown in

Figure 1-3 in the form of neighbour joining tree constructed using the ClustalW2 alignment programme (Larkin et al., 2007; Thompson et al., 1994). This thesis deals in particular with cytosolic GSTs from L. cuprina.

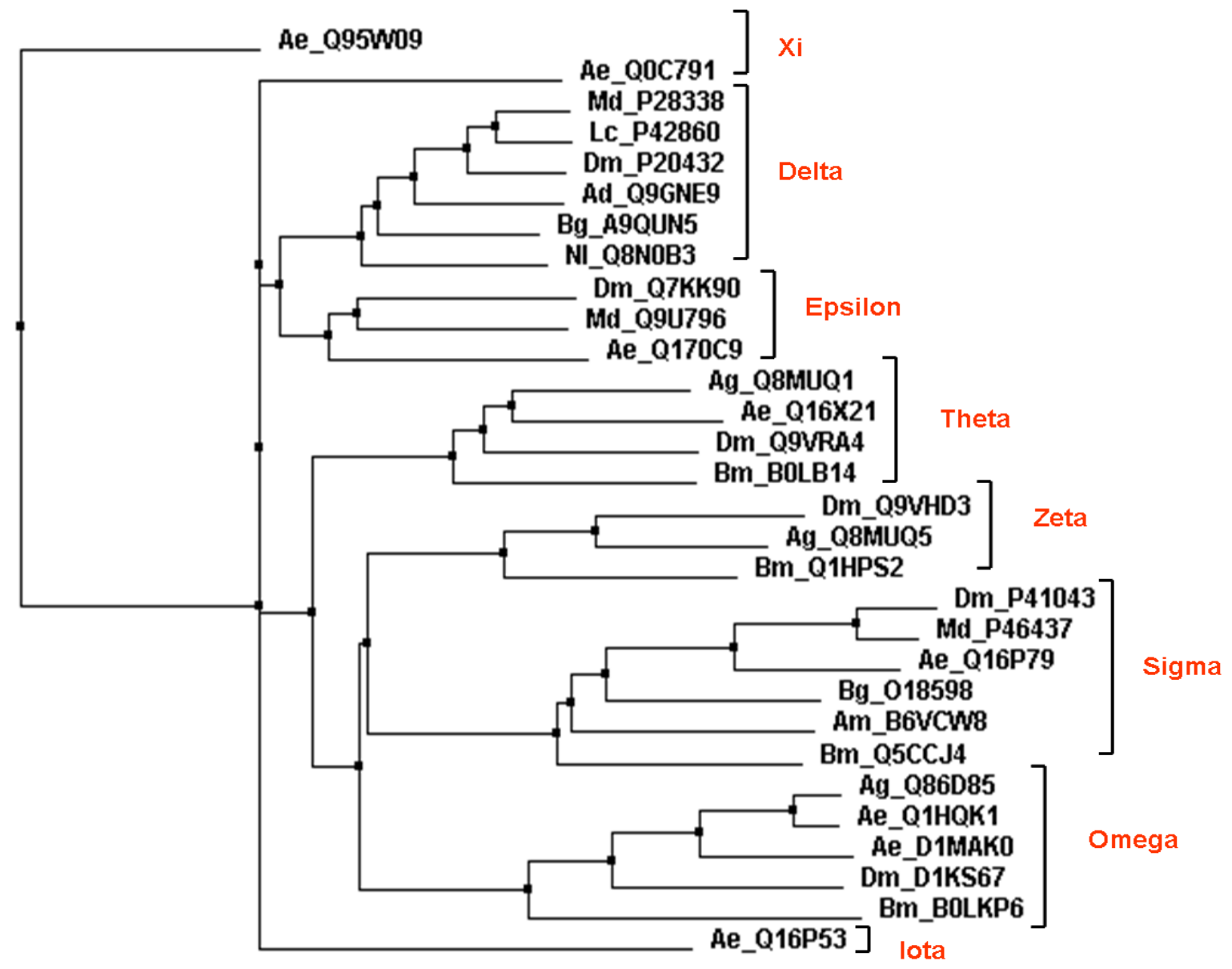

Figure 1-3: Phylogenetic tree of insect GST classes.

Phylogenetic tree of different GST classes demonstrating the relationships of the various insect GSTs to one another. Amino acid sequences were aligned using ClustalW2 (www.ebi.ac.uk/clustalW2 ) and a neighbour-joining tree was generated using BLOSUM62 in Jalview. The accession numbers of proteins are as per UniProtKB (http://www.uniprot.org/uniprot/). Ag = Anopheles gambiae, $\mathrm{Ad}=$ Anopheles dirus, $\mathrm{Ae}=$ Aedes aegypti, $\mathrm{Dm}=$ Drosophila melanogaster, $\mathrm{Bm}=$ Bombyx mori, $\mathrm{Md}=$ Musca domestica, $\mathrm{Bg}=$ Blattella germanica, $\mathrm{Lc}=$ Lucilia cuprina, $\mathrm{Nl}=$ Nilaparvata lugens 


\subsection{Structure of GSTs}

Microsomal GSTs are trimeric membrane-bound proteins and each subunit is composed of approximately 150 amino acids that assemble into four transmembrane alpha helices (Schmidt-Krey et al., 2000). The cytosolic GSTs are subject to significant genetic polymorphisms and are heterodimeric or homodimeric proteins (Boyer, 1989). Each subunit is composed of between 200 and 250 amino acid residues with typical molecular masses ranging from 20-28 kDa (Armstrong, 1991). Structural studies have revealed that the different GST classes share a common threedimensional polypeptide fold (Wilce and Parker, 1994). Each subunit adopts a canonical GST fold of seven or eight alpha helices and four beta sheets to produce two distinct domains, the $\mathrm{C}$-terminal and $\mathrm{N}$-terminal domains respectively. The $\mathrm{N}$ terminal domain (residues 1-80) is highly conserved between the different GSTs. The C-terminal domain exhibits much more structural variation than the $\mathrm{N}$-terminal domain.

GST subunits have two distinct binding sites: the G site that binds glutathione and the substrate binding or $\mathrm{H}$ site. The $\mathrm{G}$ site is mainly composed of amino acids in the N-terminal domain, including the active site residue that interacts with and activates the sulfhydryl group of glutathione to generate the catalytically active thiolate anion (Armstrong, 1991). The high level of diversity in the $\mathrm{H}$ site (a hydrophobic ligand binding site) confers, in part, the specificity of the GSTs for a broad range of electrophilic substrates (Mannervik and Danielson, 1988). The structures of the microsomal GST and cytosolic GSTs are shown in Figure 1-4 (A) and the Sigma and Delta GST monomer subunits from Drosophila melanogaster and Anopheles dirus respectively shown in Figure 1-4 (B). The structures of mammalian GSTs with their unique features are shown in Figure 1-5. 


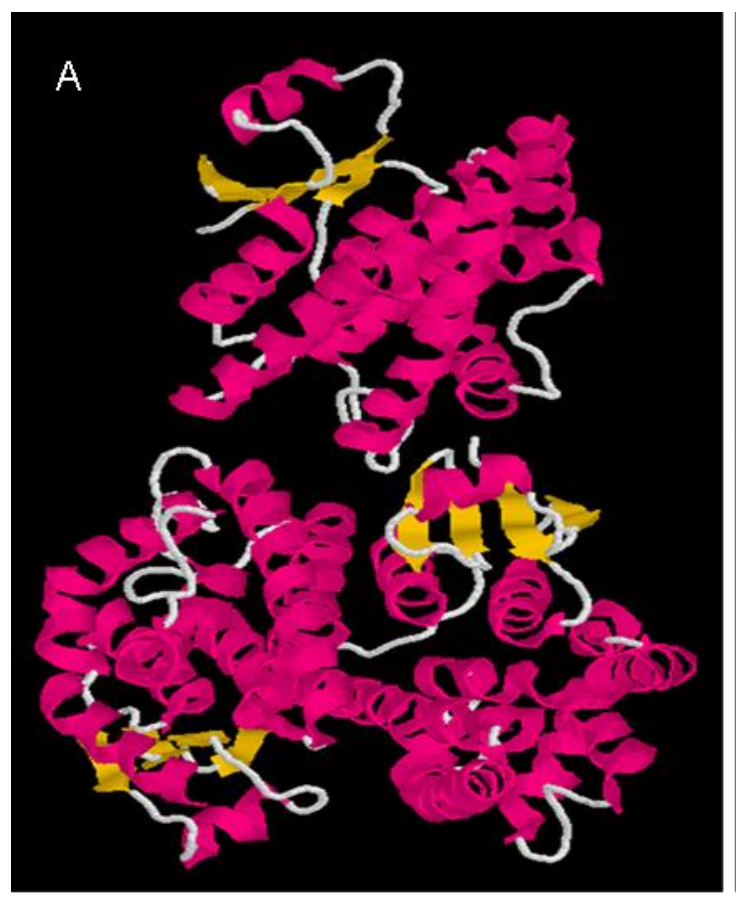

Microsomal GST (2H8A)

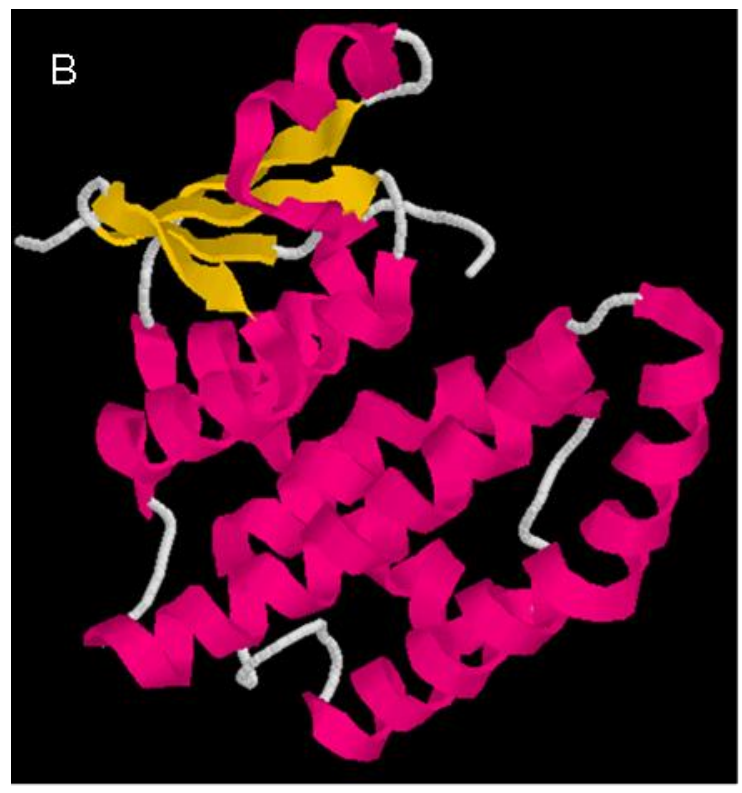

D. melanogaster Sigma GST (1MOU)

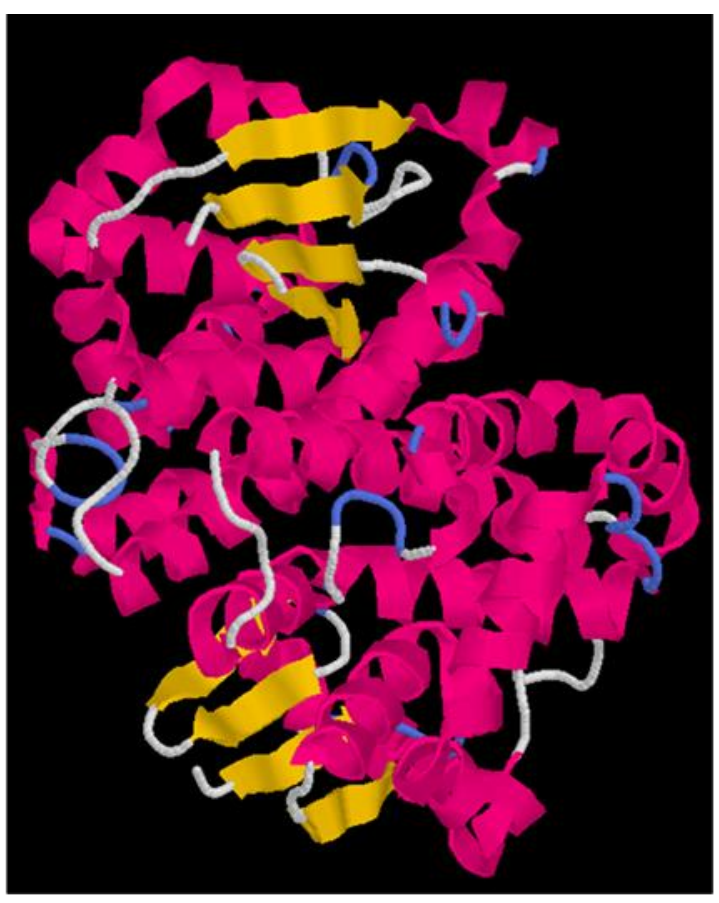

Human GST Pi dimer (1PGT)

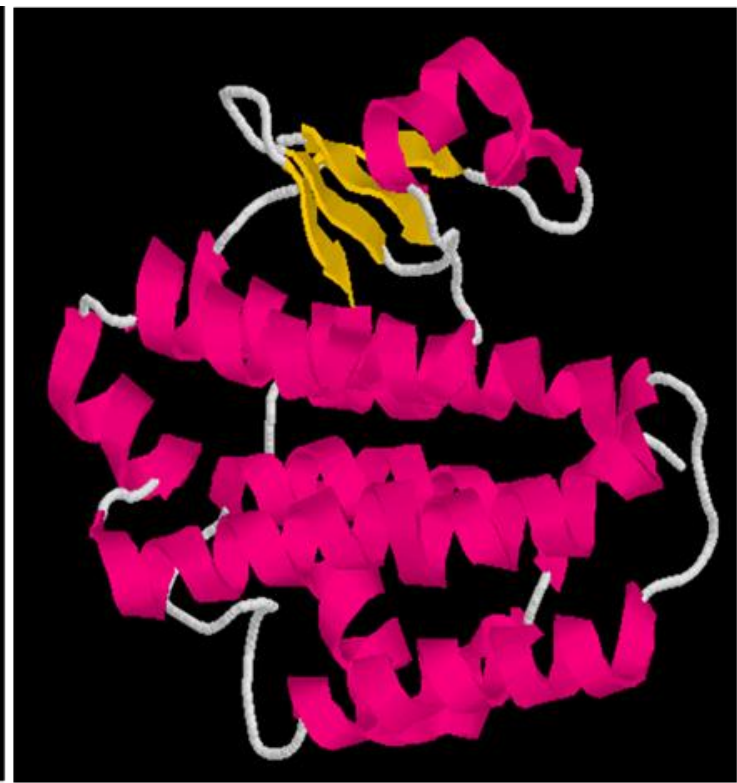

A. dirus Delta GST (3F63)

Figure 1-4: The 3-D structures of GSTs obtained from the RCSB Protein Data Bank.

A) The typical trimeric microsomal GST and dimeric cytosolic GST structures. B) The monomer structure of cytosolic D. melanogaster Sigma and A. dirus Delta GST. The pdb id for each structure is shown in brackets and these are screen shots of RasMol images. The Nterminal domain beta sheets of GSTs are shown in yellow and the alpha helices are in pink. 


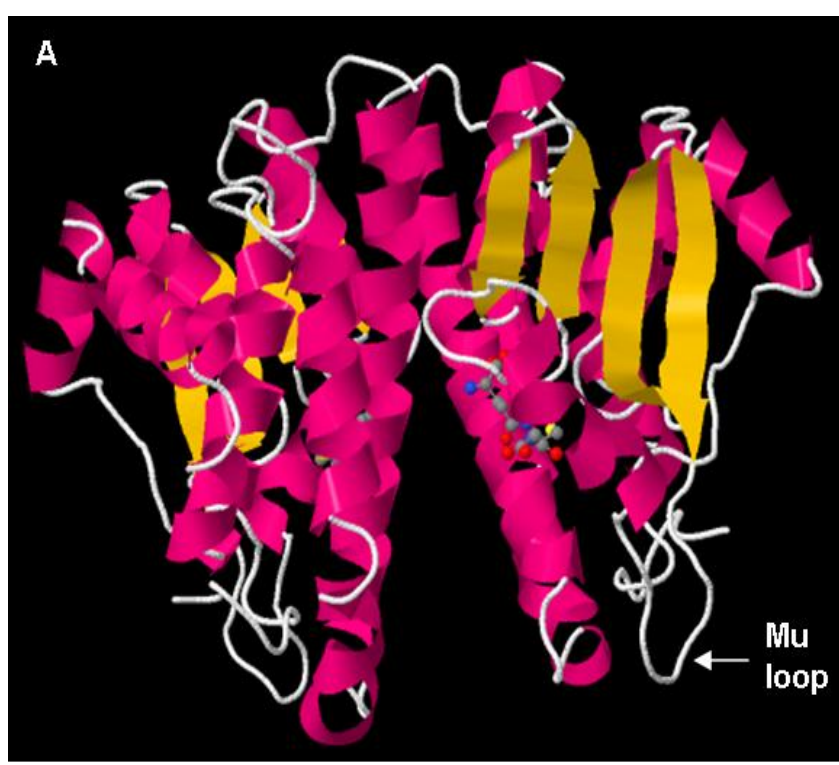

Rat Mu class (6GST)

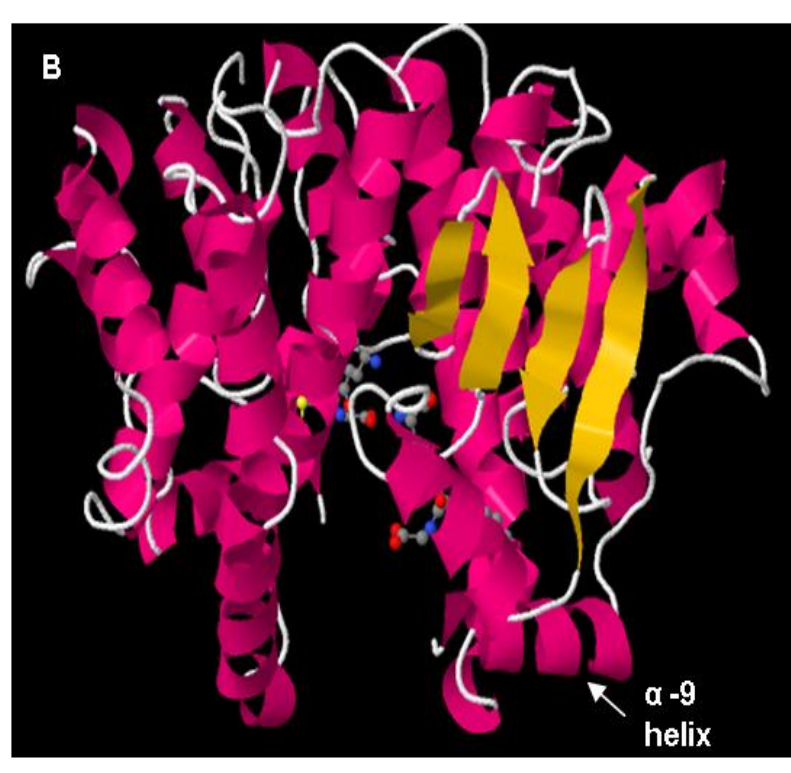

Human Alpha class (1GUH)

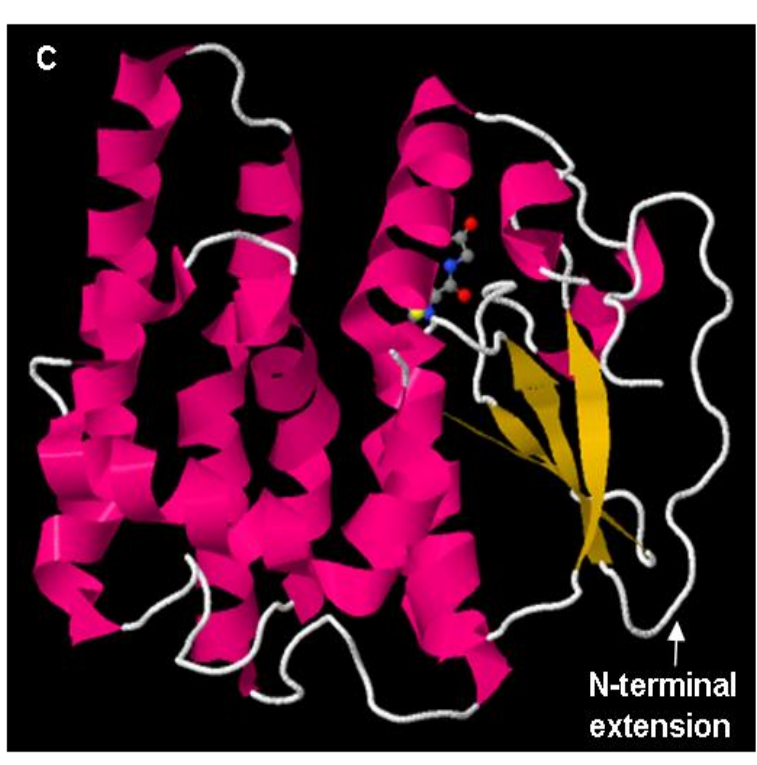

Human Omega GST (1EEM)

Figure 1-5: The 3-D structures of GSTs obtained from the RCSB Protein Data Bank.

A) Mu-loop, the unique feature of Mu class GST. B) $\alpha-9$ helix, the unique feature of Alpha class GST. C) N-terminal domain extension, the unique feature of Omega GST. The pdb id for each structure is shown in brackets and these are screen shots of RasMol images. The N-terminal domain beta sheets of GSTs are shown in yellow and the alpha helices are in pink. The ligand (GSH) attached is shown as ball-and-stick structures. 


\subsection{Mechanism of action of GSTs}

The glutathione S-transferases catalyze the conjugation of glutathione to a wide variety of electrophilic substrates and as a result produce water soluble compounds thus aiding in excretion (Boyland and Chasseaud, 1969). Characteristically, most species express multiple forms of GST having overlapping substrate specificities. As previously mentioned, GSTs catalyze the nucleophilic attack by the thiol group of reduced glutathione (GSH) on a wide range of lipophilic and electrophilic compounds, the basic theory for all the various catalytic activities of GST involves the ability of the enzyme to lower the $\mathrm{pK}_{\mathrm{a}}$ of the sulfhydryl group of reduced glutathione from 9.0 in aqueous solution to about 6.5 when bound in the active site (Armstrong, 1994). The glutathione exists as the thiolate (GS ${ }^{-}$) anion at neutral $\mathrm{pH}$ when complexed with GST (Graminski et al., 1989). It is proposed that once the $\mathrm{GS}^{-}$is formed in the active site of the GST, it becomes capable of reacting spontaneously, by nucleophilic attack, with electrophilic xenobiotics that are situated in close proximity (Jakoby, 1978a). Thus catalysis by GST depends upon the combined ability of the enzyme to promote the formation of $\mathrm{GS}^{-}$and to bind hydrophobic electrophilic compounds at a closely adjacent site. The glutathione binding site (G-site) exhibits a high specificity (Adang et al., 1989) whereas, by contrast, the second substrate binding site (H-site) displays a broad specificity towards hydrophobic compounds. The G-site residues tend to be highly conserved within GST classes but differ between classes. In most of the mammalian GSTs, the active site residue responsible for the GSH thiol residue activation in catalysis appears to be a tyrosine (Sheehan et al., 2001) but in Delta and Epsilon insect GST classes (Ranson and Hemingway, 2005; Udomsinprasert et al., 2005) and in mammalian Theta and possibly the Zeta classes, this role is carried out by a serine 
residue (Sheehan et al., 2001). In the Omega and Beta classes a mixed disulphide is formed with a cysteine residue (Sheehan et al., 2001). Although each subunit has a kinetically independent active site, their quaternary structure is essential for their activity (Danielson and Mannervik, 1985).

\subsection{Functions of GSTs}

The primary function of many GSTs is to catalyze the detoxification of both endogenous and exogenous compounds directly or indirectly by reacting with the oxidised metabolites produced by the cytochrome P450 family.

\subsubsection{Glutathione conjugation and detoxification}

All GSTs possess the ability to conjugate GSH with compounds containing an electrophilic centre. The most common group of reactions involves attack on electrophilic carbon sites (Boyland and Chasseaud, 1969; Chasseaud, 1973; Jakoby, 1978b) which may be provided by saturated carbon atoms or unsaturated carbon atoms including aromatic carbon. In addition, electrophilic sulphur is the site in disulphide exchanges (Keen and Jakoby, 1978) (equation 1). Other sites of reactivity for GSTs include nitrogen (Prohaska et al., 1977) and electrophilic oxygen (Prohaska, 1980) (equations 2 and 3 respectively).

$$
\begin{gathered}
\mathrm{GSH}+\mathrm{R}^{\prime} \mathrm{SSR} \stackrel{\mathrm{GST}}{\rightleftarrows} \mathrm{GSSR}^{\prime}+\mathrm{RSH} \\
\mathrm{R}-\mathrm{O}-\mathrm{NO}_{2} \underset{\mathrm{GST}}{\stackrel{\mathrm{GSH}}{\longrightarrow}}\left[\mathrm{GSNO}_{2}\right] \stackrel{\mathrm{GSH}}{\longrightarrow} \mathrm{GSSG}^{\mathrm{N} \mathrm{NO}_{2}^{-}} \\
\mathrm{R}-\mathrm{O}-\mathrm{OH} \underset{\mathrm{GST}}{\stackrel{\mathrm{GSH}}{\longrightarrow}}[\mathrm{GS}-\mathrm{OR}] \stackrel{\mathrm{GSH}}{\longrightarrow} \mathrm{GSSG}+\mathrm{ROH}
\end{gathered}
$$


The most commonly used substrate to study GSTs is 1-chloro-2, 4dinitrobenzene (CDNB). CDNB conjugates with GSH and gives $S$-(2,4dinitrophenyl)glutathione, which possesses an absorbance spectrum sufficiently different from that of CDNB to allow a simple spectrophotometric assay at $340 \mathrm{~nm}$ (Clark et al., 1973; Habig and Jakoby, 1981). For some years, the efficiency of cytosolic GSTs in using certain substrates and their sensitivity to some inhibitors were parameters for determining the class of GSTs. Although ranges of activities which correlated with GST class were proposed for substrates and inhibitors, it has been demonstrated that GSTs have a broad and overlapping substrate specificity. This makes it difficult to assign a class based on substrate specificity but this knowledge remains useful as a way of understanding their properties. For this reason, with mammalian GSTs, compounds such as Bromosulphothalein (BSP, Mu class), 1,2-dichloro-4-nitrobenzene (DCNB, Mu class), trans-4-phenyl-3-buten-2-one (PBO, $\mathrm{Mu}$ class), ethacrynic acid (EA, Pi class), 1,2-epoxy-3-(p-nitrophenoxy)propane (EPNP, Theta class), dehydro ascorbic acid (DHA, Omega class) and cumene hydroperoxides $\left(\mathrm{CuH}_{2} \mathrm{O}_{2}\right.$, Alpha class) are still used as class markers (Chemale et al., 2006; Hayes et al., 2005; Ketterer, 1986; Kim et al., 2006; Mannervik, 1985). Some of the substrates used for the study of GSTs are shown in Figure 1-6 and Figure 1-7. 
1

2<smiles>O=C1OC(c2ccc(O)c([N+](=O)[O-])c2)(c2ccc(O)c([N+](=O)[O-])c2)c2c(Br)c(Br)c(Br)c(Br)c21</smiles>

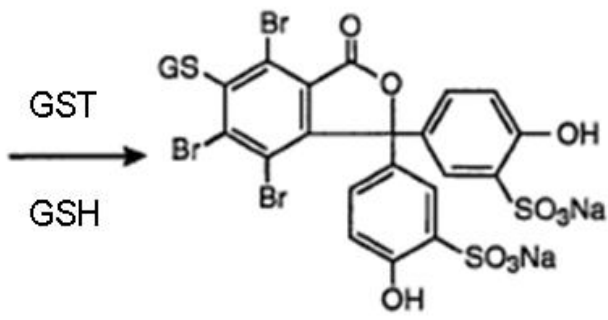

3<smiles>O=[N+]([O-])c1ccc(Cl)c(Cl)c1</smiles><smiles>O=[N+]([O-])c1ccc([SeH])c(Cl)c1</smiles>

4<smiles>C=C(CC)c1ccc(OC(=O)O)c(Cl)c1Cl</smiles><smiles>CCC(CS)C(=O)c1ccc(OC(=O)O)c(Cl)c1Cl</smiles>

5<smiles>O=[N+]([O-])c1ccc(OCC2CO2)cc1</smiles>

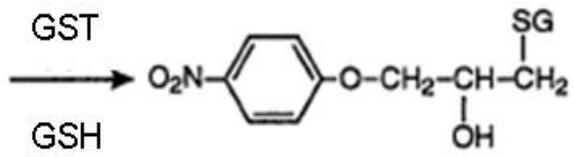

6<smiles>O=S(=O)(O)Cc1cccc2ccccc12</smiles><smiles>[Se]Cc1cccc2ccccc12</smiles>

7<smiles>O=[N+]([O-])c1ccc(Cl)cc1</smiles>

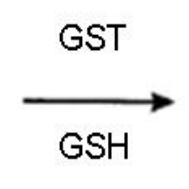<smiles>O=[N+]([O-])c1ccc([SeH])cc1</smiles>

Figure 1-6: Model substrates used in the study of GST (Hayes and Pulford, 1995).

(1) 1-chloro-2,4-dinitrobenzene; (2) Bromosulfophthalein; (3) 1,2-dichloro-4nitrobenzene; (4) Ethacrynic acid; (5) 1,2-epoxy-3-(p-nitrophenoxy)propane; (6) 1menaphthyl sulphate; (7) 4-nitrobenzyl chloride 
8<smiles>CC(=O)Oc1ccc([N+](=O)[O-])cc1</smiles>

9<smiles>O=[N+]([O-])c1ccc(CC[SeH])cc1</smiles>

10

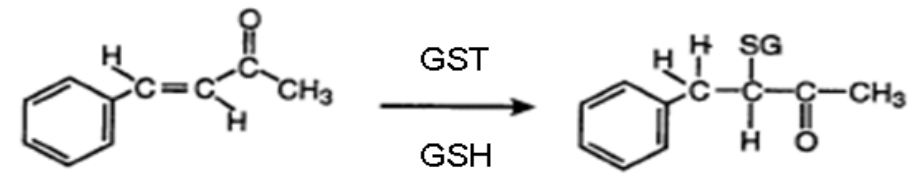

11

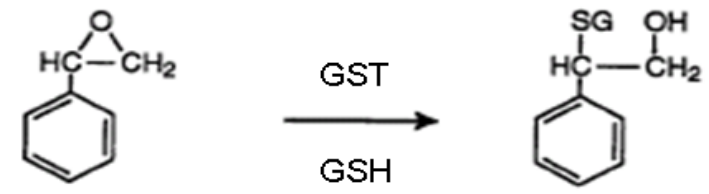

12
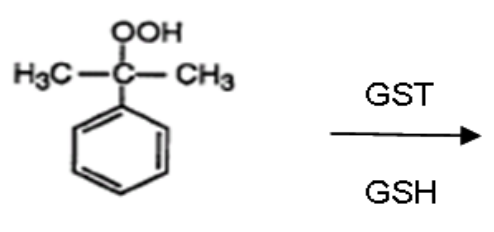<smiles>CC(C)(O)c1ccccc1</smiles>

$+(\mathrm{GSOH}) \longrightarrow \mathrm{GSSG}+\mathrm{H}_{2} \mathrm{O}$

13<smiles>CCCCCC(O)/C=C/C=O</smiles>

14<smiles>CCCCCC/C=C/C=O</smiles>

15<smiles>O=C1OC([C@H](O)CO)C(=O)C1=O</smiles><smiles>O=C1OC([C@H](O)CO)C(O)=C1O</smiles>

Figure 1-7: Model substrates used in the study of GSTs (Ayyadevara et al., 2007; Hayes and Pulford, 1995; Kim et al., 2006; Singh et al., 2001; Yang et al., 2003).

(8) 4-nitrophenyl acetate; (9) (4-nitrophenyl)ethylbromide (10) trans-4-phenyl-3-buten-2one; (11) styrene-7,8-oxide; (12) cumene hydroperoxide; (13) 4-hydroxynonenal; (14) trans2-nonenal; (15) dehydroascorbic acid. 
GSTs also detoxify herbicides and pesticides such as alachlor, atrazine, DDT, lindane, diazinon, methyl parathion, tetrachlorvinphos and chlorpyrifos (Figure 1-8). The role of GSTs in insecticide resistance will be discussed later in this chapter.

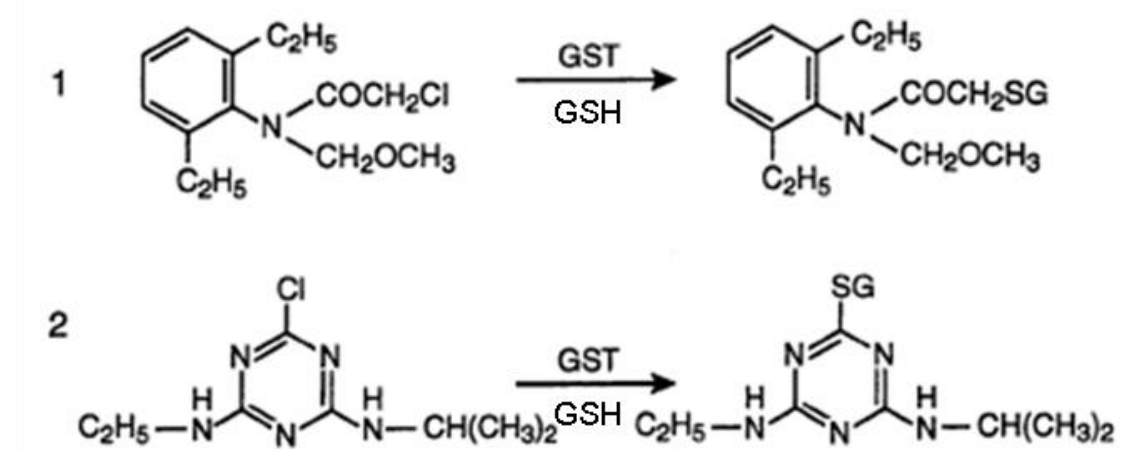

3<smiles>ClC(Cl)=C(c1ccc(Cl)cc1)c1ccc(Cl)cc1</smiles>

4

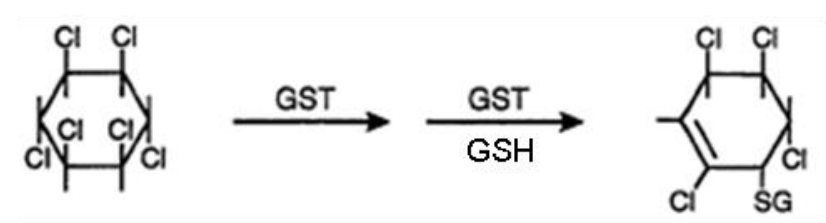

5<smiles>COP(=S)(OC)Oc1ccc(N(OP(=S)(OC)Oc2ccc([N+](=O)[O-])cc2)[Ge](Cl)(Cl)Cl)cc1</smiles>

Figure 1-8: Detoxification of Herbicides and Insecticides (Hayes and Pulford, 1995; Oppenoorth et al., 1979; Wilson and Clark, 1996).

(1) alachlor; (2) atrazine; (3) DDT; (4) lindane; (5) methyl parathion

Most of the GST substrates are either xenobiotics or products of oxidative stress. However, GSTs can also metabolise a small number of endogenous 
compounds, such as leukotriene $\mathrm{A}_{4}$ (Anuradha et al., 2000; Nicholson et al., 1993), prostaglandin $\mathrm{H}_{2}$ (Beuckmann et al., 2000a; Chang et al., 1987) and prostaglandin $\mathrm{D}_{2}$ (Urade et al., 1987) as part of their normal biosynthetic pathways and these are hence not considered as detoxification reactions. The role of GSTs in steroid isomerisation and tyrosine metabolism will be discussed in section 1.4.3.

The formation of a thioether bond between electrophiles and GSH almost always yields a conjugate that is less reactive than the parental compound (Chasseaud, 1979) and therefore the actions of GST generally result in detoxification. Once formed, the conjugates can be transported from the cell by ATPdependent glutathione $S$-conjugate efflux pumps. Several glutathione $S$-conjugate pumps have been described in mammalian (Homolya et al., 2003; Ishikawa, 1992; Leier et al., 1994; Srivastava et al., 2002; Zimniak et al., 1992) and plant cells (Martinoia et al., 1993). Ishikawa has characterized an ATP-dependent glutathione $S$-conjugate pump, now called the multidrug resistance-associated protein (MRP), from rat heart and demonstrated it can transport oxidized glutathione (GSSG), leukotriene $\quad \mathrm{C}_{4} \quad\left(\mathrm{LTC}_{4}\right)$, and $\quad S$-(2,4-dinitrophenyl)glutathione (Ishikawa, 1989; Ishikawa, 1992; Jedlitschky et al., 1994; Leier et al., 1994). The most common of the efflux transporter families are the ABC (ATP-binding cassette) transporters which include Pgp, MRPs and $\mathrm{ABCG}_{2}$ (also known as mitoxantrone-resistant protein) (Chan et al., 2004; Meijerman et al., 2008). Beyond the considerable functional redundancy between these transporters, the substrate selectivity of the pumps differs markedly. In particular MRPs are involved in the transport of GSH, glucuronate or sulphate conjugates of organic anions that arise from detoxification reactions by phase II conjugating enzymes such as GSTs, sulfotransfearses and UDPglucuronosyltransferases (Morrow et al., 2006; Sau et al., 2010) 


\subsubsection{Peroxidase activity of GSTs}

Lipid peroxidation products in general have been associated with toxicity. Apart from catalysing the conjugation between GSH and electrophilic compounds, a significant number of the GST isoenzymes also possess glutathione peroxidase activity and catalyze the reduction of organic hydroperoxides to their corresponding alcohols. According to Habig et al. (1983) this type of reaction proceeds via nucleophilic attack by GSH on an electrophilic oxygen.

It is believed to involve two steps, only one of which is catalytic, and to proceed via formation of the sulfenic acid of glutathione as follows:

1. $\mathrm{ROOH}+\mathrm{GSH} \longrightarrow \mathrm{ROH}+[\mathrm{GSOH}] \quad \ldots \ldots .$. enzymatic

2. $[\mathrm{GSOH}]+\mathrm{GSH} \longrightarrow \mathrm{GSSG}+\mathrm{H}_{2} \mathrm{O}$ …..... spontaneous

Overall the reaction is

1. $\mathrm{ROOH}+2 \mathrm{GSH} \longrightarrow \mathrm{ROH}+\mathrm{GSSG}+\mathrm{H}_{2} \mathrm{O}$ (Hayes and Pulford, 1995).

Fatty acid, phospholipid and DNA hydroperoxides can be reduced by GSTs. As these compounds are generated by lipid peroxidation and oxidative damage to DNA, it has been proposed that GST, as well as other GSH-dependent enzymes, help combat oxidative stress (Boyland and Chasseaud, 1969). The microsomal GSTs (membrane bound) and soluble GSTs (cytosolic) differ in their ability to protect against reactive oxygen species (Jakoby and Habig, 1980). The microsomal GSTs detoxify the lipid hydroperoxides in situ, whereas detoxification of lipid hydroperoxides by cytosolic GSTs requires prior release of fatty acid hydroperoxides by phospholipase $\mathrm{A}_{2}$ (Armstrong, 1994; Graminski et al., 1989). In humans, a physiological function suggested for Omega GSTs is that they may play a role in 
protection against oxidative stress by removing thiol adducts from proteins (Board et $a l ., 2000)$. GSTs are found to be involved in the management of toxic products of lipid oxidation and $S$-glutathiolated proteins generated by oxidative stress (Awasthi et al., 2004; Listowsky, 2005). Recent studies showed that deficiency of Zeta GST in mice causes oxidative stress and activation of antioxidant response pathways (Blackburn et al., 2006).

In insects, exposure to insecticides induces oxidative stress (Abdollahi et al., 2004) and insect GSTs may contribute to anti-oxidant defence by direct glutathione peroxidase activity preventing and repairing the damage of secondary products generated by reactive oxygen species (ROS) and by direct conjugation of 4-HNE, one of the major end-products of lipid peroxidation (Ding et al., 2005; Sawicki et al., 2003; Singh et al., 2001; Vontas et al., 2001). The Delta, Epsilon and Sigma classes of GSTs have been reported to exhibit peroxidase activity (Ding et al., 2005; Lumjuan et al., 2005; Ortelli et al., 2003; Singh et al., 2001; Vontas et al., 2001). Theta GSTs in D. melanogaster were identified and were suggested to have functions in oxidative stress response (Toung et al., 1993). Theta GST from the silkworm was reported as a defense mechanism against oxidative stress and in the metabolism of lipid peroxidation products (Yamamoto et al., 2005).

\subsubsection{Isomerisation by Zeta class GSTs}

The Zeta GSTs are found to be present in many different species of plants, insects and mammals, and their sequence motif SSCXWRVIAL is highly conserved at the N-terminus in these species (Board et al., 1997). This highly conserved structure of this protein suggests its important role in housekeeping, and in this context, GSTZ1 catalyses the cis-trans isomerisation of maleylacetoacetic acid to 
fumarylacetoacetic acid, an important step in the tyrosine degradation pathway (Keen and Jakoby, 1978). Small numbers of Zeta GST isoenzymes possess ketosteroid isomerase activity and catalyze the conversion of $\Delta^{5}$-3-ketosteroids to $\Delta^{4}$ 3-ketosteroids.
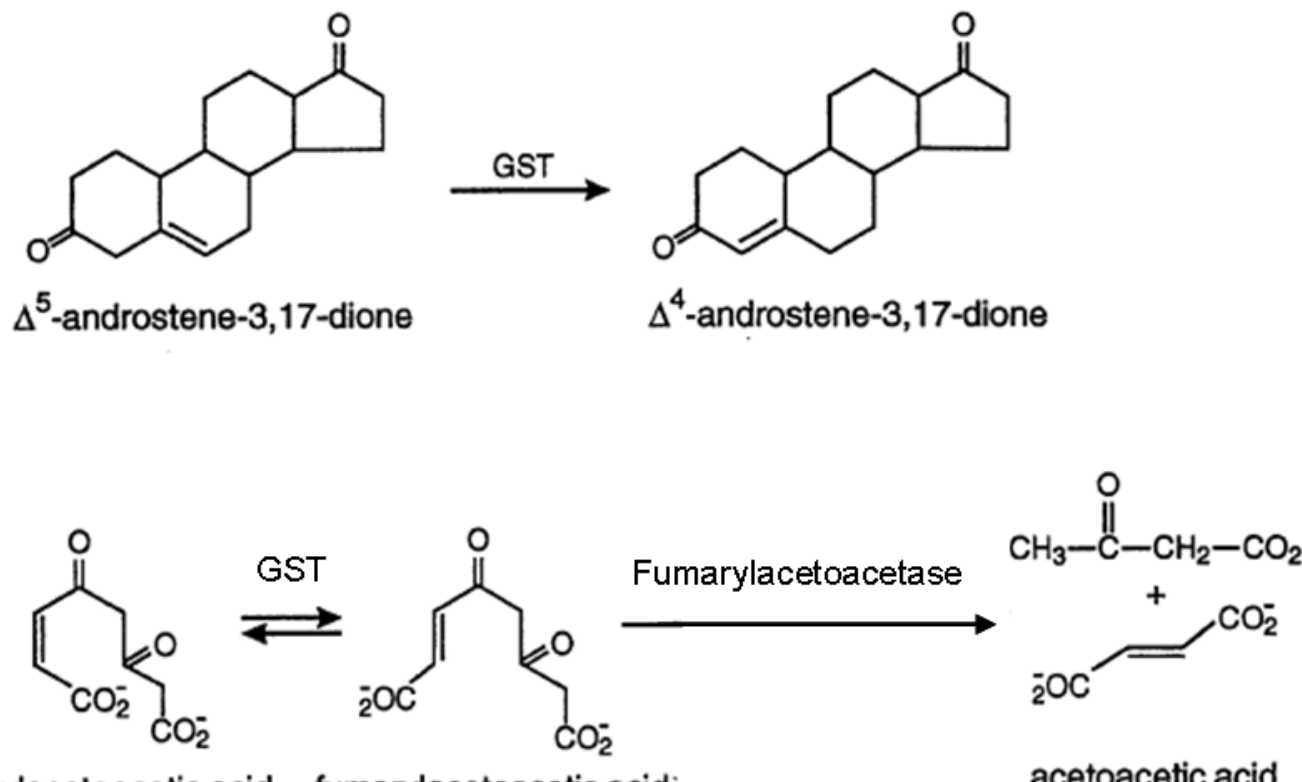

maleylacetoacetic acid fumarylacetoacetic acid

acetoacetic acid fumaric acid

Figure 1-9: Isomerization of $\Delta^{5}$-androstene-3,17-dione and maleylacetoacetic acid catalysed by GST (Hayes and Pulford, 1995).

\subsubsection{GSTs and the processing of odorant signals}

In insects and other terrestrial animals, their olfactory system is directly exposed to xenobiotics and odorants such as volatile plant compounds and pheromones. Odorant molecules enter olfactory sensilla located within the insect antennae and interact with receptor molecules to trigger an appropriate response. The odorant molecules must ultimately be degraded to terminate the sensory response, and GSTs play an important role in this process. In the sphinx moth Manduca sexta an olfactory specific GST (GST-msolf1) has been identified in male and female 
antennae and shown to modify trans-2-hexenal, an olfactory system-stimulating plant-derived aldehyde (sex pheromone). It has been proposed that GST-msolf1 may play a dual role in the antenna by contributing to the detoxification of compounds that might interfere with sex pheromone detection and to the signal termination of aldehyde sex pheromone odorants (Rogers et al., 1999). Recently mouse olfactory GST has been characterised during the acute phase response (Weech et al., 2003).

\subsubsection{Eye pigment synthesis}

The D. melanogaster eye colour mutant sepia (se') is defective in PDA (6acetyl-2-amino-3-7,8,9-tetrahydro-4H-pyrimido[4,5-b]-[1,4]diazepin-4-one or pyrimidodiazepine) synthase involved in the conversion of 6-PTP (2-amino-4-oxo-6pyruvoyl-5,6,7,8-tetrahydropteridine; also known as 6-pyruvoyltetrahydropterin) into PDA, a key intermediate in drosopterin biosynthesis ((Kim et al., 2006). Five genes predicted to encode GSTs (CG6781, CG6662, CG6673A, CG6673B, and CG6776) were isolated from the presumed $D$. melanogaster eye colour mutant sepia locus (region 66135 on chromosome 3L). All five cloned and expressed candidates exhibited relatively high thiol transferase and dehydro-ascorbate reductase activities, characteristic of Omega class GSTs. Despite the strong homology between the five Omega GSTs tested, only CG6781 catalyzed the synthesis of PDA in vitro (Kim et $a l ., 2006)$. Thus, they reported that an Omega class GST (CG6781) of $D$.

melanogaster is involved in pteridine metabolism in particular in the biosynthesis of red eye pigments. It is shown that the role of CG6781 is restricted to drosopterin biosynthesis, since it is expressed only in the head in adults, and in the late pupa. Despite the strong homology between the five Omega GSTs tested, only the CG6781 protein had PDA synthase activity (Kim et al., 2006). 


\subsubsection{Ligandin function}

In addition to binding of substrates, many GSTs are able to bind a wide range of hydrophobic chemicals covalently and noncovalently. In mammals, the compounds covalently bound by GST appear mainly to be the reactive metabolites formed from carcinogens such as dimethylaminoazobenzene and 3methylcholanthrene (3-MC) (Ketterer et al., 1967). It is thought that the covalent binding of these compounds represents a 'suicide' reaction by GST, serving to prevent genotoxic electrophiles from interacting with DNA (Jakoby and Keen, 1977). However, not all compounds that are bound covalently by GSTs are carcinogens.

Among the noncatalytic reversibly bound ligands of GSTs are steroids and thyroid hormones, bile acids, bilirubin, heme, fatty acids and penicillin (Hayes and Mantle, 1986a; Hayes and Mantle, 1986b; Ishigaki et al., 1989; Kirsch et al., 1975; Listowsky et al., 1993). Recently the non-catalytic interactions between GSTs and naturally occurring nitroalkenes, nitrolinoleic $\left(\mathrm{NO}_{2}-\mathrm{LA}\right)$ and nitrooleic $\left(\mathrm{NO}_{2}-\mathrm{OA}\right)$ fatty acids are shown to modulate nitroalkene-mediated activation of PPAR $\gamma$ (peroxisome proliferator-activated receptor $\gamma$ ), a pathway which regulates adipocyte

differentiation, glucose homeostasis, and inflammatory responses (Bates et al., 2009). Though the biological significance of this noncovalent binding-activity of GSTs has been the subject of much debate (Bates et al., 2009; Hayes and Pulford, 1995), it is worth noting that because of the large amount of GSTs in most tissues, these proteins do provide substantial intracellular-binding capacity for lipophilic ligands. It was proposed that the binding of steroid hormones, bilirubin and the bile acid lithocholate may contribute to the transport of such lipophilic compounds across the liver and facilitate their elimination into bile (Agellon and Torchia, 2000; Trauner 
and Boyer, 2003). Similarly GST in the kidney and small intestine may be involved in the transport of lipophilic compounds (Chan et al., 2004; Hayes and Pulford, 1995). GSTs are also present on mucosal surfaces (Samiec et al., 2000). The presence of this detoxication enzyme in the extracellular mucus layer provides a novel mechanism as a ligandin for preventing direct contact of potentially toxic dietary electrophiles with the intestinal enterocytes. GSTs also constitute a high capacity intracellular binding pool for hormones; they might function as a binding reserve in target organs, possibly serving a "buffering' role to minimize the effects of transient fluxes in extracellular hormone levels (Johansson and Mannervik, 2001; Listowsky et al., 1993). The ligandin function of GSTs has been suggested as contributing to defence against pyrethroid insecticides in Tenebrio molitor as a passive way of detoxification by binding the molecules in a sequestering mechanism (Kostaropoulos et al., 2001a).

\subsubsection{GSTs as regulators of the MAP kinase pathway}

c-Jun N-terminal kinases (JNKs) are mitogen-activated protein kinases responsive to stress stimuli, such as cytokines, ultraviolet irradiation, heat shock, and osmotic shock, and are involved in T cell differentiation and apoptosis (David et al., 2005; Laborde, 2010; Widmann et al., 1999). JNK pathway components and GSTs are evolutionarily conserved across mammals and insects. Different mammalian GST classes such as GST Pi and GST Mu have been reported to interact with different stress kinase proteins in the JNK pathway. For example, GST Pi is a JNK regulatory protein, and its association with JNK maintains a low basal level of JNK activity in the non-stressed cell (Adler et al., 1999). The lack of GST Pi increased constitutive JNK activity in vivo and, therefore, regulated the expression of genes that were 
specific downstream targets of the JNK pathway (Elsby et al., 2003). In contrast, GST Mu interacts with ASK1 (apoptosis signal-regulating kinase 1), an upstream activating kinase of JNK that participates in cell death (Cho et al., 2001).

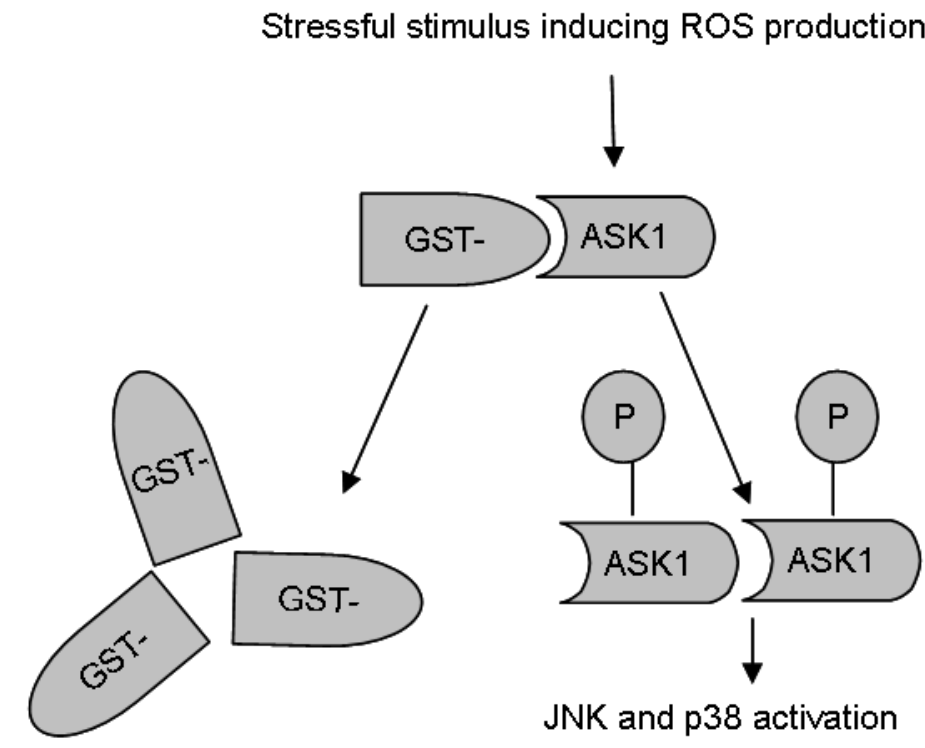

Figure 1-10: Interaction of GST Mu with ASK1

GSTM1 binds to and inhibits MAP kinase kinase kinase ASK1. Radical oxygen species are produced in response to stressful stimuli and induce GST-M1 oligomerization. In turn ASK1 is released, oligomerizes and is activated through autophosphorylation and activates downstream kinases such as JNK and p38 pathways to trigger cell death by apoptosis. (Solary et al., 2009)

Recently the interaction of GST and kinase proteins in a Dipteran system using four different spliceforms of Anopheles dirus Delta class GSTs and two different D. melanogaster kinase proteins has been reported (Udomsinprasert et al., 2004). This study revealed an interaction between the mosquito GSTD1-1, GSTD22, GSTD3-3 and GSTD4-4 spliceforms and the JNK pathway components, JNK and HEP (hemipterous). They showed using the standard CDNB assay, that GSTD1-1 was not inhibited, whereas the remaining GSTs were inhibited by their preincubation with JNK proteins. GSTs from both the insects interacted with protein kinases and this interaction with the kinase could change the GST conformation and 
results in different GST enzymic activity. The different GST isoforms appeared to possess different regulatory mechanisms in the JNK pathway and JNK interaction also affects GST activities (Udomsinprasert et al., 2004).

\subsubsection{GSTs in drug resistance}

As described before, GSTs catalyse the conjugation of glutathione to mutagens, carcinogens and chemotherapeutic agents including alkylating agents and anthracyclines (Hayes et al., 2005; Lo and Ali-Osman, 2007; Petros et al., 2005; Yang et al., 2006). It is plausible that GSTs serve two distinct roles in the development of drug resistance via direct detoxification as well as acting as an inhibitor of the MAP kinase pathway. Hence, it is not surprising that high levels of GSTs have been reported in a large number of tumor types (Tew, 1994). GSTs may also be important drug targets in disease states other than cancer. For example, many parasitic organisms showed the presence of GSTs homologous to mammalian GST isozymes and these are believed to provide targets for intervention in the human host. When many of the standard drugs used to treat such diseases are frequently ineffective, these new targeting directions provide a good opportunity. Many antiparasitic drugs (for example, chloroquinone, an antimalarial agent) form free radicals that may be inactivated by parasitic GST-mediated conjugation to GSH (Davioud-Charvet et al., 2001). Alpha, Mu, Pi and Theta class of GSTs are known to be involved in tumor drug resistance (Lo and Ali-Osman, 2007).

\subsubsection{GSTs in sperm viability}

The immunoreactive and enzymatically active $\mathrm{Mu}$ and Pi class of GSTs have been shown to be present on goat sperm surface where they serve as oocyte binding proteins (Gopalakrishnan et al., 1998; Gopalakrishnan and Shaha, 1998). A study on 
male rats demonstrated the presence of these two GST isoforms on sperm serving as detoxification enzymes (Hemachand et al., 2002). The germ cells are more susceptible to oxidative stress due to their intimate association with the free radical generating phagocytic sertoli cells (Bauche et al., 1994) and they contain higher amounts of polyunsaturated fatty acids (Beckman and Coniglio, 1979) that are vulnerable to oxidation by free radicals. Thus GSTs give protection against products of oxidative stress-induced apoptosis (Gopalakrishnan et al., 1998; Hemachand et al., 2002; Rao and Shaha, 2000).

\subsubsection{GSTs involved in haematin binding and in wound healing}

Lumjuan et al. (2007) identified two isoforms of GSTs (GSTX2-2) in Aedes aegypti which showed an affinity for haematin which may indicate a role for these enzymes in protecting haematophagous insects against heme toxicity during blood feeding.

There are reports available indicating that the GSTs in vertebrates are released from platelets (Kura et al., 1996). Li et al. found the involvement of GSTs in haemolymph clotting in Galleria mellonella while studying the interaction between the coagulation system and the prophenoloxidase activating cascade (Li et al., 2002).

\subsection{Regulation of GST expression}

In non-insect species, many GSTs are regulated in response to various inducers or environmental signals or in tissue- or developmental-specific manner (Desmots et al., 2002; Tee et al., 1992; Werle-Schneider et al., 2006). A similar complex expression pattern is also expected for insect GSTs. In insects, the effect of various dietary compounds, insecticides and laboratory inducers on general GST 
expression has been reviewed (Clark, 1989; Yu, 1996). Variation in the level of GST activity throughout the life stage of insects and in different insect tissues has been reported (Alias, 2006; Clayton et al., 1998; Ding et al., 2003; Hazelton and Lang, 1983). In cases where the variation in activity is attributed to individual enzymes, such studies can provide valuable insights into the functions of different GSTs.

\subsection{Insecticide resistance and role of GSTs}

Resistance to different group of insecticides such as organochlorine, organophosphate (OP) and carbamates can be explained in terms of several biochemical mechanisms. These include target-site resistance, penetration, efflux and detoxification-based resistance (Hemingway et al., 2002). In the former the target has reduced affinity for the insecticides and the latter occurs when enhanced levels or modified activities of esterases, oxidases, or GSTs occur. Over-production of carboxylesterase in response to organophosphorus and carbamate insecticides selection pressure has been documented in numerous arthropod species (Hemingway and Karunaratne, 1998; Khajehali et al., 2010; Rodriguez et al., 2010). In OP susceptible insects, the active oxon analogues of the insecticides act as esterase inhibitors whereas in resistant insect, esterases are more reactive with xenobiotics and sequester the oxon analogues and protect the acetyl cholinesterase target site (Karunaratne et al., 1995). The elevated levels of cytochrome P450-dependent monooxygenases are also reported in many resistant insects and their action generally results in the detoxification of insecticides, although the activation of OP insecticides from the phosphorothionate to the more toxic oxon form is a notable exception (Hemingway et al., 2004; Kasai and Scott, 2000; Komagata et al., 2010; Vulule et al., 1999). The other important metabolic enzymes are GSTs. The role of 
GSTs in the detoxification of organophosphates has been well known for many years (Motoyama and Dauterman, 1980). The detoxification of organophosphates occurs by the conjugation of GSH to OP insecticides via two distinct pathways: an $O$ dealkylation and $O$-dearylation conjugation (Figure 1-11).

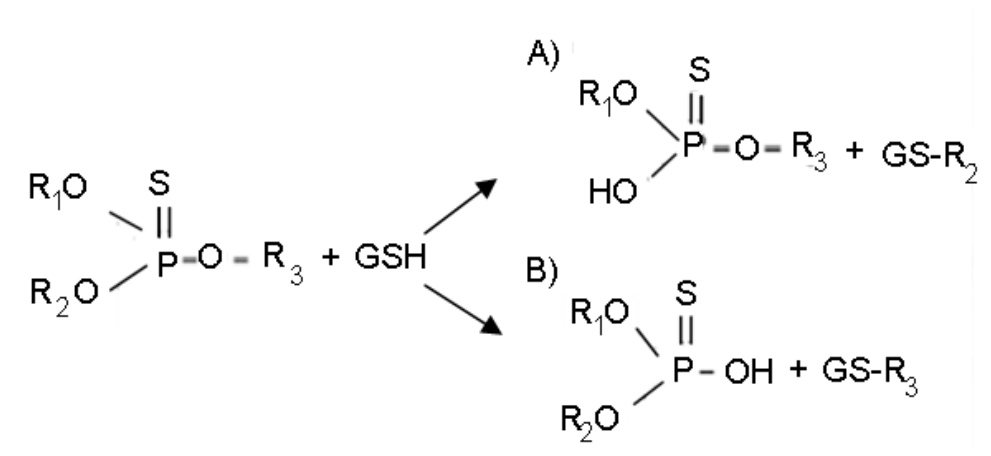

Figure 1-11: GST-mediated detoxification of organophosphate insecticides (Che-Mendoza et al., 2009)

A) $O$-dealkylation and B) $O$-dearylation conjugation; $\mathrm{R}_{1}$ and $\mathrm{R}_{2}$ represent the alkyl (either methyl or ethyl portion); and $\mathrm{R}_{3}$ is an aryl or alkyl group.

The GSTs often act as a secondary resistance mechanism in conjunction with esterase- and cytochrome P450- mediated resistance mechanisms (Hemingway et al., 1991a). The relative importance of these enzyme systems varies with species and strain of insect and insecticides. Recently, the comparative study on a susceptible laboratory strain and OP resistant field strain of locusts revealed an interesting finding on the involvement of these major detoxification enzymes in resistance. The field strain was significantly resistant to malathion (57.5-fold), but marginally resistant to chlorpyrifos (5.4-fold). The esterases and GST activities in resistant strain were 2.1- to 3.2-fold and 1.2- to 2.0-fold respectively higher than those in susceptible strain. However, there was no significant difference found between cytochrome P450 activities between the strains. Acetyl cholinesterase from the field strain showed 4.0fold higher activity in response to malathion but less activity to chlorpyrifos and 
phoxim (Yang et al., 2009). Another study on codling moth, Cydia pomonella, also evaluated the susceptibility to chlorpyrifos-ethyl and azinphos-methyl and the activity of esterases, mixed function oxidases and GSTs in larvae and adult. The activity of all these enzymes was higher in larvae of field strains. In adults, mixed function oxidases and GSTs were implicated (Rodriguez et al., 2010).

Although insecticide resistance may involve many different mechanisms as discussed above, in the present work the emphasis is on the role of GSTs. Besides organophosphates, GSTs are also involved in resistance against organochlorines such as DDT (Clark and Shamaan, 1984; Lumjuan et al., 2005; Prapanthadara et al., 2002; Ranson et al., 1997; Sternberg and Kearns, 1952), Lindane (Kristensen, 2005) and the cyclodienes (Rufingier et al., 1999; Sharif et al., 2007). The GSTs catalyse two detoxification reactions of halogenated hydrocarbons: dehydrochlorination and GSH conjugation (Tang and Tu, 1994). The catalytic reaction of GSTs with DDT and lindane is shown in Figure 1-8 $(3,4)$. Their involvement in developing resistance to pyrethroid insecticides by both indirect (Vontas et al., 2001) and direct (Mouatcho et al., 2009; Yamamoto et al., 2009a; Yamamoto et al., 2009b) mechanisms has been demonstrated. They may be involved in the resistance to insect growth regulators such as chlorfluazuron and diflubenzuron (Sonoda and Tsumuki, 2005) and to carbamate insecticides in both Anopheles and Aedes mosquitoes (Lumjuan et al., 2005; Mouatcho et al., 2009).

Among the insect GST classes, Delta and Epsilon are the largest classes based on the number of isoforms, comprising over $65 \%$ of the total complement of cytosolic GSTs in Drosophila melanogaster and Aedes aegypti (Ranson et al., 2002). It is interesting to note that elevated levels of Delta class GSTs have been implicated 
in resistance to all the major classes of insecticides (Tang and Tu, 1994; Vontas et al., 2002; Wang et al., 1991). Members of the Epsilon class have also been implicated in the detoxification of insecticides (Huang et al., 1998; Ortelli et al., 2003; Wei et al., 2001). In addition, some mosquitos Epsilon GSTs have peroxidase activity and may be important in protection against secondary effects of oxidative stress (Lumjuan et al., 2005; Ortelli et al., 2003). A structural role has been suggested for the insect Sigma GSTs; as in Drosophila melanogaster and Musca domestica, these proteins are found predominantly in the indirect muscles in close association with troponin-H. In both these species, a single Sigma GST exists with a proline/alanine-rich $\mathrm{N}$-terminal extension that may aid attachment to the flight muscle (Clayton et al., 1998). The A. gambiae Sigma GST lacks this extension (Ranson and Hemingway, 2005). Insect Sigma GSTs (with or without the N-terminal extension) have been shown to be catalytically active (Singh et al., 2001). They have low levels of activity with typical GST substrates but a high affinity for the lipid peroxidation product, 4-hydroxynonenal (4-HNE). The high levels of Sigma GSTs found in the flight muscle may be necessary to protect this metabolically highly active tissue against by-products of oxidative stress rather than having a structural function. The Omega, Theta and Zeta classes have a much wider taxonomic distribution and appear to play essential housekeeping roles (Board et al., 2000; Wildenburg et al., 1998). However, recent reports suggest that any GST isoform may be recruited to a detoxification function. Thus a Zeta GST appears to be responsible for the dechlorination of and resistance to permethrin in the Silk Moth, Bombyx mori (Yamamoto et al., 2009b), Omega GST appears to be associated with resistance to the organophosphate fenitrothion in a different strain of the same organism (Yamamoto et al., 2009a) and a $\mathrm{Xi}$ isoform is associated with DDT 
resistance in a South American strain of Aedes aegypti (Grant and Hammock, 1992; Lumjuan et al., 2007). Moreover, members of a particular family are likely to have broad and overlapping substrate specificities so that collectively they offer an extraordinary wide protection against toxic agents.

\subsection{Lucilia cuprina - an economically significant fly}

The sheep blowfly $L$. cuprina is a pest of economic significance to the meat and wool industries in Australasia. It has evolved to become a parasite which inflicts significant trauma via cutaneous myiasis. The condition is also known as blowfly strike. Sheep are not struck randomly. Urine or rain wetted fleece allows bacteria, principally Pseudomonas aeruginosa, to proliferate in an infection known as fleece rot (Burrell et al., 1982). This condition is highly attractive to L. cuprina seeking oviposition sites. Pheromones released during ovipositing attract other flies to lay eggs in the affected area and cause further waves of strike. L. cuprina causes over $90 \%$ of fly strikes and is the only species which breeds preferentially on living sheep. This fly strike not only causes severe discomfort or stress to sheep but also will cause death if left untreated (Plant, 2006). The long-term use of organophosphorus (OP) insecticides to control fly strike has resulted in the development of resistance to this class of insecticides (Arnold and Whitten, 1976; Hughes and Raftos, 1985). In Australia L. cuprina is known to have developed resistance to all the major classes of insecticides (Hughes, 1981). Ever since the introduction of L. cuprina in New Zealand apparently in the late 1970s (Heath et al., 1991), resistance to insecticides has become an increasing problem in the control of fly strike. Overall blowflies have become of major animal welfare concern and an important cause of illness and death 
in affected animals. Since GSTs have been implicated in the expression of insecticide resistance in these insects, it is of interest to study the GSTs of L. cuprina.

\subsection{Present investigation}

Different classes of GST exhibit overlapping specificity towards different insecticides as described previously. Clearly this complexity represents a major obstacle for one attempting to overcome GST-based resistance in insect (or any other invertebrate) pests. Conventional approaches to overcome metabolic resistance include identification of a single detoxication enzyme for which a specific inhibitor may be designed. A single GST might be over expressed in glutathione-dependent metabolism but, on the other hand, resistance could be the result of combined activity from multiple GSTs from disparate families. Historically, much of the work on GST involvement in resistance has depended on measurement of GST activities with model substrates. However, this approach alone cannot be enough to characterise the GSTs precisely. Our current work, described here, offers a much more direct approach. The laboratory has been developing methods for the affinity isolation of insect GSTs for many years (Clark et al., 1977). Introduction of proteomics methods has made this approach a powerful one for not only isolating GSTs but also identifying them (Alias and Clark, 2007). The quantitative and qualitative changes in protein expression can be analysed by using $2 \mathrm{D}$ electrophoresis and identification can be made by advanced mass spectrometry and peptide mass fingerprinting. Recent advances in bioinformatic technology enable one to analyse and correlate proteomic data from studies in all cell types. This proteomic approach is aimed at characterising, for a given organism and under given circumstances of development or selection, the expression of as many different GSTs as is possible. The number 
and class of over-expressed GSTs may, in principle, be quickly determined. This proteomic technique may be applied to any given organism, even without prior knowledge of its genome. In such cases where the genome of the insect in question has been established, a rapid assessment of the extent of transcription of detoxication genes may be made by use of microarray technology (David et al., 2005; Vontas et al., 2007). However, there are instances on record (Shen et al., 2003; Tang and Tu, 1995) where production of a GST protein in insects appears to be under translational or post-translational rather than transcriptional control so methods measuring mRNA production only may lead to misleading interpretations. For example, the $D$. melanogaster Delta GST showed abnormal migration (change in pI) on 2D gel indicating its post-translational modification (Alias and Clark, 2007).

The GST superfamily has diverse important roles in the normal functions of cells in addition to the purely toxicological roles as described previously. This suggests that, as opposed to being only defence mechanisms, some GSTs might themselves, because of their critical metabolic role, constitute sites of vulnerability to chemical attack and might represent new targets for chemical control. Hence the detailed study of GSTs is very useful to determine their role in development, physiology and insecticide resistance in any pest species. In the present investigation, affinity purification and proteomic methods have been applied to a well-studied species $D$. melanogaster and to L. cuprina for which there is currently no genetic database available.

The overarching aim of this research is to undertake the first proteomic study of L. cuprina GSTs, their tentative identification and their possible importance in insecticide metabolism. This can be broken down to four major objectives: 
1. Purify and identify GSTs from D. melanogaster and L. cuprina.

- To establish effective affinity purification techniques for the isolation of as many GSTs as possible.

- To compare the GST proteome of both the insects to study their similarity and dis-similarity in expression of GSTs.

- To identify L. cuprina proteins using D. melanogaster as a model insect.

- In addition to develop a consensus sequence-based approach to tentatively characterise $L$. cuprina proteins.

- To separate GST isoforms from a partially affinity-purified mixture to characterise individual classes of enzyme.

2. Examine the qualitative and quantitative variation of GSTs during the developmental stages of L. cuprina and also in the main body parts of the adult fly.

- To isolate GSTs from egg, larval, pupal and adult stage using a combination of affinity matrices and comparison of their GST proteomes.

- To study the ontogenic pattern of GST activity using model substrates.

- To study the proteome of GSTs isolated from head, thorax and abdomen.

3. Investigate the involvement of GSTs in the development of insecticide resistance in L. cuprina.

- To isolate GSTs from organophosphate susceptible and resistant strains of $L$. cuprina and to compare their proteomes. 
- To study the ability of GSTs to metabolise organophosphate insecticides in vitro using an HPLC method.

4. Determine the nature of post-translational modification of D. melanogaster Delta GST. 


\section{Materials}

\subsection{Insects}

The adult flies of $D$. melanogaster, mixed strain were obtained from the Victoria University insectary. The L. cuprina eggs, larvae, pupae and adults (OP susceptible strains NSW and CSIRO (Australian standard laboratory strains) and diazinonresistant New Zealand field strain PY81 (38x resistant, G. Lindsay, unpublished data) initially were supplied by Victoria University insectary and subsequently by Microcosmos ${ }^{\mathrm{TM}}$ Ltd. These strains had originally been obtained from Wallaceville Agricultural Research Centre, Wellington. Larvae were reared on a combined diet of processed pet-food and minced ox-liver. The standard rearing conditions for adults were feeding ad libitum on sugar and water; however, some flies were fed on protein enriched diet. All were stored at $-20^{\circ} \mathrm{C}$ or for longer periods at $-80^{\circ} \mathrm{C}$.

\subsection{Chemicals and disposables}

All reagents were of analytical grade purity or equivalent unless otherwise stated.

\section{Ajax Chemicals Ltd, Sydney-Melbourne, Australia}

Acetonitrile, HPLC grade

Ethanol 95\%

Glacial acetic acid

Heptane, HPLC grade

Hydrochloric acid 36\%

Methanol, HPLC grade

Orthophosphoric acid, $81 \%$ 
Amersham Biosciences AB, Uppsala, Sweden

QAE- Sephadex A-25, Sephadex G-75

2D Quant kit

Columns- Tricorn ${ }^{\mathrm{TM}} 5 / 50$, Tricorn ${ }^{\mathrm{TM}} 5 / 200$, HiTrap ${ }^{\mathrm{TM}}$ Desalting column (5 ml)

American National Can ${ }^{\mathrm{TM}}$, Menashaw, USA

Parafilm

Axygen Scientific Inc., California, USA

Graduated Micro tubes $(0.65 \mathrm{ml}, 1.5 \mathrm{ml})$

Pipette tips $(10 \mu \mathrm{l}, 200 \mu \mathrm{l}, 1000 \mu \mathrm{l})$

\section{British Drug Houses Chemicals Ltd., Poole, England}

Bromophenol blue

Collidine

Deoxycholic acid

Ethylenediamine tetra-acetic acid, tetrasodium salt (EDTA)

Glycerol

Phenyl thiourea (PTU)

Bio-Rad Laboratories, Richmond, USA

40\% Acrylamide/Bis Solution

Protein dye reagent (Bradford Assay)

\section{Fluka Chemicals Ltd., New Zealand}

Ammonium bicarbonate

Sodium hydroxide

Trifluoroacetic acid (TFA) 


\section{GE Healthcare, Auckland, New Zealand}

Electrode Wicks

Immobiline pH Gradient buffer (IPG Buffer), 3-10

Immobiline Dry Strip pH 4-7, PH 3-10 Linear, 7 cm

Iodoacetamide

Polybuffer 74

Invitrogen New Zealand Ltd., Auckland

BENCHMARK ${ }^{\mathrm{TM}}$ Protein ladder

Molecular Probes, Inc.

Pro- $\mathrm{Q}^{\circledR}$ Diamond Phosphoprotein gel stain kit

Sypro ${ }^{\circledR}$ Ruby Protein gel stain kit

\section{May \& Baker Ltd., Dagenham, England}

Sodium acetate

Triethanolamine

\section{Merck Ltd., New Zealand}

Trichloroacetic acid

Thin Layer Chromatography (TLC) plates - Aluminium sheets, Silica gel 60, F254

Millipore Corporation, Bedford USA

$\operatorname{ZipTip}^{\mathrm{TM}}\left(\mathrm{C}_{18}\right)$

ZipTip $_{M C}$ (for phosphopeptide enrichment)

\section{PE Biosystems, Forster City, CA}

Sequazyme $^{\mathrm{TM}}$ peptide mass standards kit- Calibration Mixture 2

\section{Riedel-de Haën, United States}

Diazinon

Methyl Parathion 
Thiourea

Urea

\section{Roche Diagnostic GmbH, Germany}

Phosphatase inhibitor tablets

\section{Sigma-Aldrich New Zealand Ltd., Auckland}

1, 4- nitrophenyl acetate (NPA)

1-chloro-2, 4-dinitrobenzene (CDNB)

3, 4-dichloronitrobenzene (DCNB)

3-[(3-Cholamidopropyl)dimethylammonio]-1-propanesulfonate (CHAPS)

Albumin, bovine serum (BSA)

Ammonium persulfate

Ammonium sulfate

Bis-Tris

Coomassie Brilliant Blue G-250

Cysteine

Dehydroascorbic acid (DHA)

Disodium hydrogen phosphate

Dithiothreitol

Epichlorhydrin

Glutathione

Glycine

$N, N, N^{\prime}, N^{\prime}$-tetramethylenediamine (TEMED)

Ninhydrin

Phenylmethylsulfonyl fluoride (PMSF)

Polybuffer 96

Potassium chloride 
Protease Inhibitor Cocktail

Sephadex ${ }^{\mathrm{TM}} \mathrm{G}-75$

Sepharose 6-B

Sodium bicarbonate

Sodium chloride

Sodium dihydrogen phosphate

Sodium dodecyl sulphate

Trans-2-nonenal (TNE)

Trans- 4-phenyl-3-buten-2-one

Trizma base (Tris[hydroxymethyl]aminomethane)

Trypsin Proteomic Grade

a-cyano-4-hydroxycinnamic acid (CHCA)

Shell New Zealand Ltd., Wellington, NZ

Ondina oil 15

Vivascience AG Hannover, Germany

Vivaspin 6 and $30 \mathrm{ml}$ concentrators (MWCO: 5K)

\subsection{Instrumentation}

Auto vortex mixer - Chiltern

Centrifuge - Beckmann XL 80 and SORVAL ${ }^{\circledR}$ RC 5B

Centrivap concentrator - Labconco

Easypure UV (Distillation) - Barnstead

Electrophoresis power supply model, 1000/500 Constant voltage - Bio-Rad

Electrophoresis Unit, Mini-PROTEAN ${ }^{\circledR}$ II - Bio-Rad

Fast Peptide Liquid Chromatography (ÄKTA FPLC) - Amersham Bioscience FLA-5100 scanner - FujiFilm

High Performance Liquid Chromatography (HPLC) - HP Agilent 1100 
Homogeniser - Polytron Kinematica GmbH, Kriens-Luzern, Switzerland

Hot plate Magnetic Stirrer - Contherm

IEF power supply, EPS 35000 XL - Pharmacia Biotech

Isoelectric Focusing (IEF) cell, Multiphore II - Pharmacia Biotech

Laminar Air Flow workstation - AES Environmental Private Limited

Liquid Chromatography (LC-MS/MS), Dionex UltiMate ${ }^{\mathrm{TM}} 3000$ LC system- LC Packings, Netherlands

Magnetic Stirrer - Chiltern

MALDI-TOF work station, Voyager-DE ${ }^{\mathrm{TM}}$ PRO Biospectrometry ${ }^{\mathrm{TM}}$ work station Applied Biosystems

Peristaltic pump - GE Healthcare

Personal Densitometer SCSI interfaceable Scanner - Molecular Dynamics SI ${ }^{\mathrm{TM}}$

Rotavapor-R - BÜCHI B-169 Vacuum system

Thermo Finnigan LTQ mass spectrometer- Thermo Electron Corporation, USA

UV-Visible Spectrophotometers, Cary 300 Bio, Cary 1E- Varian,

VERSAmax Microplate Reader, SOFTmax Pro - Molecular Devices

Water Bath - Julabo EM

Weighing Balance - Mettler AE240

\subsection{Software}

ClustalW2 alignment software (http://www.ebi.ac.uk/Tools/clustalw2/index.html)

Data Explorer 4.0.0.0 - Applied Biosystems

Image Reader FLA 5000 series V1.0 - FujiFilm

ImageQuaNT V2003.02 - Molecular Dynamics

Jalview multiple alignment editor (http://www.jalview.org/)

Voyager Instrument Control Panel V5.10.2 - Applied Biosystems

PEAKS V5.1 - Bioinformatics Solutions Inc. 


\section{General Methods}

\subsection{Introduction to Methods: basic principles underlying experimental methods}

\subsubsection{Chromatographic techniques}

\subsubsection{Affinity chromatography}

Proteins exhibit their biological functions based on the specificity and strong adsorptive properties they have towards other substances. Affinity chromatography makes use of this property. The protein to be purified is specifically and reversibly bound onto the stationary phase. The stationary phase consists of a ligand (which shows specificity towards the protein) bound to a matrix (supporting phase) with the help of spacer arms. Once the protein-ligand binding is established, the bound target protein is recovered by eluting the column with salt or with a chemical having sufficient affinity for the protein to displace the ligand or by changing the $\mathrm{pH}$ of the solution.

\subsubsection{2 lon exchange chromatography}

This chromatographic method separates the charged protein molecule depending on the anionic or cationic state of the packing material. The protein of interest is eluted from the column by a change in ionic strength or $\mathrm{pH}$.

\subsubsection{Chromatofocusing}

Chromatofocusing (CF) is a column chromatographic method for separating proteins according to their isoelectric points (Radola et al., 1977; Sluyterman and Wijdenes, 1978). CF involves the elution of ion exchangers solely by the change of pH. Specific buffers like Polybuffer 74 and Polybuffer 96 and media substituted with 
charged, buffering amines such as PBE 118 or PBE 94 are needed to form a linear $\mathrm{pH}$ gradient. In anionic $\mathrm{CF}$, proteins are bound to an anion exchanger at high $\mathrm{pH}$. As the $\mathrm{pH}$ on the column descends due to a $\mathrm{pH}$ gradient, protein's negative charge becomes less (less column-attractive). The opposite situation prevails in cationic CF. In either case, $\mathrm{pH}$ conditions in the column eventually reach a point where a given protein's net interaction with the column becomes zero, and it elutes (Gagnon, 1999).

\subsubsection{Proteomics}

The term "Proteome" was coined by Mark Wilkins in 1994 as a part of his $\mathrm{PhD}$ thesis and he defined it as the entire complement of proteins expressed by a genome, cell, tissue or organism (Wilkins et al., 1996). More specifically, it is the set of proteins expressed at a given time under a particular set of conditions (Wasinger et $a l .$, 1995). The study of the proteome is called "proteomics" and the goal of proteomics is to analyze the structure and function of biological systems. Since all functions involve the actions of proteins in a cell, proteomics has become a very important tool in understanding biological processes. Proteomics uses a combination of techniques to resolve (e.g., two-dimensional polyacrylamide gel electrophoresis (2D PAGE)), quantitate (e.g., scanning) and identify (e.g., mass spectrometry linked to database searching) proteins produced by an organism under defined circumstances (Lee, 2001; Patterson, 1995; Patterson, 2000; Pennington et al., 1997)

There are five steps to identify proteins in gel-based proteomics: sample preparation, separation, digestion, mass spectrometry and informatics. The sample preparation involves the extraction of the proteins from cells. The second step, separation, has usually been carried out by $2 \mathrm{D}$ gel electrophoresis. In the first dimension the proteins are separated according to their charge by isoelectric focusing 
and in the second dimension proteins are separated by molecular weight using SDSPAGE. Then the separated proteins on the gel are commonly visualized by Coomassie Blue staining; there are a number of staining protocols available to suit the need of the experiment. The digestion of proteins into peptides is usual in the next step since peptides are easier to identify than proteins and collectively they also contain more mass information than the intact proteins. In the fourth step, mass spectrometry is used to detect peptides and peptides fragments. Finally, the sequence of the protein is determined by interpreting all the data obtained by matching the peptide masses against those predicted for known proteins in a variety of databases or through sequence comparison if tandem mass spectrophotometric methods have been applied.

\subsubsection{2D electrophoresis}

The 2D electrophoresis involves the separation of proteins by two means. First is isoelectric focusing (IEF) in which proteins are separated according to their isoelectric points. An immobilised $\mathrm{pH}$ gradient (IPG / Immobiline ${ }^{\mathrm{TM}}$ Dry strip) is used as the first dimension and an electric potential is applied across the gel to make one end more positive than the other. Proteins will be charged at every $\mathrm{pH}$ except their pI. If they are positively charged, they will migrate towards the more negative end of the gel and if they are negatively charged they will move to the more positive end of the gel. Hence proteins applied in the first dimension will move along the gel and will finally settle systematically at their pI with a neutral charge. The second dimension is SDS-PAGE, which further separates proteins based on size. 


\subsubsection{SDS-PAGE}

Gel electrophoresis is the technique used for the separation of DNA, RNA and protein molecules (Berg et al., 2002). Sodium dodecyl sulphate- polyacrylamide gel electrophoresis (SDS-PAGE) is used to separate proteins according to their electrophoretic mobility. In this technique, SDS is added to the proteins to denature the secondary and tertiary structure of the proteins and to apply a negative charge to each protein in proportion to its mass (Laemmli, 1970; Weber and Osborn, 1969). The protein sample is also usually heated with a reducing agent (DTT or mercaptoethanol, generally added into the sample buffer) before loading on to the gel to ensure the reduction of disulfide bonds and so facilitate unfolding of the tertiary structure of the protein. Smaller proteins will migrate far down the gel, while larger ones will remain closer to the point of origin. For the visualisation of the separated proteins, the gel is usually stained with Coomassie Blue though other stains may be used.

\subsubsection{Matrix Assisted Laser Desorption/lonisation -Time Of Flight (MALDI- TOF) analysis}

Proteomics research has led an increasing number of biological and medical researchers to the technique of protein identification by mass spectrometry. In MALDI-TOF, a co-precipitate of light-absorbing matrix and bio-molecule is irradiated by a short laser pulse and the generated ions are detected after traversing a fixed distance (Karas and Hillenkamp, 1988). The traditional low resolution and mass measurement accuracy of TOF analyzers has largely been overcome by 'delayed extraction' (DE), in which the ions are extracted after a predetermined time delay following the laser pulse (Brown and Lennon, 1995; Mann and Talbo, 1996). 
MS experiments for protein identification are mainly based on peptide analysis. The mass of intact proteins can also be measured by the mass spectrometry. Cohen and Chait (1997) reported a methodology for the mass spectrometry of whole proteins eluted from SDS-PAGE gels and they successfully eluted picomole amounts of proteins from gels for MS analysis and performed identification. Thus knowledge of the accurate molecular mass of the intact protein along with information from digestions and peptide mapping can lead to more definitive results. This approach was used in the characterization of endogenous human leptin (Cohen et al., 1996). However, the sensitivity of the mass spectrometer for proteins is much lower than for peptides; moreover, the mass of whole proteins often cannot be measured accurately enough for identification purposes; finally, proteins are difficult to handle and most solubilising conditions are not compatible with MS (Salzano and Crescenzi, 2005).

The peptides are obtained by digesting the protein with different proteases. The cleavage specificities of some proteases are shown in Table 3-1. Trypsin, the most commonly used protease, is very stable and efficient and specifically cleaves at the C-terminal side of lysine and arginine residues. A problem with the use of proteases can be that they are capable of self-digestion. Nowadays, modified trypsin is available commercially where selective methylation of the lysines limits the autolytic activity to the arginine cutting sites (Rice et al., 1977). There are a number of other proteases like endoprotease Lys-C (Jekel et al., 1983; Patterson, 1995), GluC (Houmard and Drapeau, 1972; Scheler et al., 1998) and Asp-N (Wang et al., 2005) have been used for the in-gel digestion. These proteases cut specifically at only one amino acid e.g. Asp-N cuts at the $\mathrm{N}$-terminal side of aspartic acid residues (Michalski and Shiell, 1999) and hence a small number of longer peptides is obtained. The peptides obtained after the digestion have to be extracted from the gel 
matrix and incorporated with MALDI matrix for further analysis by MALDI-TOF to obtain the MALDI spectra. The next step is Peptide Mass Fingerprinting, which involves identification of proteins after the peptide mixture has been analyzed by MS. Proteins are identified by matching a list of experimental peptide masses, measured with high accuracy, with the theoretically calculated peptide masses obtained from an in silico digestion of all proteins present in a given database, taking into account the specificity of the protease employed (Henzel et al., 1993; Mann et al., 1993; Pappin et al., 1993).

Mass Spectrometry is also a general method for post-translational modification analysis, because PTMs lead to a mass increase or decrease with respect to the molecular weight expected on the basis of amino acid sequence.

Table 3-1: Proteases and their cleavage specificities

\begin{tabular}{ll}
\hline Enzyme & Cleavage specificity \\
\hline Trypsin & $\begin{array}{l}\text { /K-X... C terminal and /R-X...C terminal, where X is } \\
\text { any amino acid except proline (Walsh, 1970) }\end{array}$ \\
Endoprotease Glu C & $\begin{array}{l}\text { /E-X and /D*-X, where X is any amino acid except } \\
\text { proline (Sorensen et al., 1991) }\end{array}$ \\
Endoprotease Asp N & $\begin{array}{l}\mathrm{X}-/ \mathrm{D}, \text { where X is any amino acid (Drapeau, 1980) } \\
\text { Endoprotease Lys C }\end{array}$ \\
& /K-X, where X is any amino acid except proline (Jeno et \\
&
\end{tabular}

* Cleavage after aspartate and glutamate in sodium phosphate buffer, $\mathrm{pH} 7.8$; otherwise cleavage only after glutamate 


\subsubsection{Reverse-phase high performance liquid chromatography}

HPLC has gained importance as an analytical tool in peptide chemistry because of its exquisite speed and resolving power (Hearn et al., 1988). In reversephase HPLC, separation of substances that are sparingly soluble or insoluble in water is carried out by nonpolar bonded phases with hydro-organic eluants. Reverse-phase chromatography is so called because the polarity of the eluant is greater than that of the stationary phase. The term "reverse-phase chromatography' was originally suggested by Howard and Martin (Howard and Martin, 1950). The reverse-phase HPLC method predominates over the other HPLC methods like ion-exchange chromatography and size-exclusion chromatography and has become popular due to its relative simplicity and reproducibility (Snyder et al., 1974). In reverse-phase HPLC, the stationary phase is commonly a finely divided organo-silica with octadecyl moieties covalently bound to the solid surface (C18 columns), and aqueous acetonitrile is used as the mobile phase. This technology has been widely used for the study of metabolism of drugs as many of the compounds which are made as pharmaceuticals are of a relatively low relative molecular mass and can be successfully chromatographed (Marten and Ruane, 1980).

Since many pesticides are thermally labile and might not survive the GC process, HPLC is the best alternative. A reverse-phase ion-pair HPLC method was used to separate and quantify not only methyl parathion but also six of its hepatic biotransformation products: methyl paraoxon; desmethyl parathion; desmethyl paraoxon; $p$-nitrophenol; $p$-nitrophenyl glucuronide and $p$-nitrophenyl sulphate (Anderson et al., 1992). 


\subsubsection{Phosphopeptide enrichment}

There are a number of phosphopeptide enrichment methods which have been reported for use when a limiting amount of protein is available. These include the use of phosphospecific antibodies (Pandey et al., 2000), phosphospecific chromatographic methods (Cao and Stults, 2000; Posewitz and Tempst, 1999; Stensballe et al., 2001) and chemical modification methods (Jaffe et al., 1998; Oda et al., 2001). The present investigation proceeded with the use of chromatographic phosphopeptide enrichment before MALDI-TOF analysis. The use of immobilized metal ion affinity chromatography (IMAC) utilises the high affinity of phosphate groups towards a metal-chelated stationary phase, especially $\mathrm{Cu}^{2+}, \mathrm{Fe}^{3+}$, and $\mathrm{Ga}^{3+}$. Under acidic conditions, $(\mathrm{pH} 2.5$ to 5.5) the phosphate and metal ion form a complex which is broken at alkaline $\mathrm{pH}$.

\subsubsection{PEAKS: a tandem mass spectrometry software package}

The software package PEAKS (Bioinformatics Solutions Inc., Canada) has many applications to determine protein and peptide sequences and respective quantitative properties. It can perform auto de novo sequencing (Ma et al., 2003), protein identification using an internal database search engine (Rogers et al., 2004), post-translational modification using PTM finder and protein quantification (Xin et al., 2009). Using this software, tandem mass spectra are searched against the internal database and $\mathrm{PEAKS}^{\mathrm{TM}}$ de novo sequencing results are used to automatically discover variable PTMs. To identify modifications, PEAKS provides a library of approximately 30 common PTMs and it is also possible to create custom PTMs in the system. It takes into account the specified modification of interest while performing the identification of proteins from tandem MS spectra. 


\subsection{Methodology}

\subsubsection{Homogenate preparation}

Frozen insects were homogenized with a Polytron ${ }^{\mathrm{TM}}$ Kinematic sonicator (GmbH, Kriens-Luzern, Switzerland) in ice cold 0.05 M sodium phosphate buffer, $\mathrm{pH} 7.4$ containing $0.1 \mathrm{mM}$ phenyl thiourea, $0.1 \mathrm{mM}$ dithiothreitol, $0.1 \mathrm{mM} \alpha$-toluene sulfonyl fluoride and $1 \mathrm{mM}$ EDTA. A protease inhibitor cocktail (Sigma) containing lyophilized powder was dissolved in $100 \mathrm{ml}$ of deionised water as per the manufacturer's guideline and aliquots of $1 \mathrm{ml}$ were prepared and stored at $-20^{\circ} \mathrm{C}$. This protease inhibitor cocktail $(1 \mathrm{ml} / 0.5 \mathrm{~g}$ of flies $)$ and cysteine $(2 \mathrm{mg} / 0.5 \mathrm{~g}$ of flies) were added to avoid protein degradation and oxidative darkening of the homogenate respectively. After homogenisation, the extract was centrifuged at $4^{\circ} \mathrm{C}$ for 15 minutes at $10,000 \times \mathrm{x}$ and the supernatant was filtered through glass wool. The filtered supernatant was re-centrifuged at $4^{\circ} \mathrm{C}$ at $100,000 \times \mathrm{g}$ for $1 \mathrm{hr}$. The clear supernatant was designated the crude enzyme and used for further purification by affinity chromatography.

\subsubsection{Affinity chromatography}

The present study has involved the use of glutathione as well as $S$-2, 4dinitrophenylglutathione (DNP-GSH) and S-2-chloro-4-nitrophenylglutathione (CNP-GSH) matrices synthesised in laboratory as affinity chromatography media for the partial separation of GSTs from the different classes. The sequential use of affinity matrices permits the isolation of more different classes of GST than is possible with one matrix. 


\subsubsection{Preparation of affinity matrices}

The preparation of affinity matrices involved the linking of glutathione or a glutathione conjugate to Sepharose 6B activated with epichlorhydrin.

\subsection{GSH matrix}

Sepharose 6B linked glutathione matrix was prepared according to the method described by Clark et al. (1990). Briefly, Sepharose 6B was washed with several volumes of $0.9 \%$ sodium chloride $(\mathrm{w} / \mathrm{v})$ in a sintered glass funnel. Excess liquid was removed and caked gel was weighed. The gel was re-suspended in 1.6 volumes (compared to gel volume) of $1 \mathrm{M} \mathrm{NaOH}$ with 0.2 volume (compared to gel volume) of epichlorhydrin. The flask was stoppered and the mixture was brought to $60^{\circ} \mathrm{C}$ in a water bath with gentle rotating of the flask for 2 hours. The activated gel was removed from the mixture by filtration. The gel was washed with $0.05 \mathrm{M}$ sodium phosphate buffer $\mathrm{pH} 7.4$ (5 volumes). This washed and activated gel (1 volume) was mixed with glutathione $(10 \mathrm{~g}$ per $100 \mathrm{ml}$ gel $)$ in $0.05 \mathrm{M}$ sodium phosphate buffer, $\mathrm{pH} 7.0$ (2 volumes). Nitrogen gas was bubbled through the mixture for $20 \mathrm{~min}$. The mixture was then incubated at $37^{\circ} \mathrm{C}$ for 12 hours with gentle shaking. After that, the unconjugated glutathione was filtered off, the gel was washed with five volumes of water and the unreacted epoxide groups were blocked by incubating the gel with $1 \mathrm{M}$ ethanolamine/ $\mathrm{HCl}$ buffer $\mathrm{pH} 9$ ( 2 volumes) for 12 hours at $4^{\circ} \mathrm{C}$. The blocking solution was removed by filtration and the gel was washed five times alternately with $0.1 \mathrm{M}$ sodium acetate containing $0.5 \mathrm{M} \mathrm{NaCl}$ and $0.1 \mathrm{M}$ sodium borate containing $0.5 \mathrm{M} \mathrm{NaCl}$. Finally this gel was equilibrated with the appropriate buffer (usually $0.05 \mathrm{M}$ phosphate buffer, $\mathrm{pH} 7.4$ ) and stored with $20 \%$ ethanol in 0.05 M phosphate buffer, $\mathrm{pH}$ 7.4. 
Determination of matrix substitution:

The GSH in this matrix was linked via its thiol group. The extent of substitution was therefore estimated by measuring the concentration of free amino groups bound to the gel. This was performed by reacting these groups with the chromogenic TNBS (2,4,6-trinitrobenzenesulfonic acid) (Sigma), according to the method of Cuatrecasas (Cuatrecasas.P and Parikh, 1972). One ml of packed GSHsubstituted gel was mixed with $1 \mathrm{ml}$ of saturated disodium tetraborate solution and $100 \mu 1$ of $3 \%(w / v)$ TNBS. This mixture was shaken well and incubated at $40{ }^{\circ} \mathrm{C}$ for 30 minutes. After incubation, excess reagent was removed by filtration and the gel was washed with $0.9 \% \mathrm{NaCl}$. Then $2 \mathrm{ml}$ of concentrated $\mathrm{HCl}$ was added and the mixture was boiled until the gel dissolved ( $<30$ seconds). The solution was rapidly diluted with water and adjusted to $\mathrm{pH} 9.6$ with $\mathrm{NaOH}$. The final volume was made up to $50 \mathrm{ml}$ and the extinction was measured at $400 \mathrm{~nm}$. The assay procedure was standardized using $S$-hexyl-GSH as a suitable $S$-blocked glutathione derivative (TNBS reacts strongly with thiol groups). Controls were carried out using unsubstituted epichlorhydrin-activated Sepharose.

\subsection{DNP-GSH and CNP-GSH matrix}

The solutions $(200 \mathrm{ml})$ of $100 \mathrm{mM} \mathrm{CDNB}$ and $100 \mathrm{mM}$ reduced glutathione were prepared in ethanol and distilled water respectively. The CDNB solution (200 ml) was added to GSH solution dropwise over 5-10 minutes and the solution was continually adjusted to $\mathrm{pH} 9.6$ with $1 \mathrm{M} \mathrm{NaOH}$ solution. The mixture was allowed to react for 24 to 36 hours at room temperature. After incubation, ethanol was evaporated from the mixture by use of a rotary evaporator. The conjugate DNP-GSH was repeatedly precipitated by decreasing the $\mathrm{pH}$ from 9.6 to $\mathrm{pH}$ 3. Thin Layer Chromatography (TLC) was performed to detect the purity of the 
DNP-GSH conjugate formed. TLC was carried out in butanol: acetic acid: water (4:1:5), where the upper phase was used to run the TLC plate spotted with the conjugate. The conjugate was identified by spraying ninhydrin-collidine solution (50 $\mathrm{ml} 0.1 \%$ ninhydrin in ethanol $+2 \mathrm{ml}$ collidine $+15 \mathrm{ml}$ glacial acetic acid). The precipitation procedure was repeated until a single conjugate spot was obtained without any GSH contamination. This conjugate was kept in a vacuum desiccator. It was used to react with epichlorhydrin activated Sepharose 6B by the same procedure used to link glutathione with Sepharose 6B but at pH 9 (Clark et al., 1990). The same procedure was followed for the synthesis of CNP-GSH conjugate. A longer reaction time was required for CNP-GSH conjugate formation (usually 2-3 days) as DCNB reacts slowly with glutathione.

A<smiles>O=C([O-])CNC(=O)C(CC(=O)NCC(O)COC1CCCC1)C(=O)[O-]</smiles>

B

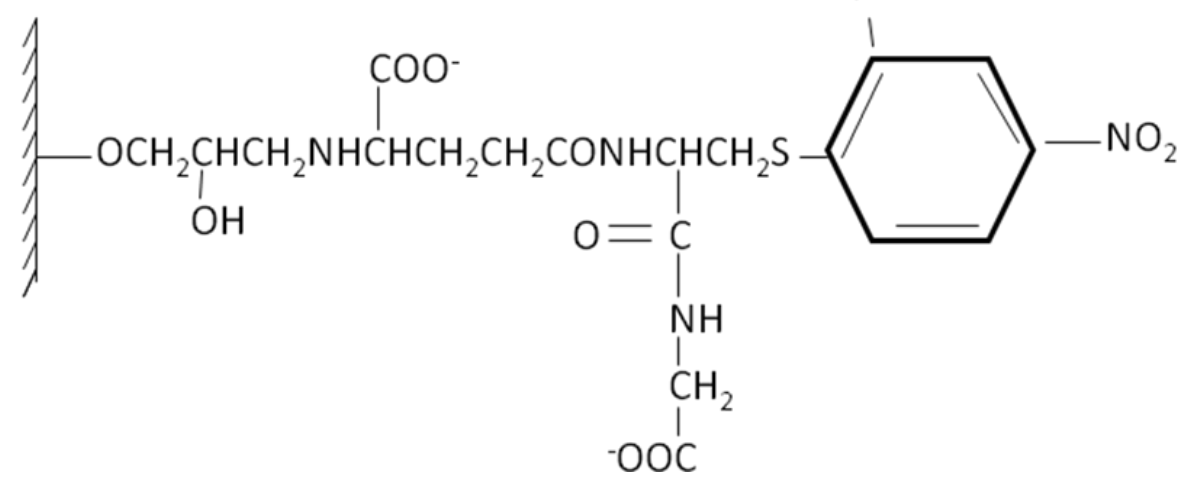

Figure 3-1: Proposed structure of A) DNP-GSH conjugate and B) CNP-GSH conjugate covalently linked to epichlorhydrin activated Sepharose 6B. 
Determination of matrix substitution:

The extent of substitution of DNP-GSH and CNP-GSH with Sepharose 6B was measured spectrophotometrically. The extinction coefficients of DNP-GSH and CNP-GSH conjugates were taken to be 10,400 and 9,300 at 340 and $344 \mathrm{~nm}$ respectively (Habig et al., 1974). Newly made DNP-GSH or CNP-GSH substituted matrix (1 ml) was mixed with $2 \mathrm{ml}$ of concentrated $\mathrm{HCl}$ and boiled until the gel dissolved ( $<30$ seconds). The solution was rapidly diluted with water and adjusted to $\mathrm{pH} 9.6$ with $\mathrm{NaOH}$. The final volume was made up to $50 \mathrm{ml}$ and the extinction was measured at the relevant wavelength. Activated Sepharose 6B was used as a control.

\subsubsection{Chromatographic techniques using FPLC}

FPLC Tricorn ${ }^{\mathrm{TM}} 5 / 50$ columns were packed with the glutathione, DNP-GSH or CNP-GSH conjugated matrices and used for the affinity chromatography using the Amersham ÄKTA FPLC system. Alternatively for preliminary experiments, the prepared matrix $(5 \mathrm{ml})$ was packed in a $10 \mathrm{ml}$ disposable syringe fitted with a plastic sinter. Buffers and sample were pumped by a peristaltic pump. All purification experiments were performed in a cold room at $4^{\circ} \mathrm{C}$.

\subsection{Desalting / de-pigmenting method protocol}

A Pharmacia HiTrap desalting column $(1.6 \times 2.5 \mathrm{~cm}, 5 \mathrm{ml})$ was used to remove "pigments" from the large volume $(10-30 \mathrm{ml})$ of crude homogenate. In some experiments, a column $(5 \times 26 \mathrm{~cm}, 500 \mathrm{ml})$ was packed with Sephadex ${ }^{\circledR} \mathrm{G}-75$ for the complete removal of low M.W. proteins and pigments from the sample. It was observed during routine experiments that use of a HiTrap desalting column gave a similar percentage recovery of GST activity to the use of the larger Sephadex ${ }^{\circledR}$ G-75. Since the former procedure was much quicker, in most of the experiments the crude 
extract was de-pigmented rather than subjected to a complete desalting. The column was equilibrated with several volumes of the $0.05 \mathrm{M}$ sodium phosphate buffer $\mathrm{pH}$ 7.4. The crude homogenate was injected using a super loop (10 $\mathrm{ml}$ or $50 \mathrm{ml})$ and the flow rate was fixed at $1.0 \mathrm{ml} / \mathrm{min}$. The FPLC was programmed to wash out the sample with phosphate buffer. Fractions of $3 \mathrm{ml}$ were collected. The GST activity of all the fractions was checked using CDNB as substrate according to the method of (Habig and Jakoby, 1981). The catalytically active fractions were pooled and subjected to further purification.

\subsection{Affinity chromatography protocol}

Active enzyme fractions obtained from the desalting or de-pigmenting column were loaded onto a Tricorn ${ }^{\mathrm{TM}} 5 / 50$ column, which was packed with affinity matrix. In some of the experiments, a commercially available $5 \mathrm{ml}$ column of immobilized glutathione with a $\mathrm{C}_{12}$ arm S-linked to GSH (GSTrap $^{\mathrm{TM}} \mathrm{FF}$, GE Healthcare-Amersham Bioscience) was used. The substitution of GSH in GSTrap column is $7-15 \mu \mathrm{mol} / \mathrm{ml}$ according to GE Healthcare. DNP-GSH and CNP-GSH substituted matrices $(9.55 \mu \mathrm{mol} / \mathrm{ml}$ and $9.65 \mu \mathrm{mol} / \mathrm{ml}$ respectively) made following method 3.2.1.1.2 were also employed for subsequent isolation of GSTs. All the columns used in FPLC had previously been equilibrated with $0.05 \mathrm{M}$ sodium phosphate buffer, $\mathrm{pH}$ 7.4. After application of the sample, the unbound material was washed out with 5 column volumes of the above phosphate buffer. A two-phase gradient of 0-20 mM GSH buffer, pH 9.6 (first phase $20 \mathrm{ml}$ of 0-100\% $20 \mathrm{mM} \mathrm{GSH}$ linear gradient for $20 \mathrm{~min}$ and second phase $20 \mathrm{ml}$ of $100 \% 20 \mathrm{mM} \mathrm{GSH}$ for $20 \mathrm{~min}$ ) was formed to elute the bound proteins. The flow rate was $1.0 \mathrm{ml} / \mathrm{min}$. Fractions of 3 $\mathrm{ml}$ were collected for unbound void fractions and $2 \mathrm{ml}$ for the GSH gradient. UV absorbance, conductivity and $\mathrm{pH}$ were recorded. Initially all the fractions were 
analysed for enzyme activity with CDNB. The enzymatically active fractions were pooled and the activities with DCNB, p-nitrophenyl acetate (NPA), trans-2-nonenal (TNE), and dehydroascorbic acid (DHA) were routinely measured. The activity with ethacrynic acid (EA) and trans-4-phenyl-3-butene-2-one (PBO) was also measured in some experiments.

\subsubsection{Chromatofocusing}

FPLC chromatofocusing involved the use of a Pharmacia Mono P column 5/50 GL. The chromatofocusing was performed in $\mathrm{pH}$ ranges 7-4 using $0.025 \mathrm{M}$ imidazole- $\mathrm{HCl}$ buffer $\mathrm{pH} 7.4$ as a start buffer and the polybuffer 7-4, $\mathrm{pH} 4.0$ as an elution buffer. Equilibration of the sample against the start buffer was carried out either by dialysis or by using a Sephadex G-25 column. The $\mathrm{pH}$ and conductivity of the sample were then checked to ensure they matched those of the start buffer. The sample was then injected into the chromatofocusing column pre-equilibrated with the start buffer. The flow rate was $0.5 \mathrm{ml} / \mathrm{min}$. The fraction size was $3 \mathrm{ml}$ for unbound fractions and $2 \mathrm{ml}$ for the $\mathrm{pH}$ gradient elution. All fractions were analysed for enzyme activity with CDNB according to the method of (Habig and Jakoby, 1981) and active fractions were pooled for further analysis.

\subsubsection{Enzyme assays}

The glutathione S-transferase activity with different model substrates was measured spectrophotometrically on a Varian Cary IE or Cary 300 Bio Spectrophotometer with the kinetic software "Cary Win UV". The enzyme assay conditions used are summarised in Table 3-2. 
Table 3-2: GST enzyme assay conditions

\begin{tabular}{|c|c|c|c|c|c|c|}
\hline \multirow[t]{2}{*}{ Substrates } & \multicolumn{2}{|c|}{ Concentration } & \multirow[t]{2}{*}{ Buffer } & \multirow{2}{*}{$\begin{array}{l}\text { Molar extinction } \\
\text { coefficient } \\
\left(\mathrm{M}^{-1} \mathbf{c m}^{-1}\right)\end{array}$} & \multirow{2}{*}{$\begin{array}{c}\text { Absorbance } \\
\text { at wavelength } \\
\qquad(\mathbf{n m})\end{array}$} & \multirow[t]{2}{*}{ Reference } \\
\hline & $\begin{array}{c}\text { Substrate } \\
(\mathbf{m M})\end{array}$ & $\begin{array}{l}\text { GSH } \\
(\mathbf{m M})\end{array}$ & & & & \\
\hline 3,4-Dichloronitrobenzene & 0.4 & 4 & $\mathrm{~B}$ & 8400 & 344 & Motoyama, 1977 \\
\hline 1, 4-Dinitrophenyl acetate & 0.3 & 0.5 & $\mathrm{C}$ & 8790 & 400 & Habig et al., 1974 \\
\hline trans-2 Nonenal & 0.025 & 1 & $\mathrm{~A}$ & -19200 & 225 & Brophy and Barrett, 1989 \\
\hline Dehydro ascorbic acid & 1 & 1 & $\mathrm{~A}$ & 1400 & 265 & Kim et al., 2006 \\
\hline
\end{tabular}

Buffer A- 0.1 M Phosphate, pH 6.5; Buffer B- 0.1 M Tris, pH 9.0; Buffer C- 0.1 M Phosphate pH 7.0 


\subsubsection{Protein measurement}

\subsubsection{Protein concentration}

Enzymatically active pooled fractions were concentrated using Vivaspin $6 \mathrm{ml}$ or $20 \mathrm{ml}$ concentrators ( $<10,000$ Da cut off) (Vivascience AG Hannover, Germany) by centrifuging at $3000 \times \mathrm{x}$ for 30-60 minutes depending on the degree of concentration required (usually 5-6-fold).

\subsubsection{Protein estimation}

\subsection{Bradford assay}

Protein estimation was carried out by the Bradford assay (Bradford, 1976; Spector, 1978). The commercially available Bradford dye reagent was used in 1:5 dilution (dye reagent: deionised water). Bovine Serum Albumin (BSA) was used as the standard. Aliquots of the BSA stock $(1 \mathrm{mg} / \mathrm{ml})$ were pipetted $(20,40,60$ and 80 $\mu \mathrm{l}$ corresponded to $20,40,60,80 \mu \mathrm{g}$ of BSA) and buffer was added to make up 100 $\mu 1$ in each tube. The reagent blank was prepared by addition of appropriate buffer. Unknown samples were prepared with dilution (2-5-fold) or without dilution as required. To each tube including standards and samples, $5 \mathrm{ml}$ of Bradford dye reagent was added, followed by vortexing. After a 5 minute incubation at room temperature, the absorbance was read at $595 \mathrm{~nm}$ in a Cary 1E or Cary $300 \mathrm{UV}$ Visible spectrophotometer. The standard curve was plotted by linear regression and the protein content of the unknown samples was calculated from the linear regression equation.

\subsection{Microtiter plate assay}

BSA stock $(10 \mathrm{mg} / \mathrm{ml})$ was prepared and subsequently diluted to a concentration of $1 \mathrm{mg} / \mathrm{ml}$. Out of this stock, dilutions of $0.05,0.1,0.2,0.3,0.4$ and 
$0.5 \mathrm{mg} / \mathrm{ml}$ working standards were prepared. Of each of these working standards 10 $\mu 1$ were pipetted into assigned wells, corresponding to $0.5,1,2,3,4$ and $5 \mu \mathrm{g}$ of BSA per well in a 96-well plate using a multi channel pipette. A reagent blank used $10 \mu 1$ of the buffer. Unknown samples $(10 \mu \mathrm{l})$ with or without dilution were pipetted into the wells. Bradford reagent $(200 \mu \mathrm{l})$ was added in each well. Each sample and blank was prepared in triplicate. The absorbance was read at $595 \mathrm{~nm}$ after 5 minutes using a Versamax Microplate Reader (Molecular Devices, USA).

\subsection{D Quant kit (Ettan ${ }^{\top \mathrm{M}}$ sample preparation kit and reagents)}

BSA standard (2 $\mathrm{mg} / \mathrm{ml})$, colour reagent $\mathrm{A}$, reagent $\mathrm{B}$, copper reagent, precipitant and co-precipitant were supplied in this 2D Quant kit (Amersham Bioscience, USA). The protein assay was performed according to the manufacturer's instructions. In brief, different concentrations of BSA standard were used to plot a standard curve $(5,10,15,20,25 \mu$ l corresponding to $10,20,30,40,50 \mu \mathrm{g}$ of BSA). These and the unknown samples $(10 \mu \mathrm{l})$ were pipetted into Eppendorf tubes. To all of these tubes including standards and samples, $500 \mu 1$ of precipitant was added. The solutions were mixed and incubated for 2-3 min at room temperature. Co-precipitant $(500 \mu \mathrm{l})$ was then added and mixed by vortexing. Tubes were centrifuged at 10,000 $\mathrm{x}$ $g$ for $5 \mathrm{~min}$. The supernatant was discarded. The copper reagent $(100 \mu \mathrm{l})$ and deionised water $(400 \mu \mathrm{l})$ were added to the pellet. Then $1 \mathrm{ml}$ working colour reagent [1:100 (colour reagent B: colour reagent A)] was added in each tube and incubated at room temperature for 15-20 min. The absorbance was measured at $480 \mathrm{~nm}$ by Varian Cary IE or Cary 300 Bio Spectrophotometer. The colour intensity varies inversely with protein concentration. The protein content of unknown samples was measured from the standard curve regression equation. This method was used when the protein 
content of the sample was very small and could not be determined by the Bradford assay.

\subsubsection{Protein precipitation of samples for electrophoresis}

To $1 \mathrm{ml}$ of protein sample, $0.1 \mathrm{ml}$ of $0.15 \%(\mathrm{w} / \mathrm{v})$ deoxycholic acid was added in an Eppendorf tube. The mixture was vortexed and was allowed to stand at room temperature for $10 \mathrm{~min}$. Then $0.1 \mathrm{ml}$ of $72 \%(\mathrm{w} / \mathrm{v})$ trichloroacetic acid (TCA) was added. The mixture was vortexed and centrifuged at $10,000 \times \mathrm{x}$ for $15 \mathrm{~min}$. The supernatant was carefully discarded and $1 \mathrm{ml}$ of acetone was added to the pellet. The tube was incubated at $4^{\circ} \mathrm{C}$ for $10 \mathrm{~min}$. It was again centrifuged at $10,000 \times \mathrm{g}$ for 10 $\min$ at $4^{\circ} \mathrm{C}$. The acetone was poured off and pellet was allowed to air-dry. Protein precipitation was performed to prepare samples for electrophoresis.

\subsubsection{Proteomics}

\subsubsection{SDS-PAGE}

One dimensional SDS-PAGE was performed using Mini-PROTEAN $^{(\mathrm{R})}$ Electrophoresis units with a Bio-Rad model $1000 / 500$ power supply. The assembly and preparation of the electrophoresis apparatus was as described in the instruction manual. The plates were cleaned with acetone and Kimwipes before casting the gel. Mini gels were prepared using $1 \mathrm{~mm}$ spacers. The $12 \%$ separating gel and $4 \%$ stacking gel were prepared according to the manufacturer's manual (see Appendix 10.1- reagent preparation).

The gel was cast on the casting unit using $5 \mathrm{ml}$ of separating gel mixture (see Appendix 10.1-Reagent preparation). 1\% SDS was layered on top of the gel and polymerisation was allowed to proceed for 30-40 minutes. Once the separating gel was polymerised, the stacking gel mixture was poured on top of the separating gel 
and a Teflon comb was inserted for sample well formation. The gel was allowed to polymerise for $30-40 \mathrm{~min}$.

The running buffer (see Appendix 10.1- Reagent preparation) was diluted 10 $\mathrm{x}$ from the stock prior to use and poured in the electrophoretic chamber. In each well $15 \mu \mathrm{l}$ of the protein sample (5-15 ug/lane) was loaded using gel loading tips (for sample preparation, see Appendix 10.1 - Reagent Preparation). Five $\mu 1$ of standard molecular weight marker (Benchmark ${ }^{\mathrm{TM}}$ protein ladder) was loaded on to each gel. A voltage of $150-200 \mathrm{~V}$ was applied to run the gel until the boundary of Bromophenol Blue in the samples moved to the end of the gel. The gel was routinely stained with colloidal Coomassie Blue G-250 stain (see Appendix 10.1- Reagent preparation) overnight in a plastic container on a shaker. The stain was prepared freshly to avoid the loss of sensitivity. Destaining was carried out by washing the gel twice with water and then shaking the gel with $25 \%$ methanol in water for $10-15$ minutes.

\subsubsection{Two dimensional (2D) electrophoresis}

For one-dimensional isoelectric focusing, Immobiline ${ }^{\mathrm{TM}}$ Dry strips, $7 \mathrm{~cm}, \mathrm{pI}$ 3-10, linear and pI 4-7, linear were used in the Multiphor ${ }^{\mathrm{TM}} \mathrm{II}$ Electrophoresis Unit.

\section{Rehydration of the gel strip}

A rehydration solution was prepared containing $8 \mathrm{M}$ urea, 2\% (w/v) CHAPS, $15 \mathrm{mM}$ DTT, $30 \mathrm{mM}$ thiourea and 2\% ampholyte, pH 3-10. (See Appendix 10.1Reagent preparation). Protein was precipitated and mixed well with $100 \mu \mathrm{l}$ of rehydration buffer. Once the protein was dissolved completely, its concentration was estimated to determine the amount of protein to load on the strip. Protein load was adjusted according to the instruction manual for the strip, in the range of 50-100 $\mu \mathrm{g}$. 
This loading mixture was adjusted to $125 \mu \mathrm{l}$ with rehydration buffer and was applied carefully to the IPG strip (gel side down) placed in a $2 \mathrm{ml}$ plastic pipette, which was cut in half and sealed with Parafilm at one end. After sealing off the other end of the pipette, the strip was left overnight at room temperature for rehydration.

\section{First dimensional isoelectric focusing}

A Multiphor ${ }^{\mathrm{TM}}$ II Electrophoresis unit was used for isoelectric focusing. Shell Ondina oil was poured on the cooling plate and the temperature was set to $20^{\circ} \mathrm{C}$. The Immobiline ${ }^{\mathrm{TM}}$ Dry Strip tray was placed on the top of the cooling plate and oil was placed in the tray. The strip aligner was placed on the sample tray ensuring that no air bubbles were trapped. The strips were then placed in the grooves of the aligner tray with the gel side up using tweezers. The acidic end of the strip was positioned near the anode while the basic end was at the cathode. If more than one IPG strip was used in first dimension IEF, then care needed to be taken to line up the anodic gel edges. Then two IEF electrode strips $(11 \mathrm{~cm}$ each) were soaked with distilled water. Excess water was removed by blotting with tissue paper. These electrode strips were placed perpendicularly at each end of the aligned strips to ensure the contact between the gel and electrode strips. The electrodes were then placed on top of the electrode strips. The Shell Ondina Oil was again poured on to the tray to completely cover the strips. IEF was run using an EPS 3501 XL power supply. Runs were programmed in a gradient mode modified from the manufacturer's guidelines.

The three phases of the voltage programme were as below:

$\begin{array}{lll}\text { Phases } & \text { Voltage } & \text { Time } \\ \text { Phase I } & 200 \mathrm{~V} & 0.017 \mathrm{~h} \\ \text { Phase II } & 200-3500 \mathrm{~V} \text { (ascending) } & 1.30 \mathrm{~h} \\ \text { Phase III } & 3500 \mathrm{~V} \text { (steady) } & 1.30 \mathrm{~h}\end{array}$




\section{Equilibration of the focused IPG strip}

The focused IPG strips then needed to be equilibrated with SDS buffer for the second dimension in SDS-PAGE. The equilibration procedure consists of two steps. The first step was the reduction all of the cysteines present in the protein with DTT and the second step was treatment with iodoacetamide. For this, the stock equilibration solution was prepared with $0.5 \mathrm{M}$ Tris-HCl, $\mathrm{pH} 6.8$, urea, glycerol, SDS and deionised water (see Appendix 10.1- Reagent preparation) and divided into two tubes. In one tube $0.25 \%(\mathrm{w} / \mathrm{v})$ DTT (solution 1) and in another $4.5 \%(\mathrm{w} / \mathrm{v})$ iodoacetamide and traces of bromophenol blue (solution 2) were added. Each strip required about $2.5 \mathrm{ml}$ of each of the equilibration solutions for $15 \mathrm{~min}$.

\section{Second dimension (SDS-PAGE)}

The second dimension separation was performed using the MiniPROTEAN ${ }^{\mathrm{TM}}$ II Electrophoresis units. The equilibrated IPG strip was dipped in the SDS electrophoresis buffer to lubricate it and placed along the top of a pre-formed acrylamide gel. The PAGE was carried out in the same way as described before in 1D SDS PAGE, except a differently designed comb was placed in the stacking gel to form wells appropriate to accommodate the strip and molecular weight marker along side. The gel was run at a constant 150 Volts using Bio-Rad Model 1000/500 constant voltage power supply. The gel was stained overnight with colloidal Coomassie Blue G-250 and destained with $25 \%$ methanol in deionised water.

\subsubsection{Gel scanning}

The Coomassie Blue stained gels were scanned by a Molecular Dynamics Personal Densitometer $\mathrm{SI}^{\mathrm{TM}}$ with the Molecular Dynamics Scanner Control application version 4.0. Gels were then visualized and analyzed using the Molecular 
Dynamics ImageQuaNT application (version 5.2). The pixel size and digital resolution were fixed at 50 micron and 12 bit, respectively. Quantitative differences in terms of spot volume and area of GST spots during the development of the insect and in susceptible and resistant L. cuprina flies were analysed by ImageQuaNT software.

\subsubsection{Destaining of the gel spots and in-gel digestion}

The gels, containing a protein band (1D gel) or spot (2D gel) of interest to be analysed, were initially washed twice with deionised water. Gels were placed on a glass surface, cleaned with acetone, and protein spots were individually excised with a sterile scalpel, cut into small pieces and placed in Eppendorf tubes. The gel pieces were destained using $100 \mathrm{mM}$ ammonium bicarbonate: acetonitrile (50:50) with shaking. Destaining was carried out for as long as required for the gel pieces to be completely colourless. The destain solution was changed every 45 minutes. When fully destained, the gel fragments were dehydrated with two washes of $100 \mu$ of $100 \%$ acetonitrile $(\mathrm{ACN})$ whereupon they turned bright white. The gel pieces were then dried in the Speed-Vac for 5 min to evaporate the ACN.

The protein was then cleaved enzymatically into peptides. For this, the proteomics grade trypsin (Sigma, $20 \mu \mathrm{g}$ ) in a vial was dissolved in $40 \mu \mathrm{l}$ of HPLC grade water. Aliquots of $5 \mu \mathrm{l}$ of dissolved trypsin were pipetted into small Eppendorf tubes $(0.6 \mathrm{ml})$ and were dried in the Speed-Vac. For the digestion of proteins, the dried trypsin was reconstituted by dissolving in $40 \mu \mathrm{l}$ of $50 \mathrm{mM}$ ammonium bicarbonate, $\mathrm{pH}$ 8.0. Trypsin solution $(2 \mu \mathrm{l})$ was added to make direct contact with dried gel pieces and incubation was carried out for 1-3 hours at room temperature. The gel pieces were fully re-swollen after tryptic digestion. Again $30 \mu 1$ of $50 \mathrm{mM}$ 
ammonium bicarbonate was added to the gel pieces and they were left overnight at room temperature to allow for the diffusion of the peptides from the gel. This peptide-containing solution was transferred to new small Eppendorf tubes. To the gel pieces, $30 \mu \mathrm{l}$ of $50 \mathrm{mM}$ ammonium bicarbonate was added and they were shaken for 30 min. The supernatant was transferred to the same small Eppendorf tubes. To the gel pieces $35 \mu 1$ of $0.2 \%$ trifluoroacetic acid (TFA) was added and diffusion was allowed for 2-3 hours. This solution was also transferred to the digest solution in the Eppendorf tubes. ACN: $0.2 \%$ TFA (1:1) $(30 \mu \mathrm{l})$ was then added to the gel pieces. The tubes were incubated for 2-3 hours. This solution was also removed and added to the digest solution. Finally $30 \mu \mathrm{l}$ of ACN was added and tubes were incubated for 23 hours at room temperature. After incubation this solution was transferred to the accumulated solution. The digest solution was lyophilised in the Speed-Vac at $35^{\circ} \mathrm{C}$ until fully dried. The dried peptides were stored at $-20^{\circ} \mathrm{C}$ for subsequent MALDI analysis.

\subsubsection{Matrix preparation}

The matrix used in the study was $\alpha$-cyano-4-hydroxycinnamic acid (CHCA). To reconstitute the dried peptides obtained as above, matrix solution was prepared using $50 \%$ acetonitrile, $0.2 \%$ trifluoroacetic acid (TFA), CHCA to saturation and deionised water $(498 \mu \mathrm{l} \mathrm{ACN}+2 \mu \mathrm{l} \mathrm{TFA}+500 \mu 1$ deionised water + CHCA $)$. The solution was vortexed and centrifuged before using the supernatant.

\subsubsection{MALDI sample preparation}

The MALDI-plate was cleaned using acetone and Kimwipes followed by rinsing the plate with water and acetone in succession. Subsequently the plate was rinsed with ultra pure water followed by acetone. The remaining acetone was 
removed using Kimwipes and the plate was dried in a laminar flow hood. To the dried peptide sample, $2 \mu$ l of matrix solution was added. The generated matrix/peptide solution was mixed and immediately spotted onto the MALDI-plate. For each sample, two spots were applied the MALDI plate, without touching the pipette tip to the plate. The external calibration mixture was generated by adding $1 \mu 1$ of Calmix 2 calibration mixture to $99 \mu 1$ Matrix Solution followed by vortexing. This was immediately spotted onto the MALDI-plate. The following figure describes the spot pattern on the MALDI-plate. One spot represents $1 \mu 1$ of external calibration mixture. The spots were left to dry in the laminar airflow hood before inserting the plate into the mass spectrometer.

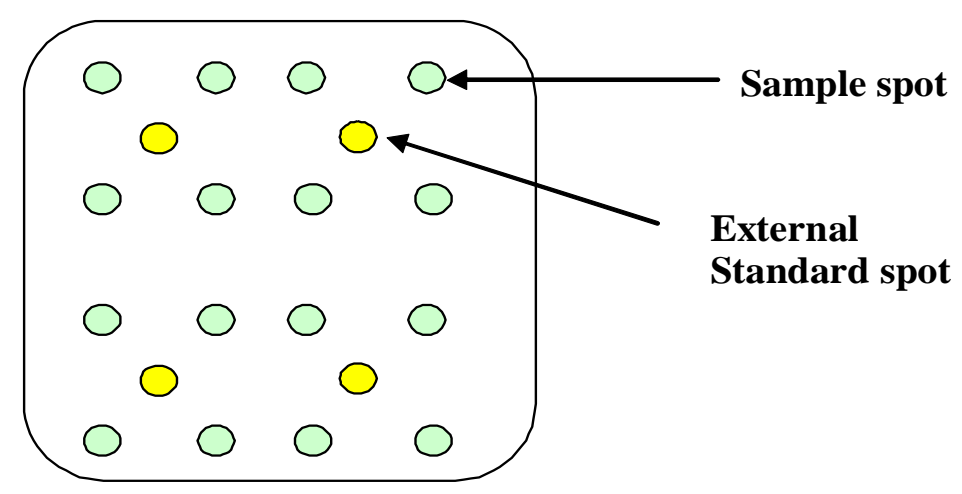

Figure 3-2: MALDI-plate spot pattern (Invitrogen Protocols)

Each external standard spot was placed in the middle of four sample spots in order to minimize the distance between sample and external calibration spots and therefore to minimize the time between the recording of a sample spectrum and an external calibration spectrum. Thus the effect of change of the conditions within the MALDI-TOF MS apparatus was reduced to a minimum. 


\subsubsection{Peptide Mass Fingerprinting}

Following the incorporation of peptides into the MALDI matrix a mass spectrum was recorded in order to obtain the protein specific peptide mass fingerprint (PMF). After drying, the sample was subjected to MALDI-TOF mass spectrometry analysis using an Applied Biosystem Voyager-DE $^{\mathrm{TM}}$ PRO Biospectrometry Workstation. A peptide mass map was generated by the Voyager Instrument Control Panel software (version 5.0). Mass spectra were recorded ten minutes after loading the MALDI-plate into the Bio Spectrometry Workstation to ensure best vacuum conditions within the flight tube of the MALDI-TOF MS apparatus. The spectra were obtained in reflector mode with an extraction delay time of $180 \mathrm{~ns}$. The instrument was used in positive polarity and set to an accelerating voltage of $20,000 \mathrm{~V}, 75 \%$ grid voltage, $0.002 \%$ guide wire and laser intensity in the range of 1700-2100. Spectra of samples were recorded using 200 laser pulses per spectrum and a mass range setting of 750-3500 Da.

Internal mass calibration was performed using trypsin auto digestion products. (i.e. autoproteolytic peptide masses of trypsin: 805.4167 and 2163.0566 present in the spectra). External calibration was only used when trypsin peaks were inadequate and was performed using the Calmix 2, which consists of peptides with monoisotopic masses of 1296.683 (angiotensin I), 2093.0868 (ACTH) and 2465.1989 (ACTH) (See Appendix Figure 10-1). This calibration procedure was carried out by the mass spectra analysis program Data Explorer. However, a manual examination and, if necessary, correction of the labelling of monoisotopic peaks was conducted. 
Fragmentation spectra cannot be used to derive a full peptide sequence, i.e. the sequence of a peptide cannot be determined de novo in this MS experiment. However, the individual PMFs were subsequently searched against the National Centre for Biotechnology Information non-redundant (NCBInr) protein database using Profound (http://prowl.rockefeller.edu/prowl-cgi/profound.exe) (Zhang and Chait, 2000) as the search algorithm in order to identify the selected proteins. ProFound is a tool for searching a protein sequence collection with peptide mass maps. It uses a Bayesian algorithm to rank the protein sequences in the database according to their probability of producing the peptide map. $\mathrm{Z}$ scores obtained correspond to the percentile of the search in the random match population. For instance, a $\mathrm{Z}$ score of 1.65 for a search means that the search is in the 95 th percentile. In other words, there are about $5 \%$ of random matches that could yield higher $\mathrm{Z}$ scores than this search. Hence, a $\mathrm{Z}$ score of 1.65 or lower indicates that the candidate is likely to be a random match. Such comparisons against the databases allow the identity of peptides that subsequently are used to compile a protein hit list. Usually a protein can be confidently identified by two or three peptides. If only a single peptide is used to identify a protein, further confirmation is needed (Salzano and Crescenzi, 2005).

\subsubsection{Protein identification}

The parameters used initially in the programme Profound were as follows: Database: NCBInr, Taxonomy: Drosophila melanogaster (fruit fly), enzyme: Trypsin, peptide mass tolerance: $0.5 \mathrm{Da}$, missed cleavage: 1 , carbamidomethylated cysteine as fixed modification, oxidation of methionine as a variable modification, charge state: $\mathrm{MH}+$, Monoisotopic masses. 
Protein identity was assigned when the algorithm came up with a significant identification result. Furthermore the calculated pI value and the molecular mass were compared to the experimental data from the gel electrophoresis. In this context protein fragments, adducts and post-translational modifications were considered. Identifications in D. melanogaster were made with a high degree of confidence whereas in $L$. cuprina they were necessarily much less sure. Some of the L. cuprina spots were matched against the $D$. melanogaster database to see the similarities between two species. In cases of poor identification scores using the D. melanogaster database the taxonomy was expanded to "Other Metazoa".

Following the protein identification by their corresponding peptide mass fingerprints using Profound, further information about the identified proteins was acquired using the Swiss-Prot and TrEMBL database hosted by the ExPASy Proteomics Server, URL: http://www.expasy.ch. This additional information included: primary accession number, protein name, gene name, computed $\mathrm{pI}$ and computed molecular mass.

\subsubsection{Reverse phase HPLC}

To determine the in vitro conjugation of insecticides by GSTs, reverse phase HPLC was employed as per the methodology of (Kostaropoulos et al., 2001b). The specific activities of affinity-purified GSTs from the three strains of L. cuprina (NSW, PY81 and CSIRO) towards methyl parathion and diazinon were determined. For this purpose, assays were carried out with $0.1 \mathrm{M}$ Tris $\mathrm{HCl}, \mathrm{pH} 8.0$ and insecticide $(5.5 \mu \mathrm{M}), \mathrm{GSH} 4 \mathrm{mM}$ and enzyme $100-200 \mu \mathrm{g}$ in $1 \mathrm{ml}$ assay mixture in screw-cap culture tubes. The reaction was stopped by addition of $1 \mathrm{ml}$ of heptane after 4 hours of incubation at $37^{\circ} \mathrm{C}$. Afterwards the reaction mixture was subjected to vigorous 
vortexing and centrifugation, and a portion of the resulting heptane extract $(20 \mu \mathrm{l})$ was subjected to HPLC analysis.

Analysis was performed on an Agilent HPLC 1100 series system (Agilent Technologies, Australia) equipped with Quat Pump (G1311A) and UV/Visible detector (G1314A). The injections were made by the use of an automated injection compartment, model G1329A. The injection volume was set to $20 \mu 1$. The system was operated in the reversed-phase mode and the analysis was carried out using a $\mathrm{C}_{18}$ Nucleosil 100, $4.6 \mathrm{~mm}$ X $250 \mathrm{~mm}, 5 \mu \mathrm{m}$ analytical column (Waters Corporation, USA). The flow rate was $1 \mathrm{ml} \mathrm{min}^{-1}$. Non-conjugated insecticide was eluted with a gradient of aqueous mobile phase (A) consisted of $0.1 \%$ TFA in water while the organic phase (C) consisted of $0.1 \%$ TFA in acetonitrile. TFA is used because of its excellent ion pairing and solvating characteristics. A 20 min gradient was used with a column temperature $30^{\circ} \mathrm{C}$.

\begin{tabular}{ccc}
\hline Time(min) & \%A & \% C \\
\hline 0 & 85 & 15 \\
5 & 85 & 15 \\
25 & 15 & 85 \\
35 & 15 & 85 \\
\hline
\end{tabular}

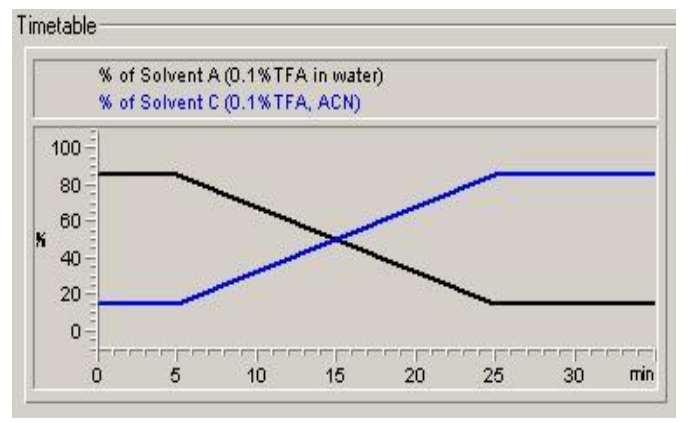

Figure 3-3: HPLC mobile phase gradient

The detection wavelength was set to $280 \mathrm{~nm}$ for methyl parathion and $254 \mathrm{~nm}$ for diazinon. Quantitative measurements for each insecticide were made using respective standard calibration curves. For each insecticide, the remaining amount, as detected by HPLC, was subtracted from the initial amount of the insecticide added to the 
assay in order to determine the enzyme's specific activity. For each assay, three controls were used: (1) insecticide without enzyme or GSH, to check the spontaneous chemical degradation of the insecticide; (2) insecticide and GSH without enzyme in order to determine the non-enzymatic reaction between GSH and the insecticide; (3) insecticide and enzyme without GSH, for the case of insecticide binding to enzyme without being conjugated to GSH or for hydrolytic degradation of an insecticide.

\subsubsection{Techniques to determine post-translational modifications}

\subsubsection{Pro- $Q^{\circledR}$ Diamond phosphoprotein gel stain}

Pro-Q Diamond stain was employed to study the possible phosphorylation of the Delta class of GSTs as per the manufacturer's protocol. The stain allows detection of 1-16 ng of phosphoprotein per band or spot depending on the phosphorylation state of the protein. This stain can be used for 1D SDS-PAGE gels or 2D gels and it is fully compatible with mass spectrometry.

The PeppermintStick ${ }^{\mathrm{TM}}$ phosphoprotein molecular weight standard was run alongside the sample on the gel. This phosphoprotein-specific standard contains a mixture of phosphorylated and nonphosphorylated proteins ranging from 14,400 to 116,250 Da. Separation by SDS PAGE resolves two phosphorylated and four nonphosphorylated proteins which serve as the control. For loading on minigels (8 $\mathrm{cm} \times 10 \mathrm{~cm}), 1 \mu 1$ of standard was mixed with $6 \mu 1$ of SDS gel loading buffer. After finishing the electrophoresis, the gels were transferred into $100 \mathrm{ml}$ of fixing solution $(50 \%(\mathrm{v} / \mathrm{v})$ methanol, $10 \%(\mathrm{v} / \mathrm{v})$ acetic acid, in ultra pure water) and incubated at room temperature with gentle agitation for 30 minutes. This fixation step was repeated twice to ensure removal of SDS from the gel. Sometimes, the gel was left in the fixing solution overnight. The fixing solution was then discarded and the gels 
were washed three times with ultra pure water for 30 minutes. It is important to completely remove the methanol and acetic acid from the gel as residues of these interfere with the Pro-Q Diamond phosphoprotein stain. After washing, the gel was incubated in $60 \mathrm{ml}$ of Pro-Q Diamond phosphoprotein gel stain (supplied in the kit) with gentle agitation in the dark for 60-90 minutes. Destaining was carried out by immersing the gel in destain solution $(20 \%(\mathrm{v} / \mathrm{v})$ acetonitrile, $50 \mathrm{mM}$ sodium acetate, $\mathrm{pH} 4.0$ in ultrapure water) for 30 minutes with gentle agitation in the dark. This step was repeated two more times. Destaining is important to reduce the gel background signal. Finally, the gel was washed twice with deionised water for 5 minutes each wash. According to manufacturer's manual, the Pro-Q Diamond stain works well in conjunction with SYPRO Ruby protein gel stain.

\subsubsection{SYPRO ${ }^{\circledR}$ Ruby protein gel stain}

SYPRO $^{\circledR}$ Ruby protein gel stain is a fluorescent stain used for the detection of proteins in $1 \mathrm{D}$ and $2 \mathrm{D}$ gels. It was used mostly as a post-stain in this study. As the gel was previously fixed in Pro-Q Diamond staining, it was directly incubated in 60 $\mathrm{ml}$ of SYPRO Ruby gel stain. The gel was left in the stain overnight, with gentle agitation, in the dark. The next day the gel was transferred to a clean container and washed in $100 \mathrm{ml}$ of wash solution (10\% methanol, $7 \%$ acetic acid in ultra pure water) for 30 minutes. Before imaging, the gel was rinsed in ultra pure water twice for at least 5 minutes each.

\subsubsection{Fluorescent gel scanning}

The scanning of fluorescent gels was conducted using the Fujifilm FLA-5100 imaging system. For phosphopeptide detection, images were generated using a laser wavelength of $532 \mathrm{~nm}$ and a LPG/0575 emission filter. The SYPRO Ruby stained gel images were scanned using a laser wavelength of $473 \mathrm{~nm}$ and a BPG1/570DF20 
emission filter. All gels were scanned at $50 \mu \mathrm{m}$ resolution and recorded as 16 bit TIFF files. While scanning an individual gel the other gels were stored at room temperature in darkness. The generated TIFF files were converted into image GEL files using the ImageQuant ${ }^{\mathrm{TM}}$ software. The fluorescence intensity of the Pro-Q Diamond signal and SYPRO Ruby signal in the gel was measured and ratiometric analysis (Diamond /Ruby) was performed for the standard proteins in the marker as well as the proteins of interest. The ratio should be higher for phosphoproteins (e.g., 8.0) compared to non-phosphorylated proteins (e.g., $~ 0.05$ ) according to the manual.

\subsubsection{Enrichment strategies for phosphopeptide analysis}

The procedure involved five steps: First the trypsin-digested dried peptides were re-suspended in $5 \mu$ l of $0.1 \%$ acetic acid or $0.01 \%$ formic acid. Secondly, ZipTipMC tips were charged and equilibrated. For this the tips were washed three times with $10 \mu$ l of wash solution I ( $0.1 \%$ acetic acid). Then the tips were charged by 10 aspirate and dispense cycles with $10 \mu \mathrm{l}$ of metal solution $(100 \mathrm{mM}$ cupric sulphate). Charged tips were washed five times with $10 \mu$ l deionised water and wash solution II ( $0.1 \%$ acetic acid with $50 \% \mathrm{ACN})$ subsequently. This step was to remove the unbound metal ions from the column. The tips were finally equilibrated with the binding solution $(0.1 \%$ acetic acid with $10 \% \mathrm{ACN})$ by $3-5$ aspirate and dispense cycles. The third step involved binding the peptides to the column. The sample was diluted with $2 \mu \mathrm{l}$ of the binding solution to ensure an acidic $\mathrm{pH}$. The sample (5-10 $\mu \mathrm{l})$ was bound to the tip by aspirating and dispensing 5-10 times. The tip was then washed with $10 \mu \mathrm{l}$ each of wash solution II and deionised water three times. The fourth step involved the elution of phosphopeptides. The eluent $(2 \mu 1,0.3 \mathrm{~N}$ ammonium hydroxide solution) was aspirated and dispensed through the Zip Tip 4 to 
6 times. Finally, the eluted phosphopeptides in the eluent were immediately neutralized by $1 \mu \mathrm{l}$ of $2 \%$ TFA. These neutralised phosphopeptides $(2 \mu \mathrm{l})$ were then mixed with CHCA matrix $(2 \mu \mathrm{l})$ and spotted on the MALDI plate. MALDI spectra were obtained and manual inspection was carried out to track phosphopeptides.

\subsubsection{Tandem mass spectrometry}

\subsubsection{Sample preparation for LC-MS/MS analysis}

The tandem MS spectra were obtained using LC-MS/MS for the Delta 1 GST spots which appear to have been modified. To prepare the sample, the Delta GST spots from the 2D gel were excised, destained and digested with trypsin as described previously. Finally the extracted peptide mixture was dried in a Speed-Vac. The dried peptides were resuspended in $10 \mu \mathrm{l} 0.25 \%$ TFA. The ZipTip ${ }^{\mathrm{TM}} \mathrm{C}_{18}$ tip (Millipore Corporation, USA) was washed thrice with $10 \mu \mathrm{l}$ of $100 \%$ acetonitrile and then with $10 \mu \mathrm{ACN}: 0.5 \%(\mathrm{v} / \mathrm{v})$ aqueous TFA (1:1) and lastly with $10 \mu \mathrm{l}$ of $0.25 \%$ aqueous TFA. The sample was drawn up and down in the Zip-Tip several times. The liquid was then discarded. At this point the sample was bound to the $\mathrm{C}_{18}$ surface in the Zip-Tip. The Zip-Tip was washed again with $10 \mu \mathrm{l}$ of $0.25 \%$ TFA three times. The sample was eluted in $5 \mu$ of $0.1 \%$ formic acid in $70 \%(\mathrm{v} / \mathrm{v})$ acetonitrile solution by aspirating 15 times. Sample eluent was stored at $-20^{\circ} \mathrm{C}$ until analysis.

\subsubsection{LC-MS/MS analysis}

LC-MS/MS was carried out using a Dionex UltiMate ${ }^{\mathrm{TM}} 3000$ LC system and a Thermo Finnigan LTQ mass spectrometer equipped with a nanospray ion source. Peptides were separated on a $75 \mu \mathrm{m} \times 15 \mathrm{~cm}$ PepMap C18 column $(3 \mu \mathrm{m}, 300 \AA$ Dionex) at a constant flow rate of $200 \mathrm{~nL} / \mathrm{min}$ using the gradient constructed from 
$0.1 \%$ formic acid in water (solvent $\mathrm{A}$ ) and $0.1 \%$ formic acid in $80 \% \mathrm{ACN}$ (solvent $\mathrm{B}$ ): 0-10\% B for $10 \mathrm{~min}$; 10-28\% B for $60 \mathrm{~min}$; 28-55\% B for $20 \mathrm{~min}$; 55-100\% B for 15 min; $100 \%$ B for 5 min. The LTQ was operated in data-dependent tandem MS mode where the 5 most abundant precursor ions detected in a single MS scan from m/z 400 to $\mathrm{m} / \mathrm{z} 2000$ were dynamically selected for subsequent MS/MS scans with the collision energy set to $35 \%$, simultaneously incorporating the dynamic exclusion option to prevent reacquisition of MS/MS spectra of the same peptides. This procedure was kindly performed by Dr. Lifeng Peng.

\subsubsection{PEAKS - de novo software}

The MS/MS spectra in RAW format were loaded on the software PEAKS. The parameters for the analysis of post-translational modification of Delta GST will be described in detail in Chapter 7.

\subsubsection{Statistical analysis}

All statistical analysis in this thesis was performed using SPSS 16.0 for Windows $^{\circledR}$ (SPSS, Chicago, IL, http://www.spss.com/). Where only two means were compared, Student's $t$-test was used to determine differences between means and their significance. 


\section{$4 \quad$ Purification and identification of GSTs from Drosophila melanogaster and Lucilia cuprina}

\subsection{Introduction}

The aim of the work presented in this chapter was to purify the GSTs from $D$. melanogaster and L. cuprina using combinations of different affinity matrices and to characterise them based on model substrate specificity. This chapter also attempted to compare the GST proteome of both insects and to identify the purified GSTs from D. melanogaster, for which there is a genetic database, and from L. cuprina, which lacks a genetic database. The separation of the GST isoenzymes such as Sigma and Delta from partially purified affinity extracts was also carried out.

Purification based on targetted affinity interactions offers high selectivity and quick purification of biomolecules (Chase, 1998; Wils et al., 1997). A good ligand offers increased adsorbent selectivity for the target relative to other proteins. Due to its high selectivity, affinity chromatography is a preferred tool in the isolation and purification of proteins (Narayanan and Crane, 1990). Affinity media might be used to achieve purification of GSTs in order to study their properties. Sequential use of different affinity media might allow isotype specific (or selective) isolation of GSTs in order to compare and contrast the properties of GSTs from different species. To fulfil the need for rapid, simple and efficient GST purification methods, a number of affinity matrices have been constructed. Glutathione, glutathione conjugates or compounds that are GST inhibitors but do not contain glutathione have been chosen as the affinity ligands. The ligands that have been used for purification of GSTs are BSP (Wolkoff et al., 1979), BSP-GSH (Clark et al., 1977), Cholic acid (Pattinson, 
1981), GSH (Simons and Vanderjagt, 1977), S-hexyl-GSH (Guthenberg and Mannervik, 1979), S-octyl-GSH (Sugimoto et al., 1987) and thyroxine (Ishigaki et $a l .$, 1989). Indeed, GST isolation through affinity purification has become so reliable, GST fusion protein tags have been commonly used to purify recombinant proteins (Rebay and Fehon, 2009a; Rebay and Fehon, 2009b).

Among several affinity matrices, glutathione coupled to Sepharose has been widely used (Clark et al., 1977; Simons and Vanderjagt, 1977). Enzymes bound selectively to the matrices can be then eluted by GSH (Clark et al., 1977). In mammals it is well known that different classes of GST have differing preferences for different substrates (Mannervik, 1985), therefore they will tend to produce different conjugates. The question is posed, if one type of conjugate is bound to a matrix, would it have a preference for a specific class of enzyme? As CDNB and DCNB are the most common substrates used for the detection of GST activity due to their remarkably different activities with different isoforms (Habig and Jakoby, 1981), the matrices prepared by linking the GSH conjugate of CDNB or DCNB to Sepharose (DNP-GSH matrix or CNP-GSH matrix respectively) should be useful for the separation of GSTs that do not bind to a GSH matrix (Clark, personal communication). Literature suggests that very few people have used the DNP-GSH matrix as an affinity support. Awasthi et al. has used it for the purification of DNPGS ATPase (Awasthi et al., 1998) and Grant and Matsumura have purified GSTs from resistant larvae of Aedes aegypti (Grant and Matsumura, 1989) however, so far, the use of CNP-GSH matrix for the purification of GSTs has not been reported. The present study explores for the first time the possibility of using CNP-GSH affinity matrix for GST purification in addition to DNP-GSH affinity matrix. 
The literature shows that multiple forms of GSTs with different isoelectric points have also been isolated using isoelectrofocusing (Clark et al., 1986; Clark et $a l ., 1973$ ) or chromatofocusing (Keeran and Lee, 1987). Purification by affinity chromatography followed by isoelectricfocusing also revealed the existence of multiple forms of GSTs (Clark and Dauterman, 1982) in the housefly strains Rutgers, Cornell R and Hirokawa. The expression of multiple isoenzymes of GSTs has also been reported in other species like Aedes aegypti (Grant et al., 1991), Galleria mellonella (Baker et al., 1994), Plutella xylostella (Chiang and Sun, 1993), Tenebrio molitor (Kostaropoulos et al., 1996) and Drosophila melanogaster (Alias and Clark, 2007).

Insect genomes contain diverse GST genes, the products of which share functionality with those present in lower and higher animals (Enayati et al., 2005). The complexity of GST genes found within an insect species is best exemplified by the genomes of D. melanogaster (Toung et al., 1993; Tu and Akgul, 2005), A. gambiae (Ranson and Hemingway, 2005; Ranson et al., 2001), M. domestica (Fournier et al., 1992; Zhou and Syvanen, 1997) and honey bee (Corona and Robinson, 2006) which are characterised by multigene GST families. Though the GSTs from both D. melanogaster and $L$. cuprina have been studied in the present work, the main focus is on L. cuprina GSTs. D. melanogaster is not a major crop pest or a primary target for commercial application of insecticides whereas $L$. cuprina, being an ecto-parasite of sheep, is exposed to insecticides and has been reported to have developed resistance against a number of organophosphate pesticides as described in section 1.7. Thus, the present work is designed to probe the role of GSTs in resistance in $L$. cuprina. The DNA microarrays provide an important means to assess the impact of xenobiotic exposure on cytochrome P450, esterase and 
GST gene expression (Belleville et al., 2004; David et al., 2005; Kitagawa et al., 2002; Le Goff et al., 2003; Le Goff et al., 2006; Vontas et al., 2007) however, the creation of microarrays requires detailed knowledge of the genome. Further, alterations in biochemical pathways due to post-transcriptional modification, translational control and modification of gene products may go undetected. In contrast, proteomics can reveal changes at the level of protein production that do not rely solely on mRNA expression. We have therefore chosen to use a proteomic approach for the study of GSTs from L. cuprina using the D. melanogaster proteome as a model. As described in the general introduction section 1.4.1, insect GSTs have been shown to be active toward numerous electrophilic xenobiotics including halogenated compounds (e.g., CDNB), nitro compounds (e.g., p-nitrophenyl acetate), $\alpha, \beta$-unsaturated carbonyl compounds (e.g., trans-4-phenyl-3-buten-2-one), isothiocyanates (e.g., allyl isothiocyanate), organothiocyanates (e.g., benzylthiocyanate), oxides (e.g., styrene oxide), organophosphates (e.g., diazinon), and organic hydroperoxides (cumene hydroperoxide) (Yu, 1996). However, very little is known about substrate specificity of individual GST isozymes in insects. This knowledge is very important for understanding the molecular mechanisms of detoxification in insects. Therefore, in addition to developing methods to purify and identify GSTs, the purpose of this chapter was also to study the substrate specificity of various GST isozymes isolated from L. cuprina and D. melanogaster using model substrates such as CDNB, DCNB, NPA, TNE, DHA, EA and PBO. The purification and characterization methodology described in this chapter will be applied in chapters 5 and 6, which describe changing profiles of GST isolates during development of $L$. cuprina, in different body parts of the adult fly and in susceptible and resistant strains of L. cuprina. 


\subsubsection{Drosophila melanogaster as a model insect}

The fruitfly, D. melanogaster has been a general testing ground for genetic concepts and techniques that have applications for both vector biology and pest control. (Foster et al., 1981) reported the possible homologies of chromosomes and genetic maps of D. melanogaster with other dipteran insects including L. cuprina. These chromosomal homology studies help to predict the location of the gene of interest in other insects based on D. melanogaster information. The D. melanogaster genome sequence (Adams et al., 2000), including the annotation and prediction of protein sequences (Misra et al., 2002) has also enabled studies of the fruitfly's proteome and comparison with other insects. Comparison of the genomes and proteomes of the two diptera Anopheles gambiae and Drosophila melanogaster revealed considerable similarities and numerous differences (Zdobnov et al., 2002). Almost half of the genes in both genomes were interpreted as orthologs and showed an average sequence identity of about $56 \%$. D. melanogaster proteome analysis offers unique possibilities for studying the function of individual molecules and protein classes and their role in innate immunity (Engstrom et al., 2004). The reference proteome map of reproductive organs of $D$. melanogaster has been constructed for the comprehensive understanding of D. melanogaster reproduction to use it as a model to study insect reproductive biology (Takemori and Yamamoto, 2009). The response of the GST proteome of adult D. melanogaster to chemical challenge has also been studied (Alias and Clark, 2007; Alias and Clark, 2010). Recently, the potential for atrazine to affect insecticide susceptibility and the activity of detoxification enzymes in D. melanogaster has been studied using proteomic analysis (Thornton et al., 2010). Thus these studies provide valuable information for the study of insecticide resistance in other insects. In the present study, $D$. 
melanogaster is well suited as a model insect as D. melanogaster and L. cuprina both belong to the same class, order and suborder.

\section{D. melanogaster}

$\begin{array}{ll}\text { Kingdom: } & \text { Animalia } \\ \text { Phylum: } & \text { Arthropoda } \\ \text { Class: } & \underline{\text { Insecta }} \\ \text { Order: } & \text { Diptera } \\ \text { Suborder: } & \text { Brachycera } \\ \text { Family: } & \text { Drosophilidae } \\ \text { Genus: } & \text { Drosophila }\end{array}$

(Fallén, 1823)

\section{L. cuprina}

Kingdom: $\quad$ Animalia

Phylum: Arthropoda

Class: $\quad \underline{\text { Insecta }}$

Order: $\quad$ Diptera

Suborder: Brachycera

Family: $\quad$ Calliphoridae

Genus: $\quad$ Lucilia

The complete genomic database for D. melanogaster is reported to include 39 GST genes (http://flybase.org/) which makes the identification of GST isoenzymes in D. melanogaster straightforward. L. cuprina, however, has limited GST gene information. The only known $L$. cuprina Theta GST has been reclassified as a member of the Delta class (Board et al., 1997) and its sequence enabled the confirmed identification of L. cuprina Delta GSTs in the present study. Previous work at our laboratory suggested that the GSH-affinity eluents of both $D$. melanogaster and L. cuprina yield quite similar classes of GST isoenzymes identified on 2D gels (Alias and Clark, 2007). In the present study, the Drosophila melanogaster database, version dated 11/12/2007 has been used to characterise GSTs expressed in L. cuprina. No doubt, there must be dis-similarities between these two insect GST proteomes, so this chapter also deals with comparative analysis throughout. To support further the tentative identification of $L$. cuprina GSTs, the matched L. cuprina GST peptide sequences have been aligned with consensus GST 
sequences from the other metazoa species using the ClustalW2 alignment programme, an approach which is described later in this section.

\subsection{Objectives:}

- To establish effective affinity purification techniques to isolate as many classes of GSTs as possible from D. melanogaster and L. cuprina

- $\quad$ To compare the GST proteome of D. melanogaster and L. cuprina

- To characterise L. cuprina GSTs tentatively in the absence of a specific genetic database by developing a consensus sequence-based approach

- To separate Sigma and Delta GSTs by chromatofocusing or ion exchange chromatography in order to study their substrate specificities

\subsection{Results}

\subsubsection{Similarity of $L$. cuprina Delta GST with $D$. melanogaster GSTs}

To find out the percentage similarities or identities of the GSTs between these two species, ClustalW2 alignment programmes (www.ebi.ac.uk/clustalW2) and a BLAST, similarity search proteomic tool available at ExPASy (proteomic server http://www.expasy.ch/tools/blast) were used. The sequence alignment of L. cuprina Delta GST against the D. melanogaster database clearly showed its similarity with D. melanogaster Delta GSTs. L. cuprina Delta GST sequence obtained from UniProt (http://www.uniprot.org/uniprot/P42860) was aligned with all D. melanogaster GST sequences using ClustalW2 and the result is shown in the form of a phylogenetic tree in Figure 4-1. The result of matching L. cuprina Delta GST against the $D$. melanogaster Delta GST sequence using BLAST found it to be $83 \%$ identical to DmGSTD1 (Figure 4-2). 


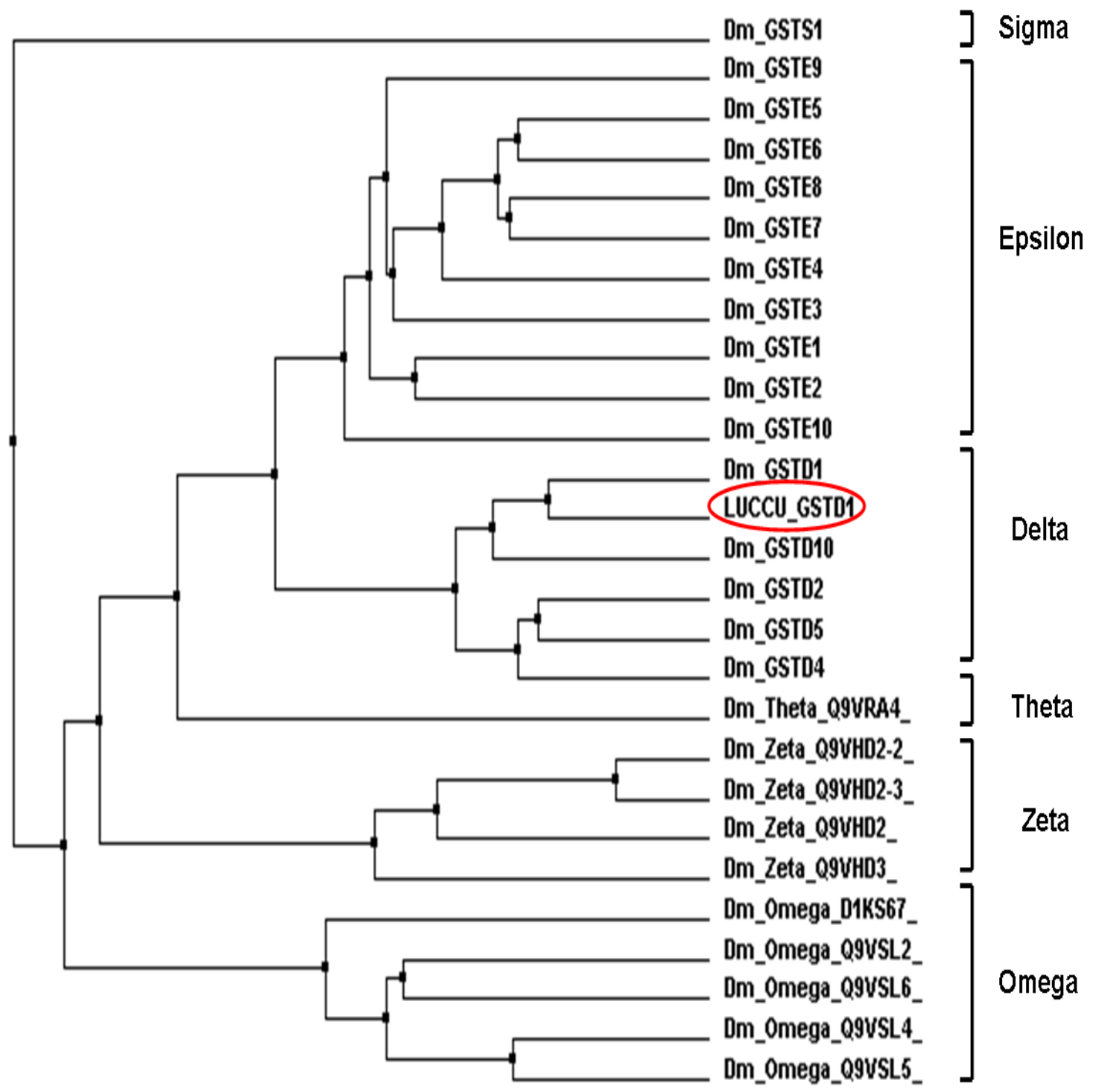

Figure 4-1: Phylogenetic relation of L. cuprina Delta GST with D. melanogaster GSTs.

Phylogenetic tree demonstrating the relationships of the $L$. cuprina Delta GST to $D$. melanogaster GSTs. Amino acid sequences were aligned using ClustalW2 and an average distance tree was generated using BLOSUM62 in Jalview.

$\mathrm{Dm}=$ D. melanogaster, $\mathrm{LUCCU}=$ L. cuprina . The accession numbers of proteins are as per UniProtKB (http://www.uniprot.org/uniprot/). 


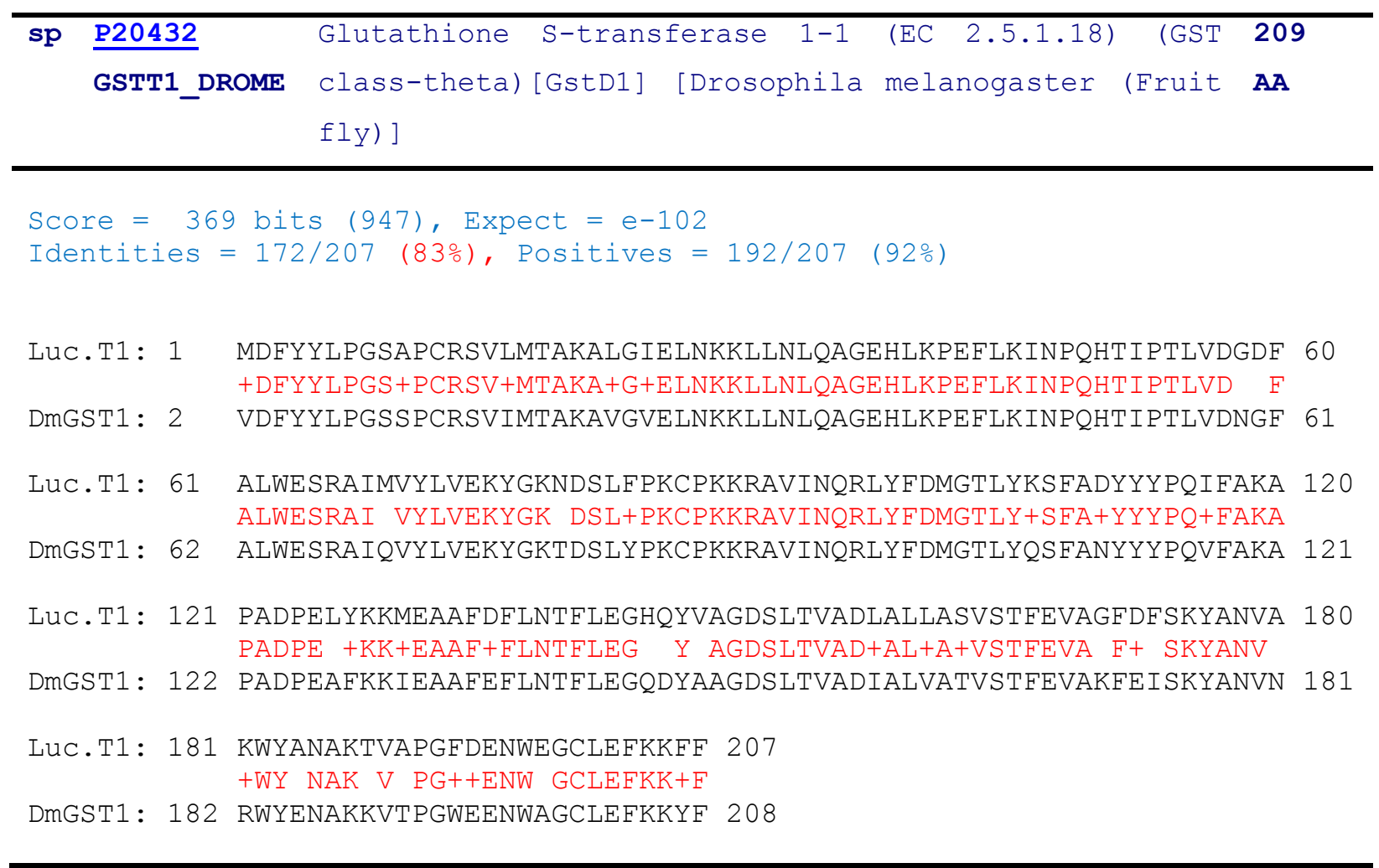

Figure 4-2: The BLAST result of alignment of GST sequence of D. melanogaster and $L$. cuprina.

The L. cuprina Delta GST (Luc.T1) sequence was entered into BLAST which was used to search for similarity against the $D$. melanogaster database. The result shows the alignment of the sequences and BLAST statistics for the highest similarity of L. cuprina Delta GST to $D$. melanogaster Delta GST1.

The close identity of the L. cuprina and D. melanogaster sequences provides confidence that matching peptides between orthologous sequences, a key technique used in 4.3 .3 will provide reliable results.

\subsubsection{Purification of GSTs}

Initially the GSTs were purified using a desalting column (G-75 Sephadex) (not de-pigmenting using HiTrap desalting column) and affinity chromatography on glutathione affinity matrix as described in section 3.2.2.2. In order to get effective purification of GSTs, three different GSH matrices were tested. Two GSH-Sepharose 6B matrices were prepared with GSH substitution of $10 \mu \mathrm{mol} / \mathrm{ml}$ and $37.5 \mu \mathrm{mol} / \mathrm{ml}$ 
(highly substituted, gift from Dr. Alan Clark). The third matrix was the commercially available GSTrap column with GSH substitution of approximately $7-15 \mu \mathrm{mol} / \mathrm{ml}$. For this initial preparation, $9 \mathrm{~g}$ of $L$. cuprina (PY81 strain) flies were homogenised in $100 \mathrm{ml}$ of homogenisation buffer, centrifuged and desalted using a G-75 Sephadex (5 x $26 \mathrm{~cm}, 500 \mathrm{ml}$ ) column. Out of this, $30 \mathrm{ml}$ of sample was applied to each of these three matrices and bound proteins were eluted with $20 \mathrm{mM}$ glutathione in $0.05 \mathrm{M}$ phosphate buffer, $\mathrm{pH} 9.6$ using FPLC. It was observed repeatedly in several experiments that a significant proportion of the CDNB activity was not retained by any of these GSH affinity matrices. However, considerable differences were observed in GST activity between affinity eluents of each (Table 4-1).

Table 4-1: Purification of GSTs from L. cuprina by three different GSH matrices.

The calculation of $\%$ yield and fold purification was made considering actual volume of application on each column. Values are mean \pm SD taken from three independent experiments with triplicate measurements within each experiment.

\begin{tabular}{lccccc}
\hline Procedure & $\begin{array}{c}\text { Total protein } \\
(\mathbf{m g})\end{array}$ & $\begin{array}{c}\text { Total activity } \\
(\mu \mathrm{mol} / \mathrm{min})\end{array}$ & $\begin{array}{c}\text { Specific activity } \\
(\mu \mathrm{mol} / \mathrm{min} / \mathrm{mg})\end{array}$ & $\begin{array}{c}\text { Yield } \\
(\%)\end{array}$ & $\begin{array}{c}\text { Purification } \\
(\text { fold })\end{array}$ \\
& & CDNB & CDNB & & \\
\hline Crude & $275 \pm 21$ & $43.0 \pm 1.5$ & $0.156 \pm 0.02$ & 100 & 1 \\
Desalted extract & $270 \pm 10$ & $42.0 \pm 2.0$ & $0.155 \pm 0.01$ & 97 & 0.99
\end{tabular}

The desalted extract was divided into three equal portions $(30 \mathrm{ml})$ and applied individually on each column

\begin{tabular}{|c|c|c|c|c|c|}
\hline Actual application & $85.0 \pm 3.50$ & $13.0 \pm 0.70$ & $0.153 \pm 0.02$ & 100 & 1 \\
\hline \multicolumn{6}{|l|}{ GSH $10 \mu \mathrm{mol}$} \\
\hline Unbound & $60.0 \pm 2.50$ & $8.30 \pm 2.70$ & $0.138 \pm 0.01$ & 64 & 0.90 \\
\hline Bound & $0.60 \pm 0.20$ & $3.20 \pm 1.00$ & $5.333 \pm 1.00$ & 24 & 34.8 \\
\hline \multicolumn{6}{|l|}{ GSTrap 7-15 $\mu \mathrm{mol}$} \\
\hline Unbound & $70.0 \pm 1.00$ & $9.60 \pm 0.20$ & $0.137 \pm 0.002$ & 74 & 0.89 \\
\hline Bound & $0.40 \pm 0.02$ & $5.20 \pm 1.50$ & $13.80 \pm 4.05$ & 40 & 90.2 \\
\hline \multicolumn{6}{|l|}{ GSH $37.5 \mu \mathrm{mol}$} \\
\hline Unbound & $60.0 \pm 3.50$ & $8.60 \pm 4.30$ & $0.143 \pm 0.03$ & 66 & 0.93 \\
\hline Bound & $0.50 \pm 0.18$ & $3.20 \pm 0.50$ & $6.400 \pm 3.00$ & 25 & 41.8 \\
\hline
\end{tabular}


The specific activity of the GSTs purified by the GSTrap column was the highest $(13.8 \pm 4.0)$ compared to the others. The fold purification (90.2) and \% yield (40) was higher than with the lab-made GSH columns. It may be that the long linker arm (C12) of GSTrap column gives easier access of the GSTs to the ligand. It was expected to recover higher GST activity in the affinity eluents of the $37.5 \mu \mathrm{mol} \mathrm{GSH}$ matrix due to its higher substitution, however there was not much difference in the $\%$ yield and fold purification between the laboratory-made matrices.

The affinity-purified fractions of all the matrices contained at least two proteins detectable by SDS-PAGE. These proteins have M.W. values of approximately 30,000 Da and 23,000 Da and were identified as Sigma and Delta GSTs on a 2D gel respectively by MALDI-TOF as described later. GSTs from the GSH $10 \mu \mathrm{mol} / \mathrm{ml}$ affinity eluents showed many faint bands and an additional band was also observed in the GSTrap affinity eluents which has not been identified by MALDI-TOF in the present study.

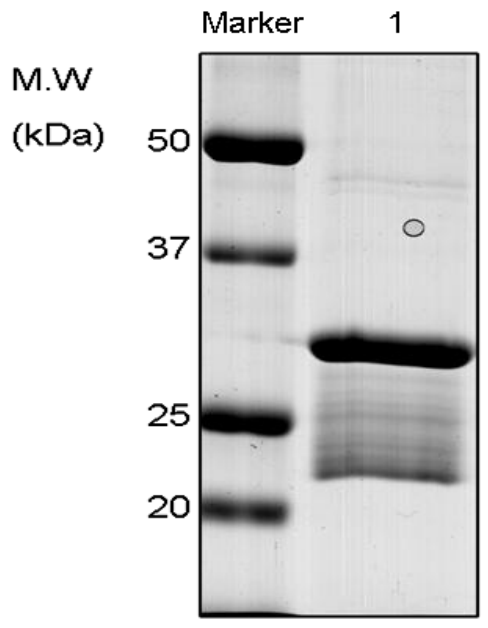

A

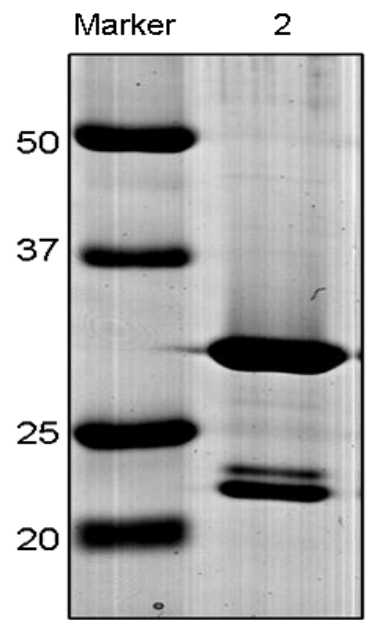

$\mathrm{B}$

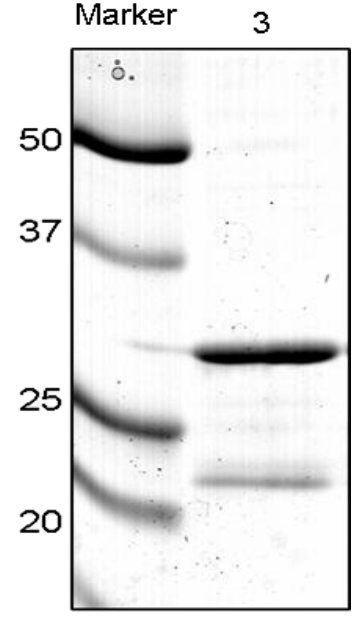

C

Figure 4-3: SDS PAGE gels of different GSH affinity-purified GSTs from L. cuprina.

(A) GSH $10 \mu \mathrm{mol} / \mathrm{ml}$, (B) GSTrap 7-15 $\mu \mathrm{mol} / \mathrm{ml}$ and (C) GSH $37.5 \mu \mathrm{mol} / \mathrm{ml}$; Lanes 1,2 and 3 show purified GSTs in all three corresponding figures. The protein M.W. marker was run along the sample. The gels were stained with Coomassie Brilliant Blue G-250. 
Whilst the $10 \mu \mathrm{mol} / \mathrm{ml} \mathrm{GSH}$ affinity matrix isolated a number of bands within the region 23-30 kDa with L. cuprina, the D. melanogaster gel produced only two clear bands of approximate M.W. $32 \mathrm{kDa}$ and $23 \mathrm{kDa}$ (Figure 4-4).

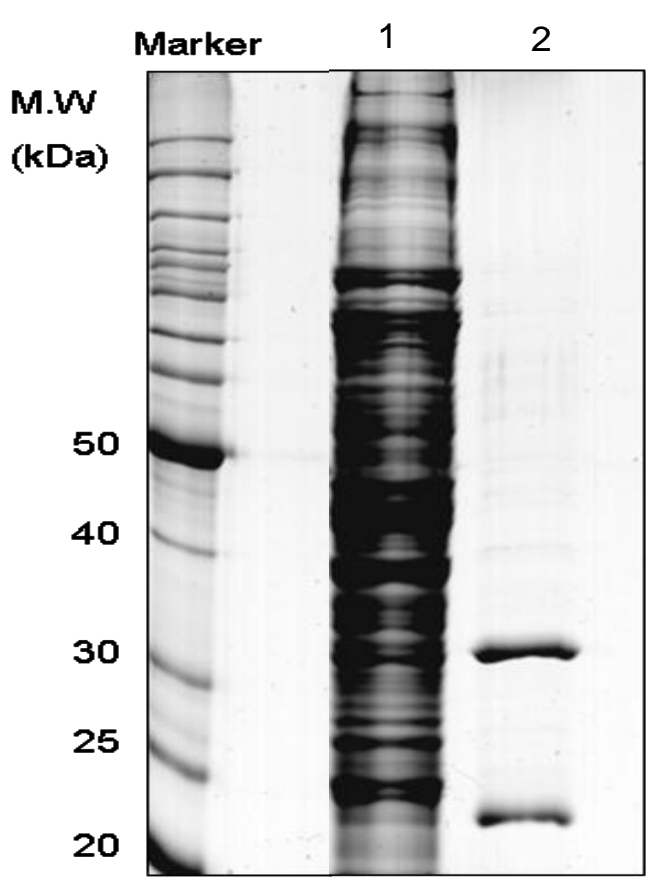

D. melanogaster

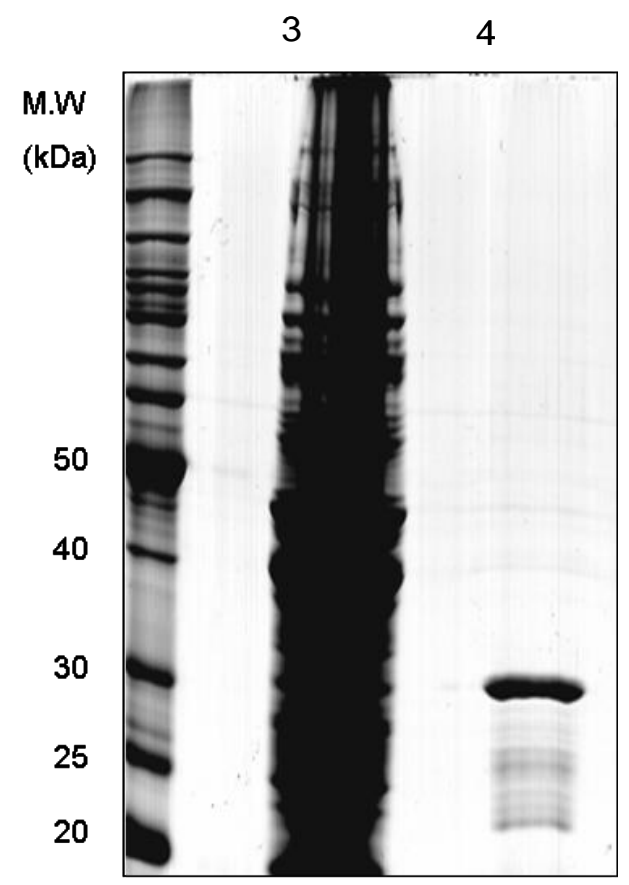

L. cuprina

Figure 4-4: SDS PAGE gel of GSH $(10 \mu \mathrm{mol} / \mathrm{ml})$ affinity-purified GSTs from $D$. melanogaster and L. cuprina.

A) D. melanogaster: Lane 1-unbound fractions of GSH affinity matrix; Lane 2bound/purified GSTs. B) L. cuprina: Lane3- unbound fractions of GSH affinity matrix; Lane 4- bound/purified GSTs. The protein M.W. marker was run along the sample. The gels were stained with Coomassie Brilliant Blue G-250.

When these purified concentrated fractions were resolved by $2 \mathrm{D}$ electrophoresis, the resulting gel was however quite similar to the D. melanogaster 2D gel (see section 4.3.3). This $1 \mathrm{D}$ result was confirmed by repeating the same experiment more than six times. To verify further this difference, all the available batches of the L. cuprina in the lab at the time were subjected to the GSH affinity chromatography and purified GSTs were resolved by SDS PAGE. 


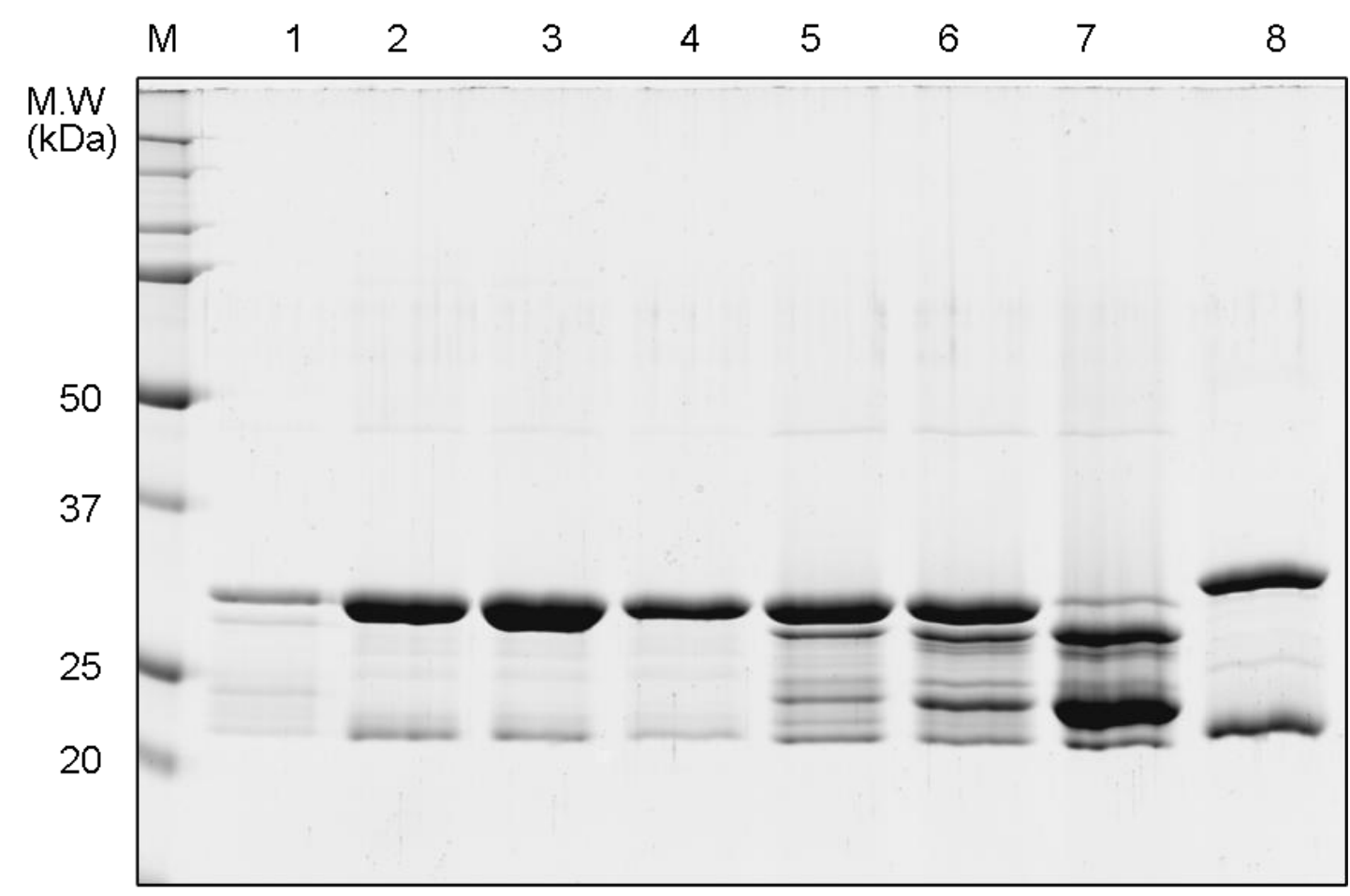

Figure 4-5: SDS PAGE gel of GSH affinity-purified GSTs from different batches and strains of L. cuprina and D. melanogaster.

Lane M is M.W. marker. Lanes 1-5 are of PY81 resistant strain of L. cuprina. Lanes 6 and 7 are of susceptible strains of L. cuprina (CSIRO and NSW strain respectively). Lane 1, Flies bred on 15th Feb 2008; Lane 2, Flies bred on 1st April 2008; Lane 3, Flies bred on 6th May 2008; Lane 4, Flies bred on 31st July 2008; Lane 5, Flies bred on 6th November 2007; Lane 6, Flies bred on 5th Feb 2008; Lane 7, Flies bred on 20th October 2008 and Lane 8, D. melanogaster. The gel was stained with Coomassie Brilliant Blue G-250.

There were remarkable differences in purified GSTs from different batches of $L$. cuprina flies which were bred in the insectary. The bands with approx. M.W. 30-32 kDa and $23 \mathrm{kDa}$ were identified as Sigma and Delta class of GST respectively by MALDI-TOF analysis. The molecular weight of the D. melanogaster Sigma and Delta class of GST appear to be slightly higher than the L. cuprina GSTs. There are other faint bands present in lanes 5-7, which also fall in the size range to that expected for GSTs. There is a noticeable difference in the insecticide susceptible 
strains (CSIRO and NSW, lane 6 and 7 respectively in Figure 4-5). It is still not confirmed what caused this change in the GST expression pattern between the batches. It is possible that the protein diet given at that time contributed to this, hence another experiment was carried out by processing only sugar fed flies of different strains of L. cuprina in similar way.

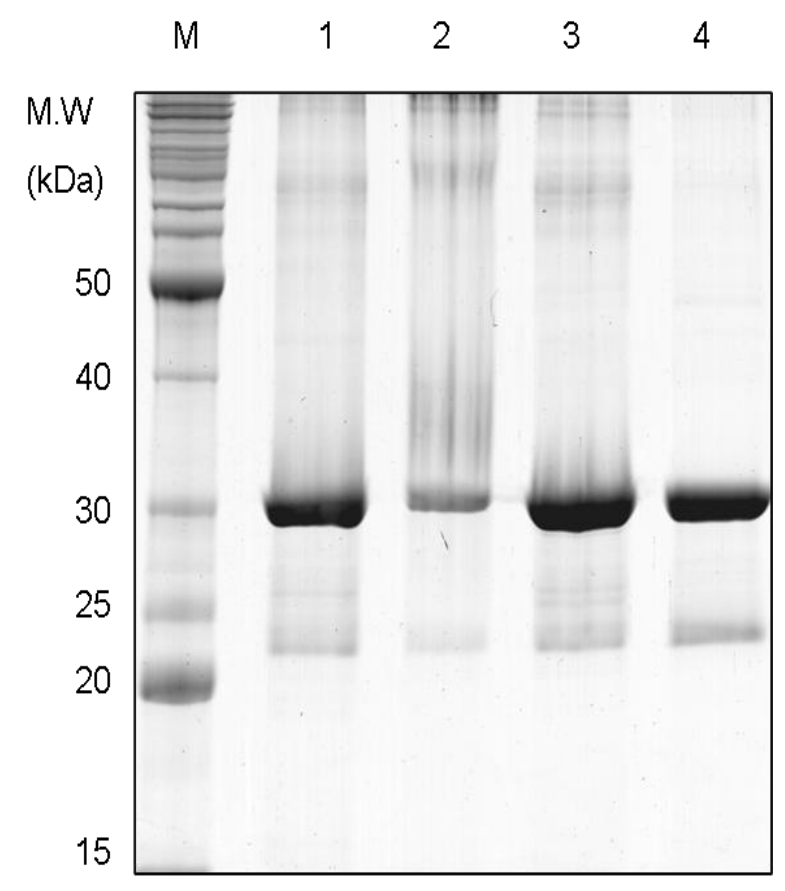

Figure 4-6: SDS PAGE gel of GSH affinity-purified GSTs from different sugar-fed strains of L. cuprina.

Lane M is M.W. marker. Lane 1 and 2 are of PY81 insecticide resistant strain of L. cuprina. Lane 3 and 4 are of susceptible strains of L. cuprina (NSW and CSIRO respectively). All flies were sugar-fed and bred in 2009.

The experiment did not show the remarkable different pattern of expression of GSTs in NSW and CSIRO (lane 3 and 4 respectively, Figure 4-6) found in previous experiments (lane 7 and 6 respectively, Figure 4-5). Some very faint bands were visible in lane 3 (Figure 4-6) but not as in lane 7 (Figure 4-5). There are only two major bands corresponding to Sigma (30-32 kDa) and Delta (23 kDa) GSTs in all three strains. The diet may clearly be a factor but the exact reasons for the 
differences in Figure 4-5 are not known. Therefore, to avoid any ambiguity, only sugar fed flies have been used in the present study. Further, to check whether there might have been an effect of storage temperature, the GSTs were purified from the flies kept at room temperature overnight and compared with those from $-20^{\circ} \mathrm{C}$. There was no difference found on separation of GSTs on a 1D gel.

A significant proportion of GSTs from the different strains of $L$. cuprina and D. melanogaster failed to bind to any of the GSH matrices employed. Lanes 1 and 3 in Figure 4-4 showing the unbound fractions of D. melanogaster and L. cuprina respectively contained many proteins, so it was not possible to detect the presence of GSTs on a gel based on M.W. Moreover, the unbound direct flow-through fractions from the glutathione matrix gave a higher total activity with DCNB compared with those eluted with GSH (Table 4-2). Therefore, the possibility of purifying the unbound material on other affinity matrices such as DNP-GSH or CNP-GSH matrix was explored. The extent of substitution was calculated to be $9.55 \mu \mathrm{mol} / \mathrm{ml}$ of gel for DNP-GSH and $9.65 \mu \mathrm{mol} / \mathrm{ml}$ of gel for CNP-GSH matrix. The general workflow of the strategies for the purification and identification of GSTs used in this study is shown in Figure 4-7. 
Table 4-2: Comparative DCNB activity with D. melanogaster and L. cuprina unbound and bound GSH affinity eluents.

GSTs from D. melanogaster and L. cuprina were partially purified by GSH affinity matrix $(10 \mu \mathrm{mol} / \mathrm{ml})$. Unbound fractions from the GSH matrix with significant GST activity towards CDNB were pooled and GST activity towards DCNB was measured. Values are means \pm S.D. taken from three independent experiments with triplicate measurements within each experiment.

\begin{tabular}{cccc}
\hline Procedure & $\begin{array}{c}\text { Total protein } \\
(\mathrm{mg})\end{array}$ & $\begin{array}{c}\text { Total activity } \\
(\mu \mathrm{mol} / \mathrm{min})\end{array}$ & $\begin{array}{c}(\mu \mathrm{mol} / \mathrm{min} / \mathrm{mg}) \\
\end{array}$ \\
& $\mathrm{DCNB}$ & $\mathrm{DCNB}$ \\
\hline
\end{tabular}

D. melanogaster

$\begin{array}{llll}\text { Unbound fractions } & 52.5 \pm 0.70 & 0.18 \pm 0.001 & 0.003 \pm 5.0 \times 10^{-05} \\ \text { Bound fractions } & 0.80 \pm 0.03 & 0.06 \pm 0.008 & 0.075 \pm 3.0 \times 10^{-03}\end{array}$

\section{L. cuprina}

$\begin{array}{llll}\text { Unbound fractions } & 60.0 \pm 0.50 & 0.40 \pm 0.01 & 0.006 \pm 2.0 \times 10^{-04} \\ \text { Bound fractions } & 0.70 \pm 0.02 & 0.08 \pm 0.03 & 0.114 \pm 1.0 \times 10^{-02}\end{array}$




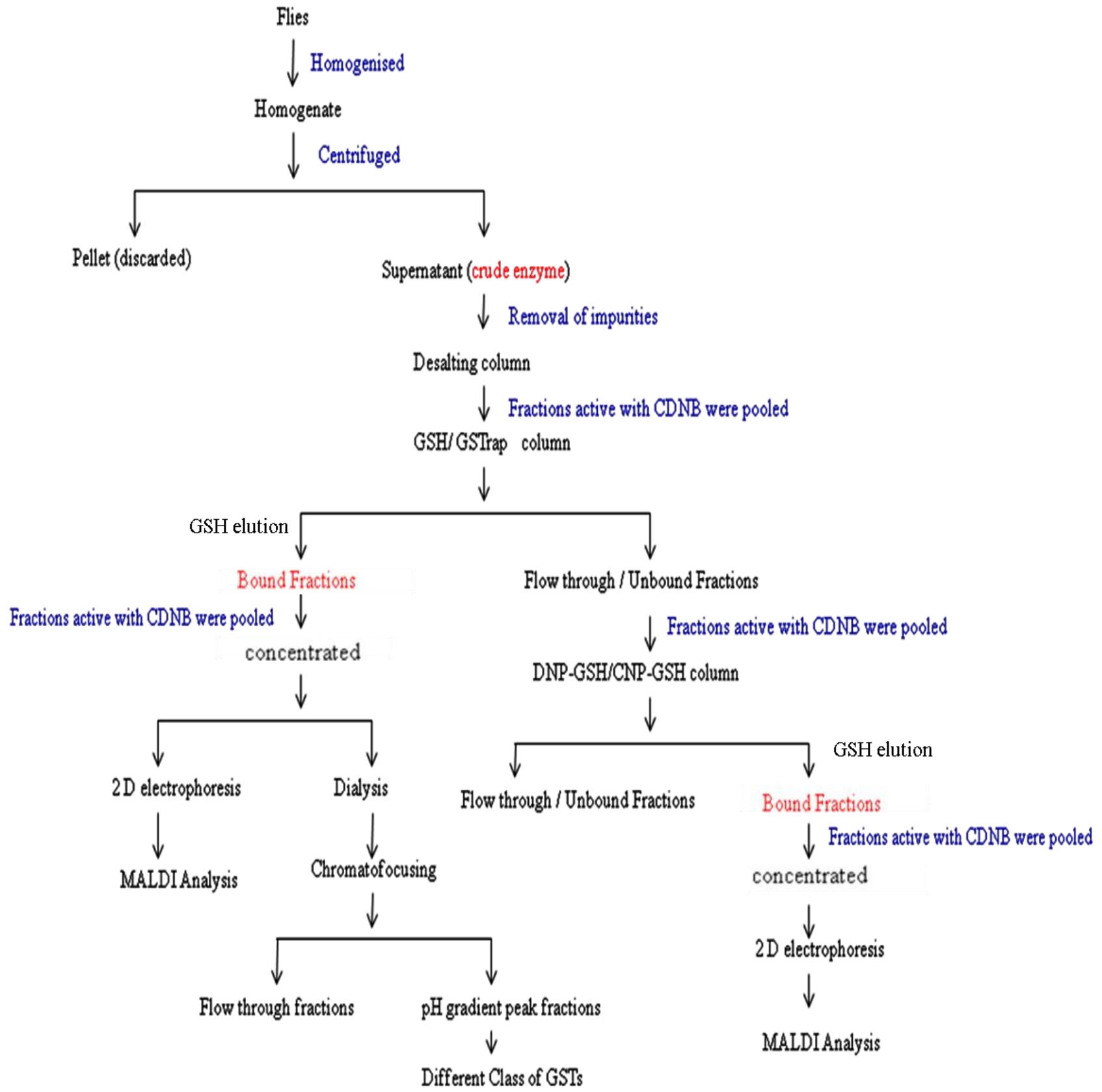

Figure 4-7: Schematic workflow of strategies for the purification and identification of GSTs used in this study. 


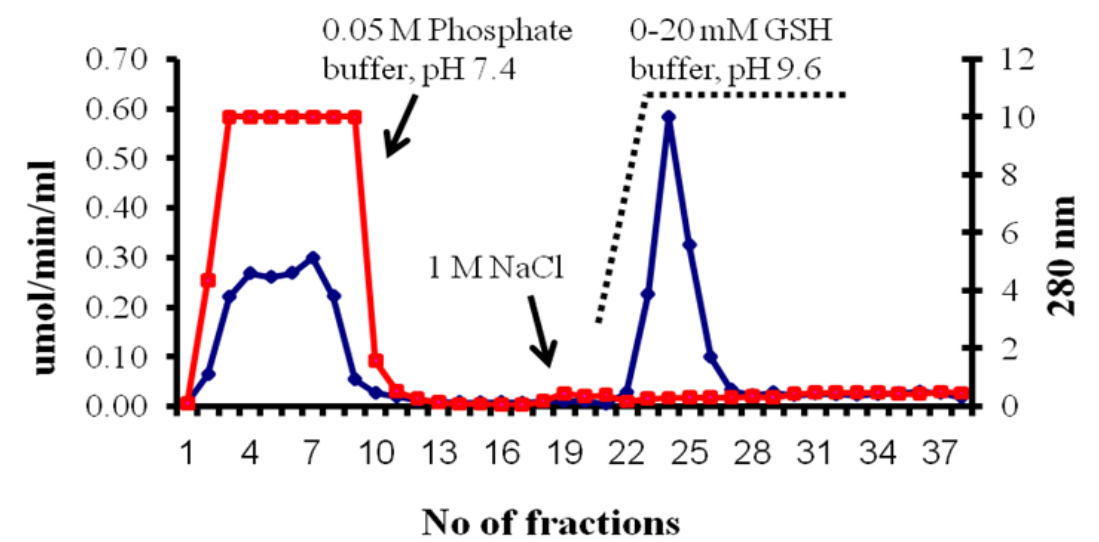

$\rightarrow$ Activity with $\mathrm{CDNB} \quad \rightarrow$ Protein $280 \mathrm{~nm}$

B

\section{DNP-GSH matrix}

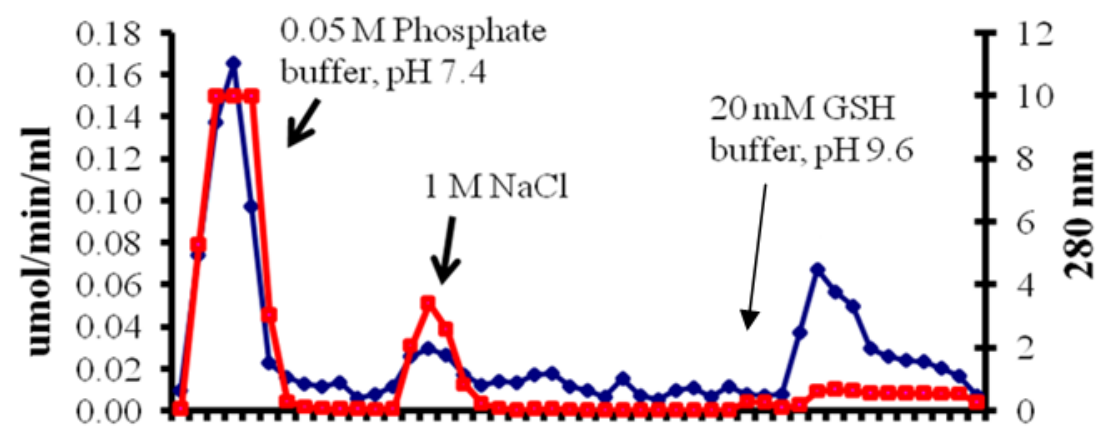

$147 \quad 10131619222528313437404346$

No of fractions

$\rightarrow$ Activity with CDNB $\rightarrow$ Protein $280 \mathrm{~nm}$

Figure 4-8: Typical chromatography elution profiles for partial purification of $D$.

melanogaster and L. cuprina GSTs by manual sequential affinity chromatography.

The de-pigmented extract was initially purified using the GSH matrix (A) and unbound fractions from the GSH matrix were pooled and subsequently applied to DNP-GSH (B) for further purification of GSTs. The elution profile for CNP-GSH matrix was similar to DNPGSH matrix. The initial peak obtained by $0.05 \mathrm{M}$ phosphate buffer, $\mathrm{pH} 7.4$ constitutes the unbound fractions, $1 \mathrm{M} \mathrm{NaCl}$ was used to remove binding of non-specific proteins and the bound GSTs were eluted by $20 \mathrm{mM}$ GSH with or without gradient. 

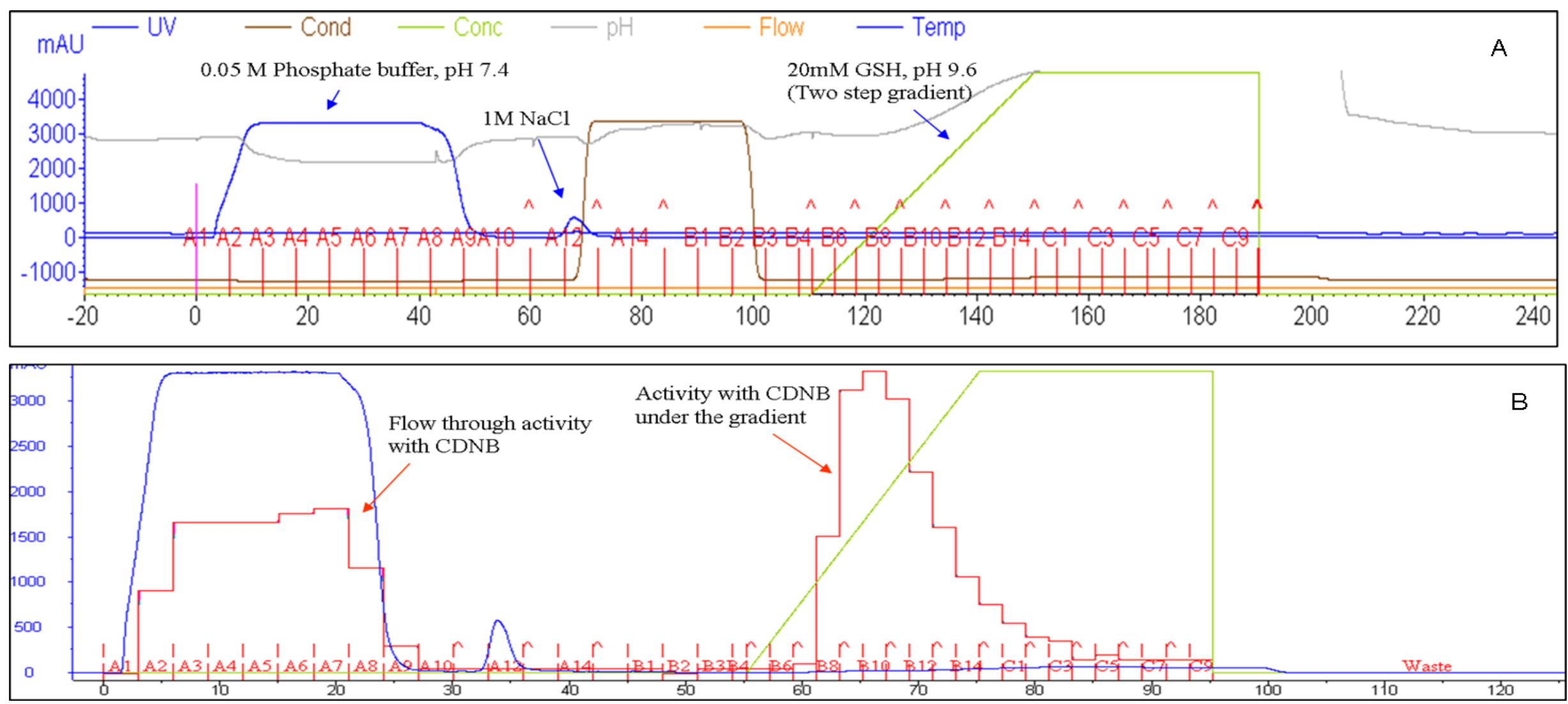

Figure 4-9: Typical elution chromatograms for the purification method of GSTs using FPLC.

(A) FPLC chromatogram with the UV absorbance of sample applied, conductivity of buffer, concentration of GSH, pH of buffer, flow rate and temperature of the experiment automatically recorded by the ÄKTA FPLC. (B) The CDNB activity of the different fractions is plotted on a FPLC chromatogram to see the correlation of activity with the unbound and bound fractions. 
The crude extract of 4-6 g of D. melanogaster was de-pigmented and applied to the GSH column as described in section 3.2.2.2. $1 \mathrm{M} \mathrm{NaCl}$ was used to remove non-specifically bound proteins from the column. Bound GSTs were eluted with 20 $\mathrm{mM}$ GSH, $\mathrm{pH} 9.6$ as shown in Figure 4-8 and Figure 4-9. The unbound fractions containing significant GST activity were pooled and divided into two portions. One half was subjected to DNP-GSH chromatography and the other half was applied to the CNP-GSH matrix for further possible purification. The elution of GSTs was carried out with $20 \mathrm{mM}$ glutathione.

Table 4-3: Glutathione S-transferase activity of D. melanogaster.

GSTs of D. melanogaster were partially purified using GSH matrix. Unbound fractions from GSH matrix with significant GST activity were pooled and applied to either DNP-GSH or CNP-GSH affinity matrix for further possible purification of GSTs. Activities were determined in triplicate of three experiments. Results are presented as mean \pm S.D.

\begin{tabular}{|c|c|c|c|c|c|}
\hline Procedure & $\begin{array}{l}\text { Total protein } \\
(\mathrm{mg})\end{array}$ & $\begin{array}{l}\text { Total activity } \\
\text { ( } \mu \mathrm{mol} / \mathrm{min}) \\
\text { CDNB }\end{array}$ & $\begin{array}{l}\text { Specific } \\
\text { activity } \\
\text { ( } \mu \mathrm{mol} / \mathrm{min} / \mathrm{mg} \text { ) } \\
\text { CDNB }\end{array}$ & $\begin{array}{l}\text { Yield } \\
\text { (\%) }\end{array}$ & $\begin{array}{l}\text { Purification } \\
\text { (fold) }\end{array}$ \\
\hline Crude & $95.0 \pm 7.5$ & $22.1 \pm 6.6$ & $0.23 \pm 0.04$ & 100 & 1 \\
\hline De-pigmented & $92.0 \pm 9.0$ & $20.8 \pm 6.4$ & $0.22 \pm 0.04$ & 94 & 0.95 \\
\hline \multicolumn{6}{|l|}{ GSH matrix } \\
\hline Unbound & $82.5 \pm 10$ & $9.82 \pm 3.5$ & $0.12 \pm 0.02$ & 44 & 0.52 \\
\hline Bound & $0.50 \pm 0.3$ & $9.61 \pm 4.2$ & $19.2 \pm 4.51$ & 43 & 83.5 \\
\hline \multicolumn{6}{|c|}{ The unbound fractions of GSH matrix were divided into two equal portions and applied individually to } \\
\hline \multicolumn{6}{|l|}{ each column } \\
\hline $\begin{array}{l}\text { Actual } \\
\text { application }\end{array}$ & $41.0 \pm 5.0$ & $5.00 \pm 1.7$ & $0.12 \pm 0.02$ & 100 & 1 \\
\hline \multicolumn{6}{|c|}{ DNP-GSH matrix } \\
\hline Unbound & $34.0 \pm 10$ & $2.00 \pm 0.9$ & $0.06 \pm 0.02$ & 40 & 0.51 \\
\hline Bound & $0.32 \pm 0.2$ & $1.80 \pm 1.2$ & $5.62 \pm 3.19$ & 36 & 46.8 \\
\hline \multicolumn{6}{|c|}{ CNP-GSH matrix } \\
\hline Unbound & $32.5 \pm 8.0$ & $1.50 \pm 0.5$ & $0.05 \pm 0.02$ & 30 & 0.42 \\
\hline Bound & $0.35 \pm 0.2$ & $1.30 \pm 0.4$ & $3.71 \pm 2.50$ & 26 & 31.0 \\
\hline
\end{tabular}


It was of note that the DNP-GSH and CNP-GSH matrices, when used subsequent to the GSH column, retained GSTs which had not previously bound to the GSH matrix and were seen in the affinity eluents from these matrices. The percentage recovery of the GST activity with CDNB was quite similar in both the matrices but the fold purification was slightly higher with the DNP-GSH matrix. The same experiment was performed using adult L. cuprina instead of D. melanogaster. Results are shown in Table 4-4.

Table 4-4: Glutathione S-transferase activity of L. cuprina.

GSTs of $L$. cuprina were partially purified using the GSH matrix. Unbound fractions from the GSH matrix with significant GST activity were then pooled, concentrated and applied to either DNP-GSH or CNP-GSH affinity matrix for further possible purification of GSTs. Activities were determined in triplicate of three experiments. Results are presented as mean \pm S.D.

\begin{tabular}{|c|c|c|c|c|c|}
\hline Procedure & $\begin{array}{c}\text { Total protein } \\
(\mathrm{mg})\end{array}$ & $\begin{array}{l}\text { Total activity } \\
\text { ( } \mu \mathrm{mol} / \mathrm{min}) \\
\text { CDNB }\end{array}$ & $\begin{array}{c}\text { Specific activity } \\
\text { ( } \mu \mathrm{mol} / \mathrm{min} / \mathrm{mg}) \\
\text { CDNB }\end{array}$ & $\begin{array}{l}\text { Yield } \\
(\%)\end{array}$ & $\begin{array}{l}\text { Purification } \\
\text { (fold) }\end{array}$ \\
\hline Crude & $100.5 \pm 3.0$ & $13.8 \pm 0.2$ & $0.138 \pm 0.002$ & 100 & 1 \\
\hline De-pigmented & $96.0 \pm 6.5$ & $12.7 \pm 1.4$ & $0.132 \pm 0.023$ & 92 & 0.95 \\
\hline \multicolumn{6}{|l|}{ GSH matrix } \\
\hline Unbound & $64.0 \pm 11$ & $6.00 \pm 3.7$ & $0.093 \pm 0.04$ & 43 & 0.67 \\
\hline Bound & $0.65 \pm 0.2$ & $7.00 \pm 1.2$ & $10.76 \pm 4.67$ & 51 & 77.9 \\
\hline \multicolumn{6}{|c|}{$\begin{array}{l}\text { The void fractions of GSH matrix were divided into two equal portions and applied individually to each } \\
\text { column }\end{array}$} \\
\hline Actual application & $32.0 \pm 5.5$ & $3.00 \pm 1.9$ & $0.093 \pm 0.04$ & 100 & 1 \\
\hline \multicolumn{6}{|l|}{ DNP-GSH matrix } \\
\hline Unbound & $27.0 \pm 0.4$ & $1.40 \pm 0.5$ & $0.051 \pm 0.02$ & 46 & 0.54 \\
\hline Bound & $0.34 \pm 0.2$ & $1.20 \pm 0.6$ & $3.529 \pm 0.09$ & 40 & 37.9 \\
\hline \multicolumn{6}{|l|}{ CNP-GSH matrix } \\
\hline Unbound & $31.5 \pm 2.00$ & $1.70 \pm 0.7$ & $0.054 \pm 0.02$ & 56 & 0.58 \\
\hline Bound & $0.44 \pm 0.01$ & $1.30 \pm 0.3$ & $2.954 \pm 0.77$ & 43 & 31.7 \\
\hline
\end{tabular}


The results show that the DNP-GSH matrix and CNP-GSH matrix showed a similar degree of purification in L. cuprina again slightly higher in DNP-GSH matrix. There was not much difference in the $1 \mathrm{D}$ and $2 \mathrm{D}$ gels of affinity preparations from both these matrices which means that the identity and number of GSTs captured by each of these matrices were also quite similar (Figure 4-10 and Figure 4-20). These matrices have a tendency to bind non-GST proteins as well (Figure 4-10). All further purification was carried out by using the DNP-GSH matrix as it is easier to prepare.

A

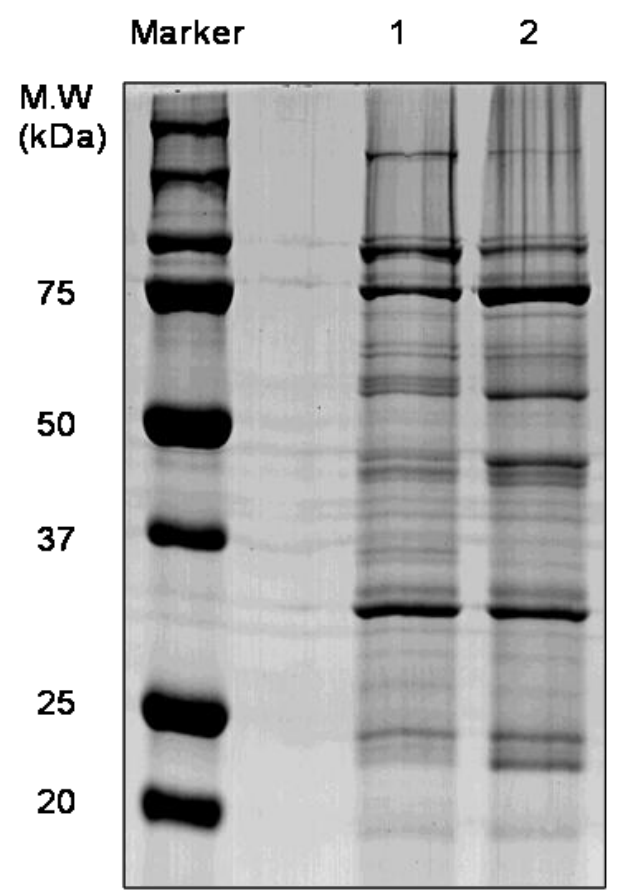

D. melanogaster
B

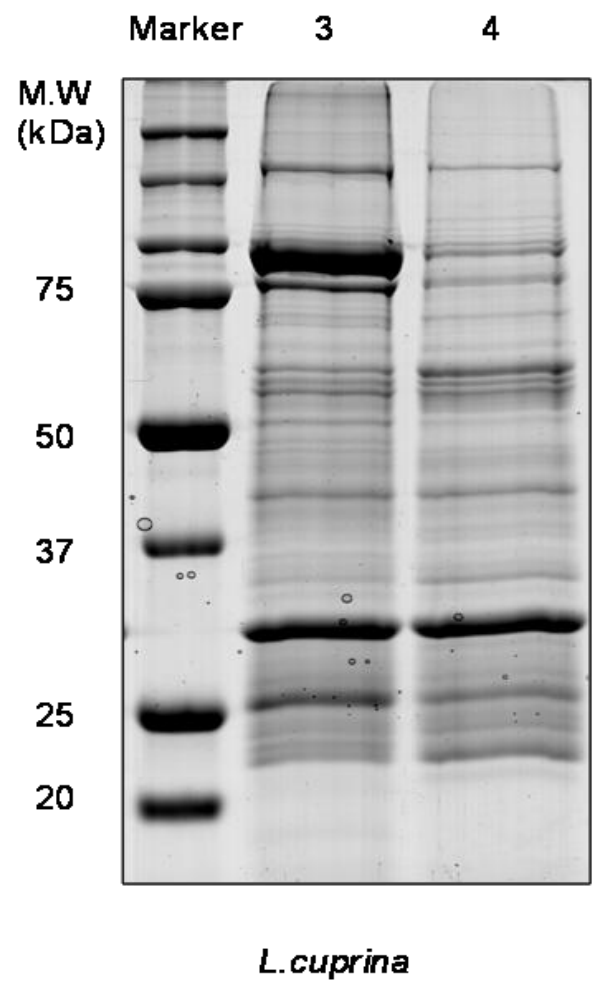

Figure 4-10: SDS PAGE gels of DNP-GSH and CNP-GSH affinity-purified GSTs from $D$. melanogaster (A) and L. cuprina PY81 strain (B).

Lane 1 and 3 - Proteins purified from fractions not bound to the GSH matrix by DNP-GSH matrix; Lane 2 and 4 - proteins purified from fractions not bound to the GSH-Sepharose matrix by CNP-GSH matrix. The M.W. marker was run along the samples. The gels were stained with Coomassie Brilliant Blue G-250. 
The total and specific activities with a range of substrates of $D$. melanogaster (Table 4-5 and Table 4-6) and L. cuprina (Table 4-7 and Table 4-8) bound and unbound fractions from each matrix are shown. The total activities of the unbound fractions from the GSH matrix with D. melanogaster extract were higher towards all the substrates employed except CDNB and EA compared to bound fractions (Figure 4-11) whereas in L. cuprina the unbound fractions from the GSH matrix had the highest activity with all the substrates employed (Figure 4-12). This indicates the presence of other classes of GST in the unbound fractions which were not adsorbed to GSH matrix. When these unbound fractions with significant activity were subsequently applied to DNP-GSH and CNP-GSH matrices, of the activities towards TNE, DCNB, NPA, EA and PBO only a fraction were retained by these columns. This again indicated need of further purification of GSTs. However, the specific activities of bound fractions from the GSH matrix were higher towards CDNB and TNE (Figure 4-13) compared to DNP-GSH or CNP-GSH affinity eluates in both insects. The specific activities towards the other substrates such as DCNB, NPA, DHA, EA and PBO were higher in DNP-GSH and CNP-GSH affinity-purified fractions than the GSH affinity-purified fractions from both insects. Some of total activities, particularly towards NPA, DHA, PBO, DCNB and EA were still present in unbound fractions of DNP-GSH and CNP-GSH affinity matrices (Figure 4-11 and Figure 4-12). There is the likelihood that other classes of GSTs which were not adsorbed to GSH, DNP-GSH or CNP-GSH matrices might be present in those unbound fractions. 


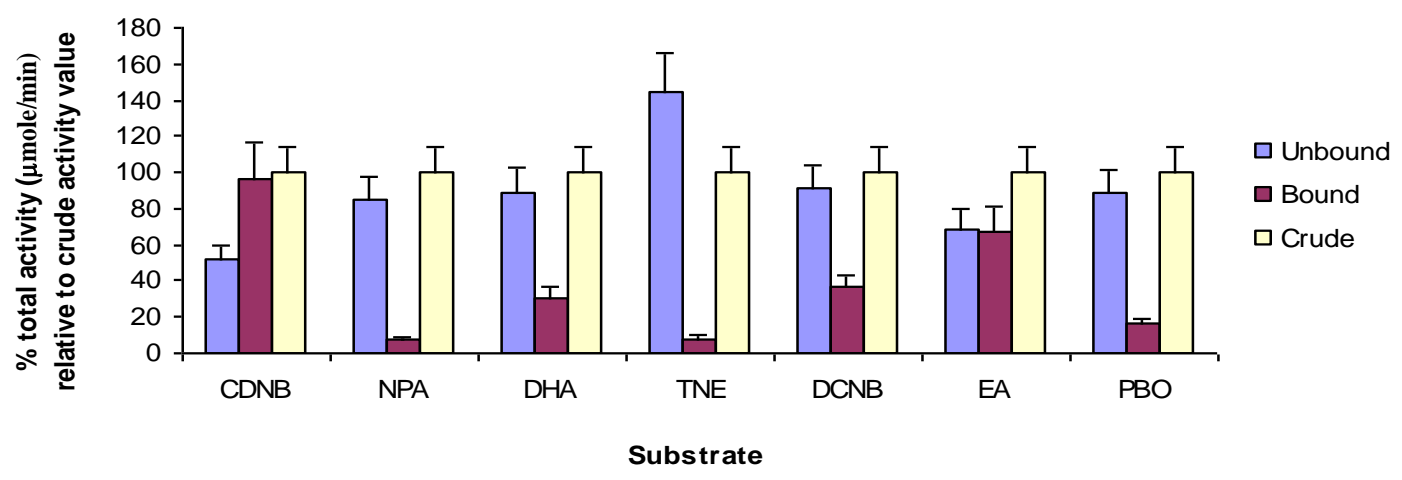

\section{DNP-GSH matrix}

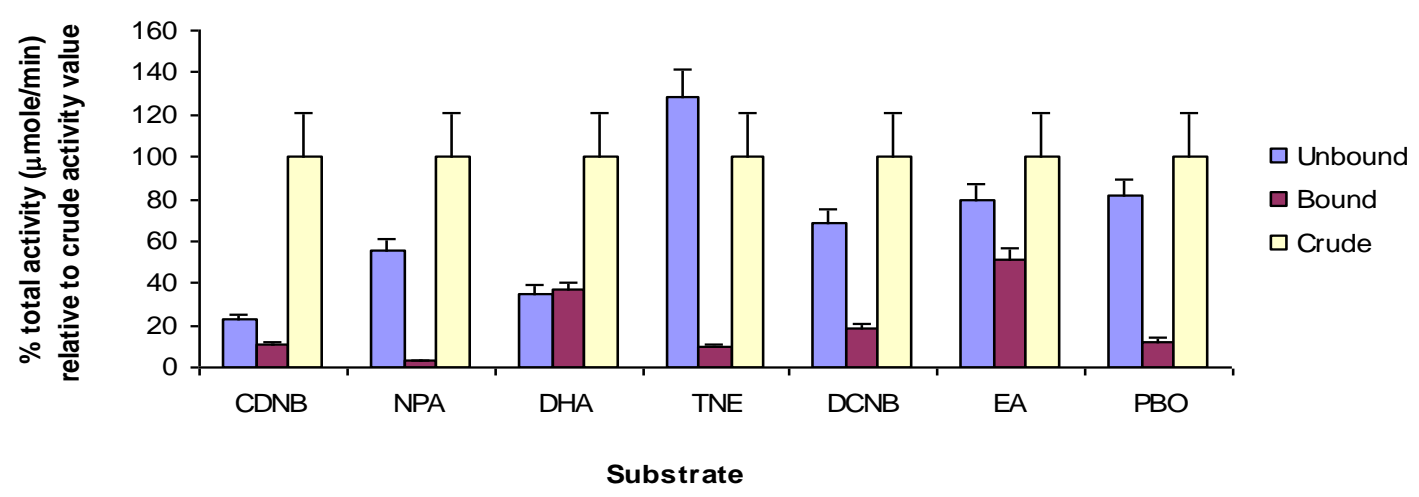

CNP-GSH matrix

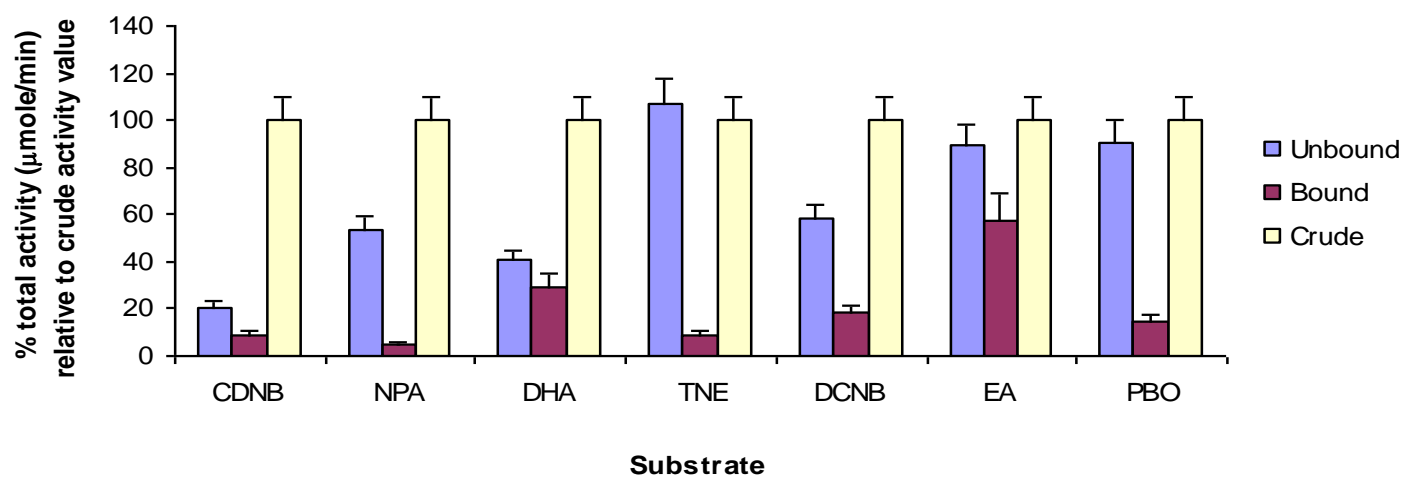

Figure 4-11: Comparison of substrate-specific total activities of unbound and bound fractions of D. melanogaster from different affinity matrices.

The unbound fractions from the GSH matrix with significant activity towards CDNB were pooled and applied equally to DNP-GSH and CNP-GSH affinity matrices. Assays were undertaken following the methods in section 3.2.4. The values of $\%$ total activity are relative to crude enzyme activity measured in triplicates from three independent experiments. Error bars represent standard deviation. 
Table 4-5: Substrate-specific total activities of GSTs from D. melanogaster.

Unbound fractions from the GSH matrix were applied equally to DNP-GSH and CNP-GSH matrices. Assays were undertaken following the methods in section 3.2.4. Activities were determined in triplicate of three experiments. Results are presented as mean \pm S.D.

\begin{tabular}{|c|c|c|c|c|c|c|}
\hline \multirow[t]{2}{*}{ Substrate } & \multicolumn{6}{|c|}{ Total activity $(\mu \mathrm{mol} / \mathrm{min})$} \\
\hline & $\begin{array}{l}\text { Unbound } \\
\text { GSH }\end{array}$ & $\begin{array}{l}\text { Bound } \\
\text { GSH }\end{array}$ & $\begin{array}{l}\text { Unbound } \\
\text { DNP-GSH }\end{array}$ & $\begin{array}{l}\text { Bound } \\
\text { DNP-GSH }\end{array}$ & $\begin{array}{l}\text { Unbound } \\
\text { CNP-GSH }\end{array}$ & $\begin{array}{l}\text { Bound } \\
\text { CNP-GSH }\end{array}$ \\
\hline CDNB & $6.28 \pm 0.102$ & $11.82 \pm 0.287$ & $2.78 \pm 1.768$ & $1.28 \pm 0.066$ & $2.44 \pm 0.13$ & $1.07 \pm 0.022$ \\
\hline DCNB & $0.20 \pm 0.098$ & $0.08 \pm 0.008$ & $0.15 \pm 0.013$ & $0.04 \pm 0.003$ & $0.03 \pm 0.004$ & $0.04 \pm 0.023$ \\
\hline NPA & $10.01 \pm 0.03$ & $0.87 \pm 0.326$ & $6.60 \pm 0.43$ & $0.34 \pm 0.024$ & $5.60 \pm 0.077$ & $0.53 \pm 0.183$ \\
\hline TNE & $1.07 \pm 0.05$ & $0.059 \pm 0.023$ & $0.95 \pm 0.966$ & $0.07 \pm 0.004$ & $0.70 \pm 0.007$ & $0.064 \pm 0.015$ \\
\hline DHA & $13.41 \pm 0.064$ & $4.57 \pm 0.315$ & $5.31 \pm 1.855$ & $5.55 \pm 0.168$ & $6.19 \pm 0.002$ & $4.42 \pm 0.150$ \\
\hline EA & $7.24 \pm 0.059$ & $7.08 \pm 0.574$ & $8.33 \pm 0.014$ & $5.34 \pm 0.406$ & $9.38 \pm 0.217$ & $6.02 \pm 0.001$ \\
\hline PBO & $0.38 \pm 0.003$ & $0.07 \pm 0.004$ & $0.35 \pm 0.008$ & $0.053 \pm 0.003$ & $0.39 \pm 0.007$ & $0.063 \pm 0.006$ \\
\hline
\end{tabular}

Table 4-6: Substrate-specific specific activities of GSTs from D. melanogaster.

Unbound activity from GSH matrix was applied equally to DNP-GSH and CNP-GSH matrices. Assays were undertaken following the methods in section 3.2.4. Activities were determined in triplicate of three experiments. Results are presented as mean \pm S.D.

\begin{tabular}{|c|c|c|c|c|c|c|}
\hline \multirow[t]{2}{*}{ Substrate } & \multicolumn{6}{|c|}{ Specific activity $(\mu \mathrm{mol} / \mathrm{min} / \mathrm{mg}$ protein $)$} \\
\hline & $\begin{array}{l}\text { Unbound } \\
\text { GSH }\end{array}$ & $\begin{array}{l}\text { Bound } \\
\text { GSH }\end{array}$ & $\begin{array}{l}\text { Unbound } \\
\text { DNP-GSH }\end{array}$ & $\begin{array}{c}\text { Bound } \\
\text { DNP-GSH }\end{array}$ & $\begin{array}{l}\text { Unbound } \\
\text { CNP-GSH }\end{array}$ & $\begin{array}{c}\text { Bound } \\
\text { CNP-GSH }\end{array}$ \\
\hline CDNB & $0.10 \pm 0.002$ & $38.5 \pm 0.94$ & $0.076 \pm 0.02$ & $14.2 \pm 0.72$ & $0.064 \pm 0.004$ & $12.0 \pm 0.25$ \\
\hline DCNB & $0.004 \pm 0.002$ & $0.26 \pm 0.02$ & $0.004 \pm 0.002$ & $0.42 \pm 0.04$ & $0.002 \pm 0.001$ & $0.45 \pm 0.26$ \\
\hline NPA & $0.15 \pm 0.0002$ & $2.83 \pm 1.06$ & $0.178 \pm 0.04$ & $3.80 \pm 0.26$ & $0.152 \pm 0.004$ & $6.00 \pm 2.06$ \\
\hline TNE & $0.016 \pm 0.008$ & $1.91 \pm 0.07$ & $0.02 \pm 0.006$ & $0.82 \pm 0.04$ & $0.02 \pm 0.0002$ & $0.64 \pm 0.16$ \\
\hline DHA & $0.21 \pm 0.190$ & $14.8 \pm 1.03$ & $0.23 \pm 0.24$ & $61.2 \pm 14.8$ & $0.264 \pm 0.004$ & $49.6 \pm 1.70$ \\
\hline EA & $0.11 \pm 0.004$ & $23.1 \pm 1.87$ & $0.494 \pm 0.012$ & $58.8 \pm 4.50$ & $0.40 \pm 0.002$ & $67.6 \pm 0.01$ \\
\hline PBO & $0.004 \pm 0.002$ & $0.24 \pm 0.01$ & $0.022 \pm 0.002$ & $0.55 \pm 0.03$ & $0.016 \pm 0.002$ & $0.63 \pm 0.06$ \\
\hline
\end{tabular}




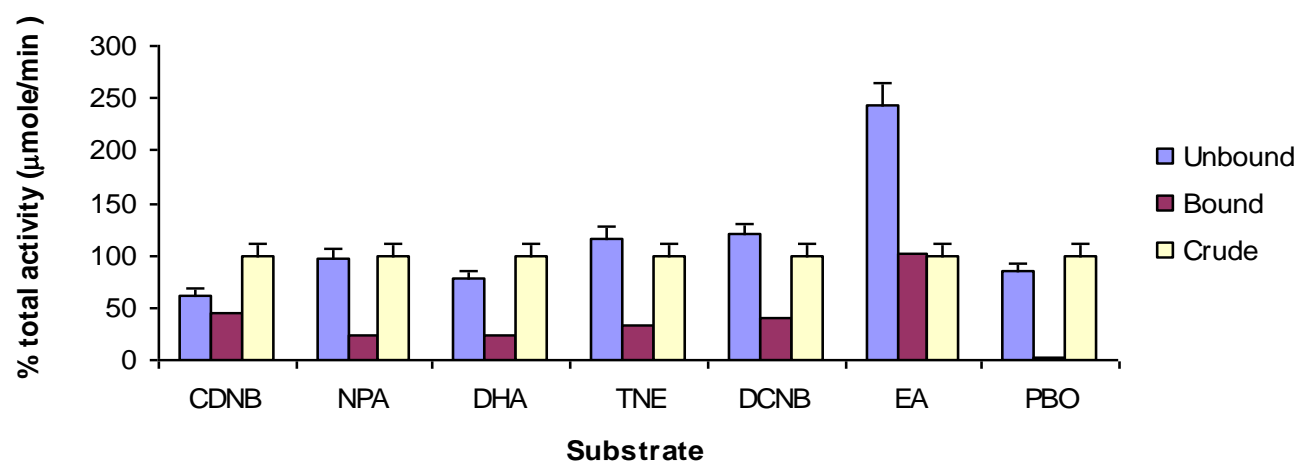

DNP-GSH matrix

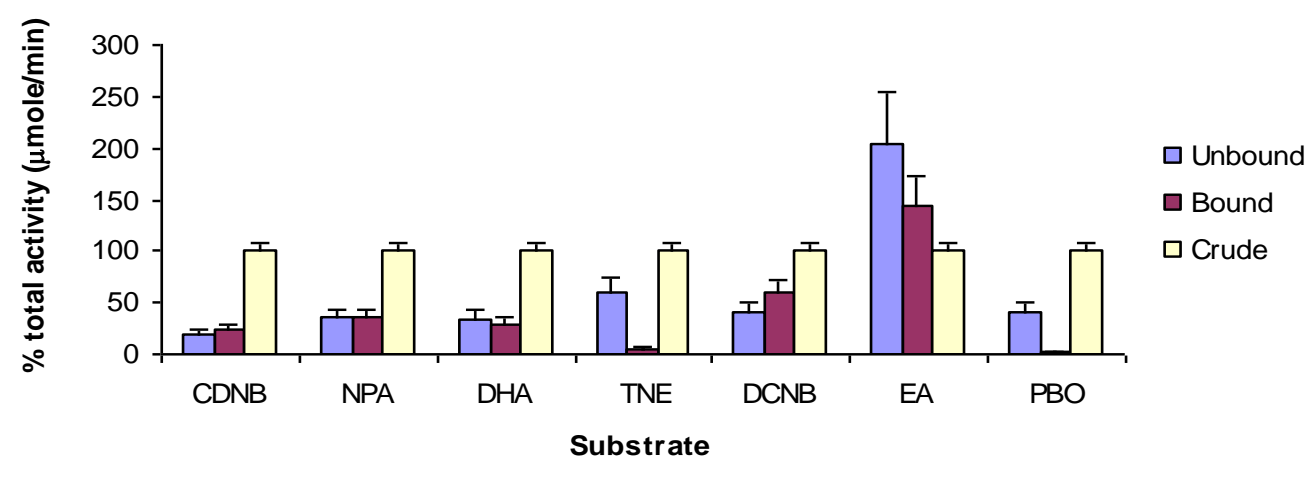

CNP-GSH matrix

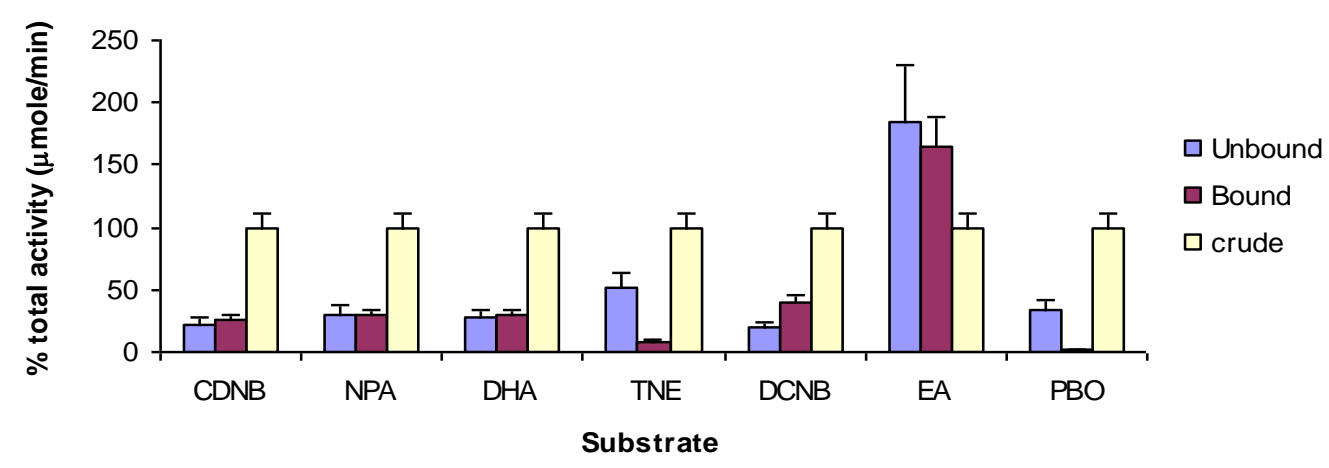

Figure 4-12: Comparison of substrate-specific total activities of unbound and bound fractions of $L$. cuprina from different affinity matrices.

The unbound fractions from the GSH matrix with significant activity towards CDNB were pooled and applied equally to DNP-GSH and CNP-GSH affinity matrices. Assays were undertaken following the methods in section 3.2.4. The values of $\%$ total activity calculated relative to the value of crude enzyme measured in triplicates from three independent experiments. Error bars represent standard deviation. 
Table 4-7: Substrate-specific total activity of GSTs from L. cuprina.

Unbound activity from the GSH matrix was applied equally to DNP-GSH and CNP-GSH matrices. Assays were undertaken following the methods in section 3.2.4. Activities were determined in triplicate of three experiments. Results are presented as mean \pm S.D.

\begin{tabular}{lcccccc}
\hline Substrate & \multicolumn{5}{c}{ Total activity $(\mu \mathrm{mol} / \mathbf{m i n})$} \\
\cline { 2 - 7 } & $\begin{array}{c}\text { Unbound } \\
\text { GSH }\end{array}$ & $\begin{array}{c}\text { Bound } \\
\text { GSH }\end{array}$ & $\begin{array}{c}\text { Unbound } \\
\text { DNP-GSH }\end{array}$ & $\begin{array}{c}\text { Bound } \\
\text { DNP-GSH }\end{array}$ & DNP-GSH & CNP-GSH \\
\hline CDNB & $2.61 \pm 0.105$ & $1.83 \pm 0.024$ & $0.81 \pm 0.024$ & $1.01 \pm 0.006$ & $0.94 \pm 0.071$ & $1.06 \pm 0.002$ \\
DCNB & $0.06 \pm 0.002$ & $0.02 \pm 0.009$ & $0.02 \pm 0.001$ & $0.03 \pm 0.021$ & $0.01 \pm 0.001$ & $0.02 \pm 0.006$ \\
NPA & $3.25 \pm 0.011$ & $0.80 \pm 0.139$ & $1.18 \pm 0.026$ & $1.20 \pm 0.124$ & $1.03 \pm 0.022$ & $0.99 \pm 0.023$ \\
TNE & $0.43 \pm 0.003$ & $0.12 \pm 0.016$ & $0.22 \pm 0.003$ & $0.02 \pm 0.005$ & $0.19 \pm 0.007$ & $0.03 \pm 0.015$ \\
DHA & $12.72 \pm 0.228$ & $3.82 \pm 0.164$ & $5.54 \pm 0.078$ & $4.87 \pm 0.459$ & $4.39 \pm 0.045$ & $4.82 \pm 0.318$ \\
EA & $9.06 \pm 0.050$ & $3.82 \pm 0.305$ & $7.59 \pm 0.046$ & $5.41 \pm 0.178$ & $6.91 \pm 0.022$ & $6.17 \pm 0.851$ \\
PBO & $1.36 \pm 0.058$ & $0.05 \pm 0.009$ & $0.64 \pm 0.024$ & $0.03 \pm 0.008$ & $0.54 \pm 0.050$ & $0.04 \pm 0.004$ \\
\hline
\end{tabular}

Table 4-8: Substrate-specific specific activity of GSTs from L. cuprina.

Unbound activity from the GSH matrix was applied equally to DNP-GSH and CNP-GSH matrices. Assays were undertaken following the methods in section 3.2.4. Activities were determined in triplicate of three experiments. Results are presented as mean \pm S.D.

\begin{tabular}{|c|c|c|c|c|c|c|}
\hline \multirow[t]{2}{*}{ Substrate } & \multicolumn{6}{|c|}{ Specific activity $(\mu \mathrm{mol} / \mathrm{min} / \mathrm{mg}$ protein $)$} \\
\hline & $\begin{array}{l}\text { Unbound } \\
\text { GSH }\end{array}$ & $\begin{array}{l}\text { Bound } \\
\text { GSH }\end{array}$ & $\begin{array}{l}\text { Unbound } \\
\text { DNP-GSH }\end{array}$ & $\begin{array}{l}\text { Bound } \\
\text { DNP-GSH }\end{array}$ & $\begin{array}{l}\text { Unbound } \\
\text { DNP-GSH }\end{array}$ & $\begin{array}{l}\text { Bound } \\
\text { CNP-GSH }\end{array}$ \\
\hline CDNB & $0.1 \pm 0.02$ & $6.70 \pm 0.1$ & $0.07 \pm 0.01$ & $4.02 \pm 0.1$ & $0.07 \pm 0.004$ & $3.40 \pm 0.64$ \\
\hline DCNB & $0.004 \pm 0.002$ & $0.07 \pm 0.03$ & $0.002 \pm 0.002$ & $0.12 \pm 0.08$ & $0.002 \pm 0.002$ & $0.07 \pm 0.02$ \\
\hline NPA & $0.11 \pm 0.01$ & $2.94 \pm 0.51$ & $0.1 \pm 0.04$ & $4.80 \pm 0.49$ & $0.106 \pm 0.004$ & $3.20 \pm 0.07$ \\
\hline TNE & $0.014 \pm 0.002$ & $0.41 \pm 0.06$ & $0.014 \pm 0.004$ & $0.05 \pm 0.01$ & $0.014 \pm 0.002$ & $0.03 \pm 0.01$ \\
\hline DHA & $0.204 \pm 0.28$ & $14.0 \pm 0.6$ & $0.216 \pm 0.28$ & $19.5 \pm 1.84$ & $0.176 \pm 0.22$ & $15.6 \pm 1.03$ \\
\hline EA & $0.408 \pm 0.16$ & $14.0 \pm 1.12$ & $0.66 \pm 0.12$ & $21.6 \pm 0.71$ & $0.642 \pm 0.16$ & $20.0 \pm 2.76$ \\
\hline PBO & $0.044 \pm 0.002$ & $0.20 \pm 0.03$ & $0.04 \pm 0.001$ & $0.14 \pm 0.3$ & $0.036 \pm 0.004$ & $0.12 \pm 0.01$ \\
\hline
\end{tabular}




\section{D. melanogaster}

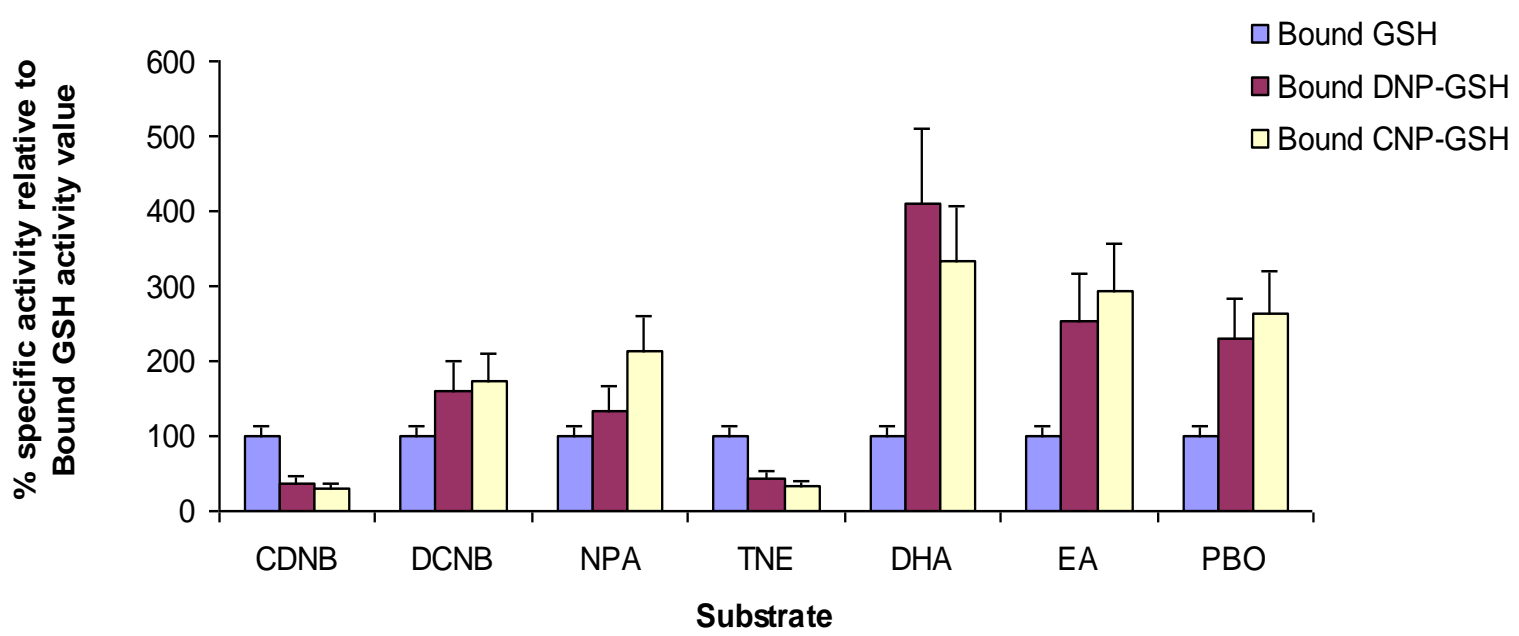

L. cuprina

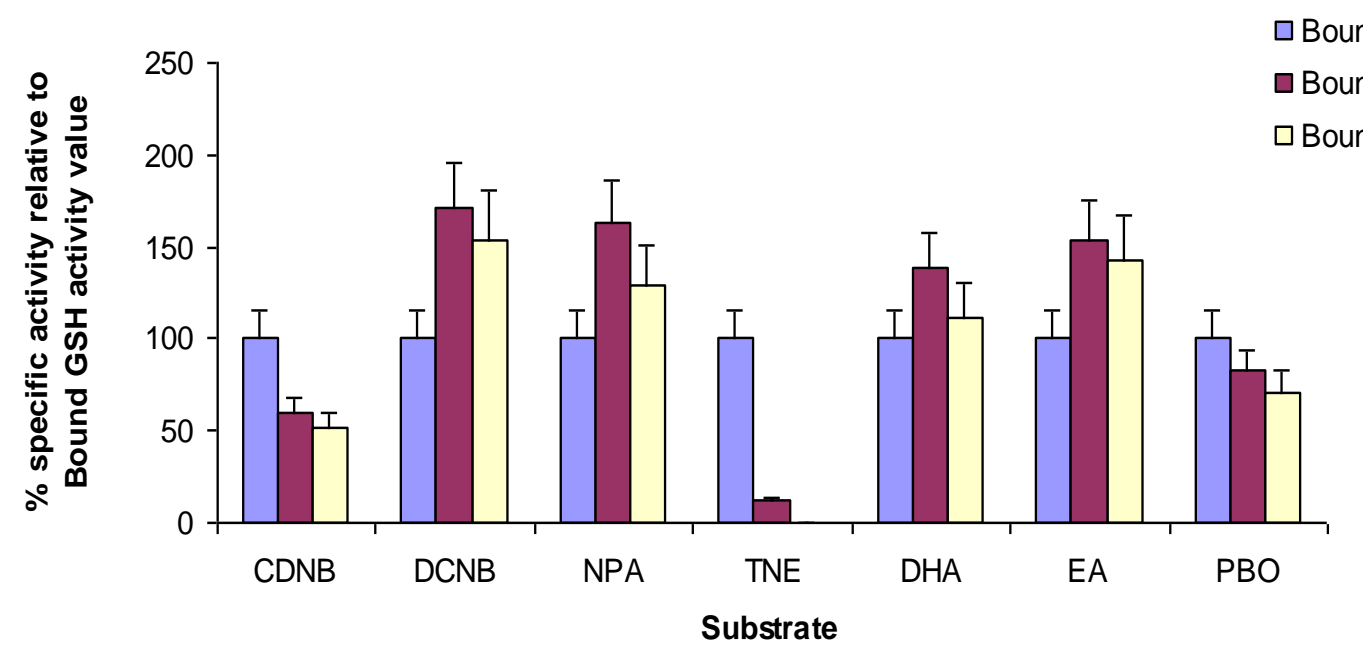

Figure 4-13: Comparison of specific activities of D. melanogaster and L. cuprina purified GSTs from different affinity matrices.

The unbound fractions from the GSH matrix with significant activity with CDNB were pooled and applied equally to DNP-GSH and CNP-GSH affinity matrices. Assays were undertaken following the methods in section 3.2.4. The \% specific activities of purified GSTs from DNP-GSH and CNP-GSH matrices were calculated relative to the value of GSH affinity-purified GSTs measured in triplicates from three independent experiments. Error bars represent standard deviation. 


\subsubsection{Identification of purified GSTs}

In order to identify the GSTs purified by the different affinity matrices, two dimensional gel electrophoresis was carried out on the concentrated samples of purified enzymes as described in the section 3.2.7.2. The GST profiles of $D$. melanogaster and L. cuprina gels were compared. Mass fingerprinting analysis was carried out on the major spots using MALDI-TOF. The spectra were analysed by the software Data Explorer and the resulting monoisotopic masses $(\mathrm{MH}+)$ were submitted to Profound in order to identify the GSTs against NCBInr database, version dated 12/08/2008. For identification of D. melanogaster proteins the taxonomy "Drosophila melanogaster" was used. The identification of L. cuprina GSTs was challenging due to lack of a specific genetic database. The GSH affinitypurified GSTs from both the insects resolved on 2D gels in a similar separation pattern (Figure 4-17 and Figure 4-18) and were identified as Sigma and Delta GSTs (Table 4-9 and Table 4-10). Previously our laboratory sent a L. cuprina Sigma GST preparation to HortResearch, Hamilton, NZ for the amino acid sequencing and in the present study in addition to MALDI-TOF identification, that deduced Sigma sequence was matched against the $L$. cuprina Sigma matched peptides to confirm its identity. The only L. cuprina GST sequence available in the NCBInr database is that for GSTD1, which helped to confirm the identification of Delta GST spots. However, the DNP-GSH affinity-purified L. cuprina GSTs were resolved on 2D gels somewhat differently than D. melanogaster GSTs purified by the same matrix (Figure 4-20 and Figure 4-21). The L. cuprina peptides were matched against the "Other Metazoa" fragmentation database and GSTs in other species were matched. However, using this approach the classification of GSTs could not be reliably determined. Therefore, to tentatively assign a class to the matched GSTs in other species, a consensus 
sequence-based approach was developed to avoid problems that might result from individual variation.

\subsubsection{Development of a consensus sequence-based approach for tentative characterisation of $L$. cuprina GSTs}

A consensus sequence is a way of representing the results of a multiple sequence alignment, where related sequences are compared to each other, and similar functional sequence motifs are found. It shows which residues are most abundant in the alignment at each position. Specific sequence motifs can function as regulatory sequences and they are thought to be conserved across long periods of evolution (McEwen et al., 2009). In the present investigation, as mentioned before, the monoisotopic masses of L. cuprina peptides (obtained after tryptic digestion) were searched in Profound for similarity against the whole metazoa fragmentation database and thus their characterisation was not limited to insects. When the $L$. cuprina peptides were identified as GSTs in other metazoa species, the matched peptides were aligned with the consensus sequences of different insect GST classes to determine their relatedness. Hence the characterization of $L$. cuprina GSTs is tentative, based on $\%$ similarity and \% identity with the GST class consensus sequences. To derive the consensus sequences for all the insect GST classes, the sequences of each class from different insect species were aligned using the ClustalW2 alignment programme with default conditions. For example, to obtain the Sigma GST consensus sequence, D. melanogaster GSTS1 sequence has been matched against the Arthropoda database using BLAST software. The BLAST result identified Sigma GST sequences in different species (Figure 4-14). Out of all these representative matched sequences, those with high scores from different species were selected from genera closely related to L. cuprina. Here the sequences from 
Drosophila melanogaster, Musca domestica, Aedes aegypti, Blattella germanica, Bombyx mori, and Apis melliferra were selected and aligned by using the ClustalW2 alignment program (Figure 4-15). These alignment data were processed in the programme JalView to determine the consensus from the combined selected sequences (Figure 4-16). The consensus sequences for all other GST classes were derived the same way. For the consensus sequence of Delta GSTs, the different Delta subclasses from D. melanogaster D1, D2, D3, D4, D5, D10 and Delta GSTs from other species such as Musca domestica, Lucilia cuprina, Anopheles dirus, Blatella germanica and Culex quinquefasciatus were selected and aligned. For the consensus sequence of the Theta GSTs, the human Theta GST sequence was matched against the Arthropoda using BLAST and the Theta sequences from Anopheles gambiae, Aedes aegypti, Drosophila melanogaster, Bombyx mori, Nilaparvatha lugens, Blatella germanica and Culex quinquefasciatus were selected. The consensus sequence for the Mu GSTs was also obtained by matching the Boophilus microplus (Tick species) Mu GST in BLAST and the sequences from other ticks and mites with more than $60 \%$ similarity score were aligned in clustalW2. The Xi and Iota GSTs are reported to be uniquely present in mosquitoes (Ding et al., 2003; Lumjuan et al., 2007), their consensus sequences were determined and used to check for similarity with L. cuprina GST matched peptides. Once the consensus sequences for all the GSTs were derived as shown in Appendix 10.4, the sequences of matched peptides of the L. cuprina spots were aligned with them using ClustalW2 to identify the level of similarity between the peptides and each of the consensus sequences of GST classes. The tentative classification of the L. cuprina GST spots reported in Table 4-11 and Table 4-14 is based on this approach. The spots which are non-GST proteins served as controls, having no significant score. 
$\square$ sp P41043

$\square \operatorname{tr} \quad$ B4OAO1

$\square$ tr $34 \mathrm{P} 8 \mathrm{H} 2$

$\square \operatorname{tr} \quad$ B 4 HMH 8

$\square \operatorname{tr}$ A4UZL5

$\square$ tr B3NP18

$\square$ tr B3MD60

$\square$ tr B4J549

$\square$ tr Q28XE0

$\square$ tr B4GIT4

$\square$ tr B4LKG9

$\square$ tr $\quad B 4 \mathrm{KQG} 2$

$\square$ tr B4N5s8

$\square \operatorname{sp} \quad$ P46437

$\square$ tr $\quad$ A 8 CWB

$\square$ tr A8CAE7

vtr 016P79

$\square$ tr Q16P78

$\square$ tr BOWFXO

$\square$ tr Q8MUR9

$\square$ sp P46428

$\square$ tr A1XSY5

$\square$ tr Q16P80

$\square$ sp vs P46428-2

$\square$ tr c3v9u8

$\square$ tr COLRA4

$\square$ tr BOWFW9

v sp 018598

$\square$ tr c3v9u6

$\square$ tr C4WTN3

v tr $05 \mathrm{CCJ} 4$

$\square$ tr Q6F436

1 tr C6ZJZ8

$\square$ tr B6VCW8

$\square$ tr 076783

$\square$ tr e3HVI8

$\square \operatorname{tr} \quad 06 \times 4 \mathrm{~T} 7$

$\square$ tr D3JYQ6

$\square \operatorname{tr} \quad \mathrm{B} 2 \mathrm{BNZ} 2$

$\square$ tr D2CSP9

$\square \operatorname{tr} \quad \mathrm{C2SOO}$

P4 6429

P46429

C3VMN1

Q4ACU 7

$\mathrm{A} 0 \mathrm{FRH} 3$

Q9U4X1

Q6UJ46

Q6F 443

096097

096096

Q4 JHJ5

C1BP72

Q49M30

Q6 JVN0

C1C1s2

B7SP22

Q6 JVM9

B7QGH2

Q2YFE4

BOKZJ1

GST1_DROME Glutathione S-transferase S1 (EC 2.5.1.18) . . 415 e-115 DROSI GD25495 [GD25495] [Drosophila simulans (Fruit fly)] 415 e-115 _DROYA GE14214 [GE14214] [Drosophila yakuba (Fruit fly)] 415 e-115 DROSE GM20005 [GM20005] [Drosophila sechellia (Fruit f... 415 e-115 DROME Glutathione S transferase S1, isoform C (EC 1.11... 415 e-115 _DROER GG22217 [GG22217] [Drosophila erecta (Fruit fly)] 415 e-115 DROAN GF13422 [GF13422] [Drosophila ananassae (Fruit f... 409 e-114 DROGR GH20881 [GH20881] [Drosophila grimshawi (Fruit f... 409 e-113 DROPS GA21422 [GA21422] [Drosophila pseudoobscura pseu... 407 e-113 _DROPE GL17264 [GL17264] [Drosophila persimilis (Fruit .. 407 e-113 DROVI GJ20716 [GJ20716] [Drosophila virilis (Fruit fly)] 405 e-112 _DROMO GI21003 [GI21003] [Drosophila mojavensis (Fruit... 404 e-112 DROWI GK17930 [GK17930] [Drosophila willistoni (Fruit .. 400 e-111 GST_MUSDO Glutathione S-transferase (EC 2.5.1.18) (GST. . 389 e-108 LUTLO Putative glutathione s-transferase [GST1] [Lutzo... $3176 e-86$ _PHLPP Glutathione s-transferase-like protein [Phleboto... $3176 e-86$ AEDAE Glutathione s-transferase [AAEL011741] [Aedes ae... 302 2e-81 _AEDAE Glutathione s-transferase [AAEL011741] [Aedes ae... 302 2e-81 CULQU Glutathione s-transferase [CpipJ CPIJ006160] [Cu... 2974 4e-80 ANOGA Glutathione S-transferase S1-2 (Fragment) [GSTs1... $2906 e-78$ GST ANOGA Glutathione S-transferase (EC 2.5.1.18) (GST .. 290 8e-78 MAYDE Sigma GST [GST-2] [Mayetiola destructor (Hessian... 289 1e-77 AEDAE Glutathione s-transferase [AAEL011741] [Aedes ae... 289 1e-7] Isoform B of Glutathione S-transferase OS=Anopheles ... $2852 e-76$ CHITE Glutathione S-transferase (EC 2.5.1.18) [GSTs4] . . 2614 4 469 AEDAL Glutathione S-transferase (Fragment) [Aedes albo... $2567 e-68$ _CULQU Glutathione-requiring prostaglandin D synthase [... $2239 e-58$ GST1 BLAGE Glutathione S-transferase (EC 2.5.1.18) (GS... $2175 e-56$ _CHITE Glutathione S-transferase (EC 2.5.1.18) [GSTs2] . . $2175 e-56$ _ACYPI ACYPI009326 protein [ACYPI009326] [Acyrthosiphon... $1852 e-46$ BOMMO Glutathione S-transferase sigma [GST sigma] [Bom... $1844 e-46$ - PLUXY Glutathione S transferase 2-like protein [Plutel... 182 2e-45 CHOFU Glutathione S-transferase 4 (EC 2.5.1.18) [Chori... 181 3e-45 APIME Glutathione S-transferase HP19 [Apis mellifera (... $1745 e-43$ 9NEOP Glutathione transferase [PigstII] [Platynota ida... 174 6e-43 _SOLIN Glutathione S-transferase [GSTS1] [Solenopsis in... 173 1e-42 SOLIN Glutathione S-transferase [GSTS1] [Solenopsis in... 172 1e-42 ANTPE Glutathione S-transferase sigma [Antheraea perny... 172 2e-42 HELAM Glutathione S-transferase GSTX01 [GSTx01] [Helic... 168 3e-41 BEMTA Glutathione-S-transferase (Fragment) [Bemisia ta... $1605 e-39$ BEMTA Glutathione-S-transferase (Fragment) [Bemisia ta... $1607 e-39$ GST2 MANSE Glutathione S-transferase 2 (EC 2.5.1.18) (... 159 1e-38 BOMMO Glutathione S-transferase 2 [Bombyx mori (Silk m... $1521 e-36$ APIME Glutathione S-transferase S1 [Apis mellifera (Ho... $1506 e-36$ HYPCU Glutathione S-transferase [gst] [Hyphantria cune... 138 4e-32 LEPDE Putative glutathione S-transferase GST3 (Fragmen... 127 5e-29 CHOFU Glutathione S-transferase (EC 2.5.1.18) [GST] [C... 122 2e-27 CORCP GST-like hemolymph protein [CHP19] [Corcyra ceph... 114 5e-25 PLUXY Glutathione S-transferase [Plutella xylostella (.. 113 1e-24 _ANTYA $24 \mathrm{kDa}$ female-specific fat body protein [Anthera... $971 e-19$ ANTPE $24 \mathrm{kDa}$ female-specific fat body protein [Anthera... 94 1e-18 9MAXI Glutathione S-transferase [GST] [Tigriopus japon... 77 1e-13 9MAXI Glutathione S-transferase P [GSTP1] [Caligus rog... 72 3e-12 ARAVE Gluthatione S-transferase [GST] [Araneus ventric... 678 e-11 HAELO Glutathione S-transferase (EC 2.5.1.18) [GST] [H... 655 -10 9MAXI Glutathione S-transferase Mu 3 [GSTM3] [Caligus ... 62 3e-09 DERVA Putative glutathione s-transferase [Dermacentor ... $618 e-09$ RHIAP Glutathione S-transferase (EC 2.5.1.18) [GST] [R... 60 1e-08 IXosC Glutathione S-transferase, putative (EC 2.5.1.18... $602 e-08$ DERPT Glutathione transferase mu class Dp7002H05 [Derm... 59 3e-08 ACASI Allergen Aca s 8 [Acarus siro (Dust mite)]

58 5e-08

Figure 4-14: BLAST result of D. melanogaster Sigma GSTS1 against the Arthropoda fragmentation database.

The sequences of GSTs marked with a tick in the left hand column were selected to obtain the consensus sequence for Sigma GST. 


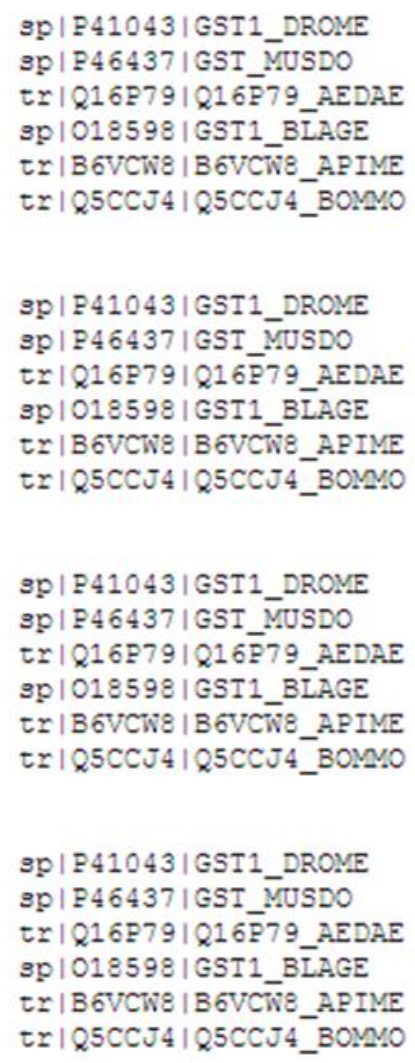

3p | P41043|GST1_DROME

sp | P46437| |GST_MUSDO

tr|Q16P79|Q16P79_AEDAE sp|018598|GST1_BIAGE tr|B6VCW8|B6VCW8_APIME tr|Q5CCJ4 4 Q 5 CCJ4_BONO

MADEA-QAPPAEG---APPAEGEAPPPAEGAEGAVEGGEAAPPAEPAEPI 46 MADEAPAAPPAEGEAPAAPAEGEAPPPAEG----------EAP---PAEPV 38 MSIQI-DIVLCHG-VIIKPLKFVVQTLINAS--------------SQNPN 34

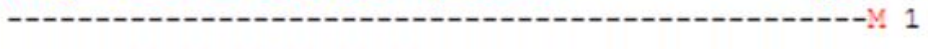

-

KHSYTLFYFNVKALAEPLRYLFAYGNQEYEDVRVTR--DEWPALKPTMPM 94 KNTYTLFYFNVKALAEPLRYLFAYGGIEYEDVRVTR--DEWPALKPTMPM 86 MPDYKVYYFIVKALGEPLRELLSYGNLPFDDIRITR--EEWPALKPTMPM 82 APSYKLTYCPVKALGEPIRFLLSYGEKDFEDYRFQE--GDWPNLKPSMPE 49 MASYKLVYFNVNGLGEPIRFLISYGGVEFEDVRITHSSEDWSNMKPTTPF 50 MPNVKFYYFPVKALGESQRLILAYGGQEFEDNRISS--ENWPEFKPKTPE 48

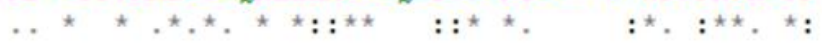

GQMPVLEVDGKRVHQSISMARELAKTVGLCGATPWEDIQIDIVVDIINDF 144 GQMPVLEVNGKRVHQSISMARFLAKTVGLCGATPWEDLQVDIVVDIINDF 136 GQMPVLSVDGKKVHQSVAMSRYLAKQVGLAGADDWENLMIDTVVDTINDE 132 GKTPVLEIDGKQTHQSVAISRYLGKQFGLSGKDDWENLEIDMIVDIISDE 99 GQLPTLEINGKVYSQTLPICRYLAKQFNLIGKTDIDTLQIDAIASALYDF 100 GQMPVLEIDGKQYAQSTAICRYLGRKYGLAGANDEEAFEIDQNVEFLNDI 98

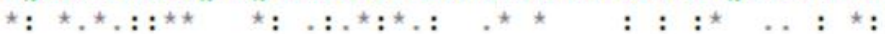

R-LKIAVVSYEPEDEIKEKKLVTLNAEVIPFYLEKLEQTVKDNDGHLALG 193 R-LKIAVVSYEPEDEIKEKKLVTLNNEVIPFYLEKLEQTVKDNDGHLALN 185 R-LKIAVVSYEPDDDVKEKKLVILNSEVIPFYLEKLDDIARDNGGHMANG 181 R-AAIANYHYDADENSKQKKWDPLKKEIIPYYTKKEDEVVKANGGYLAAG 148 RWLTISSYYRESDPVLKAKKKKVVMTRVI PFYLNKLEELAKANGGYLHGD 150 R-ASAASVHYEKDEAVKAKKKAELEETKYPFFFEKLNEILTKMNGHIALG 147 ${ }^{\star} \quad: \quad:: \quad *{ }^{*} \quad: \quad{ }^{\star}:::{ }^{\star}::: \quad{ }^{*},{ }^{\star}::$

KLTWADVYFAGITDYMVYNKR-DLIEPYPALRGVVAVNALEPIKAWIE 242 KLTWADVYFAGILDYMTYMVR-DILEQYPALRGVVDSVNAIEPIKAWIE 234 KLTWADMYFVAILDYLNYMTKS-DLVANHPNLQRVVDNVTSIDSIKAWID 230 KLTWADEYEVAILDYLNHMAKE-DLVANQPNLKALREKVLGLPAIKAWVA 197 KLSYADLFEVGISDSLNTAYES-DITNDKPYLKSLKQKILAIPNIKANVE 199 KLTWGDFYAGMYYLKAMLQKPDLEQKYPAFRKPIEAVLAIPKVKAYVD 197

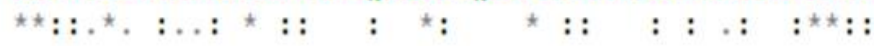

$\begin{array}{cc}\text { KRPVIEV } & 249 \\ \text { KRPQTEV } & 241 \\ \text { KRPQTEI } & 237 \\ \text { KRPPTDL } 204 \\ \text { KRPKLEF } 206 \\ \text { ARPRTEL } 204 \\ \text { * } \quad \text {. }\end{array}$

Figure 4-15: ClustalW2 alignment result of Sigma GST sequences from the different insects. The "*" means that the residues in that column are identical in all sequences in the alignment. ":" means that conservative substitutions have been observed "." means that semiconserved substitutions are observed. DROME $=D$. melanogaster, $\mathrm{MUSDO}=M$. domestica, $\mathrm{AEDAE}=A$. aegypt, $\mathrm{BLAGE}=$ B. germanica, $\mathrm{APIME}=A$. melliferra and $\mathrm{BOMMO}=$ Bombyx mori 


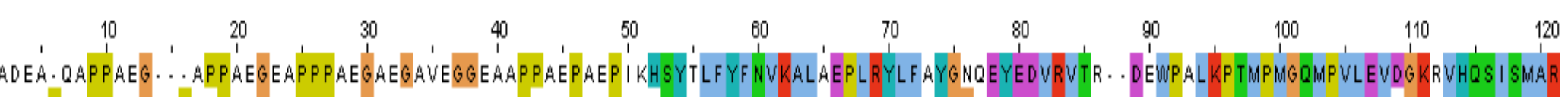

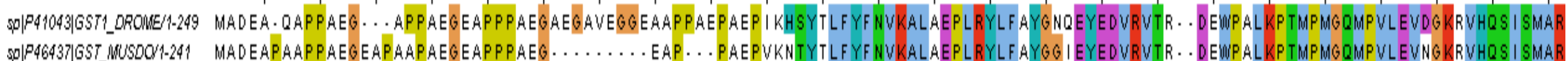

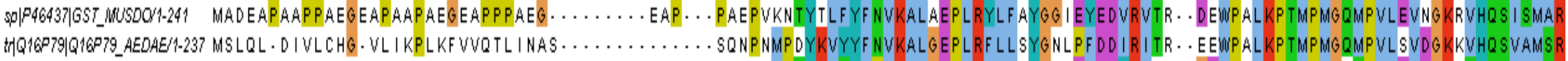

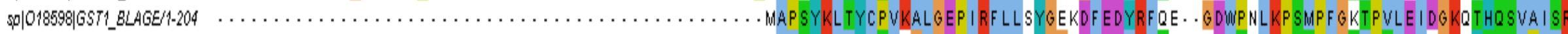

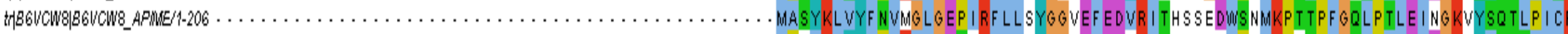

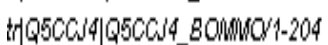
MPNVKFYYFPVKALGESQRLLLAYGGQEF EDNRISS. ENWPEFKPKTPFGQMPVLEI DGKOYAQSTAICR

120
Mo

Figure 4-16: Alignment result in Jalview.

The selected sequences from D. melanogaster (DROME), M. domestica (MUSDO), A. aegypti (AEDAE), B. germanica (BLAGE), A. melliferra (APIME) and Bombyx mori (BOMMO) were aligned in ClustalW2 and the amino acid similarity is shown in Jalview window. 


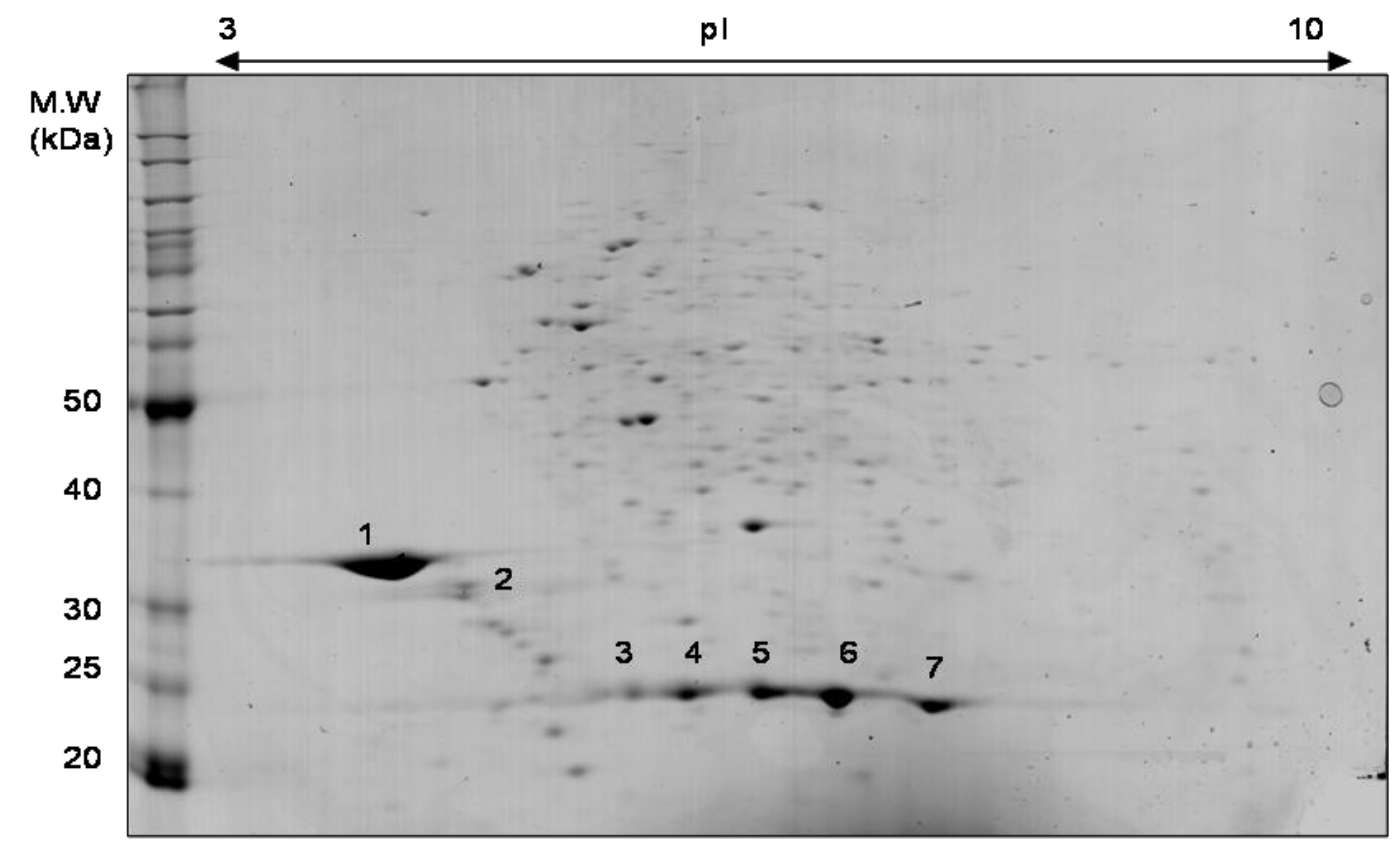

Figure 4-17: Two dimensional gel electrophoresis of GSH affinity-purified D. melanogaster GSTs.

Glutathione affinity- purified D. melanogaster GSTs were separated in the first dimension on a $7 \mathrm{~cm}$ pH 3-10 linear IPG strip and then in the second dimension on a SDS-PAGE gel. The M.W. marker was run along the sample. The gel was stained with Coomassie Brilliant Blue G-250. The identification of the numbered spots can be found in Table 4-9. 
Table 4-9: Identification of D. melanogaster GSTs purified by GSH affinity matrix.

teins identified from the GSH affinity-purified fractions of D. melanogaster separated over a pH range of 3-10 (Figure 4-17) using 2D gel electrophoresis as described in section 3.2.7.2. The proteins were identified using MALDI-TOF peptide mass fingerprinting. Protein spots are as numbered in Figure 4-17. Z score, the sequence coverage and the number of matched peptides are reported as obtained from Profound. In addition the protein name, the SwissProt/TrEMBL accession numbers, the computed molecular mass and isoelectric point are given as obtained from the ExPASy Proteomics server of Swiss Institute of Bioinformatics and the Swiss-Prot database. Identification was made by using taxonomy D. melanogaster and the NCBInr database, version dated 12/08/2008. A protein Z score greater than 1.65 is significant in Profound. The matched peptide sequences were searched against the sequences of all $D$. melanogaster GST classes and unique peptide sequences for each isoform of GSTs are listed in Appendix section 10.3.2.

\begin{tabular}{|c|c|c|c|c|c|c|c|c|c|}
\hline $\begin{array}{r}\text { Spot } \\
\text { No. }\end{array}$ & $\begin{array}{c}\text { NCBInr } \\
\text { Accession } \\
\text { (Tgi) }\end{array}$ & $\begin{array}{l}\text { Swiss-prot } \\
\text { /TrEMBL } \\
\text { Accession No. }\end{array}$ & Identified protein & $\begin{array}{l}\text { GST class } \\
\text { identified }\end{array}$ & $\begin{array}{c}\text { Theoretical } \\
\text { M.W./pI }\end{array}$ & $\begin{array}{c}\text { Experimental } \\
\text { M.W./pI }\end{array}$ & $\begin{array}{c}\text { Number of } \\
\text { matched } \\
\text { peptides }\end{array}$ & $\begin{array}{c}\text { Coverage } \\
(\%)\end{array}$ & $\mathbf{Z}$ score* \\
\hline 1 & 24654347 & P41043 & GST S1 & Sigma & $27.65 / 4.6$ & $33 / 4.6$ & 15 & 35 & 2.43 \\
\hline 2 & 24654347 & P41043 & GST S1 & Sigma & $27.65 / 4.6$ & $31 / 4.9$ & 20 & 64 & 2.43 \\
\hline 3 & 17737923 & P20432 & GST D1 & Delta & $23.89 / 6.8$ & $24 / 5.5$ & 6 & 38 & 2.43 \\
\hline 4 & 17737923 & P20432 & GST D1 & Delta & $23.89 / 6.8$ & $24 / 5.7$ & 8 & 33 & 2.43 \\
\hline 5 & 17737923 & P20432 & GST D1 & Delta & $23.89 / 6.8$ & $24 / 6.0$ & 7 & 35 & 2.43 \\
\hline 6 & 17737923 & P20432 & GST D1 & Delta & $23.89 / 6.8$ & $23.5 / 6.4$ & 8 & 33 & 2.43 \\
\hline 7 & 17737923 & P20432 & GST D1 & Delta & $23.89 / 6.8$ & $23 / 7.0$ & 5 & 38 & 2.43 \\
\hline
\end{tabular}




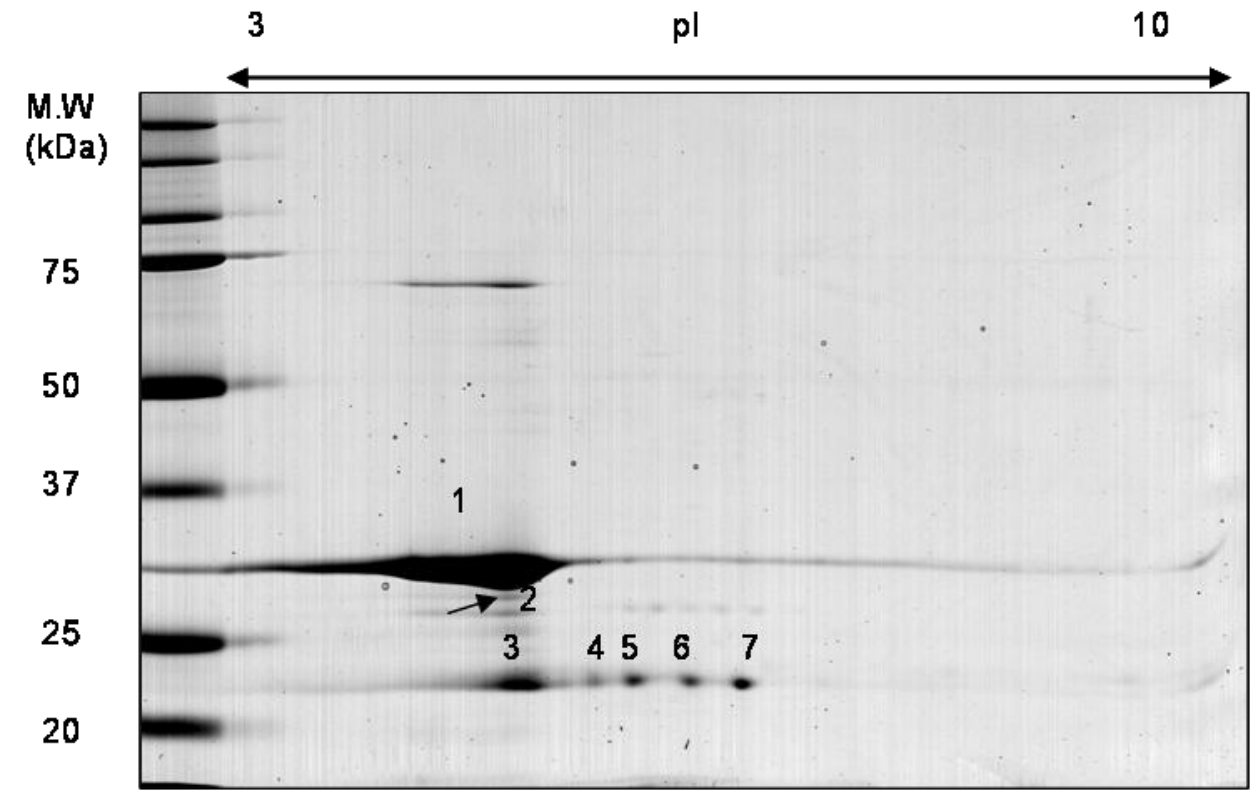

Figure 4-18: Two dimensional gel electrophoresis of GSH affinity-purified L. cuprina GSTs. Glutathione affinity-purified L. cuprina GSTs were separated in the first dimension on a 7 cm pH 3-10 linear IPG strip and then in the second dimension on a SDS-PAGE gel. The M.W. marker was run along the sample. The gel was stained with Coomassie Brilliant Blue G-250. The identification of the numbered spots can be found in Table 4-10. The arrow shows the spot 2 . 
Table 4-10: Identification of L. cuprina GSTs purified by GSH affinity matrix.

Proteins identified from the GSH affinity-purified fractions of L. cuprina separated over a pH range of 3-10 (Figure 4-18) using 2D gel electrophoresis as described in section 3.2.7.2. The proteins were identified using MALDI-TOF peptide mass fingerprinting. Protein spots are as numbered in Figure 4-18. Z score, the sequence coverage and the number of matched peptides are reported as obtained from Profound. In addition the protein name, the SwissProt/TrEMBL accession numbers, the computed molecular mass and isoelectric point are given as obtained from the ExPASy Proteomics server of Swiss Institute of Bioinformatics and the Swiss-Prot database. Identification was made by using taxonomy D. melanogaster and Other Metazoa NCBInr database, version dated 11/12/2007. A protein Z score greater than 1.65 is significant in Profound.

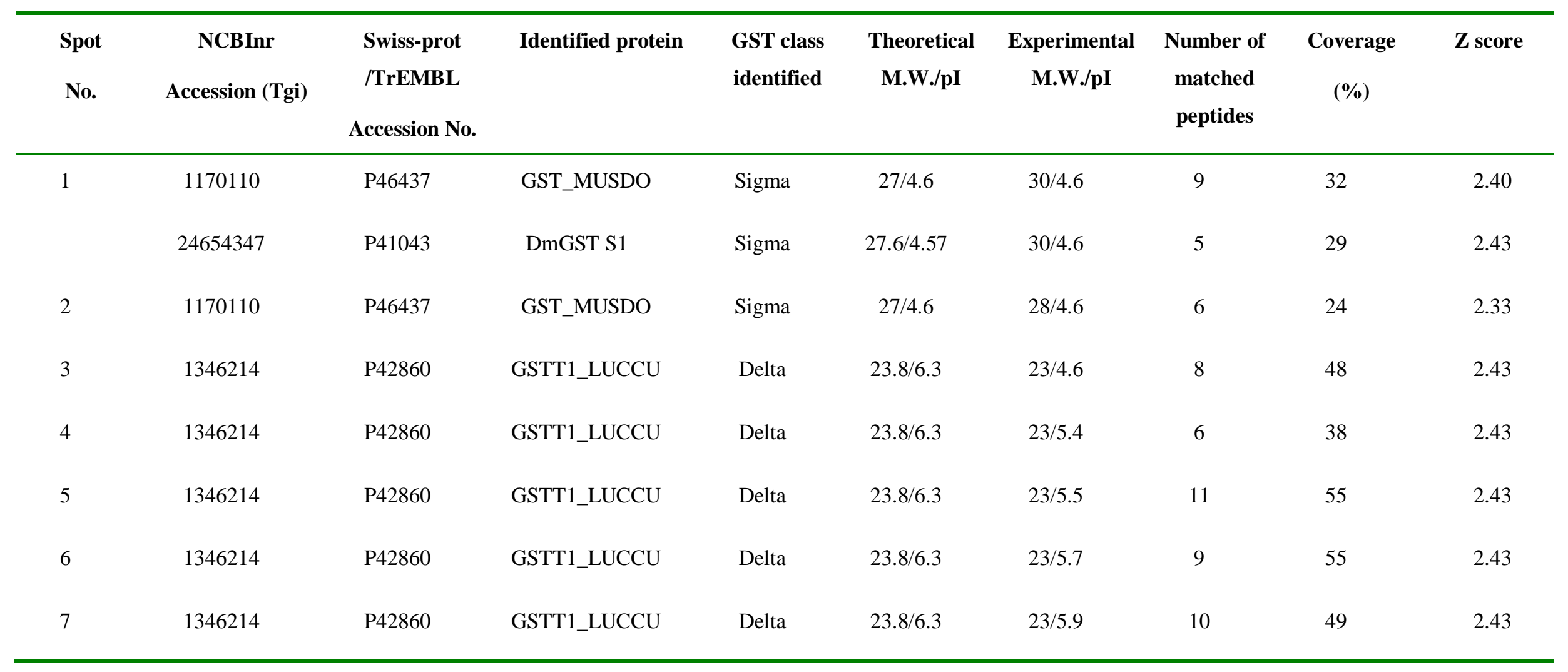


The result obtained from the proteins that bound to the GSH affinity matrix showed that at least 7 spots were detected on both the gels (Figure 4-17 and Figure 4-18) and Table 4-9 and Table 4-10 summarise the identification of each spot based on significant $\mathrm{Z}$ scores (>1.65). The spots 1 and 2 in Figure 4-17 and Figure 4-18 have been identified as Sigma GST and it appears as if spot 2 is due to degradation of a Sigma GST. The peptides from $L$. cuprina spot 1 matched against $M$. domestica and $D$. melanogaster Sigma GST. These matched peptide sequences were aligned with the deduced amino acid sequence of L. cuprina Sigma GST previously obtained by our laboratory as shown in Figure 4-19. The clustalW2 alignment score was 80 and 76 against $M$ Musca and D. melanogaster respectively confirming the identity of spot 1 as Sigma GST. The rest of the spots have been identified as Delta GSTs and particularly the D1 class of GSTs as shown in the Table 4-9 (D. melanogaster spots) and Table 4-10 (L. cuprina spots) approach (matching against the consensus sequences of GSTs) is shown in Table 4-11. 
Deduced Lucilia sequence
Lucilia peptides matched against Musca

Deduced Lucilia sequence Lucilia peptides matched against Musca

Deduced Lucilia sequence Lucilia peptides matched against Musca

Deduced Lucilia sequence Lucilia peptides matched against Drosophila

Deduced Lucilia sequence Lucilia peptides matched against Drosophila

Deduced Lucilia sequence Lucilia peptides matched against Drosophila

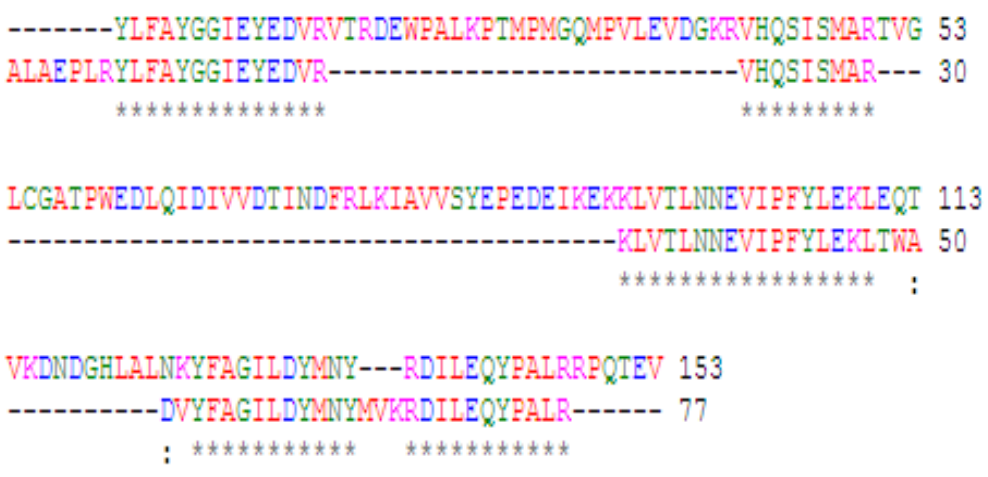

B

YLFAYGGIEYEDVRVTRDEWPALKPTMPNGQMPVIEVDGKRVHOSISMARTVGICGATPN 60 --------------------ALAEPL-------------RVHQSISMARTVGICGATPN 26

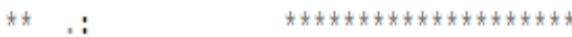

EDIQIDTWDTINDERLKIAWVYEPEDEIKEKKLVILNNEVIPFYLEKIEQTVKDNDGH 120

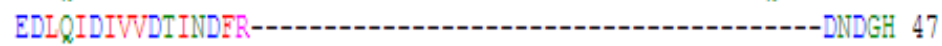
$t * t * t * t * t * t * t * k t *$

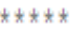

LALINK------YFAGILDYMTYRDILEQYPALRRPQIEV 153

LALGKLTWADVYFAGITDYMTY--MVK------------- 72

$\star * *, * \quad * k * t * \quad * k * k * \quad:::$

Figure 4-19: Alignment of peptide sequences of L. cuprina spot 1(Figure 4-18) matched against M. domestica (A) and D. melanogaster (B) (Table 4-10) using ClustalW2.

The list of the peptide sequences can be found in Appendix section 10.3.4. See the legend to Figure 4-15 for the details. 
Table 4-11: Assignment of class of GST using the ClustalW2 programme.

The peptides of L. cuprina GSH affinity-purified spots on 2D gel matched against "Other Metazoa" were aligned with consensus sequences of insect GSTs using the ClustalW2 alignment programme. The highest match score is highlighted in red text. The identification of each spot is shown in Table 4-10

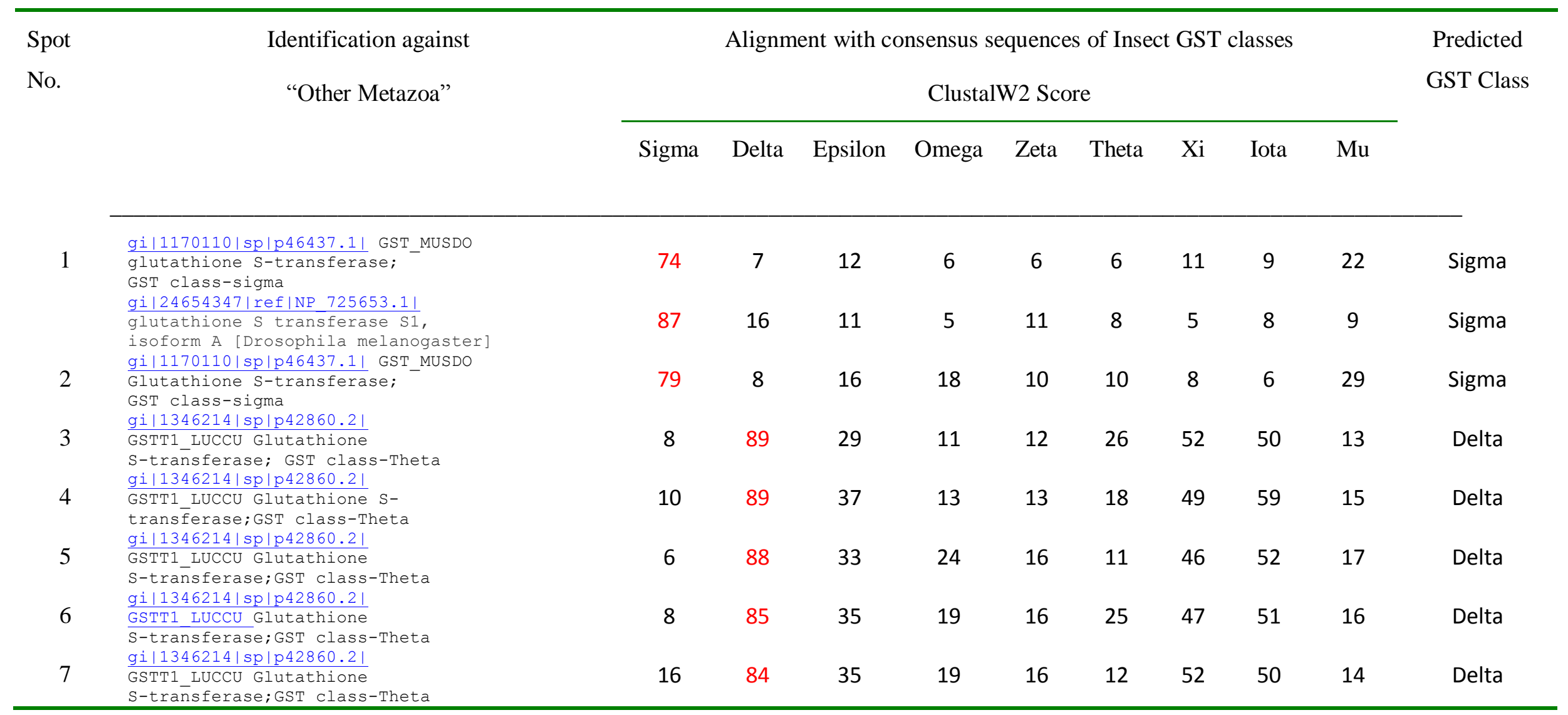


The unbound fractions of GSH matrix from $D$. melanogaster were further purified by DNP-GSH and CNP-GSH affinity matrices and purified fractions resolved by 2D electrophoresis.

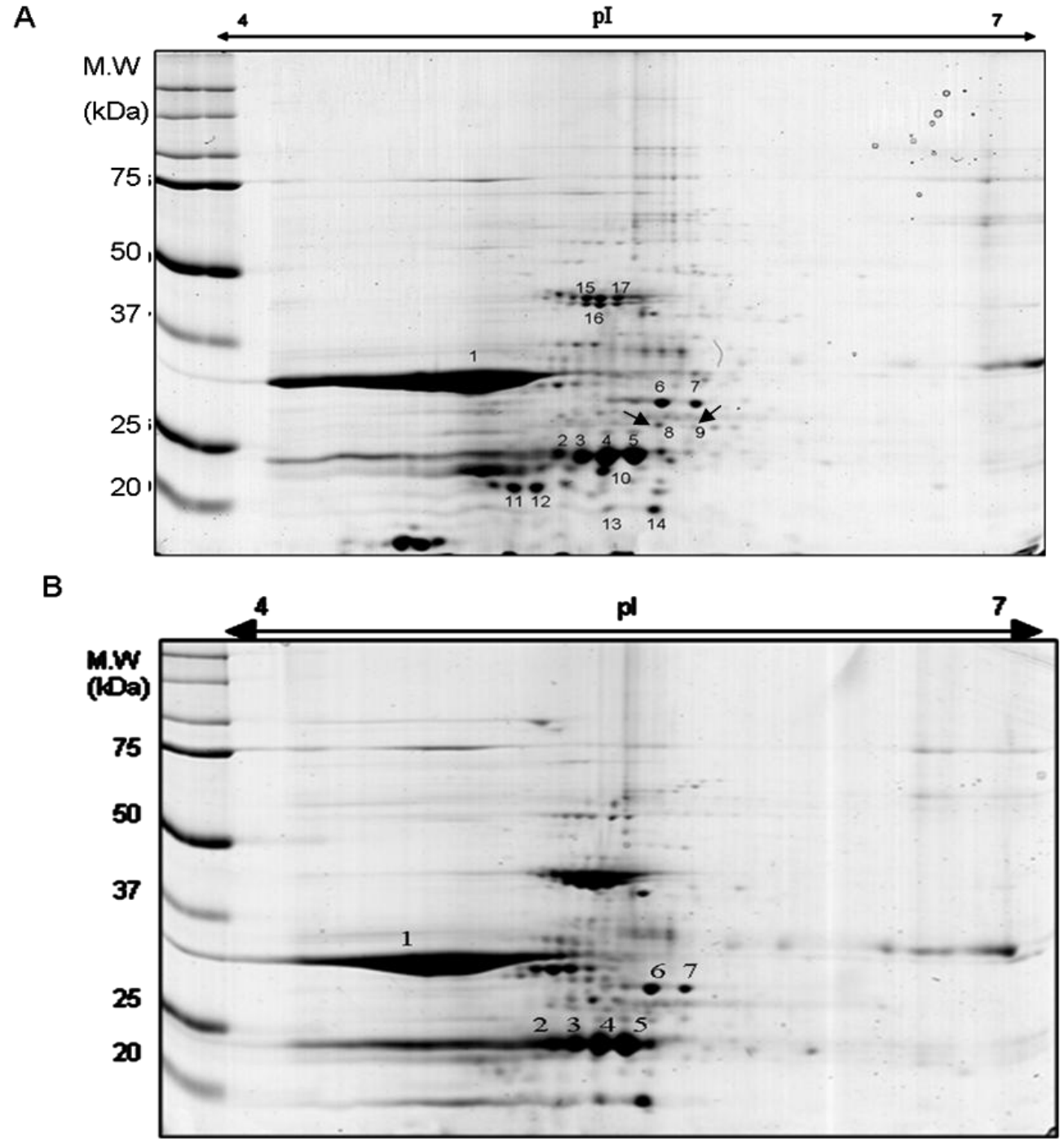

Figure 4-20: Two dimensional gel electrophoresis of DNP-GSH and CNP-GSH affinitypurified D. melanogaster GSTs after purification by GSH matrix.

A) DNP-GSH affinity-purified D. melanogaster GSTs, B) CNP-GSH affinity-purified $D$. melanogaster GSTs. The bound fractions were separated in the first dimension on a $7 \mathrm{~cm} \mathrm{pH} 4-$ 7 linear IPG strip and then in the second dimension on a SDS-PAGE gel. The M.W. marker was run along the sample. The gels were stained with Coomassie Brilliant Blue G-250. The identification of numbered spots on gel $\mathrm{A}$ is detailed in Table 4-12 and identification of some critical spots, numbered on gel B, is shown in Appendix 10.3.8 to confirm its similarity with gel A. 
Table 4-12: Identification of D. melanogaster GSTs purified by sequential DNP-GSH affinity matrix.

Proteins identified from the DNP-GSH affinity-purified fractions of D. melanogaster separated over a pH range 4-7 (Figure 4-20 A) using 2D gel electrophoresis as described in section 3.2.7.2. The proteins were identified using MALDI-TOF MS peptide mass fingerprinting. Protein spots are as numbered in Figure 4-20. Z score, the sequence coverage and the number of matched peptides are reported as obtained from Profound. In addition the protein name, the Swiss-Prot/TrEMBL accession numbers, the computed molecular mass and isoelectric point are given as obtained from the ExPASy Proteomics server of Swiss Institute of Bioinformatics and the Swiss-Prot database. Identification was made by using taxonomy D. melanogaster and NCBInr database, version dated 12/08/2008. A protein Z score greater than 1.65 is significant in Profound. The matched peptide sequences were searched against the sequences of all D. melanogaster GST classes and unique peptide sequences for each isoform of GSTs are listed in Appendix section 10.3.6.

\begin{tabular}{|c|c|c|c|c|c|c|c|c|c|}
\hline $\begin{array}{l}\text { Spot } \\
\text { No. }\end{array}$ & $\begin{array}{c}\text { NCBInr } \\
\text { Accession } \\
\text { (Tgi) }\end{array}$ & $\begin{array}{l}\text { Swiss-prot } \\
\text { /TrEMBL } \\
\text { Accession No. }\end{array}$ & Identified protein & $\begin{array}{l}\text { GST class } \\
\text { identified }\end{array}$ & $\begin{array}{c}\text { Theoretical } \\
\text { M.W./pI }\end{array}$ & $\begin{array}{c}\text { Experimental } \\
\text { M.W./pI }\end{array}$ & $\begin{array}{c}\text { Number of } \\
\text { matched } \\
\text { peptides }\end{array}$ & $\begin{array}{c}\text { Coverage } \\
(\%)\end{array}$ & $\begin{array}{c}\mathrm{Z} \\
\text { score }\end{array}$ \\
\hline 1 & 24654347 & P41043 & GST S1 & Sigma & $27.65 / 4.6$ & $33 / 4.6$ & 8 & 47 & 2.43 \\
\hline 2 & 19922932 & Q9XYZ9 & CG16936 & Epsilon & $25.45 / 5.9$ & $24 / 5.0$ & 6 & 33 & 2.43 \\
\hline 3 & 19922932 & Q9XYZ9 & CG16936 & Epsilon & $25.45 / 5.9$ & $24 / 5.1$ & 9 & 49 & 2.35 \\
\hline 4 & 19922932 & Q9XYZ9 & CG16936 & Epsilon & $25.45 / 5.9$ & $24 / 5.2$ & 8 & 47 & 2.35 \\
\hline 5 & 19922932 & Q9XYZ9 & CG16936 & Epsilon & $25.45 / 5.9$ & $24 / 5.3$ & 7 & 38 & 2.35 \\
\hline 6 & 21355763 & Q9VSL4 & CG6673 & Omega & $28.76 / 6.5$ & $28 / 5.4$ & 13 & 48 & 2.43 \\
\hline 7 & 21355763 & Q9VSL4 & CG6673 & Omega & $28.76 / 6.5$ & $28 / 5.5$ & 15 & 46 & 2.43 \\
\hline
\end{tabular}




\begin{tabular}{|c|c|c|c|c|c|c|c|c|c|}
\hline \multicolumn{10}{|c|}{ Table 4-12 continued. } \\
\hline $\begin{array}{l}\text { Spot } \\
\text { No. }\end{array}$ & $\begin{array}{c}\text { NCBInr } \\
\text { Accession } \\
\text { (Tgi) }\end{array}$ & $\begin{array}{c}\text { Swiss-prot } \\
\text { /TrEMBL } \\
\text { Accession No. }\end{array}$ & Identified protein & $\begin{array}{l}\text { GST class } \\
\text { identified }\end{array}$ & $\begin{array}{c}\text { Theoretical } \\
\text { Mr/pI }\end{array}$ & & $\begin{array}{c}\text { Number of } \\
\text { matched } \\
\text { peptides }\end{array}$ & $\begin{array}{c}\text { Coverage } \\
(\%)\end{array}$ & $\begin{array}{c}\mathbf{Z} \\
\text { score }\end{array}$ \\
\hline $8 *$ & 21355779 & Q9VSL2 & CG6776 & Omega & $27.76 / 6.5$ & $26 / 5.4$ & 5 & 24 & 1.90 \\
\hline $9 *$ & 21355779 & Q9VSL2 & CG6776 & Omega & $27.76 / 6.5$ & $26 / 5.5$ & 6 & 22 & 2.09 \\
\hline $10 *$ & 385883 & P20432 & GST D1 & Delta & $23.89 / 6.9$ & $23 / 5.2$ & 4 & 26 & 2.43 \\
\hline 11 & 6517192 & Q9U5D5 & Drab 2 & Non-GST & $23.69 / 5.8$ & $20 / 4.8$ & 7 & 37 & 2.43 \\
\hline 12 & 24641150 & Q9VZ47 & Rab GTPase 9Fa & Non-GST & $23.1 / 6.0$ & $20 / 4.9$ & 4 & 21 & 2.43 \\
\hline 13 & 20129743 & A1Z6X6 & CG1707 (Lactoyl glutathione lyase) & Non-GST & $20.14 / 6.1$ & $19 / 5.2$ & 11 & 60 & 2.37 \\
\hline 14 & 20129743 & A1Z6X6 & CG1707(Lactoyl glutathione lyase) & Non-GST & $20.14 / 6.1$ & $19 / 5.3$ & 13 & 72 & 2.39 \\
\hline 15 & 17137696 & Q9V345 & COP9 complex homolog subunit 4 & Non-GST & $46.74 / 5.9$ & $46 / 5.1$ & 11 & 33 & 1.40 \\
\hline 16 & 17137696 & Q9V345 & COP9 complex homolog subunit 4 & Non-GST & $46.74 / 5.9$ & $46 / 5.2$ & 9 & 29 & 2.43 \\
\hline 17 & 1580758 & P13706 & Glycerol 3-phosphate dehydrogenase & Non-GST & $44.75 / 6.4$ & $46 / 5.2$ & 13 & 38 & 2.00 \\
\hline
\end{tabular}

* Spots were identified from a replicate gel.

Spots 1-12 are in the range of theoretical M.W. of GSTs (22-40 kDa). 
The DNP-GSH and CNP-GSH affinity matrices were still able to bind the Sigma and Delta GSTs which were binding to GSH matrix. As the number of protein spots detected were similar in both the matrices, full characterisation was taken only on prominent D. melanogaster protein spots on the gel of DNP-GSH affinity fractions, which have been positively identified and reported in the Table 4-12. Some critical spots on the CNP-GSH affinity gel (numbered on gel B, Figure 4-20) were also identified to check their similarities in identification with DNP-GSH affinity gel spots. This is reported in Appendix Table 10-1. Both these gels have confirmed the expression of the same GSTs. Approximately $80 \%$ of the proteins isolated in the M.W. range $22-40 \mathrm{kDa}$ and focusing in the $\mathrm{pH}$ range $4-7$ were identified as belonging to the GST superfamily. However, there are other non-GST proteins also identified on the gel. An interesting result was that spots 2, 3, 4 and 5 have been identified as CG16936, which is reported in the D. melanogaster database as a putative uncharacterised protein having glutathione transferase activity. The sequence of CG16936 protein is close to the Epsilon class of GSTs (47\%) when aligned with the consensus sequences of GSTs using ClustalW2 alignment programme. The spots 6 and 7 from the D. melanogaster DNP-GSH gel have been identified as CG6673 and spots 8 and 9 have been identified as CG6776. These proteins (CG6673 and CG6776) undoubtedly belong to the Omega class of GSTs. Hence, members of four major classes of GSTs (Sigma, Delta, Epsilon and Omega) have been partially purified and identified successfully on the gel. The proteins other than the GSTs present on gel include a Rab protein, which is involved in GTPbinding and protein transport, CG1707; a protein having lactoylglutathione lyase activity, and glycerol 3-phosphate dehydrogenase, which has oxidation-reduction capability. 
Given the greater ease of preparation of the DNP-GSH matrix and its performance being at least equivalent to the CNP-GSH matrix, the unbound fractions from the GSH matrix from $L$. cuprina were only subjected to DNP-GSH affinitypurification. The fractions from the DNP-GSH matrix were resolved by $2 \mathrm{D}$ electrophoresis and are shown in Figure 4-21.

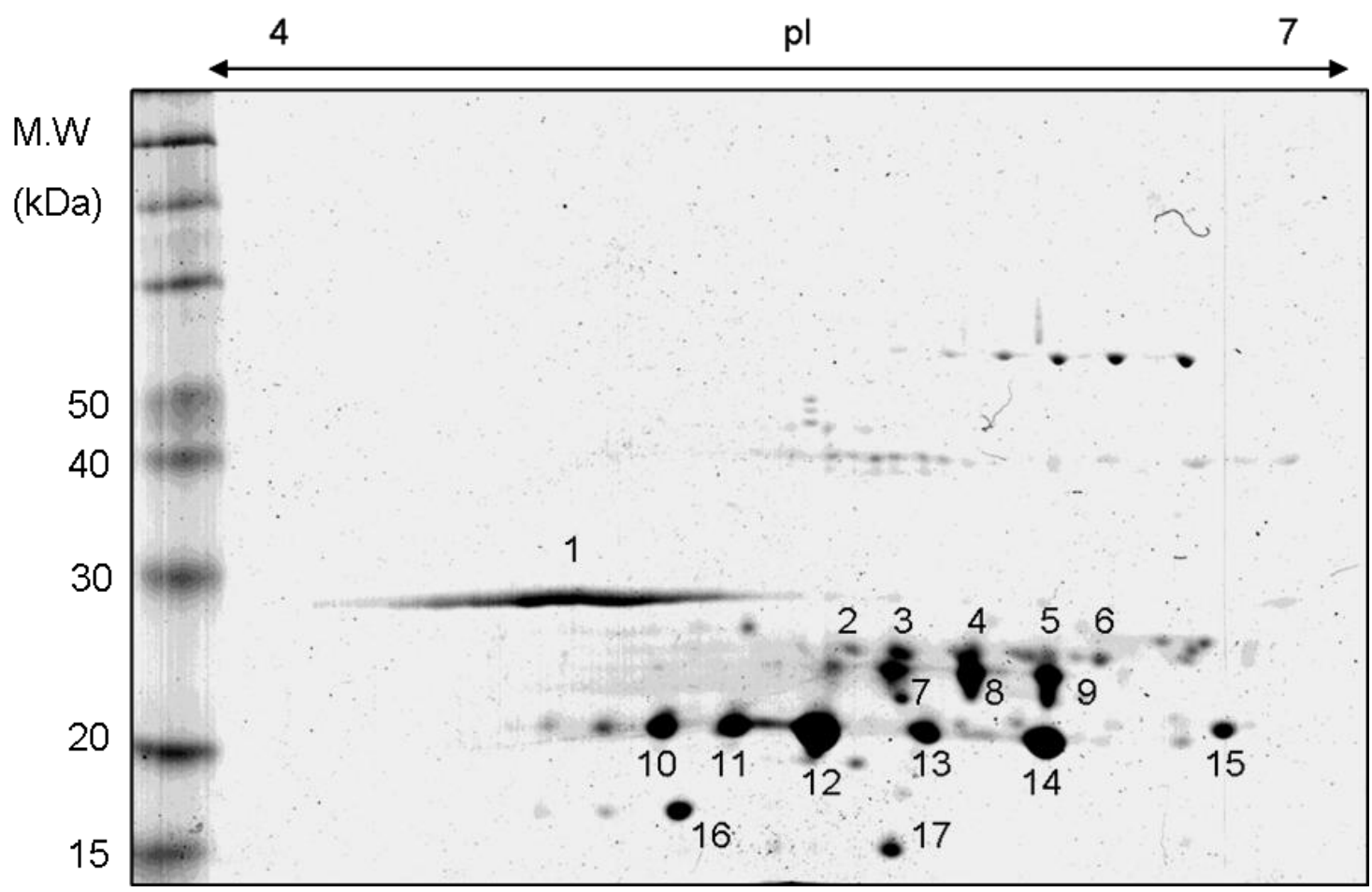

Figure 4-21: Two dimensional gel electrophoresis of DNP-GSH affinity-purified L. cuprina GSTs after purification by GSH matrix.

DNP-GSH affinity-purified L. cuprina GSTs were separated in the first dimension on a $7 \mathrm{~cm}$ pH 4-7 linear IPG strip and then in the second dimension on a SDS-PAGE gel. The M.W. marker was run along the sample. The gel was stained with Coomassie Brilliant Blue G-250. The identification of the numbered spots against "Other Metazoa" fragmentation database is shown in Table 4-13 and their tentative classification can be found in Table 4-14. 
Table 4-13: Identification of L. cuprina GSTs purified by sequential DNP-GSH affinity matrix.

Proteins identified from the DNP-GSH affinity-purified fractions of L. cuprina separated over a pH range 4-7 (Figure 4-21) using 2D gel electrophoresis as described in section 3.2.7.2. The proteins were identified using MALDI-TOF MS peptide mass fingerprinting. Protein spots are as numbered in Figure 4-21. Z score, the sequence coverage and the number of matched peptides are reported as obtained from Profound. In addition the protein name, the SwissProt/TrEMBL accession numbers, the computed molecular mass and isoelectric point are given as obtained from the ExPASy Proteomics server of Swiss Institute of Bioinformatics and the Swiss-Prot database. Identification was made by using taxonomy D. melanogaster and "Other Metazoa” NCBInr database, version dated 2/01/09. A protein $\mathrm{Z}$ score greater than 1.65 is significant in Profound.

\begin{tabular}{|c|c|c|c|c|c|c|c|c|c|}
\hline $\begin{array}{l}\text { Spot } \\
\text { No. }\end{array}$ & $\begin{array}{c}\text { NCBInr } \\
\text { Accession } \\
\text { (Tgi) }\end{array}$ & $\begin{array}{c}\text { Swiss-prot } \\
\text { /TrEMBL } \\
\text { Accession No. }\end{array}$ & Matched protein & $\begin{array}{l}\text { GST class } \\
\text { matched } \\
\text { against } D \text {. } \\
\text { melanogaster }\end{array}$ & $\begin{array}{c}\text { Theoretical } \\
\text { M.W./pI }\end{array}$ & $\begin{array}{c}\text { Experimental } \\
\text { M. W./pI }\end{array}$ & $\begin{array}{c}\text { Number of } \\
\text { matched } \\
\text { peptides }\end{array}$ & $\begin{array}{c}\text { Coverage } \\
(\%)\end{array}$ & Z score \\
\hline \multirow[t]{2}{*}{1} & 24654347 & P41043 & GST_Drosophila melanogaster & Sigma & $27.6 / 4.57$ & $30 / 4.6$ & 6 & 29 & 2.43 \\
\hline & 1170110 & P46437 & GST_Musca domestica & Sigma & $27 / 4.6$ & $30 / 4.6$ & 9 & 32 & 2.39 \\
\hline 2 & 3201479 & O61996 & GST_Bombyx mori & Delta & $25.25 / 5.1$ & $28 / 5.3$ & 8 & 48 & 2.00 \\
\hline 3 & 3511225 & O77462 & GST_Anopheles gambiae & Theta/Delta & $25.08 / 5.8$ & $28 / 5.4$ & 4 & 20 & 2.43 \\
\hline 4 & 57967586 & O77462 & GST_Anopheles gambiae & Theta/Delta & $25.08 / 5.8$ & $28 / 5.5$ & 5 & 32 & 2.43 \\
\hline 5 & 159058 & P31671 & mu GST_Fasciola hepatica & Sigma & $25.41 / 5.9$ & $28 / 5.6$ & 10 & 50 & 2.43 \\
\hline 6 & 7387485 & Q26387 & GST_Heligmosomoides polygyrus & Sigma & $24.53 / 6.6$ & $28 / 5.7$ & 9 & 56 & 1.94 \\
\hline 7 & 12007374 & Q9GPL8 & GST E2_Anopheles gambiae & Epsilon & $24.95 / 5.5$ & $27 / 5.4$ & 4 & 23 & 1.26 \\
\hline
\end{tabular}




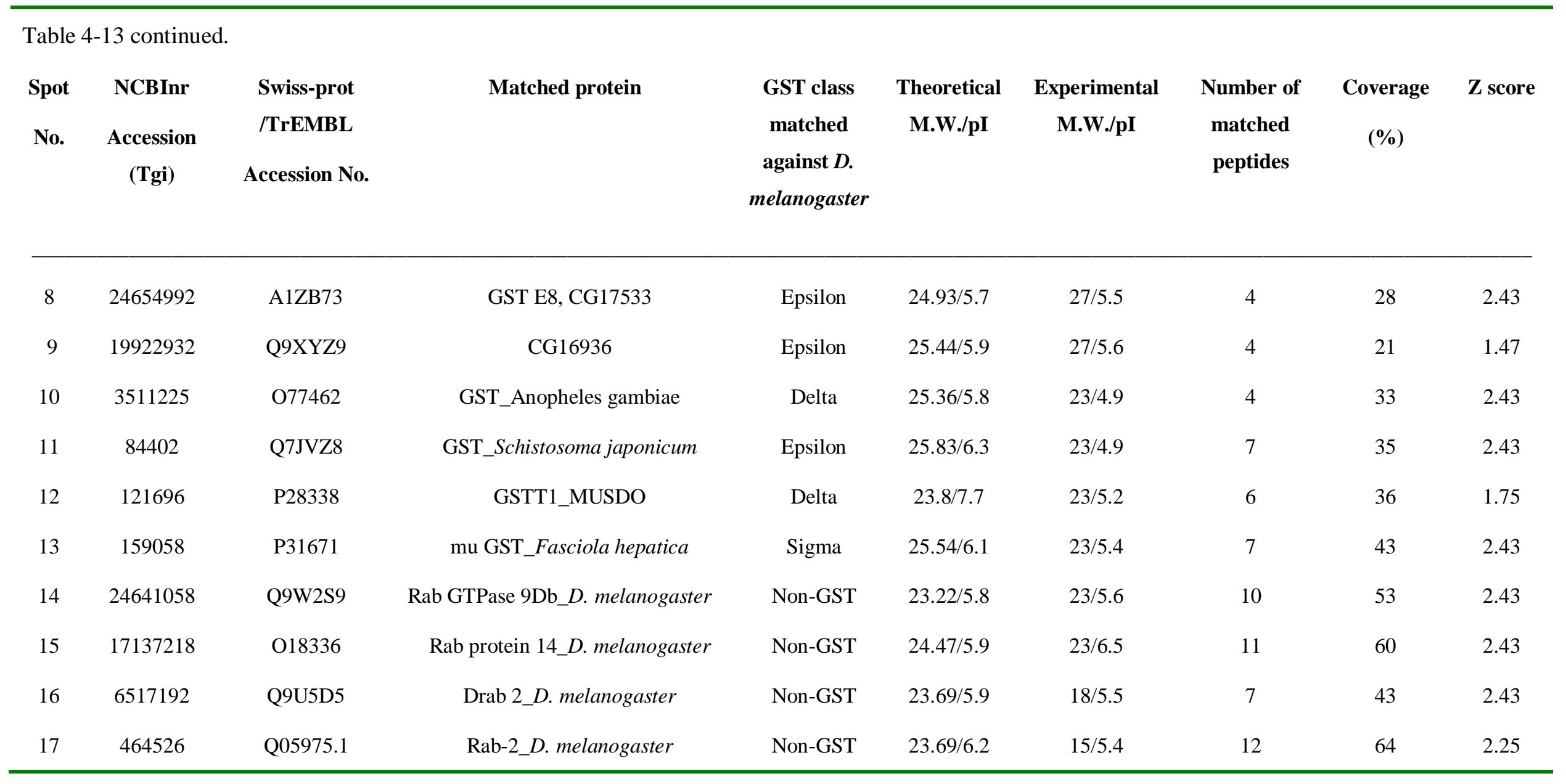

Drosophila melanogaster - Fruit fly; Musca domestica - Housefly; Bombyx mori - Silk moth; Anopheles gambiae - Mosquitoes; Fasciola hepatica - Sheep liver fluke; Heligmosomoides polygyrus - Nematode found in rodents; Schistosoma japonicum - a parasite often referred as human blood fluke 
Seventeen prominent spots were identified from the 2D electrophoresis of $L$. cuprina DNP-GSH affinity-purified fractions. Fragments from spot 1 matched the sequences in the $M$. domestica and in D. melanogaster databases for Sigma GST, much as was found with the previous L. cuprina GSH affinity gel. However, other spots which did not match with the D. melanogaster database were compared with "Other Metazoa" fragmentation database. Interestingly, thirteen spots gave fragmentation patterns which matched GSTs in other different species such as Musca domestica, Bombyx mori, Anopheles gambiae, Fasciola hepatica, Heligmosomoides polygyrus and Schistosoma japonicum and possible sequences of the peptide fragments were obtained from these databases. As seen with the D. melanogaster gel, the DNP-GSH affinity matrix also captured some non-GST proteins, mainly Rab proteins. In the M.W. range of GSTs (22-40 kDa), approximately $87 \%$ proteins were tentatively identified as GSTs. However, it was difficult to determine the class of the GSTs in L. cuprina with great confidence. Therefore, another tentative classification approach was used to propose the identity by aligning the sequences of the peptides that matched L. cuprina peptide mass fingerprinting data, with consensus sequences of different GST classes which were created as described earlier in this section (Table 4-14). 
Table 4-14: Assignment of class of GST using the ClustalW2 programme.

The peptides of L. cuprina DNP-GSH affinity-purified spots on 2D gel matched against "Other Metazoa" were aligned with consensus sequence of insect GSTs using the ClustalW2 alignment programme. The highest match score is highlighted in red text. The identification of each spot is shown in Table 4-13.

\begin{tabular}{|c|c|c|c|c|c|c|c|c|c|c|c|}
\hline \multirow{3}{*}{$\begin{array}{l}\text { Spot } \\
\text { No. }\end{array}$} & \multirow{3}{*}{$\begin{array}{l}\text { Identification against } \\
\text { "Other Metazoa" }\end{array}$} & \multicolumn{9}{|c|}{ Alignment with consensus sequences of Insect GST classes } & \multirow{3}{*}{$\begin{array}{l}\text { Predicted } \\
\text { GST Class }\end{array}$} \\
\hline & & \multicolumn{9}{|c|}{ ClustalW2 Score } & \\
\hline & & Sigma & Delta & Epsilon & Omega & Zeta & Theta & $\mathrm{Xi}$ & Iota & $\mathrm{Mu}$ & \\
\hline \multirow[t]{2}{*}{1} & $\begin{array}{l}\frac{\text { gi|24654347 }}{\text { Glutathione S-transferase-S1 }} \\
\text { CG8938-PA, isoform A }\end{array}$ & 87 & 16 & 11 & 5 & 11 & 8 & 5 & 8 & 9 & Sigma \\
\hline & $\begin{array}{l}\frac{\text { gi|1170110|sp| p46437.1| }}{\text { GST MUSDO }} \\
\text { Glutathione S-transferase; } \\
\text { GST class-sigma }\end{array}$ & 74 & 7 & 12 & 6 & 6 & 6 & 11 & 9 & 23 & Sigma \\
\hline 2 & 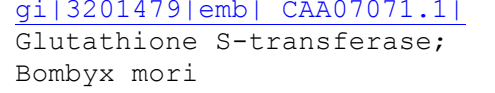 & 17 & 54 & 44 & 10 & 12 & 28 & 37 & 49 & 4 & Delta (high M.W.) \\
\hline 3 & $\begin{array}{l}\frac{\text { gi|3511225|gb| AAC79992.1| }}{\text { Glutathione S-transferase; }} \\
\text { Anopheles gambiae }\end{array}$ & 18 & 76 & 20 & 6 & 11 & 9 & 30 & 53 & 23 & Delta(high M.W.) \\
\hline 4 & $\begin{array}{l}\frac{\text { gi| } 57967586 \mid \text { ref |XP } 562680.1 \mid}{\text { Glutathione S-transferase }} \\
\text { Anopheles gambiae str.pest }\end{array}$ & 8 & 52 & 30 & 16 & 18 & 25 & 36 & 39 & 9 & Delta(high M.W.) \\
\hline 5 & $\begin{array}{l}\frac{\text { gi|159058|gb| AA29139.1| }}{\text { mu-glutathione transferase }} \\
\text { (Fasciola hepatica) }\end{array}$ & 26 & 12 & 13 & 13 & 13 & 4 & 11 & 17 & 48 & $\mathrm{Mu}$ \\
\hline 6 & $\begin{array}{l}\frac{\text { gi|7387485|gb| AAB33637.2| }}{\text { Glutathione S-transferase }} \\
\text { (Heligmosomoides polygyrus) }\end{array}$ & 8 & 5 & 7 & 4 & 5 & 4 & 7 & 5 & 5 & Not determined \\
\hline
\end{tabular}




\begin{tabular}{|c|c|c|c|c|c|c|c|c|c|c|c|}
\hline \multicolumn{12}{|c|}{ Table 4-14 continued. } \\
\hline 7 & $\begin{array}{l}\frac{\text { gi|12007374 |gb| AAG45164.1| }}{\text { Glutathione transferase E2 }} \\
\text { (Anopheles gambiae) }\end{array}$ & 10 & 32 & 58 & 18 & 12 & 24 & 30 & 32 & 20 & Epsilon \\
\hline 8 & $\begin{array}{l}\text { gi|24654992|ref| NP 611330.21 } \\
\text { Glutathione transferase E8 } \\
\text { CG17533-PA (Drosophila } \\
\text { melanogaster) }\end{array}$ & 22 & 42 & 79 & 22 & 28 & 22 & 36 & 38 & 17 & Epsilon \\
\hline 9 & $\begin{array}{l}\frac{\text { gi|19922932|ref| NP } 611964.1 \mid}{\text { CG16936-PA }} \\
\text { (Drosophila melanogaster) }\end{array}$ & 12 & 36 & 55 & 12 & 8 & 42 & 46 & 44 & 17 & Epsilon \\
\hline 10 & $\begin{array}{l}\frac{\text { gi|3511225|gb|AAC79992.1| }}{\text { glutathione S-transferase }} \\
\text { [Anopheles gambiae] }\end{array}$ & 9 & 69 & 33 & 8 & 4 & 36 & 29 & 56 & 11 & Delta (low M.W.) \\
\hline 11 & $\begin{array}{l}\frac{\text { gi|84402|pir||A26484 }}{\text { glutathione transferase - fluke }} \\
\text { (Schistosoma japonicum) (fragment) }\end{array}$ & 15 & 22 & 12 & 12 & 7 & 25 & 6 & 22 & 39 & $\mathrm{Mu}$ \\
\hline 12 & $\begin{array}{l}\text { gi|121696|sp|P28338.1|GSTT1 MUSDO } \\
\text { RecName: FulleGlutathione } \\
\text { S-transferase } 1 \text {; AltName: } \\
\text { Full=GST class-theta }\end{array}$ & 17 & 85 & 37 & 20 & 22 & 21 & 42 & 52 & 14 & Delta (low M.W.) \\
\hline 13 & $\begin{array}{l}\frac{\text { gi|159058|gb|AAA29139.1| }}{\text { mu-glutathione transferase }} \\
\text { [Fasciola hepatica] }\end{array}$ & 23 & 10 & 7 & 6 & 6 & 11 & 12 & 18 & 30 & $\mathrm{Mu}$ \\
\hline 14 & $\frac{\text { gi|24641058|ref|NP_572642.1| }}{\text { Rab GTPase 9Db }}$ & 7 & 1 & 7 & 9 & 9 & 5 & 9 & 7 & 3 & Non-GST \\
\hline 15 & $\begin{array}{l}\frac{\text { gi|17137218|ref|NP } 477171.1 \mid}{\text { Rab-protein } 14, \text { isoform A }} \\
\text { [Drosophila melanogaster] }\end{array}$ & 2 & 5 & 5 & 6 & 3 & 8 & 6 & 11 & 3 & Non-GST \\
\hline 16 & $\frac{\text { gi } 16517192 \text { | dbj| BAA87878.1| }}{\text { Drab2 [Drosophila melanogaster] }}$ & 5 & 8 & 9 & 8 & 10 & 6 & 7 & 5 & 10 & Non-GST \\
\hline 17 & $\begin{array}{l}\text { gi|464526|sp|005975.1|RAB2 LYMSTRe } \\
\text { cName: Full=Ras-related protein } \\
\text { Rab-2 }\end{array}$ & 11 & 8 & 10 & 2 & 7 & 13 & 6 & 5 & 12 & Non-GST \\
\hline
\end{tabular}




\subsubsection{Separation of Sigma and Delta class GSTs}

\subsubsection{Chromatofocusing}

D. melanogaster GSTs partially purified by GSH affinity chromatography, were separated by chromatofocusing over a range of $\mathrm{pH} 7-4$. For this, the purified GSTs from the GSH column were equilibrated with the start buffer $(0.025 \mathrm{M}$ imidazol-HCl buffer, $\mathrm{pH}$ 7.4) and applied to the chromatofocusing Mono $\mathrm{P}$ column as described in section 3.2.3. The flow rate was $0.5 \mathrm{ml} / \mathrm{min}$. The column was preequilibrated with the start buffer and $40 \%$ of the CDNB activity eluted from the GSH matrix was applied and eluted with the polybuffer 74, $\mathrm{pH} 4$ and $1 \mathrm{M} \mathrm{NaCl}$.

Three major peaks were obtained at different $\mathrm{pH}$ values. Peak I was obtained at $\mathrm{pH} 7.28$ and contained a single protein; (i.e. a single GST form which did not bind to the Mono P column (Figure 4-24, lane 1) and accounted for $64 \%$ of the total GST activity (measured with CDNB) applied to the Mono P chromatofocusing column. The peaks (Peak II) which were eluted under the $\mathrm{pH}$ gradient (Figure 4-23) accounted for $6 \%$ of the total GST activity and comprised many small peaks eluted at different $\mathrm{pH}$ values from 5.2- 4.4. The protein eluted by $1 \mathrm{M} \mathrm{NaCl}$ formed peak III. Due to a column blockage total protein comprising that peak could not be determined. Instead values for protein from half the peak were calculated and are shown in Table 4-15. The half peak III contained only $1.09 \%$ of the starting GST activity. The activity towards CDNB is shown in Figure 4-22. A loss of the activity towards CDNB was detected in dialysed or equilibrated sample due to the overnight equilibration process before the chromatofocusing (Table 4-15). 


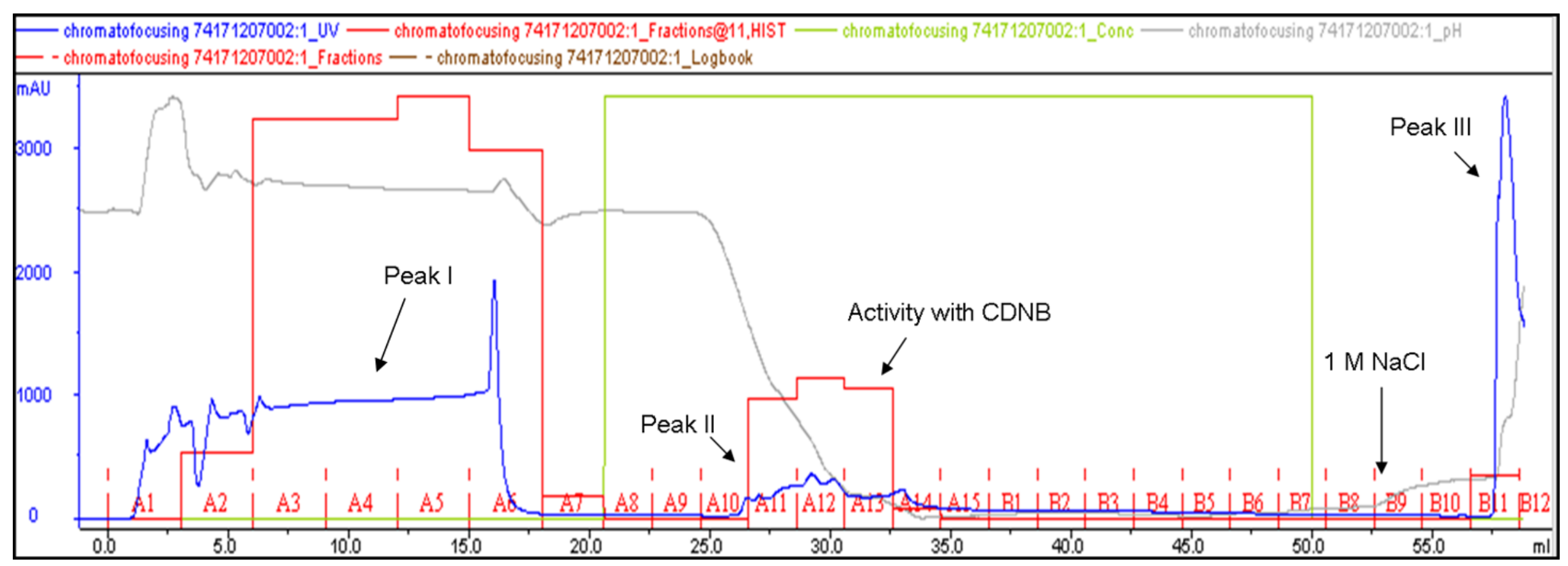

Figure 4-22: Typical FPLC chromatogram, for the separation of D. melanogaster Delta GSTs by Mono P HPLC chromatofocusing at pH 7-4.

GSTs partially purified by GSH affinity chromatography were equilibrated with $25 \mathrm{mM}$ imidazole/ $\mathrm{HCl}$, pH 7.4 and then applied to a Mono P 5/50 GL column equilibrated with the same buffer. The GSTs were eluted with polybuffer $\mathrm{pH} 4$ as described in the text. The flow rate was $0.5 \mathrm{ml} / \mathrm{min}$ and fractions of $3 \mathrm{ml}$ were collected for unbound material and $2 \mathrm{ml}$ for the material eluted under the $\mathrm{pH}$ gradient. $\mathrm{UV}$, $\mathrm{pH}$, concentration of sample applied were recorded and shown in different colours above. The histogram plotted in red colour displays the GST activity towards CDNB in corresponding fractions. 


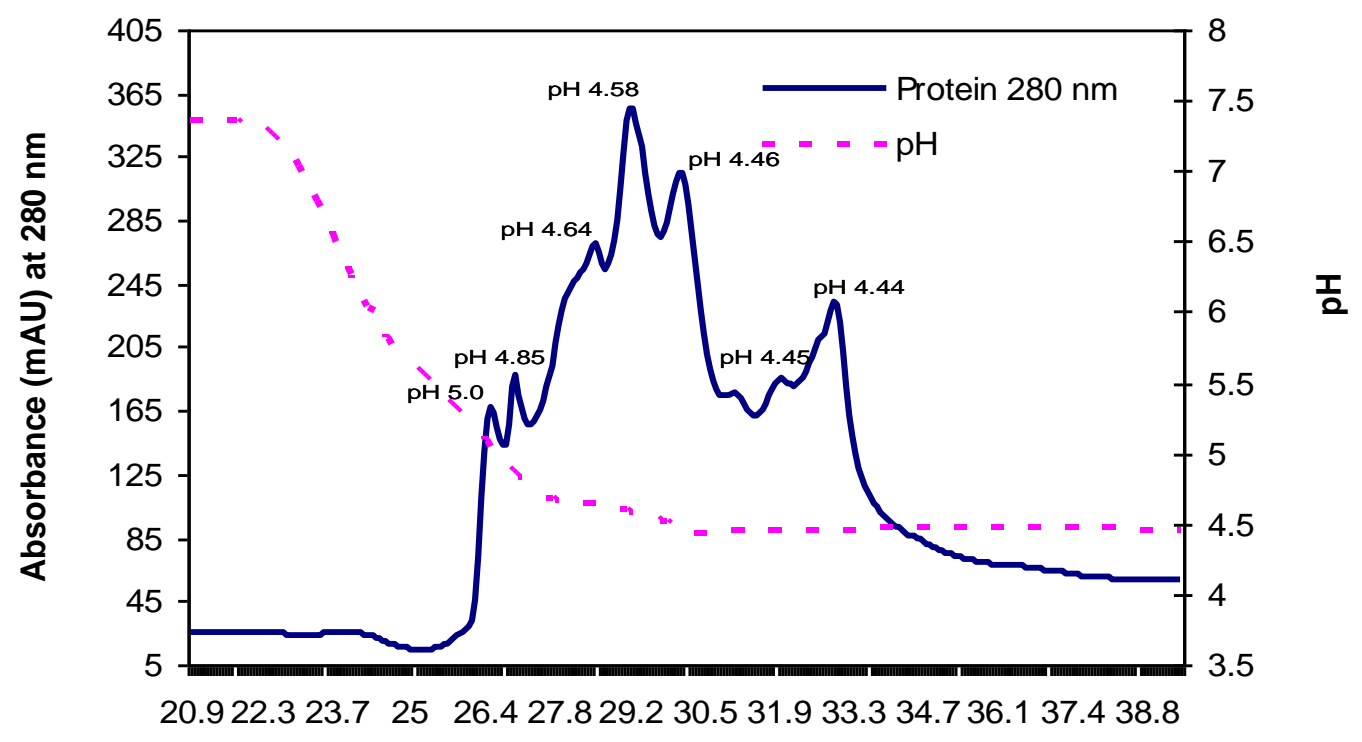

Time (min)

Figure 4-23: Magnified image of peak II of Figure 4-22 showing separation of $D$. melanogaster Delta GSTs by Mono P HPLC chromatofocusing at pH 7-4. 
Table 4-15: Purification of GSTs from D. melanogaster.

The purification procedure is as given in this section. GST activity is that towards CDNB from a single experiment. $2.5 \mathrm{~g}$ of adult $D$. melanogaster flies were used.

\begin{tabular}{|c|c|c|c|c|c|}
\hline Step & $\begin{array}{l}\text { Total } \\
\text { protein } \\
\quad \text { mg }\end{array}$ & $\begin{array}{l}\text { Total } \\
\text { activity } \\
\text { (Units) }\end{array}$ & $\begin{array}{l}\text { Specific } \\
\text { activity } \\
\text { (Units/mg) }\end{array}$ & $\begin{array}{c}\text { Yield } \\
\%\end{array}$ & $\begin{array}{c}\text { Purification } \\
\text { fold }\end{array}$ \\
\hline Crude & 60.5 & 3.94 & 0.065 & 100 & 1 \\
\hline De-pigmented & 54.5 & 3.23 & 0.059 & 82 & 0.90 \\
\hline Unbound GSH & 39.1 & 2.33 & 0.059 & 59 & 0.90 \\
\hline Bound GSH & 2.30 & 1.58 & 0.687 & 40 & 10.56 \\
\hline \multicolumn{6}{|c|}{ Part of the bound GSH enzyme was taken aside for gel analysis and activity check. The rest was equilibrated } \\
\hline \multicolumn{6}{|c|}{ and applied to Mono P column for the separation of GST isoforms } \\
\hline Equilibration & 2.25 & 0.55 & 0.24 & 100 & 1 \\
\hline Peak I & 1.88 & 0.36 & 0.19 & 65 & 0.79 \\
\hline Peak II & 0.18 & 0.03 & 0.16 & 5.4 & 0.66 \\
\hline Peak III & 0.04 & 0.006 & 0.15 & 1.1 & 0.62 \\
\hline
\end{tabular}

The fractions forming the peak I, II and III were pooled and resolved by SDS PAGE and identification of protein bands was made by MALDI-TOF. The peak I and peak II were identified as the Delta GSTs eluted at different $\mathrm{pH}$ and the salt eluted peak III was identified as the Sigma class of GST. 


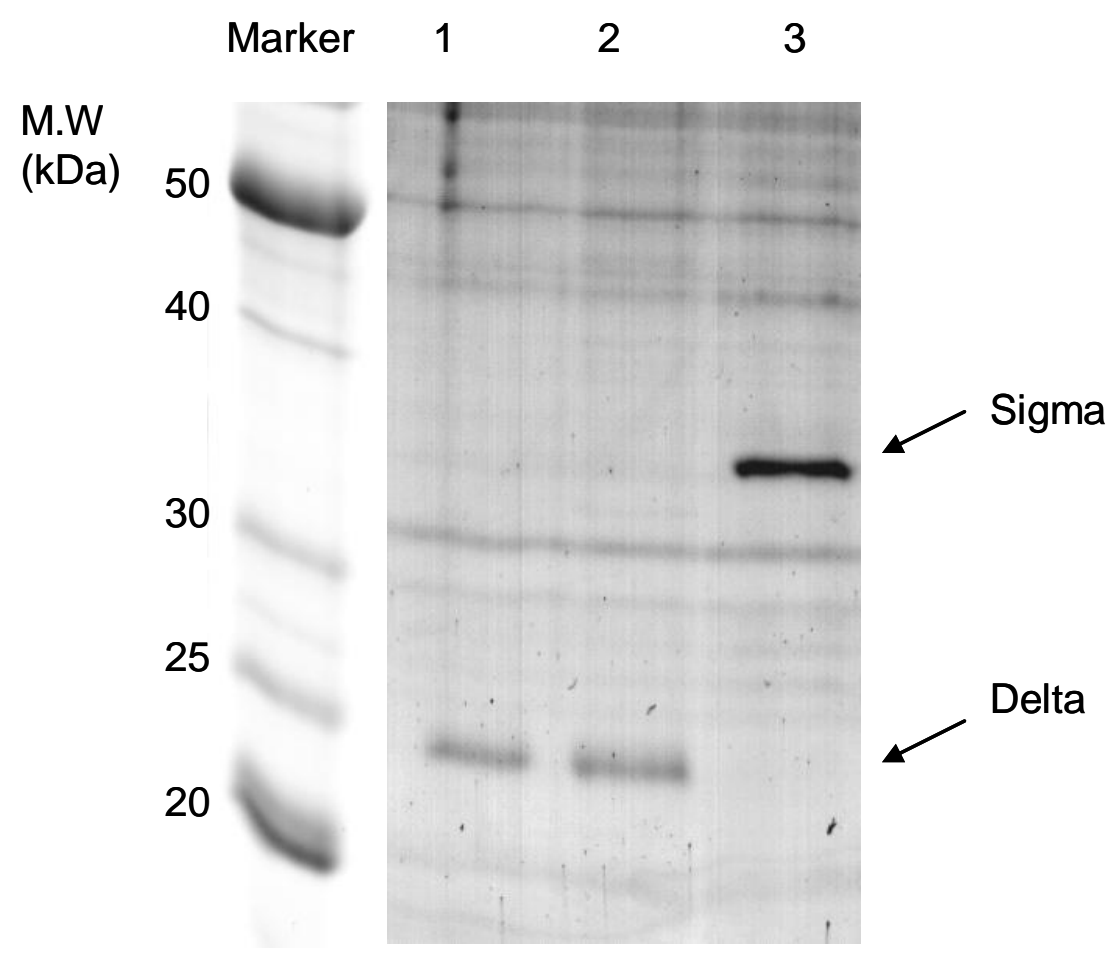

Figure 4-24: Characterization of D. melanogaster GST subunits by SDS PAGE.

Lane 1, 2 and 3 respectively, show D. melanogaster GST separated in peak I, II and III during the chromatofocusing 74. The gel was stained by Coomassie Brilliant Blue G 250 .

One interesting observation is that the Delta class of GSTs which were eluted at different $\mathrm{pH}$ conditions $(\mathrm{pH} 7.28$ and under the $\mathrm{pH}$ gradient 5.2- 4.46) could be different sub classes/isoforms. The separation result with the 2D gel (Figure 4-17) also showed different spots of Delta class GSTs having the same molecular weight but different pIs. However, they all were identified as Delta D1 not as different isoforms of Delta GST. The magnified image of peak II (Figure 4-23) shows presence of many small peaks eluted at different $\mathrm{pH}$, however it was not feasible to collect each individual peak in different fractions so all the fractions comprising peak II were pooled and used for the analysis. Thus chromatofocusing $\mathrm{pH}$ 7-4 was able to separate the Sigma and Delta GSTs from the partially purified mixture. 


\subsubsection{2 lon exchange chromatography}

Another way to separate Sigma and Delta GSTs from the partially purified mixture is use of a combination of affinity chromatography and ion exchange chromatography. The GSH-Sepharose matrix and QAE Sephadex A-25 packed in Tricorn $^{\mathrm{TM}} 5 / 200$ were both used for this experiment. A crude extract of $D$. melanogaster was de-pigmented using HiTrap desalting column $(5 \mathrm{ml}, 1.6 \times 2.5 \mathrm{~cm})$. CDNB active fractions were passed through the GSH column and the column was washed with $0.05 \mathrm{M}$ phosphate buffer, $\mathrm{pH} 7.4$ and $1 \mathrm{M} \mathrm{NaCl}$. The QAE Sephadex A25 packed in a Tricorn ${ }^{\mathrm{TM}} 5 / 200$ column was equilibrated with $0.05 \mathrm{M}$ phosphate buffer, $\mathrm{pH} 7.4$ and connected to the GSH column at the time of elution of the bound GSTs with 0-20 mM glutathione gradient, $\mathrm{pH}$ 9.6. GSTs eluted from the GSH column were passed immediately through the QAE-Sephadex A-25 column. The Delta class of GSTs, which are active towards CDNB, were not retained by QAESephadex A-25 column and were collected in the unbound fractions. However, the Sigma class GST was bound to the QAE Sephadex A-25 and eluted separately using 0.5 M NaCl. Sample application, washing and elution were programmed using ÄKTA FPLC. The purification steps are summarized in Figure 4-25 and Table 4-16.

The specific activities of GSH affinity-purified GSTs and QAE Sephadex A25 purified GSTs were 27-fold and 2-fold higher than that of crude homogenate respectively. The GST activity eluted from the GSH affinity chromatography and ion exchange chromatography has been shown in Figure 4-25 (B and C respectively). The SDS PAGE analysis revealed the presence of a single protein band from both peaks, which were identified as Sigma and Delta GSTs by MALDITOF mass spectrometry (Figure 4-25 D). The same procedure was applied to separate Sigma and Delta GSTs from L. cuprina. 
A

\section{Crude extract}

Depigmented

HiTrap desalting column
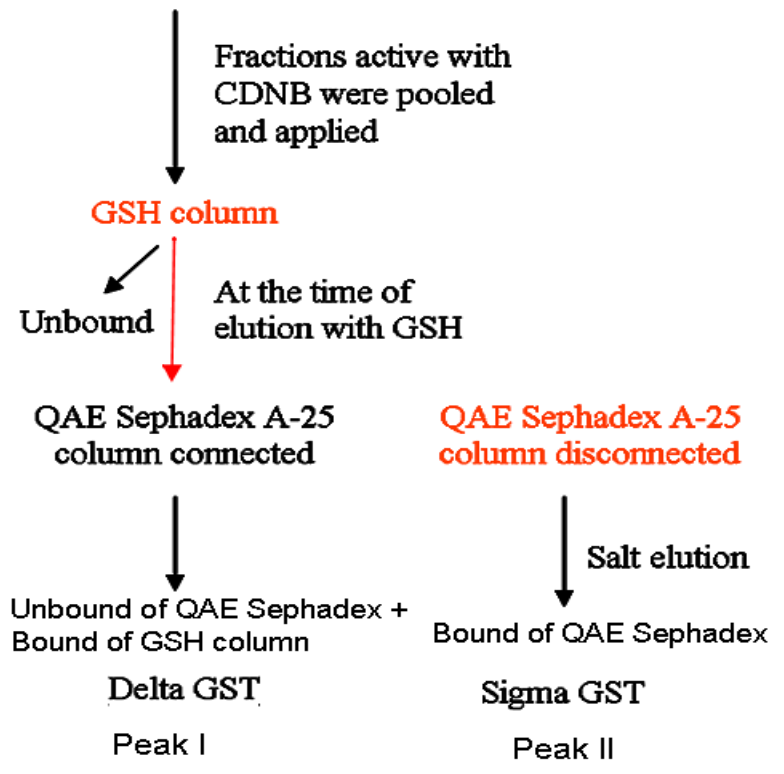

B

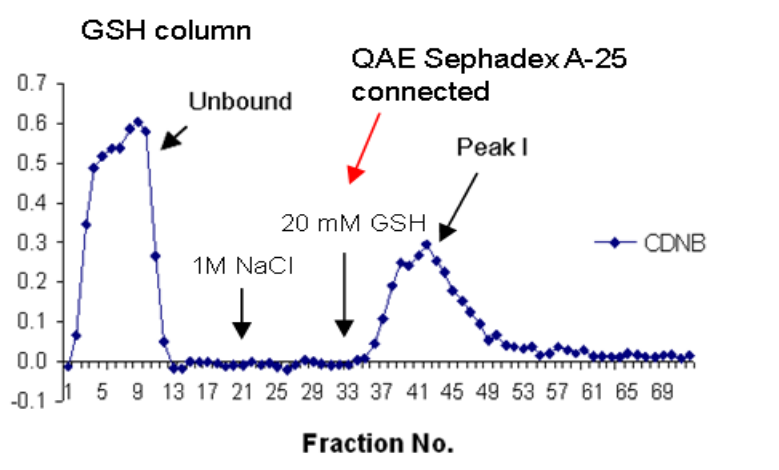

C

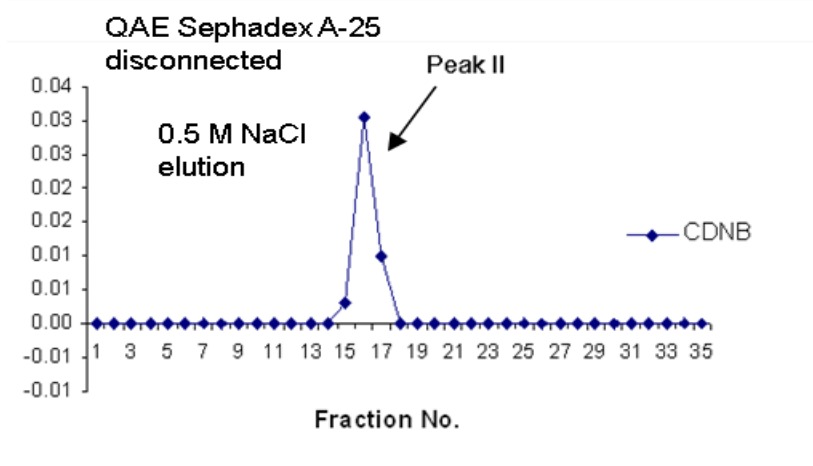

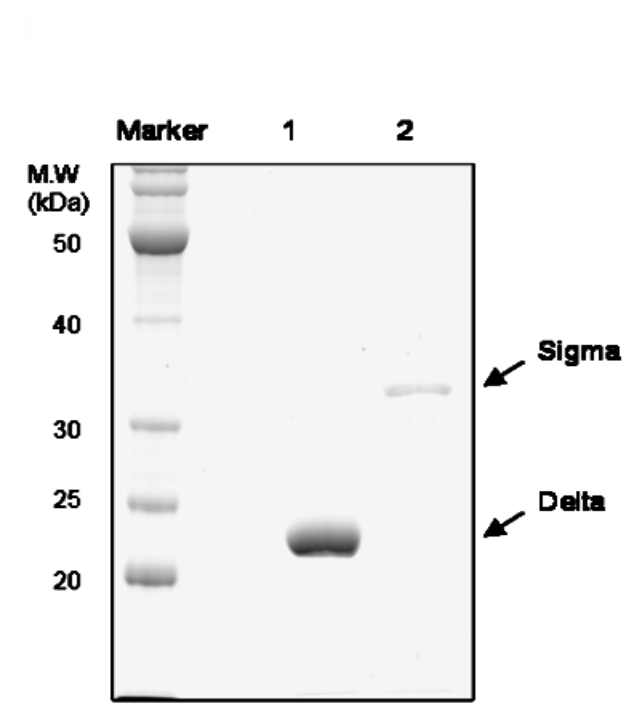

Figure 4-25: Separation of Sigma and Delta GSTs by ion exchange chromatography.

(A) The methodology of GST separation. (B) The GST activity eluted from the GSH column which was connected to QAE Sephadex A-25 ion exchange column. (C) Elution of GSTs retained by QAE-Sephadex A-25 ion exchange column and (D) SDS PAGE analysis on the fractions of peak I and II in lanes 1 and 2 respectively. The gel was stained with Coomasssie Brilliant Blue G-250. 
Table 4-16: Purification of D. melanogaster GSTs by ion exchange chromatography.

The purification procedure is given in the text. Activity is towards CDNB. $3.5 \mathrm{~g}$ of adult flies were used. The values are means \pm S.D. of three independent experiments with triplicate measurements in each.

\begin{tabular}{|c|c|c|c|c|c|}
\hline Procedure & $\begin{array}{c}\text { Total } \\
\text { protein } \\
\text { mg }\end{array}$ & $\begin{array}{c}\text { Total } \\
\text { activity } \\
\text { (Units) }\end{array}$ & $\begin{array}{l}\text { Specific } \\
\text { activity } \\
\text { (Units/mg) }\end{array}$ & $\begin{array}{c}\text { Yield } \\
\%\end{array}$ & $\begin{array}{c}\text { Purification } \\
\text { fold }\end{array}$ \\
\hline Crude & $76.5 \pm 0.3$ & $17.0 \pm 0.2$ & $0.22 \pm 0.003$ & 100 & 1 \\
\hline De-pigmented & $77.0 \pm 6.0$ & $16.2 \pm 0.1$ & $0.21 \pm 0.01$ & 94.8 & 0.94 \\
\hline Unbound GSH & $68.0 \pm 1.0$ & $10.0 \pm 0.8$ & $0.14 \pm 0.01$ & 58.5 & 0.65 \\
\hline $\begin{array}{l}\text { Bound GSH + } \\
\text { Unbound QAE }\end{array}$ & $1.20 \pm 0.01$ & $7.25 \pm 0.35$ & $6.04 \pm 0.3$ & 42.4 & 27 \\
\hline Sephadex & & & & & \\
\hline Bound QAE & $0.40 \pm 0.07$ & $0.20 \pm 0.02$ & $0.5 \pm 0.03$ & 1.2 & 2.27 \\
\hline
\end{tabular}

The peaks containing Sigma and Delta GSTs were resolved by 2D gel electrophoresis. Although the Delta GST present as a single band on SDS PAGE, it is separated in six different spots on 2D gel (Figure 4-26) and all spots were identified as DmGSTD1. 

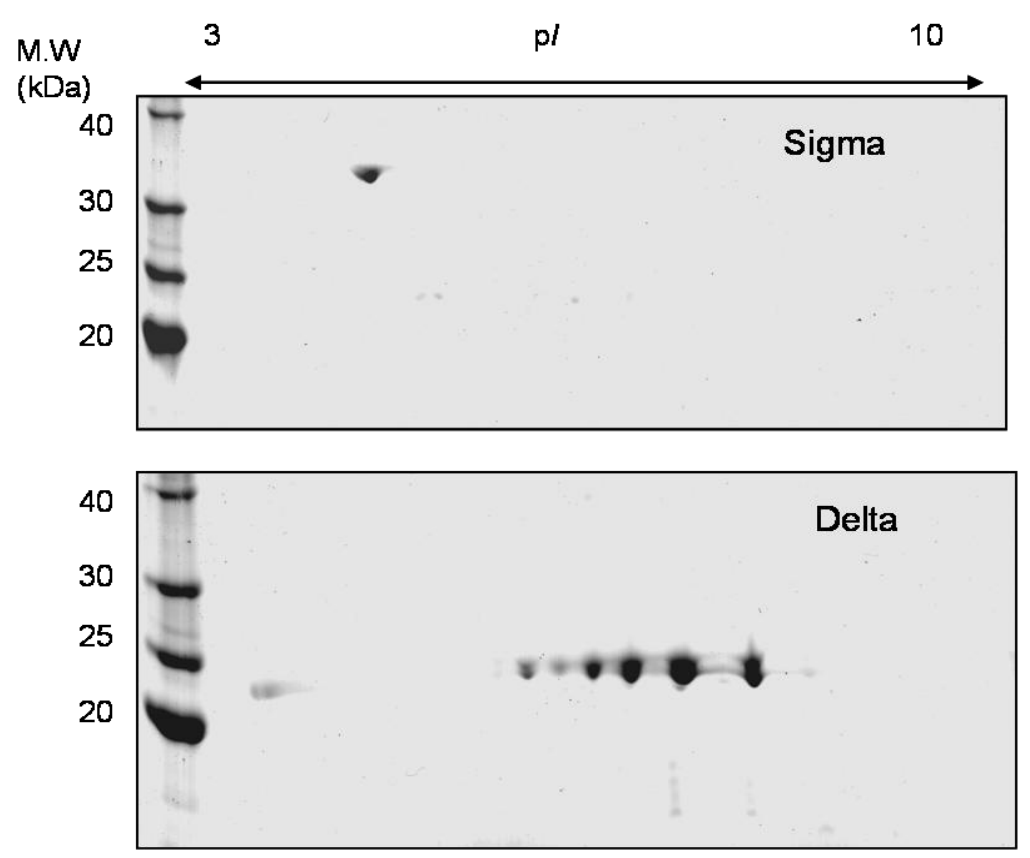

Figure 4-26: Resolution of Sigma (peak I) and Delta GSTs (peak II) from D. melanogaster by $2 \mathrm{D}$ electrophoresis.

The fractions of peak I and peak II in Figure 4-25 were separately pooled, concentrated and separated in the first dimension on a $7 \mathrm{~cm} \mathrm{pH} \mathrm{3-10} \mathrm{linear} \mathrm{IPG} \mathrm{strip} \mathrm{and} \mathrm{then} \mathrm{in} \mathrm{the} \mathrm{second}$ dimension on a SDS-PAGE gel. The M.W. marker was run along the sample. The gel was stained with Coomassie Brilliant Blue G-250.

Table 4-17: Substrate specificities of Sigma and Delta GSTs from D. melanogaster and $L$. cuprina. Four $\mathrm{g}$ of flies were used. The specific activity is from three individual experiments with triplicate measurements in each and values represent mean $\pm S$. D.

\begin{tabular}{llllc}
\hline \multirow{2}{*}{ Substrate } & \multicolumn{4}{c}{ Specific activity $(\mu \mathrm{mol} / \mathrm{min} / \mathrm{mg}$ protein) } \\
\cline { 2 - 5 } & \multicolumn{3}{c}{ D. melanogaster } & L. cuprina \\
& Sigma & Delta & Sigma & Delta \\
\hline CDNB & $7.11 \pm 0.6$ & $58.7 \pm 1.7$ & $2.63 \pm 0.3$ & $4.75 \pm 1.3$ \\
DCNB & $0.07 \pm 0.01$ & Nil & Nil & $0.05 \pm 0.1$ \\
NPA & $8.36 \pm 0.3$ & $1.75 \pm 0.9$ & $2.85 \pm 0.2$ & $4.46 \pm 1.3$ \\
DHA & $11.6 \pm 0.7$ & Nil & $5.35 \pm 0.4$ & $13.5 \pm 4.3$ \\
TNE & $0.69 \pm 0.1$ & $4.78 \pm 0.3$ & $0.27 \pm 0.01$ & $0.85 \pm 0.2$ \\
EA & $25.6 \pm 1.5$ & Nil & $13.1 \pm 0.5$ & Nil \\
PBO & Nil & Nil & Nil & $0.06 \pm 0.1$ \\
\hline
\end{tabular}




\subsection{Discussion}

It is evident from the affinity purification experiments that a significant proportion of the CDNB activity from both insects is not retained by a GSH affinity matrix. In a study of the GSTs of the housefly, (Clark et al., 1990) also found a similar result. This also confirms the result of (Alias and Clark, 2007) purifying GSTs from D. melanogaster using a GSH matrix. In an attempt to characterise those isoenzyme(s) from D. melanogaster and L. cuprina responsible for the activity in the unbound fractions, the DNP-GSH and CNP-GSH affinity matrices were employed in the present study. When the CDNB activities of proteins not bound to the GSH matrix from either D. melanogaster or L. cuprina were applied to DNP-GSH and CNP-GSH matrices, the recoveries using the DNP-GSH matrix were $36 \%$ and $40 \%$ respectively whereas with the CNP-GSH matrix they were $26 \%$ and $43 \%$ respectively (Table 4-3 and Table 4-4). The GSTs purified from both insects by the GSH affinity matrix appeared to contain polypeptides of two different M.W. (23 and 30-32 kDa) when separated by one dimensional gel electrophoresis. These were respectively identified as Delta and Sigma GSTs. The use of GSTrap column for the purification of L. cuprina GSTs showed the presence of an additional band on a 1D gel compared to the other GSH matrices (Figure 4-3). A previous study on purification of GSTs from D. melanogaster identified it as CG16936, a putative uncharacterised Epsilon class GST (Alias and Clark, 2007). This might explain the higher CDNB activity obtained with GSTrap matrix compared to with other GSH matrices. When the unbound fractions from the GSH column with significant GST activity towards CDNB were purified using CNP-GSH and DNP-GSH matrices, these matrices still captured the Sigma and Delta GSTs. The GSH matrix did not bind all the Sigma and Delta GSTs from the crude extract. The possible explanation 
for this could be that the GSH matrix might have become saturated or the presence of the endogenous inhibitors in crude extract might have impeded binding as the 'depigmented' preparation may have still contained some potentially inhibitory pigments (Motoyama et al., 1978). The GSH affinity-purified fractions resolved by 2D electrophoresis revealed that the Sigma remains one dominant spot whereas the Delta GST is composed of at least five spots of comparable size and intensity. This is consistent with observations of the housefly GSTs- GST1 (Delta, $28 \mathrm{kDa}$ ) and GST2 (Sigma, $32 \mathrm{kDa}$ ) when purified on a GSH affinity matrix, which yielded a similar pattern on a 2D gel (Fournier et al., 1992). This multispot appearance of Delta GST on 2D electrophoresis gel may indicate their post-translational modifications (PTMs) as the post-translational modifications such as phosphorylation, acetylation, deamidation, and cysteine oxidation cause changes in the $\mathrm{pI}$ of the protein which can be detected on a $2 \mathrm{D}$ gel. A detailed study on which type of PTM might be involved with Delta GST is described in chapter 7.

Most of the GSH affinity-purified GSTs from the D. melanogaster gel in the present study were identified by Alias (Alias and Clark, 2007). The use of BSP-GSH conjugate as a ligand used in that study resulted in the isolation of many GSTs including CG16936 (a putative uncharacterized GST) and a number of Epsilon GSTs (E3, E6, E7, E9) along with many non-GST proteins. However, the DNP-GSH affinity matrix appeared to be less effective in binding Epsilon isoforms than BSPGSH matrix but did bind two isoforms of Omega GSTs: CG6673 and CG6776 (Figure 4-20, Spots 6-9). It is reported in FlyBase (a database of D. melanogaster genes and genomes, http://flybase.org/) that the CG6673 has two annotated transcripts (A and B) that generate two different polypeptides of much the same M.W. but with different pI values. Spots 6 and 7 in Figure 4-20 in the present study 
correspond to those. The gene responsible for the expression is referred in FlyBase as DmeACG6673 (CG6673, FBgn0035906). There is experimental evidence that it is involved in biological processes such as determination of adult lifespan and response to oxidative stress (Kim et al., 2007). However, spots 8 and 9 in Figure 4-20 were identified as CG6776 which has only one annotated transcript and one polypeptide according to FlyBase and it may be involved in biological process similar to those involving CG6673. The reason for the expression of two CG6776 spots on gel may be due to post-translational modification. Neither of these Omega GST isoforms are involved in eye pigment synthesis (Kim et al., 2006). The CNP-GSH matrix purified fractions were resolved in a similar pattern to those from the DNP-GSH matrix and identification of spots also confirmed their similarity with each other (See Table 4-12 and Appendix Table 10-1). Therefore the DNP-GSH matrix was used subsequently throughout due to its reliably higher GST binding and easier preparation compared to CNP-GSH matrix.

The identification of the L. cuprina Delta GST spots on the gel was straightforward due to the availability of the sequence of this L. cuprina Delta GST. However, to characterise other L. cuprina GSTs by MALDI-TOF was challenging. The literature suggests that genome annotation methods are also commonly used for identification of protein-coding genes and analysis of protein sequences (Koonin and Galperin, 2003). The goal of the annotation process is to assign as much information as possible to the raw sequence of complete genomes with an emphasis on the location and structure of the genes. This can be accomplished by gene finding, by identifying homologies to known genes from other organisms, by the alignment of full-length or partial sequences to the sequence of known identity or through combinations of such methods (Reese et al., 2000). This approach has been used to 
develop the complete Arabidopsis GST database (Crowe et al., 2003), in identification of Epsilon and Delta class GSTs from D. melanogaster (Sawicki et al., 2003), in genomic organization and developmental expression of GST genes of the diamondback moth, Plutella xylostella (Sonoda et al., 2006) and to identify novel genes within the GST clusters (Kim et al., 2009). However, many researchers still view genome annotation as a notoriously unreliable and inaccurate process. That may be because it produces a considerable number of errors (Nakashita et al., 2000; Natale et al., 2000). Another approach to identify proteins is based on the de novo sequencing. A novel Sigma-class GST of the fall webworm, Hyphantria cunea has been identified by aligning its $\mathrm{N}$-terminal amino acid sequence with other insect GST N-terminal sequences (Yamamoto et al., 2007). In the Hessian fly Mayetiola destructor, two new Delta and Sigma GSTs were similarly characterized. The deduced amino acid sequences for the two M. destructor Delta GSTs (MdesGST-1 and MdesGST-3) and Sigma GST (MdesGST-2) showed high similarity with other insect GSTs including the conserved glutathione and substrate binding sites (Mittapalli et al., 2007). Two GST clones of Manduca sexta were sequenced and identified (Snyder et al., 1995). As the present study proceeded with proteomics, no genomics study was carried out. Some de novo sequences from a few critical spots of the proteins resolved on 2D gel were obtained from Centre for Protein Research (CPR), at University of Otago, but with minimal success. Only 4-6 peptides of lengths between 4 and 8 amino acids long were sequenced from three spots. Only three fragments DDKLYPK, F(M)CPYAHR and GEH(I/L)TPEF(I/L)K matched to D. melanogaster GSTs and that was not sufficient to identify the class of L. cuprina GSTs. Instead, an approach for the tentative classification of L. cuprina GSTs based on matching the sequences of peptides fitting the mass fingerprinting data from $L$. 
cuprina with consensus sequences of other insect GST classes was developed. The search of these peptides against the "D. melanogaster" database and "Other Metazoa" fragmentation database provided the provisional characterisation of each spot. When the peptides of spot 1 in Figure 4-18 were searched against the $D$. melanogaster database, it was identified as Sigma GST (pI 4.6, $30 \mathrm{kDa}$ ) and when searched against the "Other Metazoa" fragmentation database, the identified protein was still GST S1 with a high confidence score but in Musca domestica. When a sequence for the L. cuprina Sigma GST obtained by our laboratory from HortResearch (Hamilton) aligned with Musca domestica and Drosophila melanogaster Sigma GST sequences, it was found to be $94 \%$ and $91 \%$ similar respectively (Figure 4-27). The long deletion in the sequence of L. cuprina Sigma GST is due to the lack of the N-terminal sequence compared to Drosophila melanogaster and Musca domestica. The L. cuprina sequence is not complete with an absence of sequence data at some positions as shown in Figure 4-27.

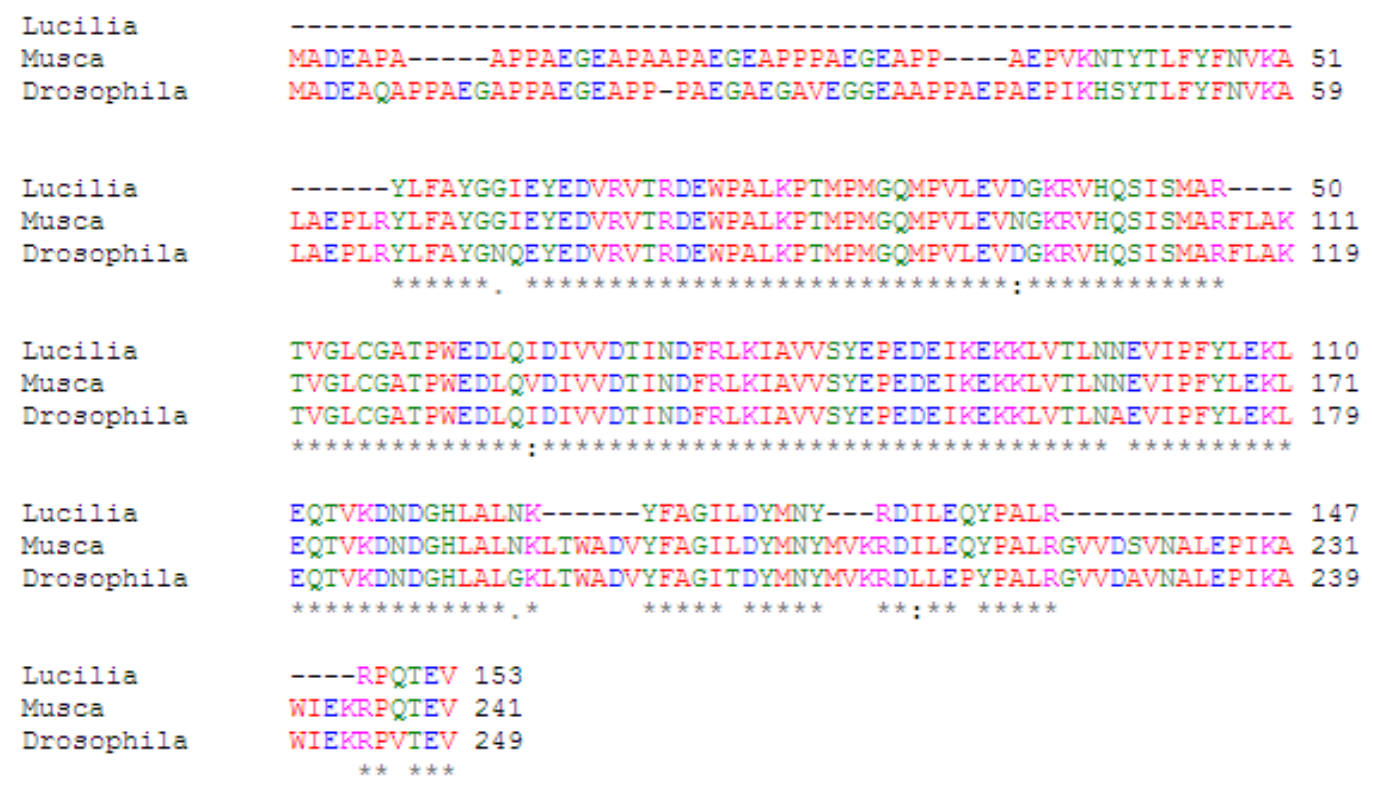

Figure 4-27: Alignment of L. cuprina Sigma GST sequence with other known insect Sigma GST sequences from Musca domestica and Drosophila melanogaster using ClustalW2. 
Thus, the relatedness of $L$. cuprina Sigma GST with other insect Sigma GST was confirmed by a combination of cross-species database matching and de novo sequencing. Two dimensional gels of GSH affinity-purified fractions from both insects in the present study showed a similar pattern of migration of GSTs (Figure 4-17 and Figure 4-18).

As the DNP-GSH affinity matrix resulted in the isolation of the widest range of GSTs, the identification of each spot was attempted by aligning the matched $L$. cuprina peptides with the consensus sequence of each class of GSTs. This approach yielded a tentative classification of $L$. cuprina GSTs.

Spot 2 (Figure 4-21) best matched a GST from Bombyx mori and BLAST search against D. melanogaster database showed its similarity with CG17639, a protein having glutathione transferase activity. It is worth noting that according to FlyBase CG17639 has also two annotated transcripts (A, B) generating two polypeptides in D. melanogaster with M.W. 27,841 Da (243 a. a.) and 25,429 Da (222 a. a.). The L. cuprina peptides of spot 2 matched with the higher M.W. CG17639 isoform. Similarly, spots 3 and 4 were best matched to a GST from Anopheles gambiae but BLAST against the D. melanogaster database showed strong similarity with GST D1 with M.W. 25,203 Da. When the alignment with the GST consensus sequences was performed in ClustalW2, spots 2, 3 and 4 confirmed their similarity with the Delta GSTs (pI 5.3, $28 \mathrm{kDa}$; pI 5.4, $28 \mathrm{kDa}$ and pI 5.5, $28 \mathrm{kDa}$ respectively). Interestingly, the D. melanogaster gel did not show the presence of the high M.W. form of CG17639 Delta GST (Figure 4-20 A). The analysis of spots 5 and 6 resulted in uncertainty of the protein identification. Spot 5 matched a Mu GST in Fasciola hepatica (Sheep liver fluke) and BLAST against D. melanogaster 
indicated its similarity with Sigma GST. There is not any evidence of Mu GSTs in insects (Enayati et al., 2005; Ranson et al., 2001). Spot 6 most closely matched Heligmosomoides polygyrus (a common nematode) GST when searched against "Other Metazoa" database. Despite good sequence coverage, alignment with consensus sequences of GSTs did not predict it to belong to any specific GST class at all. Spots 7 compared well with Anopheles gambiae GST (pI 5.4, $27 \mathrm{kDa}$ ) and spots 8 and 9 compared well with CG17533 (pI 5.5, $27 \mathrm{kDa}$ ) and CG16936 (pI 5.6, 27 $\mathrm{kDa}$ ) in D. melanogaster respectively and aligned well with the consensus sequence of Epsilon class GSTs. Spots 10 and 12 were identified as Delta GSTs with low molecular weight (pI 4.9, $23 \mathrm{kDa}$ and $\mathrm{pI} 5.25,23 \mathrm{kDa}$ respectively). Thus the DNPGSH affinity matrix captured both the Delta isoforms with low and high M.W. in $L$. cuprina. The Spots 11 and 13 matched GST in Fasciola hepatica (Sheep liver fluke) and Schistosoma japonicum (human blood fluke responsible for Schistosomiasis infection) and showed a similarity with Mu GSTs (pI 5.1, $23 \mathrm{kDa}$ and pI 5.45, 23 $\mathrm{kDa}$ respectively). As mentioned before, insects are reported to lack Mu GSTs but the alignment score and MALDI-TOF identification showed them as Mu-like GSTs. However, these similarity scores are not markedly higher than for other GST classes therefore their classification is uncertain. The DNP-GSH affinity fractions from both the insects contained some non-GST proteins as well. Spots 11 and 12 in $D$. melanogaster and spots 14, 15, 16 and 17 in L. cuprina gels appeared to be Rab proteins which have a significant role in GTP binding and in protein trafficking in the cell. Spots 13 and 14 in D. melanogaster were identified as lactoylglutathione lyase. It is not surprising that lactoyl glutathione lyase (which is also known as glyoxalase I) binds to DNP-GSH matrix as it uses a glutathione conjugate as its substrate (Thornalley, 2003). Glycerol 3-phosphate dehydrogenase was also present 
on D. melanogaster gel. Since during the course of the purification the column was washed with $1 \mathrm{M} \mathrm{NaCl}$ or $\mathrm{KCl}$, it is unlikely that these proteins are bound due to ionic interactions with the DNP-GSH matrix. Therefore their presence suggests specific interactions with the ligand or a close relation with the adsorbed GSTs, although neither are commonly reported artefacts in isolation of GST fusion proteins. The members of the Omega and Zeta GST classes may have a lower affinity for glutathione (Board et al., 1997; Rouimi et al., 2001) and thus may not bind well to the GSH affinity matrix. When fractions that did not bind to the GSH matrix were applied subsequently to the DNP-GSH matrix, it retained the Omega GSTs in $D$. melanogaster preparations but not those from L. cuprina. It is possible they may be present on the L. cuprina gels but could not be identified by mass fingerprinting. However, when a few spots from the L. cuprina DNP-GSH gel were sent to the Centre for Protein Research (CPR), at University of Otago for de novo sequencing, one of the peptides- $\mathrm{F}(\mathrm{M}) \mathrm{CPYAHR}$ from spot 5, which has uncertain tentative identification (Mu-like GST- pI 5.6, $28 \mathrm{kDa}$ ) in the present study, matched to the Omega GST sequence in D. melanogaster database. In the absence of detailed sequence information for L. cuprina GSTs other than the Delta class GST, the strategy approached here is a way of getting a preliminary view of what class the proteins might belong to. This is by no means a certain identification and there appears to be no rigorous statistical method for estimating the confidence of the characterisation. Even if this way of identification gives an apparently reliable assignment to a class, it is still difficult to know how good an assignment is without the gene or protein sequence information.

Based on the specific activities towards the model substrates, the GSH affinity-purified fractions and DNP-GSH affinity-purified enzymes were clearly 
distinguishable from one another. The GSH affinity-purified GSTs showed marked preferences for CDNB and very low activity towards DCNB compared to DNP-GSH purified GSTs. The DNP-GSH purified extract also showed thiol transferase and dehydroascorbate reductase activity, a characteristic of presence of Omega class GSTs (Board et al., 2000; Girardini et al., 2002). The activity with TNE, a lipid peroxidation product under conditions of oxidative stress (Agianian et al., 2003; Singh et al., 2001) was higher in GSH affinity-purified fractions. Higher TNE activity is associated with the presence of Sigma and Delta GSTs. Singh et al. investigated aspects of the catalytic activity of Sigma GST and they found that recombinant or native Sigma GST had significant glutathione-conjugating activity towards 4-HNE, whereas the activity was low for typical GST substrates such as aryl and alkyl halides, epoxides and nitro-aromatic compounds (Singh et al., 2001). In D. melanogaster, it has been shown that there are at least seven distinct GSTs including six Delta-class GSTs, and one Epsilon-class GST with significant activity for 4-HNE conjugation (Sawicki et al., 2003). We were unable to detect activities of Sigma GSTs towards DCNB and PBO (see Table 4-17)

The Delta GSTs from D. melanogaster and L. cuprina had higher activity with CDNB and TNE compared to Sigma GSTs but almost no activity with DCNB, EA and PBO. The specific activities of Sigma GSTs from both the insects were higher with EA. The D. melanogaster Sigma GST and L. cuprina Delta GSTs showed higher specificity for NPA and DHA (Table 4-17).

In summary, the work presented in this chapter attempted to purify and identify the GSTs from L. cuprina, using D. melanogaster as a model insect. It was observed that the affinity purification matrices successfully purified four major GST classes: Sigma, Delta, Epsilon and Omega in D. melanogaster however no Omega 
GST was identified in L. cuprina. A similar separation pattern of GSH affinitypurified GSTs was observed on 2D gels from both the insects. However, the differences of DNP-GSH affinity-purified GSTs separation on 2D gels between both the insects led us to develop an approach to tentatively characterise the L. cuprina GSTs. After affinity chromatography, the majority of proteins from D. melanogaster falling into the experimental M.W. range of 22-40 kDa identified as GSTs. This gave us confidence that cross-database matching of mass finger printing data from $L$. cuprina proteins in that M.W. range that showed up as GSTs were likely to be valid. By aligning the matched $L$. cuprina peptides against GST consensus sequences, we have assigned the class of GSTs. However, this approach is tentative and needs further confirmation. Some of the L. cuprina peptides identified as Mu-like GSTs, which is very intriguing because there is no Mu GST class known in insects. The high M.W. Delta GSTs were found only in L. cuprina but not in D. melanogaster suggesting a different role or properties in L. cuprina.

Given this information about L. cuprina GSTs, the next questions were:

- Does the GST proteome vary during the development of L. cuprina?

- Is GST expression tissue-specific in this insect?

The answers to these questions will add to our knowledge of the role of $L$. cuprina GSTs in the detoxification of various xenobiotics including insecticides. Chapter 5 addresses these questions using proteomics. 


\section{Examination of qualitative and quantitative variation of GSTs during the life span of Lucilia cuprina and in main body parts of the adult fly}

\subsection{Introduction}

This chapter describes the qualitative and quantitative variation in the expression of GSTs in developmental stages and in main body parts (head, thorax and abdomen) of L. cuprina as revealed using proteomics. The ontogenic pattern of GST activities towards model substrates has also been studied.

Age-dependent alteration of GST activities has been demonstrated in both vertebrates (Gregus et al., 1985) and invertebrates (Hazelton and Lang, 1983; Kotze and Rose, 1987; Wood et al., 1986). Literature suggests that GSTs are differentially regulated with specific enzymes being expressed in different tissues during each developmental stage and in response to various xenobiotics (Ranson et al., 1997). The varied susceptibility of insects towards insecticides during different developmental stages has been reported in Drosophila melanogaster (Hunaiti et al., 1995), Apis mellifera macedonica (Papadopoulos et al., 2004a) and Tenebrio molitor (Kostaropoulos et al., 1996; Kostaropoulos et al., 2001b). Resistance mechanisms to organophosphorus insecticides have been proposed to include decreased penetration and enhanced detoxification by esterases and GSTs in Plutella xylostella larvae (Maa and Chuang, 1983; Sun and $\mathrm{Ku}, 1994)$. It has also been proposed that resistance to certain selected pesticides in insects may be related to changes in their GST expression (Clark and Dauterman, 1982; Hayaoka and Dauterman, 1983). These studies have reported the purification of limited GSTs or GSTs from limited developmental stages of insects and characterised them based on the activity with 
model substrates, with insecticides or inhibitors. The changes in the proteins' characteristics (such as substrate specificity, sensitivity to certain inhibitors, physicochemical properties and kinetic parameters) during development may be explained either by post-translational modification of the proteins during the development (Rattan, 1996) or by variation in the expression of certain GST isoenzymes (Clark and Dauterman, 1982; Hayaoka and Dauterman, 1983). Ontogenic patterns of cytosolic GST activity towards model substrates have been determined in Aedes aegypti (Hazelton and Lang, 1983), L. cuprina (Kotze and Rose, 1987) and Tenebrio molitor (yellow mealworm beetle) (Kostaropoulos and Papadopoulos, 1998) including four developmental stages i.e. egg, larvae, pupae and adults. An attempt to relate the presence of GSTs in L. cuprina to its role in the detoxification of various xenobiotics including insecticides requires knowledge of level of these enzymes in different developmental stages of the fly. To our knowledge, so far no one has scrutinized the detailed changes in the GST proteome which may occur during the development of L. cuprina.

Expression of GSTs is often tissue specific (Voss and van Bladeren, 1990). In insects, dissected tissues have been used to locate GSTs (Kotze and Rose, 1987). Detection of GSTs in $M$. domestica haemolymph cells, indirect flight muscles and the cell bodies of the central nervous system has been undertaken using immunohistology (Franciosa and Berge, 1995). A comparison of the GSTs' location between an insecticide-susceptible strain of housefly and resistant ones was studied and no variation due to resistance found (Franciosa and Berge, 1995). However, over- expression of GSTs found when the head, thorax and abdomen of the GG strain of Aedes aegypti, a strain having $\geq 4$-fold higher total GST activity compared to the wild-type lab strain, was studied. These results suggested that elevated GST activity 
in the GG strain is due to constitutive overexpression of GST-2 (Sigma) and GST-1a (Delta) that expressed in tissue-specific manner (Grant et al., 1991). The GSTs in head, thorax and abdomen were detected through their activities with limited model substrates such as CDNB and DCNB (Franciosa and Berge, 1995; Grant et al., 1991; Kotze and Rose, 1987). However, the substrates alone are not sufficient to distinguish precisely the different isoforms of GSTs due to their overlapping specificity (Habig and Jakoby, 1981). This could explain discrepancies and disagreements over GST location reported by various groups (Hunaiti et al., 1995; Kotze and Rose, 1987). Therefore, a re-examination of GST location seemed appropriate, and is analysed in this chapter through a proteomic approach using protein extracts from main body parts such as head, thorax and abdomen due to the presence and abundance of proteins and ease of preparation of these parts.

\subsection{Objectives:}

- To isolate the GSTs from egg, larval, pupal and adult stages of L. cuprina and to compare their GST proteomes.

- To study the ontogenic pattern of GST activity in the developmental stages using different model substrates.

- To study the GST proteomes of head, thorax and abdomen of adult $L$. cuprina. 


\subsection{Results:}

\subsubsection{Purification of GSTs from eggs, larvae, pupae and adult}

The biochemical comparison of GSTs from various developmental stages of L. cuprina (PY81 strain) was carried out following methods described in section 3.2. The data in Table 5-1 showed that the crude extracts prepared from $L$. cuprina pupae displayed significantly higher total and specific activity towards CDNB $(\mathrm{p}<0.05)$ than the enzyme of eggs, larvae and adults. To further characterize the GST activity, the crude preparation of egg, larval, pupal and adult stages were separately applied to the GSH affinity matrix (10 $\mu \mathrm{mol} / \mathrm{ml}, S$-linked to Sepharose) initially and then unbound fractions from the GSH matrix were further purified using the DNP-GSH affinity matrix $(9.55 \mu \mathrm{mol} / \mathrm{ml})$ as described in section 3.2. As shown in the previous chapter, the GSH affinity matrix specifically binds to only Sigma and Delta GSTs and therefore it was used first to purify Sigma and Delta GSTs from different developmental stages of $L$. cuprina. The purification of unbound fractions from the GSH matrix was then subsequently carried out using the DNP-GSH matrix as it binds a wide range of proteins, allowing the isolation of proteins other than Sigma and Delta GSTs. The results of such purification schemes are summarised in Table 5-1. The total protein recovered from the crude preparation of larvae, pupae and adult was significantly $(\mathrm{p}<0.05)$ almost 2 -fold higher than that of egg crude preparation. Of GSH affinity-purified GSTs, the specific activity of the pupal GSTs towards CDNB was significantly different $(\mathrm{p}<0.05)$ from other stages being 3-, 2.4- and 3.4fold higher than egg, larvae and adult respectively. However, the specific activity of DNP-GSH affinity-purified GSTs of the adult stage was higher than other stages. 
Table 5-1: Purification of GSTs from developmental stages of L. cuprina.

GSTs of eggs, larvae, pupae and adults ( $3 \mathrm{~g}$ each) were partially purified using a GSH affinity matrix. Fractions that did not bind to the GSH matrix with significant GST activity towards CDNB were pooled, concentrated and applied to a DNP-GSH affinity matrix for further possible purification of GSTs. The values represent mean \pm S.D. of three individual experiments measured in triplicates. The \% yield and fold purification of DNP-GSH matrix fractions are based on the activity in the crude extract.

\begin{tabular}{|c|c|c|c|c|c|c|}
\hline Stage & Purification step & $\begin{array}{l}\text { Total } \\
\text { protein } \\
\text { conc. } \\
\text { (mg/16 } \\
\mathrm{ml} \text { ) } \\
\end{array}$ & $\begin{array}{l}\text { Total } \\
\text { activity } \\
\text { ( } \mu \mathrm{mol} / \mathrm{min})\end{array}$ & $\begin{array}{c}\text { Specific } \\
\text { activity } \\
\left(\begin{array}{c}\mu \mathrm{mol} / \mathrm{min} / \\
\mathrm{mg})\end{array}\right.\end{array}$ & $\begin{array}{l}\text { Yield } \\
(\%)\end{array}$ & Fold \\
\hline \multirow[t]{6}{*}{ Egg } & Crude & $25.0 \pm 1.5$ & $2.20 \pm 0.9$ & $0.09 \pm 0.04$ & 100 & 1.00 \\
\hline & De-pigmented & $20.5 \pm 0.4$ & $2.10 \pm 0.5$ & $0.10 \pm 0.02$ & 95.4 & 1.11 \\
\hline & Unbound GSH & $13.5 \pm 0.5$ & $1.40 \pm 0.2$ & $0.10 \pm 0.01$ & 63.6 & 1.11 \\
\hline & Bound GSH & $0.30 \pm 0.1$ & $1.40 \pm 0.1$ & $4.66 \pm 1.18$ & 63.6 & 51.7 \\
\hline & Unbound DNP-GSH & $10.0 \pm 1.0$ & $0.78 \pm 0.2$ & $0.08 \pm 0.02$ & 35.4 & 0.88 \\
\hline & Bound DNP-GSH & $1.30 \pm 0.1$ & $0.77 \pm 0.1$ & $0.59 \pm 0.07$ & 35.0 & 6.55 \\
\hline \multirow[t]{6}{*}{ Larvae } & Crude & $51.0 \pm 0.5$ & $2.70 \pm 1.0$ & $0.05 \pm 0.02$ & 100 & 1.00 \\
\hline & De-pigmented & $45.0 \pm 0.3$ & $2.40 \pm 0.4$ & $0.05 \pm 0.01$ & 88.8 & 1.00 \\
\hline & Unbound GSH & $33.5 \pm 1.0$ & $1.40 \pm 0.1$ & $0.04 \pm 0.003$ & 51.8 & 0.80 \\
\hline & Bound GSH & $0.3 \pm 0.01$ & $1.50 \pm 0.2$ & $5.01 \pm 1.3$ & 55.5 & 100 \\
\hline & Unbound DNP-GSH & $29.0 \pm 2.0$ & $0.96 \pm 0.1$ & $0.03 \pm 0.003$ & 35.5 & 0.60 \\
\hline & Bound DNP-GSH & $1.9 \pm 0.03$ & $0.57 \pm 0.1$ & $0.30 \pm 0.05$ & 21.1 & 6.00 \\
\hline \multirow[t]{6}{*}{ Pupae } & Crude & $51.5 \pm 1.0$ & $7.50 \pm 1.8$ & $0.14 \pm 0.04$ & 100 & 1.00 \\
\hline & De-pigmented & $50.0 \pm 2.0$ & $7.20 \pm 0.7$ & $0.14 \pm 0.02$ & 96.0 & 1.00 \\
\hline & Unbound GSH & $36.0 \pm 0.5$ & $2.25 \pm 0.4$ & $0.06 \pm 0.01$ & 30.0 & 0.42 \\
\hline & Bound GSH & $0.40 \pm 0.1$ & $5.80 \pm 0.7$ & $14.5 \pm 3.80$ & 77.3 & 103 \\
\hline & Unbound DNP-GSH & $31.5 \pm 5.0$ & $1.40 \pm 0.1$ & $0.04 \pm 0.003$ & 18.6 & 0.28 \\
\hline & Bound DNP-GSH & $2.10 \pm 0.1$ & $0.80 \pm 0.1$ & $0.38 \pm 0.04$ & 10.6 & 2.71 \\
\hline \multirow[t]{6}{*}{ Adult } & Crude & $46.0 \pm 0.5$ & $3.90 \pm 0.8$ & $0.08 \pm 0.02$ & 100 & 1.00 \\
\hline & De-pigmented & $43.0 \pm 1.0$ & $3.70 \pm 0.5$ & $0.08 \pm 0.01$ & 94.8 & 1.00 \\
\hline & Unbound GSH & $26.0 \pm 0.5$ & $2.40 \pm 0.1$ & $0.09 \pm 0.003$ & 61.5 & 1.12 \\
\hline & Bound GSH & $0.6 \pm 0.02$ & $2.50 \pm 0.7$ & $4.16 \pm 1.16$ & 64.1 & 52.0 \\
\hline & Unbound DNP-GSH & $20.0 \pm 1.0$ & $1.48 \pm 0.1$ & $0.07 \pm 0.005$ & 38.0 & 0.87 \\
\hline & Bound DNP-GSH & $1.7 \pm 0.04$ & $1.1 \pm 0.03$ & $0.64 \pm 0.017$ & 28.2 & 8.00 \\
\hline
\end{tabular}




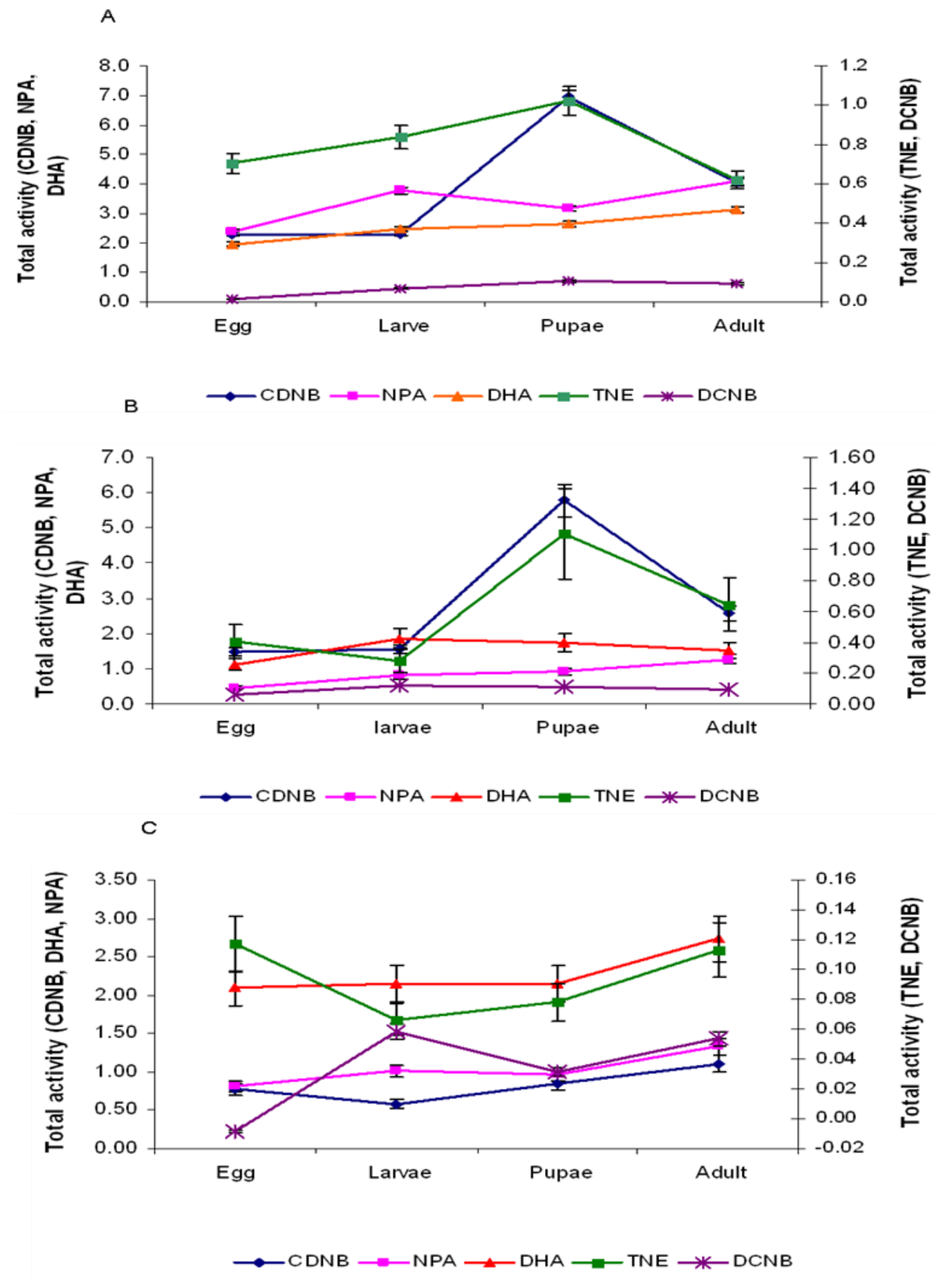

Figure 5-1: Substrate-specific total activity of crude and affinity-purified GSTs from different developmental stage of L. cuprina towards the model substrates.

A) Crude GSTs, B) GSH affinity-purified GSTs, C) DNP-GSH affinity-purified GSTs. Whole body homogenates ( $3 \mathrm{~g}$ ) were used as the crude enzyme source and purified by GSH affinity matrix and subsequently by DNP-GSH affinity matrix. Total activity values are given as $\mu \mathrm{mol} / \mathrm{min}$. Assays were undertaken following the methods in section 3.2.4. The values are from three independent experiments with triplicate measurements within each experiment. Error bars represent S.D. 


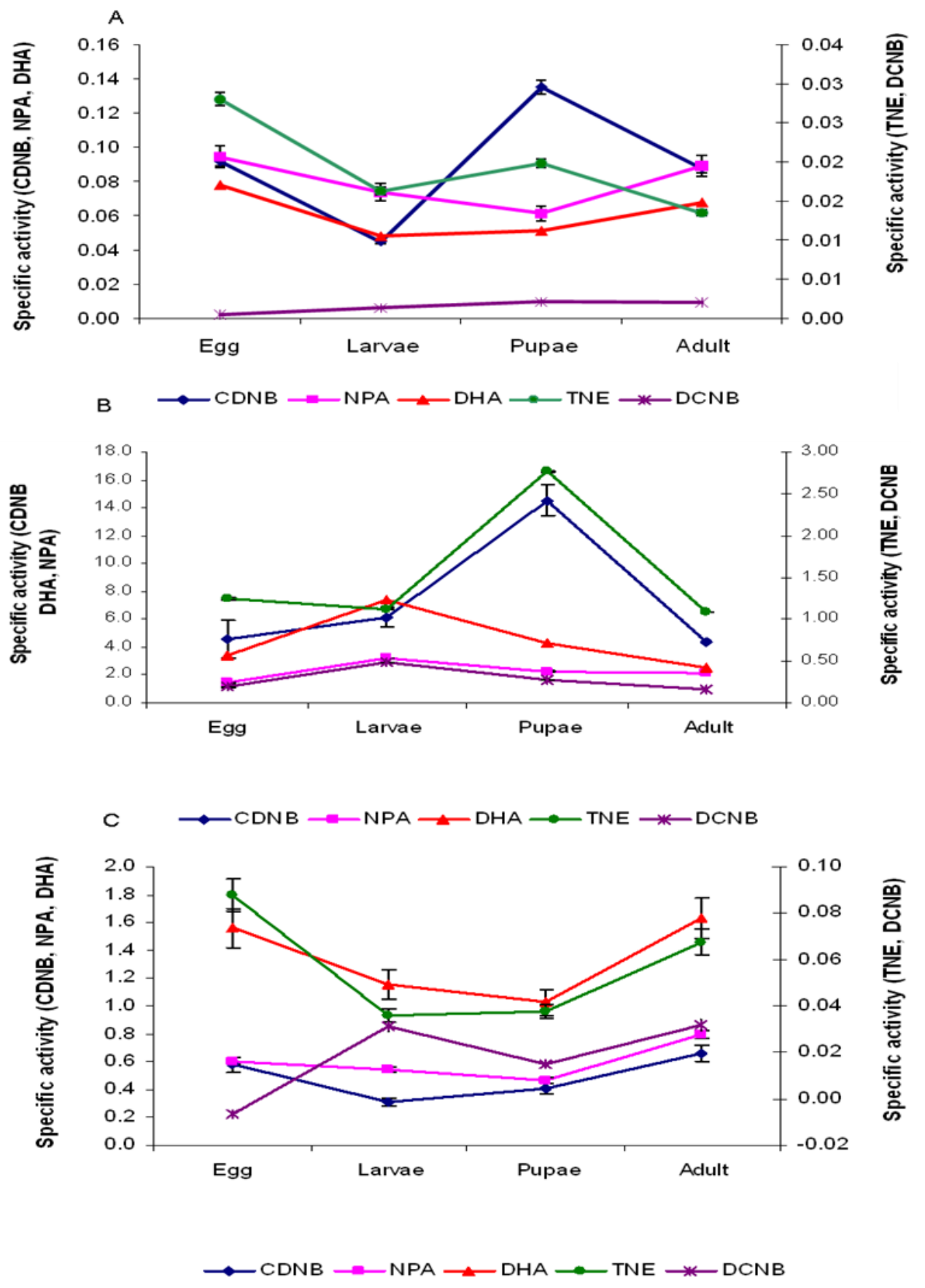

Figure 5-2: Substrate-specific specific activity of crude and affinity-purified GSTs from different developmental stage of $L$. cuprina towards the model substrates.

A) Crude GSTs, B) GSH affinity-purified GSTs, C) DNP-GSH affinity-purified GSTs. Whole body homogenates ( $3 \mathrm{~g}$ ) were used as the crude enzyme source and purified by GSH affinity matrix and subsequently by DNP-GSH affinity matrix. Specific activity values are given as $\mu \mathrm{mol} / \mathrm{min} / \mathrm{mg}$ of protein. Assays were undertaken following the methods in section 3.2.4. The values are from three independent experiments with triplicate measurements within each experiment. Error bars represent S.D. 
The total activity of crude and affinity-purified GSTs towards the model substrates CDNB, DCNB, NPA, DHA, and TNE during the life span of the $L$. cuprina is shown in Figure 5-1. The ontogenic total activity profile for CDNB, DCNB and TNE were similar i.e., the activity of crude enzyme reached a maximum at the pupal stage. Thereafter, the activity started declining steadily in the adult phase. The ontogenic activity profile observed for the substrates NPA and DHA was quite different. The maximum activity was found in adult however NPA activity was quite similar in the larval stage and there was not much difference in DHA activity in larval and pupal stages. GSH affinity-purified GSTs from the pupal stage had the significantly highest total activity with CDNB and TNE ( $\mathrm{p}<0.05)$. The DNP-GSH affinity-purified GSTs showed increased activity in the adult stage with all the substrates used except DCNB and TNE. Total DCNB activity was the highest in the larval stage.

The specific activities of crude and affinity-purified GSTs towards the model substrates are shown in Figure 5-2. The crude enzyme from eggs showed the highest activity towards NPA, DHA and TNE but not significantly compared to other stages. The specific activities determined in the GSH affinity-purified GSTs from pupae were significantly $(\mathrm{p}<0.05)$ higher towards CDNB and TNE. An interesting finding was that larval GSH affinity-purified GSTs showed higher activity towards DCNB, DHA and NPA. DNP-GSH affinity-purified GSTs from the eggs showed high activity towards TNE. It is noteworthy the activity towards DCNB in DNPGSH affinity-purified GST from larvae and adult was significantly higher than egg and pupal GSTs.

While the substrate profiling confirms that changes in GST activities occur during development, it is far from definitive and more specific methods for 
identifying GST isoforms from different developmental stages of the insect is needed. To investigate further, the GSH and DNP-GSH affinity-purified fractions were studied using proteomic methods.

\subsubsection{Proteomic analysis}

The sequential purification scheme presented in section 3.2.2.2 allowed the isolation of many GSTs from the different developmental stages of L. cuprina and facilitated their comparison. A typical SDS-PAGE analysis of GSH affinity-purified GSTs from the developmental stages of $L$. cuprina is presented in Figure 5-3.

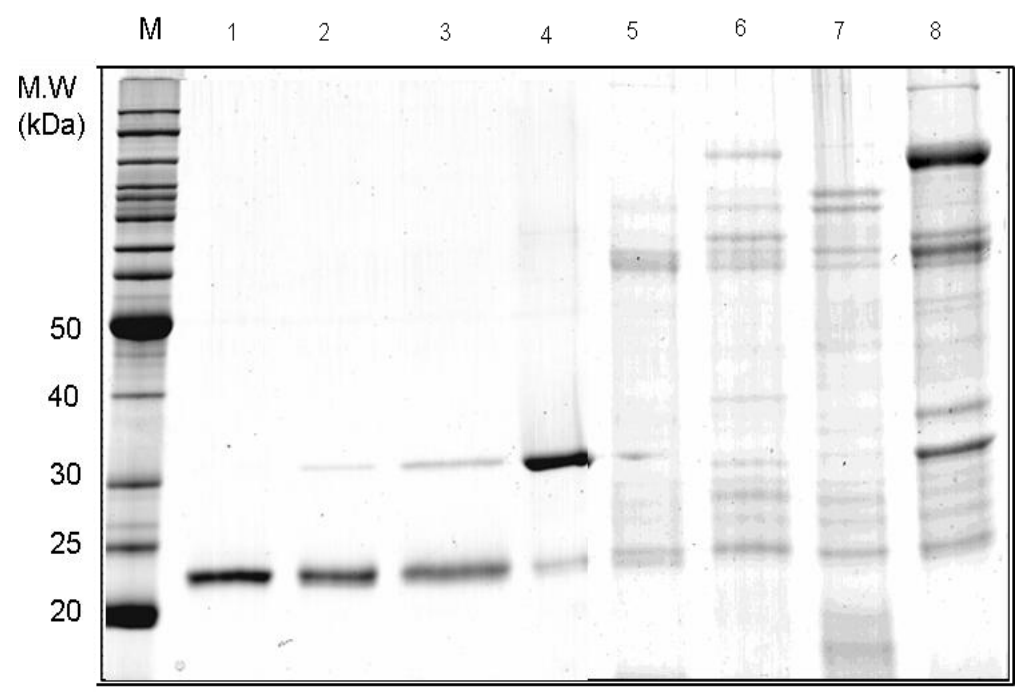

Figure 5-3: A typical SDS PAGE of eluents from fractions that bound to GSH and DNPGSH affinity matrices from different developmental stages of L. cuprina.

Lane M is M.W. marker. Lanes 1-4 show bound GSH fractions of egg, larvae, pupae and adult respectively ( $3 \mathrm{~g}$ of each insects). The unbound fractions from GSH affinity were then purified on a DNP-GSH affinity column. Lanes 5-8 are of bound DNP-GSH fractions of egg, larvae, pupae and adult respectively. The gel was stained with Coomassie Brilliant Blue G250 . 
One protein band with approximate M.W. 23,000 Da was always obtained in SDS gel electrophoresis of the GSH affinity-purified enzymes from egg, larval, pupal and adult stages as shown in Figure 5-3. It was identified as Delta GST by MALDITOF as described in section 4.3.3. Another protein band, with approximate M.W. 30,000 Da started appearing from the larval stage and is abundantly present in the adult stage. This was identified as a Sigma GST by MALDI-TOF as described in section 4.3.3. As shown by the 1D gel (Figure 5-3), the bound DNP-GSH fractions contained many high M.W. proteins due to non-specific binding of proteins to the DNP-GSH matrix. In chapter 4, it was shown that the Delta GST, though present as a single band on a $1 \mathrm{D}$ gel, was resolved into at least 6 spots on a $2 \mathrm{D}$ gel. To see whether this was the same case for Delta GSTs in the different developmental stages, 2D gel electrophoresis was carried out on GSH affinity-purified fractions (Figure 5-4). The DNP-GSH affinity-purified extract was also separated by $2 \mathrm{D}$ gel for the better resolution (Figure 5-6). 


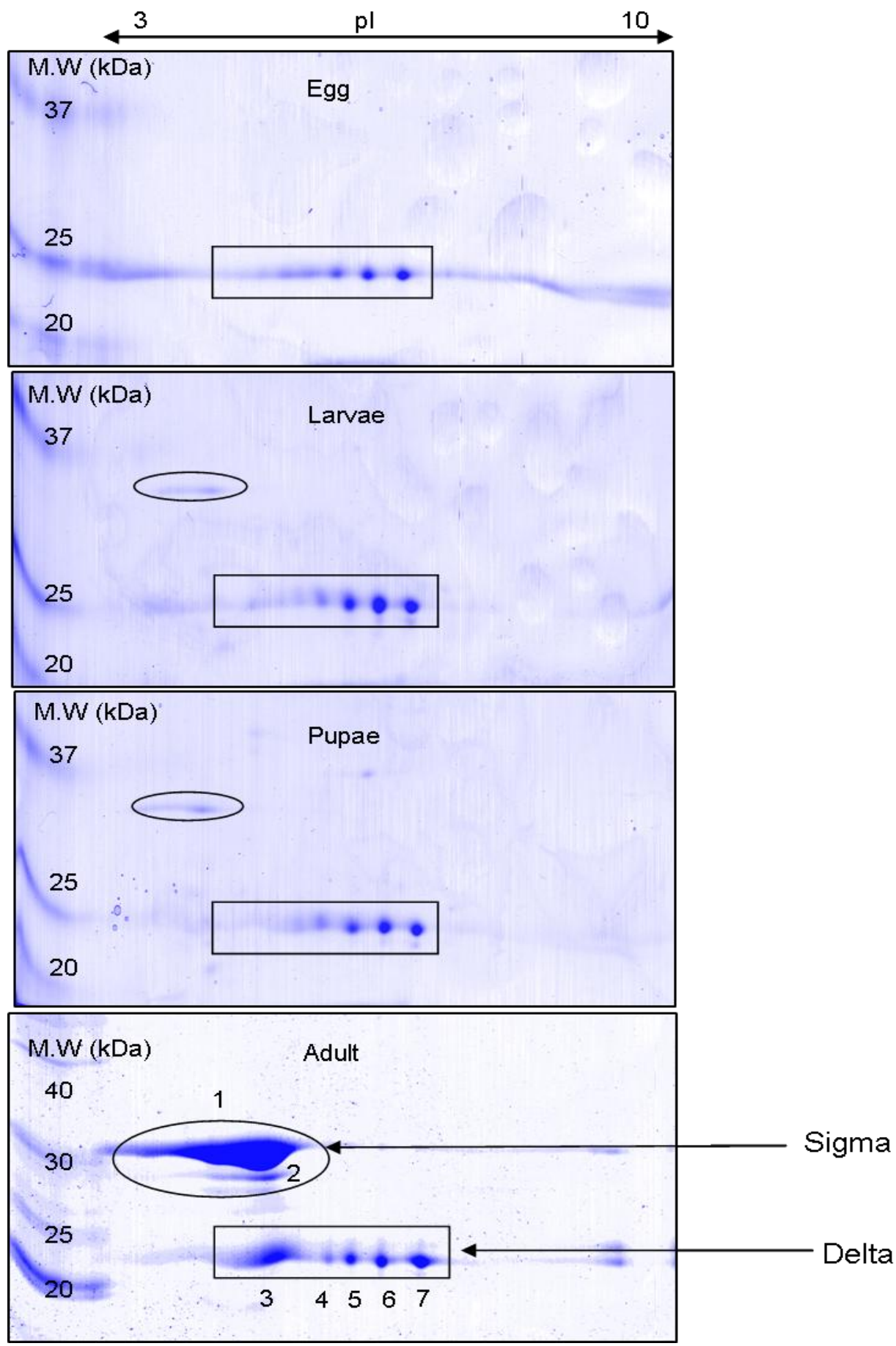

Figure 5-4: Two dimensional electrophoresis gels of GSH affinity-purified fractions from egg, larvae, pupae and adult stages of L. cuprina.

GSH affinity-purified GSTs from each developmental stage were separated in the first dimension on a $7 \mathrm{~cm} \mathrm{pH} \mathrm{3-10} \mathrm{linear} \mathrm{IPG} \mathrm{strip} \mathrm{and} \mathrm{then} \mathrm{in} \mathrm{the} \mathrm{second} \mathrm{dimension} \mathrm{on} \mathrm{a} \mathrm{SDS}$ PAGE gel. The M.W. marker was run along the sample. The gels were stained by Coomassie Brilliant Blue G-250. The protein load on each gel was from an equal weight of insect ( $3 \mathrm{~g}$ each). The identification of the numbered spots can be found in Table 4-10. 


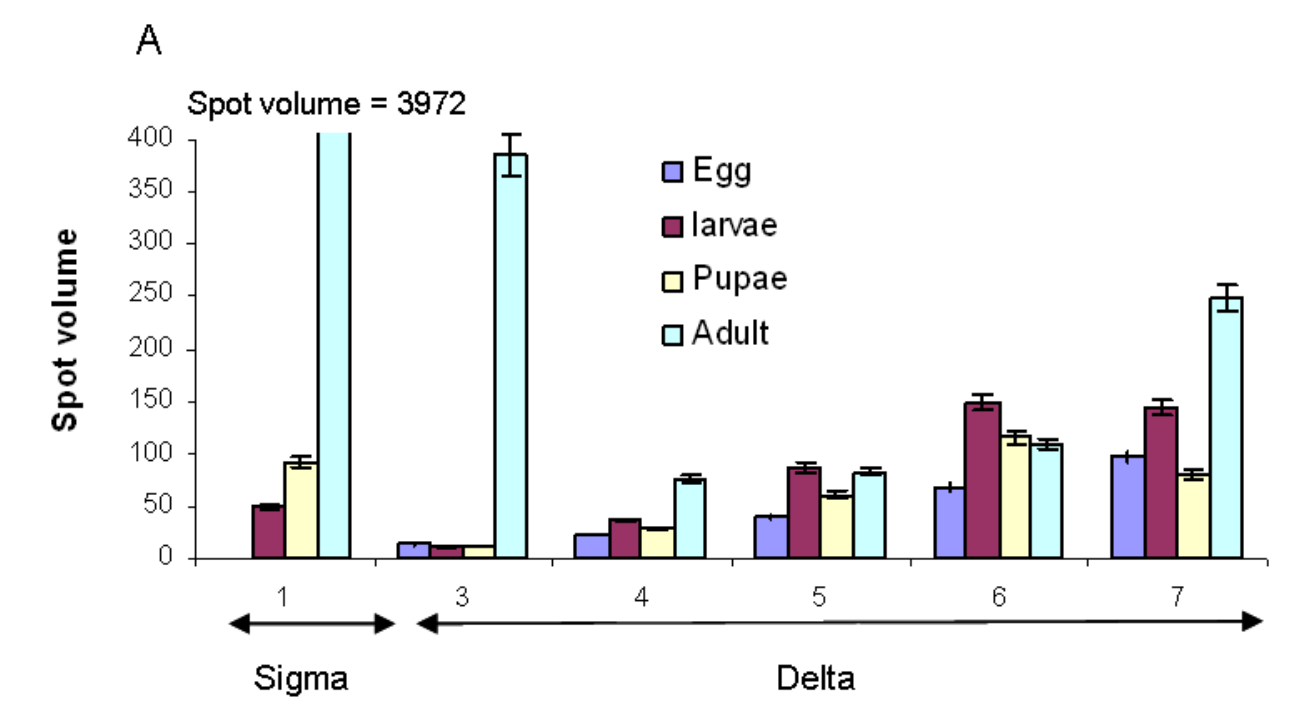

\section{B}

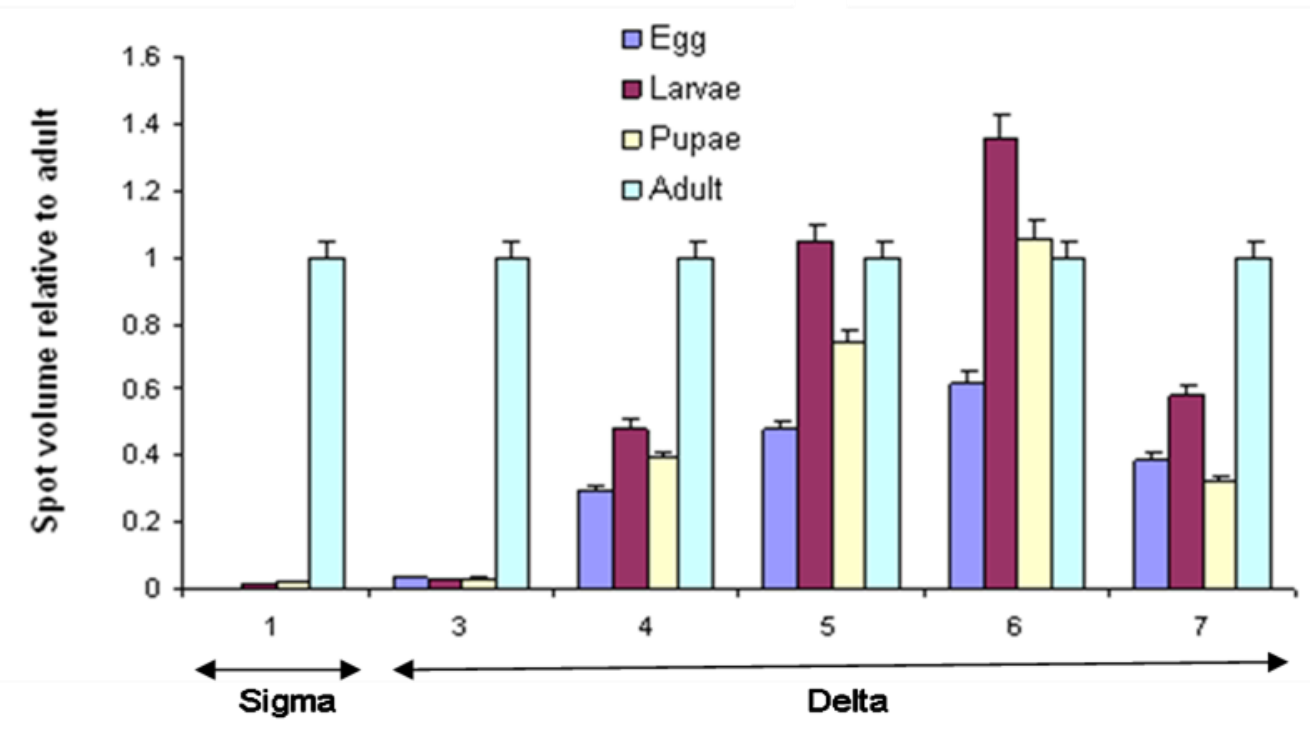

Figure 5-5: Quantification of the GSH affinity-purified protein spots expressed in different developmental stages of L. cuprina.

Quantitative analysis of the digitized images was carried out using ImageQuant (Version 5.2, Molecular Dynamics) software. A) Spot volume and B) Spot volume relative to adult value. Values are the mean of at least three independent experiments with three measurements in each. Error bars represent standard deviation. Spot $1=$ Sigma GST (pI 4.6, $30 \mathrm{kDa})$ and spot 3-7 are Delta GSTs with M.W. $23 \mathrm{kDa}$ and pI 4.9, 5.4, 5.5, 5.7 and 5.9 respectively. Numbers assigned to GSTs correspond to those in Figure 5-4. Spot 2 appears to be a degradation product of the Spot 1 and is not included in the analysis. 
GSH affinity-purified GSTs resolved on a 2D gel from each developmental stage of L. cuprina were identified by MALDI-TOF and their identity confirmed with the previous reported identification of GSTs purified from the GSH affinity matrix in section 4.3.3 (Table 4-10 and Table 4-11). Spots 1 and 2 were identified as Sigma GST and spots 3-7 were assigned as the same Delta GST1 (Figure 5-4). The proteomic results show that the Delta GSTs are expressed in all life stages of flies. However, the Sigma GST (spot 1) only starts expressing from the larval stage and is most abundantly present in adult stage. While in the larval and pupal stages the Delta GST was expressed at a significantly higher level than Sigma GST, in the adult stage, the relative amounts were changed dramatically. Significant $(p<0.05) 80$ - and 44-fold differences for Sigma GST were determined between larvae and pupae respectively compared to the adult. Eggs lack the Sigma GST. The Delta GSTs also vary quantitatively during the developmental stages (Figure 5-5). The interesting observation was that the spot 3 (Delta, pI 4.9, $23 \mathrm{kDa})$ had significantly $(\mathrm{p}<0.05)$ higher 27-30-fold expression in adult stage compared to other stages. In the adult stage the volume of spot 3 was 5.1-, 4.6-, 3.5- and 1.6-fold significantly higher $(\mathrm{p}<0.05)$ compared to spots $4,5,6$ and 7 respectively. This may suggest that the there is some physiological processing of Delta GSTs going on during the development of an insect. The higher volume and distorted shape of spot 3 also suggests that it may be a group of more than one spot but not resolved on a 2D gel. The expression of Delta GSTs in adult was 3.7-, 2.1- and 3.0-fold higher than the Delta GSTs of egg, larvae and pupae. However, the expression of Delta GSTs did not significantly vary between the different developmental stages as did the Sigma GSTs. The DNP-GSH affinity-purified fractions from each stage were also resolved by $2 \mathrm{D}$ electrophoresis. 


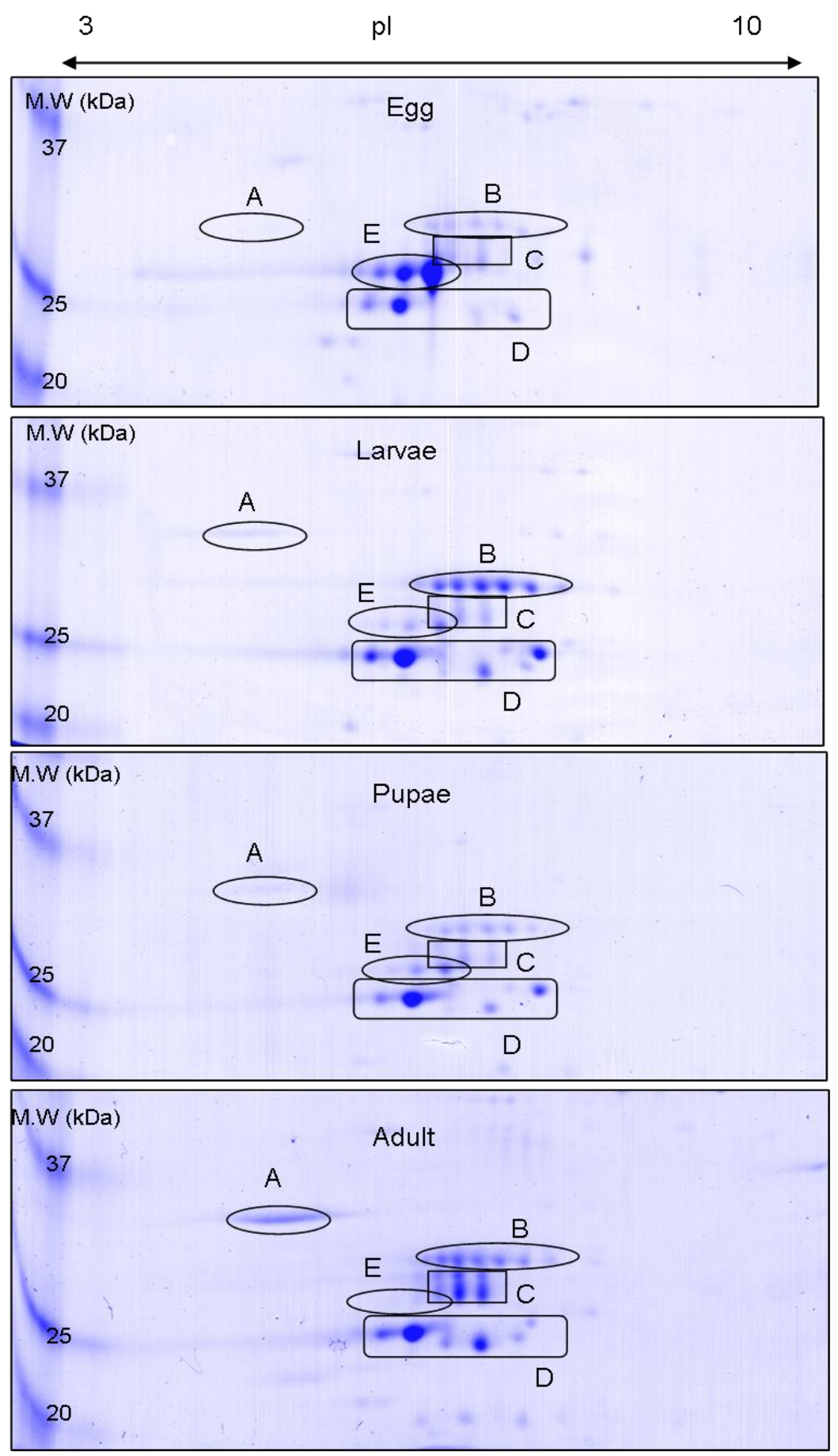

Figure 5-6: Two dimensional electrophoresis gels of DNP-GSH affinity-purified fractions from egg, larvae, pupae and adult stages of L. cuprina.

DNP-GSH affinity-purified GSTs from each developmental stage were separated in the first dimension on a $7 \mathrm{~cm} \mathrm{pH} \mathrm{3-10} \mathrm{linear} \mathrm{IPG} \mathrm{strip} \mathrm{and} \mathrm{then} \mathrm{in} \mathrm{the} \mathrm{second} \mathrm{dimension} \mathrm{on} \mathrm{a} \mathrm{SDS}$ PAGE gel. The M.W. marker was run along the sample. The gels were stained by Coomassie Brilliant Blue G-250. The protein load on each gel was from an equal weight of insect (3 g each). 
A

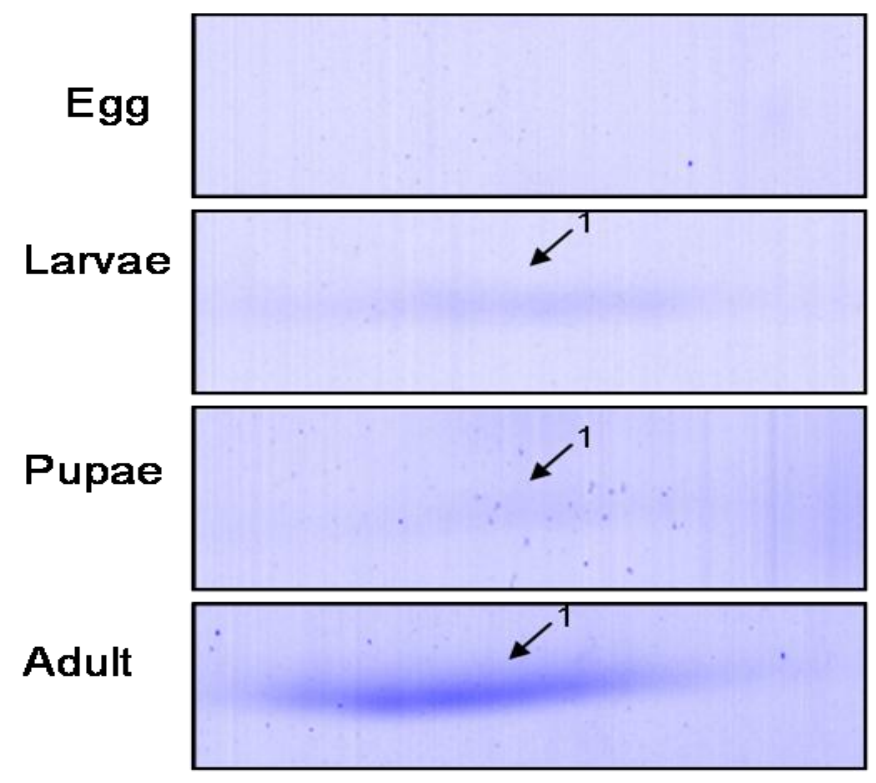

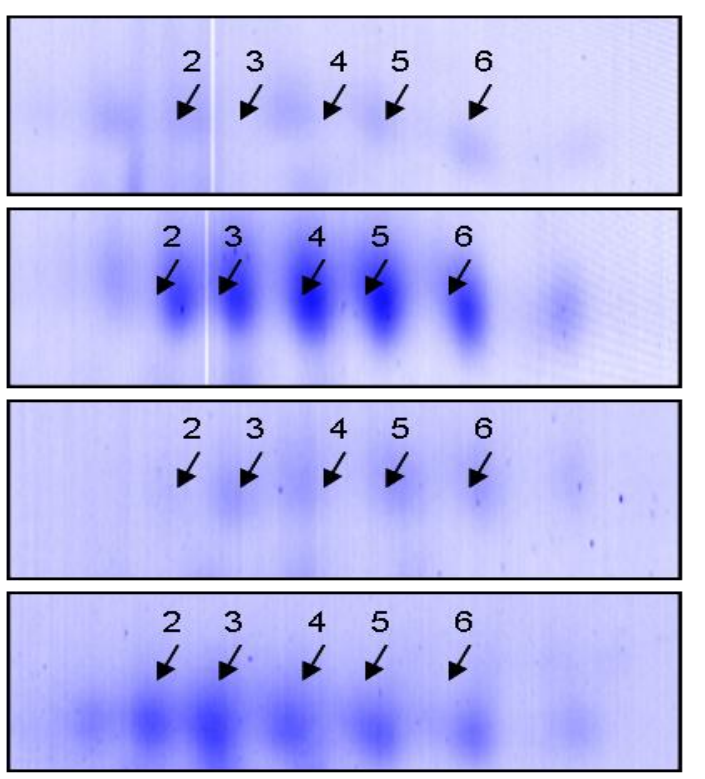

C

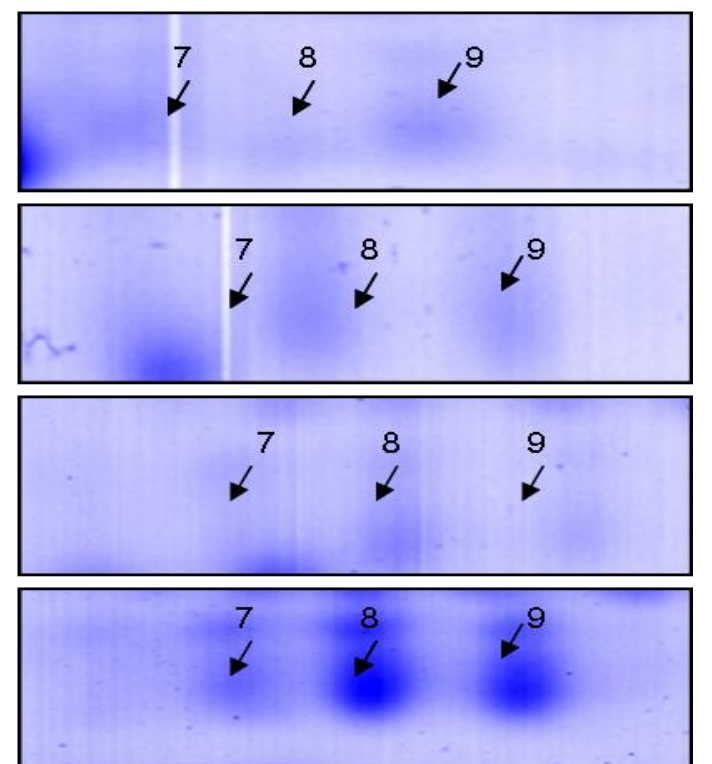

Figure 5-7: Changes in expression of DNP-GSH affinity-purified proteins in different developmental stages of L. cuprina.

Zones A, B and C from Figure 5-6 are shown in enlarged images to give the clear numbering of the spots. The numbered spots were identified by MALDITOF and confirmed their identity with the previous identification of DNP-GSH affinity-purified proteins from adult L. cuprina reported in section 4.3.3, Table 4-13 and for the tentative classification of numbered spots of Zone A, B and C details refer to section 4.3.3, Table 4-14 accordingly. The proteins expressed in zone A were identified as Sigma GST (spot 1). Zone B proteins were tentatively identified as high M.W. Delta class GSTs (spots 2-4) and Mu-like GST (spot 5). Spot 6 - not characterised. Zone $C$ proteins were identified as the Epsilon GSTs. 


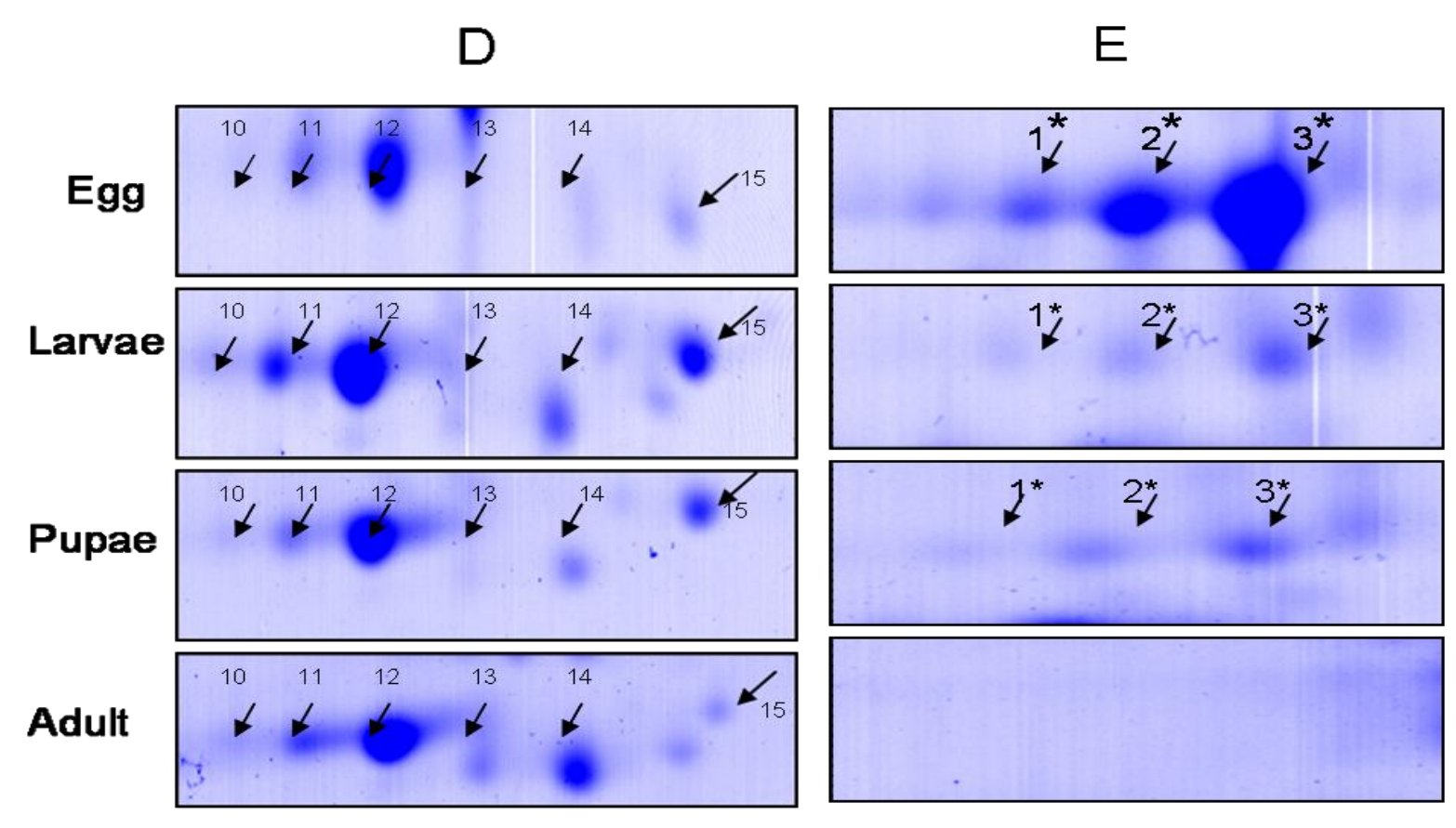

Figure 5-8: Changes in expression of DNP-GSH affinity-purified proteins in different developmental stages of L. cuprina.

Zones D and E from Figure 5-6 are shown in enlarged images to give the clear numbering of the spots. The numbered spots were identified by MALDI-TOF and confirmed their identity with the previous identification of DNP-GSH affinity-purified proteins from adult L. cuprina reported in section 4.3.3, Table 4-13 and for the tentative classification of numbered spots of zone D details, refer to section 4.3.3, Table 4-14 accordingly. The proteins expressed in zone D were tentatively identified as the Delta class of GSTs with lower M.W. (spot 10 and 12), Mu-like GSTs (spot 11 and 13) and Rab proteins (spot 14 and 15). Proteins in zone $\mathrm{E}$ were not characterised previously as adult did not show their expression on $2 \mathrm{D}$ gel. For the identification and tentative characterisation of zone $\mathrm{E}$ proteins, refer to Table 5-2 and Table 5-3. The sequence alignment with matched GST class is shown in Figure 5-9 and Figure 5-10. 
Table 5-2: Identification of spots present in Zone E in Figure 5-8.

The proteins in zone E were obtained from the DNP-GSH affinity-purified fractions of $L$. cuprina separated over a pH range of 4-7 (Figure 5-6) using 2D gel electrophoresis as described in section 3.2.7.2. The proteins were identified using MALDI-TOF MS peptide mass fingerprinting. Protein spots are as numbered in Figure 5-8 E. Z score, the sequence coverage and the number of matched peptides are reported as obtained from Profound. In addition to the protein name, the Swiss-Prot/TrEMBL accession numbers, the computed and experimental molecular mass and isoelectric point are given. Identification was made by using D. melanogaster and Other Metazoa NCBInr fragmentation databases. A protein Z score greater than 1.65 is significant in Profound. The tentative classification of GSTs was assigned by aligning the matched peptides of each spot from mass-fingerprinting data against GST consensus sequences (see section 4.3.3) and clustalW2 scores obtained. See Table 5-4 and Figure 5-9 and Figure 5-10 for sequences and alignment.

\begin{tabular}{|c|c|c|c|c|c|c|c|c|}
\hline $\begin{array}{l}\text { Spot } \\
\text { No. }\end{array}$ & $\begin{array}{c}\text { NCBInr } \\
\text { Accession (Tgi) }\end{array}$ & Matched protein & $\begin{array}{l}\text { GST class } \\
\text { predicted }\end{array}$ & $\begin{array}{l}\text { Predicted } \\
\qquad \mathrm{Mr} / \mathrm{p} I\end{array}$ & $\begin{array}{c}\text { Experimental } \\
\qquad \mathrm{Mr} / \mathrm{pI}\end{array}$ & $\begin{array}{c}\text { Number of matched } \\
\text { peptides }\end{array}$ & $\begin{array}{c}\text { Coverage } \\
(\%)\end{array}$ & $Z$ score \\
\hline $1^{*}$ & \multicolumn{8}{|c|}{ No Identification was obtained due to the low intensity of spot 1} \\
\hline $2 *$ & 221116269 & $\mathrm{GST}_{-}$Hydra magnipapillata & $\mathrm{Mu}$ & $22.09 / 5.5$ & $26 / 5.5$ & 5 & 33 & 1.05 \\
\hline $3 *$ & 170586932 & $\mathrm{GST}_{-}$Brugia malayi & $\mathrm{Mu}$ & $24.44 / 6.8$ & $26 / 5.6$ & 4 & 16 & 2.43 \\
\hline & 28630830 & GST_Wuchereria bancrofti & $\mathrm{Mu}$ & $24.44 / 6.8$ & $26 / 5.7$ & 4 & 16 & 2.43 \\
\hline
\end{tabular}

The $\mathrm{Z}$ score for spot 2 is low. 
Table 5-3: Assignment of class of GST using the ClustalW2 programme.

The peptides of L. cuprina DNP-GSH affinity-purified zone E spots on 2D gel matched against "Other Metazoa" were aligned with consensus sequences of insect GSTs using the ClustalW2 alignment programme. The highest match score is highlighted in red text. The identification of each spot is shown in Table $5-2$.

\begin{tabular}{|c|c|c|c|c|c|c|c|c|c|c|c|}
\hline \multirow{3}{*}{$\begin{array}{l}\text { Spot } \\
\text { No. }\end{array}$} & \multirow{3}{*}{$\begin{array}{l}\text { L. cuprina Spots on 2D gel identified } \\
\qquad \text { in other metazoa }\end{array}$} & \multicolumn{9}{|c|}{ Alignment with consensus sequences of Insect GST classes } & \multirow{3}{*}{$\begin{array}{l}\text { Predicted } \\
\text { GST Clas: }\end{array}$} \\
\hline & & \multirow[b]{2}{*}{ Sigma } & \multicolumn{5}{|c|}{ ClustalW2 Score } & \multirow[b]{2}{*}{$\mathrm{Xi}$} & \multirow[b]{2}{*}{ Iota } & \multirow[b]{2}{*}{$\mathrm{Mu}$} & \\
\hline & & & Delta & Epsilon & Omega & Zeta & Theta & & & & \\
\hline $1^{*}$ & No Identification was obtained. & & & & & & & & & & \\
\hline $2^{*}$ & $\begin{array}{l}\text { gi|221116269|ref|XP_002156386.1] PREDICTED: } \\
\text { similar to mu-class glutathione s-transferase } \\
\text { [Hydra magnipapillata] }\end{array}$ & 26 & 20 & 20 & 17 & 14 & 9 & 22 & 22 & 53 & $\mathrm{Mu}$ \\
\hline \multirow[t]{2}{*}{$3 *$} & $\begin{array}{l}\text { gi|170586932|ref|XP_001898233.1] } \\
\text { transferase [Brugia malayi] }\end{array}$ & 16 & 11 & 6 & 9 & 11 & 11 & 13 & 13 & 23 & $\mathrm{Mu}$ \\
\hline & $\begin{array}{l}\text { gi|28630830|gb|AAO45827.1| } \\
\text { glutathione S-transferase [Wuchereria bancrofti] }\end{array}$ & & & & & & & & & & \\
\hline
\end{tabular}


A

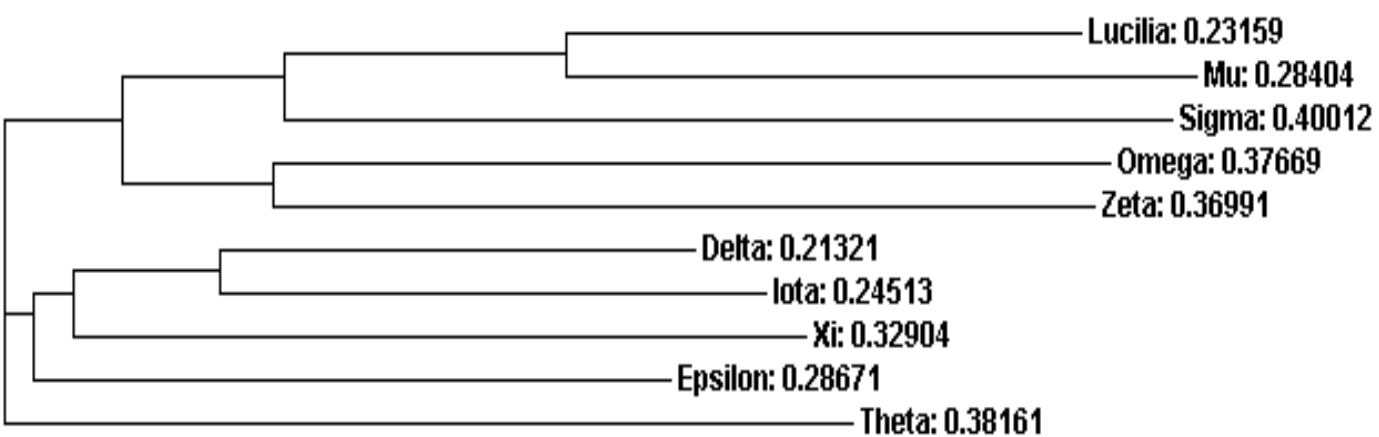

B

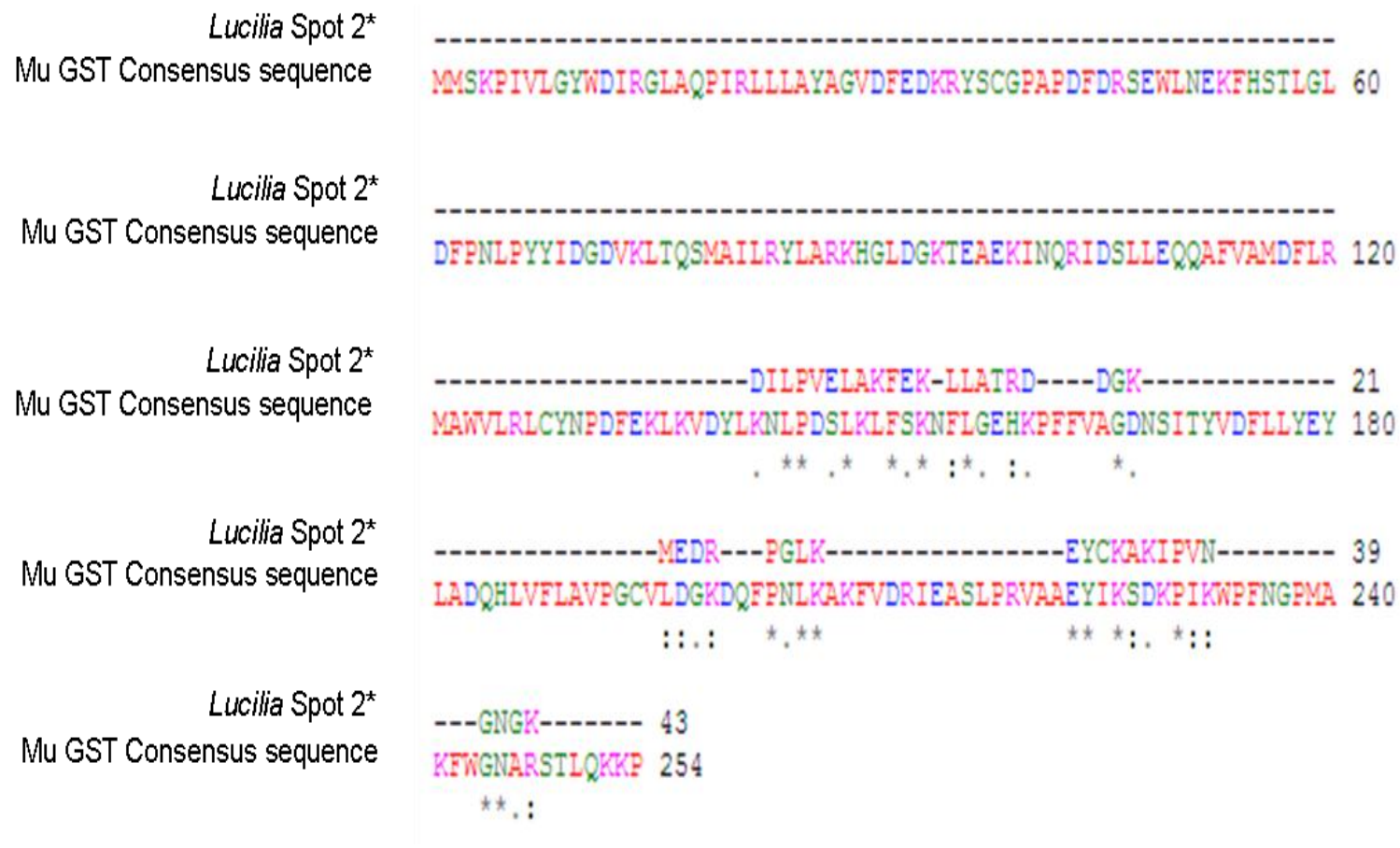

Figure 5-9: A tentative approach to classify the spot $2 *$ GST by matching to consensus sequences of GST classes from other species.

A) The peptide sequences of matched GST of spot $2 *$ in Hydra magnipapillata (a fresh water polyp, commonly known as Hydra) when aligned with the consensus sequences of GST classes from other species, were matched to Mu GSTs. Sequences were aligned using ClustalW2 and the result is presented as phylogram tree showing the distance figures. B) The alignment of matched peptide sequences of spot $2 *$ with Mu GST consensus sequence. 
A

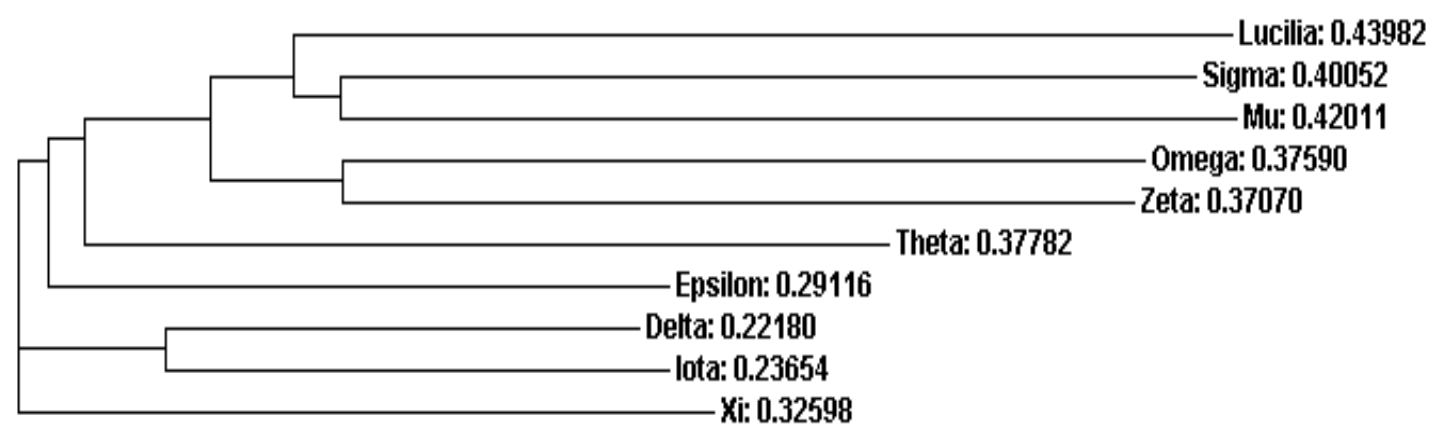

B

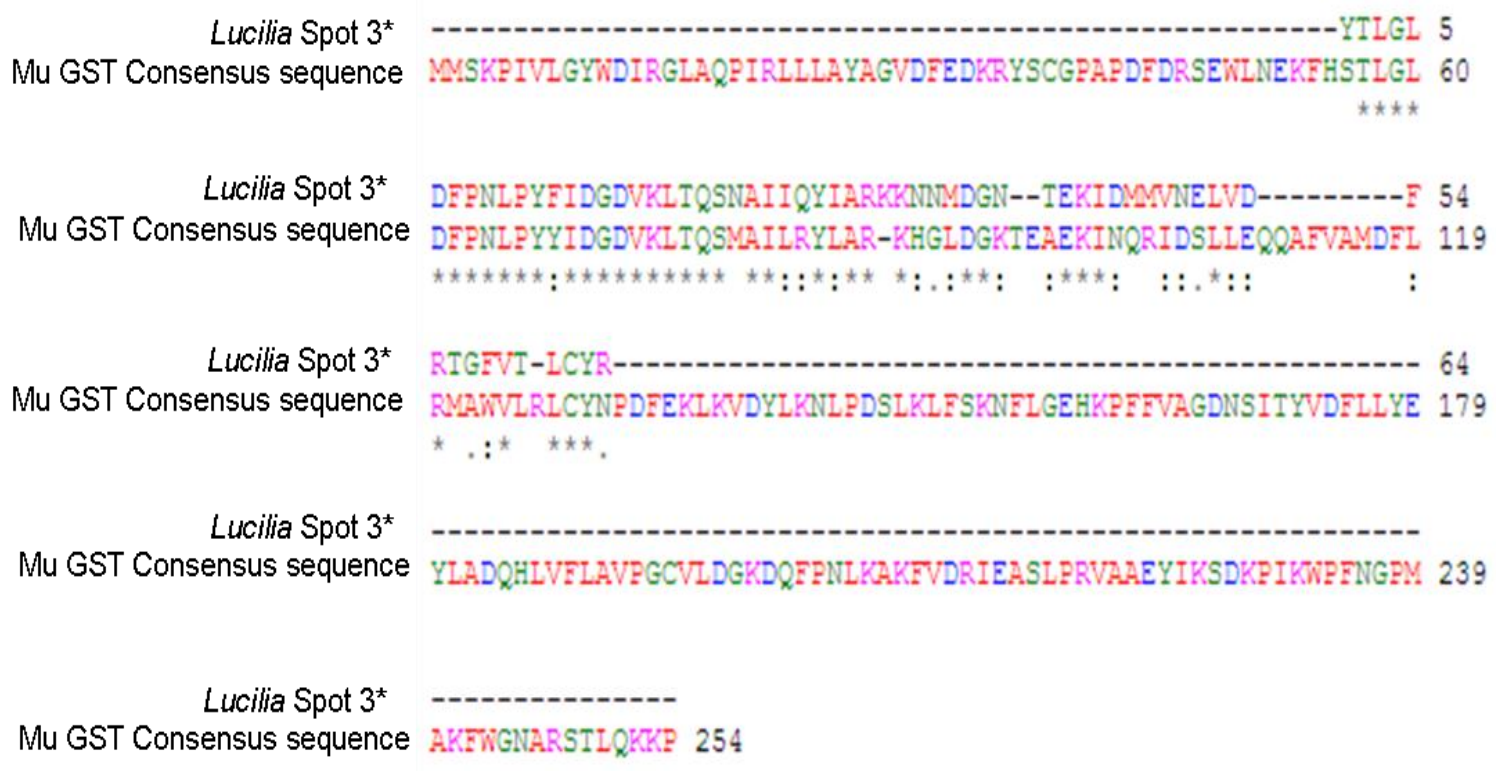

Figure 5-10: A tentative approach to classify the spot $3^{*}$ GST by matching to consensus sequences of GST classes from other species.

A) The peptide sequences of matched GST of spot $3 *$ in Brugia malayi or Wuchereria bancrofti (Nematodes, causative agents of lymphatic filariasis in humans) when aligned with the consensus sequences of GST classes from other species, were closely matched to $\mathrm{Mu}$ GSTs. Sequences were aligned using ClustalW2 and the result is presented as phylogram tree showing the distance figures. B) The alignment of matched peptide sequences with $\mathrm{Mu}$ GST consensus sequence. 

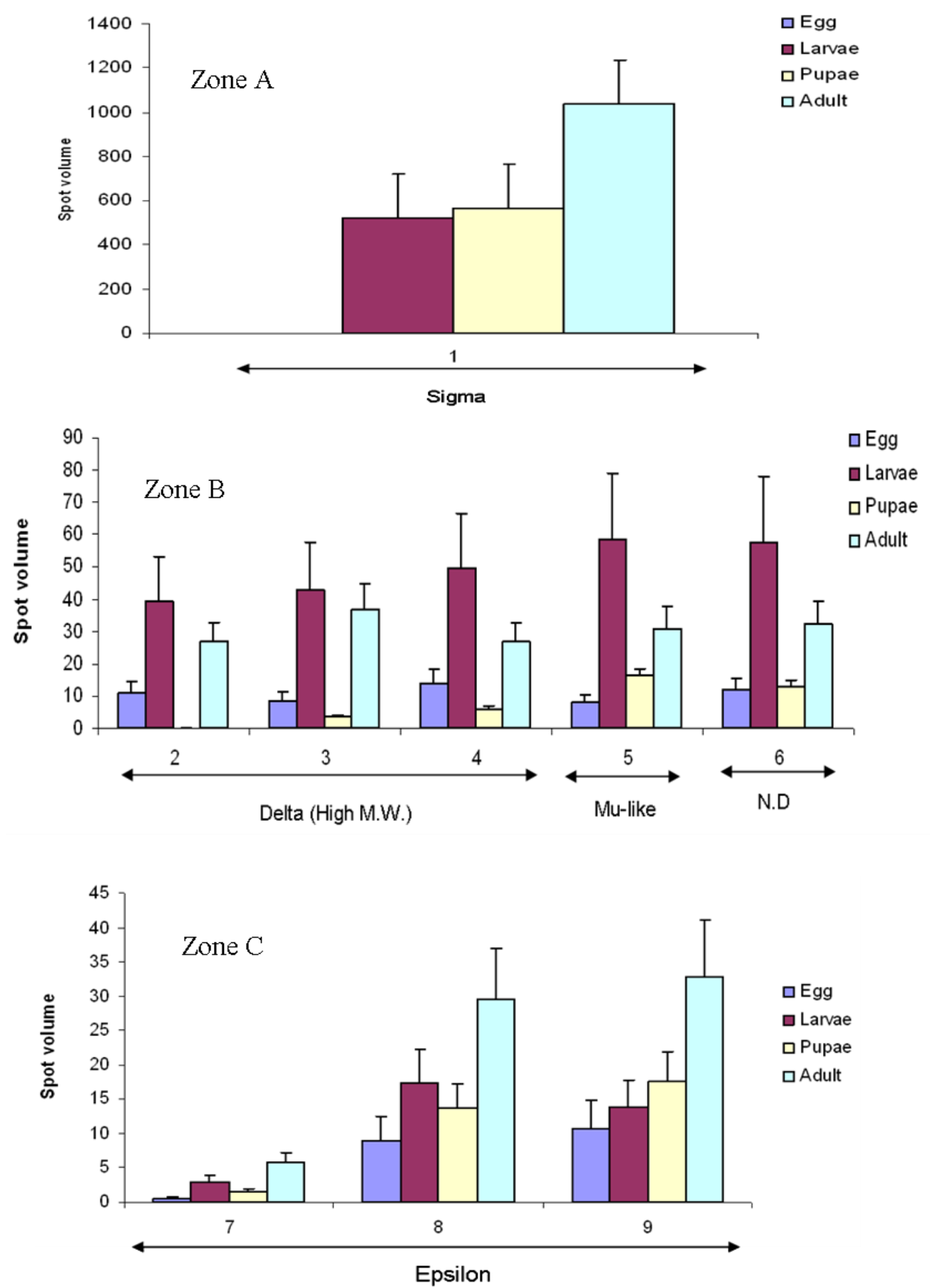

Figure 5-11: Quantitation of differentially expressed DNP-GSH purified GSTs in developmental stages of L. cuprina.

Quantitative analysis of digitized images was carried out using ImageQuant (Version 5.2, Molecular Dynamics) software. Values are the mean of at least three independent experiments with three measurements in each. Error bars represent standard deviation. The spot numbers of each zone are as per Figure 5-7. N. D = not determined. 

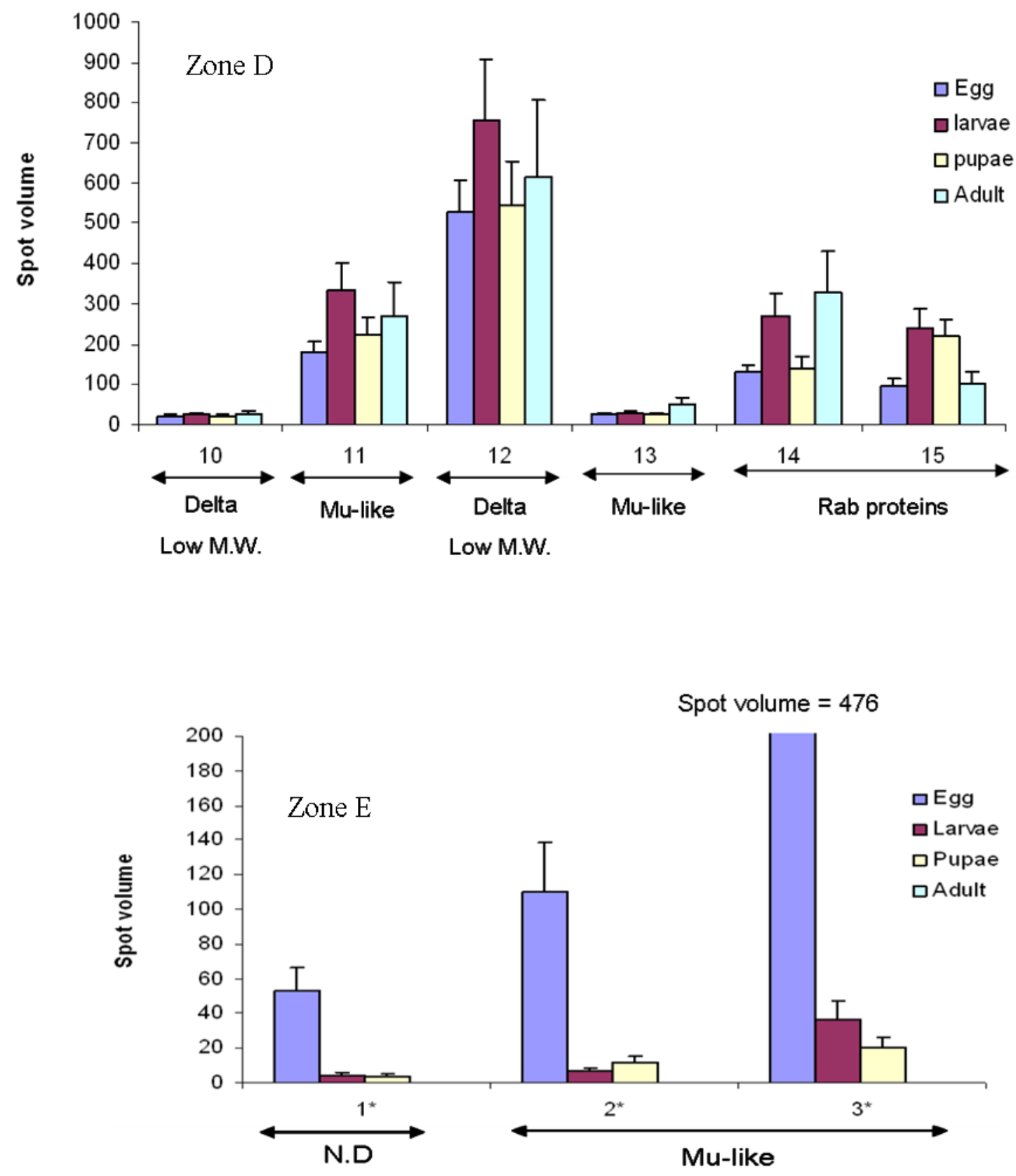

Figure 5-12: Quantitation of differentially expressed DNP-GSH purified GSTs in developmental stages of L. cuprina.

Quantitative analysis of digitized images was carried out using ImageQuant (Version 5.2, Molecular Dynamics) software. Values are the mean of at least three independent experiments with three measurements in each. Error bars represent standard deviation. The spot numbers of each zone are as per Figure 5-8. N. D = not determined 
Sigma GST found in zone A, was undoubtedly present abundantly in the adult stage. It was interesting to note that in DNP-GSH purified GSTs, zone B GSTs (Delta GSTs with high M.W. and possibly Mu-like GST) were expressed in much greater quantity in the larval stage $(\mathrm{p}<0.05)$ than egg and pupal stages but there was no significant difference between larvae and adult $(\mathrm{p}>0.05)$ for the spots 2 and 3 (high M.W. Delta). The total Epsilon GSTs in zone C (spots 7, 8 and 9) were highly expressed in the adult stage having a significant $(\mathrm{p}<0.05)$ difference of 2 -fold compared to all other stages. In zone D, the only spots expressed differently between stages were spots 14 and 15 (Figure 5-8), which were identified as Rab proteins. The volume of spot 14 was significantly $(\mathrm{p}<0.05)$ greater in larvae and adult than other stages whereas the spot 15 was expressed significantly $(\mathrm{p}<0.05)$ high in quantity in larvae and pupae. Zone E GSTs were expressed in much greater quantity in the egg stage having significant $(\mathrm{p}<0.05) 14$ - and 18 -fold difference for total spots $1^{*}, 2^{*}$ and $3^{*}$ compared to larvae and pupae respectively. Zone E GSTs were absent in the adult phase. These GSTs have been tentatively identified as Mu-like GSTs (Table 5-2). 


\subsection{Insect body distribution of GSTs}

The head, thorax and abdomen were dissected from the adult L. cuprina (PY81 strain) flies in the laboratory on a glass plate on ice. The dissected body parts were homogenised separately and GSTs were purified using affinity chromatography as described in the section 3.2. Initially the GSH affinity matrix was used and the unbound fractions from the GSH column were applied to DNP-GSH affinity matrix for the further purification of GSTs. To measure the mass proportion of head, thorax and abdomen of an adult fly, 20 adult flies were dissected and head, thorax and abdomen were weighed which found to be $15 \%, 53 \%$ and $32 \%$ respectively of the whole body mass of the adult $L$. cuprina. In the current set of experiments, the purification of GSTs was carried out using an equal mass of the tissue of head, thorax and abdomen ( $1 \mathrm{~g}$ each) to compare the expression of GSTs. However, in a whole fly the distribution of GST varies due to the different proportions of the head, thorax and abdomen of the insect (1:3.53:2.13 respectively). One gram of $L$. cuprina heads was obtained from dissection of approximately 1000 flies; the thorax and abdomen were taken during this dissection. Usually for $1 \mathrm{~g}$ of thorax, approximately 285 flies and for $1 \mathrm{~g}$ of abdomen approximately 485 flies were required.

Enzyme activity distribution in these main body regions of the adult fly is shown in Table 5-4. GST activity with CDNB found in crude and affinity-purified fractions of thorax was significantly higher than abdomen $(\mathrm{p}<0.05)$ and head GSTs $(\mathrm{p}<0.05)$. The abdomen also showed slightly higher levels of GST activity with CDNB than head region. 
Table 5-4: Distribution of the total GST activity in adults of L. cuprina towards CDNB.

Values represent mean \pm S.D. of three individual experiments.

\begin{tabular}{llcl}
\hline Insect body part & \multicolumn{3}{c}{ Distribution (\%) } \\
\cline { 2 - 4 } & Crude & B. GSH & B. DNP-GSH \\
Head & $15 \pm 3.0$ & $15 \pm 7.0$ & $21 \pm 7.4$ \\
Thorax & $65 \pm 14$ & $66 \pm 14$ & $48 \pm 19$ \\
Abdomen & $20 \pm 5.0$ & $19 \pm 3.2$ & $30 \pm 4$ \\
Whole body & 100 & 100 & 100 \\
\hline
\end{tabular}

The result of affinity-purification of GSTs from different body parts is shown in Figure 5-13. When the comparison was made between the amount of protein expressed in equal mass of body parts ( $1 \mathrm{~g}$ each), the thorax values were significantly $(\mathrm{p}<0.05)$ higher than for abdomen but there was no significant difference between the quantity of expressed proteins in head and thorax (Figure 5-13, A, Total protein). However, unsurprisingly the result changes if the figures are expressed as function of the mass of flies ( $1 \mathrm{~g})$ with different body proportions. The thorax expresses significantly $(\mathrm{p}<0.05)$ higher amount of GSTs than head and abdomen (Figure 5-13, B, Total protein). The total GST activity of the thorax crude preparation was higher than in head or abdomen irrespective of the comparison but the specific activity was higher in abdomen GSTs. The total activity of GSH affinity-purified fractions from thorax showed significantly $(\mathrm{p}<0.05)$ higher activity towards CDNB compared to head and abdomen. However, the specific activity of GSH-affinity-purified fractions from the head showed significantly $(\mathrm{p}<0.05)$ higher activity towards CDNB compared to thorax and abdomen. The specific activity of DNP-GSH purified-GSTs from head and thorax was higher than from abdomen but no significant difference was found (Figure 5-13). 
A

Values from equal mass of body parts ( $1 \mathrm{~g}$ each)
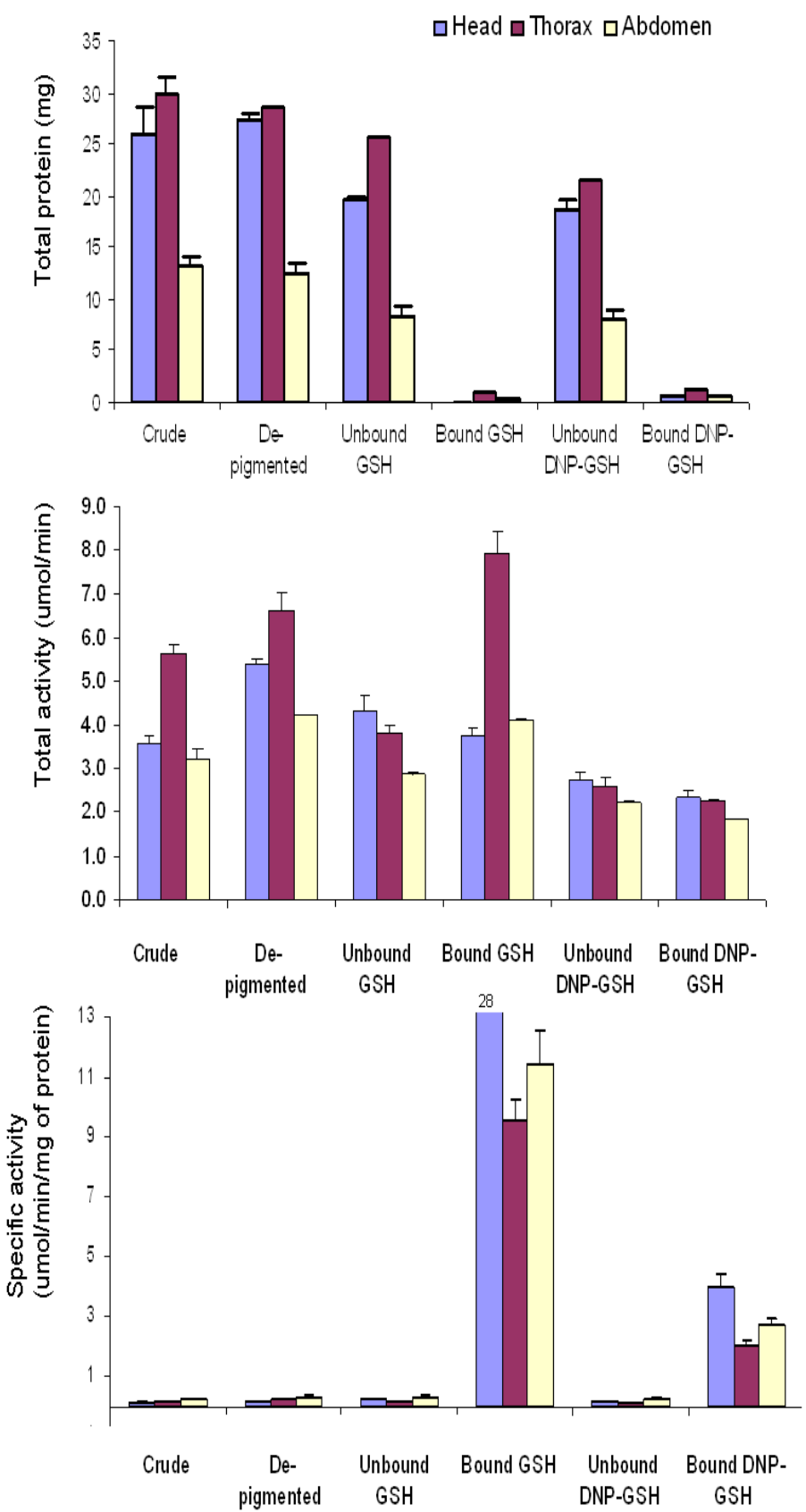

B

Values from body parts of $1 \mathrm{~g}$ flies
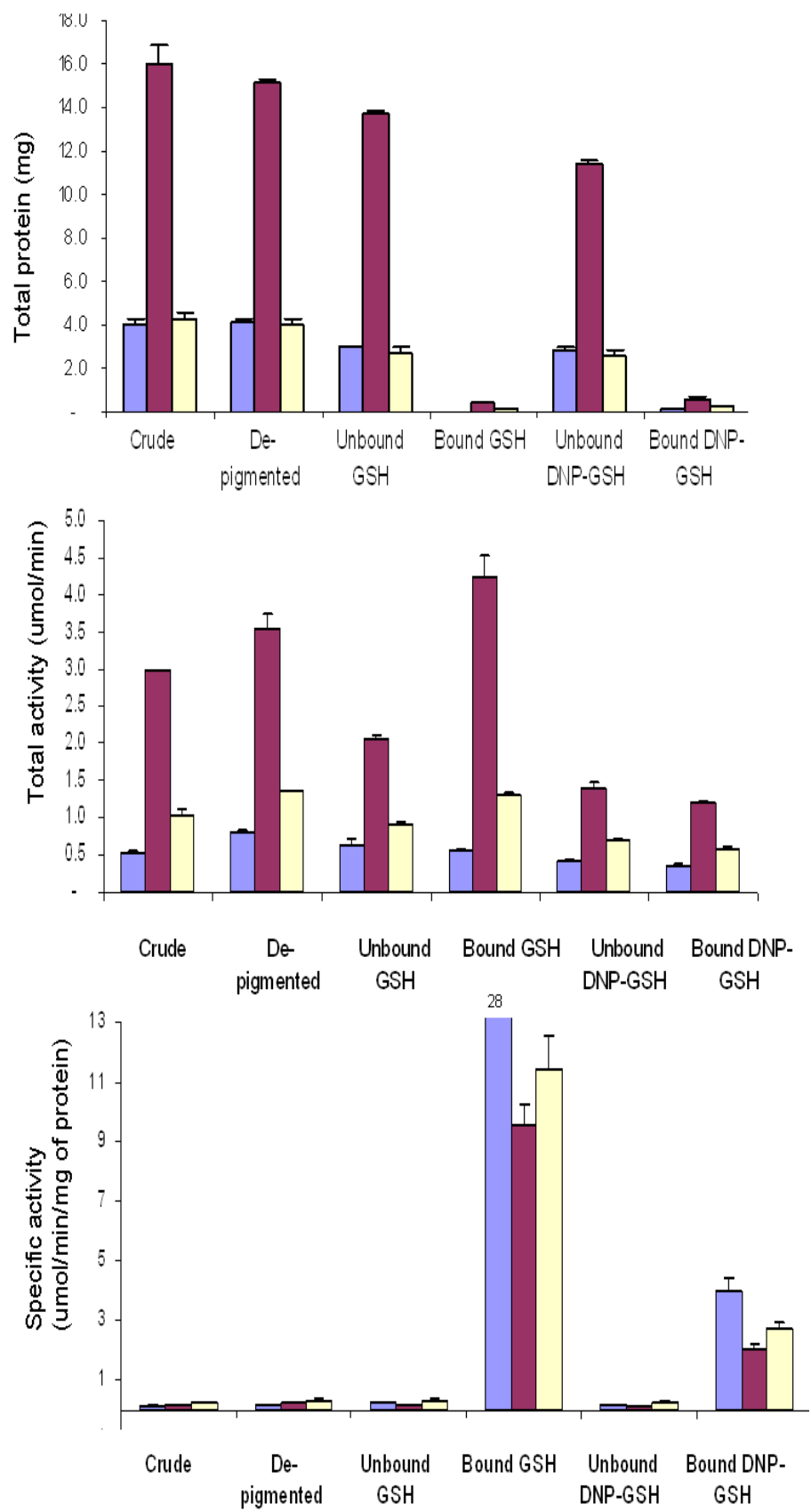

Figure 5-13: Comparative study of GST production and activity towards CDNB from different stages of purification of head, thorax and abdomen.

A) Values from the equal mass of body parts (1 $\mathrm{g}$ each), B) Values adjusted to $1 \mathrm{~g}$ of adult flies which contain different masses of head, thorax and abdomen. Assays were undertaken following the methods in section 3.2.4. The values are from three independent experiments with triplicate measurements within each experiment. Error bars represent standard deviation. 

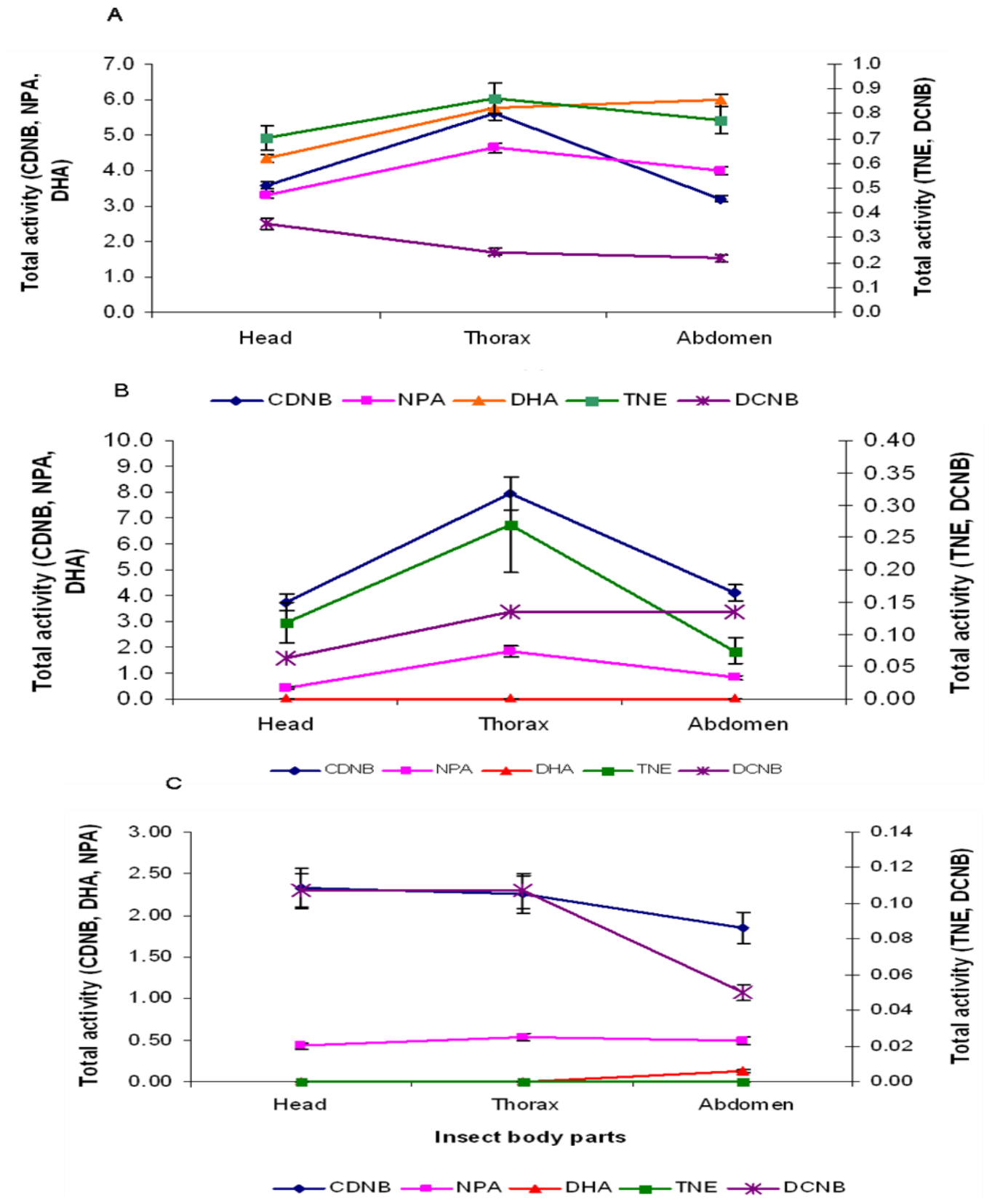

Figure 5-14: Substrate-specific total activity of crude and affinity-purified GSTs from head, thorax and abdomen towards the model substrates.

A) Crude GSTs, B) GSH affinity-purified GSTs. C) DNP-GSH affinity-purified GSTs.

Homogenates of head, thorax and abdomen (1g each) were used as the crude enzyme source and purified by GSH affinity matrix and subsequently by DNP-GSH affinity matrix. Total activity values are given as $\mu \mathrm{mol} / \mathrm{min}$. Assays were undertaken following the methods in section 3.2.4. The values are from three independent experiments with triplicate measurements within each experiment. Error bars represent standard deviation. 
A
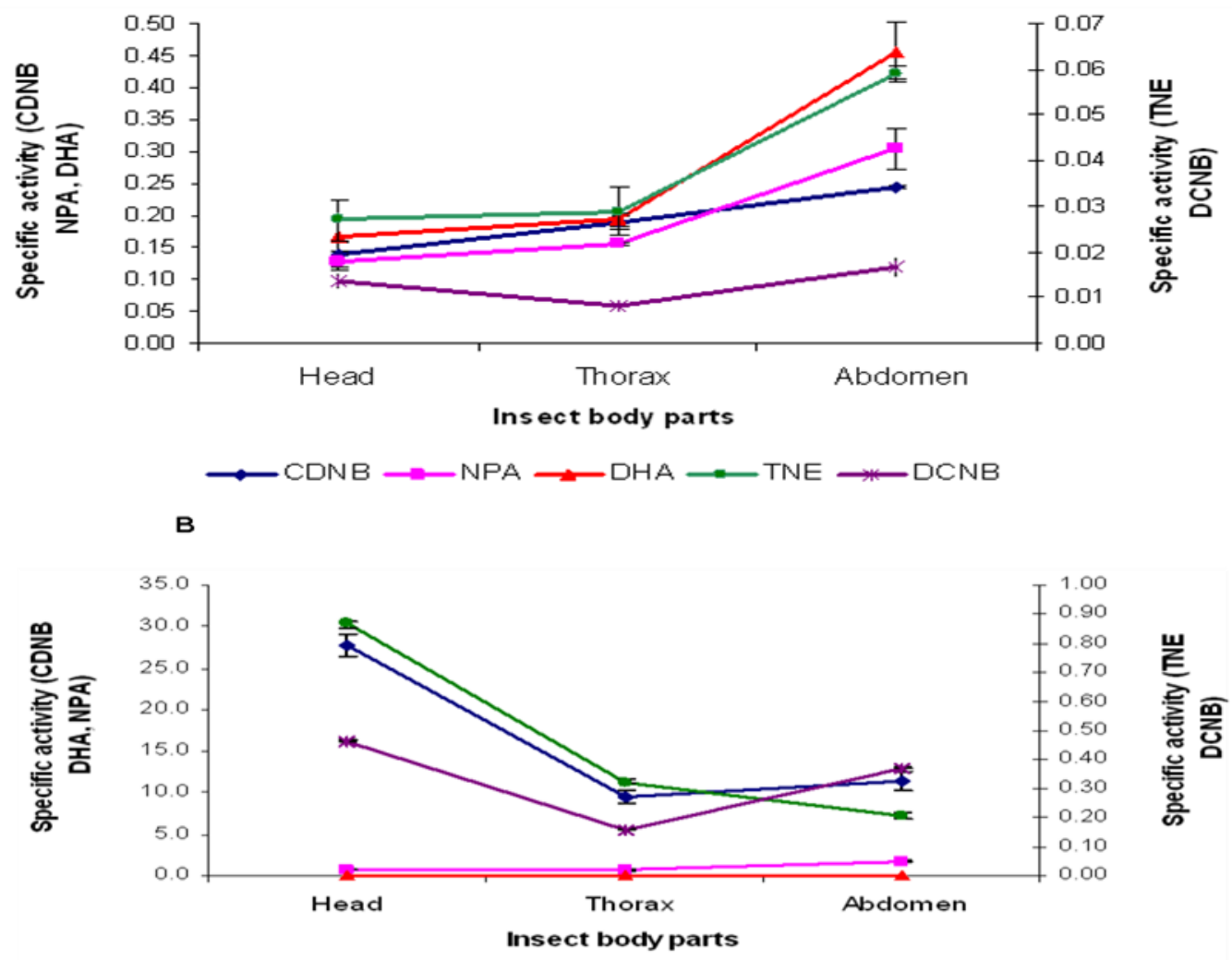

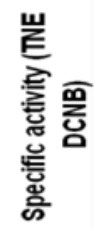

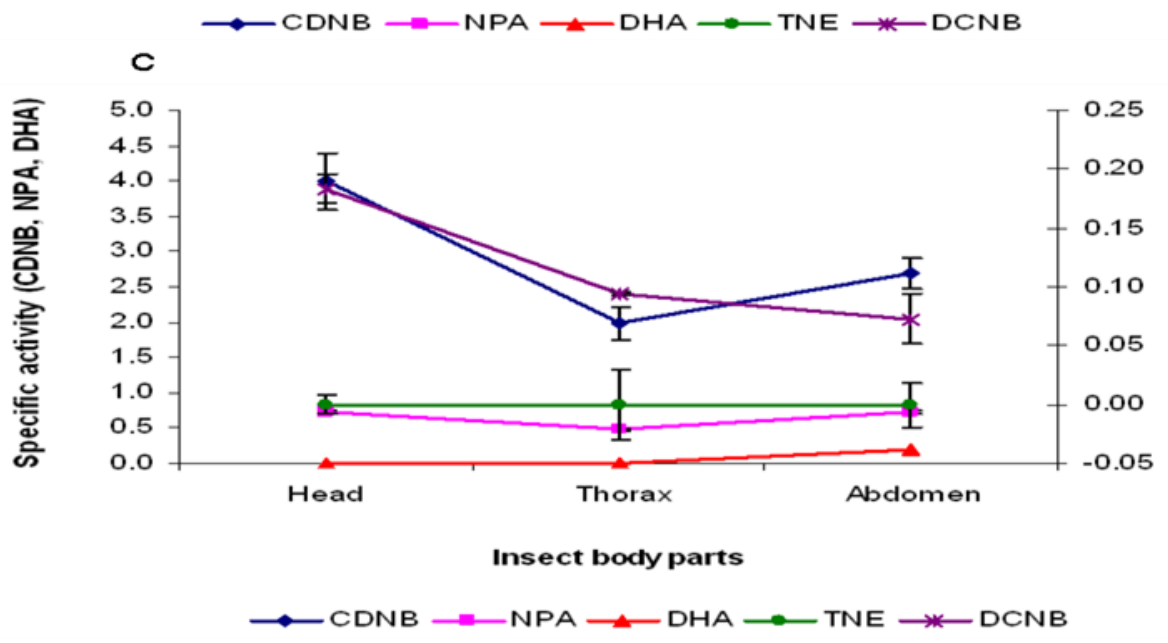

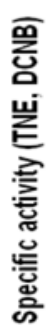

Figure 5-15: Substrate-specific specific activity of crude and affinity-purified GSTs from head, thorax and abdomen towards the model substrates.

A) Crude GSTs, B) GSH affinity-purified GSTs, C) DNP-GSH affinity-purified GSTs.

Homogenates of head, thorax and abdomen (1g each) were used as the crude enzyme source and purified by GSH affinity matrix and subsequently by DNP-GSH affinity matrix. Specific activity values are given as $\mu \mathrm{mol} / \mathrm{min} / \mathrm{mg}$ of protein. Assays were undertaken following the methods in section 3.2.4. The values are from three independent experiments with triplicate measurements within each experiment. Error bars represent standard deviation 
The GST activities towards the model substrates provided valuable information about GSTs expressed in different body parts of the fly. The cytosolic GSTs from thorax had the highest total activity with the substrates CDNB, NPA and TNE compared to head and abdomen GSTs. The GSH affinity-purified GSTs from thorax showed the highest activity with CDNB, TNE and NPA. There was not much difference in DNP-GSH purified GSTs from the head, thorax and abdomen except the activity towards DCNB was higher in head and thorax compared to abdomen.

However in terms of specific activity, the cytosolic GSTs from the abdomen showed the highest activity towards most of the substrates employed. There was significant $(\mathrm{p}<0.05)$ difference in activities towards DHA, TNE and NPA but no significant difference was found towards CDNB and DCNB. The head GSTs purified from the GSH affinity matrix showed significantly $(p<0.05)$ higher specificity towards CDNB compared to thorax and abdomen GSTs. However, there was almost no activity towards NPA and DHA found from the GSH affinity-purified head GSTs. Interestingly, the abdomen GSH-affinity purified GSTs showed higher activity towards DCNB compared to head and thorax. The DNP-GSH purified GSTs from head showed higher activity towards CDNB and DCNB compared to thorax and abdomen. It is difficult to characterise the type of GSTs expressed, due to their overlapping substrate specificity from the above results. Therefore the protein extracts from all three body parts were individually subjected to electrophoresis to characterise the GSTs expressed as shown in Figure 5-16. 


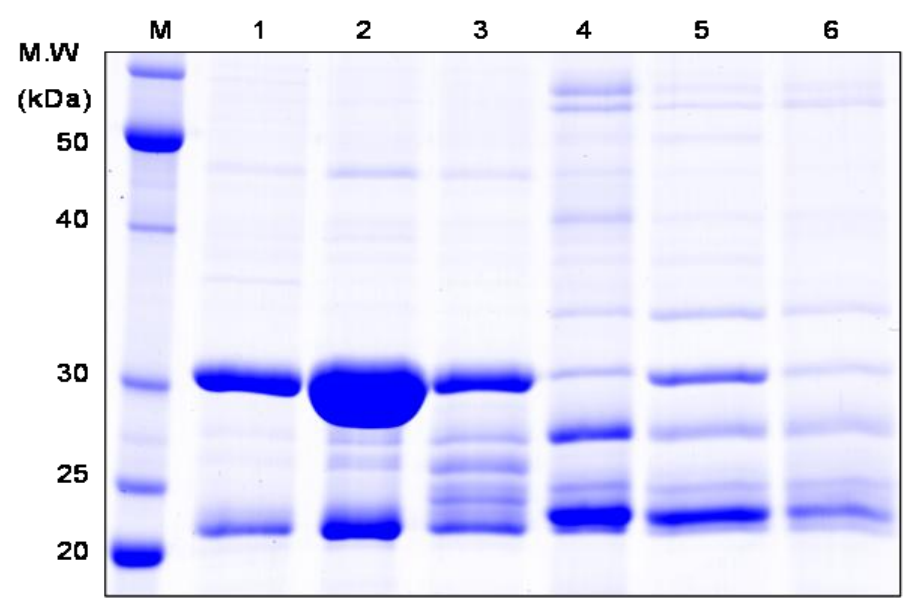

Figure 5-16: SDS PAGE of affinity-purified fractions of head, thorax and abdomen of $L$. cuprina.

Lane M is a M.W. marker. Lanes 1-3 are bound GSH fractions and Lanes 4-6 are of bound DNP-GSH fractions from head, thorax and abdomen respectively. Homogenates of head, thorax and abdomen (1g each) were used as the crude enzyme source and purified by GSH affinity matrix and subsequently by DNP-GSH affinity matrix. The protein load on the gel was according to equal volume of purified extracts from equal mass of tissue ( $1 \mathrm{~g}$ each). The gel was stained with Coomassie Blue G-250.

The GSH affinity-purified fractions showed that thorax expresses a significantly higher amount of proteins in the form of Sigma ( M.W. $30 \mathrm{kDa})$ and Delta ( M.W. $23 \mathrm{kDa}$ ) GSTs (p<0.05; 5.7- and 3.2-fold) compared to head and abdomen respectively. The abdomen fractions however showed the presence of many bands in the M.W. range of the GSTs that may be due to the degradation of Sigma GSTs. The DNP-GSH fractions showed expression of two additional bands other than Sigma and Delta GSTs in all three extracts with varying intensity. However the difference in amount of protein recovered from the DNP-GSH matrix was not significant between head and thorax but it was significantly higher $(\mathrm{p}<0.05 ; 1.2$-fold $)$ in thorax compared to abdomen. As observed before, the DNP-GSH matrix showed affinity towards proteins of high M.W. as seen in lanes 4-6 in Figure 5-16. The GSH 
and DNP-GSH affinity-purified fractions were further resolved by 2D electrophoresis.

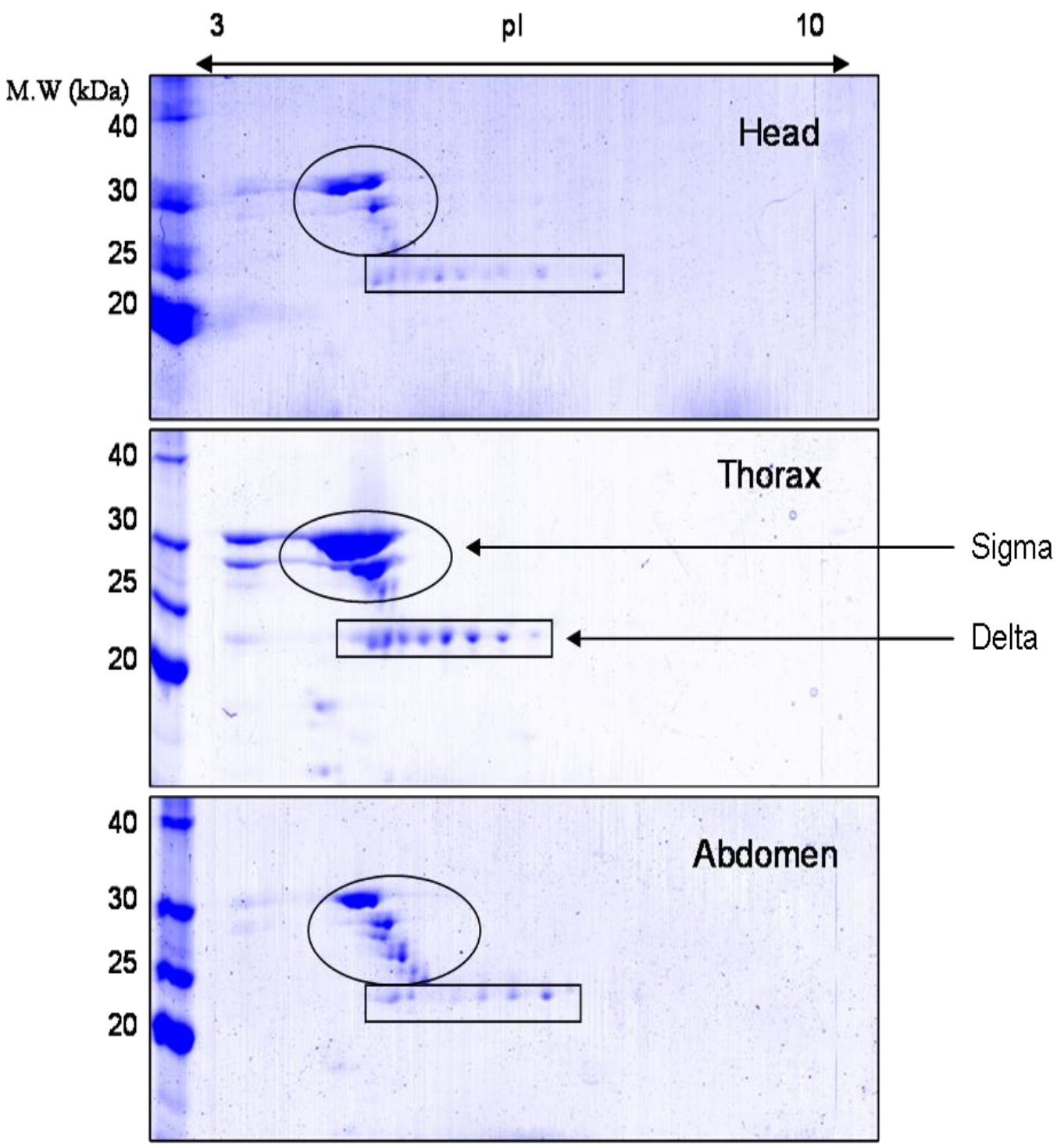

Figure 5-17: Two dimensional electrophoresis gels of GSH affinity-purified fractions from head, thorax and abdomen of L. cuprina.

GSH affinity-purified GSTs from head, thorax and abdomen (1g each) were separated in the first dimension on a $7 \mathrm{~cm} \mathrm{pH} \mathrm{3-10} \mathrm{linear} \mathrm{IPG} \mathrm{strip} \mathrm{and} \mathrm{then} \mathrm{in} \mathrm{the} \mathrm{second} \mathrm{dimension} \mathrm{on} \mathrm{a}$ SDS-PAGE gel. The gels were stained by Coomassie Brilliant Blue G-250. The protein load on the gels was according to equal volume of purified extracts from the equal tissue ( $1 \mathrm{~g}$ each) of head, thorax and abdomen. 


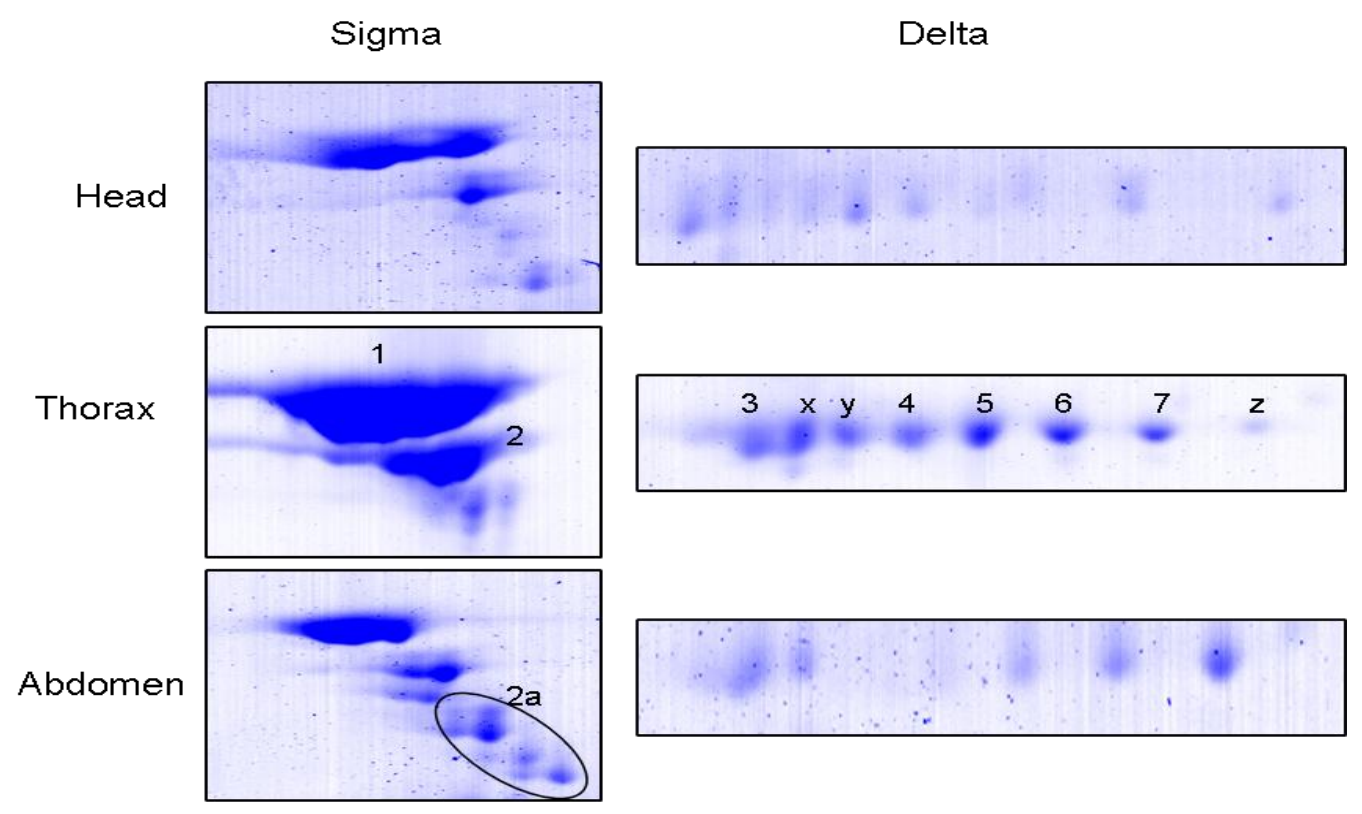

Figure 5-18: Differential expression of Sigma and Delta GSTs on 2D gel of GSH affinitypurified extracts from head, thorax and abdomen.

The images of Sigma and Delta GST zones from head, thorax and abdomen of Figure 5-17 are enlarged for the clear numbering of the spots. The numbered spots were identified by MALDI-TOF and their identity confirmed with the previous identification of GSH affinity-purified proteins from adult L. cuprina reported in section 4.3.3, Table 4-10. The additional spots $\mathrm{x}, \mathrm{y}, \mathrm{z}$ were assumed as Delta GSTs based on the M.W.

The number of Delta spots produced in the GSH affinity-purified fractions from different body parts ( 8 spots, additional spots $x, y, z$, Figure 5-18) is more than the whole adult fly (5 spots, Figure 5-4). The possible explanation for this could be that minor Delta spots do not get resolved significantly when whole adult flies are processed on the 2D electrophoresis gel. The intensity of the Delta GST zones varies among the different parts of the body. The abdomen shows an increase in the intensity with increase in the pI whereas this does not happen with the Delta GSTs of the thorax. In the Sigma zone, additional spot 2a represents a collection of small spots resulting, possibly, from spot 1 or from spot 2 degradation (Alias and Clark, 2007). 
It was interesting to see that when comparison was made with the equal mass of body parts ( $1 \mathrm{~g}$ each), the abdomen always expressed a lesser quantity of GSTs on a gel compared to head and thorax. However, this result will differ if $1 \mathrm{~g}$ of whole flies were used. To understand the actual quantitative expression of GSTs in the fly, the analysis was carried out using ImageQuant software and the values of equal mass of body parts ( $1 \mathrm{~g}$ each) were normalised according to body parts of $1 \mathrm{~g}$ of flies.

A
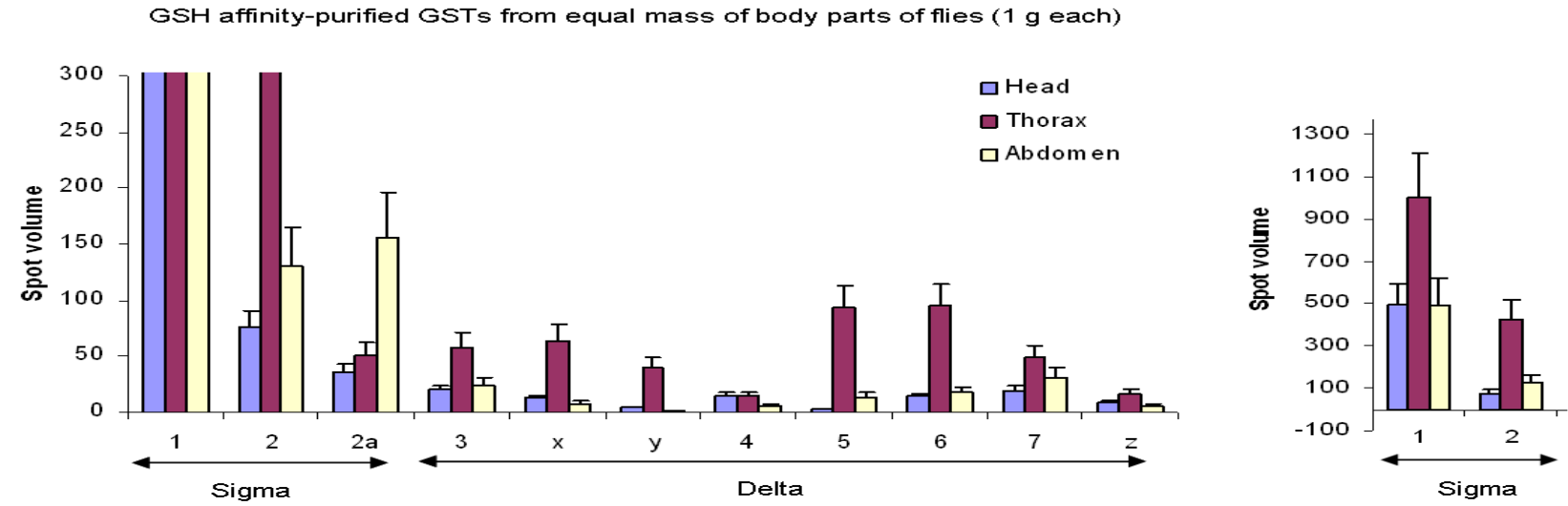

$\mathrm{B}$
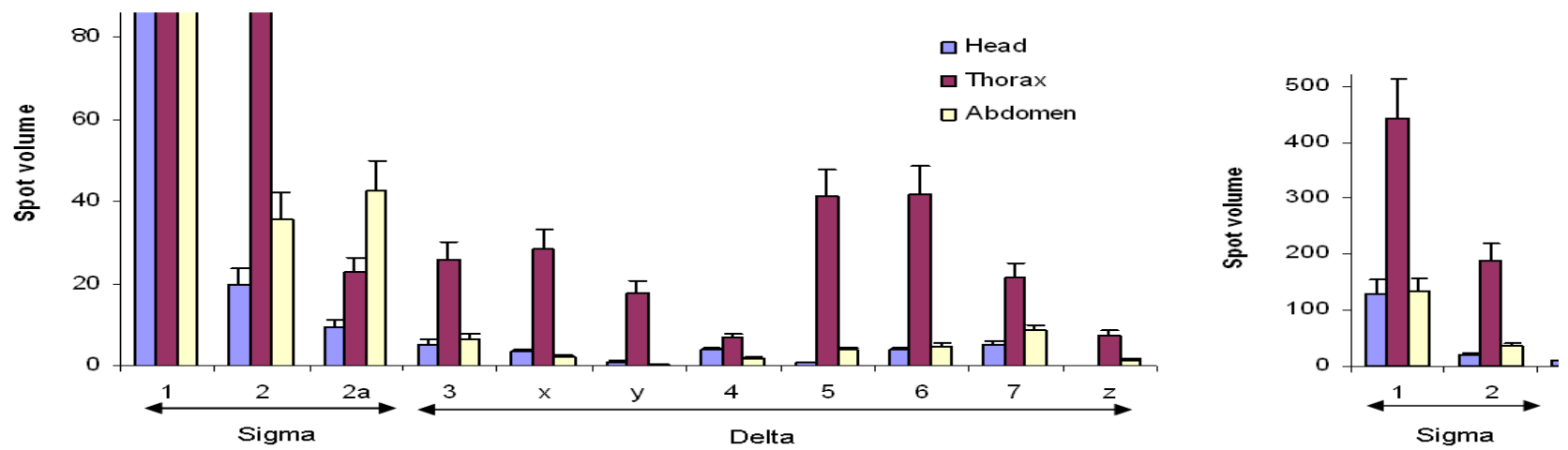

Figure 5-19: Quantification of the GSH affinity-purified protein spots expressed in head, thorax and abdomen of L. cuprina

Quantitative analysis of the digitized images was carried out using ImageQuant (Version 5.2, Molecular Dynamics) software. A) Spot volume from equal mass of each body parts head, thorax and abdomen $(1 \mathrm{~g}$ each). B) Spot volume from the body parts of mass of flies $(1 \mathrm{~g})$. Values are the mean of at least three independent experiments with three measurements in each. Error bars represent standard deviation. The spot numbers are as per Figure 5-18. Spots 1 and 2 are shown alongside to full scale. The value on top of the bar shows corresponding volume of the spot. 
This quantitative analysis indicated a higher expression of GST proteins in the thorax followed by abdomen and head. In the Sigma zone, spots 1 and 2 were quantitatively highest in the thorax but the region $2 \mathrm{a}$ in the abdomen showed many spots possibly suggesting increased degradation of the Sigma GST (Figure 5-19).

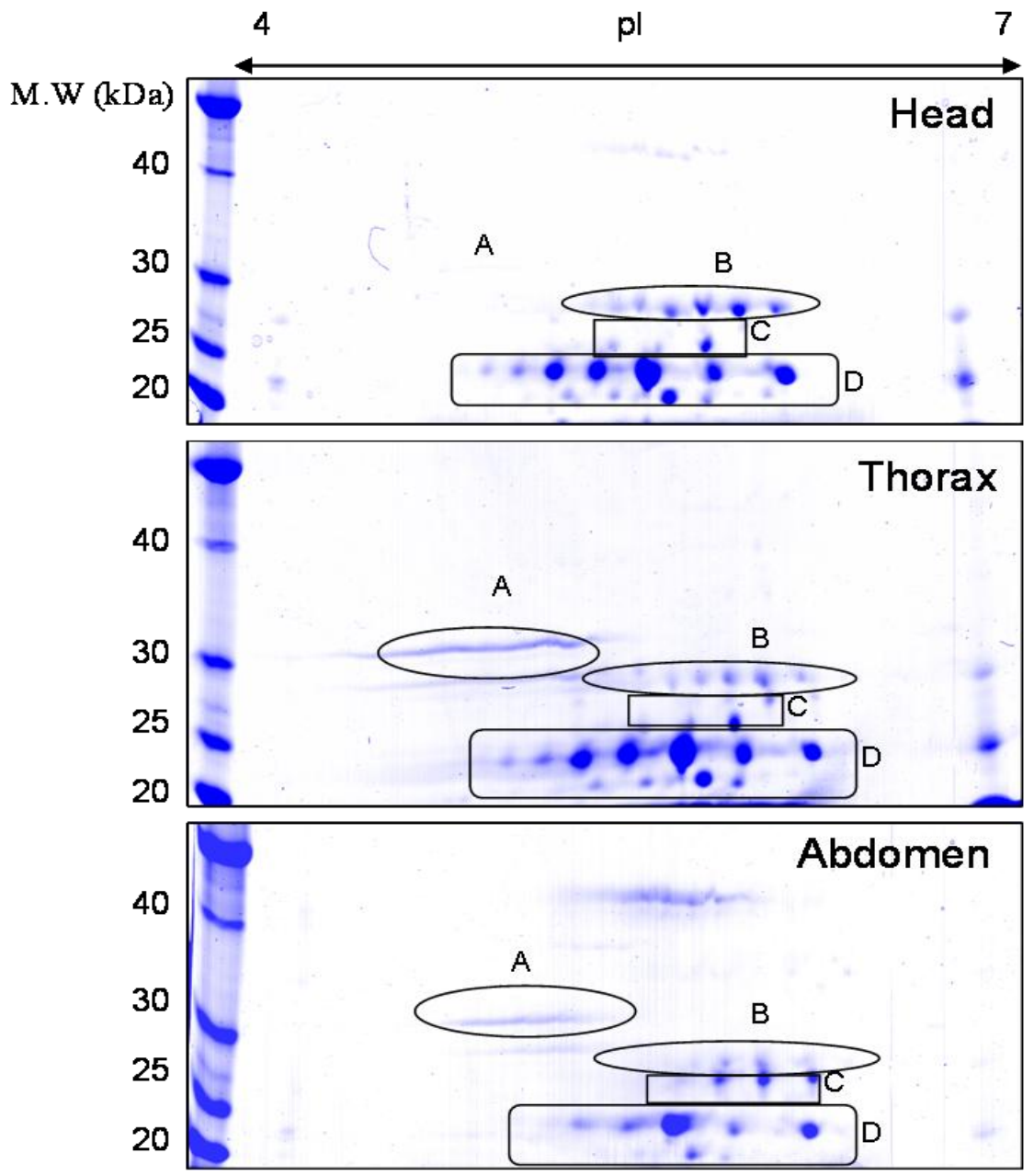

Figure 5-20: Two dimensional electrophoresis gels of DNP-GSH affinity-purified fractions of head, thorax and abdomen of L. cuprina.

DNP-GSH affinity-purified GSTs from head, thorax and abdomen were separated in the first dimension on a $7 \mathrm{~cm} \mathrm{pH} \mathrm{4-7} \mathrm{linear} \mathrm{IPG} \mathrm{strip} \mathrm{and} \mathrm{then} \mathrm{in} \mathrm{the} \mathrm{second} \mathrm{dimension} \mathrm{on} \mathrm{a} \mathrm{SDS-}$ PAGE gel. The gels were stained by Coomassie Brilliant Blue G-250. The protein load on above gel was obtained from an equal volume of purified extracts from equal masses of tissue of head, thorax and abdomen (1 $\mathrm{g}$ each). The spots of zone A, B, C and D are as numbered in Figure 5-7 and Figure 5-8 and their tentative identification details are as in section 4.3.3 (Table 4-13 and Table 4-14). 
A DNP-GSH affinity-purified GSTs from equal mass of body parts of flies ( $1 \mathrm{~g}$ each)

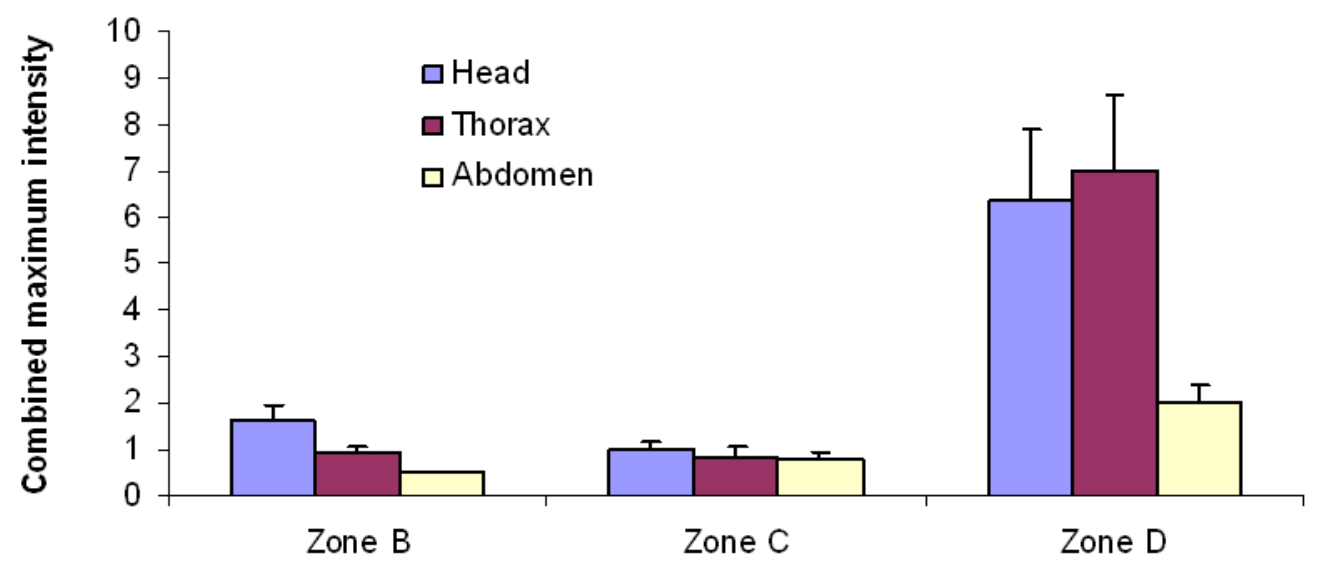

Total protein spots of each zone

$\mathrm{B}$

DNP-GSH affinity-purified GSTs from body parts of $1 \mathrm{~g}$ flies

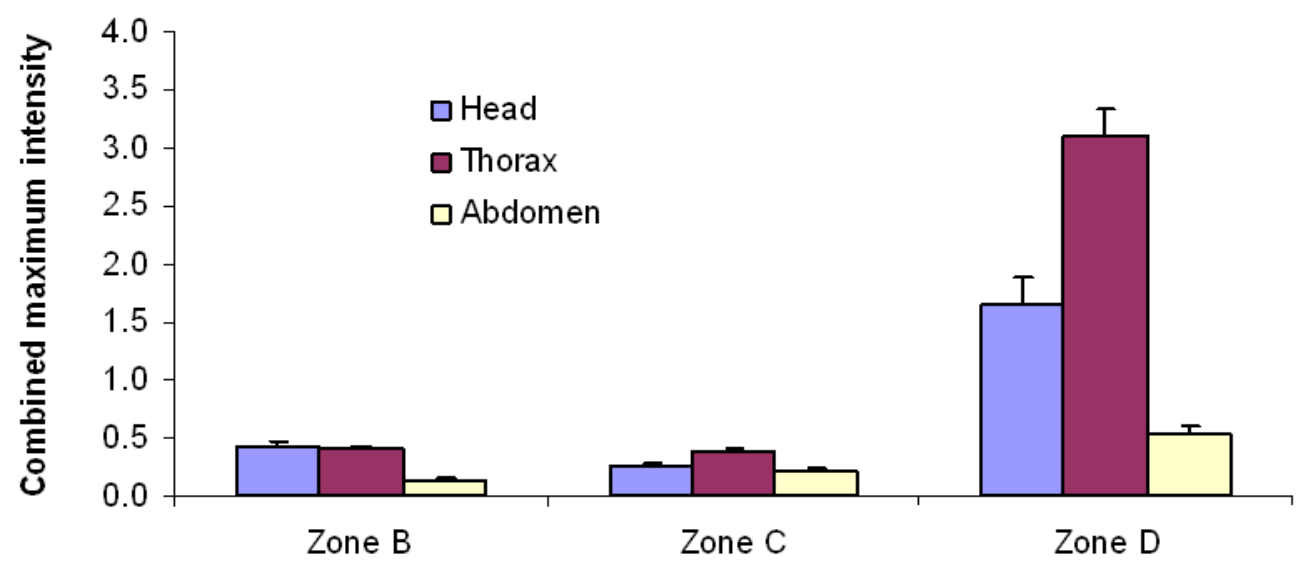

Total protein spots of each zone

Figure 5-21: Quantification of the DNP-GSH affinity-purified protein spots expressed in head, thorax and abdomen of L. cuprina.

Quantitative analysis of the digitized images shown in Figure 5-20 was carried out using ImageQuant (Version 5.2, Molecular Dynamics) software. A) Spot volume from equal mass of each body parts head, thorax and abdomen ( $1 \mathrm{~g}$ each). B) Spot volume from the body parts of $1 \mathrm{~g}$ of flies. Values are the mean of at least three independent experiments with three measurements in each. Error bars represent standard deviation. The values in B have been calculated according to the proportion of each main region of the fly. 
There were notable differences in the expression of GSTs that were purified by the DNP-GSH affinity matrix from head, thorax and abdomen (Figure 5-20). The zone B GSTs, which include those tentatively identified as Delta GSTs with high M.W. and Mu-like GST, were more strongly observed on the 2D gel of the head region compared to thorax and abdomen on the basis of equal mass of each body part in head, thorax, abdomen. The comparison with the body parts of $1 \mathrm{~g}$ flies shown in Figure 5-21 also showed the higher expression of these GSTs in the head region. On the basis of equal masses of body parts, the tentatively identified Epsilon GSTs (zone C) were expressed in slightly higher quantity in abdomen compared to head and thorax. However, on the basis of $1 \mathrm{~g}$ of flies, the thorax expressed more Epsilon GSTs (zone C). On the basis of equal mass of body parts (1 g each), Zone D proteins, which included tentatively identified Delta GSTs with low M.W., Mu-like GSTs and Rab proteins were expressed significantly higher in thorax compared to head and abdomen ( $\mathrm{p}<0.05 ; 1.1$ - and 2.5-fold respectively) but when compared with body parts from $1 \mathrm{~g}$ of flies, the thorax expressed zone D proteins significantly higher than head and abdomen ( $\mathrm{p}<0.05 ; 4$ - and 4.2-fold respectively). After GSH affinity chromatography, the remaining Sigma GST (zone A) was found only in thorax and abdomen fractions. The reason for the poor resolution of Sigma GST on the pI scale of these 2D gels is not clear. It could be due to experimental handling during sample preparation but then other spots were resolved precisely, so the reason is not known. 


\subsection{Discussion}

The ontogenic pattern of GST activity towards five substrates was studied. GST isoenzymes expressed during the development of L. cuprina were purified by GSH and DNP-GSH affinity chromatography as described in section 3.2. Purified GSTs were resolved by 2D electrophoresis. The importance of this procedure is that different activity patterns against three to five substrates are indicative of the existence of multiple isoenzymic forms and the use of proteomics confirms that.

It is suggested in several reports that an alteration of the specific characteristics of each GST isoenzyme may occur during different developmental stages of the insect (Hazelton and Lang, 1983; Kostaropoulos et al., 1996; Kostaropoulos and Papadopoulos, 1998; Kotze and Rose, 1987; Wood et al., 1986). On the basis of substrate specificities several ontogenic studies have also been reported for mammals (Gregus et al., 1985) and for embryonic stages of the frog Bufo bufo (Del Boccio et al., 1987). The result of the present study is in agreement with the ontogenic model study of $L$. cuprina by Kotze and Rose (1987) who showed the cytosolic GST activity towards CDNB is highest in the pupae compared to other stages. Kotze and Rose studied day-wise developmental stages of L. cuprina in detail. They reported that the cytosolic GST activity increased rapidly through the larval stage and reached a peak in the early pupae. It then decreased through the first 6-7 days after emergence of the adult. Activity was then approximately constant for the remainder of the adult stage. In the present study eggs, larvae, pupae and adult were of the mixed ages and the day of emergence of adults was not determined. $A$. aegypti, L. cuprina and T. molitor have been shown to exhibit a similar ontogenic pattern, i.e highest GST activity is found in the pupal stage while in the adult the 
activity is very low (Hazelton and Lang, 1983; Kostaropoulos et al., 1996; Kotze and Rose, 1987). A somewhat similar pattern of GST activity with age was reported by Hazelton and Lang in Aedes aegypti (Hazelton and Lang, 1983). In mosquitoes, conjugating activities for CDNB and DCNB increased rapidly during the larval and pupal stages to reach a peak in the newly emerged adult and then declined sharply over the 5 days after emergence (Hazelton and Lang, 1983). In the present work, the GSH affinity-purified GSTs from pupae also showed the highest specific activity towards CDNB and TNE, a lipid peroxidation product formed under conditions of oxidative stress (Agianian et al., 2003; Singh et al., 2001). This may be an evolutionary adaptation because pupae are immobile and therefore more vulnerable to unfavourable environmental conditions (Gillott, 1980) including the presence of toxic substances. Another reason may be the elevated biosynthesis and formation of adult tissues in the pupal stage (Doctor and Fristrom, 1985) and therefore high specific activity could be maintained to protect important biosynthetic pathways from inhibition by toxic substances both endogenous and exogenous.

The relatively low level of enzyme activity towards CDNB and DCNB in the adult stage compared to other developmental stages is also an important feature of the ontogenic models; this seems to be quite common in mammals (Fujita et al., 1985; Gregus et al., 1985) and insects (Kotze and Rose, 1987; Stenersen et al., 1987). Few exceptions are observed, although the insect Triatoma infestans and honeybee exhibit maximum GST activity in adults (Smirle and Winston, 1987; Wood et al., 1986). The differences in the specific activity could be attributed to the different isoenzymes present in the different developmental stages of the insect and 
their preference for CDNB as well as the presence of some inhibitors that manifest their action in some stages more than in others (Hunaiti et al., 1995).

The reported specific activity towards CDNB for the L. cuprina larvae in the present study is higher than that of the fruit-fly D. melanogaster (Hunaiti et al., 1995) and lower than that of Wiseana cervinata (Clark and Drake, 1984), insect Apis mellifera macedonica (Papadopoulos et al., 2004a) and the yellow-fever mosquito Aedes aegypti (Hazelton and Lang, 1983). Likewise, the specific activity of the $L$. cuprina adult enzyme is higher than adult D. melanogaster $(0.08$ vs 0.015 $\mu \mathrm{mol} / \mathrm{min} / \mathrm{mg})($ Hunaiti et al., 1995) but lower than reported for the adult housefly (0.08 vs $0.23 \mu \mathrm{mol} / \mathrm{min} / \mathrm{mg}$ ) (Clark and Dauterman, 1982), the yellow-fever mosquito (0.08 vs $2.7 \mu \mathrm{mol} / \mathrm{min} / \mathrm{mg}$ ) (Hazelton and Lang, 1983) and the Apis mellifera macedonica (0.08 vs $0.6 \mu \mathrm{mol} / \mathrm{min} / \mathrm{mg}$ ) (Papadopoulos et al., 2004a).

Few studies have been reported for insect pupae. Hazelton and Lang (1983) reported a much higher specific activity for the pupae of the yellow-fever mosquito (5.3 $\mu \mathrm{mol} / \mathrm{min} / \mathrm{mg})$ and Papadopoulos et al., (2004a) also reported higher specific activity for the pupae of Apis mellifera macedonica $(0.6 \mu \mathrm{mol} / \mathrm{min} / \mathrm{mg})$ than observed for $L$. cuprina pupae $(0.14 \mu \mathrm{mol} / \mathrm{min} / \mathrm{mg})$ in the present study. The specific activity of L. cuprina eggs $(0.09 \mu \mathrm{mol} / \mathrm{min} / \mathrm{mg})$ was similar to the eggs of Apis mellifera macedonica $(0.1 \mu \mathrm{mol} / \mathrm{min} / \mathrm{mg})$.

The present work also studied the specific activities towards model substrates other than CDNB. The specific activities of GSH affinity-purified GSTs from pupae were significantly $(\mathrm{p}<0.05)$ higher towards CDNB and TNE. Interestingly, the larval GSH affinity-purified GSTs showed significantly $(\mathrm{p}<0.05)$ higher activity towards 
DCNB and DHA. The specific activity towards DCNB was highest in the DNP-GSH affinity-purified larval and adult GSTs. The observed changes in the substrate specificity during the development of the insect may be explained in two ways, either by assuming alterations to the structure of the protein by post-translational modifications or by variation in the expression of different isoenzymes so that the insect meets the continuously changing demands of its development. Therefore proteomics was used to investigate the isoenzyme pattern expressed in the developmental stages.

GSH affinity-purified fractions from eggs showed the presence of only Delta GST on the 1D gel. Sigma GST starts developing from the larval stage and is expressed abundantly in the adult stage. The literature suggests the involvement of Sigma GSTs in flight muscle (Clayton et al., 1998) and also in testis (Takemori and Yamamoto, 2009) in the adult D. melanogaster fly. This could be the reason for the massive expression of Sigma GST in the adult stage. The consistent presence of Delta GSTs in all developmental stages (egg, larvae, pupae and adult) suggests a role for Delta GSTs in the general defence against toxic chemicals that they might be exposed to. While studying the developmental stages of D. melanogaster, Alias (2006) also found a higher expression of Sigma GST in the adult and showed the relative expression of GSTS1 among the developmental stages. However, (Hunaiti et al., 1995) reported the presence of Sigma GST only in the adult stage of $D$. melanogaster which is in contrast to our findings. The result reported here was also supported by the finding that gstS1 mRNA was detectable in developmental stages by Beall et al., 1992 . 
The GSTs expressed in all stages differ quantitatively. The DNP-GSH affinity chromatography showed the presence of tentatively identified Mu-like GSTs (Zone E in Figure 5-6) which are present in egg, larvae and pupae but totally absent in the adult stage. However, literature suggests that Mu GSTs are not found in insects (Enayati et al., 2005) and a firm characterisation of these will need further research. The identification of these Mu-like GSTs will be discussed in section 8.1.2. The tentatively identified Epsilon GSTs are more prominent in the adult stage compared to other developmental stages. Thus multiple GST isoenzymes have been shown to be expressed differentially in different developmental stages of $L$. cuprina.

Interestingly, expression of particular GSTs is often confined to specific tissues. So far most of the studies on the location of invertebrate GSTs have been carried out using protein extracts from dissected tissues and GSTs have been detected through their catalytic activities against the model substrates like CDNB and DCNB (Franciosa and Berge, 1995; Kotze and Rose, 1987). In the present study, in addition to CDNB and DCNB, three other substrates DHA, NPA and TNE were also used to study the catalytic activity of GSTs expressed in different tissues. The GSTs from head, thorax and abdomen were purified by GSH and DNP-GSH affinity chromatography. The protein found in the crude preparation of thorax as well as in the GSH affinity chromatography isolates had the highest total GST activity towards CDNB compared to head and abdomen (Figure 5-14). This may be explained by the important role of the thorax GSTs in flight muscles as well as detoxification (Clayton et al., 1998). However, crude enzymes from abdomen showed the highest specific activity compared to head and thorax towards all the substrates employed except DCNB (Figure 5-15). There was no significant difference in DCNB activity found 
between cytosolic GSTs of head, thorax and abdomen. This result presented here is not in agreement with the study by Kotze and Rose (1987). They studied GST activity towards DCNB in the main body regions of the adult $L$. cuprina female fly and reported the abdomen is more active compared to head and thorax. Similarly, Saleh et al. (1978) reported that $60-65 \%$ of activity towards DCNB was localised in the abdomen in the 6-day-old housefly.

In the present study, the GSH affinity-purified GSTs from the head showed the highest specific activity towards CDNB and TNE. The DNP-GSH affinitypurified fractions from head showed the highest specific activity towards CDNB and DCNB.

Although, substrate-specific profiling leads to interesting observations, it alone cannot distinguish between GST classes. Therefore, the location of the GSTs of L. cuprina was examined through proteomic methods. Two dimensional electrophoresis on GSH affinity-purified GSTs revealed interesting isoenzymic patterns. Two GST isoenzymes (Sigma and Delta) were always present in all main regions however the intensity of the spots varied markedly in different parts. The "laddering behaviour" (possibly due to degradation) of the Sigma GST suggests the possibility of proteolytic physiological processing (Alias and Clark, 2007). Additional spots (x, y, and $\mathrm{z}$ ) of supposed Delta GSTs were resolved on 2D gel of head and thorax; however abdomen appeared to lack some of the Delta GSTs. The DNP-GSH affinity-purified GSTs also showed variation in expression of proteins of each zone. It is notable that the high M.W. Delta GSTs (which resembled $D$. melanogaster CG17639) were present in greatest quantity in the head. Literature suggests that in Manduca sexta, this type of GST was found in antennae and may 
play an important role in processing of odorant signals (Rogers et al., 1999) and this is consistent with the results in L. cuprina. Furthermore, these isoforms are expressed most in larvae and adult life stages where an olfactory response may be important. There are reports available suggesting the presence of Delta GST in the central nervous system and indirect flight muscle cells (Franciosa and Berge, 1995) and in invertebrates, GSTs were also detected in the same tissues (Cammer et al., 1989; Campbell et al., 1990; Singhal et al., 1991).

In summary, the work presented in this chapter attempted to find out if the GST proteome changes during the developmental stages of $L$. cuprina and between different body parts of an adult fly. It was observed that the GST isoenzymes are expressed very differently in different developmental stages qualitatively and quantitatively. Sigma GST is absent in eggs whereas eggs express some Mu-like GSTs which are totally absent in the adult stage. The abdomen shows the highest specific activity towards TNE, suggesting an important role of abdomen GSTs in detoxification of products of oxygen toxicity.

With this information of the GST expression profile in L. cuprina, our next step was to study role of GSTs in insecticide resistance, aiming to answer the following questions:

- Is the expression of the L. cuprina GST proteome elevated in insecticide resistant strains?

- Do the L. cuprina GSTs from resistant insects have greater capacity to metabolise insecticides in vitro?

Chapter 6 attempts to answer these questions by studying GSTs in organophosphate susceptible and resistant strains of L. cuprina. 


\section{Involvement of glutathione S-transferases in the development of insecticide resistance in Lucilia cuprina}

\subsection{Introduction}

The aim of this chapter was to study the proteome of GSTs in the context of development of insecticide resistance in L. cuprina. The GST proteome of organophosphate (OP) susceptible and resistant strains have been compared and ability of the GSTs to metabolise insecticides in vitro has been studied.

Insecticide resistance can be explained in terms of several biochemical mechanisms. As described earlier, these include target-site resistance, penetration, efflux and detoxification-based resistance (Hemingway et al., 2002). In the former the target has reduced affinity for the insecticides and the latter occurs when enhanced levels or modified activities of phosphatases (Srinivas et al., 2004), esterases (Hemingway and Karunaratne, 1998; Rodriguez et al., 2010) oxidases (Vulule et al., 1999; Hemingway et al., 2004; Komagata et al., 2010), or GSTs (Motoyama and Dauterman, 1980; Clark and Shamaan, 1984; Lumjuan et al., 2005) destroy the insecticide reducing its concentration at its site of action. Trying to determine whether a specific GST is involved in the development of resistance to a particular insecticide is difficult, as it has been shown that most organisms have multiple GSTs and that ability to detoxify an insecticide may derive from increased levels of only a minor GST (Clark, 1989) or a combination of GSTs (Motoyama and Dauterman, 1980). Increases in the amount of GST enzymes have been reported to result from gene amplification or more commonly through increases in transcriptional rate, rather than expression of new isoforms (Ranson et al., 2004). 
Increased GST activity in resistance was first identified in OP resistance (Hayes and Wolf, 1988) and so far GSTs have been implicated in OP resistance in many insect species. The involvement of GSTs in the metabolism of organophosphate pesticides was shown for the first time by (Fukami and Shishido, 1966). They found demethylation of methyl parathion by glutathione catalyzed by the cytosolic fraction of rat liver or insect homogenates and the direct product of this reaction, $S$-methyl glutathione was subsequently identified. Benke et al. also reported that methyl parathion is biotransformed via a glutathione-dependent pathway in rat and mouse liver fractions (Benke et al., 1974; Benke and Murphy, 1975). Recombinant GST enzymes from the diamondback moth and housefly have verified the role of these enzymes in OP metabolism (Cho et al., 2001; Ichijo et al., 1997). In organophosphates, detoxification may occur via $O$-dealkylation or $O$-dearylation reactions. In $O$-dealkylation, glutathione is conjugated with the alkyl portion of the insecticide (Oppenoorth et al., 1979) whereas the reaction of glutathione with an aromatic leaving group is an $O$-dearylation reaction (Chiang and Sun, 1993) (see Figure 1-11). Some of the examples of these reactions with OP insecticides are shown in Figure 6-1.
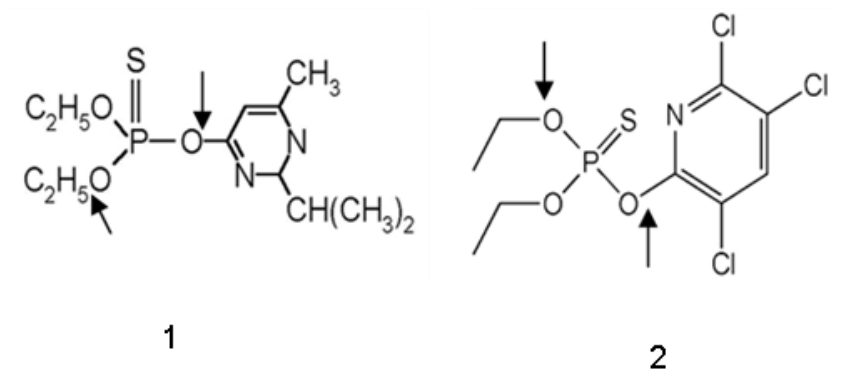

Figure 6-1: OP insecticides being metabolised by GSTs via dealkylation and dearylation reactions.

1) Diazinon; 2) Chlorpyrifos. The arrows show the site of attack by GST. 
Resistance to parathion, diazinon and diazoxon in a resistant strain of housefly was caused by an increase in GST activity via deethylation of these insecticides (Lewis, 1969; Lewis and Sawicki, 1971). GSTs can also catalyze detoxification of the secondary metabolites of OP insecticides. This insecticide class is often applied in its non-insecticidal phosphorothionate form and activated to the insecticidal oxon form by the action of cytochrome P450s in the insect (Hemingway et al., 2004). GSTs that can detoxify the active oxon analogue have been described in mosquitoes and elevated activity of these enzymes is a cause of OP resistance in Anopheles subpictus (Hemingway et al., 1991b).

High levels of GST activity towards DCNB and CDNB have been shown to be associated with OP resistance in resistant insects. Ottea and Plapp (1981) found out that GST activity toward DCNB was higher in the Rutgers diazinon-R housefly strain than the susceptible SBO strain. Elevated GST activity (DCNB) was consistently associated with azinphosmethyl resistance in the tufted apple bud moth, Platynota idaeusalis (Carlini et al., 1995) and light brown apple moth (Armstrong and Suckling, 1990). In several field strains of the fall armyworm, increased GST activity towards both $\mathrm{DCNB}$ and $\mathrm{CDNB}$ was associated with resistance to pyrethroid, organophosphorus and carbamate insecticides (Yu, 1992). Cochrane et al. documented that malathion resistance in D. melanogaster was associated with elevated levels of two GST isoenzymes (Cochrane et al., 1992). Higher DCNB activity was also detected in the multi-resistant Rutgers strain of housefly (Motoyama and Dauterman, 1977) and in resistant field strains of the sheep blowfly, L. cuprina (Wilson, 1999; Wilson and Clark, 1996). 
Clark and Shamaan (1984) reported that the detoxification of DDT by dehydrochlorination is catalyzed by a GST in Musca domestica. Initially the enzymes catalysing this reaction had not been identified as GSTs because of the lack of DDT dehydrochlorinase activity in some purified GSTs. However, strong evidence was provided when three enzymes with CDNB conjugation activity were isolated from a DDT resistant strain of housefly. Out of these, two were active with DDT as a substrate. The DDT dehydrochlorination reaction was proposed to proceed via a base abstraction of hydrogen, catalyzed by the thiolate anion generated in the active site of the GST, leading to the elimination of chloride from DDT, generating DDE (1,1-bis-(4-chlorophenyl)-2,2-dichloroethene) (Clark and Shamaan, 1984). An increased rate of dehydrochlorination confers resistance to DDT in Aedes aegypti (Grant et al., 1991) and Anopheles gambiae (Prapanthadara et al., 1993).

In the metabolism of pyrethroid insecticides, previous reports suggested that GSTs detoxify the lipid peroxidation products induced by pyrethroids and thus confer indirect resistance (Vontas et al., 2001). They may also protect against pyrethroid toxicity in insects by sequestering the insecticides (Kostaropoulos et al., 2001a; Prapanthadara et al., 1993). However, a recent report suggests that GSTs may also be responsible for the direct dechlorination of and resistance to permethrin in the Silk Moth, Bombyx mori (Yamamoto et al., 2009b). Pyrethroid resistance related to GSTs has been reported in mosquitoes (Liu et al., 2006; Singh et al., 2002), houseflies (Liu and Yue, 2001) and cotton bollworm (Martin et al., 2002).

Different GST isoforms may be involved in detoxification functions in different species. Generally it has been observed that the isoenzymes responsible for these detoxification reactions come from the Delta and Epsilon families. There are 
several reports available demonstrating that elevated levels of Delta class GSTs have been implicated in resistance to all the major classes of insecticides in Drosophila melanogaster, in the rice brown planthopper, Nilaparvata lugens and in Musca domestica (Tang and Tu, 1994; Vontas et al., 2002; Wang et al., 1991). Epsilon GSTs also play a very important role in detoxification of insecticides in the Diamondback Moth, Plutella xylostella, in the malaria vector Anopheles gambiae and in Blattella germanica (Huang et al., 1998; Ortelli et al., 2003; Wei et al., 2001). Insect Sigma GST has been shown to be catalytically active with the lipid peroxidation product, 4-hydroxynonenal (4-HNE) (Singh et al., 2001). The Delta and Epsilon classes of GSTs have also been reported to exhibit peroxidase activity in Nilaparvata lugens (Ortelli et al., 2003; Vontas et al., 2001) and in Anopheles gambiae (Ortelli et al., 2003). Zeta GST appears to be responsible for the resistance to permethrin in the Silk Moth, Bombyx mori (Yamamoto et al., 2009b), Omega GST appears to be associated with resistance to the organophosphate fenitrothion in a different strain of Bombyx mori (Yamamoto et al., 2009a). Recently, a Xi isoform has been identified uniquely in mosquitoes and appears to be associated with DDT resistance in a South American strain of Aedes aegypti (Grant and Hammock, 1992; Lumjuan et al., 2007). In addition to the above complexity, members of a particular family are likely to have broad and overlapping substrate specificities so that collectively they offer an extraordinarily wide protection against chemical agents in insects. 


\subsubsection{The Lucilia cuprina enzyme system and insecticide resistance}

The sheep blowfly, L. cuprina, is a pest of economic significance to the sheep meat and wool industries and its development of resistance to insecticides is a matter of concern. This insect has developed resistance towards organophosphate insecticides and organochlorine insecticides including dieldrin and aldrin in New Zealand (Gleeson et al., 1994; Hart, 1961) and also towards the chitin synthesis inhibitors (Batterham et al., 2006; Kotze et al., 1997). Diazinon resistance was not detected until 1965. By 1970 about 95\% of flies were diazinon resistant in Australia (Levot, 1995). Many years before, in Australia, L. cuprina had demonstrated resistance to cyclodienes and carbamates (Hughes and McKenzie, 1987; Shanahan, 1959). In the first instance, OP resistance has been shown to be due to the action of mutated carboxyl esterase (the E3 esterase) which acquires the ability to hydrolyse phosphate triester insecticides (Hughes and Raftos, 1985). In L. cuprina, the major gene responsible for the resistance against OPs is the Rop-1 gene, present on chromosome 4 , that belongs to $\alpha$-esterase gene family and encodes carboxylesterase E3. The diazinon resistant strains from $L$. cuprina were found to have an amino acid substitution in the active site of the E3 enzyme, Gly ${ }^{137} \rightarrow$ Asp (Newcomb et al., 1997a; Newcomb et al., 1997b). Later, in a malathion-resistant strain, it was found that a novel amino acid substitution $\operatorname{Trp}^{251} \rightarrow$ Leu in the active site of the enzyme was responsible for the resistance that developed (Campbell et al., 1998). Besides the esterases mediating resistance in insects, the monooxygenases also contribute towards resistance against insecticides. Kotze et al. studied resistant strains of $L$. cuprina larvae and showed a correlation between diazinon resistance and in vitro aldrin epoxidase activity (Kotze and Sales, 1995). Also, other studies in L. cuprina 
have produced the evidence indicating strong correlations between resistance and the activity of glutathione S-transferases (Wilson and Clark, 1996).

Proteomic analysis is a powerful tool to study alterations in protein expression in response to chemical selection. Therefore the expression of GSTs in the OP susceptible strains NSW and CSIRO (Australian standard laboratory strains) and the Diazinon-resistant strain PY81 (38x resistant, G. Lindsay and A.G. Clark, unpublished data) of L. cuprina has been studied in this chapter using proteomics with a view to evaluating the use of such techniques in this context. In parallel to that, the in vitro metabolism of insecticides by cytosolic and affinity-purified GSTs from susceptible and resistant strains was also studied using reverse-phase HPLC in order to assess the significance of GST as a mechanism of insecticide detoxification in these strains of L. cuprina.

\subsection{Objectives:}

- To profile the GST proteome of laboratory OP susceptible strains (NSW and CSIRO) and a resistant strain (PY81) of L. cuprina.

- To assess the in vitro interaction of GSTs with insecticides using reversephase HPLC. 


\subsection{Results:}

\subsubsection{Purification of GSTs from susceptible and resistant strains of Lucilia cuprina}

A comparative analysis was performed between organophosphate susceptible (NSW and CSIRO) and resistant (PY81) strains of L. cuprina. The crude enzyme preparations from adult flies of each strain were subjected to a GSH and DNP-GSH affinity matrices sequentially as described in the section 3.2. The crude cytosolic enzyme preparation of the PY81 strain had 2.2- and 1.7-fold significantly higher total activity towards CDNB ( $\mathrm{p}<0.05)$ compared to NSW and CSIRO strains respectively (Table 6-1). The GSH affinity-purified GST isoenzymes from the resistant strain had 1.3- and 1.1- fold higher total activity towards CDNB in comparison with NSW and CSIRO respectively. The total activity of DNP-GSH purified GSTs of PY81 strain was almost 2-fold greater compared to both the susceptible ones.

The amount of the protein recovered in the DNP-GSH affinity eluents of PY81 strain was significantly 2.5 -fold and 2.0 -fold higher $(\mathrm{p}<0.05)$ than the NSW and CSIRO strains (Figure 6-2). The difference in amount of protein recovered from the resistant and susceptible insects accounts for the majority of elevated total GST activity observed (Table 6-1) indicating that elevated total GST activity is mainly associated with a quantitative change in the enzyme. This suggests the possibility that the DNP-GSH affinity-purified GSTs may be involved in the OP resistance. 


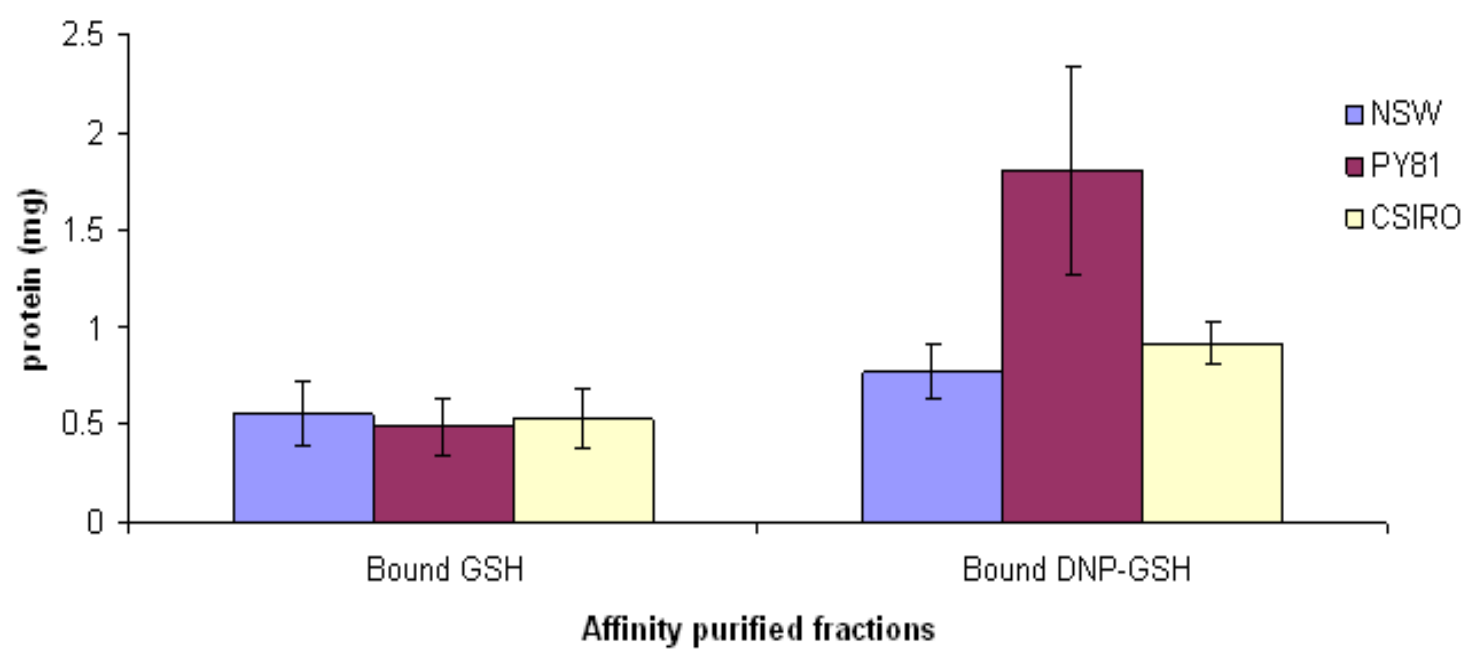

Figure 6-2: Amount of protein recovered from the GSH and DNP-GSH affinity matrices.

The crude enzyme preparations from equal masses ( $3 \mathrm{~g}$ ) of susceptible (NSW and CSIRO) and resistant (PY81) strains were de-pigmented using HiTrap desalting column (5 ml) and applied to the GSH affinity column. The unbound fractions from the GSH affinity column having GST activity towards CDNB were pooled and subsequently applied to the DNP-GSH column. The bound proteins on both the columns were eluted with $20 \mathrm{mM}$ glutathione in $0.05 \mathrm{M}$ phosphate buffer, $\mathrm{pH} 9.6$ as described in the section 3.2.2. Values are the mean of at least three independent experiments with three measurements in each. Error bars represent standard deviation. 
Table 6-1: Purification of GSTs from susceptible (NSW and CSIRO) and resistant strain (PY81) of L. cuprina.

The calculation of $\%$ yield and fold purification was made considering the actual volume of application on each column. Values are mean \pm SD taken from three independent experiments with triplicate measurements within each experiment. For the purification details of GSTs from flies, refer the legend of Figure 6-2.

\begin{tabular}{|c|c|c|c|c|c|c|}
\hline Strain & Purification step & $\begin{array}{l}\text { Total } \\
\text { protein } \\
(\mathrm{mg})\end{array}$ & $\begin{array}{l}\text { Total } \\
\text { activity } \\
(\mu \mathrm{mol} / \mathrm{min})\end{array}$ & $\begin{array}{c}\text { Specific } \\
\text { activity } \\
(\mu \mathrm{mol} / \mathrm{min} / \\
\mathrm{mg})\end{array}$ & $\begin{array}{l}\text { Yield } \\
\text { (\%) }\end{array}$ & $\begin{array}{l}\text { Purification } \\
\text { fold }\end{array}$ \\
\hline \multirow[t]{6}{*}{ NSW } & Crude & $37.0 \pm 0.5$ & $3.30 \pm 0.1$ & $0.09 \pm 0.002$ & 100 & 1.00 \\
\hline & Desalt & $30.0 \pm 0.5$ & $3.20 \pm 0.2$ & $0.1 \pm 0.006$ & 97 & 1.11 \\
\hline & Unbound GSH & $29.0 \pm 3.5$ & $1.20 \pm 0.4$ & $0.04 \pm 0.02$ & 36 & 0.44 \\
\hline & Bound GSH & $0.60 \pm 1.5$ & $1.80 \pm 0.2$ & $3.51 \pm 0.9$ & 54 & 39.0 \\
\hline & Unbound DNP-GSH & $27.0 \pm 2.0$ & $1.00 \pm 0.1$ & $0.03 \pm 0.003$ & 30 & 0.33 \\
\hline & Bound DNP-GSH & $0.70 \pm 0.1$ & $0.90 \pm 0.2$ & $1.28 \pm 0.3$ & 27 & 14.2 \\
\hline \multirow[t]{6}{*}{ PY81 } & Crude & $52.0 \pm 7.0$ & $7.40 \pm 1.6$ & $0.14 \pm 0.03$ & 100 & 1.00 \\
\hline & Desalt & $50.0 \pm 10$ & $6.60 \pm 1.4$ & $0.13 \pm 0.02$ & 89 & 0.92 \\
\hline & Unbound GSH & $43.5 \pm 5.0$ & $3.40 \pm 2.1$ & $0.08 \pm 0.04$ & 46 & 0.57 \\
\hline & Bound GSH & $0.50 \pm 0.1$ & $2.30 \pm 0.4$ & $4.60 \pm 2.7$ & 31 & 32.8 \\
\hline & Unbound DNP-GSH & $39.0 \pm 6.0$ & $2.30 \pm 1.1$ & $0.06 \pm 0.02$ & 31 & 0.42 \\
\hline & Bound DNP-GSH & $1.80 \pm 0.7$ & $1.90 \pm 0.6$ & $1.05 \pm 0.1$ & 25 & 7.50 \\
\hline \multirow[t]{6}{*}{ CSIRO } & Crude & $41.0 \pm 2.5$ & $4.30 \pm 0.3$ & $0.10 \pm 0.007$ & 100 & 1.00 \\
\hline & Desalt & $35.5 \pm 2.0$ & $3.70 \pm 0.1$ & $0.10 \pm 0.003$ & 86 & 1.00 \\
\hline & Unbound GSH & $31.5 \pm 1.0$ & $1.20 \pm 0.5$ & $0.04 \pm 0.02$ & 28 & 0.40 \\
\hline & Bound GSH & $0.50 \pm 0.1$ & $2.10 \pm 0.2$ & $4.26 \pm 1.2$ & 49 & 42.6 \\
\hline & Unbound DNP-GSH & $29.0 \pm 1.5$ & $1.00 \pm 0.1$ & $0.03 \pm 0.003$ & 23 & 0.35 \\
\hline & Bound DNP-GSH & $0.92 \pm 0.1$ & $1.00 \pm 0.1$ & $1.08 \pm 0.10$ & 23 & 10.8 \\
\hline
\end{tabular}


The crude enzyme and affinity-purified GSTs of each strain were tested for activity with a number of model substrates in order to give a detailed characterisation of enzyme activity. The substrates used were CDNB, DCNB, NPA, DHA and TNE. Since the absolute activities varied widely among the strains, activity was normalised relative to the CSIRO strain. The results are shown in the form of histograms in Figure 6-3 (total activity) and Figure 6-4 (specific activity).

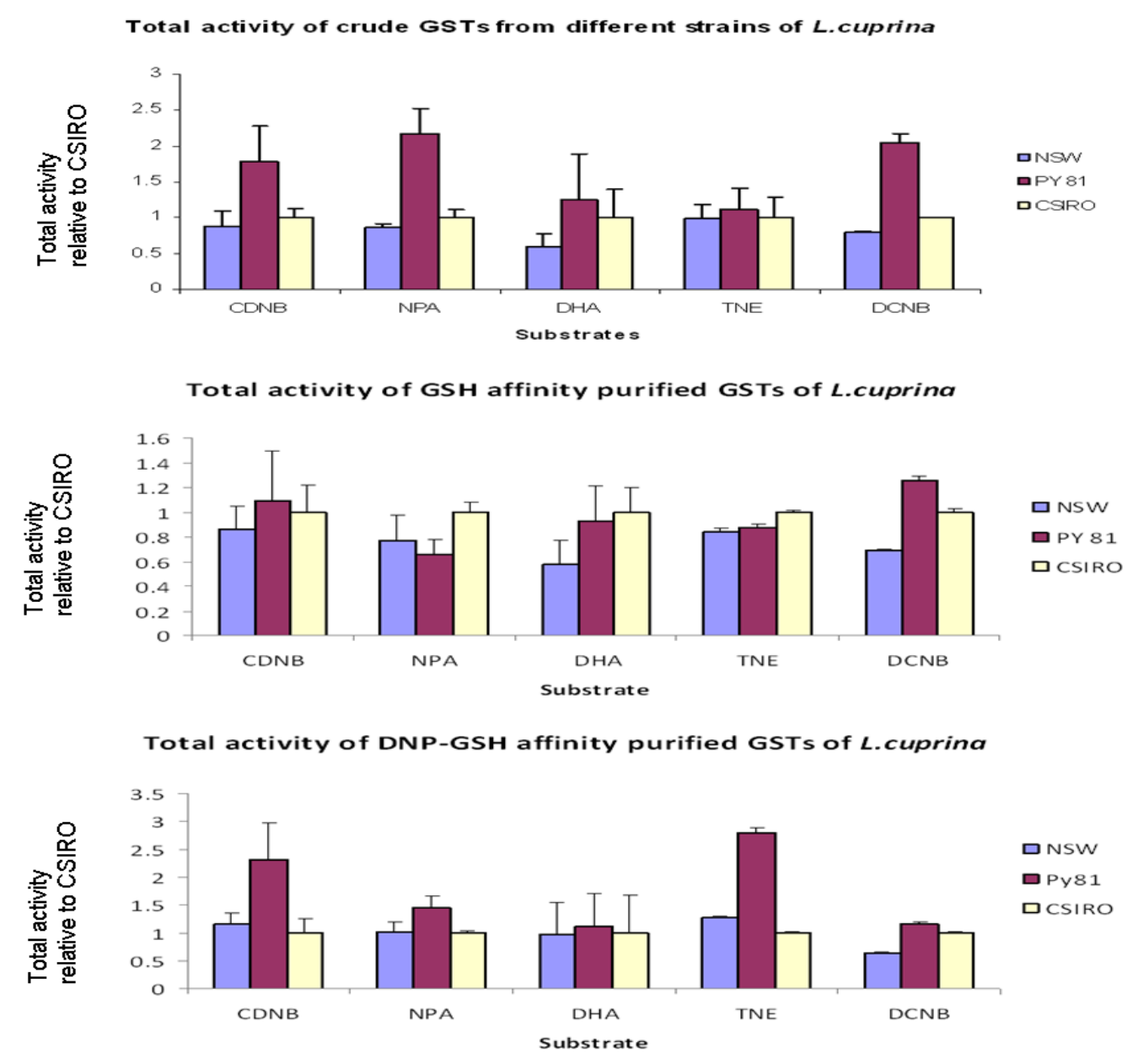

Figure 6-3: Substrate-specific total activity of crude GSTs and affinity-purified GSTs with different model substrates relative to CSIRO strain

GST activity assays were performed as described in section 3.2.4. The volume of both affinity-purified fractions of each strain was $20 \mathrm{ml}$. Total activity is in Units ( $\mu \mathrm{mol} / \mathrm{min}$ ). Values are the mean of at least three independent experiments with three measurements in each. Error bars represent standard deviation. For the purification details of GSTs from flies, refer to the legend of Figure 6-2. 
The crude extract of the PY81 resistant strain has the highest total activity with all the substrates compared to NSW and CSIRO susceptible strains (Figure 6-3) but the difference is not significant with TNE and DHA substrates. The data show that the GSH affinity-purified fractions of the PY81 strain has the highest total activity with CDNB and DCNB. The total activity of DNP-GSH affinity fractions of the PY81 strain have significantly higher activity with CDNB and TNE compared to susceptible ones $(\mathrm{p}<0.05)$. The specific activity of the cytosolic GSTs from PY81 was also higher with the model substrates except TNE compared to other strains (Figure 6-4). Interestingly, the GSH affinity-purified fractions from the PY81 strain showed significantly higher activity towards DCNB compared to NSW and CSIRO strains $(p<0.05)$ though there was no elevated amount of purified GSTs in the GSH affinity-purified fractions (Figure 6-2) of the PY81 strain. The specific activities of DNP-GSH affinity-purified fractions from the PY81 strain were less than those of the NSW and CSIRO strains due to the greater amount of protein recovered. It is worth noting that the total activity gives a clear indication that the PY81 strain GSTs purified from the DNP-GSH matrix had a significantly higher activity with TNE - an electrophilic product of lipid peroxidation $(\mathrm{p}<0.05)$ and the specific activity of the PY81 GSTs from GSH affinity-purified fractions have significantly higher activity with DCNB $(\mathrm{p}<0.05)$ compared to susceptible strains. This implies that the GSTs isolated with either affinity matrix might have important roles in metabolism of insecticides in L. cuprina or in protecting against the effects of insecticide poisoning. 


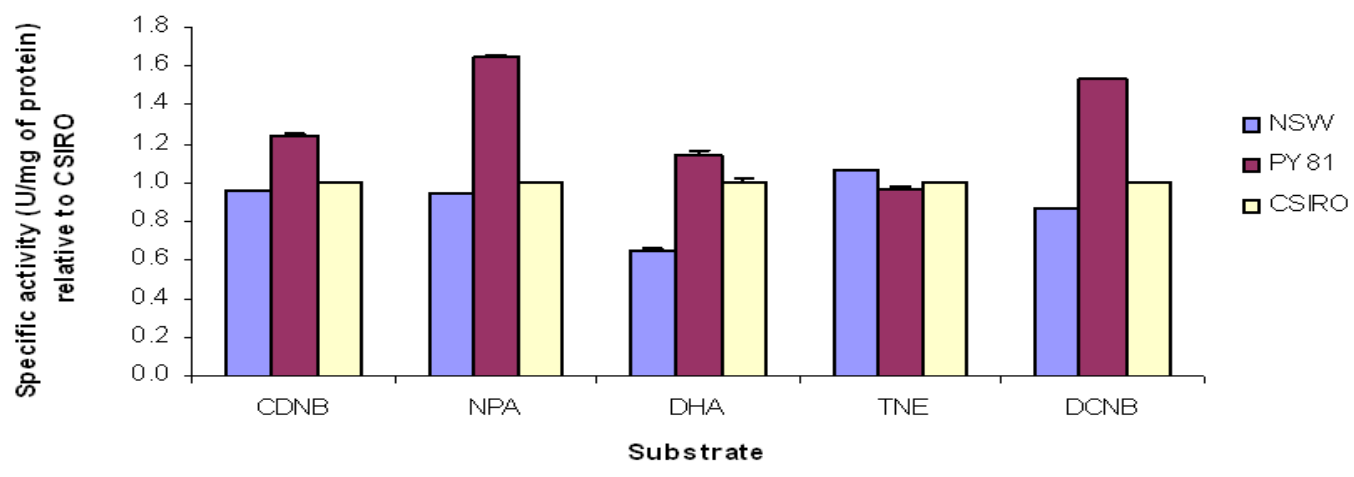

Specific activtiy of GSH affinity purified GSTs of L. cuprina

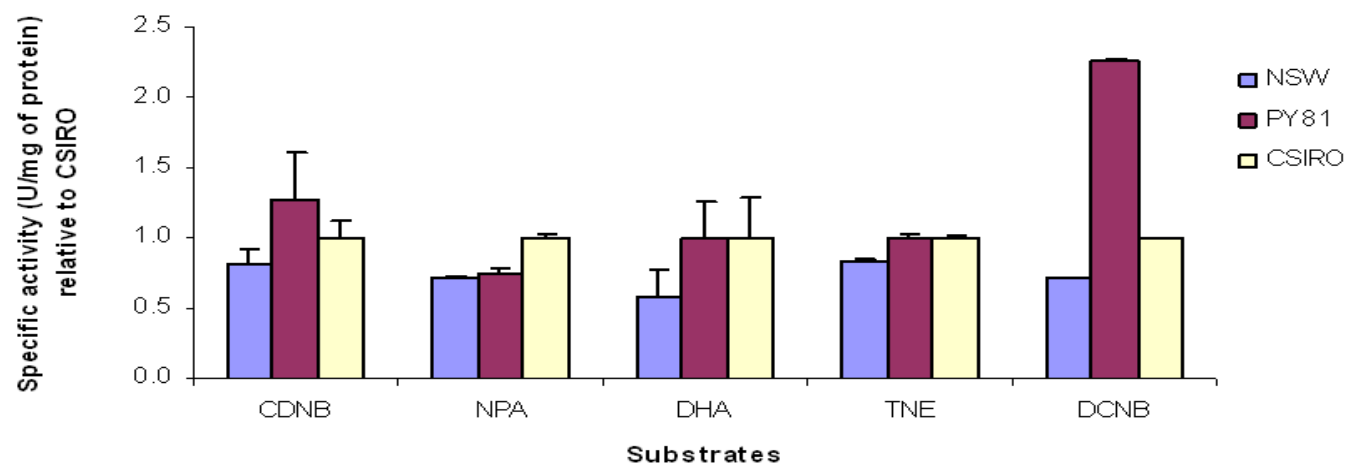

Specific activity of DNP-GSH affinity purified GSTs of $L$. cuprina

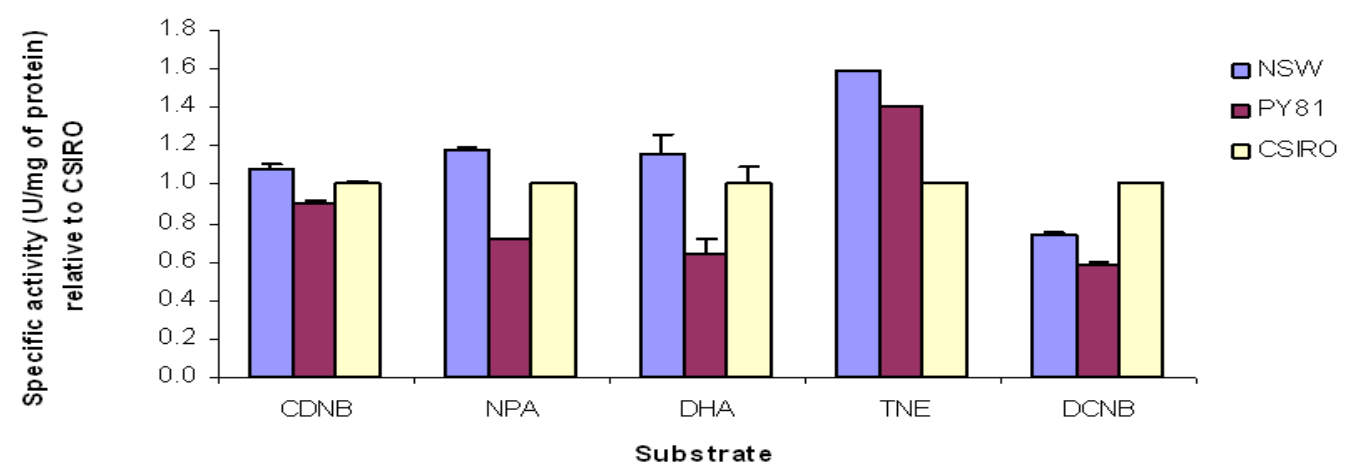

Figure 6-4: Substrate-specific specific activities of crude GSTs and affinity-purified GSTs with different model substrates relative to CSIRO strain.

GST activity assays were performed as described in section 3.2.4. The volume of both affinity-purified fractions of each strain was $20 \mathrm{ml}$. Specific activity is in Units/mg of protein. Values are the mean of at least three independent experiments with three measurements in each. Error bars represent standard deviation. For the purification details of GSTs from flies, refer to the legend of Figure 6-2. 


\subsubsection{Proteomic analysis of GSTs from susceptible and resistant strains}

The following one dimensional gel shows that GSTs other than the Sigma and Delta from all three strains have been captured by DNP-GSH affinity matrix when used in sequence with the GSH matrix. The DNP-GSH purified fractions do not show the binding of non-specific proteins (Figure 6-5) perhaps due to use of matrix that had been used for several previous preparations. Newly prepared DNP-GSH matrix always binds to non-specific proteins along with the GSTs as shown in Figure 4-10 and Figure 5-3. Fewer bands were purified relative to new DNP-GSH matrix.

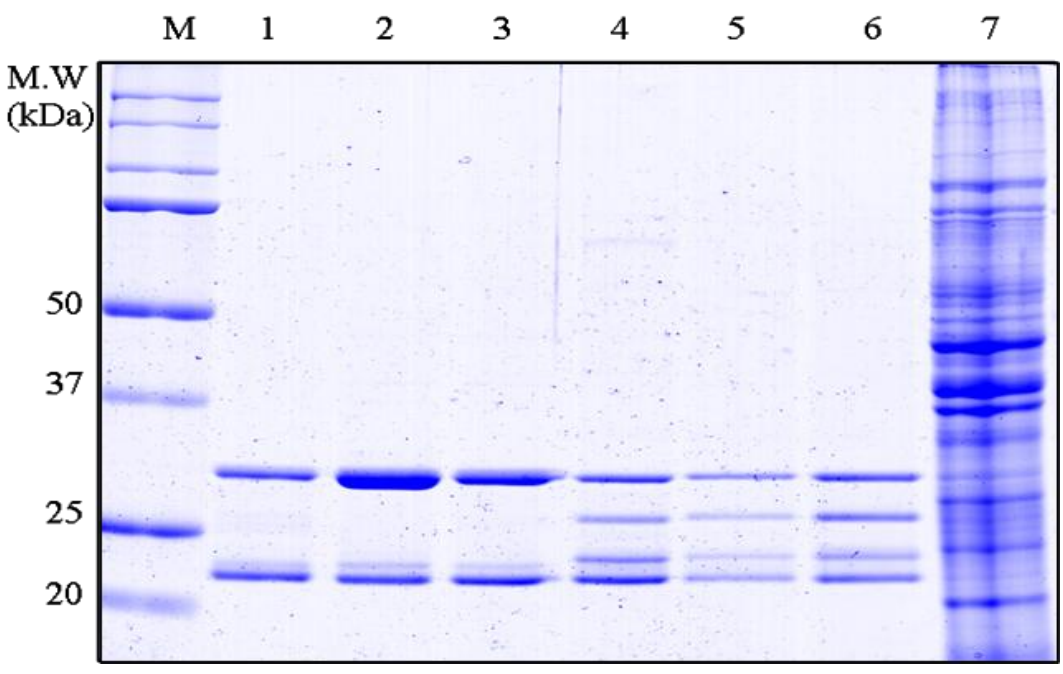

Figure 6-5: SDS PAGE on bound GSH and bound DNP-GSH affinity-purified fractions of NSW, PY81 and CSIRO strains.

The crude enzyme was prepared from $3 \mathrm{~g}$ of NSW, PY81 and CSIRO strain of L. cuprina and purified by GSH and DNP-GSH matrix as described in the section 3.2. Lane M is M.W. marker. Lanes 1-3 show bound fractions of GSTrap column from NSW, PY81 and CSIRO respectively. Lanes 4-6 are of bound DNP-GSH fractions of NSW, CSIRO and PY81 respectively. Lane 7 is of crude extract of PY81 strain. The gel was stained with Coomassie Brilliant Blue G-250. 

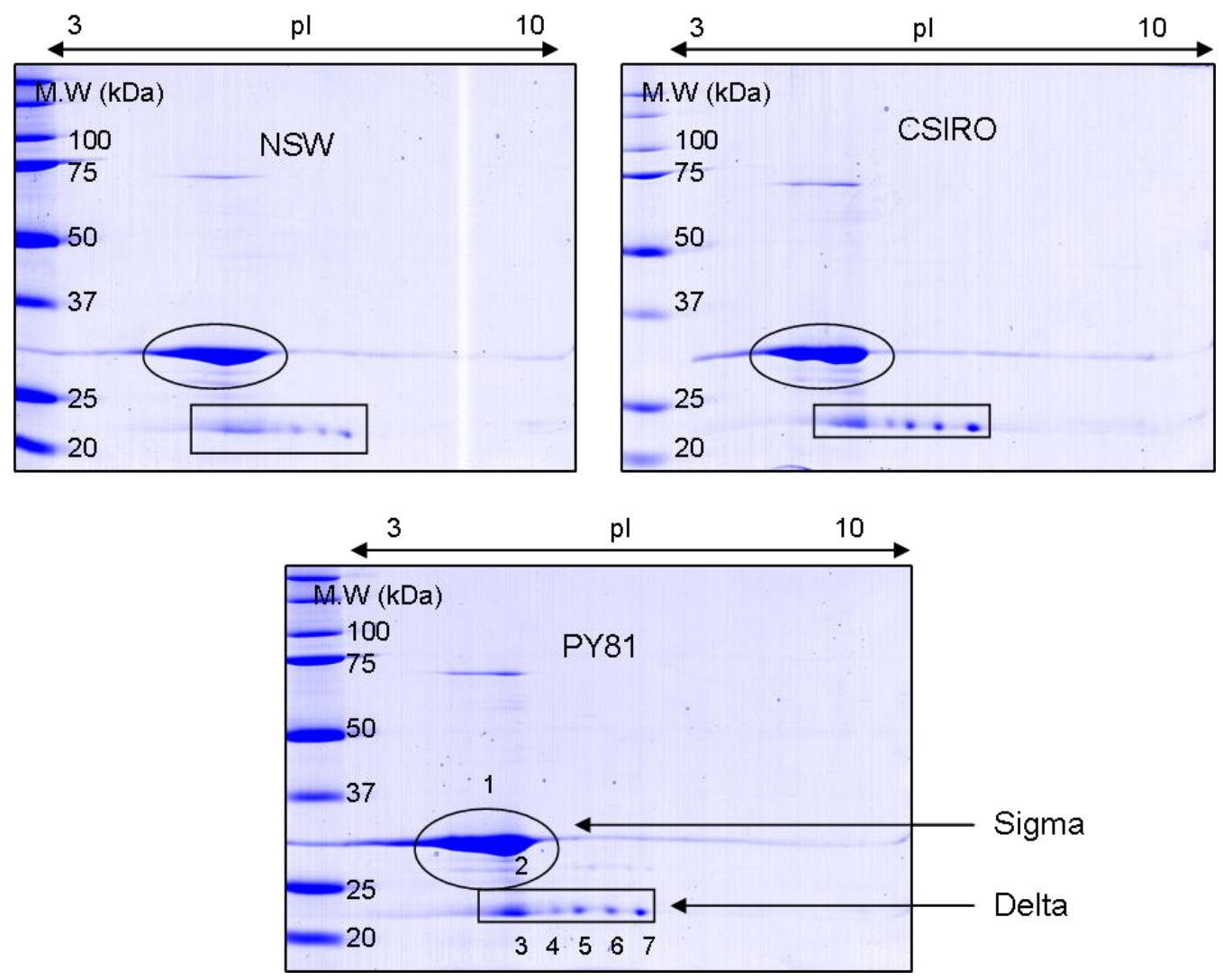

Figure 6-6: Two dimensional electrophoresis gels of the GSH affinity-purified fractions from susceptible and resistant strains of L. cuprina.

The GSH affinity-purified fractions of each strain were separated in the first dimension on a $7 \mathrm{~cm} \mathrm{pH} \mathrm{3-10} \mathrm{linear} \mathrm{IPG} \mathrm{strip} \mathrm{and} \mathrm{then} \mathrm{in} \mathrm{the} \mathrm{second} \mathrm{dimension} \mathrm{on} \mathrm{a} \mathrm{SDS-PAGE} \mathrm{gel.} \mathrm{The}$ gel was stained with Coomassie Brilliant Blue G-250. The protein load on each gel was obtained from the same mass of flies ( $3 \mathrm{~g})$. Spot numbers are as referred to the Figure 4-18 and their tentative identification can be found in Table 4-11.

The Sigma GST was expressed in slightly higher quantity in the PY81 strain compared to NSW and CSIRO, but not significantly higher. The Delta GSTs in NSW strain appear to be expressed in a slightly lower quantity when visualised on the $2 \mathrm{D}$ gel but the 1D gel showed no difference at all. However, the quantitative differences are not significant except in spot 7 between NSW and PY81. The quantitative analysis of these results is in Figure 6-7. 


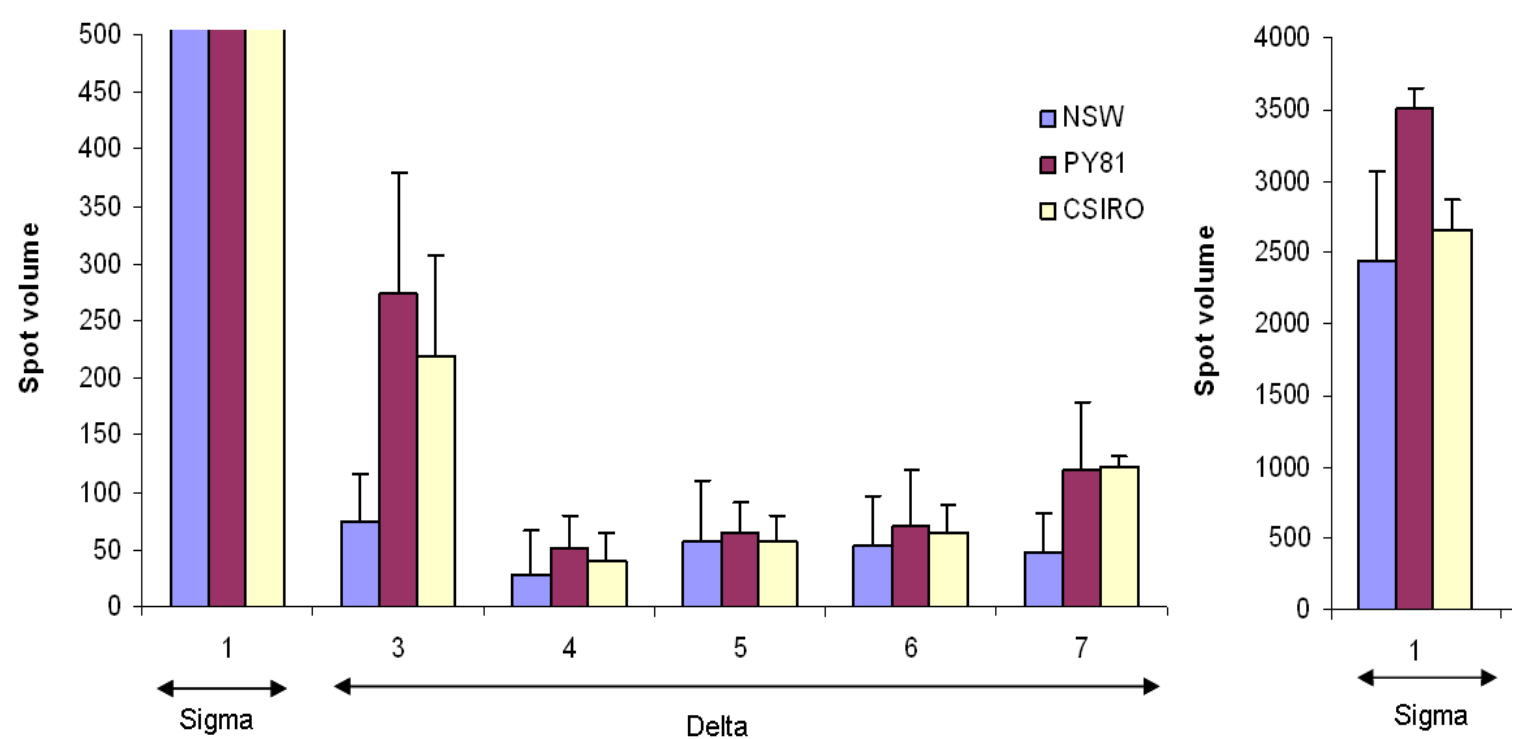

Figure 6-7: Quantitative analysis of GSH affinity-purified GSTs in different L. cuprina strains.

Quantitative analysis of numbered spots of the digitized images of 2D gels from NSW, PY81 and CSIRO strains shown in Figure 6-6 was carried out using ImageQuant (Version 5.2, Molecular Dynamics) software. The data represent mean of three independent experiments with triplicate measurements in each and error bars represent standard deviation. Spot 2 is appeared to be a degradation product of the Spot 1 and not included in the analysis. Spot 1 is shown alongside to full scale.

The DNP-GSH affinity fractions were also subjected to 2D electrophoresis to determine if there was any quantitative or qualitative difference between the strains. The results are shown in the Figure 6-8. 


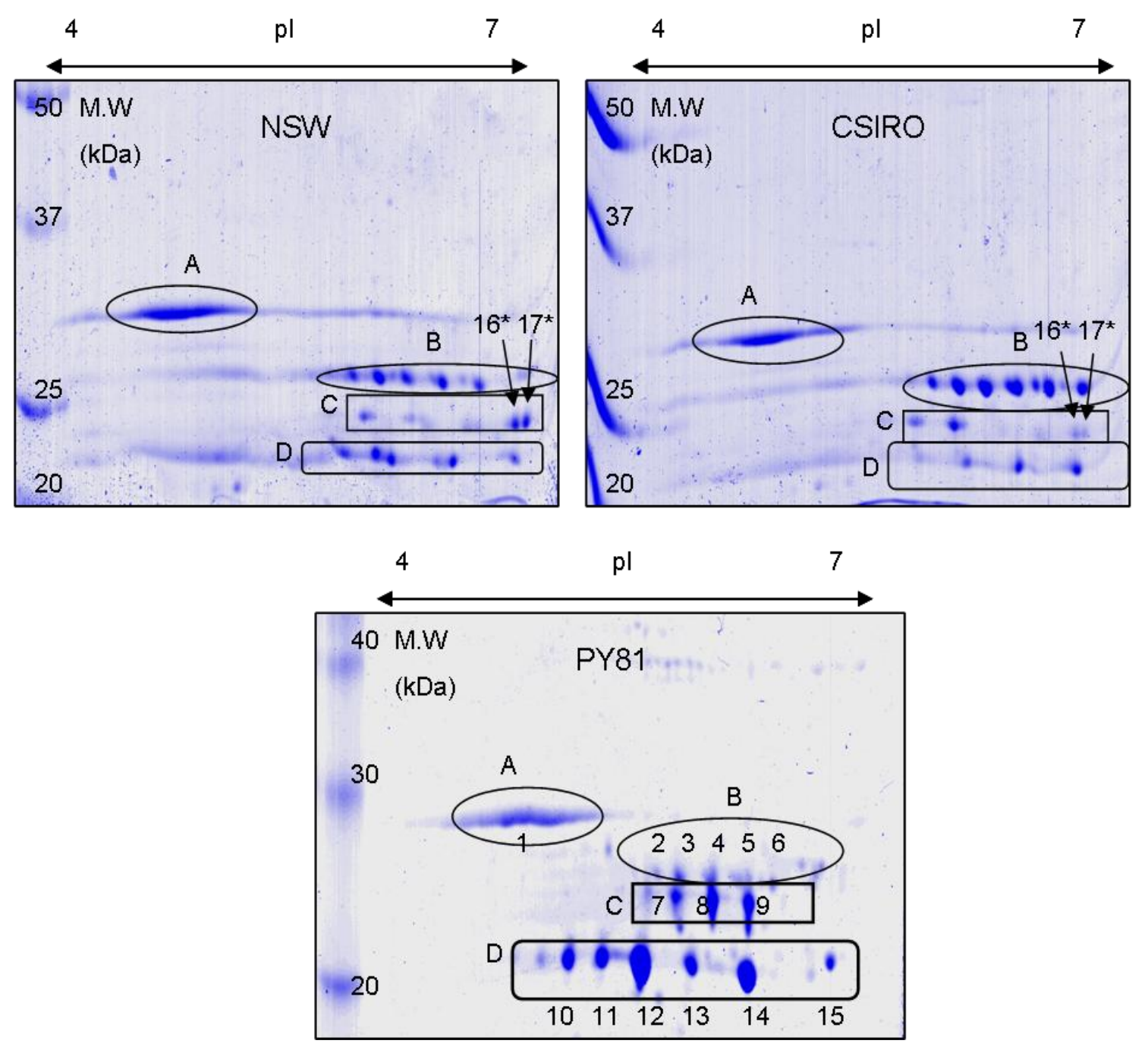

Figure 6-8: Two dimensional electrophoresis gels of the DNP-GSH affinity-purified fractions from susceptible and resistant strains of L. cuprina.

The DNP-GSH affinity-purified fractions of each strain were separated in the first dimension on a $7 \mathrm{~cm} \mathrm{pH} \mathrm{4-7} \mathrm{linear} \mathrm{IPG} \mathrm{strip} \mathrm{and} \mathrm{then} \mathrm{in} \mathrm{the} \mathrm{second} \mathrm{dimension} \mathrm{on} \mathrm{a} \mathrm{SDS-PAGE} \mathrm{gel.}$ The gel was stained with Coomassie Brilliant Blue G-250. The protein load on each gel was obtained from the same mass of flies $(3 \mathrm{~g})$. Spot numbers are as referred in Figure 4-21 except two new spots $16^{*}$ and $17^{*}$ in NSW and CSIRO strains. The tentative identification of spots 1-15 can be found in Table 4-13 and Table 4-14 according to the numbers. The new spots $16^{*}$ and $17^{*}$ were tentatively identified as Epsilon GSTs by MALDI-TOF and identification can be found in Appendix section 10.3.10. 
The Figure 6-8 shows the typical gel pattern for the separation of DNP-GSH affinity-purified GSTs from susceptible and resistant strains of $L$. cuprina. The spot volume data from the three independent experiments has been quantified and shown in Figure 6-9. Different pI variants of Delta and Epsilon GSTs are noted. As evident from Figure 6-9, the only significant $(\mathrm{p}<0.05)$ differences between PY81 and the susceptible strains were in the Epsilon (zone C) and low M.W. Delta and Mu-like GSTs (zone D). Interestingly there was also a significantly higher abundance of spot 14, tentatively identified as Rab protein (zone D). There are even quantitative differences between both the susceptible strains. The proteins in zone D (tentatively identified as Delta GSTs and Rab proteins) in NSW has six spots whereas in CSIRO there are only four spots. The tentatively identified Delta GSTs with high M.W. (zone B) in PY81 have not been expressed as prominently as in the NSW and CSIRO strains. The Epsilon GSTs (zone C) from NSW and CSIRO differ in the pI compared to the PY81 strain and have expressed in much greater quantity in the PY81 strain compared to susceptible strains. The Sigma GST (zone A) does not appear to vary amongst all three strains. 


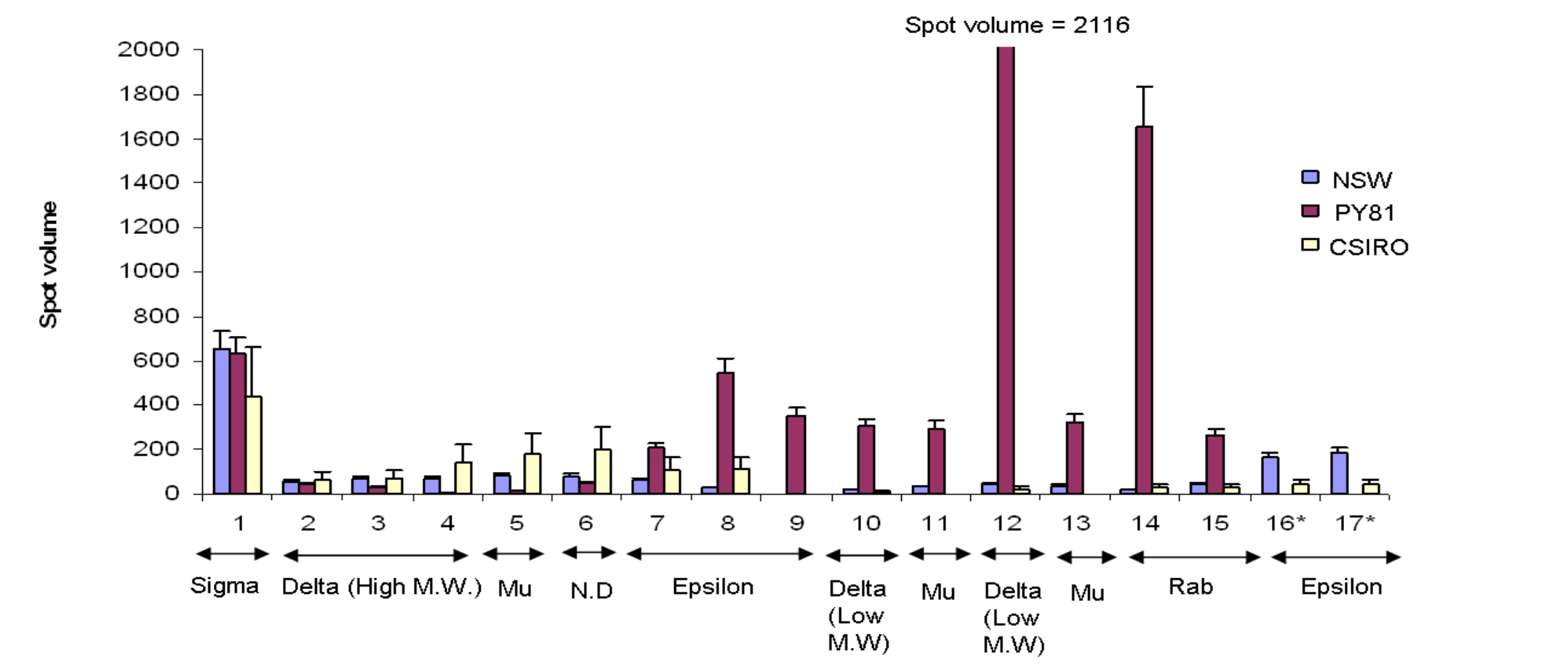

Figure 6-9: Quantitative analysis of DNP-GSH affinity-purified GSTs from different $L$. cuprina strains.

Quantitative analysis of numbered spots of digitized images of 2D gels from NSW, PY81 and CSIRO strains shown in Figure 6-8 was carried out using ImageQuant (Version 5.2, Molecular Dynamics) software. The data represent mean of three independent experiments with triplicate measurements in each and error bars represent standard deviation. The Epsilon GSTs (zone C) and some of Delta (low M.W.), Mu and Rab proteins (zone D) are significantly higher in PY81 than NSW and CSIRO(p<0.05). N. D = isoform class not determined. 


\subsection{In vitro conjugation of insecticides with GSTs determined by reverse phase HPLC}

GSTs from L. cuprina were purified by GSH affinity chromatography. The unbound fractions from the GSH matrix active with CDNB were further purified using DNP-GSH affinity matrix as described in section 3.2.2. The organophosphates methyl parathion and diazinon were tested for GSH dependent degradation in vitro by GSTs as described in the section 3.2.8. In brief, the reaction mixture $(1 \mathrm{ml})$ containing insecticide, the enzyme and GSH in tris buffer was incubated for $4 \mathrm{hrs}$ at $37^{\circ} \mathrm{C}$ and extracted with $1 \mathrm{ml}$ of heptane by vortexing the reaction mixture for 30 seconds. Then $20 \mu \mathrm{l}$ of each extract was injected into the HPLC in reverse phase mode and the remaining insecticide was eluted under the gradient. Controls using only tris buffer $\mathrm{pH} 8.0$, only heptane and tris buffer with methanol and glutathione extracted with heptane were also tested (Figure 6-11). Glutathione conjugates were not directly measured. Since glutathione conjugates are not extracted into heptane, the extent of conjugation was determined on the basis of reduction in insecticide concentration, measured through the area under the insecticide peak. The calculation of amount of insecticide remaining in the reaction mixture was made using a standard curve for each insecticide. Figure 6-10 shows the calibration curve for methyl parathion as an example. The peaks with the retention time corresponding to methyl parathion and diazinon under the employed gradient are shown in Figure 6-11. The change in the peak area of the remaining methyl parathion in the reaction mixture after incubation with and without enzyme is shown in Figure 6-12 as an example to determine the conjugation of insecticide. The change in concentration of diazinon was measured in the same way. 
A
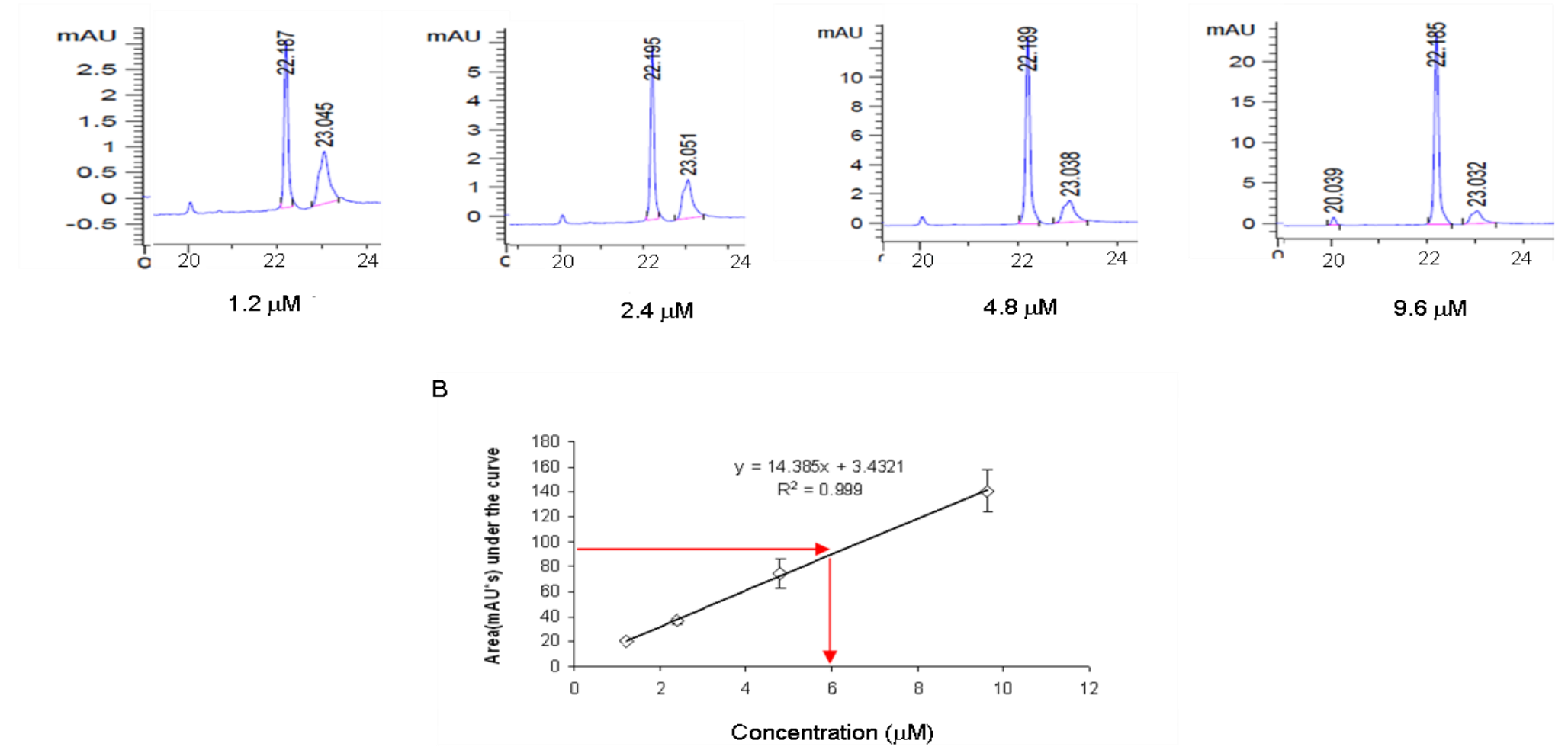

Figure 6-10: Calibration curve for methyl parathion.

A) Typical HPLC traces which were obtained with different concentrations of methyl parathion (retention time $22.2 \mathrm{~min}$ ) at $280 \mathrm{~nm}$ as discussed in the text. The peak with the retention time 23 min was always present and was in proportion to the methyl parathion. B) The calibration curve for methyl parathion. The data represent the mean of three independent experiments with error bars showing standard deviation. 

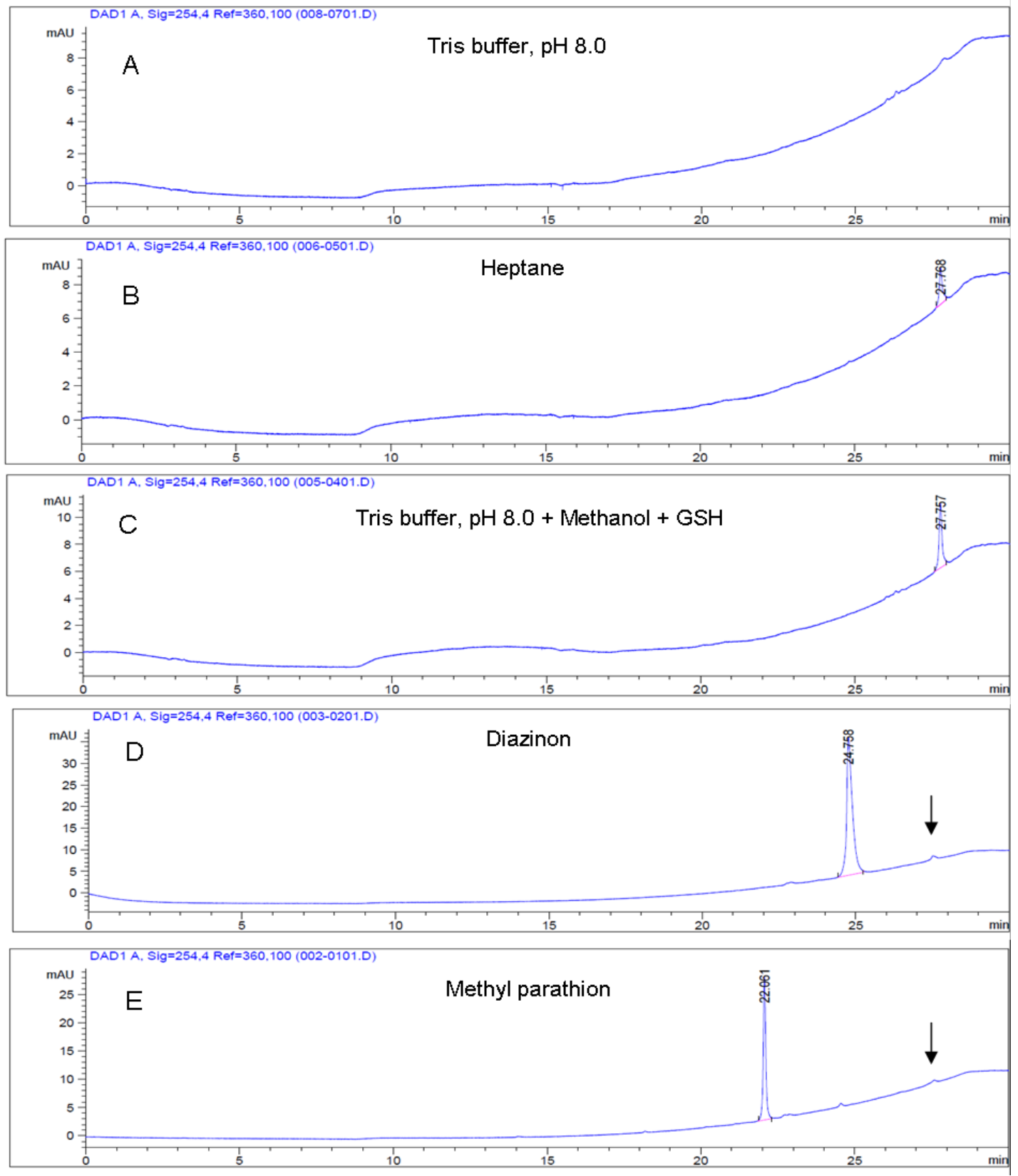

Figure 6-11: Typical HPLC chromatograms under the given gradient condition at $254 \mathrm{~nm}$.

A) Control chromatogram from the extraction of tris buffer, $\mathrm{pH} 8.0$ with heptane. B) Control chromatogram showing heptane contains a small impurity (retention time $27.8 \mathrm{~min}$ ).

C) Control chromatogram from the heptane extraction of glutathione and methanol dissolved in tris buffer, $\mathrm{pH}$ 8.0. D) Control chromatogram from the heptane extraction of diazinon (retention time $24.8 \mathrm{~min}$ ). E) Control chromatogram from the heptane extraction of methyl parathion (retention time $22 \mathrm{~min}$ ). The arrows show the peak of impurity obtained at 27.8 retention time. 

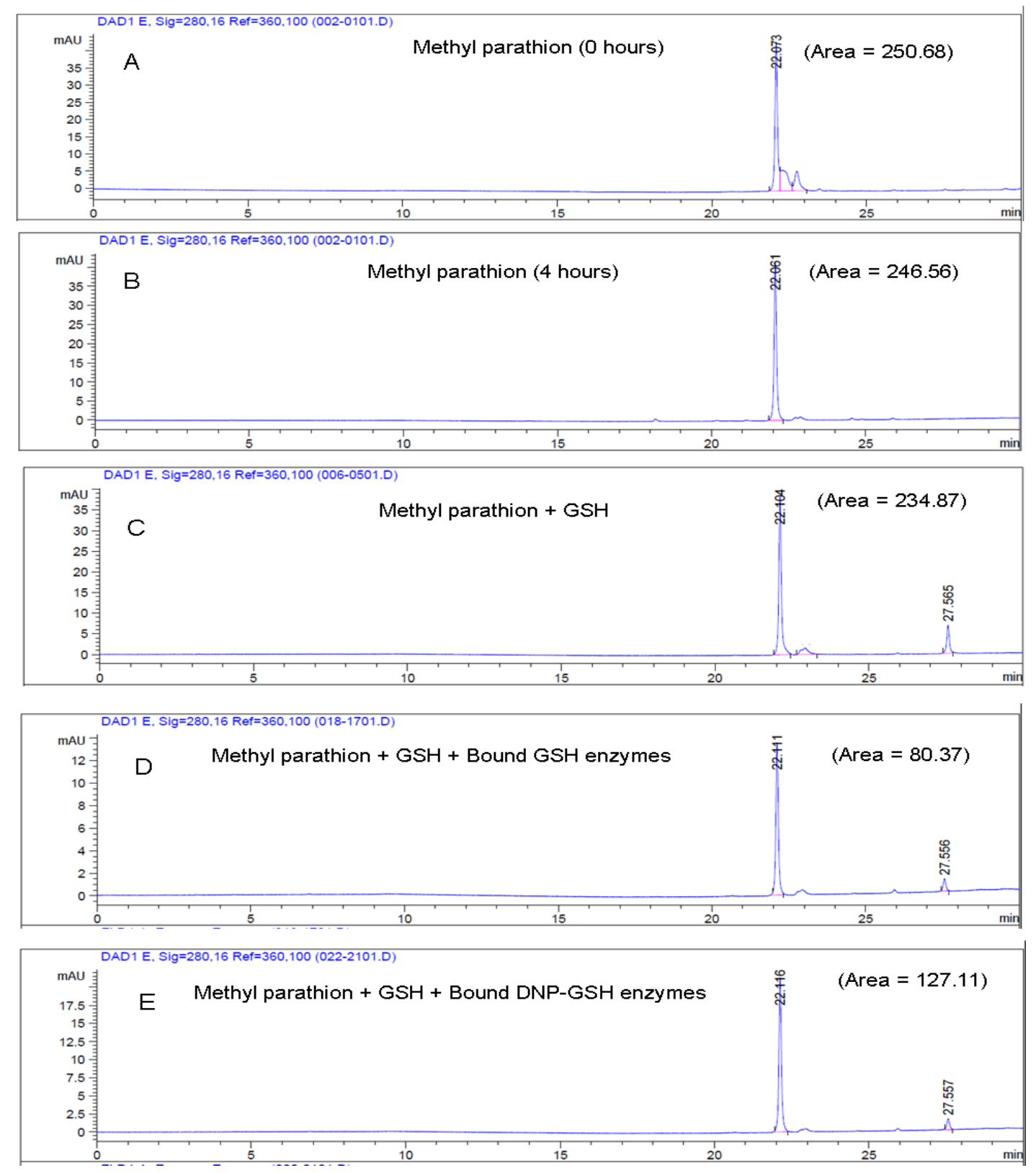

Figure 6-12: Typical HPLC chromatograms of methyl parathion with and without GSH and affinity-purified enzymes from L. cuprina (PY81 strain) at $280 \mathrm{~nm}$.

A) Chromatogram from the heptane extraction of methyl parathion at 0 hours. B) Chromatogram from the heptane extraction of methyl parathion after $4 \mathrm{~h}$ at $37^{\circ} \mathrm{C}$. C) Chromatogram from the heptane extraction of methyl parathion and glutathione after $4 \mathrm{~h}$ at $37^{\circ} \mathrm{C}$. D) Chromatogram from the heptane extraction of methyl parathion, glutathione and GSH affinity-purified extract from $L$. cuprina after $4 \mathrm{~h}$ at $37^{\circ} \mathrm{C}$. E) Chromatogram from the heptane extraction of methyl parathion, glutathione and DNP-GSH affinity-purified extract from L. cuprina after $4 \mathrm{~h}$ at $37^{\circ} \mathrm{C}$. 

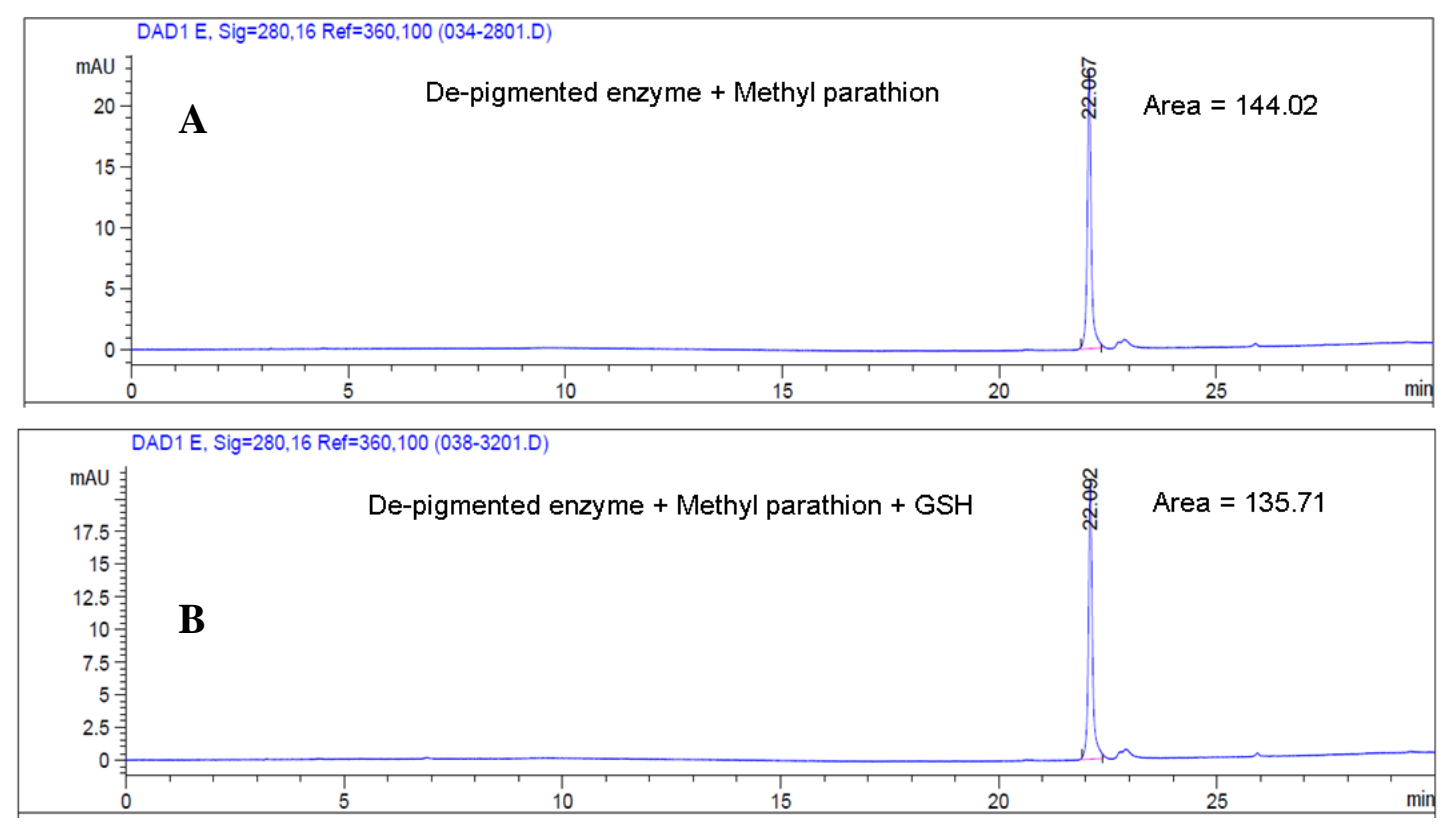

Figure 6-13: Typical HPLC chromatograms of conjugation of methyl parathion by depigmented enzyme from L. cuprina (PY81 strain) at $280 \mathrm{~nm}$ with and without GSH.

A) Chromatogram from the heptane extraction of methyl parathion and de-pigmented enzyme after $4 \mathrm{~h}$ at $37^{\circ} \mathrm{C}$. B) Chromatogram from the heptane extraction of methyl parathion and de-pigmented enzyme with GSH after $4 \mathrm{~h}$ at $37^{\circ} \mathrm{C}$.

To determine the GST activity in metabolism of methyl parathion and diazinon, the crude enzyme preparation from $4 \mathrm{~g}$ of $L$. cuprina flies in $20 \mathrm{ml}$ was prepared. The crude GSTs were then de-pigmented by HiTrap desalting column and purified using the GSH then DNP-GSH matrix as described in section 3.2.2. The purified extracts from both the matrices were concentrated and used to study insecticide metabolism. The insecticide $(5.5 \mathrm{nmole})$ was incubated in $1 \mathrm{ml}$ assay with enzyme (crude- $0.25 \mathrm{ml}$ and affinity-purified GSTs- $0.3 \mathrm{ml}$ ) at $37^{\circ} \mathrm{C}$ for $4 \mathrm{~h}$. Quantitative measurements for each insecticide were made and insecticide concentrations determined using respective standard calibration curve. For each 
insecticide, the remaining amount, as detected by HPLC, was subtracted from the initial amount of the insecticide added to the assay in order to determine the GST total and specific activities. For each assay, three controls were used: (1) Insecticide without enzyme and GSH, to check the spontaneous chemical degradation of the insecticide at $0 \mathrm{~h}$ and after $4 \mathrm{~h}$ incubation Figure 6-12, A, B); no chemical degradation was observed, the recovery being always $\geq 98 \%$. (2) Insecticide and GSH without enzyme (Figure 6-12, C) in order to determine the non-enzymatic reaction between GSH and the insecticide. This value was subtracted from the value of the initial assay mixture (1, above). (3) Insecticide and enzyme without GSH, for the case of insecticide binding to enzyme without being conjugated to GSH (Figure 6-13). The quantity of non-conjugated insecticide in this control was significantly lower than in the initial assay mixture suggesting binding of insecticides to enzyme. This control was not performed for the GSH and DNP-GSH affinity-purified enzymes. The total activity and specific activity of GSTs in conjugating methyl parathion and diazinon is calculated and shown in Table 6-2 and Table 6-3. 
Table 6-2: Total activities of affinity-purified GSTs and crude enzyme from L. cuprina (PY81 strain) towards insecticides determined by HPLC.

The experimental conditions are as stated in the text. The insecticide (5.5 nmole) was incubated in $1 \mathrm{ml}$ assay with enzyme (de-pigmented enzyme- $0.25 \mathrm{ml}$ and affinity-purified GSTs- $0.3 \mathrm{ml}$ ) at $37^{\circ} \mathrm{C}$ for $4 \mathrm{~h}$. The values were scaled to the total volume of de-pigmented enzyme (20ml) and total volume of concentrated affinity-purified enzyme $(5 \mathrm{ml})$. The data represent the mean of three independent experiments with triplicate measurements and standard deviation.

Total activity (nmole insecticide degraded / total volume of enzyme / h)

\begin{tabular}{lllll}
\hline Insecticide & $\begin{array}{l}\text { De-pigmented } \\
\text { enzyme }\end{array}$ & $\begin{array}{l}\text { De-pigmented } \\
\text { enzyme +GSH }\end{array}$ & Bound GSH & $\begin{array}{l}\text { Bound } \\
\text { DNP-GSH }\end{array}$ \\
\hline Methyl parathion & $24.2 \pm 5.7$ & $29.7 \pm 1.5$ & $11.1 \pm 4.5$ & $7.0 \pm 1.7$ \\
Diazinon & $65.3 \pm 3.3$ & $64.6 \pm 3.7$ & $2.9 \pm 1.6$ & $3.3 \pm 1.7$ \\
\hline
\end{tabular}

Table 6-3: Specific activities of affinity-purified GSTs and crude enzyme from L. cuprina (PY81 strain) towards insecticides determined by HPLC.

The experimental conditions are as stated in the text. The insecticide (5.5 nmole) was incubated in $1 \mathrm{ml}$ assay with enzyme at $37^{\circ} \mathrm{C}$ for $4 \mathrm{~h}$. The values represent the mean of three independent experiments with triplicate measurements and standard deviation.

Specific activity (nmole insecticide degraded / mg protein / h)

\begin{tabular}{lllll}
\hline Insecticide & $\begin{array}{l}\text { De-pigmented } \\
\text { enzyme }\end{array}$ & $\begin{array}{l}\text { De-pigmented } \\
\text { enzyme +GSH }\end{array}$ & Bound GSH & $\begin{array}{l}\text { Bound } \\
\text { DNP-GSH }\end{array}$ \\
\hline Methyl parathion & $0.19 \pm 0.04$ & $0.23 \pm 0.01$ & $4.96 \pm 1.9$ & $2.50 \pm 0.62$ \\
Diazinon & $0.51 \pm 0.02$ & $0.51 \pm 0.03$ & $1.31 \pm 0.7$ & $1.18 \pm 0.63$ \\
\hline
\end{tabular}


The preliminary data indicate that the organophosphates methyl parathion and diazinon were conjugated in vitro by GSH and DNP-GSH affinity-purified GSTs from L. cuprina. The degradation of methyl parathion was 1.2- fold higher by the crude enzyme in the presence of GSH compared to without GSH whereas diazinon was degraded almost equally by crude enzyme in presence or absence of GSH. The activity of crude enzyme in absence of GSH in the reaction mixture implies a role of metabolic enzymes (such as esterases) other than GSTs. This suggests that diazinon is much more degraded by these enzymes compared to methyl parathion. The specific activity of crude cytosolic GSTs was almost 2-fold higher in the catabolism of diazinon compared to methyl parathion. However, incubation with the affinitypurified GSTs revealed interesting findings. Methyl parathion was conjugated by GSH affinity-purified fractions 2 times more than by DNP-GSH affinity-purified fractions whereas with diazinon, activities with both preparations were the same. The specific activity of both the affinity-purified enzymes was higher with methyl parathion compared to diazinon (Table 6-2 and Table 6-3). Thus methyl parathion served as the best substrate to study the GST-based insecticide metabolism.

Further to explore the ability of GSTs in metabolising methyl parathion, different concentrations of purified enzyme and GSH were incubated with methyl parathion. The result is shown in Figure 6-14, which shows the amount of methyl parathion metabolised after incubation with different concentrations of affinitypurified enzymes in the presence of GSH (Figure 6-14). With higher amounts of enzyme, the conjugation of methyl parathion was higher, so the residual amount of chemical is lower and the metabolised amount of chemical is higher. 


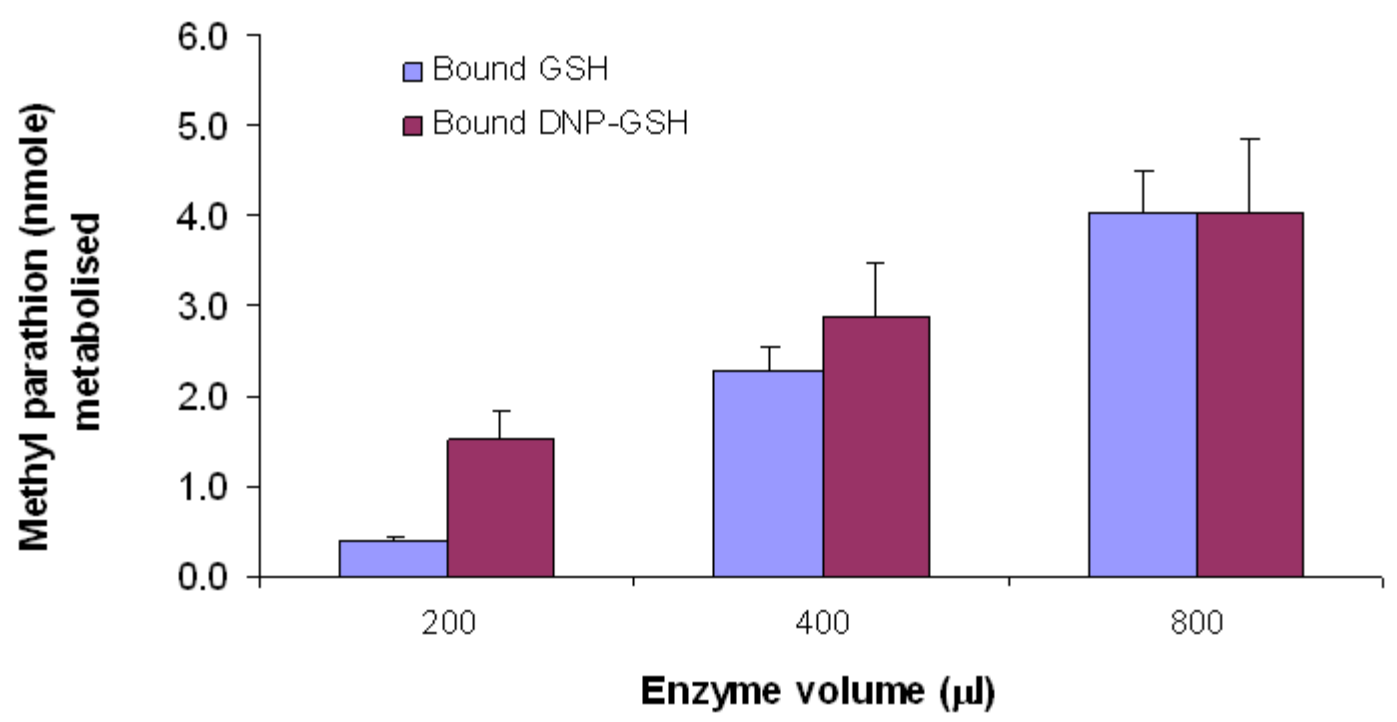

Figure 6-14: Metabolism of methyl parathion by affinity-purified GSTs.

The different concentration of GSH and DNP-GSH affinity GSTs (200, 400 and $800 \mu \mathrm{l})$ were incubated with methyl parathion and unchanged methyl parathion was measured using HPLC to calculate the methyl parathion metabolised. $100 \mu 1$ of bound GSH enzyme corresponds to $24 \mu \mathrm{g}$ and $100 \mu \mathrm{l}$ of bound DNP-GSH enzyme corresponds to $34 \mu \mathrm{g}$.

The data showed that the GSH affinity-purified fractions have a higher capability in metabolising the methyl parathion than the DNP-GSH affinity-purified enzymes. As the GSH affinity fractions contain a mixture of Sigma and Delta GSTs and they can be separated easily by one of the methods described previously in section 4.3.4, the attempt was made to determine which isoform is responsible for higher metabolising activity. To perform this experiment, Sigma and Delta GSTs were separated by ion exchange chromatography and $50 \mu \mathrm{g}$ of each purified enzyme was incubated with an equal amount of insecticide as per section 3.2.8. The result is shown in Figure 6-15. The Delta GSTs and bound DNP-GSH GSTs contribute significantly $(\mathrm{p}<0.05)$ in metabolising methyl parathion, almost $10-12$-fold higher than the Sigma GST. 


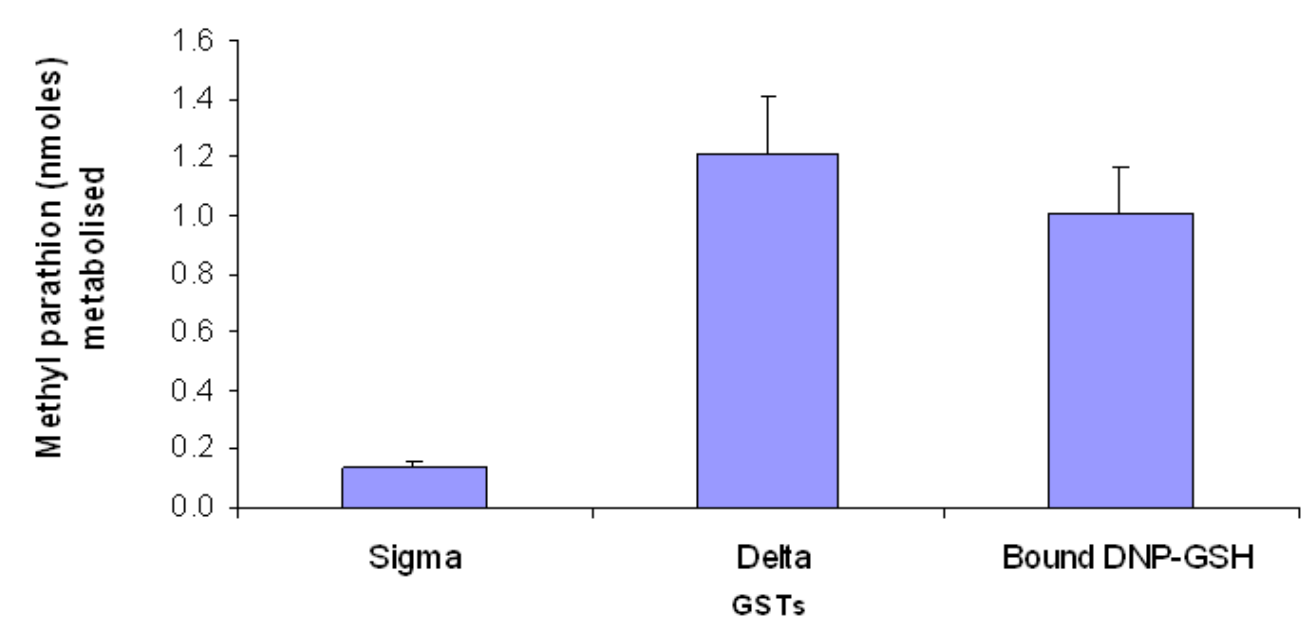

Figure 6-15: Metabolism of methyl parathion by GST isoforms and partially purified DNPGSH eluents.

The Sigma and Delta GSTs were separated by ion exchange chromatography as described in section 4.3.4. Fifty $\mu \mathrm{g}$ of each isoform and DNP-GSH affinity eluent was incubated with 5.5 nmoles of methyl parathion as described in the text. The unchanged methyl parathion was measured using HPLC to calculate the methyl parathion metabolised. Results were corrected for non-catalytic degradation of methyl parathion.

Considerable differences were observed in GST activity between susceptible and resistant strains of $L$. cuprina with the model substrates CDNB, DCNB, NPA, DHA and TNE as described previously in this chapter. Therefore the difference in GST activity in conjugating insecticides among the susceptible and resistant strains was also explored. The activity of GSH affinity-purified and DNP-GSH affinity purified GSTs in metabolism of methyl parathion and diazinon is shown in Figure 6-16. There was no significant difference in GST activity noted amongst all three strains. To determine the insecticide metabolising activity not accounted for by the affinity-purified GSTs, in a final experiment, GSH dependent degradation of the insecticides was determined in fractions not bound to either matrix (Table 6-4). However, there was no significant difference found between the strains. 

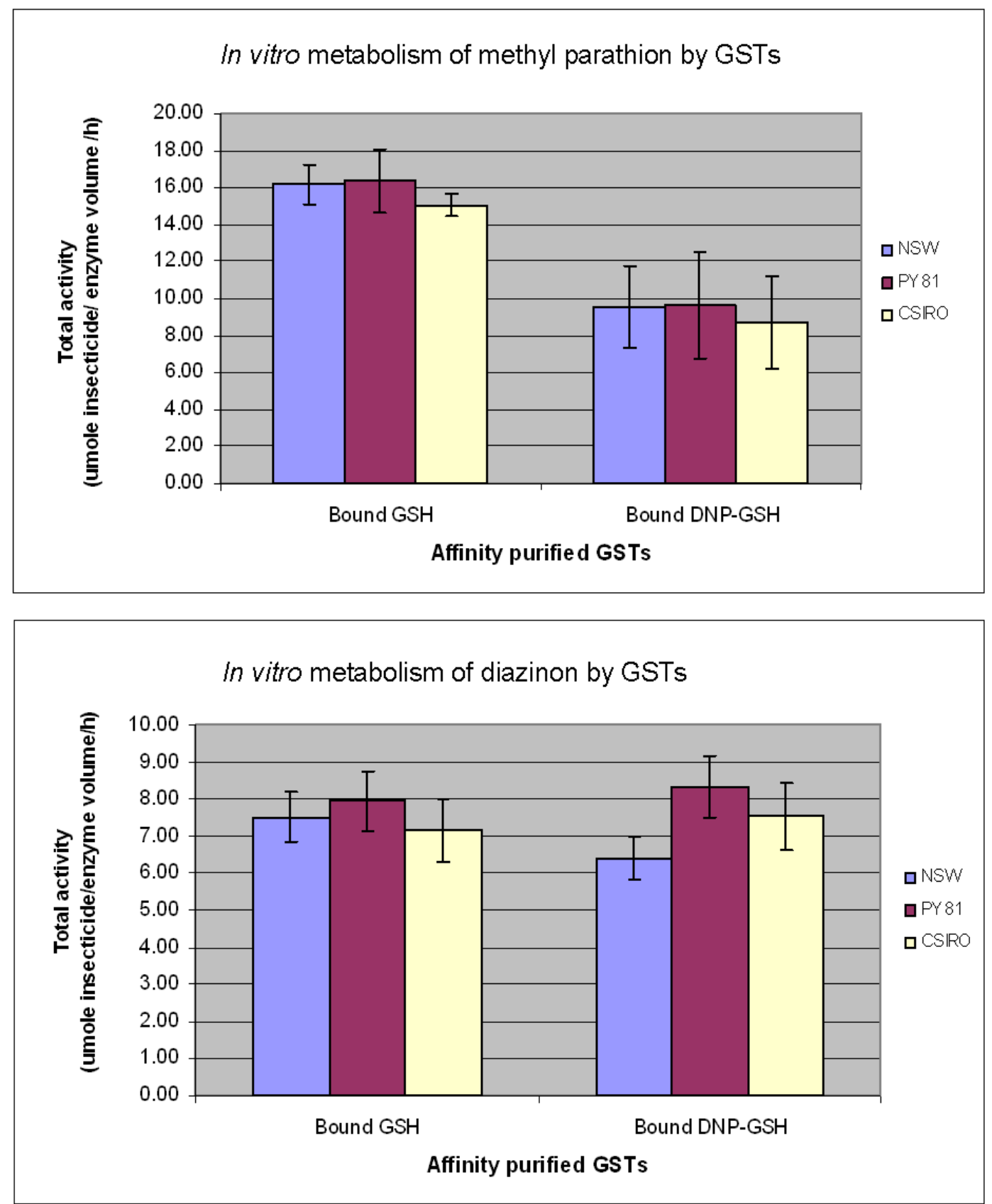

Figure 6-16: GST activity in metabolising OPs from different strains of $L$. cuprina.

The partially purified GSTs were concentrated and an equal volume of enzyme from equal mass of insects $(1.5 \mathrm{~g})$ was incubated with the insecticide. The unchanged methyl parathion was measured using HPLC to calculate the methyl parathion metabolised. Results were corrected for non-catalytic degradation of methyl parathion. Activity from the resistant strain is not significantly higher than susceptible strains. 
Table 6-4: Metabolism of methyl parathion and diazinon by NSW, PY81 and CSIRO strains of L. cuprina.

The values were calculated as per total volume of crude enzyme $(6 \mathrm{ml})$ and total volume of affinity unbound enzyme $(8 \mathrm{ml})$ from $1 \mathrm{~g}$ of flies. The data represent the mean of two measurements in one experiment and standard deviation. Method details as per Table 6-2.

\begin{tabular}{|c|c|c|c|c|}
\hline L. cuprina strain & crude enzyme & $\begin{array}{l}\text { crude enzyme } \\
\text { +GSH }\end{array}$ & $\begin{array}{l}\text { Unbound of } \\
\text { GSH+ DNP- } \\
\text { GSH matrices } \\
\text { with no GSH in } \\
\text { assay }\end{array}$ & $\begin{array}{l}\text { Unbound of } \\
\text { GSH+ DNP- } \\
\text { GSH matrices } \\
\text { with GSH in } \\
\text { assay }\end{array}$ \\
\hline
\end{tabular}

Methyl parathion metabolism

Total activity (nmole of methyl parathion degraded / total volume of enzy me / h)

$\begin{array}{lllll}\text { NSW } & 17.4 \pm 4.4 & 19.7 \pm 1.3 & 11.0 \pm 7.8 & 11.2 \pm 6.6 \\ \text { PY81 } & 18.0 \pm 6.6 & 14.0 \pm 1.8 & 6.60 \pm 10 & 10.8 \pm 6.1 \\ \text { CSIRO } & 15.0 \pm 4.5 & 10.0 \pm 1.8 & 4.60 \pm 8.9 & 5.80 \pm 10\end{array}$

Specific activity (nmole of methyl parathion degraded / total mg of protein / h)

$\begin{array}{lllll}\text { NSW } & 0.50 \pm 0.13 & 0.57 \pm 0.04 & 0.24 \pm 0.1 & 0.25 \pm 0.2 \\ \text { PY81 } & 0.53 \pm 0.2 & 0.41 \pm 0.05 & 0.15 \pm 0.2 & 0.24 \pm 0.1 \\ \text { CSIRO } & 0.40 \pm 012 & 0.25 \pm 0.3 & 0.10 \pm 0.1 & 0.12 \pm 0.3\end{array}$

Diazinon metabolism

Total activity (nmole of diazinon degraded / total volume of enzyme / h)

$\begin{array}{lllll}\text { NSW } & 13.0 \pm 1.6 & 17.4 \pm 1.2 & 16.0 \pm 1.2 & 12.5 \pm 0.7 \\ \text { PY81 } & 17.0 \pm 0.6 & 16.8 \pm 2.1 & 8.00 \pm 4.1 & 7.10 \pm 3.3 \\ \text { CSIRO } & 11.0 \pm 4.2 & 15.7 \pm 1.2 & 12.7 \pm 6.3 & 8.40 \pm 3.2\end{array}$

Specific activity (nmole of diazinon degraded / total mg of protein / h)

$\begin{array}{lllll}\text { NSW } & 0.37 \pm 0.05 & 0.50 \pm 0.03 & 0.35 \pm 0.03 & 0.27 \pm 0.01 \\ \text { PY81 } & 0.50 \pm 0.02 & 0.50 \pm 0.06 & 0.18 \pm 0.1 & 0.16 \pm 0.08 \\ \text { CSIRO } & 0.37 \pm 0.11 & 0.42 \pm 0.03 & 0.26 \pm 0.1 & 0.17 \pm 0.07\end{array}$




\subsection{Discussion}

The aim of the present investigation was to understand and evaluate the significance of $L$. cuprina GSTs in the defence against organophosphorus insecticides. To assess that, GSTs from resistant (PY81) and susceptible strains (NSW and CSIRO) of L. cuprina were partially purified by sequential GSH and DNP-GSH affinity chromatography. The purification procedures for GST isoenzymes from the three strains were the same. However, it was quite striking that the yield of the DNP-GSH affinity-purified proteins from the resistant strain was 2fold higher than that in the susceptible strains. (Vontas et al., 2002) have also reported an increase in the amount of the GST proteins in a resistant strain of rice brown planthopper, Nilaparvata lugens and while studying GST-based pyrethroid resistance and thus the majority of elevated GST activity is associated with the quantitative change in the amount of enzyme produced. The DNP-GSH affinitypurified fractions from the resistant strain, had significantly $(\mathrm{p}<0.05)$ higher total activity with $\mathrm{CDNB}$ and especially with TNE, an electrophilic product of lipid peroxidation. The higher activity towards TNE indicates an important role in protection from the products of oxidative damage (Esterbauer et al., 1991). As discussed previously in this chapter, evidence has been published to suggest that high levels of GST activity towards DCNB and CDNB are associated with OP resistance in resistant insects (Cochrane et al., 1992; Motoyama and Dauterman, 1977; Wilson and Clark, 1996; Yu, 1992). In the present investigation, both the crude cytosolic GSTs and the GSH affinity-purified GSTs of the PY81 strain showed elevated GST activity towards DCNB, suggesting a possible significance in resistance. 
The results from 2D gel electrophoresis also suggested significant quantitative differences in proteins including GSTs among the susceptible and resistant flies obtained from similar purification procedures. These proteins (zones $\mathrm{C}$ and $\mathrm{D}$ in Figure 6-8) have been tentatively classified to belong to Epsilon and low M.W. Delta class of GSTs and Mu-like GSTs along with Rab proteins. In many of the studies in the literature, effort has been concentrated on analysis of resistance mechanisms in one or two highly resistant strains of insect. In such cases, gene amplification often plays an important role (Oppenoorth, 1984). Oppenoorth found a massive production of GSTs while studying a series of 12 housefly strains in which activity towards DCNB varied over a 30-fold range correlating with resistance and the GSTs apparently accounting for up to $6 \%$ of total soluble protein in the most resistant strain. This led him to correlate it with the established examples of gene amplification. The situation in the present work might be similar but no analysis was done at the genetic level and also the DCNB activity of the resistant strain (PY81) was a modest 2-fold higher compared to susceptible strains which is a quite different result from that obtained in the study of housefly by Oppenoorth. In the present study, though there was no significant quantitative difference in GSH affinitypurified fractions between the strains, it is possible that they might also contribute to resistance based on the higher specific activity with DCNB. Attempts were made to find out if there is any difference in metabolising the insecticides by susceptible and resistant strains in vitro as there are many reports available suggesting GSTs can produce resistance to a range of insecticides by conjugating reduced glutathione to the insecticide or its primary toxic metabolic products (Kostaropoulos et al., 2001a; Kostaropoulos et al., 2001b). So in this study the interaction of GSTs with the organophosphate insecticides methyl parathion and diazinon was tested by means of 
HPLC. The technique showed that both the affinity-purified enzymes from all these strains were able to conjugate with methyl parathion and diazinon. The de-pigmented preparation of enzyme catalysed the reaction with both the insecticides whereas when the ability of the de-pigmented enzyme to conjugate with the insecticides was tested in presence of GSH, the specific activity was 1.2-fold higher with methyl parathion whereas it was not much different with diazinon. Activity in degrading diazinon was apparently higher in the crude preparation without the presence of GSH which suggests a significant role of esterases and other metabolic enzymes. The GSH affinity-purified GSTs which include Sigma and Delta GSTs were more active in conjugation with methyl parathion and diazinon compared to DNP-GSH affinitypurified fractions.

Although DNP-GSH affinity-purified GSTs were expressed at twice the level in the resistant strain, there was no significant difference in conjugation with insecticides in vitro between the susceptible and resistant strains (Figure 6-16). There was no significant difference found in metabolism of methyl parathion and diazinon by crude and unbound enzymes from GSH and DNP-GSH affinity matrices of susceptible and resistant strains (Table 6-4). In a single experiment, unbound fractions of PY81 strain showed the higher total and specific activity in metabolising methyl parathion than the other strains but due to limited data, it was not confirmed. However, the GSH and DNP-GSH affinity-purified enzymes from PY81 showed no significant increase in metabolism of methyl parathion or diazinon in replicated experiments. Thus there may be a possibility that the GSTs active in metabolising insecticides are not binding to our affinity matrices or in L. cuprina the resistance is not due to GSTs but to other mechanisms. While studying the larvae and adults from field isolates of L. cuprina and Lucilia sericata, Wilson et al. (1996) observed the 
absence of the correlation between levels of GST conjugating activity with respect to the model substrate DCNB and resistance to diazinon in L. sericata. However, a strong relationship was found between the GST conjugating activity towards DCNB and resistance to diazinon in both adult and larval strains of L. cuprina. From the data of Wilson et al., which are very scattered, it is quite likely that an individual strain of blowfly may have high resistance but relatively low GST activity and vice versa (Wilson, 1999; Wilson and Clark, 1996).

There is a possibility that the substantially elevated expression of GSTs in PY81 strain (zones C and D) such as Epsilon, Delta and Mu-like GSTs are a part of a platform of stress responses but are not involved in detoxification of these specific insecticides. However, in the present study the situation is complex as there are differences of expressed GSTs even between the susceptible strains. The Mu-like GSTs in zone D were absent in CSIRO but present in NSW. The Epsilon GSTs expressed in PY81 strain has a different pI than the Epsilon of the NSW and CSIRO strain. The Zone D GSTs though expressed in greater quantity in PY81 strain did not show any significantly higher specificity with model substrates and insecticides.

In the present study, the insects were not exposed to insecticides. The possibility that resistance is due to increased ability to induce detoxication enzymes by the insecticides cannot be excluded. However, literature suggests that when insects are exposed to insecticides in laboratory, the constitutive over-expression of metabolic genes play a much greater role in insect survival rather than the induction of members of these gene families (Willoughby et al., 2006). 
When separated classes of GSTs were tested for their ability to conjugate with insecticides, the current study strongly suggests that Delta GST and the enzymes present in DNP-GSH affinity fractions (mainly Delta and Epsilon class) are more strongly involved in conjugation with insecticides than Sigma GST, which has almost no activity.

In summary, the work presented in this chapter attempted to study the involvement of L. cuprina GSTs in insecticide resistance. The comparison of GST proteomes of the susceptible and resistant strains showed interesting findings with variable expression of Epsilon and Low M.W. Delta GSTs and Mu-like GSTs. It was observed that the L. cuprina GSTs can conjugate insecticides in vitro. Delta GST and DNP-GSH affinity-purified GSTs can metabolise insecticides at a much greater rate than Sigma GST. However, there was no significant difference found between the susceptible and resistant strains of $L$. cuprina in metabolising the insecticides. It is proposed that resistance in this particular strain of $L$. cuprina may be due to other mechanisms.

During the proteomic analysis a number of distinct Delta GST spots with different pIs on 2D gels from both D. melanogaster and L. cuprina was found. Out of curiosity, it was studied whether this pattern of separation of Delta GST on 2D gel was due to post-translational modification and if so, what type of modification it could be? Chapter 7 attempts to study the nature of post-translational modifications of D. melanogaster Delta GSTs due to availability of its genetic database. 


\section{The nature of post-translational modification of Delta GST}

\subsection{Introduction}

The aim of this chapter was to determine the type of post-translational modification involved in producing the multiple forms of D1-type GSTs in $D$. melanogaster.

The overwhelming majority of proteins are modified after synthesis, a process known as post-translational modification and more than 200 modifications of amino acid residues have been reported to occur in vivo (Krishna and Wold, 1997). Post-translational modifications (PTMs) are often covalent modifications that regulate protein functions, determining their activity state, cellular location and interactions with other proteins (Boatright and Salvesen, 2003; Chiu et al., 2003; Khidekel and Hsieh-Wilson, 2004; Manning et al., 2002). Hence, knowledge of the nature of such modifications seen in the present study will facilitate our understanding of the cellular processes in which the GSTs may be regulated.

The literature suggests that GSTs may be subject to phosphorylation (Nicholson et al., 1993; Taniguchi and Pyerin, 1989), methylation (Johnson et al., 1992), glycosylation (Kuzmich et al., 1991) and auto-oxidation (Hayes and Clarkson, 1982). The human pi GST may be glycosylated (Kuzmich et al., 1991) and human leukotriene C-4 synthase, a member of the GST family, contains a potential glycosylation site (Nicholson et al., 1993). Much of the data suggesting that these enzymes may be post-translationally modified have been obtained from in vitro experiments and therefore the in vivo biological significance of such modifications is unclear. 
A large number of these PTMs may be observed on 2D electrophoresis gels as distinct protein isoform spots. The PTMs such as phosphorylation, acetylation, deamidation, alkylation and cysteine oxidation cause changes in the mass and pI of the protein by adding, removing or changing titratable groups and 2D gel electrophoresis is able to resolve many PTM-induced isoforms. As depicted in Figure 4-17 and Figure 4-18 in section 4.3.3, Delta GSTs (D1) from both D. melanogaster and L. cuprina exhibited multiple forms of varying pI when observed on a 2D gel, presumably as a result of PTMs. Since no M.W. changes were evident observing the 2D gel, one can easily suggest that the protein has become modified. However, it is not easy to detect which type of modification is most likely to have occurred.

The analysis of PTMs has encountered some biological and analytical limitations as they are often transient and occur in vivo (Salzano and Crescenzi, 2005). In spite of these difficulties, this chapter attempts to determine the type of modifications found in D. melanogaster D1 GST.

\subsection{Objective:}

- To determine the type of PTM generating the different pI variants of DmGSTD1 using MALDI-TOF and tandem mass spectrometric sequencing.

\subsection{Results:}

\subsubsection{Phosphorylation as a possible PTM of Delta GSTs}

The most frequently occurring protein PTM appears to be phosphorylation. To test whether this was likely in the present study, initially the GSTD1 sequence in FASTA format was submitted to NetPhos 2.0 server. This is a post-translational modification prediction tool available at Center for Biological Sequence analysis 
(CBS) (http://www.cbs.dtu.dk/services/NetPhos/). It produces neural network predictions for serine, threonine and tyrosine phosphorylation sites in eukaryotic proteins. The prediction result showed five possible sites at serine, three at threonine and four at tyrosine which can be phosphorylated (Figure 7-1). This prediction suggested that it was well worth pursuing further the possibility of phosphorylation.

To explore this, the GSH affinity-purified fractions from D. melanogaster were separated on 1D and 2D gels (Figure 7-2). These gels were stained with the ProQ Diamond phosphoprotein gel stain (as per section 3.2.9) which is reported to provide a high sensitivity approach for the selective detection of phosphoproteins separated by polyacrylamide gel electrophoresis (Steinberg et al., 2003). SYPRO Ruby gel stain was used as a post stain to calculate the fluorescence intensity ratio (Diamond/SYPRO Ruby) to determine the presence and relative abundance of phosphorylated protein. The ratio is higher for phosphoproteins (e.g., 8.0) compared to non-phosphorylated proteins (e.g., 0.05 ) according to the manual of Pro-Q Diamond phosphoprotein gel stain. Ovalbumin and BSA were used as a positive and negative control on the gel respectively. The peppermint-stick phosphate marker containing negative ( $\beta$-galactosidase and BSA) and positive (Ovalbumin and $\beta$-casein) controls was run alongside the sample. 
$233 \mathrm{sP} \mathrm{P20432} \mathrm{G}$

LAMELANXGASTERGN̄GSTDPESVMVFYYLPGSSPCRSVIMTAKAVGVELNKKLLNLQAGEHLKPEFLKINPQHTIPTL VDNGFALWESRAIQVYLVEKYGKTDSLYPKCPKKRAVINQRLYFDMGTLYQSFANYYYPQVFAKAPADPEAFKKIEAAFE FLNT FLEGQDYAAGDSLTVADIALVATVST FEVAKFE ISKYANVNRWYENAKKVT PGWEENWAGCLE FKKYFE

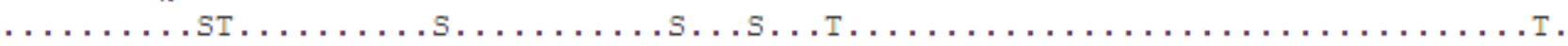

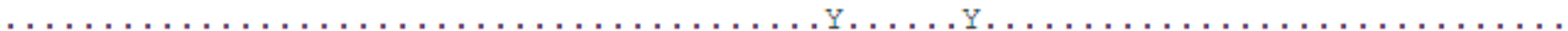

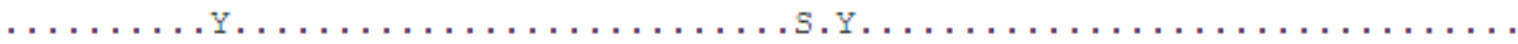

………

Ser: 5 Thr: 3 Tyr: 4

B

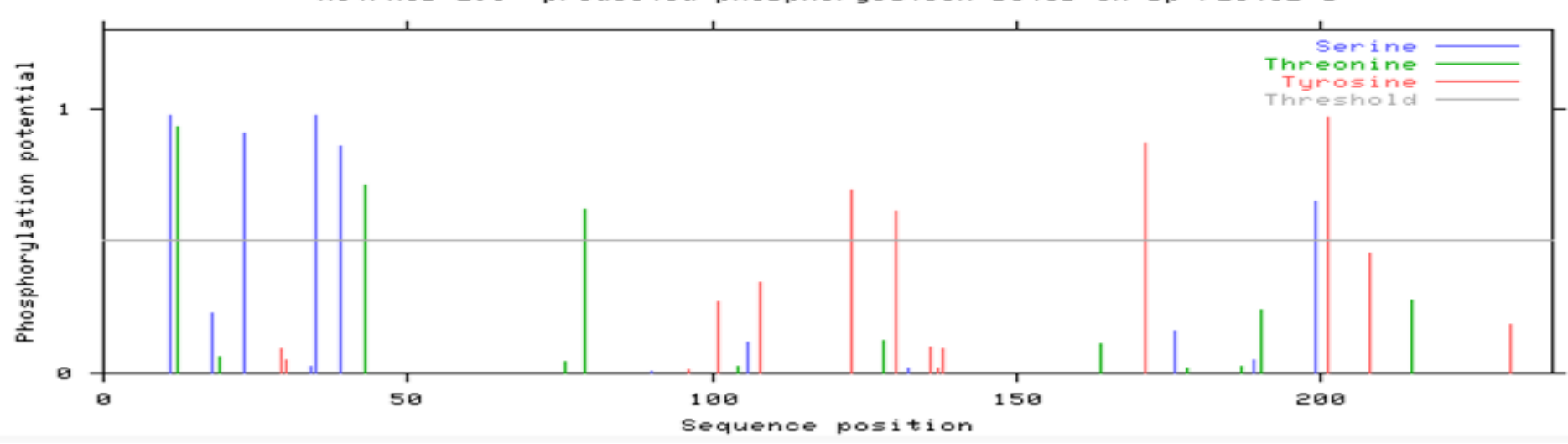

Figure 7-1: NetPhos 2.0 Server prediction results.

The DmGSTD1 sequence was submitted to NetPhos 2.0 Server and possible phosphorylation sites on the sequence were predicted.

A) The DmGSTD1 sequence and most probable phosphorylation sites identified. B) Graphically demonstrates the proposed phosphorylation sites and the predicted likelihood of phosphorylation. 
A

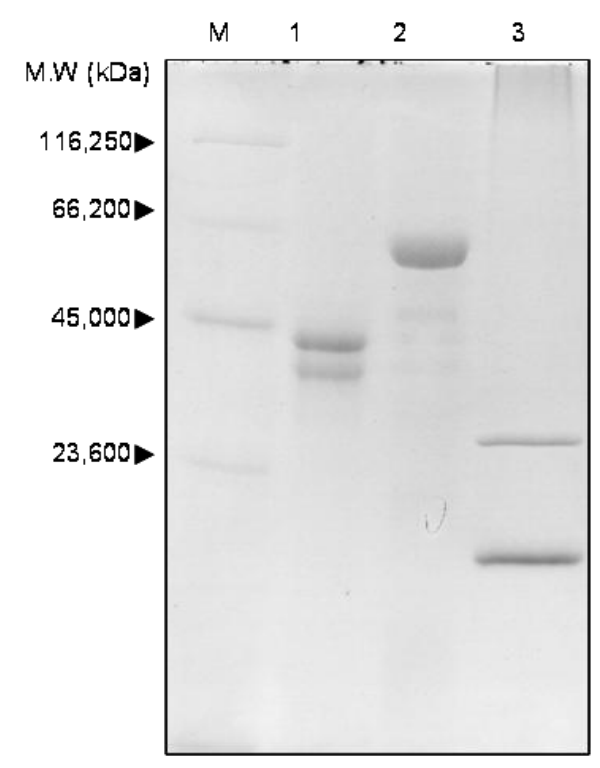

Pro-Q Diamond stain

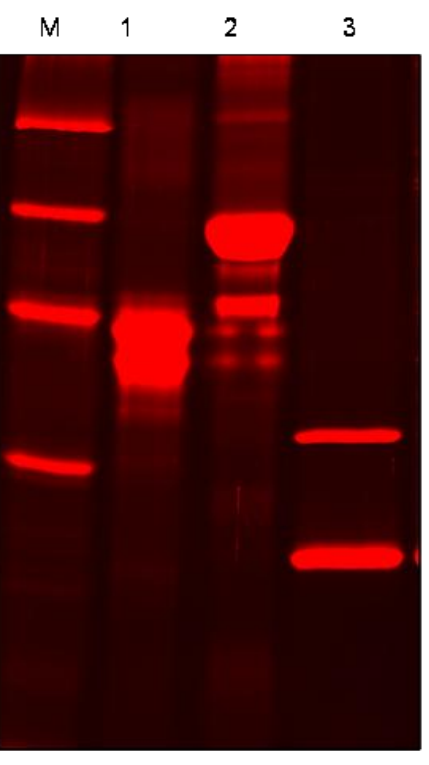

SYPRO Ruby stain
B

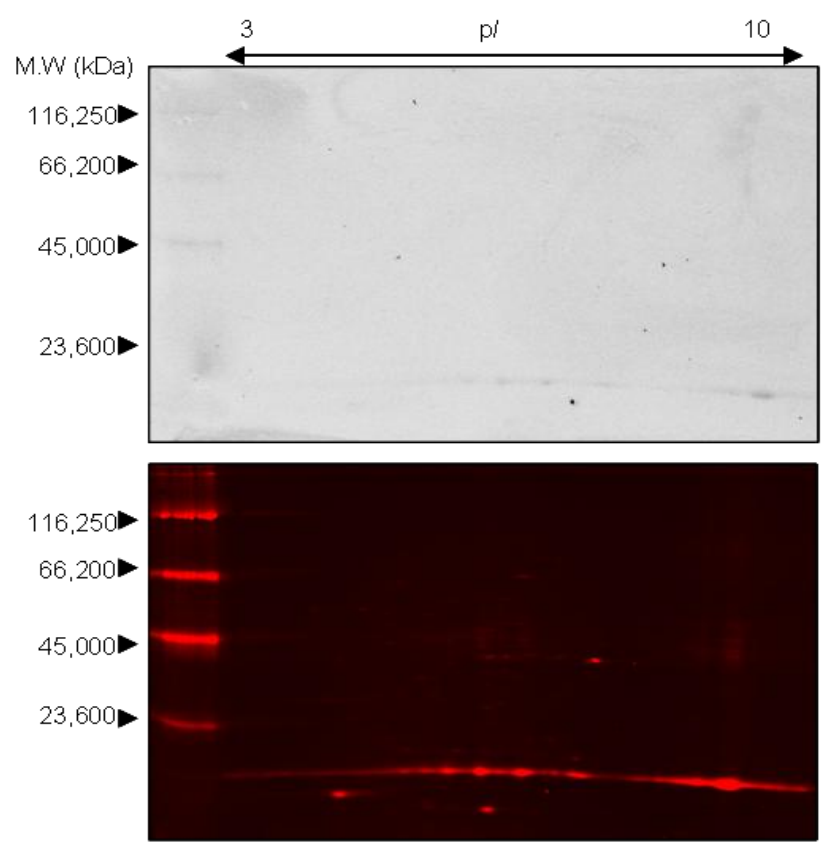

Figure 7-2: Pro-Q Diamond and SYPRO Ruby gel staining.

A) SDS PAGE on GSH affinity-purified fractions of D. melanogaster. Lane M-Peppermint-stick phosphate marker, lane 1-Ovalbumin (positive control), lane 2-BSA (negative control) and lane 3- GSH affinity-purified GSTs. B) 2D gel electrophoresis of Delta GSTs from D. melanogaster. The first dimension separation on a $7 \mathrm{~cm} \mathrm{pH} \mathrm{3-10} \mathrm{linear} \mathrm{IPG} \mathrm{strip} \mathrm{and} \mathrm{then} \mathrm{in} \mathrm{the} \mathrm{second} \mathrm{dimension} \mathrm{on} \mathrm{a} \mathrm{SDS-PAGE} \mathrm{gel.} \mathrm{Both} \mathrm{1D} \mathrm{and} \mathrm{2D} \mathrm{gels} \mathrm{were} \mathrm{stained} \mathrm{by} \mathrm{Pro-Q} \mathrm{Diamond}$ stain and post-stained by SYPRO Ruby dye. The fluorescence intensity of SYPRO stain gel was 100 times higher than the intensity of Pro-Q Diamond stained gel. 
A

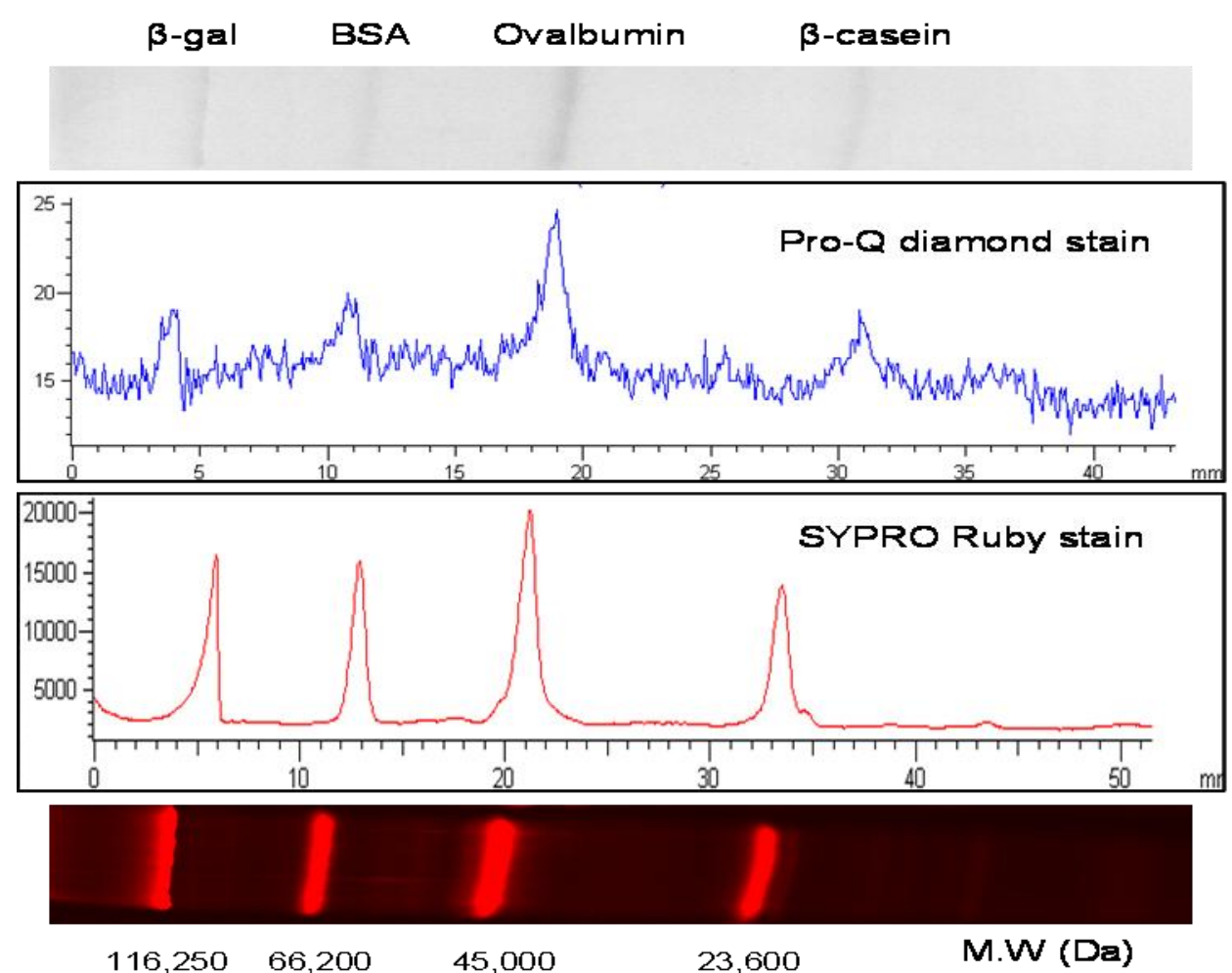

B

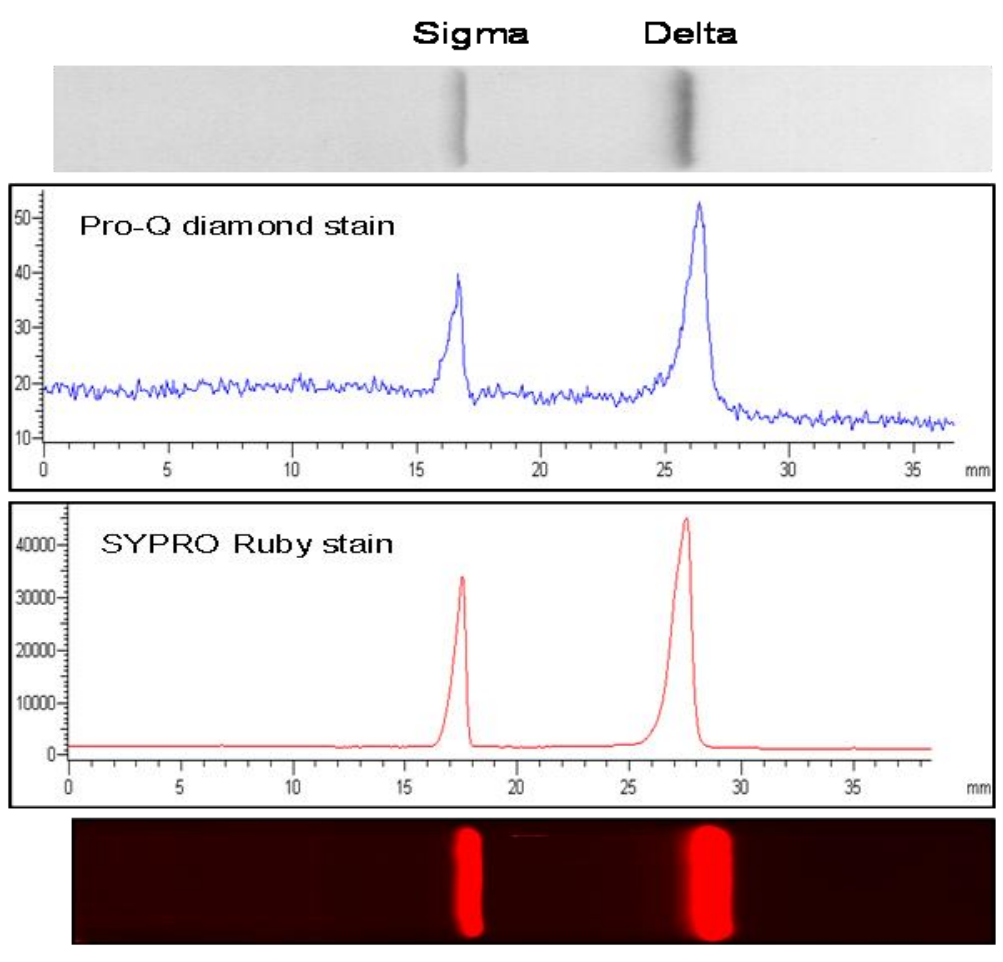

Figure 7-3: Analysis of a SDS-PAGE gel using ImageQuant version 5.2 software.

A) Fluorescence intensity of the peppermint-stick phosphate marker with negative ( $\beta$-galactosidase and BSA) and positive (Ovalbumin and $\beta$-casein) controls. B) Sigma and Delta GSTs separated on SDS PAGE. 
The results indicate that the negative control BSA (Figure 7-2, lane 2) was also stained by Pro-Q Diamond stain which was not expected. Even negative controls in the peppermint-stick phosphate marker were stained by the Pro-Q Diamond stain. The gels shown in Figure 7-2 were optimised by setting the pixel display range value in the ImageQuant software. The pixel value for Pro-Q Diamond stained gels was 100 whereas it was 10000 for the SYPRO ruby stained gels. This was done because at 100 pixels, the SYPRO Ruby stained gel was too intense to measure any intensity and at 10000 pixels no bands on the Pro-Q Diamond gel were visible. The fluorescence intensity chromatograms at the different pixel ranges are displayed in Figure 7-3. Therefore to calculate the fluorescence intensity ratio, the value of SYPRO Ruby stained gel was normalised by dividing by 100 to get the equal intensity measures for both the gels. The Diamond/Sypro ratio for the positive control ovalbumin and negative control BSA (lanes 1 and 2 in Figure 7-2) was 0.12 and 0.13 respectively. Even the ratios for the positive (ovalbumin and $\beta$-casein) and negative ( $\beta$-galactosidase and BSA) proteins in the peppermint-stick marker were 0.124 and 0.143 for positive and 0.088 and 0.124 for negative controls respectively. Since the stains were not differentiating the controls, it could not be determined whether Sigma and Delta GSTs were phosphorylated.

Due to the ambiguous result with these stains, another approach was considered. This was to compare the MALDI-spectra of each Delta spot as some studies have demonstrated mass differences corresponding to changes in the presence of a phosphate group, from the peptides detected by a difference of 80 or $98 \mathrm{Da}$ in the mass (Annan and Carr, 1996; Kinumi et al., 2000; Neubauer and Mann, 1999). 
For this analysis, the D. melanogaster Sigma and Delta GST were separated from the partially purified mixture obtained from the GSH affinity chromatography and both the isoforms were separated using ion exchange chromatography as described in section 4.3.4.2. The Delta GST fractions were pooled, concentrated and subjected to 2D electrophoresis. The 2D gel is shown in Figure 7-4.

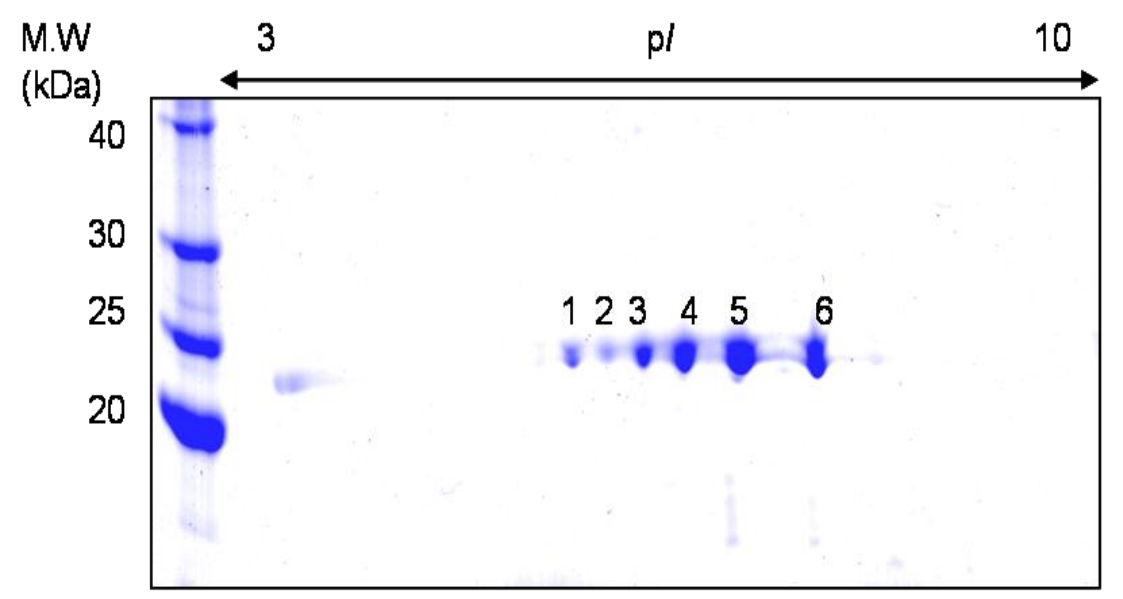

Figure 7-4: D. melanogaster Delta GST separation by 2D gel electrophoresis.

The Delta GST fractions separated by ion exchange chromatography were separated by 2D electrophoresis in the first dimension on a $7 \mathrm{~cm}$ pH 3-10 linear IPG strip and then in the second dimension on a SDS-PAGE gel. The gel was stained by Coomassie Brilliant Blue G-250.

The spots 3, 5 and 6 were excised from the gel, destained and digested with trypsin as per section 3.2.7.4. The peptide fragments were dried and reconstituted in the CHCA matrix to prepare for the MALDI-TOF analysis as per method section 3.2.7.6. The spectra were obtained with the parameters setting as described in section 3.2.7.7. The MALDI-TOF spectra for all three spots are shown in Appendix Figure 10-8. All these spots were identified as D. melanogaster D1 GST. The MALDI-TOF spectra of spot numbers 3, 5 and 6 were compared with each other manually. No mass difference corresponding to $\mathrm{H}_{3} \mathrm{PO}_{4}$ or $\mathrm{HPO}_{3}$ was detected between all Delta GST MALDI-spectra. 
To avoid the possibility of not getting the phosphopeptides as intense peaks, especially in the presence of other nonphosphorylated peptides owing to ionic suppression, a phosphopeptide enrichment strategy (ZipTip MC $_{\text {tips) }}$ was also employed as per section 3.2.10. MALDI spectra with and without enrichment were compared manually (Figure 7-5). The list of the masses of the peptides obtained in the software "Data explorer" after enrichment was also compared with the masses of native GSTD1 peptides. No significant change was found that can lead us to identify a site of phosphorylation. A possible reason for that is that the phosphopeptides are negatively charged whereas acquisition of spectra is generally performed in the positive mode (Mann et al., 2002). Therefore a final approach was considered: using LC-MS to separate peptides and to sequence them using the newly installed software, PEAKS. 


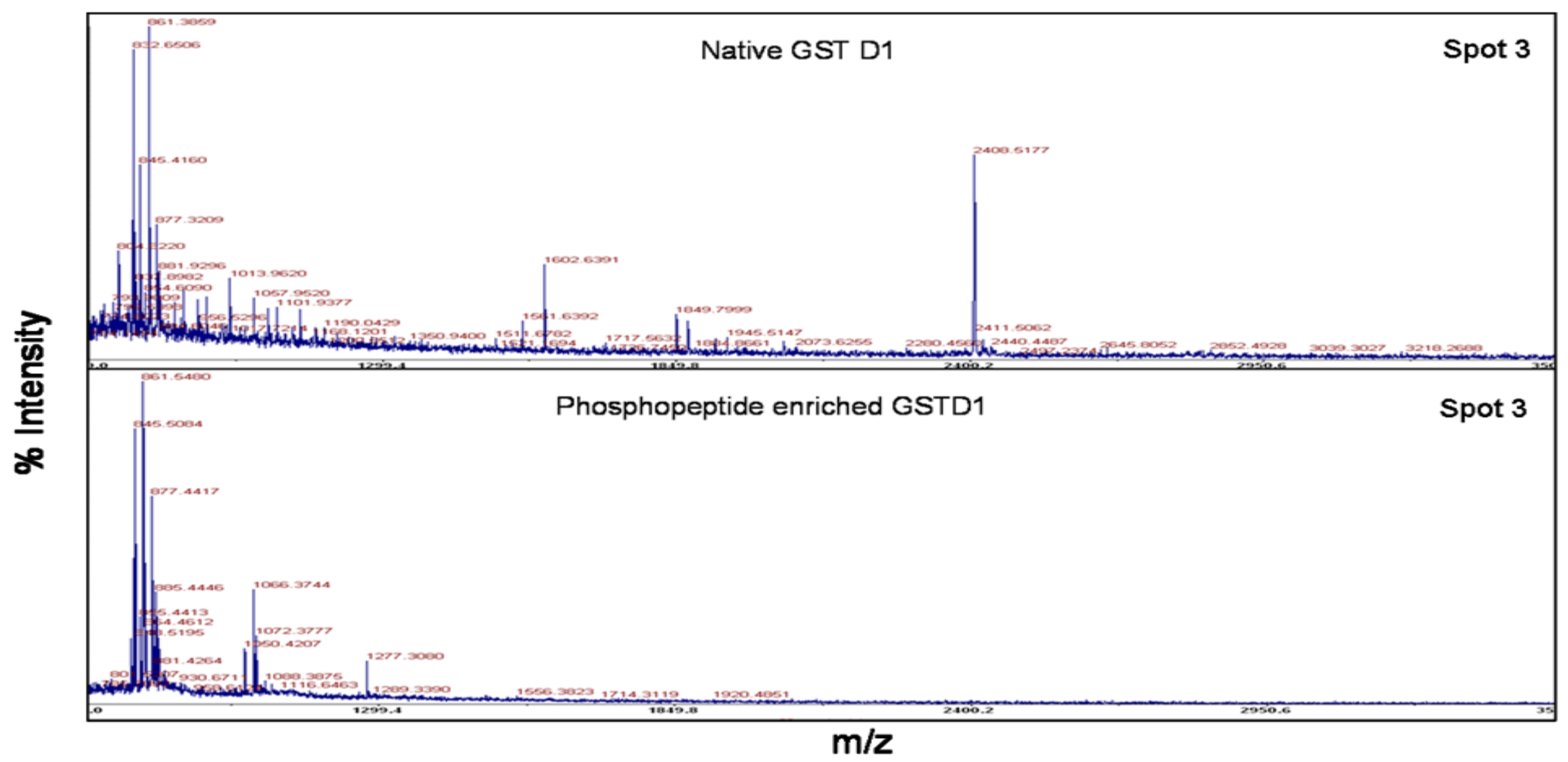

Figure 7-5: Comparison of MALDI-TOF spectrum of native GSTD1 and phosphopeptide enriched GST D1 (spot 3) as shown in Figure 7-4. 


\subsubsection{Use of PTMFinder in PEAKS software}

The D. melanogaster Delta GST spots from a 2D PAGE gel were excised and digested with trypsin. The sample was prepared using ZipTip ${ }^{\mathrm{TM}} \mathrm{C}_{18}$ tips as described in the section 3.2.11 and MS/MS spectra were obtained. The raw LC-MS/MS data from the DmGSTD1 spots 3, 5 and 6 (Figure 7-4) were loaded as a project on PEAKS and pre-processed to perform the de novo sequencing. Pre-processing involves noise filtering and peak centering, as well as deconvolution of the doubly and triply charged species to singly charged ions. This step is very important for the interpretation of MS/MS data by PEAKS. The auto de novo sequencing was performed on processed data by setting parameters as shown in Table 7-1 A. Following de novo sequencing, a PEAKS search was performed in order to identify the protein. The parameters were set as shown in Table 7-1 B. All the spots which were subjected to the analysis were correctly identified as GST D1, DmGST1 \{EC 2.5.1.18 (D. melanogaster, peptide 208 a. a) with PEAKS score of 99.1\%. The PTMFinder was run on the PEAKS search result by allowing fixed and variable modifications listed in Table 7-1 C. 
Table 7-1: Setting parameters for each application in PEAKS.

A) The setting parameters for the auto de novo sequencing. B) The setting parameters for the PEAKs search (for identification of protein). C) The setting parameters for the PTMFinder. The type of PTMs searched were variable modifications such as acetylation [2], deamidation [3], oxidation [4] and phosphorylation [5].

\begin{tabular}{|ll|}
\hline Denovo details: Trypsin(f: Carbamidomethylation) \\
\hline & \\
Parent Mass Error Tolerance & $0.1 \mathrm{Da}$ \\
Fragment Mass Error Tolerance & $0.8 \mathrm{Da}$ \\
Enzyme & Trypsin \\
Semi (enzyme) & false \\
Fixed Modification & {$[1] 57.021465 @[C]$} \\
Max variable PTM per peptide & 3 \\
Report \# peptides & 1 \\
Preprocess data & false \\
& \\
\hline
\end{tabular}

$\mathbf{A}$

\begin{tabular}{|ll|}
\hline PEAKS Search details: Dro NCBInr & \\
\hline & \\
Parent Mass Error Tolerance & $0.1 \mathrm{Da}$ \\
Fragment Mass Error Tolerance & $0.8 \mathrm{Da}$ \\
Precursor Mass Search Type & Mono \\
Enzyme & Trypsin \\
Semi (enzyme) & false \\
Fixed Modification & {$[1] 57.021465 @[C]$} \\
Max variable PTM per peptide & 3 \\
Database & NCBInr \\
Taxon & Drosophila melanogaster (fruit fly) \\
Max Missed Cleavages & 1 \\
Preprocess Data & false \\
\hline
\end{tabular}

B

\begin{tabular}{|ll|}
\hline \multicolumn{2}{|l|}{ PEAKS Search details: dro delta PTMFinder } \\
\hline & \\
Parent Mass Error Tolerance & $0.1 \mathrm{Da}$ \\
Fragment Mass Error Tolerance & $0.8 \mathrm{Da}$ \\
Precursor Mass Search Type & Mono \\
Enzyme & Trypsin \\
Semi (enzyme) & false \\
Fixed Modification & {$[1] 57.021465 @[\mathrm{C}]$} \\
Variable Modification & {$[2] 42.010567 @[\mathrm{~K}]$} \\
Variable Modification & {$[3] 0.984016 @[\mathrm{NQ}]$} \\
Variable Modification & {$[4] 15.994915 @[\mathrm{M}]$} \\
Variable Modification & {$[5] 79.96633 @[S T \mathrm{~T}]$} \\
Max variable PTM per peptide & 3 \\
Database & NCBInr \\
Max Missed Cleavages & 1 \\
Preprocess Data & false \\
\hline
\end{tabular}

C 


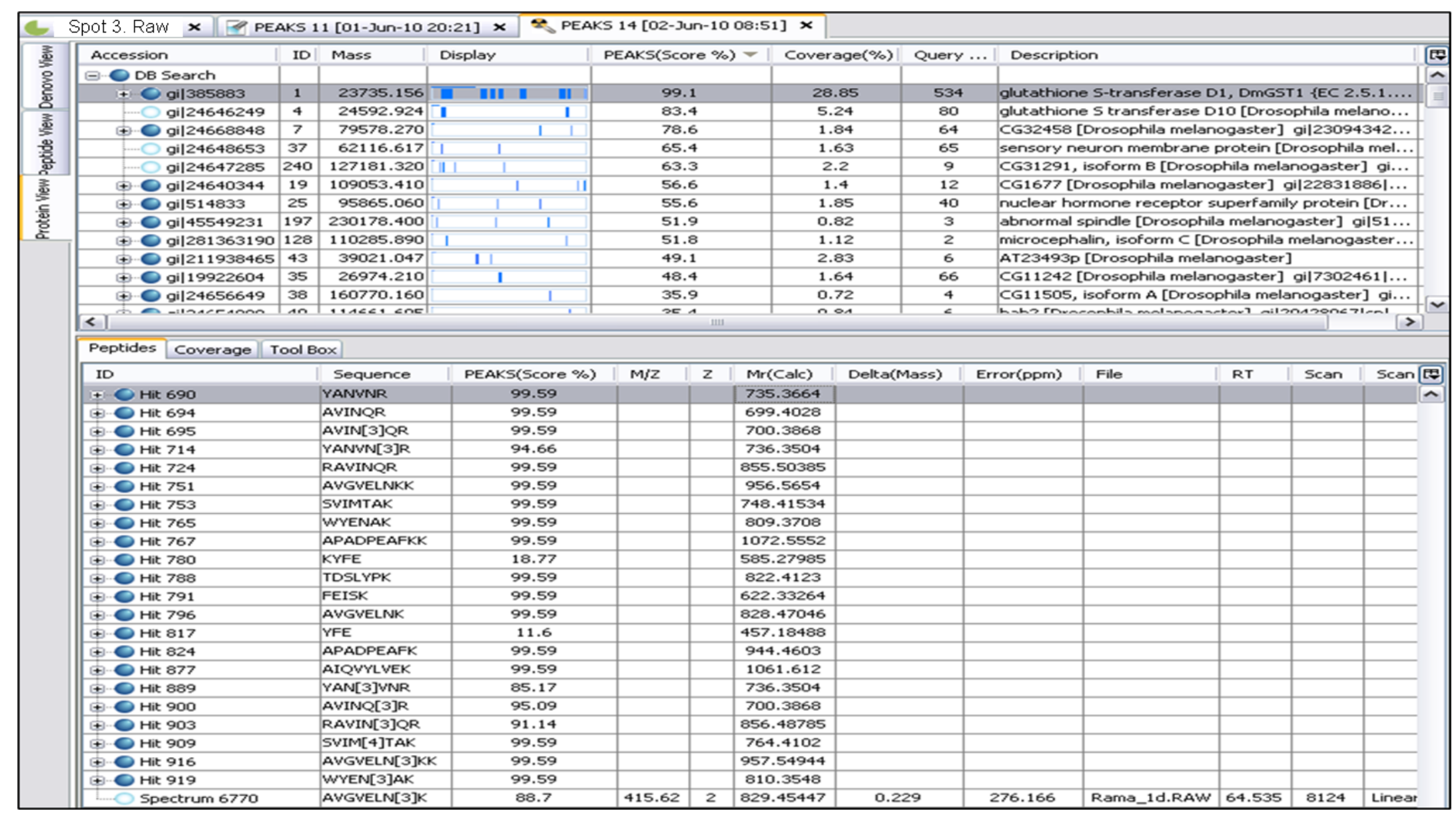

Figure 7-6: PTMFinder result with identification of protein spot 3 in Figure 7-4.

The upper window shows the identification of the protein with PEAKS score. The lower window shows the matched peptides. The numbers [3], [4] in the peptide sequence represent the modification on the amino acid and type of modification as shown in Table 7-1 C. 


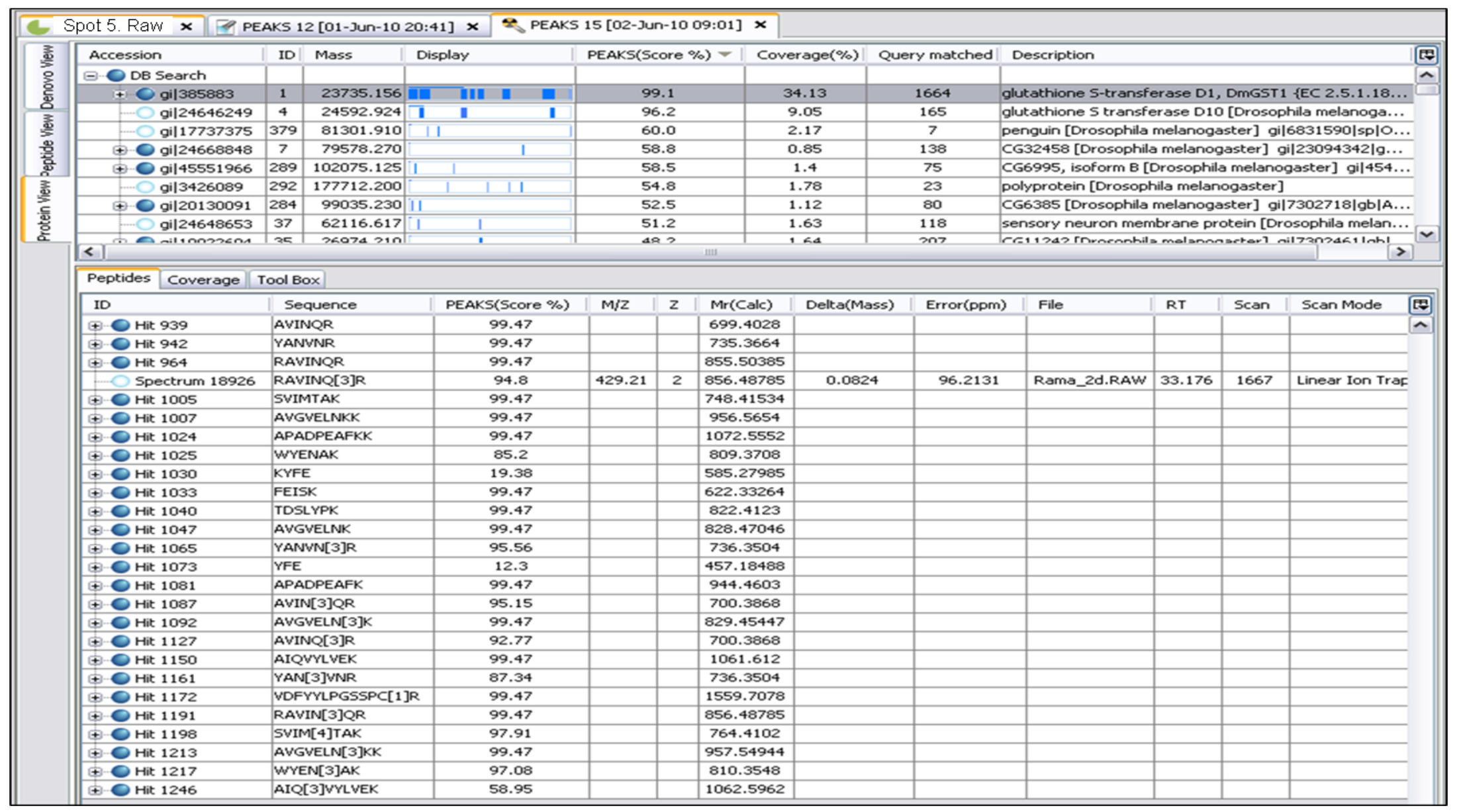

Figure 7-7: PTM Finder result with identification of protein spot 5 in Figure 7-4.

The upper window shows the identification of protein with PEAKS score. The lower window shows the matched peptides. The numbers [1], [3], [4] in the peptide sequence represent the modification on the amino acid and type of modification as shown in Table 7-1 C. 


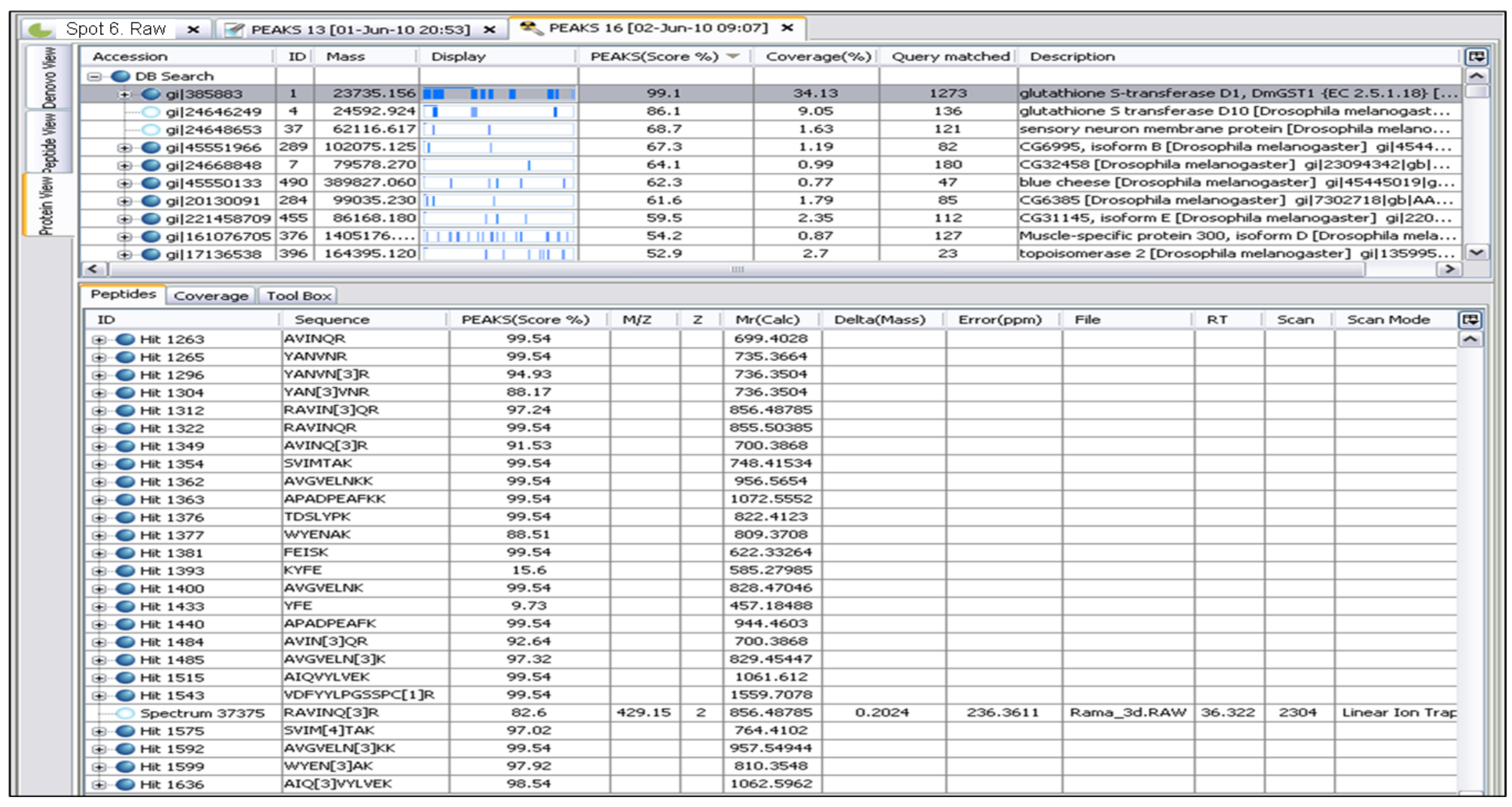

Figure 7-8: PTM Finder result with identification of protein spot 6 in Figure 7-4.

The upper window shows the identification of protein with PEAKS score. The lower window shows the matched peptides. The numbers [1], [3], [4] in the peptide sequence represent the modification on the amino acid and type of modification as shown in Table 7-1 C. 
Table 7-2: List of modified peptides suggested by PTMFinder.

The sequences of peptides of spots 3, 5 and 6 with indicated post-translational modifications from Figure 7-6, Figure 7-7 and Figure 7-8 are listed.

\begin{tabular}{ccc}
\hline \multicolumn{2}{c}{ Modified peptides of Delta GST suggested by PTMFinder } \\
\hline Spot 3 & Spot 5 & Spot 6 \\
pI 5.7 & pI 6.4 & pI 7.0 \\
\hline AVIN[3]QR & AVIN[3]QR & AVIN[3]QR \\
AVINQ[3]R & AVINQ[3]R & AVINQ[3]R \\
YANVN[3]R & YANVN[3]R & YANVN[3]R \\
YAN[3]VNR & YAN[3]VNR & YAN[3]VNR \\
RAVIN[3]QR & RAVIN[3]QR & RAVIN[3]QR \\
& RAVINQ[3]R & RAVINQ[3]R \\
SVIM[4]TAK & SVIM[4]TAK & SVIM[4]TAK \\
AVGVELN[3]KK & AVGVELN[3]KK & AVGVELN[3]KK \\
WYEN[3]AK & WYEN[3]AK & WYEN[3]AK \\
AVGVELN[3]K & AVGVELN[3]K & AVGVELN[3]K \\
& AIQ[3]VYLVEK & AIQ[3]VYLVEK \\
\hline
\end{tabular}

(A-alanine, V-valine, I-isoleucine, N-asparagine, Q-glutamine, R-arginine, Ytyrosine, S-serine, M-Methionine, K-lysine, G-glycine, E-glutamic acid, Wtryptophan) 


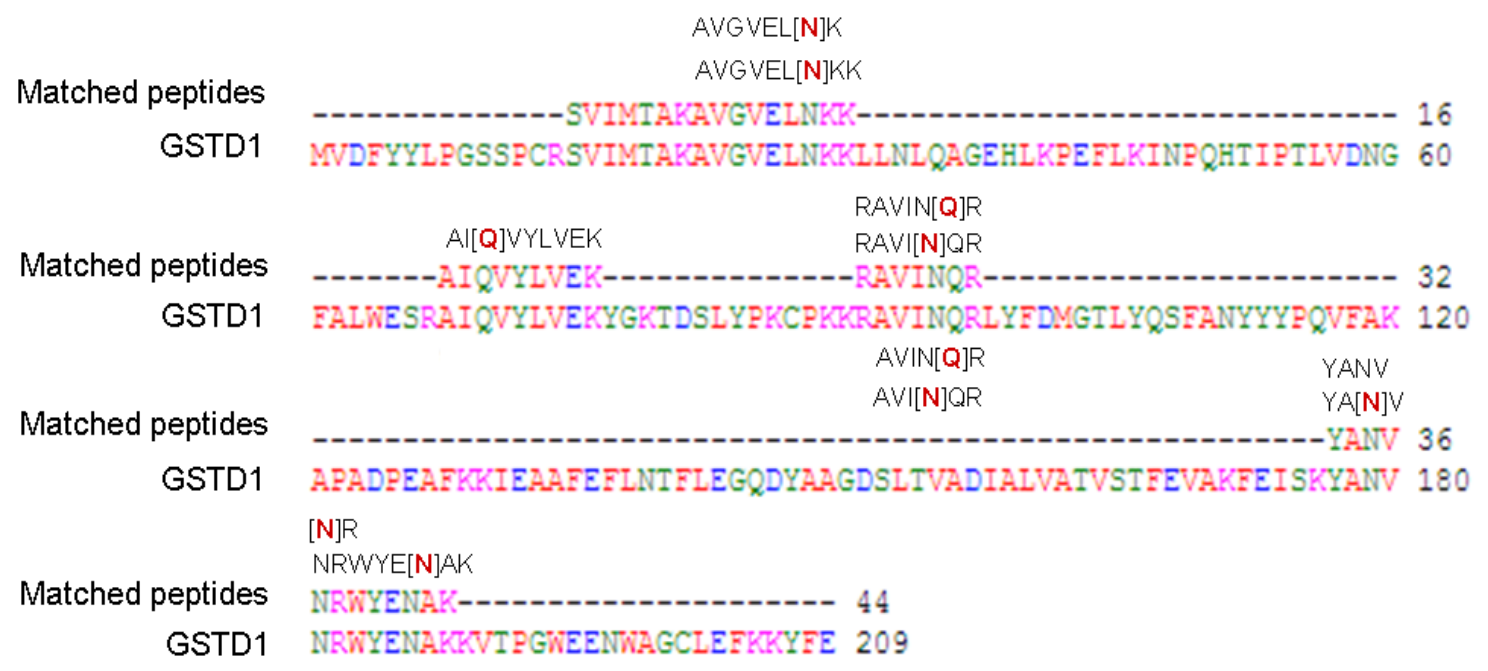

Figure 7-9: Alignment of matched deamidated peptides with DmGSTD1 sequence.

Sites of deamidation determined by PTM finder are shown in brackets [].

The results with identified matched peptides are shown in Figure 7-6, Figure 7-7 and Figure 7-8 for the spot 3, spot 5 and spot 6 on Figure 7-4 respectively and all matched peptides are separately shown in Table 7-2. The alignment of matched deamidated peptides with the DmGSTD1 sequence is shown in Figure 7-9. The number of matched peptides in all three spots was similar except the additional peptides AIQVYLVEK and VDFYYLPGSSPCR were present in spots 5 and 6 which resulted in the increase of percent coverage (34.13 compared to 28.85 of spot 3) of the protein identified. It was interesting to see that despite the possible variable PTMs allowed for in PTMFinder, the result indicated mostly deamidation (modification [3]) of asparagine and glutamine of a few matched peptides as listed in Figure 7-6, Figure 7-7 and Figure 7-8. There was also the presence of oxidation of methionine on a single matched peptide SVIM[4]TAK in all the spots. This should not affect the pI. The software identifies all the matched peptides both with and without modification in all spots. For example the peptide AVGVELNKK and its deamidated equivalent AVGVELN[3]KK were both detected in all spots. This is 
because in an LC-MS coupled device, these peptides can be separated, analyzed simultaneously and distinguished as the deamidated and amidated peptides usually co-elute with acidic eluents (Lindner and Helliger, 2001). Figure 7-10 displays the spectra corresponding to the AVGVELNKK peptide and its deamidated product AVGVELN[3]KK. The unmodified peptide co-elutes with the modified one, hence its peaks are found in the modified spectrum, but not attributed. All these modified pairs are consistently present in all three spots except the pair AIQVYLVEK and AIQ[3]VYLVEK, deamidation at glutamine was apparently absent in spot 3. In spot 3, two peptides have deamidation at two different sites: in AVIN[3]QR and AVINQ[3]R, YANVN[3]R and YAN[3]VNR. Therefore a total of six peptides containing deamidation sites were present along with one oxidation on SVIM[4]TAK in spot 3. In spot 5 and spot 6 three peptides were modified at two different sites. Those are AVIN[3]QR and AVINQ[3]R, RAVINQ[3]R and RAVIN[3]QR and YANVN[3]R and YAN[3]VNR. Therefore a total of seven deamidated peptides were identified along with one oxidation on SVIM[4]TAK. So the PTMFinder result suggests that in spot 3 out of 14 matched peptides, 6 peptides were deamidated with two peptides having two deamidation sites on the same peptide and in spot 5 and spot 6 out of 15 matched peptides, 7 peptides showed deamidation with three peptides having two deamidation sites on the same peptide. It is not surprising to find both amidated and deamidated versions of a peptide. Any one copy of the protein may have suffered one (or more) deamidation events. Over multiple copies of the protein each deamidation site will be represented by a combination of amidated and deamidated peptides. Within any one spot, with each protein having the same $\mathrm{pI}$, each protein molecule is likely to have the same number of deamidations, not necessarily at the same sites. 

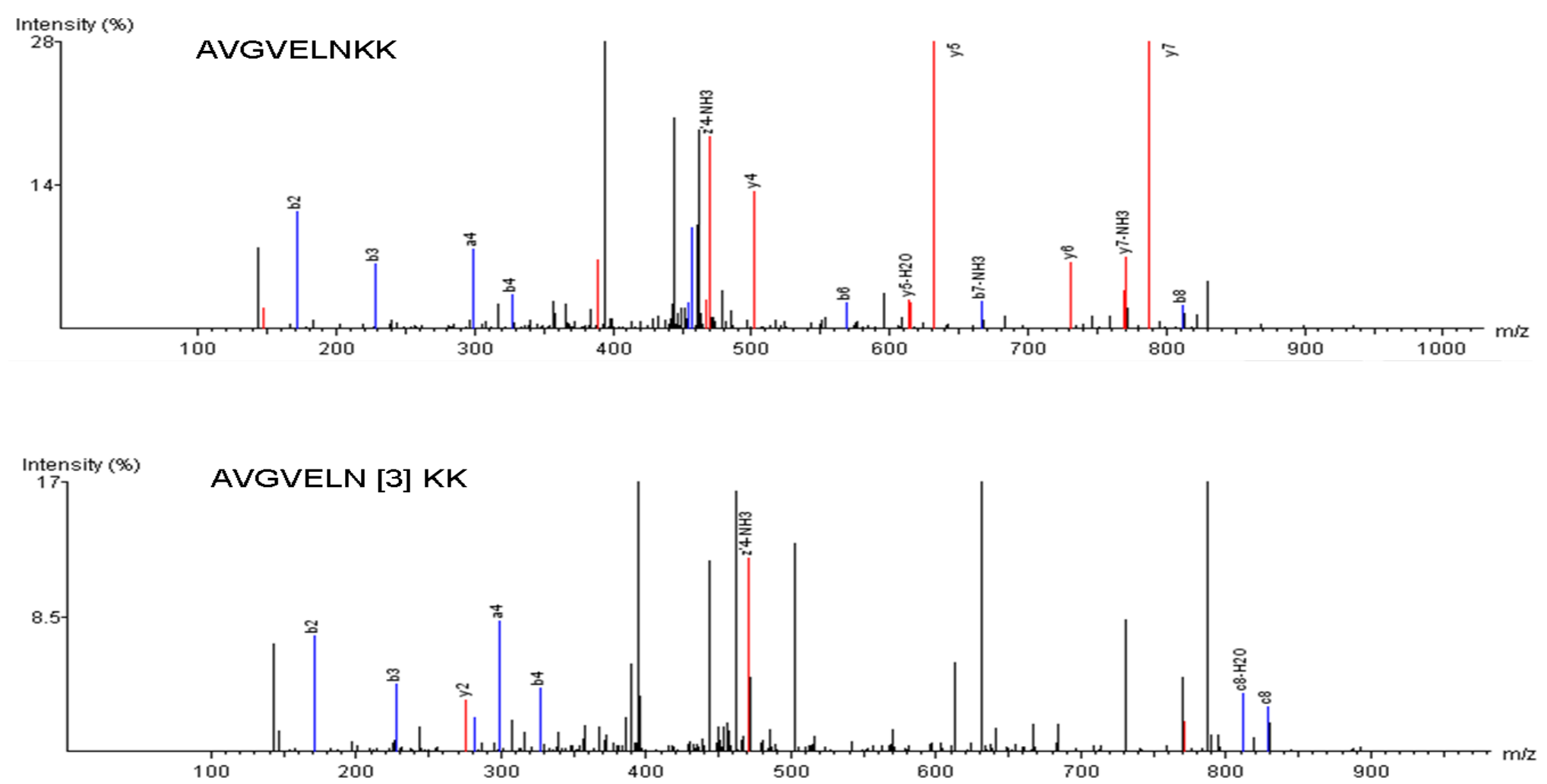

Figure 7-10: Comparison of the mass spectra of the peptide AVGVELNKK and modified peptide AVGVELN[3]KK suggested by PTMFinder in PEAKS.

The different colours of the peaks show the confidence scores. Red represents very high confidence (greater than 90\%), Blue represents medium confidence (60-80\%) and black represents low confidence (less than $60 \%$ ). Letters show the fragment ions during the collision induced dissociation in LC-MS/MS. 


\subsection{Discussion}

The routine proteomic technique used in this work has given a clear indication of the presence of PTMs on Delta 1 GSTs from both $L$. cuprina and $D$. melanogaster. This is seen in 2D electrophoresis analysis. Initially, it was assumed that the Delta GST might be variably phosphorylated and a fluorescent phosphosensor dye (Pro-Q Diamond dye) was used to probe this. Unfortunately, the positive control and negative controls could not be distinguished in these experiments and therefore, the phosphorylation on Sigma or Delta GSTs could also not be determined.

MALDI-TOF has been successfully used for identification of proteins by peptide mass fingerprinting. However, analysis of phosphopeptides is not as straightforward as the identification of proteins for several reasons: (1) selective suppression of ionization/detection efficiencies of phosphopeptides in the presence of large amounts of unphosphorylated peptides, and (2) lower detection efficiencies of phosphopeptides as compared with their unphosphorylated forms (Steen et al., 2006). It is sometimes possible to identify phosphopeptides based on a characteristic mass shift owing to loss of phosphate (80 Da or $98 \mathrm{Da}$ or multiples) after treatment with phosphatases (Annan and Carr, 1996). In the present study the comparison of the MALDI-TOF spectra of different Delta spots did not provide any support for phosphorylation as a PTM. MALDI-TOF can be valuable if performed on peptide mixtures that are first purified on IMAC columns to enrich for phosphorylated peptides. Therefore, IMAC columns/ZipTip ${ }_{M C}$ tips were used for the phosphopeptide enrichment. IMAC generally enriches for phosphorylated serine, threonine and tyrosine residues and has been successfully used in the detection of phosphopeptides 
using MS (Cao and Stults, 2000; Cleverley et al., 1998; Posewitz and Tempst, 1999; Stensballe et al., 2001; Zhou et al., 2000). This method enables the visualization of peptides that are otherwise not observed in the Mass spectrum because of suppression effects. However using MALDI and comparing the masses of peptides obtained with and without enrichment, there was no phosphorylated peptide detected.

Tandem mass spectrometry (MS/MS) has also been used for the identification of phosphopeptides by precursor-ion scanning as on fragmentation by collisioninduced dissociation (CID), phosphopeptides not only produce sequence-specific fragments but also fragments that are specific for phosphate groups (Carr et al., 1996; Wilm et al., 1996). In the present study, MS/MS spectra, when subjected to PEAKS for PTM analysis, showed no phosphorylation in any peptide (Table 7-2). There is also a recent report published on the study of phosphorylation in developing $D$. melanogaster embryos using the mass spectrometry based proteomics (Zhai et al., 2008). In total 13,720 different phosphorylation sites were discovered from 2702 proteins including GSTs, however there was no evidence of any phosphorylation sites on Delta GST. The GSTs CG6673 (Omega) and GST-containing FLYWCH zinc-finger protein were shown to be phosphorylated. Therefore our study, along with other research, supports the view that Delta GST is not phosphorylated.

The analysis of LC-MS/MS raw data of tryptic digests of the Delta GST spots by the software package PEAKS revealed interesting findings. PEAKS contains a library of $\sim 30$ common PTMs. The PTMFinder analysis was run on three spots from the Delta GST regions of the 2D gel of the D. melanogaster GST isolate. The proteins were all identified as DmGSTD1 and indicated deamidation of asparagine and glutamine as a possible post-translational modification of the Delta GST peptides. 
A single deamidation would be expected to shift the $\mathrm{pI}$ from around 6.75 to 6.2 and a second to 5.8. Spot 6 (pI 7.0) has the closest pI to that predicted for DmGSTD1. Therefore spots 5 and 3 might be expected to show the presence of additional acidic residues. Spot 6 would make for a less acidic protein so the presence of deamidation in spot 6 may suggest post-gel or at least post IPG (first dimension) degradation during the experiments. Although several possible deamidations are shown for each spot, it is their number that will determine the overall $\mathrm{pI}$ of the protein.

There are two possible reaction pathways known for deamidation: The first is the enzymatic deamidation of asparagines and glutamines catalysed by several specific deamidases (Stewart et al., 1995; Yan and Sloan, 1987) and the second is the non-enzymatic pathway, which may occur under physiological conditions (Hochstrasser, 1998) or during sample preparation (Wright, 1991a). Investigation of several hundred model peptides have shown that under physiological conditions deamidation of asparagine can be detected ten times more often than the deamidation of glutamine (Robinson and Robinson, 1991). In the amino acid sequence of DmGSTD1, there are 11 asparagine residues and 7 glutamine residues. Out of those PEAKS identifies only 7 asparagine and 3 glutamine residues as being deamidated. The rate of non-enzymatic deamidation is promoted by chemical reaction conditions like $\mathrm{pH}$ or temperature and amino acid sequence (Wright, 1991a). Especially for asparagine- $X$ sequences where $X$ is glycine, serine or alanine or for serineasparagine, non-enzymatic deamidation is more probable (Wright, 1991b). The current result suggests that only one peptide WYEN[3]AK, is deamidated where asparagine precedes alanine. However, there is a report available that in acidic solution, the rate of deamidation of the asparagine is not affected by the amino acid sequence of the peptide (Tyler-Cross and Schirch, 1991) and, as in the present study, 
the peptides were suspended in $0.25 \%$ TFA and subjected to LC-MS/MS analysis for the separation using the gradient constructed from $0.1 \%$ formic acid in water and acetonitrile solution (see section 3.2.11), the possibility of accelerated deamidation due to amino acid sequence is minimised and the only mechanism of deamidation that appears to operate in acidic conditions is direct hydrolysis of the side-chain amide group of the asparagine residue. In the present case, deamidated asparagine and glutamine residues precede basic amino acids in the sequences AVINQ[3]R, YANVN[3]R, AVGVELN[3]KK and RAVINQ[3]R. The significance of this occurrence, if any, is unknown although at $\mathrm{pH} 7$ these basic residues might provide a local source of protons to catalyse hydrolysis. The deamidation overall introduces negative charges, which in turn may affect secondary and tertiary structure of proteins and peptides. These changes in the structure and charge of proteins may affect their biological activity, especially important in aging organisms. Deamidation has been hypothesized to serve as an in vivo biological clock controlling the rate of protein turnover (Robinson and Robinson, 1991). Proteins with a high turnover generally have a greater deamidation rate than do proteins with a lower one. It is assumed that deamidation destabilizes the proteins by making them more susceptible to proteolytic degradation (Rogers and Rechsteiner, 1988). This susceptibility could be determined by a change in protein structure and/or cellular location. Thus, in this respect deamidation triggers protein turnover and marks the protein for degradation. In young cells, the marked protein is readily recognised and eliminated, whereas it is accumulated in old cells (Lindner and Helliger, 2001). In chapter 5, the present study showed similar migration patterns of Delta GST on 2D gels of the egg, larval and pupal stages of $L$. cuprina (Figure 5-4). There may be possibility that deamidation in the developmental stages may affect the structural integrity and biological activity of 
proteins due to their association with development (Lindner and Helliger, 2001). In the present study, however there is no clear evidence for the cause of the deamidation on Delta GSTs or any role in development. It would be interesting to investigate further as these supposed deamidation sites at asparagine and glutamine residues in predicted deamidated peptides seem to be conserved (except for two places) in GSTD1 sequences from L. cuprina and Musca domestica.

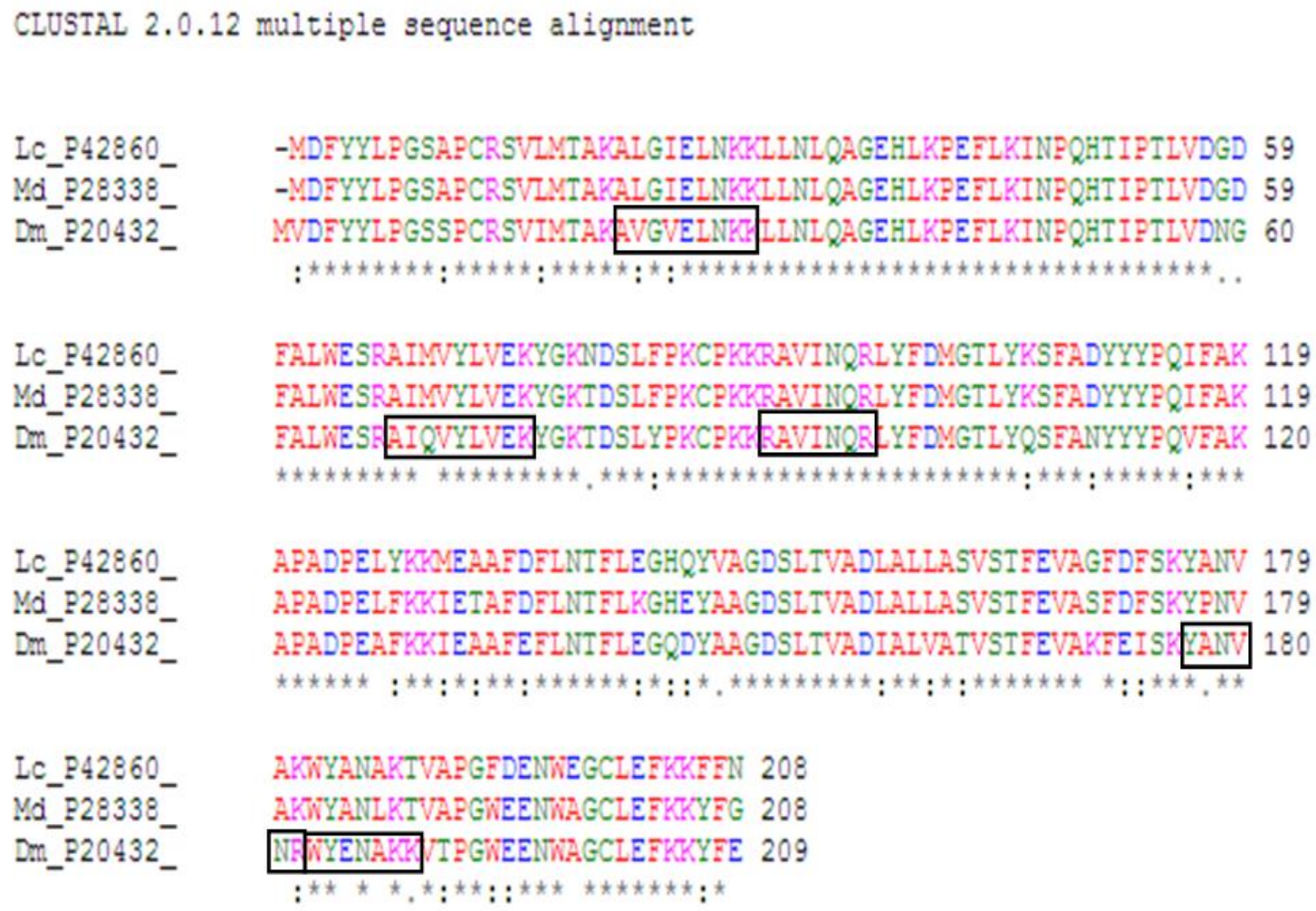

Figure 7-11: Alignment of D. melanogaster DmGSTD1 sequence with L. cuprina and Musca domestica D1 sequences using ClustalW2.

The boxes display the peptides which are predicted to be deamidated by PEAKS. The peptide RAVINQR is conserved in all three insects where as other peptides differ in $L$. cuprina and $M$. domestica by one or two amino acids compared to D. melanogaster sequence.

Lc - L. cuprina; $\mathrm{Md}-$ M. domestica. 
It is interesting to note that the deamidated peptides RAVINQR and AVGVELNKK are completely conserved in a large number of Delta GSTs from different insect species other than Musca domestica and L. cuprina. Therefore any explanation related to physiological significance of deamidation of Delta GST can apply to those insects as well.

The examination of 3D structure of the dimeric model of GSTD1-3EIAN available at RCSB protein databank in RasMol, revealed important information about the asparagines and glutamines residues. The screening for buried asparagines and glutamines showed that none of the residues anywhere in the protein are buried and 22 asparagines and 14 glutamine residues were found on the surface. Therefore, it seems quite likely hydrolysis is random and spontaneous and may not be of any functional significance. For example, Gln 70 although slightly exposed on surface is not readily accessible, so it is unlikely that its deamidation would be enzymatically controlled. However, it cannot be determined whether deamidation occurred during the sample processing or intracellularly. However, the alignment of DmGSTD1 (Figure 7-11) shows that Gln 96 (RAVIN[Q]R) and Trp64 are conserved in all three species and there is literature evidence that Gln 96 is shown to associate with Trp64 through hydrogen bonding (Low et al., 2010). This hydrogen bonding to Gln 96 might be functionally important and therefore also its deamidation, but its significance is not known. The examination of GST D1 structure also showed that there are many asparagine and glutamine residues next to basic amino acids however, the question is: why are only three peptides RAVIN[Q]R, AVIN[Q]R and YANV[N]R next to arginine deamidated? Whether it is significant in the biological processes such as aging is not clear. 
In summary, the work presented in this chapter attempted to determine the types of post-translational modifications on Delta (D1) GST. The comparison of masses of peptides of native GSTD1 and phosphopeptide enriched GSTD1 did not provide any evidence of phosphorylation. Our finding, together with literature support, suggests that GSTD1 is not phosphorylated. Instead, use of tandem mass spectrometry showed deamidation is a possible post-translational modification of Delta GST. Further work will be required to confirm that deamidation occurs physiologically, rather than being an artefact of protein handling. 


\section{General Discussion}

\subsection{Project summary}

The overall aim of this thesis was to examine the use of proteomic techniques for investigating the role of the GSTs in the development of insecticide resistance in L. cuprina. The primary requirement to achieve this goal was to purify the GSTs from the crude extract of the insect and therefore sequential affinity purification techniques were developed to purify as many GSTs as possible from L. cuprina and D. melanogaster. After the establishment of purification conditions, proteomics was chosen for the characterisation and identification of GSTs from both the insects.

The questions addressed in thesis were:

- Can proteomic and bioinformatic methods be adapted to the study of GSTs and their characterization in the most widely used and genetically best-known insect species D. melanogaster and also in L. cuprina, an insect species which lacks a genetic database?

- How is the GST proteome/expression regulated during the development of $L$. cuprina?

- Are GSTs expressed in a tissue-specific manner in L. cuprina?

- Does the GST proteome vary in an OP resistant insect compared to susceptible ones? If yes, does the resistant insect have higher capability to metabolise insecticides in vitro?

- Finally, what type of post-translational modification occurs with Delta GST? 
In Chapter 4, Partial success was demonstrated in isolation and identification of GSTs from D. melanogaster that are known from the genetic databases, including some that had not previously been known to be expressed. Thus, CG16936 (a putative uncharacterised protein with glutathione transferase activity), CG6673 and CG6776 (both Omega class of GSTs) have been identified in the D. melanogaster proteome. However, whilst this model organism has a predicted 39 GST genes, the actual number of GSTs detected is much lower. Previous proteomic studies identified the Delta isoforms D2 and D3 and the Epsilon GST isoforms - E3, E6, E7 and E9 using BSP-GSH as an affinity matrix (Alias and Clark, 2007)) and CG5224, CG30000 and CG1702 were identified using S-hexyl-GSH affinity matrix (Ding and Clark, unpublished data) which were not purified by the matrices used in the present study. The affinity matrices (GSH, DNP-GSH and CNP-GSH matrices) employed in the present study captured major classes of GSTs such as Sigma, Delta, Epsilon and Omega but failed to bind all the GST isoforms as evidenced by the amount of GST catalytic activity in unbound fractions.

In L. cuprina, the first and foremost challenge was how to identify the purified L. cuprina GSTs other than L. cuprina Delta GST. Use of D. melanogaster as a model provided a limited possibility for identification due to its combination of similarity and dis-similarity with L. cuprina proteins separated on 2D gels. The separation pattern of Sigma and Delta GSTs on 2D gels from both the insects was strikingly similar and MALDI-TOF analysis showed near identity of the proteins from the two species, which confirmed the identification of Sigma GST. Much more of a problem was to identify the DNP-GSH affinity-purified GSTs on 2D gels since entirely different patterns of separation between the two insects were seen. However, 
in D. melanogaster, within the $22-40 \mathrm{kDa}$ M.W. range, over $80 \%$ of proteins isolated on DNP-GSH matrix were firmly identified as GSTs therefore it is argued that the probability of similarly isolated L. cuprina proteins in that particular M.W. range being GSTs is high. To tentatively characterise these DNP-GSH affinity-purified $L$. cuprina GSTs, a consensus sequence-based approach was developed. To obtain consensus sequences for each GST class, selected representative sequences, obtained as discussed in section 4.3.3 were aligned in ClustalW2 and a consensus sequence obtained from the aligned sequences using Jalview. The MALDI fragmentation patterns for L. cuprina proteins were matched against proteins in the "Other Metazoa" database. The sequences of these matched peptides were then aligned against the consensus sequences using ClustalW2 and a tentative classification of GSTs was assigned. Thirteen L. cuprina spots in the 22-40 kDa M.W. range on 2D gels were matched to GSTs in "Other Metazoa" and were assigned the GST class based on this approach. Thus, the combination of interaction with appropriate affinity matrices and peptide matching gives high confidence but not certainty that these proteins are GSTs. This leads on to the question posed in the following section.

\subsubsection{How reliable is the consensus sequence-based approach for the characterisation of L. cuprina GSTs?}

Consensus sequences show which residues are conserved and which residues are variable. Use of the consensus sequences for each GST class from different species offers the broad possibility of matching the L. cuprina peptides. In the present study, the identification of $L$. cuprina Sigma GST was confirmed using this approach, in addition to its matching with the deduced amino acid sequence obtained by our laboratory (see Figure 4-19) to check the reliability of this approach. Spots 2 , 
3, 4 and Spots 7, 8, 9 in Figure 4-21 were tentatively identified as Delta (High M.W.) and Epsilon GSTs respectively with the promising ClustalW2 alignment score of $>50$. The alignment of these Delta and Epsilon GSTs with the respective class consensus sequences along with the ClustalW2 alignment score is shown in Figure 8-1 and Figure 8-2.

Clustal W2 score against Delta consensus: Spot 2-54; Spot 3-76; Spot 4-52

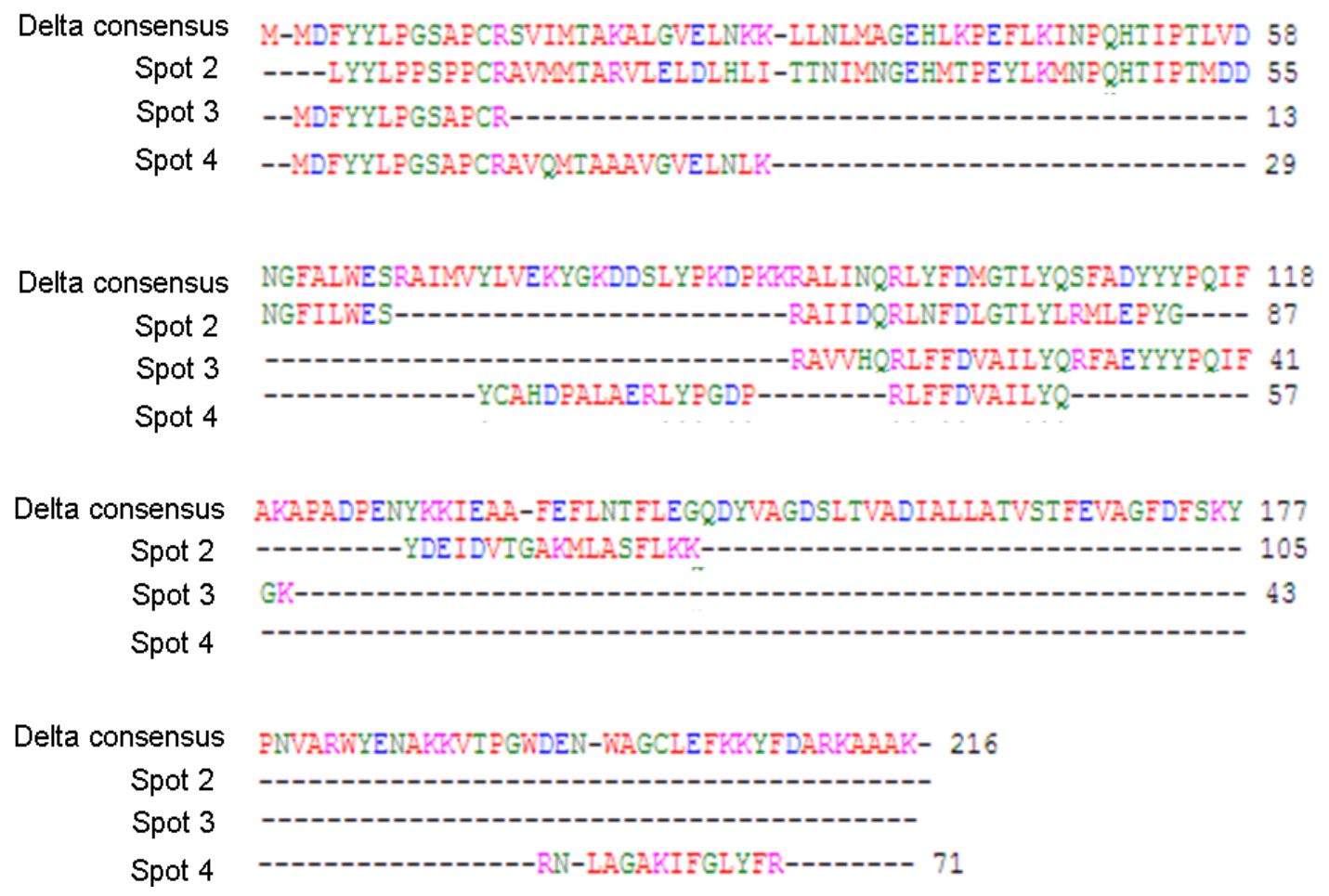

Figure 8-1: Alignment of L. cuprina peptide sequences matched against "Other Metazoa" with Delta GST consensus sequence 


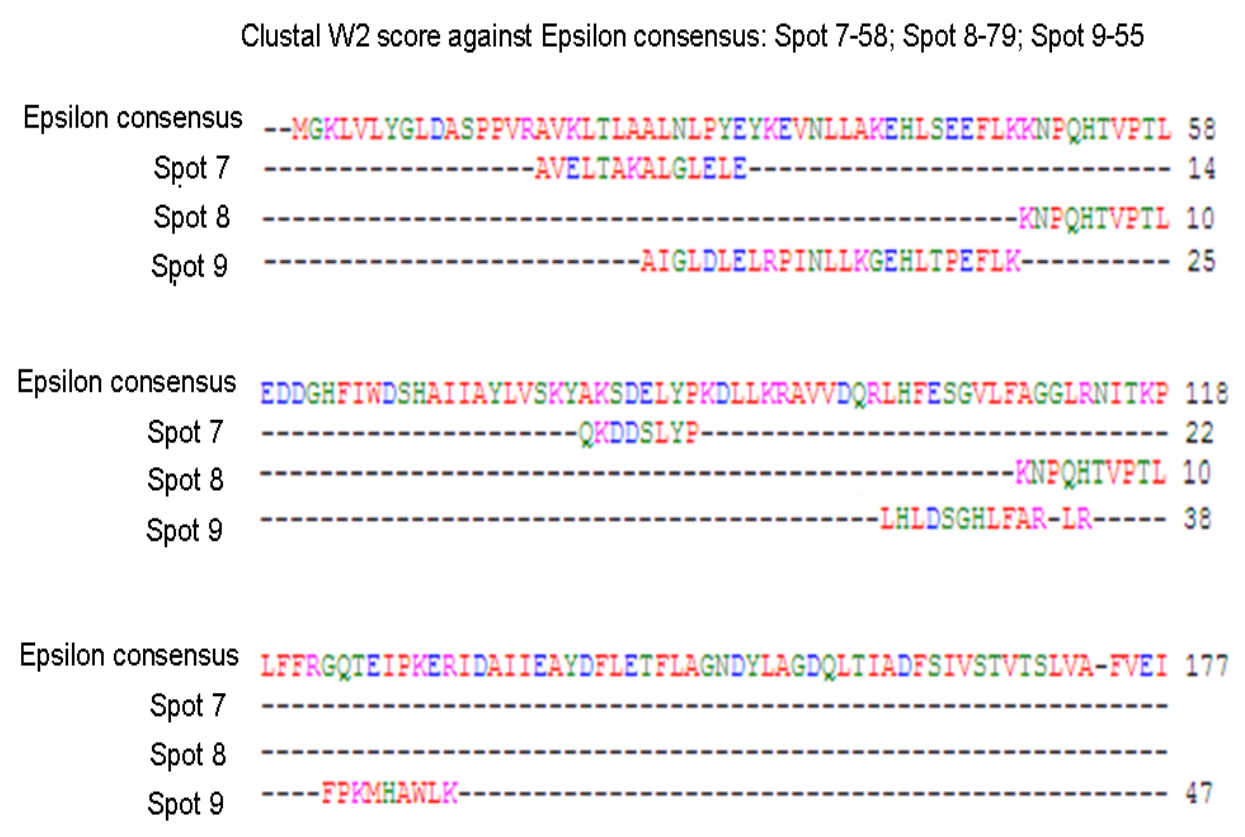

Figure 8-2: Alignment of L. cuprina peptide sequences matched against "Other Metazoa" with Epsilon GST consensus sequence

The alignment score is $>50$ for each corresponding spot with consensus sequences. Thus, classification of spots 2,3 and 4 as Delta and spots 7,8 and 9 as Epsilon GSTs and there is reasonable confidence of this classification being correct. The $L$. cuprina proteins (spots $14,15,16,17$ in Figure 4-21) did not match with the GSTs in "Other Metazoa" but identified as Rab proteins. Their peptide sequences were also aligned with the consensus sequences of all GST classes to again validate this approach. The alignment of spot 14 peptides is shown in Figure 8-3 and the result is negative as no significant score was found for any class of GSTs. 
Clustal W2 score of Spot 14 with consensus sequences:

Delta-1; Iota-7; Xi-9; Epsilon-7; Theta-5; Omega-9; Zeta-9; Sigma-7; Mu-3

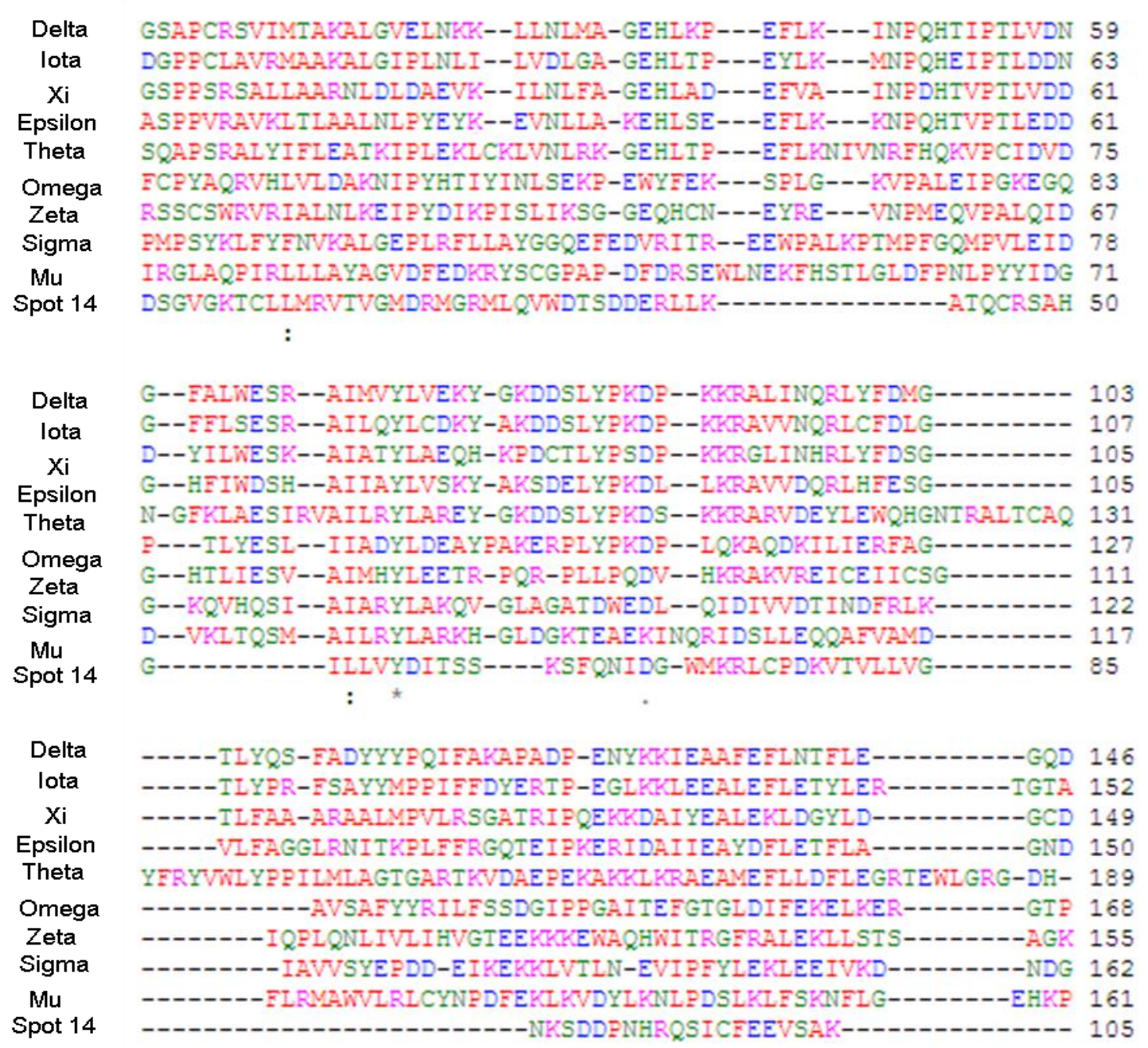

Figure 8-3: Alignment of L. cuprina peptide sequences matched against "Other Metazoa" with consensus sequences of different GST class

Thus, based on the alignment similarity, it appears that this approach gives a reliable characterisation. However, spot 5, 11 and 13 in Figure 4-21 and spots $2^{*}$ and $3^{*}$ (zone E) in Figure 5-6 in the present study were tentatively identified as Mu-like GSTs. This result is intriguing as there are reports available emphasising no similarity of Mu GSTs with insect GSTs (Enayati et al., 2005; Ranson et al., 2001). 


\subsubsection{Do insects possess Mu-like GSTs?}

Members of the Mu class GSTs are responsible for conjugating a wide variety of pesticides such as the organophosphate insecticides, the halogenated hydrocarbon insecticides and the $S$-triazine herbicides (Hayes et al., 2005) but their presence in insects is not known. While studying the relationship between D. melanogaster GSTs and mammalian GSTs from each of the evolutionary distinct GST class, (Ranson et al., 2001) found no relationship between human Alpha, Pi and Mu GSTs with $D$. melanogaster GSTs but Theta, Sigma, Omega and Zeta GSTs shared 56-58\% similarity with the respective human GST classes (Figure 8-4).

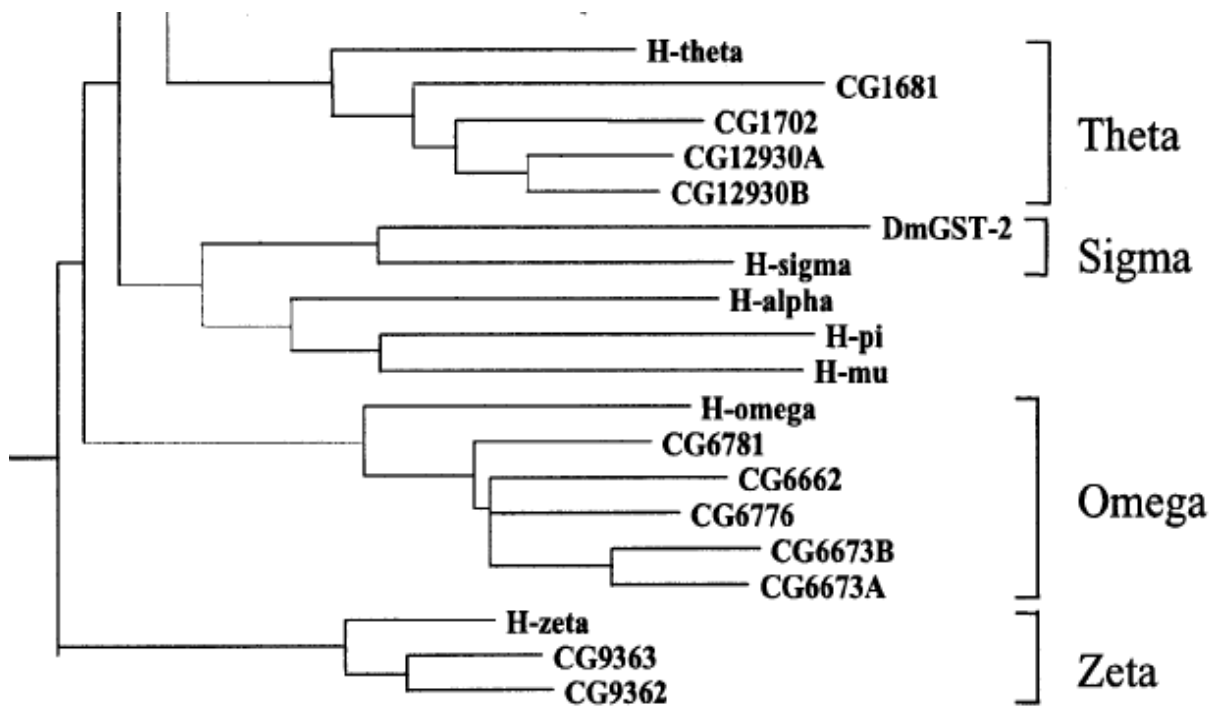

Figure 8-4: Dendrogram showing the relationship between D. melanogaster and human GSTs (Ranson et al., 2001).

Though only six GST classes: Sigma, Delta, Epsilon, Zeta, Theta and Omega have been identified in dipteran and other insect species, there is the possibility of the existence of additional or novel GSTs (Ding et al., 2003; Tu and Akgul, 2005). Thus, recently a group of cytosolic GST in A. gambiae were found and designated as 
unclassified GSTs (Ding et al., 2003). The identification of these unclassified GST orthologs in A. aegypti and their absence in D. melanogaster suggested that these GSTs are specific to mosquitoes and were named as Xi and Iota GSTs (Lumjuan et al., 2007).

In the present study, the reliability of consensus sequence- based approach for characterising L. cuprina GSTs has been tested. To confirm the identity of some spots as Mu GSTs, the location of the Mu-loop (a unique feature of Mu GST) on consensus sequence was first identified. The Mu-loop was identified through the alignment with the loop sequence of human Mu GST and was found to be very good match with an alignment score of 70 (Figure 8-5A). The identity of spot 5 as a $\mathrm{Mu}$ like GST was suggested as the score was 46 against the $\mathrm{Mu}$ consensus sequence (Figure 8-5 B). However, it is notable that whilst there are peptides that match sequences immediately adjacent to the Mu-loop, the loop itself has no match with spot 5 peptide sequences. To determine whether that is the case with all other identified Mu-like GSTs, the peptide sequences from all spots identified as Mu-like were aligned with the $\mathrm{Mu}$-loop containing $\mathrm{N}$-terminus of the $\mathrm{Mu}$ consensus sequence (Figure 8-5 C). Despite the good sequence similarity observed between the matched peptides and the consensus sequence, none of the peptides from these identified $\mathrm{Mu}-$ like GSTs matched with the Mu-loop sequence. Indeed, the longer matched peptide of spot 5 could be tentatively inferred to indicate deletion of the $\mathrm{Mu}$-loop as its $\mathrm{C}$ terminal amino acids (WLG) appear to match well the consensus sequence immediately following the Mu-loop motif. It could be that our Mu-like GSTs are not actually Mu GSTs, lacking the characteristic loop motif even though they share sufficient sequence similarity to be identified as such in this study. However, those identified Mu-like GSTs did not share any similarity with any other GST class, so 
there is the possibility that they might be novel GSTs. However, the probability that the consensus sequence-based approach is not giving reliable characterization for those spots cannot be excluded. This is one of the limitations of the approach which was taken into account for characterisation of GSTs.
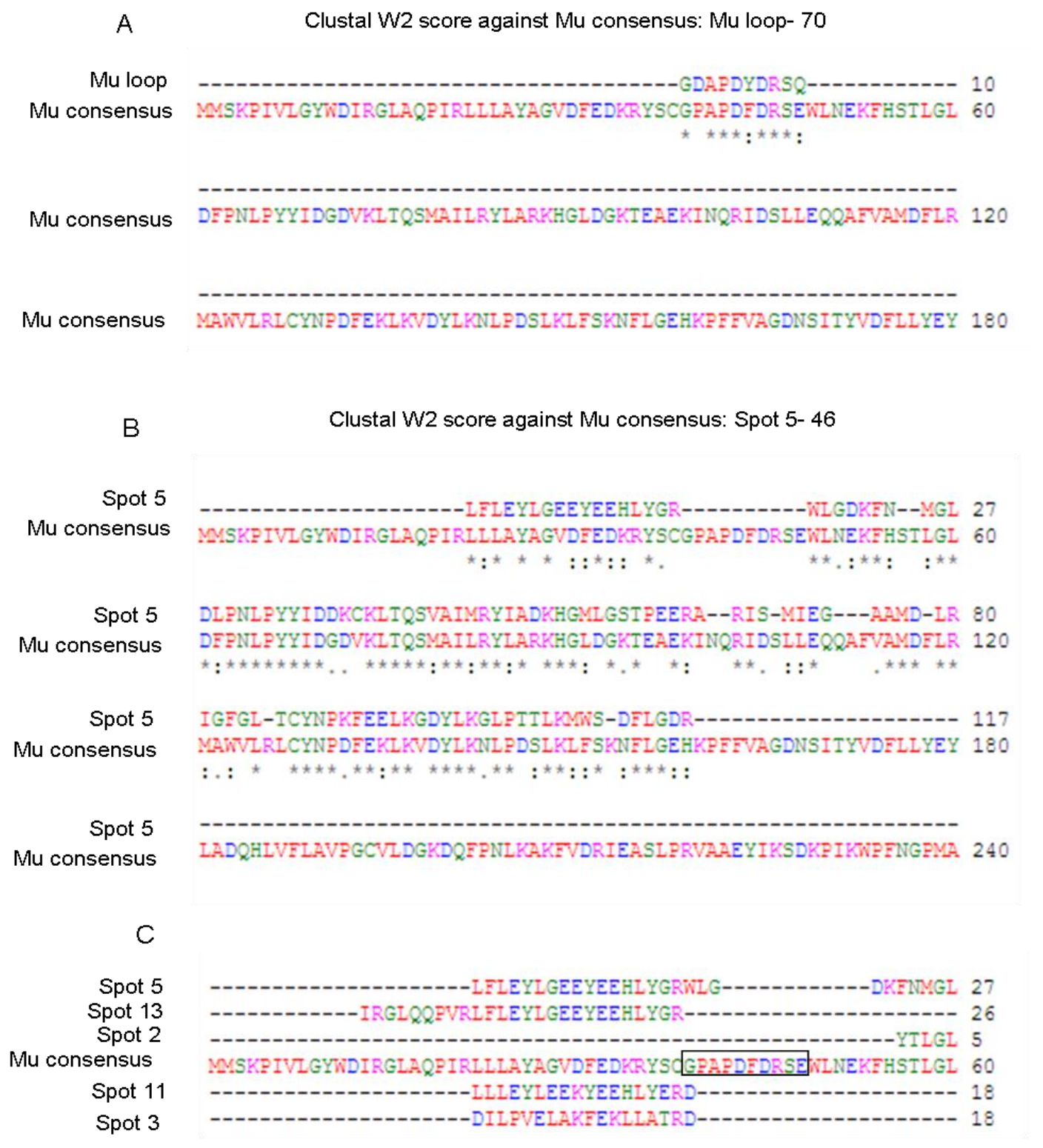

Figure 8-5: Alignment of various peptide sequences with Mu GST consensus sequence

(A) Mu-loop sequence; (B) L. cuprina Spot 5 peptide sequences matched against "Other Metazoa"; (C) Peptide sequences from other L. cuprina spots identified as $\mathrm{Mu}$, edited to include the position of the Mu-loop if present. Box represents the location identical to the Mu-loop. 


\subsubsection{A comparison of the GST proteomes of $D$. melanogaster and $L$. cuprina}

Other differences detected in chapter 4 based on proteomics, are that the high M.W. Delta (28 kDa; zone B in Figure 4-21) purified on DNP-GSH matrix were for the first time observed on the L. cuprina gel, yet were totally absent on the $D$. melanogaster gel and the Omega GSTs, though present on D. melanogaster gel, were never identified in L. cuprina. There are several possibilities: 1) the consensus sequence-based approach is characterising proteins wrongly; 2) L. cuprina and $D$. melanogaster express different GSTs as the proteomes are significantly different and 3) The L. cuprina GSTs are sufficiently different from those of D. melanogaster so that they bind differently to the affinity media.

Thus, in the absence of a genetic database of L. cuprina, this approach has its uncertainties. Our attempt to study the GSTs of an insect without a genetic database is exploratory and needs further confirmation. However, one can quickly determine which GSTs are being expressed for a given insect; the proteins produced being visualised on 2D gels. This approach of characterising the proteins may provide the useful starting points for the preliminary molecular biology study such as predicting the gene responsible for production of protein. An advantage of proteomics over genomics is that a genomics study cannot predict protein abundances or the posttranslational modification of proteins.

In Chapter 5, the changes in the GST proteome during the development and in the main body parts of an adult L. cuprina was studied. Irrespective of the identification of specific L. cuprina proteins, there were differences in proteins between eggs, larvae, pupae and adults evident from the 2D gels. The quantitative 
variations for Sigma GST and Mu-like GSTs between developmental stages were striking. The eggs lack Sigma GST whereas the adult expresses it significantly higher $(\mathrm{p}<0.05)$ than larvae and pupae. The significance of this phenomenon may lie in the involvement of Sigma GST in flight muscle of an adult and also in adult male reproductive tissues (Clayton et al., 1998). The Mu-like GSTs (Zone E in Figure 5-6) were totally absent in adult and abundantly $(\mathrm{P}<0.05)$ present in eggs compared to larvae and pupae. The role of these Mu-like GSTs in eggs is not clear. The pupal GSTs showed higher $(\mathrm{p}<0.05)$ total and specific activity towards CDNB and DCNB compared to other stages due to their involvement in biosynthesis and formation of adult tissues and in detoxification during this immobile stage.

Quantitative variation was also found in the expression of the GST proteome between the main body parts. Thorax produces a greater quantity of GSTs compared to the head and abdomen. Sigma GST was expressed in significantly higher $(\mathrm{p}<0.05)$ amount in thorax compared to head and abdomen. That again supports the role of Sigma GSTs in flight muscles. The high M.W. Delta GSTs in zone B Figure 5-20 (which resembled D. melanogaster CG17639) were present in greatest quantity in the head compared to thorax and abdomen. Literature suggests that in Manduca sexta, this type of GST was found in antennae and may play an important role in processing of odorant signals (Rogers et al., 1999) and this may be the case in $L$. cuprina as well. The specific activity of crude cytosolic GSTs of abdomen however was greater with most of the substrates including TNE, which suggests an important role in detoxification.

In Chapter 6, the variation in the GST proteome between OP susceptible and resistant strains of L. cuprina was examined. The interesting finding was that the 
resistant strain PY81 showed significantly $(\mathrm{p}<0.05)$ elevated expression of Epsilon and low M.W. Delta GSTs (23 kDa) and Mu-like GSTs (zone C and D in Figure 6-8). Along with these, the Rab proteins were also elevated in the resistant strain compared to susceptible strains NSW and CSIRO. The literature suggest that Rab proteins have been directly implicated in the regulation of vesicular transport and membrane traffic and localized to various compartments of both the biosynthetic/secretory and endocytic pathways of eukaryotic cells (Martinez and Goud, 1998). In the resistant mosquitoes, genes that are involved in protein biosynthesis, ubiquitination, and/or degradation, such as ribosomal proteins and ubiquitin dependent protein lysis in cell regulation and signal transduction, are over expressed. This suggests that the rapid regulation and turnover of proteins in the resistant mosquitoes are involved in response to resistance. Therefore, it is not surprising that genes involved in vesicular and molecular transport are also over expressed in the resistant mosquitoes (Liu et al., 2007). This could be the similar case in resistant strain PY81 of L. cuprina.

Two spots tentatively identified as Mu-like GSTs in zone D were absent in CSIRO compared to NSW. The high M.W. Delta GSTs $(28 \mathrm{kDa})$ were expressed in much lower quantity in PY81 compared to susceptible strains. The importance of this phenomenon is not known. The specific activity of the PY81 strain towards DCNB was significantly higher compared to susceptible strains indicating an important role of these GSTs in detoxification (Clark et al., 1986). However, the study of in vitro conjugation of insecticides by affinity-purified GSTs did not show any significant correlation between susceptible and resistant strains of $L$. cuprina but the likelihood is that resistance to organophosphorus insecticides in $L$. cuprina depends upon 
multiple factors. This is compatible with the literature as although(Wilson and Clark, 1996) have demonstrated a correlation between insecticide resistance in blowflies and GST activity, their data were extremely scattered, so that high resistance values could in particular cases be associated only with low GST values. In the present case, the resistant strain has expressed quantitatively higher levels of GSTs and these are active with model substrates, but it is possible that they are less active with organophosphates as substrates. Therefore, it is difficult in the present work to decide whether the elevated PY81 GSTs (Epsilon and Delta (23 kDa) and Mu-like GSTs) have any important role in insecticide resistance based on these in vitro assays. When the purified individual Delta, Sigma and partially purified DNP-GSH eluents were tested for the metabolism of methyl parathion, it suggested that the Delta and DNPGSH affinity-purified GSTs are much more active in metabolising the organophosphates compared to Sigma GST.

In Chapter 7, an attempt was made to determine the type of post-translational modification affecting D. melanogaster Delta GST. This study found no evidence for phosphorylation on Delta GSTs and this is supported by the literature (Zhai et al., 2008). LC-MS/MS and the de novo software PEAKS identified deamidation as a possible modification on some of the peptides of Delta GST. However, the deamidated peptides did not appear to clearly correlate with specific changes in $\mathrm{pI}$ on 2D gel of D. melanogaster Delta spots. The 3D structure of Delta GST was examined for buried asparagine or glutamine. If these buried residues, which might normally be expected to be protected, were deamidated, it would suggest that deamidation was an artefact resulting from the experimental processing. Since there is no buried asparagine or glutamine, it cannot be ruled out that deamidation is an 
artefact but neither can it be ruled in. However, the deamidated peptides AVINQR and AVGVELNKK are completely conserved in a large number of Delta GSTs from different insects. The potential significance of deamidation of these sequences, if any is not known. The literature suggests that Glu 96 of peptide AVINQR and Trp 64 do interact between subunits (Low et al., 2010). It is possible that this might have functional significance.

\subsection{Future Research}

The results presented in this thesis have shown that it may be possible to unravel the complex functions of the GST proteome, including a contribution to insecticide resistance in L. cuprina, using a combination of affinity purification and proteomics. Only a small part of this complex issue, the involvement of GSTs in resistance, using the laboratory susceptible and resistant strains of $L$. cuprina has been studied. However, what is needed is to carry out these experiments on larger numbers of field strains and correlate individual GSTs with resistance not only to organophosphates but also to the insecticides that are taking over from them. There is still a considerable need for future research in relation to the findings presented in this thesis.

\subsubsection{GST study at molecular genetics level}

There is no doubt that whole-genome sequencing of the L. cuprina will assist characterisation, revealing the range of the GST genes present in the genome and potentially providing information on their regulation. The tentative characterization of affinity-purified L. cuprina GSTs may then be confirmed or not from the corresponding genetic information. 
GSTs are well conserved at their N-termini, but are diverse at their C-termini (Ranson et al., 1997). The deduced $\mathrm{N}$-terminal amino acid sequencing of expressed $L$. cuprina proteins (GSTs) would also provide important information in the search for homology with other insect GSTs. The conserved N-terminal region can also be used to obtain cDNA sequences for different classes of GSTs from the L. cuprina. This may be amplified via a PCR reaction and cloned into a plasmid which can be expressed in E. coli to produce the recombinant protein. Recombinant proteins then can be characterized and their interaction with various insecticides can be studied. This approach has been used to study individual GSTs from Anopheles dirus (Ranson et al., 1997), Nilaparvata lugens (Vontas et al., 2002), Musca domestica (Wei et al., 2001), tarnished plant bug, Lygus lineolaris (Zhu et al., 2007) and Aedes aegypti (Lumjuan et al., 2005). In the present investigation, digests of some of the $L$. cuprina spots were sent to the Centre for Protein Research, Department of Biochemistry, University of Otago, Dunedin for de novo sequencing however very few peptides were sequenced from any protein spot and showed general, rather than class-specific similarity with GSTs when searched against D. melanogaster database. More extensive and successful de novo sequencing of individual $L$. cuprina protein spots would be useful to support the characterisation presented in this work. In the event of a complete genetic database being developed for $L$. cuprina, the microarray analysis of the GST transcriptome can also be useful to understand gene expression pattern and gene functions.

\subsubsection{Use of further GST purification techniques}

The combination of affinity matrices employed in this work successfully identified members of four major classes of GSTs (Sigma, Delta, Epsilon and Omega) 
in D. melanogaster however, the Theta and Zeta class of GSTs have not been seen on 2D gels. A significant fraction, 25-35\% of total activity towards CDNB, was not retained by either column (Table 4-3 and Table 4-4) suggesting the presence of GST isoenzymes in non-bound fractions. Further purification of non-bound fractions by using different specific affinity matrices or chromatofocusing and ion exchange chromatography should be used to isolate the additional GSTs. Different GST isoforms might also be separated by using longer affinity columns so that retardation as well as batch adsorption is used as the basis for isolation.

\subsubsection{Study of interaction of GSTs with insecticides}

The current work described the in vitro conjugation of only two organophosphate insecticides by L. cuprina GSTs. However the examination of conjugation with a wider range of insecticides would be useful. The in vivo direct interaction of insecticides with the enzyme can provide a better understanding of the significance of GSTs as a mechanism of insecticide detoxification. The insecticide can be applied to the adult insect or larval or pupal stage in order to determine the insecticide toxicity. Stage-dependent susceptibility of insects has been considered responsible for pest insect control failures in the past (Koehler et al., 1993). Insecticide metabolism by GSTs from different developmental stages has not been studied in the present work. Investigating the mechanisms underlying the difference in tolerance to insecticides among the developmental stages may further improve our understanding of insect defence to insecticides, as well as the proper use of insecticides in order to achieve a more successful chemical control of pest species. The reverse-phase HPLC method employed here successfully determined the loss of free insecticide. The formation of GSH-conjugates was not assayed. The enzyme- 
insecticide binding could also be studied by fluorescence spectrometry monitoring quenching of protein fluorescence by bound insecticide. Study of enzyme-insecticide binding during the reaction can be recorded. This enzyme-insecticide binding would be useful to see if there might be a significant "ligandin" factor in resistance. Enzyme kinetics can be performed using insecticides as substrates or inhibitors and direct measurement or characterisation of the GSH-conjugate can be studied.

\subsubsection{Comparative developmental study of $D$. melanogaster}

The developmental changes in the GST proteome of $L$. cuprina revealed interesting findings. Differential expression of GSTs was found in egg, larvae, pupae and adult stages of L. cuprina. A comparative developmental study of $D$. melanogaster could provide valuable additional information on the role of each GST isoenzyme (due to availability of its gene profile) and help to understand the changes in L. cuprina.

\subsubsection{Post-translational modification of Delta GSTs}

In the present investigation, it was confirmed that the D. melanogaster D1 GST is not phosphorylated and deamidation of Delta GST has been proposed. The reason for the change in $\mathrm{pI}$ on 2D gels has not been unambiguously determined and therefore its significance remains unknown. To understand further, the Delta GST spots on the 2D gel can be in-gel digested by specific proteases like Glu-C or Asp-N which cut specifically at only one amino acid, C-terminal of glutamic acid (E) or N-terminal of aspartic acid (D) respectively. The theoretical number of peptides after cleavage with Glu-C or Asp-N can be predicted from the sequence of GST. If deamidation has occurred on glutamine or on asparagine and they convert to glutamic acid or aspartic 
acid respectively, one would obtain additional cleavages which could be detected by LC-MS/MS. A gel-free approach could also be applied whereby affinity-purified GSTs can be digested by proteases directly and be subjected to tandem MS analysis. 


\section{Conclusion}

The aim of this study was to investigate how proteomics could be applied to the study of the role of $L$. cuprina GSTs in insecticide resistance. The primary objectives were to purify and identify D. melanogaster and L. cuprina GSTs, to investigate the expression of GSTs in different developmental stages of L. cuprina in order to understand their significance in detoxification, to compare the GST proteome between OP susceptible and resistant strain of $L$. cuprina and to test their ability to metabolise insecticides in vitro and finally, to determine the posttranslational modification of Delta GST.

The research undertaken has revealed notable similarities and dis-similarities between $D$. melanogaster and L. cuprina GST proteomes as visualised on 2D gels. Four major insect GST classes Sigma, Delta, Epsilon and Omega were identified in D. melanogaster whereas Omega GSTs were not identified in L. cuprina. Differences were observed between the GST proteomes in different developmental stages and in different body parts of L. cuprina. Sigma GST was present in the highest amount in adults whereas Mu-like GSTs were most abundant in eggs. The comparison of GST proteomes of resistant and susceptible strains showed the elevation of GST expression in resistant insects but there was no significant difference found between the strains in metabolism of two organophosphate insecticides in vitro. The post-translational modification study suggested that deamidation is a possible modification of D1 GST however, the change in pI on 2D gels could be explained in these terms but the assignment of actual pI values remains tentative. 
Along the way it was also discovered that our developed consensus sequencebased approach for the identification of GSTs from an insect with no genetic database appears reliable for strongly conserved proteins which may be useful for subsequent molecular biology studies. The presence of Mu-like GSTs in L. cuprina and their abundance in eggs and total absence in adult is of interest as is the association of high M.W. Delta isoforms with the mobile life stages. The elevation of the GST proteome in a resistant strain but absence of a higher capability to metabolise insecticides in vitro is also an interesting finding. The isolation by the DNP-GSH matrix and higher expression of Rab proteins in resistant insects suggest a possible functional association between these proteins and GSTs. 


\section{Appendices}

\subsection{Reagent preparation}

\subsection{Tris-HCl, pH 8.8}

Tris base $(54.54 \mathrm{~g})$ was dissolved in $160 \mathrm{ml}$ deionised water and the $\mathrm{pH}$ was adjusted to 8.8 with $\mathrm{HCl}$. The solution was made $300 \mathrm{ml}$ with deionised water and stored at $4^{\circ} \mathrm{C}$

\subsection{Tris-HCl, pH 6.8}

Tris base $(6 \mathrm{~g})$ was dissolved in $60 \mathrm{ml}$ deionised water and the $\mathrm{pH}$ was adjusted to 6.8 with $\mathrm{HCl}$. The solution was made $100 \mathrm{ml}$ with deionised water and stored at $4^{\circ} \mathrm{C}$

\section{$12 \%$ separating gel}

To prepare $10 \mathrm{ml}$ of $12 \%$ gel: $4 \mathrm{ml}$ of $30 \%$ Acrylamide/Bis (BioRad) $2.5 \mathrm{ml} 1.5 \mathrm{M}$ Tris-HCl, $\mathrm{pH}$ 8.8, $0.1 \mathrm{ml}$ 10\% SDS, $3.35 \mathrm{ml}$ deionised water were mixed gently and degassed under vacuum for at least 15 minutes. Then, 5-10 $\mu$ TEMED and 50 $\mu 110$ $\%$ APS were mixed and the mixture was poured into the electrophoresis plates for polymerisation.

\section{$4 \%$ stacking gel}

To prepare $10 \mathrm{ml}$ of $12 \%$ gel: $1.33 \mathrm{ml}$ of $30 \%$ Acrylamide/Bis, $2.5 \mathrm{ml} 0.5 \mathrm{M}$ Tris$\mathrm{HCl}, \mathrm{pH} 6.8,0.1 \mathrm{ml} 10 \% \mathrm{SDS}, 6.1 \mathrm{ml}$ deionised water was mixed gently and degassed under vacuum for at least 15 minutes. Then, 5-10 $\mu$ TEMED and 50 $\mu 1$ $10 \%$ APS was mixed and mixture was poured into the electrophoresis plates on top of the polymerised separating gel.

\section{SDS sample buffer}

The sample buffer consisted of $62.5 \mathrm{mM}$ Tris $\mathrm{HCl}, \mathrm{pH} \mathrm{6.8,20 \%} \mathrm{glycerol,} \mathrm{2 \%} \mathrm{SDS}$ and $5 \% \beta$ mercaptoethanol. To prepare sample in sample buffer, precipitated protein was diluted with sample buffer in at least 1:4 ratio. Then the sample was heated at $95^{\circ} \mathrm{C}$ for $4-5$ minutes. 


\section{SDS electrophoresis running buffer}

Running buffer was prepared by dissolving $15.1 \mathrm{~g}$ Tris, $5 \mathrm{~g}$ SDS and $72.1 \mathrm{~g}$ glycine in 5 litre deionised water. The $\mathrm{pH}$ of the buffer was adjusted.

\section{Overlay solution}

$200 \mu \mathrm{l}$ of $0.1 \%(\mathrm{w} / \mathrm{v})$ SDS solution, It was used to overlay the separating gel while polymerising.

\section{Colloidal Coomassie Blue G-250 stain}

The stain $(100 \mathrm{ml})$ was prepared in two steps. First to a final concentration of $0.075 \%$ (w/v) of Coomassie Blue G-250 was dissolved in $34 \mathrm{ml}$ of methanol. The second solution was prepared by adding $17 \mathrm{~g}$ of ammonium sulphate and $2 \mathrm{ml}$ of phosphoric acid in $66 \mathrm{ml}$ of MQ water. Both the solutions were stirred for about 5 minutes to fully dissolve and finally mixed slowly. The stain was prepared freshly to avoid the loss of sensitivity over the period.

\section{IPG strip rehydration buffer}

A solution containing $8 \mathrm{M}$ Urea, $2 \%$ (w/v) CHAPS, $30 \mathrm{mM}$ thiourea, $15 \mathrm{mM}$ DTT and 2\% IPG Buffer $\mathrm{pH}$ 3-10 and traces of bromophenol blue in deionised water was prepared and stored at $-20^{\circ} \mathrm{C}$.

\section{IPG strip equilibration solutions}

Stock solution- $50 \mathrm{mM}$ Tris $\mathrm{HCl}, \mathrm{pH} 8.0 ; 6 \mathrm{M}$ Urea; 30\% (w/v) glycerol and 2\% $(w / v)$ SDS

Solution $1-0.25 \%(w / v)$ DTT in stock solution

Solution 2- $4.5 \%(\mathrm{w} / \mathrm{v})$ iodoacetamide in stock solution + traces of bromophenol blue 


\subsection{Enzyme assay purification parameters and calculation}

\section{Equation 1:}

Catalytic activity $=(\Delta \mathrm{A} \times \mathrm{V} \times 1000) /(\varepsilon \times v \times \Delta \mathrm{d})(\mu \mathrm{mol} / \mathrm{min}$ or Units $)$

$\Delta \mathrm{A}$ is absorbance change; $\varepsilon$ is $\mathrm{L} \mathrm{x} \mathrm{mmol}^{-1} \mathrm{x} \mathrm{cm}^{-1} ; \mathrm{V}$ is assay volume in $\mathrm{L} ; \mathrm{v}$ is of sample volume; $\Delta \mathrm{d}$ in $\mathrm{cm}$; $\mathrm{t}$ in $\mathrm{min}$

\section{Equation 2:}

Catalytic activity $=(\Delta \mathrm{A} \times \mathrm{V}) /(\varepsilon \times v \times \Delta \mathrm{d} \times 1000)(\mu \mathrm{mol} / \mathrm{min} / 1$ or Units $/ \mathrm{l})$

$\Delta \mathrm{A}$ is absorbance change; $\varepsilon$ is $\mathrm{L} \mathrm{x} \mathrm{mmol}^{-1} \mathrm{x} \mathrm{cm}^{-1} ; \mathrm{V}$ is assay volume in $\mathrm{L} ; \mathrm{v}$ is of sample volume; $\Delta \mathrm{d}$ in $\mathrm{cm} ; \mathrm{t}$ in $\min$

\section{Equation 3:}

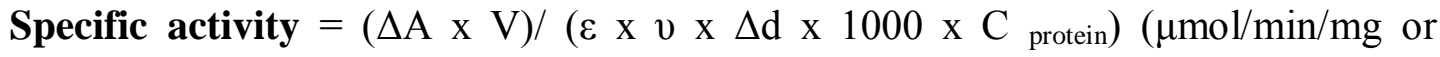
Units/mg)

$\Delta \mathrm{A}$ is absorbance change; $\varepsilon$ is $\mathrm{L} \mathrm{x} \mathrm{mmol}^{-1} \mathrm{x} \mathrm{cm}^{-1} ; \mathrm{V}$ is assay volume in $\mathrm{L} ; \mathrm{v}$ is of sample volume; $\mathrm{d}$ in $\mathrm{cm}$; $\mathrm{t}$ in $\mathrm{min} ; \mathrm{C}$ is protein concentration in $\mathrm{mg} / \mathrm{l}$ )

\section{Equation 4:}

Fold purification $=$ Specific activity of affinity-purified fraction/ Specific activity of crude enzyme

\section{Equation 5:}

$\%$ yield $=($ Total catalytic activity of purified fraction $/$ Total activity of crude enzyme) x 100 
10.3 Mass spectra and identification of spots using PROFOUND

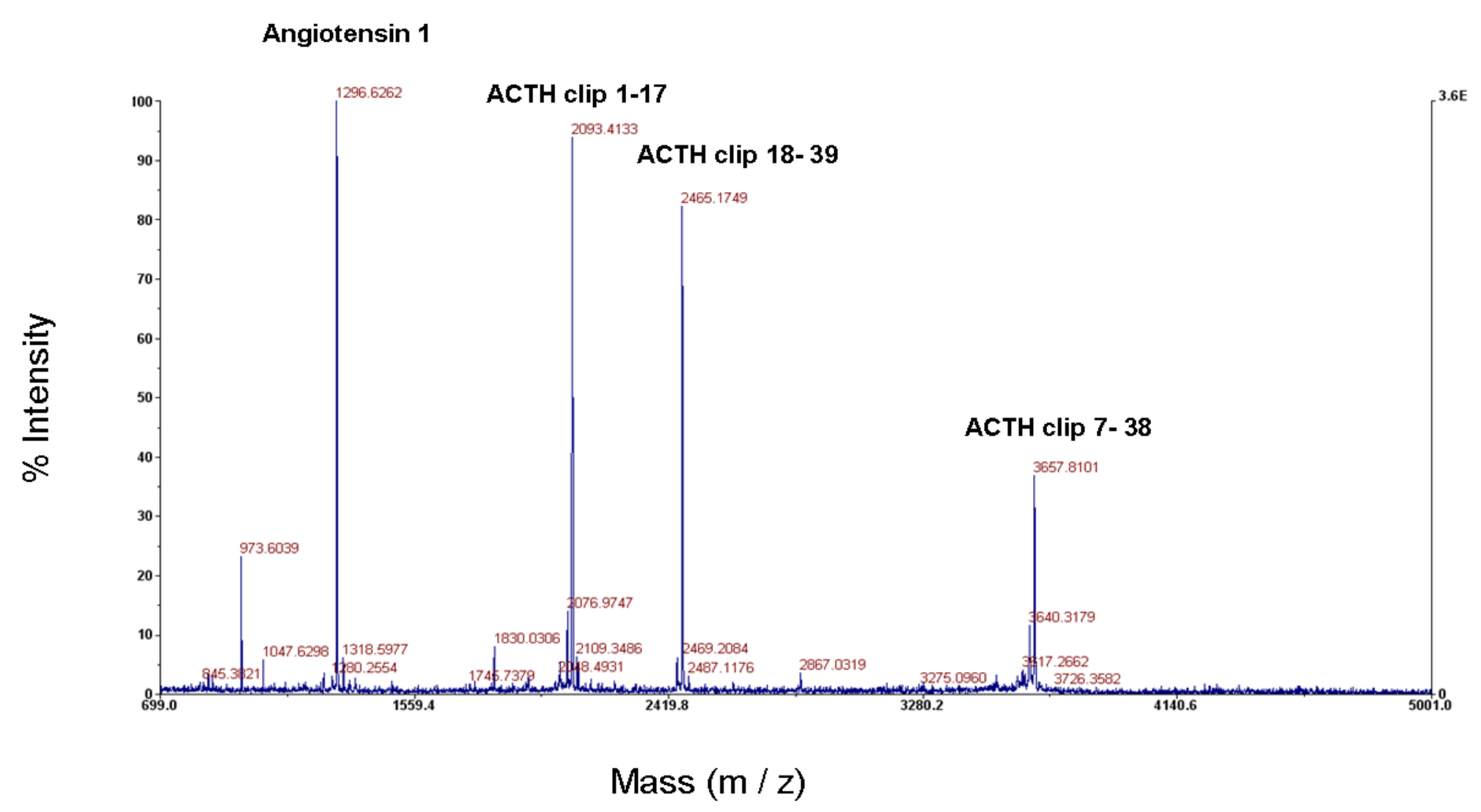

Figure 10-1: A MALDI-TOF spectrum of the external standard CalMix 2 
10.3.1 Spectra of spots labelled in Figure 4-17
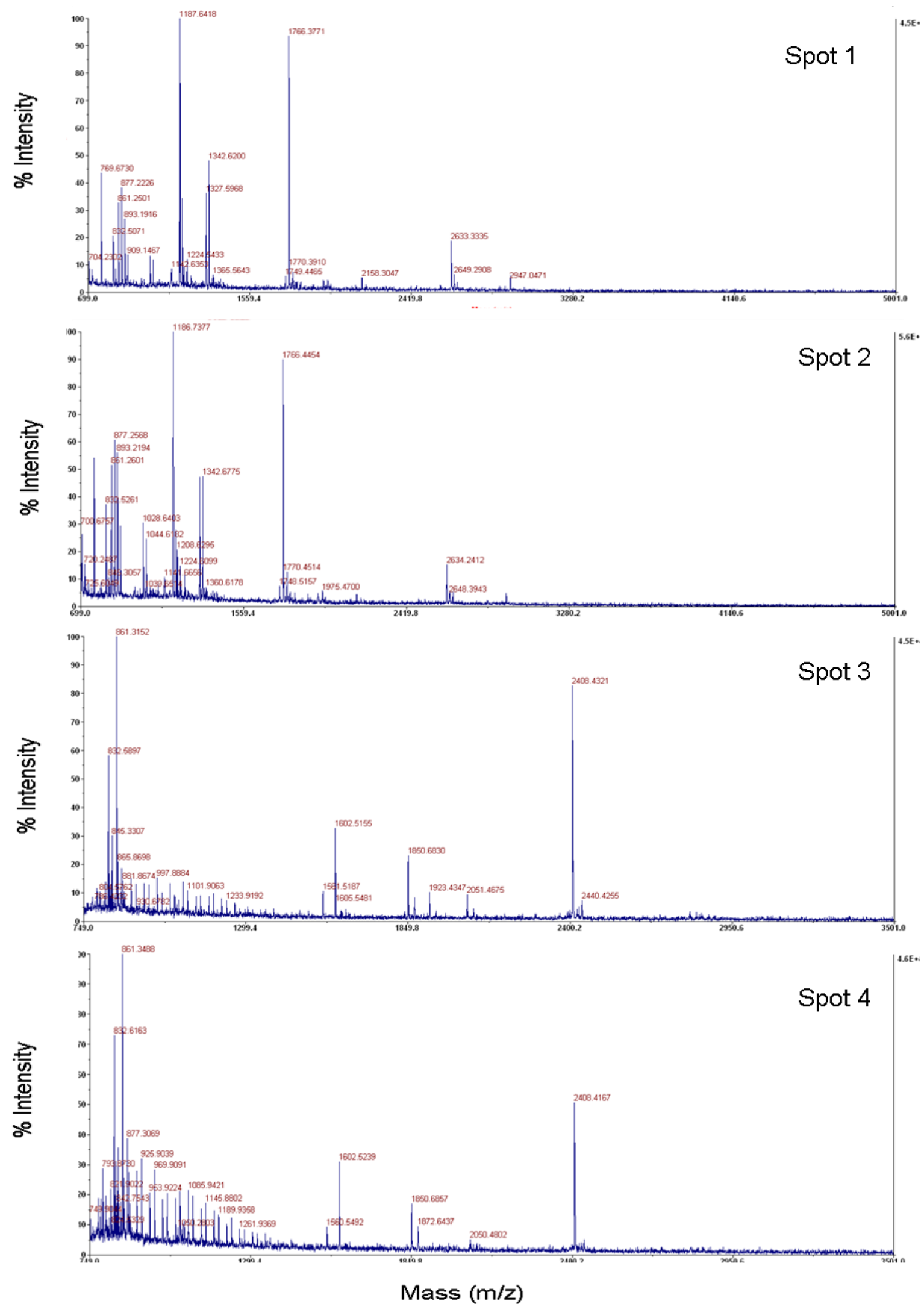

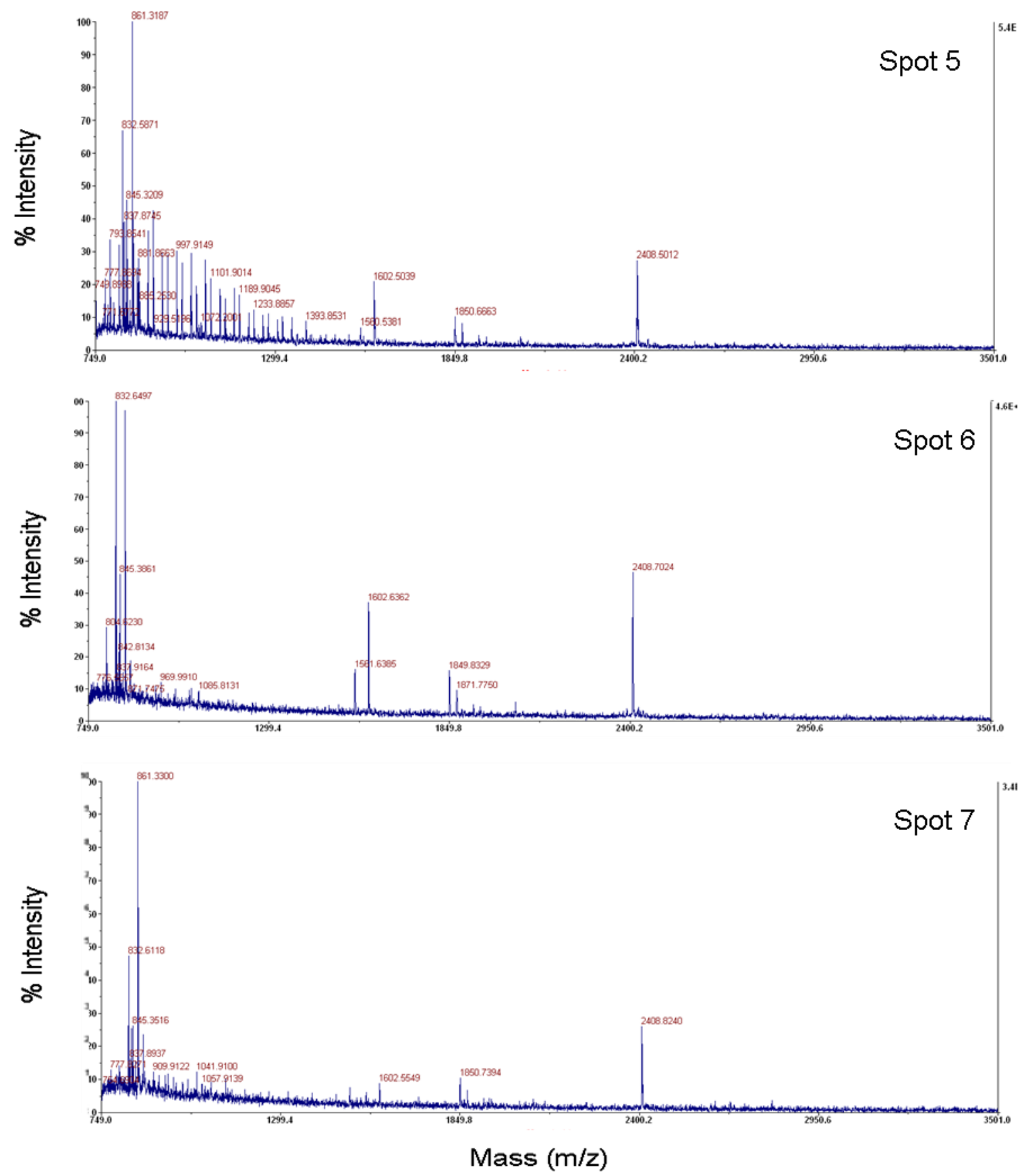

Figure 10-2: MALDI-TOF spectra of the GSH affinity-purified D. melanogaster spots on 2D gel labelled in Figure 4-17 


\subsubsection{Identification of spots labelled in Figure 4-17 using PROFOUND}

\begin{tabular}{|c|c|c|c|}
\hline Spot & 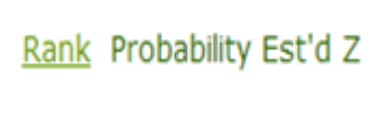 & $\begin{array}{l}\text { Protein Intormation and Sequence Analyse } \\
\qquad \text { Tools }(T)\end{array}$ & $\%$ pI $\quad \mathrm{kDa}$ \\
\hline
\end{tabular}

Measured Avg/ Computed Brror Desidues Missed

Mass (M) Mono Mass $\mathbf{D}_{(\mathrm{Da})}$ Start To Cut Peptide sequence

1

gi|24654347|ref|NP_725653.1| glutathione S

$+1 \quad 1.0 \mathrm{e}+000 \quad 2.43$ transferase S1, isoform A [Drosophila

$43 \quad 4.6 \quad 27.650$

melanogaster]

\begin{tabular}{|c|c|c|c|c|c|c|c|}
\hline $\begin{array}{l}699.304 \\
768.356\end{array}$ & $\begin{array}{l}\mathbf{M} \\
\mathbf{M}\end{array}$ & $\begin{array}{l}699.391 \\
768.449\end{array}$ & $\begin{array}{l}-0.087 \\
-0.093\end{array}$ & $\begin{array}{r}244 \\
59\end{array}$ & $\begin{array}{r}249 \\
65\end{array}$ & $\begin{array}{l}0 \\
0\end{array}$ & $\begin{array}{l}\text { RPUTEV } \\
\text { ALAEPLR }\end{array}$ \\
\hline 1027.463 & $\mathbf{M}$ & 1027.523 & -0.060 & 107 & 115 & 0 & VHQSISMAR \\
\hline 1043.429 & $\mathbf{M}$ & 1043.518 & -0.089 & 107 & 115 & 0 & $\begin{array}{l}\text { VHQSISMAR } \\
\text { (1) +0 Q }\end{array}$ \\
\hline 1183.532 & $\mathbf{M}$ & 1183.624 & -0.092 & 106 & 115 & 1 & RUHQS ISMAR \\
\hline 1185.599 & $\mathbf{M}$ & 1185.639 & -0.040 & 216 & 225 & 0 & DLLEPYPALR \\
\hline 1199.545 & $\mathbf{M}$ & 1199.619 & -0.074 & 106 & 115 & 1 & $\begin{array}{l}\text { RUHQS ISMAR } \\
\text { (1) +0 @M: }\end{array}$ \\
\hline 1323.689 & $\mathbf{M}$ & 1323.739 & -0.0 .50 & 226 & 238 & 0 & GOUDAVNALEPIK \\
\hline 1326.641 & $\mathbf{M}$ & 1326.729 & -0.089 & 239 & 249 & 1 & ADIERKRPVTEV \\
\hline 1341.655 & $\mathrm{M}$ & 1341.740 & -0.085 & 215 & 225 & 1 & PD LLE PYPALR \\
\hline 1747.844 & $\mathbf{M}$ & 1747.887 & -0.044 & 148 & 162 & 1 & IAUUSYEP EDE IREK \\
\hline 1765.741 & $\mathbf{M}$ & 1765.794 & -0.053 & 66 & 79 & 0 & YL FAYGNQ EYEDUR \\
\hline 2946.528 & $\mathbf{M}$ & 2946.442 & 0.086 & 120 & 145 & 0 & TVGLCGAT PUEDLQI \\
\hline
\end{tabular}

2

gi|24654347|ref|NP_725653.1| glutathione S

$+1 \quad 1.0 e+000 \quad 2.43$ transferase S1, isoform A [Drosophila

$47 \quad 4.6 \quad 27.650$

melanogaster]

$\begin{array}{rrrrrrll}768.335 & \text { M } & 768.449 & -0.114 & 59 & 65 & 0 & \text { ALAEPLR } \\ 1027.416 & \text { M } & 1027.523 & -0.107 & 107 & 115 & 0 & \text { VHQSISMAR } \\ 1185.513 & \text { M } & 1185.639 & -0.125 & 216 & 225 & 0 & \text { DLLEPYPALR } \\ 1417.260 & \text { M } & 1417.703 & -0.443 & 48 & 58 & 0 & \text { HSYTLFYFNVK } \\ 1747.825 & \text { M } & 1747.975 & -0.150 & 164 & 178 & 0 & \text { LVTLNAEVIPFYLEK } \\ 1747.825 & \text { M } & 1747.887 & -0.062 & 148 & 162 & 1 & \text { IAVVSYEPEDEIKEK } \\ 1765.554 & \text { M } & 1765.794 & -0.240 & 66 & 79 & 0 & \text { YLFAYGNQEYEDVR } \\ 2724.795 & \text { M } & 2724.342 & 0.453 & 83 & 106 & 1 & \text { DEWPALKPTMPMGQMPVLEVD } \\ 2946.063 & \text { M } & 2946.442 & -0.379 & 120 & 145 & 0 & \text { TVGLCGATPWEDLQIDIVVDT }\end{array}$




\begin{tabular}{lccccc}
\hline Spot & Rank Probability Est'd Z & $\begin{array}{c}\text { Protein Intormation and Sequence Analyse } \\
\text { No. }\end{array}$ & Tools (T) & pI kDa & $R$ \\
N & & &
\end{tabular}

$\begin{array}{ccccc}\text { Measured Avg/ Computed } & \text { Brror } & \text { DResidues Missed } \\ \text { Mass(M) Mono } & \text { Mass } & \text { प(Da) } & \text { Start } & \text { To Cut }\end{array}$

3

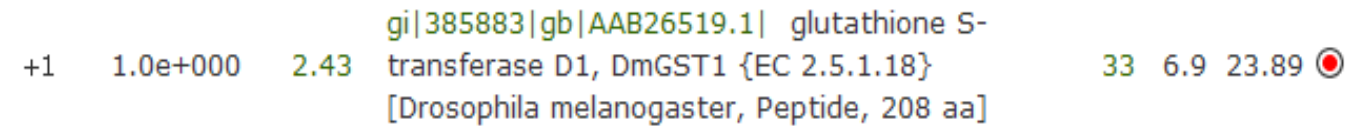

$\begin{array}{rrrrrrll}764.339 & \text { M } & 764.410 & -0.071 & 14 & 20 & 0 & \begin{array}{l}\text { SVIMTAR } \\ (1)+0 @ M ;\end{array} \\ 1061.607 & M & 1061.612 & -0.005 & 67 & 75 & 0 & \text { AIQVYLVEK } \\ 1559.799 & M & 1559.707 & 0.092 & 1 & 13 & 0 & \text { VDFYYLPGSSPCR } \\ 1849.149 & M & 1849.045 & 0.104 & 30 & 45 & 0 & \text { LLNLQAGEHLKPEFLK } \\ 1921.979 & M & 1921.866 & 0.114 & 189 & 204 & 0 & \text { VTPGWEENWAGCLEFK } \\ 1977.236 & M & 1977.140 & 0.096 & 29 & 45 & 1 & \text { KLLNLQAGEHLKPEFLK } \\ 2050.073 & M & 2049.961 & 0.113 & 188 & 204 & 1 & \text { KVTPGWEENWAGCLEFK } \\ 2050.073 & M & 2049.961 & 0.113 & 189 & 205 & 1 & \text { VTPGWENWAGCLEFKK } \\ 2407.349 & M & 2407.227 & 0.121 & 46 & 66 & 0 & \text { INPQHTIPTLVDNGFALWESR }\end{array}$

gi|194742608|ref|XP_001953793.1| GF17052

[Drosophila ananassae]

gi|17737923|ref|NP_524326.1| glutathione S

- transferase D1, isoform A [Drosophila

melanogaster]

gi|385883|gb|AAB26519.1| glutathione S-

- $\quad$ transferase D1, DmGST1 \{EC 2.5.1.18\}

[Drosophila melanogaster, Peptide, 208 aa]

\begin{tabular}{|c|c|c|c|c|c|c|c|}
\hline 1061.409 & $\mathrm{M}$ & 1061.612 & -0.203 & 68 & 76 & 0 & AIQVYLVER \\
\hline 1848.692 & M & 1849.045 & -0.353 & 31 & 46 & 0 & LLNLQAGEHLKPEFLK \\
\hline 1921.536 & M & 1921.866 & -0.330 & 190 & 205 & 0 & VTPGWEENWAGCLEFK \\
\hline 1976.768 & M & 1977.140 & -0.372 & 30 & 46 & 1 & KLLNLQAGEHLKPEFLK \\
\hline 2049.583 & M & 2049.961 & -0.377 & 189 & 205 & 1 & KVTPGWEENWAGCLEFK \\
\hline 2049.583 & M & 2049.961 & -0.377 & 190 & 206 & 1 & VTPGWEENWAGCLEFKK \\
\hline 2406.868 & M & 2407.227 & -0.359 & 47 & 67 & 0 & INPQHTIPTLVDNGFALWESR \\
\hline 2420.768 & M & 2421.152 & -0.384 & 1 & 21 & 1 & MVDFYYLPGSSPCRSVIMTAK \\
\hline
\end{tabular}




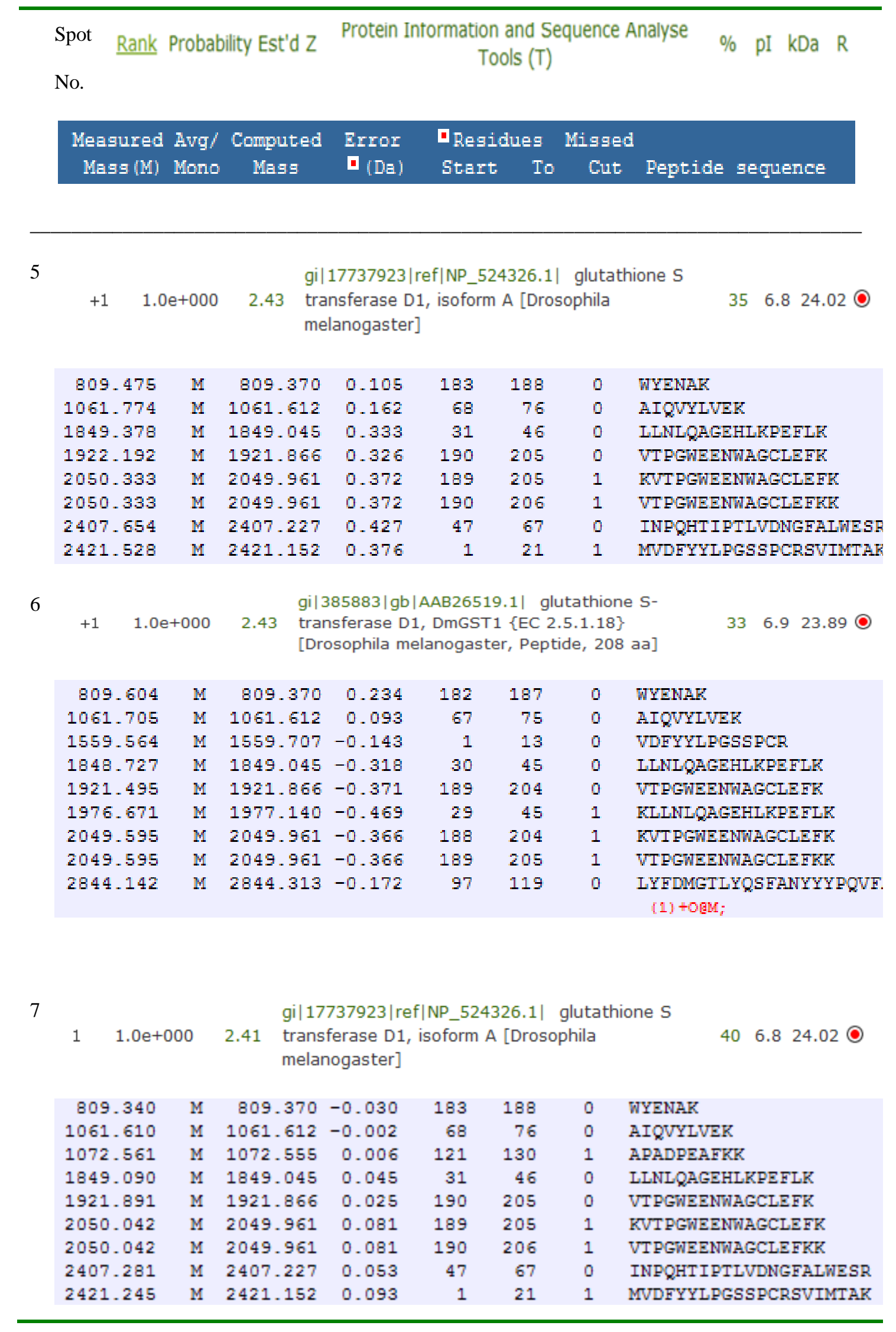




\subsubsection{Spectra of spots labelled in Figure 4-18}
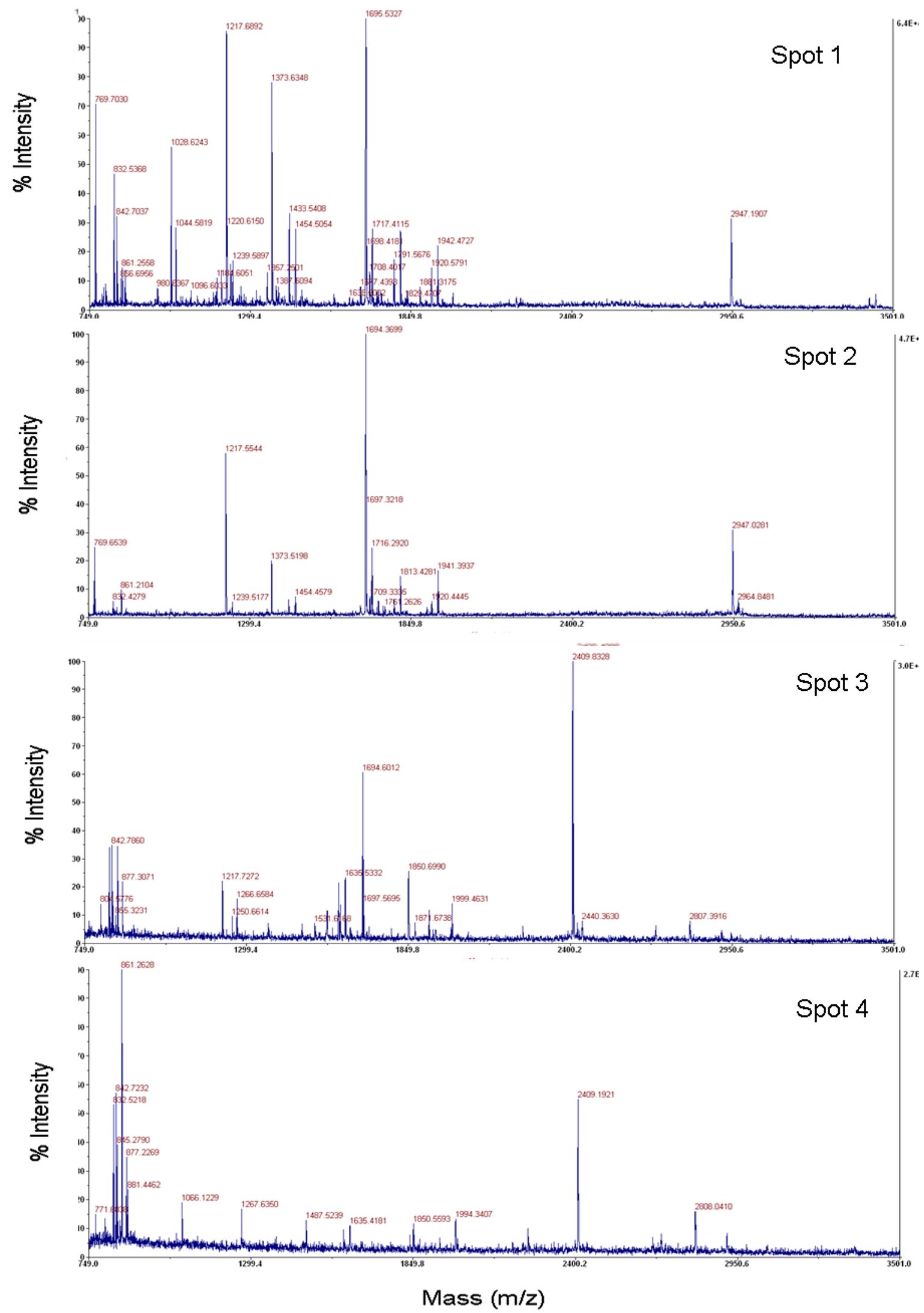

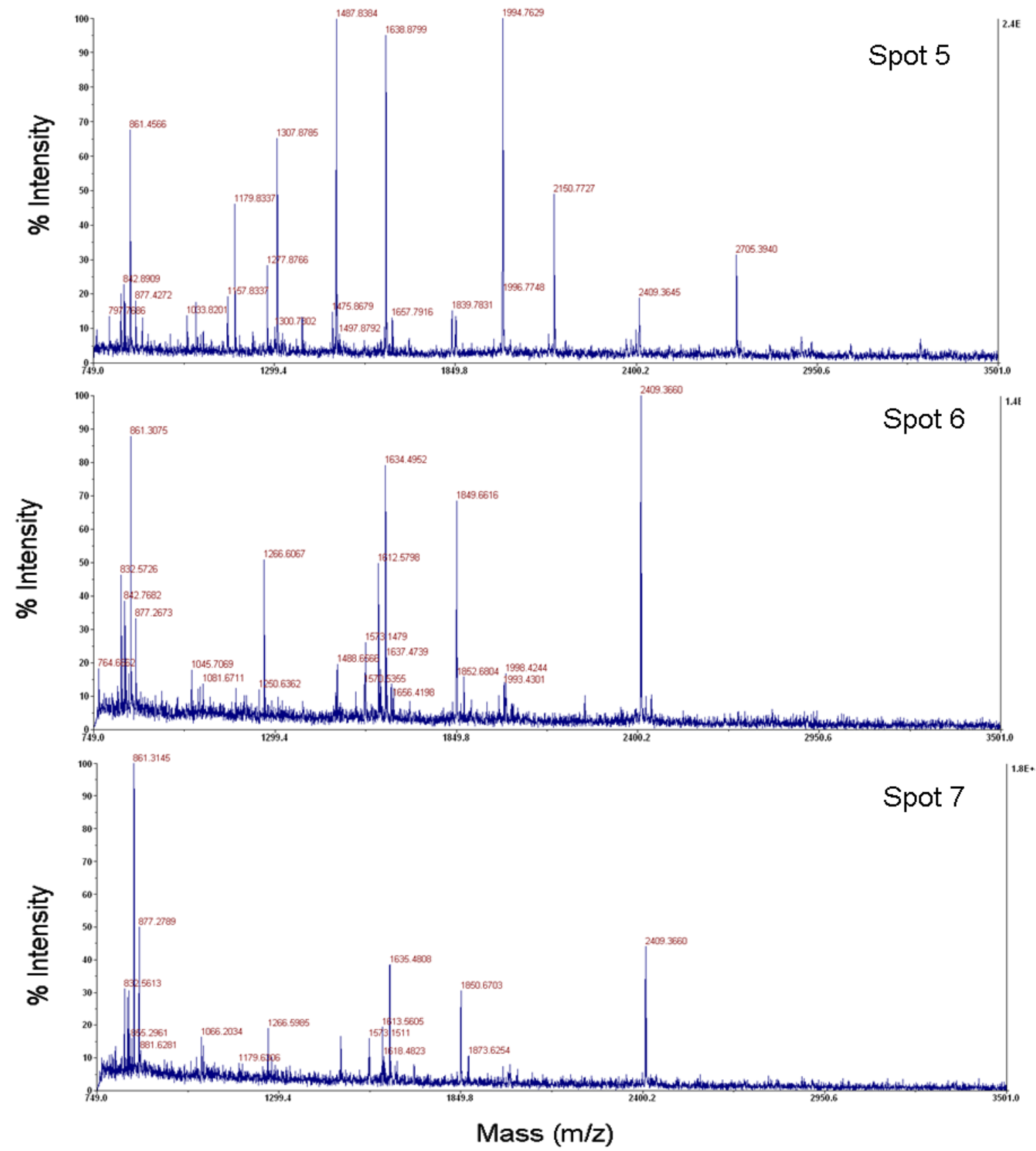

Figure 10-3: MALDI-TOF spectra of the GSH affinity-purified L. cuprina spots on 2D gel labelled in Figure 4-18 


\subsubsection{Identification of spots labelled in Figure 4-18 using PROFOUND}

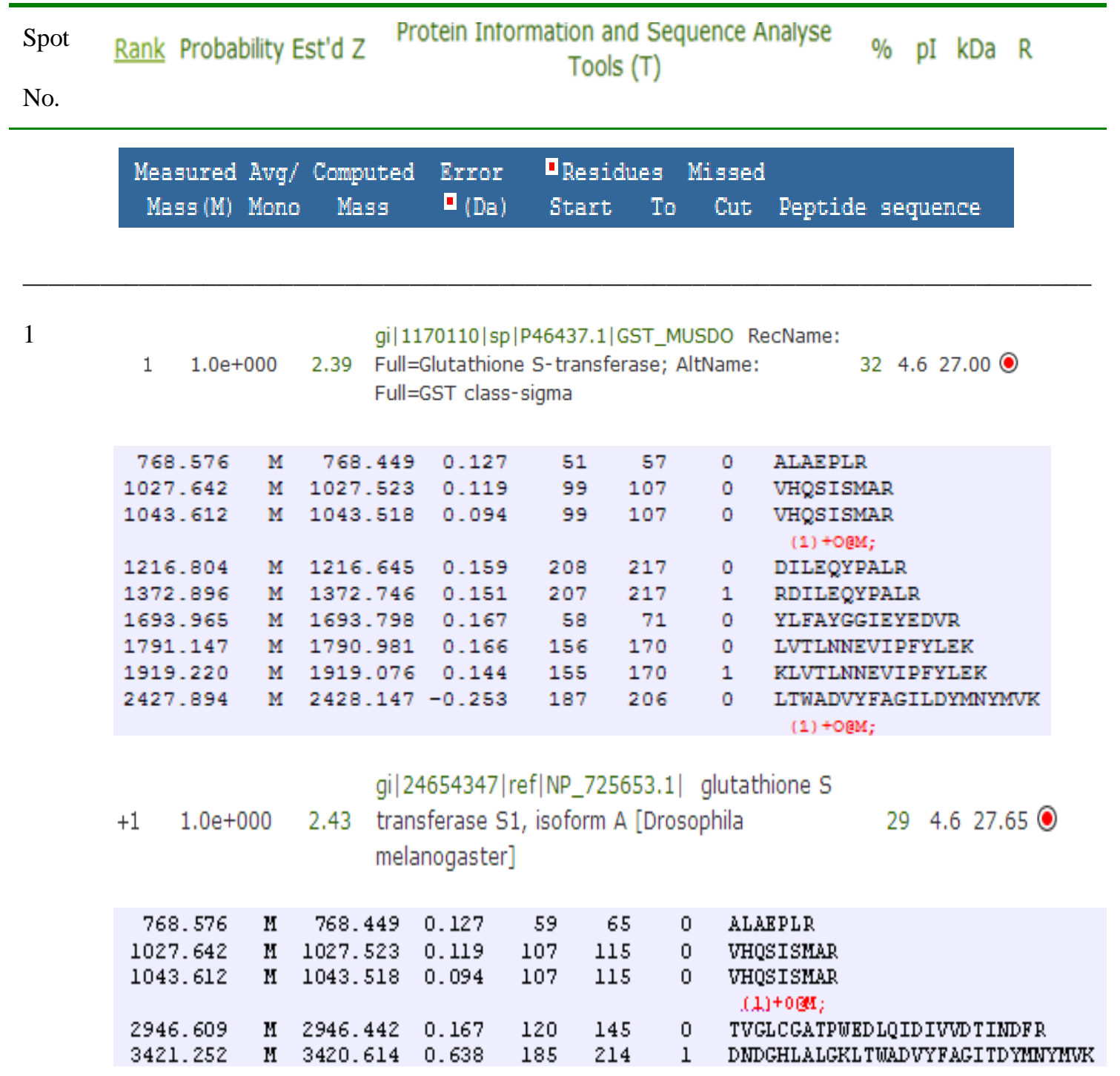

2 gi|1170110|sp|P46437.1|GST_MUSDO RecName:
$1 \quad 1.0 \mathrm{e}+000 \quad 2.18$ Full=Glutathione S-transferase; AltName: $\quad 204.627 .000$ Full=GST class-sigma

$\begin{array}{rrrrrrll}768.456 & \text { M } & 768.449 & 0.007 & 51 & 57 & 0 & \text { ALAEPLR } \\ 1216.687 & M & 1216.645 & 0.042 & 208 & 217 & 0 & \text { DILEQYPALR } \\ 1372.793 & \text { M } & 1372.746 & 0.048 & 207 & 217 & 1 & \text { RDILEQYPALR } \\ 1693.901 & \text { M } & 1693.798 & 0.103 & 58 & 71 & 0 & \text { YLFAYGGIEYEDVR } \\ 1791.097 & M & 1790.981 & 0.116 & 156 & 170 & 0 & \text { LVTLNNEVIPFYLEK } \\ 1919.213 & M & 1919.076 & 0.137 & 155 & 170 & 1 & \text { KLVTLNNEVIPFYLEK }\end{array}$




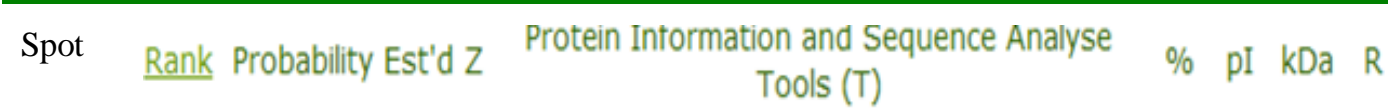 \\ No.}

$\begin{array}{ccccc}\text { Measured Avg/ Computed } & \text { Brror } & \text { DResidues Missed } \\ \text { Mass (M) Mono Mass } & \mathbf{D}(\mathrm{Da}) & \text { Start } & \text { To Cut Peptide sequence }\end{array}$

3

gi| $1346214 \mid$ sp $\mid$ P42860.2|GSTT1_LUCCU
RecName: Full=Glutathione S-transferase 1-1; $\quad 2.0 \mathrm{e}+000 \quad 2.43 \quad \begin{array}{ll}\text { Re.3 } 23.80 \\ \text { AltName: Full=GST class-theta }\end{array}$

\begin{tabular}{|c|c|c|c|c|c|c|c|}
\hline 1204.835 & M & 1204.590 & 0.245 & 79 & 88 & 1 & NDSLFPKCPK \\
\hline 1249.708 & M & 1249.605 & 0.104 & 97 & 106 & 0 & LYFDMGTLYK \\
\hline 1265.696 & M & 1265.600 & 0.096 & 97 & 106 & 0 & $\begin{array}{l}\text { LYFDMGTLYK } \\
\text { (1) +O@M; }\end{array}$ \\
\hline 1591.386 & M & 1591.679 & -0.293 & 1 & 13 & 0 & $\begin{array}{l}\text { MDFYYLPGSAPCR } \\
\text { (1) +OeM; }\end{array}$ \\
\hline 1611.891 & M & 1611.760 & 0.131 & 107 & 119 & 0 & SFADYYYPQIFAK \\
\hline 1849.188 & M & 1849.045 & 0.142 & 30 & 45 & 0 & LLNLQAGEHLKPEFLK \\
\hline 1998.067 & M & 1997.882 & 0.185 & 188 & 204 & 0 & TVAPGFDENWEGCLEFK \\
\hline 2408.451 & M & 2408.211 & 0.240 & 46 & 66 & 0 & INPQHTIPTLVDGDFALWESR \\
\hline
\end{tabular}

4

\begin{tabular}{|c|c|c|c|}
\hline $1.0 \mathrm{e}+000$ & 2.43 & $\begin{array}{l}\text { gi|409146|gb|AAA29287.1| glutathione } \\
\text { transferase [Lucilia cuprina] }\end{array}$ & 386.123 .810 \\
\hline - & - & $\begin{array}{l}\text { gi| } 1346214 \mid \text { sp |P42860.2|GSTT1_LUCCU } \\
\text { RecName: Full=Glutathione S- transferase 1-1; } \\
\text { AltName: Full=GST class- theta }\end{array}$ & $\begin{array}{lll}38 & 6.3 & 23.80\end{array}$ \\
\hline
\end{tabular}

$\begin{array}{rrrrrrll}1204.516 & \text { M } & 1204.590 & -0.074 & 79 & 88 & 1 & \text { NDSLFPKCPK } \\ 1265.735 & \text { M } & 1265.600 & 0.135 & 97 & 106 & 0 & \begin{array}{l}\text { LYFDMGTLYK } \\ (1)+0 M \text {; }\end{array} \\ 1611.958 & \text { M } & 1611.760 & 0.198 & 107 & 119 & 0 & \text { SFADYYYPIFAR } \\ 1849.289 & M & 1849.045 & 0.244 & 30 & 45 & 0 & \text { LLNLQAGEHLKPEFLK } \\ 2408.496 & M & 2408.211 & 0.285 & 46 & 66 & 0 & \text { INPQHTIPTLVDGDFALWESR } \\ 2596.377 & M & 2596.251 & 0.125 & 107 & 128 & 1 & \text { SFADYYYPQIFAKA.PADPELYK }\end{array}$

5

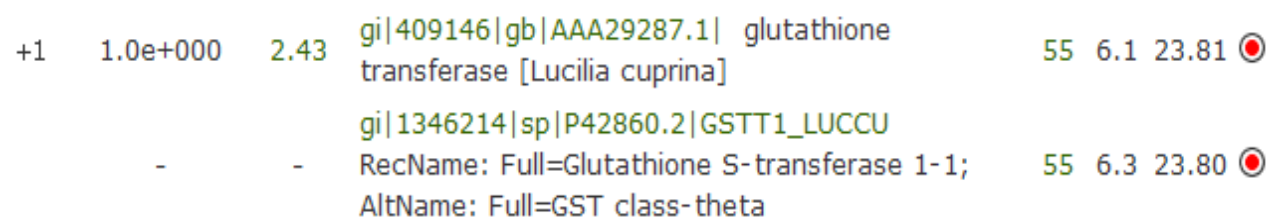

$\begin{array}{rr}21 & 29 \\ 67 & 75 \\ 67 & 75 \\ & \\ 120 & 129 \\ 79 & 88 \\ 97 & 106 \\ 97 & 106 \\ & \\ 107 & 119 \\ 30 & 45 \\ 188 & 204 \\ 46 & 66\end{array}$

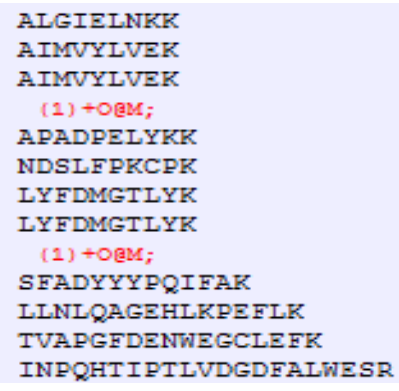




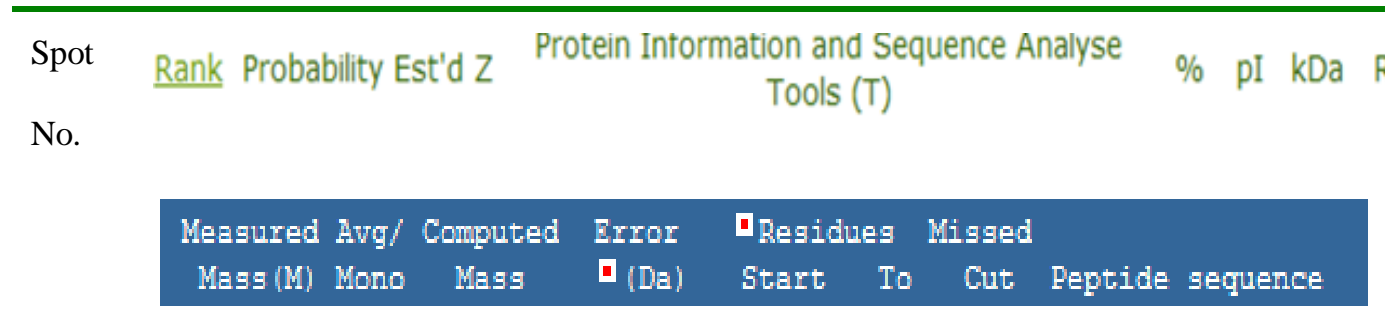

6 $+1 \quad 1.0 \mathrm{e}+000 \quad 2.43 \begin{aligned} & \text { gi|409146|gb|AAA29287.1| glutathione } \\ & \text { transferase [Lucilia cuprina] }\end{aligned} \quad \begin{array}{lll}55 & 6.123 .810\end{array}$

$\begin{array}{rrrrrrll}985.052 & M & 984.596 & 0.456 & 21 & 29 & 1 & \text { ALGIELNKK } \\ 1064.569 & M & 1064.594 & -0.024 & 67 & 75 & 0 & \text { AIMVYLVEK } \\ 1167.287 & M & 1167.592 & -0.305 & 76 & 85 & 1 & \text { YGKNDSLFPK } \\ 1265.581 & M & 1265.600 & -0.019 & 97 & 106 & 0 & \text { LYFDMGTLYK } \\ & & & & & & & \text { (1) +OGM; } \\ 1611.736 & M & 1611.760 & -0.025 & 107 & 119 & 0 & \text { SFADYYYPQIFAK } \\ 1849.039 & M & 1849.045 & -0.006 & 30 & 45 & 0 & \text { LLNLQAGEHLKPEFLK } \\ 1997.861 & M & 1997.882 & -0.021 & 188 & 204 & 0 & \text { TVAPGFDENWEGLEFK } \\ 2408.142 & M & 2408.211 & -0.069 & 46 & 66 & 0 & \text { INPQHTIPTLVDGDALWESR } \\ 2596.093 & M & 2596.251 & -0.159 & 107 & 128 & 1 & \text { SFADYYYPQIFAKA.PADPELYK }\end{array}$

7

\begin{tabular}{|c|c|c|c|c|c|c|c|}
\hline $1.0 \mathrm{e}$ & & 2.43 & $\begin{array}{l}409146 \mid g b \\
\text { nsferase }[\end{array}$ & $\begin{array}{l}\text { A29 } \\
\text { lia c }\end{array}$ & $\begin{array}{l}11 \\
\text { ha] }\end{array}$ & tathion & $\begin{array}{lll}49 & 6.1 & 23.810\end{array}$ \\
\hline 02.529 & M & 1002.502 & 0.027 & 120 & 128 & 0 & APADPELYK \\
\hline 80.562 & M & 1080.588 & -0.026 & 67 & 75 & 0 & $\begin{array}{l}\text { AIMVYLVEK } \\
\text { (1) +o@M; }\end{array}$ \\
\hline 49.575 & M & 1249.605 & -0.029 & 97 & 106 & 0 & LYFDMGTLYK \\
\hline 265.586 & $\mathrm{M}$ & 1265.600 & -0.014 & 97 & 106 & 0 & $\begin{array}{l}\text { LYFDMGTLYK } \\
\text { (1) +O@M; }\end{array}$ \\
\hline 111.805 & M & 1611.760 & 0.044 & 107 & 119 & 0 & SFADYYYPQIFAK \\
\hline 349.057 & M & 1849.045 & 0.012 & 30 & 45 & 0 & LLNLQAGEHLKPEFLK \\
\hline 1946.955 & M & 1946.992 & -0.036 & 91 & 106 & 1 & $\begin{array}{l}\text { AVINQRLYFDMGTLYK } \\
\text { (1) +O@M; }\end{array}$ \\
\hline 977.095 & M & 1977.140 & -0.045 & 29 & 45 & 1 & KLLNLQAGEHLKPEFLK \\
\hline 997.942 & M & 1997.882 & 0.060 & 188 & 204 & 0 & TVAPGFDENWEGCLEFK \\
\hline 408.218 & M & 2408.211 & 0.007 & 46 & 66 & 0 & INPQHTIPTLVDGDFALWESR \\
\hline
\end{tabular}


10.3.5 Spectra of spots labelled in Figure 4-20 (A)
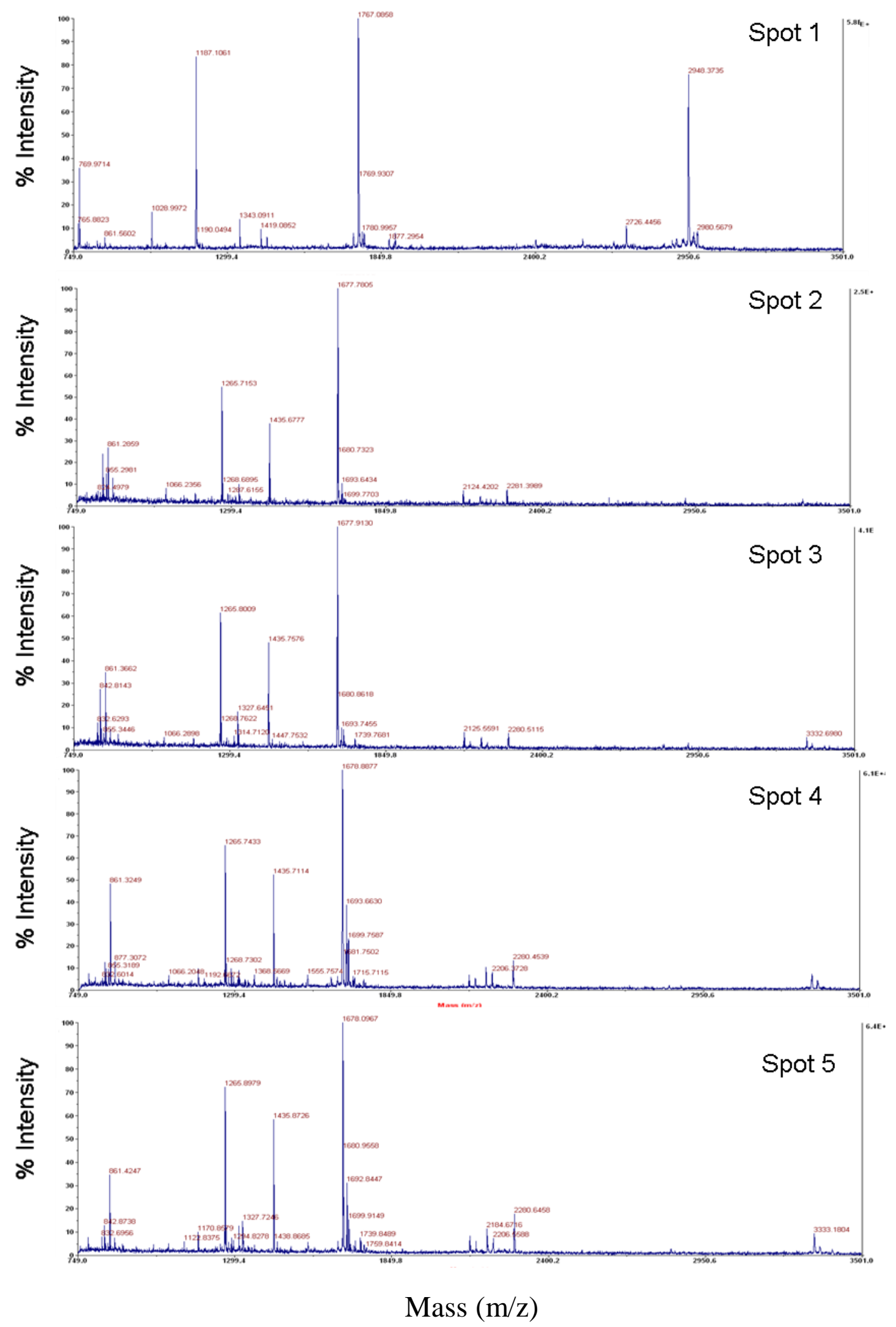

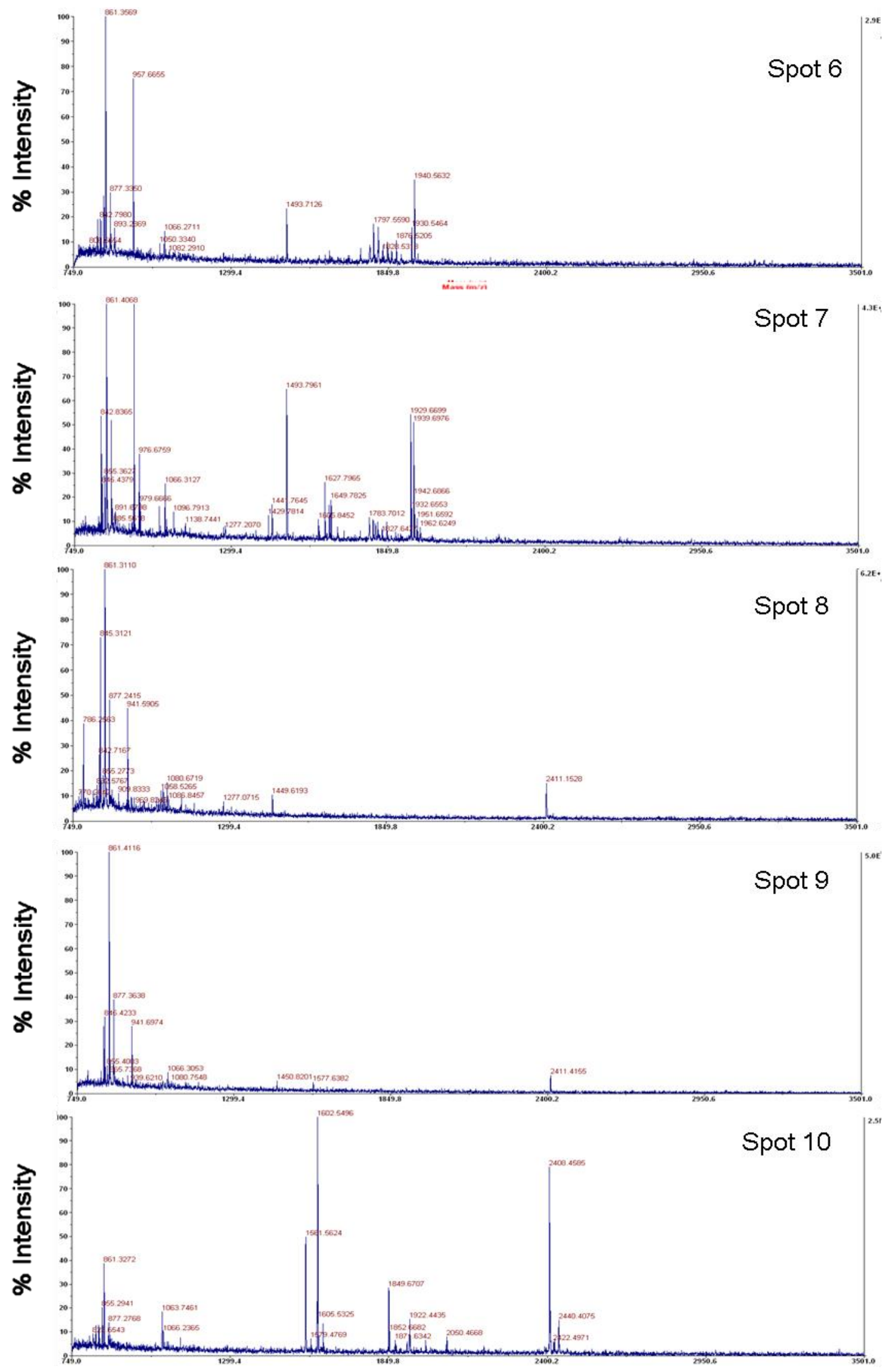

Mass (m/z) 


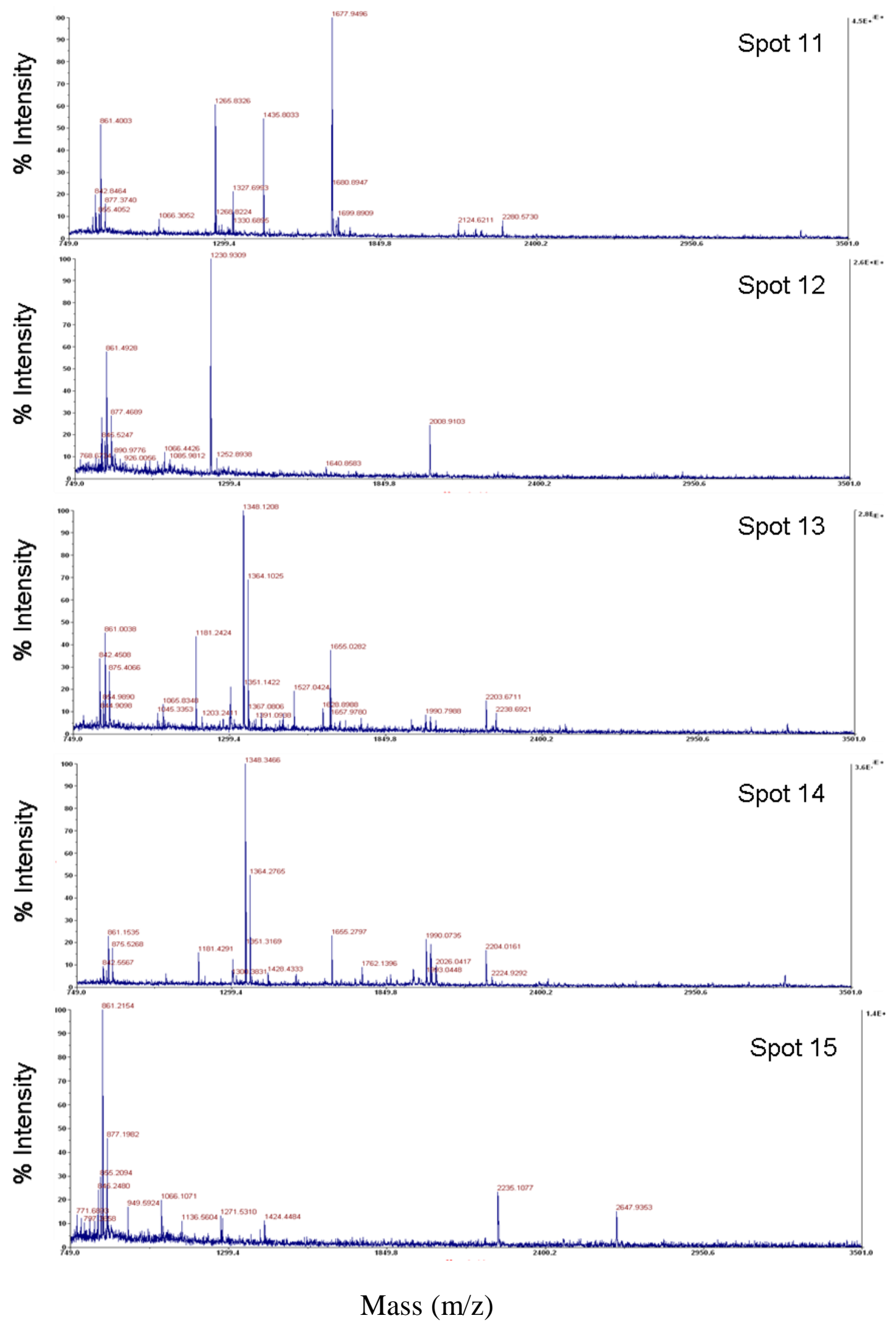




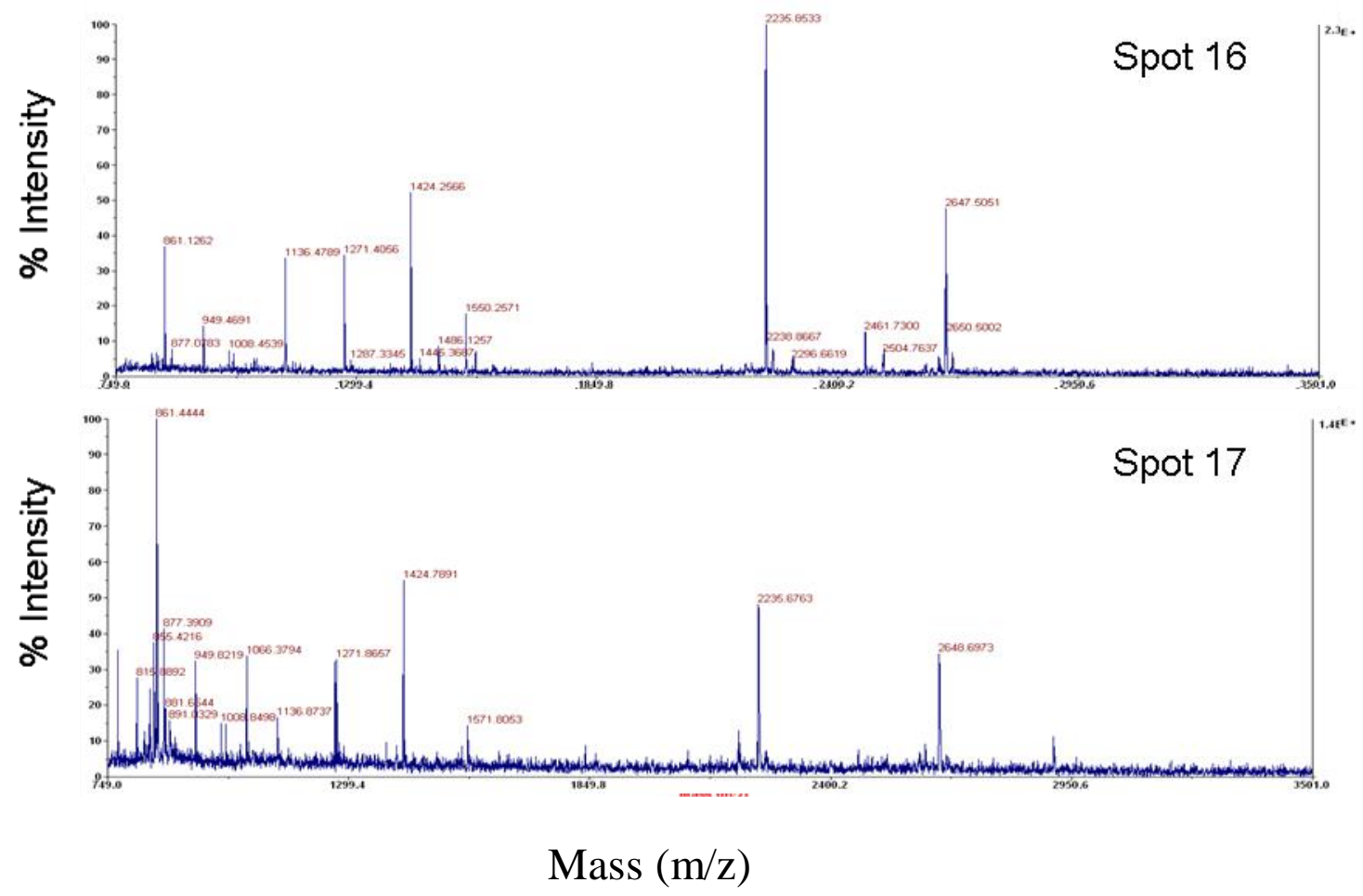

Figure 10-4: MALDI-TOF spectra of the DNP-GSH affinity-purified D. melanogaster spots on 2D gel labelled in Figure 4-20 (A). 


\subsubsection{Identification of spots labelled in Figure 4-20 (A) using PROFOUND}

\begin{tabular}{lcccc}
\hline Spot & Rank Probability Est'd $Z$ & Protein Intormation and Sequence Analyse & $\%$ & pI kDa \\
No. & Tools $(T)$ & &
\end{tabular}

Measured Avg/ Computed Brror DResidues Missed

Mass (M) Mono Mass D(Da) Start To Cut Peptide sequence

gi|24654347|ref|NP_725653.1| glutathione S

$\begin{array}{llllll}+1 & 1.0 \mathrm{e}+000 & 2.43 & \begin{array}{l}\text { transferase S1, isoform A [Drosophila } \\ \text { melanogaster] }\end{array}\end{array}$

\begin{tabular}{|c|c|c|c|c|c|c|c|}
\hline 768.335 & M & 768.449 & -0.114 & 59 & 65 & 0 & ALAEPLR \\
\hline 1027.416 & M & 1027.523 & -0.107 & 107 & 115 & 0 & VHQSISMAR \\
\hline 1185.513 & M & 1185.639 & -0.125 & 216 & 225 & 0 & DLLEPYPALR \\
\hline 1417.260 & M & 1417.703 & -0.443 & 48 & 58 & 0 & HSYTLFYFNVK \\
\hline 1747.825 & M & 1747.975 & -0.150 & 164 & 178 & 0 & LVTLNAEVIPFYLEK \\
\hline 1747.825 & M & 1747.887 & -0.062 & 148 & 162 & 1 & IAVVSYEPEDEIKEK \\
\hline 1765.554 & M & 1765.794 & -0.240 & 66 & 79 & 0 & YLFAYGNQEYEDVR \\
\hline 2724.795 & M & 2724.342 & 0.453 & 83 & 106 & 1 & DEWPALKPTMPMGQMPVLEVDGKR \\
\hline 2946.063 & M & 2946.442 & -0.379 & 120 & 145 & 0 & TVGLCGATPWEDLQIDIVVDTINDFP \\
\hline
\end{tabular}

2
$1 \quad 1.0 \mathrm{e}+000$
2.42
gi|19922932|ref|NP_611964.1| CG16936 [Drosophila melanogaster]
335.925 .440

$\begin{array}{lllrrrll}1169.616 & M & 1169.607 & 0.009 & 39 & 48 & 0 & \text { GEHLTPEFLK } \\ 1264.628 & M & 1264.667 & -0.039 & 101 & 111 & 0 & \text { LHLDSGHLFAR } \\ 1676.988 & M & 1677.018 & -0.030 & 24 & 38 & 0 & \text { AIGLDLELRPINLLK } \\ 2122.992 & M & 2123.005 & -0.013 & 192 & 210 & 0 & \text { LAELPYYQEVNGDGADELK } \\ 2182.976 & M & 2183.012 & -0.036 & 114 & 131 & 0 & \text { FLYEPILYYGSTDCSIDK } \\ 2279.084 & M & 2279.106 & -0.022 & 191 & 210 & 1 & \text { RLAELPYYQEVNGDGADELK }\end{array}$

3
$1 \quad 1.0 \mathrm{e}+000 \quad 2.35$
gi|19922932|ref|NP_611964.1| CG16936 [Drosophila melanogaster]
$49 \quad 5.925 .440$

\begin{tabular}{|c|c|c|c|c|c|c|c|}
\hline 800.462 & M & 800.450 & 0.012 & 215 & 221 & 1 & AKLAENR \\
\hline 800.462 & M & 800.400 & 0.062 & 185 & 190 & 0 & $\begin{array}{l}\text { MHAWLK } \\
\text { (1) +o@M; }\end{array}$ \\
\hline 904.909 & M & 904.465 & 0.444 & 83 & 89 & 0 & EQQLYPK \\
\hline 1156.691 & M & 1156.621 & 0.070 & 182 & 190 & 1 & FPKMHAWLK \\
\hline 1264.661 & M & 1264.667 & -0.006 & 101 & 111 & 0 & LHLDSGHLFAR \\
\hline 1676.952 & M & 1677.018 & -0.066 & 24 & 38 & 0 & AIGLDLELRPINLLK \\
\hline 2122.872 & M & 2123.005 & -0.133 & 192 & 210 & 0 & LAELPYYQEVNGDGADELK \\
\hline 2182.827 & M & 2183.012 & -0.185 & 114 & 131 & 0 & FLYEPILYYGSTDCSIDK \\
\hline 2278.946 & M & 2279.106 & -0.160 & 191 & 210 & 1 & RLAELPYYQEVNGDGADELK \\
\hline 3332.327 & M & 3331.710 & 0.617 & 49 & 78 & 0 & LNPQHTIPTLIDGEATIIDSHAICAYLVE] \\
\hline
\end{tabular}




\begin{tabular}{|c|c|c|c|}
\hline Spot & 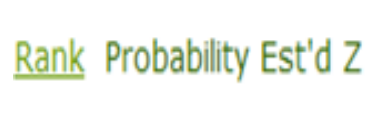 & $\begin{array}{l}\text { Protein Intormation and Sequence Analyse } \\
\qquad \text { Tools }(T)\end{array}$ & $\%$ pI kDa R \\
\hline
\end{tabular}

$\begin{array}{cccccc}\text { Measured Avg/ Computed } & \text { Brror } & \text { QResidues } & \text { Missed } \\ \text { Mass (M) Mono Mass } & \text { प(Da) } & \text { Start } & \text { To } & \text { Cut Peptide sequence }\end{array}$

4
$1 \quad 1.0 \mathrm{e}+000$
2.35
gi|19922932|ref|NP_611964.1| CG16936
[Drosophila melanogaster]
$47 \quad 5.925 .440$

1169.517
1264.537
1676.767
2122.513
2182.518
2278.465
2829.253
3331.692
M $\quad 1169.607-0.091$
M $1264.667-0.130$
M $1677.018-0.251$
M $2123.005-0.492$
M $2183.012-0.494$
M $2279.106-0.641$
$\begin{array}{lll}\text { M } & 2828.615 & 0.638\end{array}$
M $3331.710-0.018$

$\begin{array}{rrr}39 & 48 & 0 \\ 101 & 111 & 0 \\ 24 & 38 & 0 \\ 192 & 210 & 0 \\ 114 & 131 & 0 \\ 191 & 210 & 1 \\ 24 & 48 & 1 \\ 49 & 78 & 0\end{array}$
GEHLTPEFLK
LHLDSGHLFAR
AIGLDLELRPINLLK
LAELPYYQEVNGDGADELK
FLYEPILYYGSTDCSIDR
RLAELPYYQEVNGDGADELK
AIGLDLELRPINLLKGEHLTPEFLK
LNPOHTIPTLIDGEATIIDSHAICAYLVEK

5
$1 \quad 1.0 \mathrm{e}+000$
2.40
gi|19922932|ref|NP_611964.1| CG16936
[Drosophila melanogaster]
$38 \quad 5.925 .440$

$\begin{array}{llrrrrll}1169.607 & \text { M } & 1169.607 & -0.000 & 39 & 48 & 0 & \text { GEHLTPEFLK } \\ 1264.679 & M & 1264.667 & 0.011 & 101 & 111 & 0 & \text { LHLDSGHLFAR } \\ 1293.645 & M & 1293.642 & 0.003 & 138 & 147 & 0 & \text { CWEILEGFLK } \\ 1677.091 & M & 1677.018 & 0.073 & 24 & 38 & 0 & \text { AIGLDLELRPINLLK } \\ 2123.044 & M & 2123.005 & 0.040 & 192 & 210 & 0 & \text { LAELPYYQENGDGADELK } \\ 2183.094 & M & 2183.012 & 0.081 & 114 & 131 & 0 & \text { FLYEPILYYGSDCSIDK } \\ 2279.068 & M & 2279.106 & -0.038 & 191 & 210 & 1 & \text { RLAELPYYQENIGDGADELK }\end{array}$

6
$+1 \quad 1.0 \mathrm{e}+000$
2.43 gi|21355763|ref|NP_648236.1|
B [Drosophila melanogaster]
CG6673, isoform
$48 \quad 6.528 .760$

$\begin{array}{rrrrrrll}956.508 & \text { M } & 956.417 & 0.091 & 30 & 36 & 0 & \text { FCPYSQR } \\ 1095.630 & \text { M } & 1095.546 & 0.085 & 161 & 170 & 1 & \text { RGTPYFGGNK } \\ 1428.772 & \text { M } & 1428.736 & 0.036 & 209 & 220 & 1 & \text { WRDLVAQDEAVK } \\ 1492.807 & \text { M } & 1492.788 & 0.019 & 11 & 24 & 0 & \text { GSPKPEIPEDGVLR } \\ 1604.894 & \mathrm{M} & 1604.917 & -0.023 & 123 & 137 & 0 & \text { LSPAVSAIYPVLFTK } \\ 1641.793 & \mathrm{M} & 1641.788 & 0.005 & 190 & 202 & 1 & \text { YTLDEPYELDKTR } \\ 1648.901 & \mathrm{M} & 1648.889 & 0.012 & 10 & 24 & 1 & \text { RGSPKPEIPEDGVLR } \\ 1672.782 & \mathrm{M} & 1672.712 & 0.070 & 25 & 36 & 1 & \text { YYSMRFCPYSQR } \\ & & & & & & & \text { (1)+OQM; } \\ 1782.807 & \mathrm{M} & 1782.867 & -0.060 & 146 & 160 & 0 & \text { NFETALDVFEQITK } \\ 1795.859 & \mathrm{M} & 1795.875 & -0.016 & 171 & 184 & 0 & \text { IGIADYMIWPWFER } \\ 1811.855 & \mathrm{M} & 1811.870 & -0.015 & 171 & 184 & 0 & \text { IGIADYMIWPWFER } \\ & & & & & & & \text { (1) +OQM; } \\ 1928.898 & \mathrm{M} & 1928.937 & -0.039 & 235 & 250 & 1 & \text { TRHENKPDYDVAFQPL } \\ 1938.922 & \mathrm{M} & 1938.968 & -0.046 & 146 & 161 & 1 & \text { NFETALDVFEQEITKR }\end{array}$




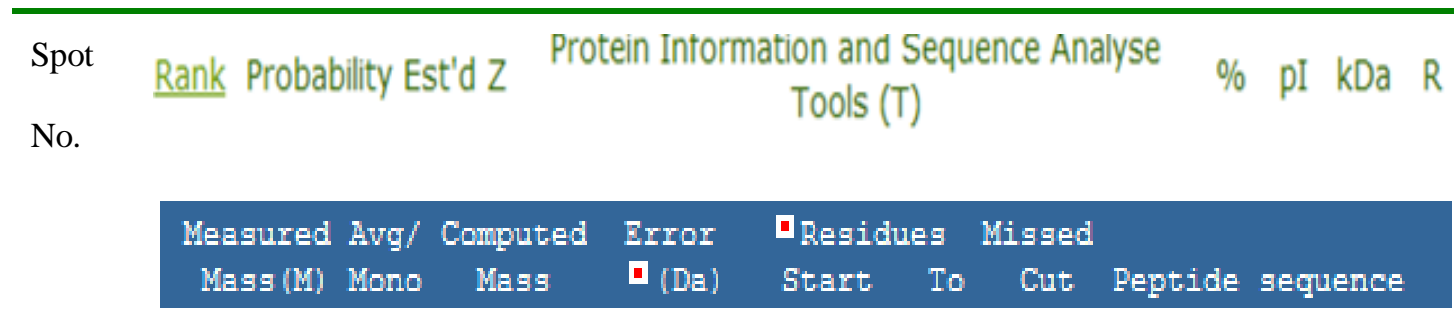

7

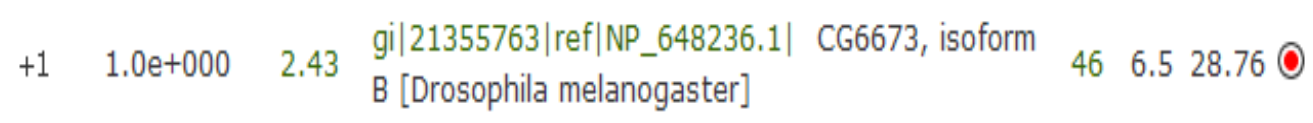

$\begin{array}{rrrrrrll}889.975 & M & 889.484 & 0.491 & 228 & 234 & 1 & \begin{array}{l}\text { IHAKFMK } \\ (1)+0 @ M ;\end{array} \\ 956.447 & M & 956.417 & 0.030 & 30 & 36 & 0 & \text { FCPYSQR } \\ 1086.814 & M & 1086.555 & 0.259 & 211 & 220 & 0 & \text { DLVAQDEAVK } \\ 1384.603 & \mathrm{M} & 1384.639 & -0.036 & 190 & 200 & 0 & \text { YTLDEPYELDK } \\ 1428.787 & \mathrm{M} & 1428.736 & 0.051 & 209 & 220 & 1 & \text { WRDLVAQDEAVK } \\ 1492.828 & \mathrm{M} & 1492.788 & 0.040 & 11 & 24 & 0 & \text { GSPKPEIPEDGVLR } \\ 1604.964 & \mathrm{M} & 1604.917 & 0.046 & 123 & 137 & 0 & \text { LSPAVSAIYPVLFTK } \\ 1641.903 & \mathrm{M} & 1641.788 & 0.115 & 190 & 202 & 1 & \text { YTLDEPYELDKTR } \\ 1648.977 & \mathrm{M} & 1648.889 & 0.088 & 10 & 24 & 1 & \text { RGSPRPEIPEDGVLR } \\ 1671.941 & \mathrm{M} & 1671.789 & 0.152 & 237 & 250 & 0 & \text { HENKPDYDVAFQPL } \\ 1782.976 & \mathrm{M} & 1782.867 & 0.109 & 146 & 160 & 0 & \text { NFETALDVFEQEITK } \\ 1795.945 & \mathrm{M} & 1795.875 & 0.071 & 171 & 184 & 0 & \text { IGIADYMIWPWFER } \\ 1811.963 & \mathrm{M} & 1811.870 & 0.093 & 171 & 184 & 0 & \text { IGIADYMIWPWFER } \\ & & & & & & & \text { (1) +OQM; } \\ 1929.012 & \mathrm{M} & 1928.937 & 0.075 & 235 & 250 & 1 & \text { TRHENKPDYDVAFQPL } \\ 1939.040 & \mathrm{M} & 1938.968 & 0.072 & 146 & 161 & 1 & \text { NFETALDVFEQEITKR }\end{array}$

$8 \quad 1 \quad 1.0 \mathrm{e}+000 \quad 1.90 \quad \mathrm{gi}|21355779|$ ref|NP_648234.1| CG6776 [Drosophila melanogaster]

246.527 .760

\begin{tabular}{rrrrrrll}
940.447 & $M$ & 940.422 & 0.025 & 29 & 35 & 0 & FCPYAQR \\
1057.465 & $M$ & 1057.446 & 0.019 & 189 & 196 & 0 & EYNFNESR \\
1084.815 & $M$ & 1084.623 & 0.192 & 112 & 120 & 1 & AQDKILLER \\
1084.815 & $M$ & 1084.700 & 0.115 & 200 & 208 & 1 & ITKWIALLK \\
1448.832 & $M$ & 1448.798 & 0.034 & 10 & 23 & 0 & GSPKPVLPDDGVLR \\
2410.246 & $M$ & 2410.097 & 0.149 & 209 & 228 & 0 & ADSVVQSFYATPEQHNEFWR \\
\hline
\end{tabular}

9

$\begin{array}{llll}1 & 1.0 \mathrm{e}+000 & 2.09 & \begin{array}{l}\text { gi|21355779|ref|NP_648234.1| CG6776 } \\ \text { [Drosophila melanogaster] }\end{array}\end{array} 226.527 .760$

$\begin{array}{rrrrrrll}940.486 & \text { M } & 940.422 & 0.064 & 29 & 35 & 0 & \text { FCPYAQR } \\ 1057.567 & \text { M } & 1057.446 & 0.121 & 189 & 196 & 0 & \text { EYNFNESR } \\ 1066.728 & \text { M } & 1066.638 & 0.089 & 70 & 79 & 0 & \text { VPALQLVAEK } \\ 1084.862 & \text { M } & 1084.700 & 0.162 & 200 & 208 & 1 & \text { ITKWIALLK } \\ 1448.938 & \text { M } & 1448.798 & 0.140 & 10 & 23 & 0 & \text { GSPKPVLPDDGVLR } \\ 1590.788 & \text { M } & 1590.743 & 0.046 & 24 & 35 & 1 & \text { LYSMRFCPYAQR }\end{array}$




\section{\begin{tabular}{ccccc}
\hline Spot $\quad$ Rank Probability Est'd $Z$ & Protein Intormation and Sequence Analyse & $\%$ pI & TDa & $R$
\end{tabular} No.}

\section{Measured Avg/ Computed Brror Desidues Missed \\ Mass (M) Mono Mass $\mathbf{D}(\mathrm{Da})$ Start To Cut Peptide sequence}

10

gi|385883|gb|AAB26519.1| glutathione S-

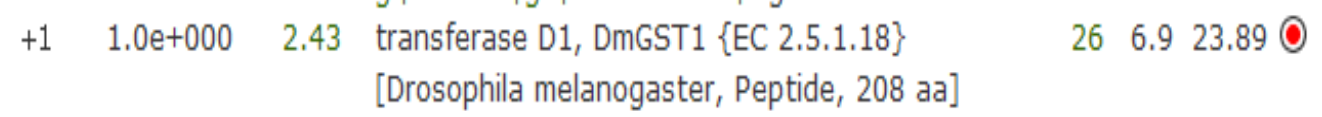

$\begin{array}{rrrrrrll}944.715 & M & 944.460 & 0.255 & 120 & 128 & 0 & \text { APADPEAFK } \\ 956.591 & M & 956.565 & 0.026 & 21 & 29 & 1 & \text { AVGVELNKK } \\ 1849.446 & M & 1849.045 & 0.401 & 30 & 45 & 0 & \text { LLNLQAGEHLKPEFLK } \\ 2407.388 & M & 2407.227 & 0.161 & 46 & 66 & 0 & \text { INPQHTIPTLVDNGFALWESR }\end{array}$

11 $+1 \quad 1.0 \mathrm{e}+000 \quad 2.43 \begin{aligned} & \text { gi| } 17137088 \mid \text { ref|NP_477090.1| Rab-protein } 2 \\ & \text { [Drosophila melanogaster] }\end{aligned} \quad \begin{array}{lll}37 & 6.023 .710\end{array}$

$\begin{array}{rrrrrrll}846.948 & \text { M } & 847.414 & -0.466 & 121 & 127 & 1 & \text { SDLDSRR } \\ 1044.506 & \text { M } & 1044.535 & -0.029 & 70 & 77 & 1 & \text { SITRSYYR } \\ 1475.067 & \text { M } & 1474.825 & 0.242 & 78 & 91 & 1 & \text { GAAGALLVYDITRR } \\ 1533.405 & \text { M } & 1533.757 & -0.352 & 57 & 69 & 0 & \text { LQIWDTAGQEAFR } \\ 1716.778 & \text { M } & 1716.828 & -0.050 & 106 & 120 & 0 & \text { QHSNSNMVIMLIGNK } \\ & & & & & & & (2)+\text { OQM; } \\ 1990.482 & \text { M } & 1991.022 & -0.539 & 57 & 73 & 1 & \text { LQIWDTAGQEAFRSITR } \\ 2422.746 & \text { M } & 2422.200 & 0.546 & 166 & 186 & 1 & \text { EIYEKIQEGVFDINNEANGIK }\end{array}$

12

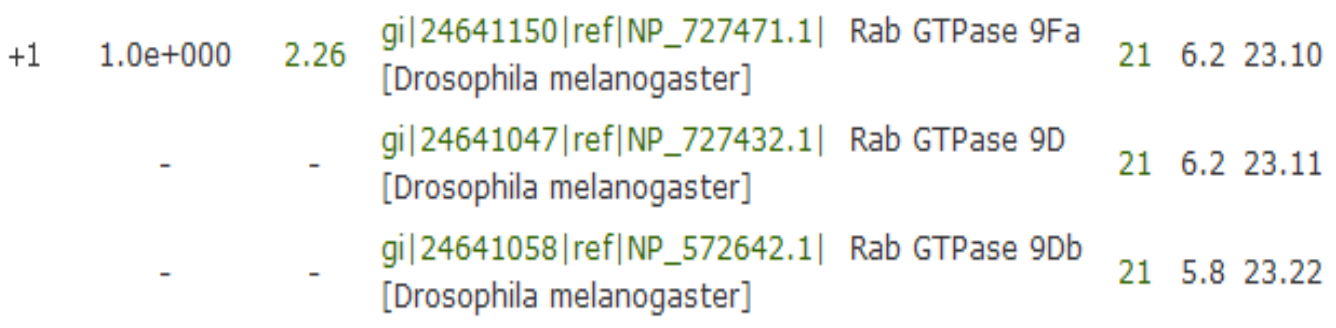

\begin{tabular}{rrrrrrrl}
787.443 & $M$ & 787.401 & 0.042 & 109 & 114 & 1 & RLCPDK \\
988.423 & $M$ & 988.548 & -0.125 & 73 & 80 & 1 & LLKATQCR \\
1638.695 & $M$ & 1638.782 & -0.087 & 96 & 108 & 1 & $\begin{array}{c}\text { SFQNIDGWMKEIR } \\
(1)+0 @ M ;\end{array}$ \\
2219.100 & $M$ & 2219.136 & -0.035 & 76 & 95 & 1 & ATQCRSAHGILLVYDITSSK \\
\hline
\end{tabular}




\begin{tabular}{|c|c|c|c|}
\hline Spot & $\underline{\text { Rank }}$ Probability Est'd Z & $\begin{array}{l}\text { Protein Intormation and Sequence Analyse } \\
\qquad \text { Tools (T) }\end{array}$ & $\%$ pI kDa R \\
\hline
\end{tabular}

Measured Avg/ Computed Brror DResidues Missed

Mass (M) Mono Masg $\mathbf{\square}(\mathrm{Da})$ Start To Cut Peptide sequence

13
$1 \quad 1.0 \mathrm{e}+000$
2.37
gi|20129743|ref|NP_610270.1| CG1707
[Drosophila melanogaster]
$\begin{array}{lll}60 & 6.120 .14 & 0\end{array}$

\begin{tabular}{|c|c|c|c|c|c|c|c|}
\hline 874.348 & M & 874.477 & -0.129 & 85 & 91 & 1 & RSWALSR \\
\hline 1180.426 & M & 1180.612 & -0.186 & 138 & 147 & 0 & FQELGVDFVK \\
\hline 1347.436 & M & 1347.628 & -0.192 & 25 & 34 & 0 & DFLFQQTMYR \\
\hline 1363.450 & M & 1363.622 & -0.172 & 25 & 34 & 0 & $\begin{array}{l}\text { DFLFQQTMYR } \\
\text { (1) +O@M; }\end{array}$ \\
\hline 1526.532 & M & 1526.747 & -0.215 & 93 & 105 & 0 & ATIELTHNWGTER \\
\hline 1628.487 & M & 1628.681 & -0.194 & 106 & 119 & 0 & DPDQNYHTGNTDPR \\
\hline 1654.617 & M & 1654.842 & -0.225 & 92 & 105 & 1 & KATIELTHNWGTER \\
\hline 1761.526 & M & 1761.799 & -0.273 & 162 & 176 & 0 & DPDGYWIEI FNAHSV \\
\hline 1989.696 & $\mathrm{M}$ & 1989.954 & -0.259 & 120 & 137 & 0 & GFGHIGIMVPDVYAACQR \\
\hline 2005.688 & M & 2005.949 & -0.261 & 120 & 137 & 0 & $\begin{array}{l}\text { GFGHIGIMVPDVYAACQR } \\
\text { (1) +O@M; }\end{array}$ \\
\hline 2202.846 & M & 2203.083 & -0.236 & 64 & 82 & 1 & FSLYFLGYENATDVPKDPK \\
\hline
\end{tabular}

14

$\begin{array}{llll}1 & 1.0 \mathrm{e}+000 & 2.39 & \begin{array}{l}\text { gi|20129743|ref|NP_610270.1| } \\ \text { [Drosophila melanogaster] }\end{array}\end{array}$

$72 \quad 6.120 .14 \bigcirc$

874.404
961.330
1180.491
1347.511
1363.483
1628.535
1654.665
1761.683
1989.768
2005.751
2202.886
2391.581
2662.828

15

$\begin{array}{lrrrrl}M & 874.477 & -0.073 & 85 & 91 & 1 \\ M & 961.465 & -0.135 & 148 & 155 & 1 \\ M & 1180.612 & -0.121 & 138 & 147 & 0 \\ M & 1347.628 & -0.116 & 25 & 34 & 0 \\ M & 1363.622 & -0.139 & 25 & 34 & 0 \\ M & 1628.681 & -0.146 & 106 & 119 & 0 \\ M & 1654.842 & -0.177 & 92 & 105 & 1 \\ M & 1761.799 & -0.116 & 162 & 176 & 0 \\ M & 1989.954 & -0.186 & 120 & 137 & 0 \\ M & 2005.949 & -0.198 & 120 & 137 & 0 \\ M & 2203.083 & -0.197 & 64 & 82 & 1 \\ M & 2391.189 & 0.392 & 156 & 176 & 1 \\ M & 2663.315 & -0.487 & 57 & 79 & 1\end{array}$

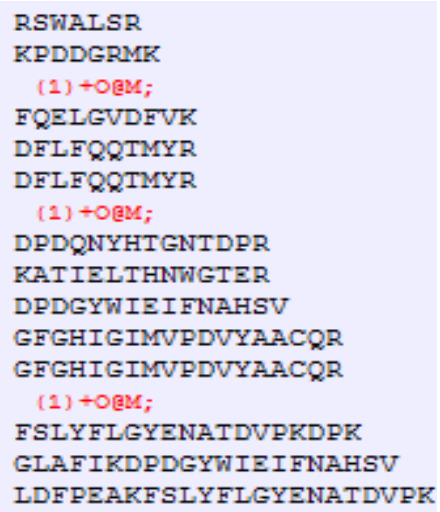

$201 \quad 206$ $207 \quad 214$ $250 \quad 258$ 236249 395407

$129 \quad 144$ $236 \quad 251$ $161 \quad 177$
VLDYRR

KFIEAAQR

SRMLATLFK

ALICTVLASAGQQR

ISVAE PDWMDNLN

(1) + ogM;

DAATVLVGI PLETGQK

ALICTVLASAGQQRSR

LYLEDNDSVQAELFINR

SQLMGLINFTGTHKDQADK

DAATVLVGI PLETGQKQYSVECK IASQMITEGRMNGHIDQISAIVH 


\section{\begin{tabular}{lccccc}
\hline Spot & Rank Probability Est'd Z & Protein Intormation and Sequence Analyse & $\%$ & pI kDa & $R$ \\
No. & Tools $(T)$ & &
\end{tabular}}

Measured Avg/ Computed Brror Desidues Missed

Mass (M) Mono Mass D(Da) Start To Cut Peptide sequence

16 gi|17137696|ref|NP_477444.1| COP9 complex $+1 \quad 1.0 \mathrm{e}+000 \quad 2.43$ homolog subunit 4, isoform A [Drosophila $\quad 295.946 .740$ melanogaster]

\begin{tabular}{|c|c|c|c|c|c|c|c|}
\hline 961.021 & M & 961.534 & -0.513 & 207 & 214 & 1 & KFIEAAQR \\
\hline 1065.025 & M & 1065.600 & -0.575 & 250 & 258 & 1 & SRMLATLFK \\
\hline 1071.043 & M & 1071.534 & -0.492 & 215 & 222 & 1 & YNELSYRK \\
\hline 1104.289 & $\mathrm{M}$ & 1104.563 & -0.274 & 90 & 98 & 0 & MLSHFTLEK \\
\hline 1104.289 & M & 1104.559 & -0.270 & 345 & 354 & 0 & IASQMITEGR \\
\hline 1570.756 & M & 1570.828 & -0.072 & 90 & 102 & 1 & MLSHFTLEKVNPR \\
\hline 1611.118 & M & 1610.887 & 0.231 & 129 & 144 & 0 & DAATVLVGIPLETGQK \\
\hline 1614.871 & M & 1614.887 & -0.015 & 235 & 249 & 1 & KALICTVLASAGQQR \\
\hline 2487.754 & $\mathrm{M}$ & 2488.255 & -0.501 & 298 & 321 & 1 & AATSDGSSILDRAVFEHNLLSASK \\
\hline 3016.833 & M & 3017.492 & -0.659 & 355 & 379 & 1 & MNGHIDQISAIVHFENRELLPQWDR \\
\hline
\end{tabular}

17 gi|1580758|emb|CAA47892.1| glycerol-3$1 \quad 1.0 \mathrm{e}+000 \quad 2.00$ phosphate dehydrogenase [Drosophila $38 \quad 6.444 .750$ melanogaster]

\begin{tabular}{|c|c|c|c|c|c|c|c|}
\hline 834.131 & $\mathrm{M}$ & 834.390 & -0.259 & 291 & 297 & 0 & $\begin{array}{l}\text { EMLNGQK } \\
\text { (1) +OQM; }\end{array}$ \\
\hline 860.036 & M & 860.448 & -0.413 & 284 & 290 & 0 & TIEELEK \\
\hline 880.298 & M & 880.422 & -0.124 & 384 & 390 & 0 & CIFSAQR \\
\hline 1179.731 & $\mathrm{M}$ & 1179.624 & 0.106 & 273 & 283 & 1 & RVSEAFVTSGK \\
\hline 1471.777 & M & 1471.836 & -0.059 & 227 & 239 & 1 & AAVIRLGLMEMIR \\
\hline 1487.863 & M & 1487.831 & 0.032 & 227 & 239 & 1 & $\begin{array}{l}\text { AAVIRLGLMEMIR } \\
\text { (1) +OQM; }\end{array}$ \\
\hline 1819.173 & M & 1819.165 & 0.009 & 105 & 121 & 1 & QLLGKIKPNAIAISLIK \\
\hline 1866.130 & $\mathrm{M}$ & 1865.961 & 0.169 & 274 & 290 & 1 & VSEAFVTSGKTIEELEK \\
\hline 2505.136 & $\mathrm{M}$ & 2505.223 & -0.086 & 291 & 312 & 1 & $\begin{array}{l}\text { EMLNGQKLQGPPTAEEVNYMLK } \\
\text { (1) +OQM; }\end{array}$ \\
\hline 2664.937 & M & 2665.250 & -0.314 & 369 & 390 & 1 & EAETFLTFLCTTENKCIFSAQR \\
\hline 3110.155 & M & 3110.562 & -0.407 & 22 & 49 & 1 & IVGANAAALPEFEERVTMFVYEELIDGK \\
\hline 3254.519 & $\mathrm{M}$ & 3254.469 & 0.050 & 144 & 173 & 0 & IPCAVLMGANLANEVAEGNFCETTIGCTDK \\
\hline 3270.421 & M & 3270.465 & -0.043 & 144 & 173 & 0 & $\begin{array}{l}\text { IPCAVLMGANLANEVAEGNFCETTIGCTDK } \\
(1) \text { +NaM. }\end{array}$ \\
\hline
\end{tabular}


10.3.7 Spectra of spots labelled in Figure 4-20 (B)
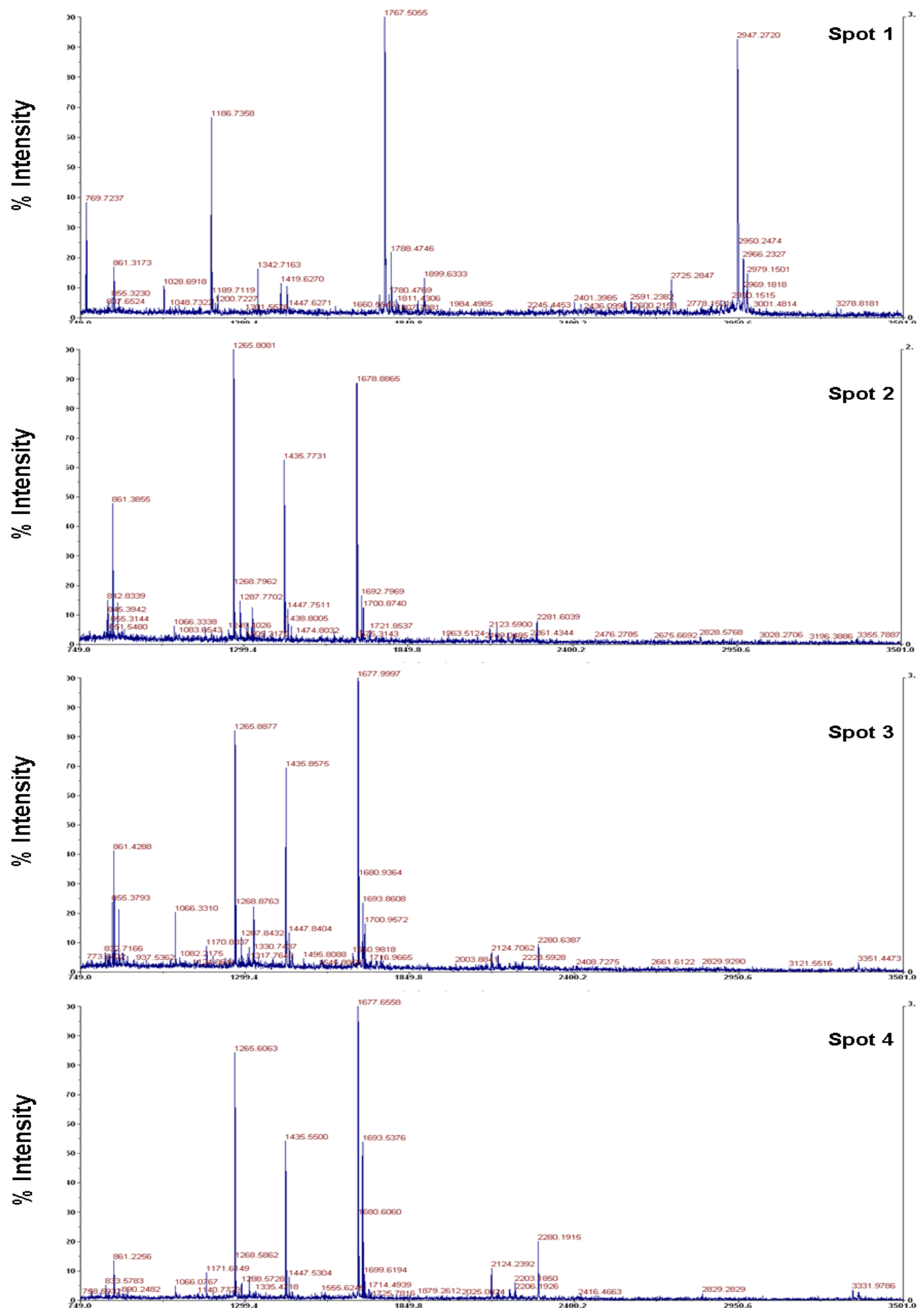

$\operatorname{Mass}(\mathbf{m} / \mathbf{z})$ 

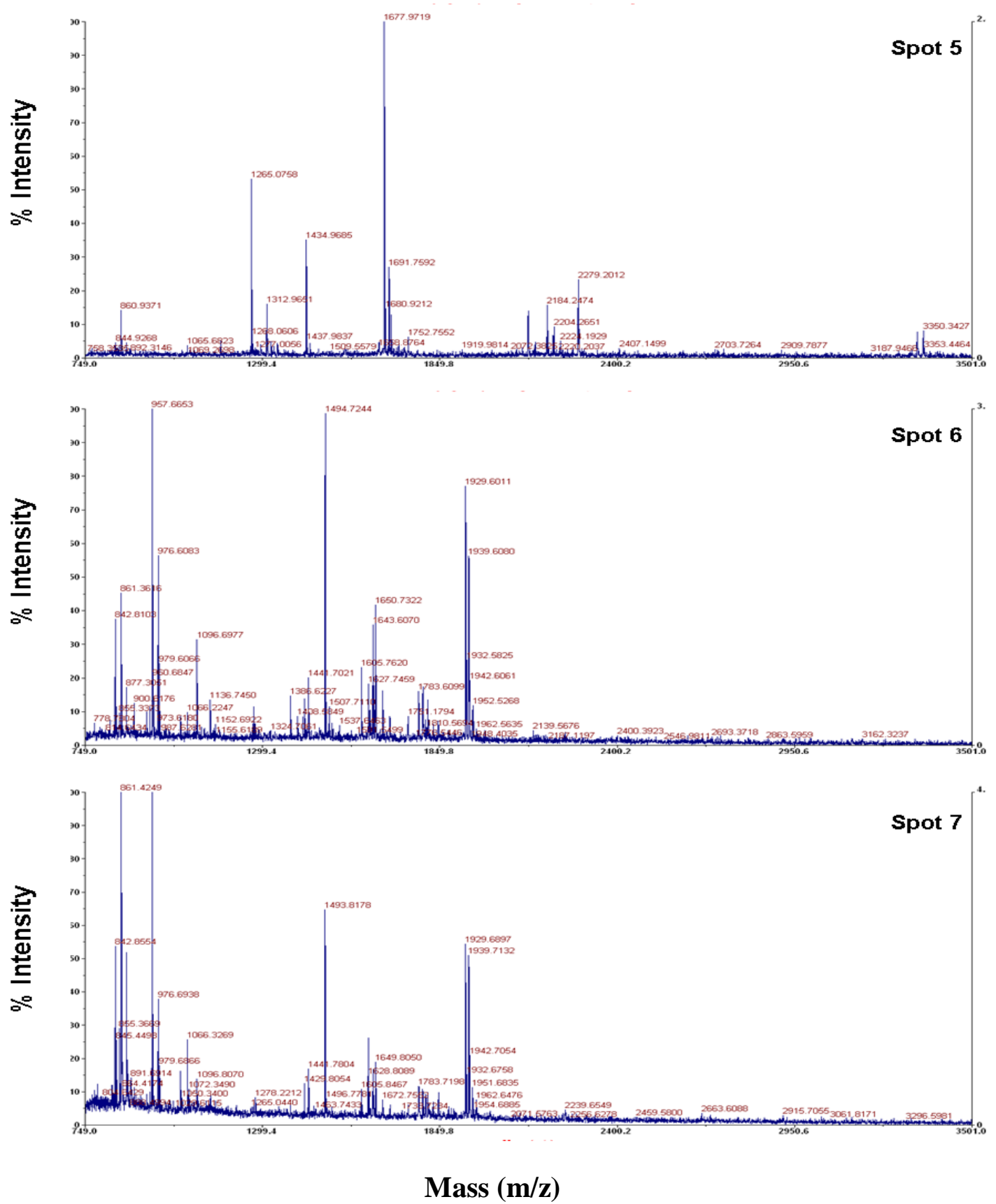

Figure 10-5: MALDI-TOF spectra of the CNP-GSH affinity-purified D. melanogaster spots on 2D gel labelled in Figure 4-20 (B) 


\subsubsection{Identification of spots labelled in Figure 4-20 (B) using PROFOUND}

\begin{tabular}{lcccc}
\hline Spot & Rank Probability Est'd $Z$ & $\begin{array}{c}\text { Protein Intormation and Sequence Analyse } \\
\text { No. }\end{array}$ & Tools (T) & pI kDa R \\
\hline
\end{tabular}

\begin{tabular}{cccccc}
\hline Measured Avg/ Computed & Brror & \multicolumn{2}{c}{ QResidues } & Missed \\
Mass (M) Mono & Mass & $\mathbf{D}(\mathrm{Da})$ & Start & To & Cut Peptide sequence
\end{tabular}

1 gi|24654347|ref|NP_725653.1| glutathione S

$\begin{array}{lllll}+11 & 1.0 \mathrm{e}+000 & 2.36 \quad \begin{array}{l}\text { transferase S1, isoform A [Drosophila } \\ \text { melanogaster] }\end{array} & 55 \quad 4.627 .650\end{array}$

\begin{tabular}{|c|c|c|c|c|c|c|c|}
\hline 768.332 & M & 768.449 & -0.117 & 59 & 65 & 0 & ALAEPLR \\
\hline 1027.369 & M & 1027.523 & -0.154 & 107 & 115 & 0 & VHQSISMAR \\
\hline 1183.505 & M & 1183.624 & -0.119 & 106 & 115 & 1 & RVHQSISMAR \\
\hline 1185.513 & M & 1185.639 & -0.126 & 216 & 225 & 0 & DLIEPYPALR \\
\hline 1341.579 & M & 1341.740 & -0.161 & 215 & 225 & 1 & RDLLEPYPALR \\
\hline 1417.526 & M & 1417.703 & -0.176 & 48 & 58 & 0 & HSYTLFYFNVK \\
\hline 1747.655 & M & 1747.887 & -0.232 & 148 & 162 & 1 & IAVVSYEPEDEIKEK \\
\hline 1747.655 & M & 1747.975 & -0.320 & 164 & 178 & 0 & LVTLNAEVIPFYLEK \\
\hline 1765.655 & M & 1765.794 & -0.139 & 66 & 79 & 0 & YLFAYGNQEYEDVR \\
\hline 2400.212 & M & 2400.116 & 0.096 & 195 & 214 & 0 & LTWADVYFAGITDYMNYMVK \\
\hline 2724.085 & M & 2724.342 & -0.257 & 83 & 106 & 1 & DEWPALKPTMPMGQMPVLEVDGKR \\
\hline 2946.245 & M & 2946.442 & -0.197 & 120 & 145 & 0 & TVGLCGATPWEDLQIDIVVDTINDFR \\
\hline
\end{tabular}

2
$+1 \quad 1.0 \mathrm{e}+000$
2.43
gi|19922932|ref|NP_611964.1| CG16936
[Drosophila melanogaster]
$33 \quad 5.925 .440$

$\begin{array}{lllrrrll}1169.616 & \text { M } & 1169.607 & 0.009 & 39 & 48 & 0 & \text { GEHLTPEFLK } \\ 1264.628 & \text { M } & 1264.667 & -0.039 & 101 & 111 & 0 & \text { LHLDSGHLFAR } \\ 1676.988 & \text { M } & 1677.018 & -0.030 & 24 & 38 & 0 & \text { AIGLDLILRPINLLK } \\ 2122.992 & \text { M } & 2123.005 & -0.013 & 192 & 210 & 0 & \text { LAELPYYQZVNGDGADELK } \\ 2182.976 & \text { M } & 2183.012 & -0.036 & 114 & 131 & 0 & \text { FLYEPILYYGSTDCSIDK } \\ 2279.084 & \text { M } & 2279.106 & -0.022 & 191 & 210 & 1 & \text { RLAELPYYQEVNGDGADELK }\end{array}$

3
$+1 \quad 1.0 \mathrm{e}+000$
2.43
gi|195353258|ref|XP_002043122.1| GM11796 [Drosophila sechellia]
$57 \quad 5.925 .370$
gi|19922932|ref|NP_611964.1| CG16936
[Drosophila melanogaster]

$\begin{array}{rrrrrrll}784.951 & \text { M } & 784.405 & 0.546 & 185 & 190 & 0 & \text { MHAWLK } \\ 1169.750 & \text { M } & 1169.607 & 0.143 & 39 & 48 & 0 & \text { GEHLTPEFLK } \\ 1264.728 & \text { M } & 1264.667 & 0.061 & 101 & 111 & 0 & \text { LHLDSGHLFAR } \\ 1676.804 & \text { M } & 1677.018 & -0.214 & 24 & 38 & 0 & \text { AIGLDLELRPINLLK } \\ 2122.449 & \text { M } & 2123.005 & -0.555 & 192 & 210 & 0 & \text { LAELPYYQEVNGDGADELK } \\ 2182.884 & \text { M } & 2183.012 & -0.128 & 114 & 131 & 0 & \text { FLYEPILYYGSTDCSIDK } \\ 2278.423 & \text { M } & 2279.106 & -0.683 & 191 & 210 & 1 & \text { RLAELPYYQEVNGDGADELK } \\ 2374.155 & \text { M } & 2373.471 & 0.684 & 17 & 38 & 1 & \text { AVLLTAKAIGLDLELRPINLLK } \\ 2828.006 & \text { M } & 2828.615 & -0.609 & 24 & 48 & 1 & \text { AIGLDLELRPINLLKGEHLTPEFLK } \\ 3331.560 & \text { M } & 3331.710 & -0.150 & 49 & 78 & 0 & \text { LNPQHTIPTLIDGEATIDSHAICAYLVEK }\end{array}$




\begin{tabular}{lcccc}
\hline Spot & Rank Probability Est'd $Z$ & $\begin{array}{c}\text { Protein Intormation and Sequence Analyse } \\
\text { No. }\end{array}$ & Tools $(T)$ & pI \\
\hline
\end{tabular}

\begin{tabular}{|c|c|c|c|c|c|c|}
\hline Measured & Avg/ & Computed & Brror & 'Residues & Missec & \\
\hline Mags (M) & Mono & Mags & $\mathbf{D}(\mathrm{Da})$ & Start & Cut & Peptide sequence \\
\hline
\end{tabular}

4

\begin{tabular}{|c|c|c|c|}
\hline$+1 \quad 1.0 \mathrm{e}+000$ & 2.43 & $\begin{array}{l}\text { gi|195353258|ref|XP_002043122.1| GM11796 } \\
\text { [Drosophila sechellia] }\end{array}$ & $\begin{array}{lll}33 & 5.925 .370\end{array}$ \\
\hline - & - & $\begin{array}{l}\text { gi|19922932|ref|NP_611964.1| CG16936 } \\
\text { [Drosophila melanogaster] }\end{array}$ & $33 \quad 5.925 .440$ \\
\hline
\end{tabular}

$\begin{array}{llllrrll}1169.635 & M & 1169.607 & 0.027 & 39 & 48 & 0 & \text { GEHLTPEFLK } \\ 1264.724 & M & 1264.667 & 0.057 & 101 & 111 & 0 & \text { LHLDSGHLFAR } \\ 1677.104 & M & 1677.018 & 0.086 & 24 & 38 & 0 & \text { AIGLDLELRPINLLK } \\ 2123.111 & M & 2123.005 & 0.106 & 192 & 210 & 0 & \text { LAELPYYQZNIGDGADELK } \\ 2183.127 & M & 2183.012 & 0.115 & 114 & 131 & 0 & \text { FLYZPILYYGSTDCSIDK } \\ 2279.198 & M & 2279.106 & 0.092 & 191 & 210 & 1 & \text { RLAELPYYQZMNGDGADELK }\end{array}$

5 $+1 \quad 1.0 \mathrm{e}+000 \quad 2.43 \begin{aligned} & \text { gi| } 19922932 \text { |ref|NP_611964.1| CG16936 } \\ & \text { [Drosophila melanogaster] }\end{aligned} \quad \begin{array}{ll}51 & 5.925 .440\end{array}$

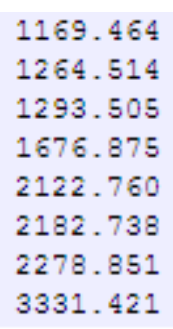

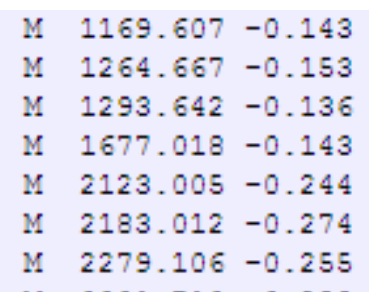

$\mathrm{M} \quad 3331.710-0.289$

$\begin{array}{rr}39 & 48 \\ 101 & 111 \\ 138 & 147 \\ 24 & 38 \\ 192 & 210 \\ 114 & 131 \\ 191 & 210 \\ 49 & 78\end{array}$

6

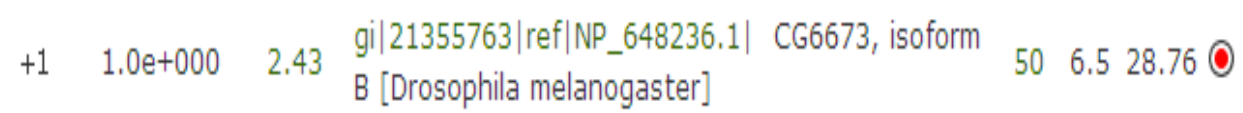

\begin{tabular}{rrrrrrrl}
824.528 & $\mathrm{M}$ & 824.439 & 0.089 & 138 & 145 & 0 & NPPADAIK \\
939.539 & $\mathrm{M}$ & 939.444 & 0.095 & 162 & 170 & 0 & GIPYFGGNK \\
956.553 & $\mathrm{M}$ & 956.417 & 0.136 & 30 & 36 & 0 & FCPYSQR \\
1095.678 & $\mathrm{M}$ & 1095.546 & 0.132 & 161 & 170 & 1 & RGTPYFGGNK \\
1384.849 & $\mathrm{M}$ & 1384.639 & 0.209 & 190 & 200 & 0 & YTLDEPYZLDK \\
1428.901 & $\mathrm{M}$ & 1428.736 & 0.165 & 209 & 220 & 1 & WRDLVAQDEAVK \\
1493.002 & $\mathrm{M}$ & 1492.788 & 0.214 & 11 & 24 & 0 & GSPKPEIPEDGVLR \\
1605.130 & $\mathrm{M}$ & 1604.917 & 0.212 & 123 & 137 & 0 & LSPAVSAIYPVLFTK \\
1642.025 & $\mathrm{M}$ & 1641.788 & 0.237 & 190 & 202 & 1 & YTLDEPYZIDKTR \\
1649.135 & $\mathrm{M}$ & 1648.889 & 0.246 & 10 & 24 & 1 & RGSPKPEIPEDGVLR \\
1672.007 & $\mathrm{M}$ & 1671.789 & 0.218 & 237 & 250 & 0 & HENKPDYDVAFQPL \\
1783.143 & $\mathrm{M}$ & 1782.867 & 0.277 & 146 & 160 & 0 & NFETALDVFEQEITK \\
1796.115 & $\mathrm{M}$ & 1795.875 & 0.240 & 171 & 184 & 0 & IGIADYMIWPWFER \\
1929.240 & $\mathrm{M}$ & 1928.937 & 0.302 & 235 & 250 & 1 & TRHENKPDYDVA.QPL \\
1939.253 & $\mathrm{M}$ & 1938.968 & 0.286 & 146 & 161 & 1 & NFETALDVFEQEITKR \\
\hline
\end{tabular}




\begin{tabular}{|c|c|c|c|}
\hline Spot & 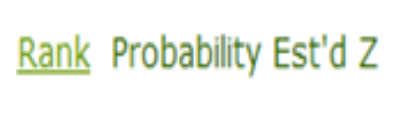 & $\begin{array}{l}\text { Protein Intormation and Sequence Analyse } \\
\qquad \text { Tools }(T)\end{array}$ & $\%$ pI $\mathrm{kDa} \quad \mathrm{R}$ \\
\hline
\end{tabular}

Measured Avg/ Computed Brror Desidues Missed

Mass (M) Mono Mass $\mathbf{D}(\mathrm{Da})$ Start To Cut Peptide sequence

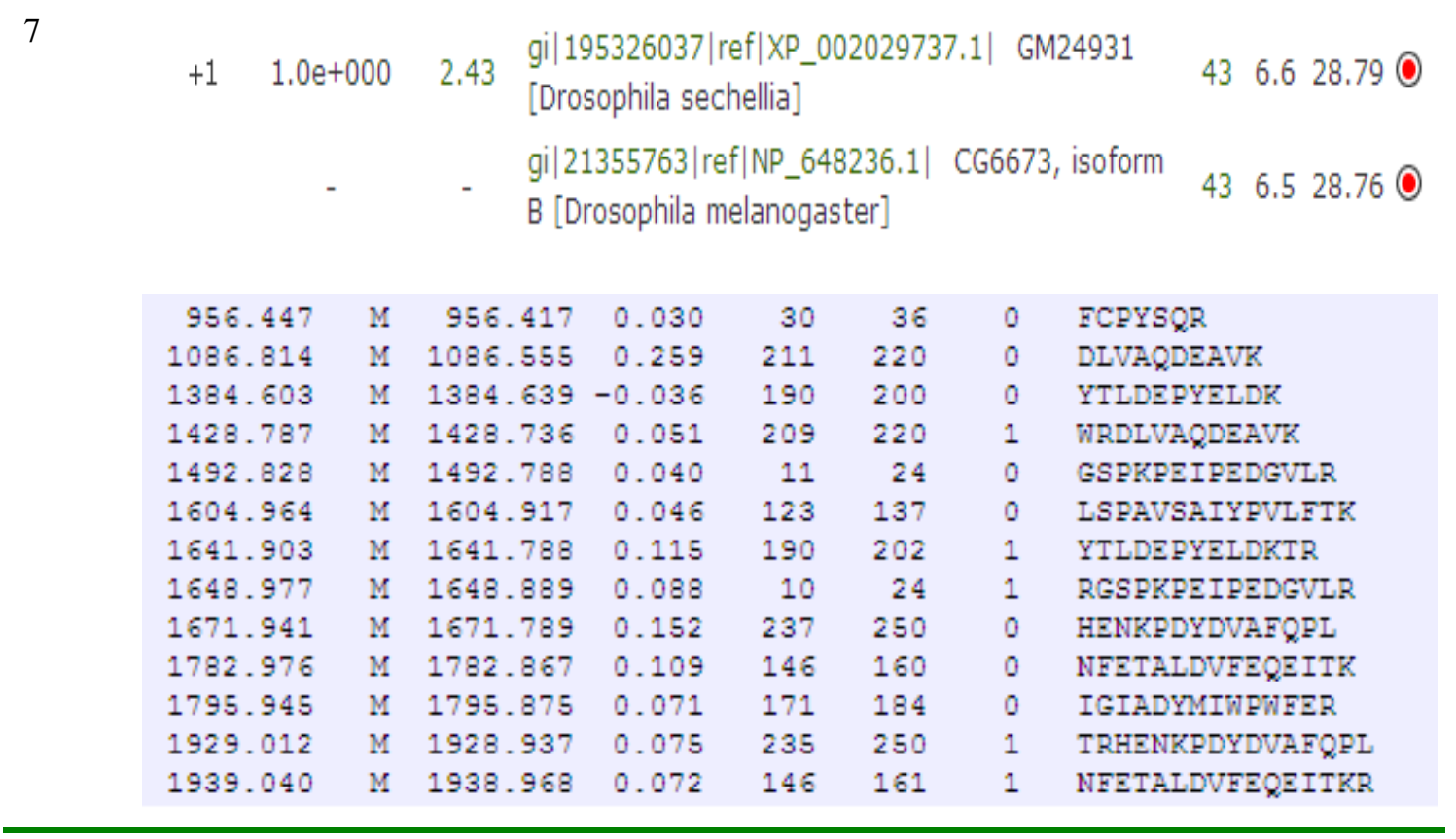


Table 10-1: Identification of D. melanogaster GSTs purified by CNP-GSH affinity matrix

Proteins identified from the CNP-GSH affinity-purified fractions of D. melanogaster separated over a pH range 4-7 (Figure 4-20 B) using 2D gel electrophoresis as described in section 3.2.7.2. The proteins were identified using MALDI-TOF MS peptide mass fingerprinting. Protein spots are as numbered in Figure 4-20. Z score, the sequence coverage and the number of matched peptides are reported as obtained from Profound. In addition the protein name, the Swiss-Prot/TrEMBL accession numbers, the computed molecular mass and isoelectric point are given as obtained from the ExPASy Proteomics server of Swiss Institute of Bioinformatics and the Swiss-Prot database. Identification was made by using taxonomy D. melanogaster and NCBInr database, version dated 12/08/2008. A protein Z score greater than 1.65 is significant in Profound.

\begin{tabular}{|c|c|c|c|c|c|c|c|c|c|}
\hline $\begin{array}{l}\text { Spot } \\
\text { No. }\end{array}$ & $\begin{array}{c}\text { NCBInr } \\
\text { Accession } \\
\text { (Tgi) }\end{array}$ & $\begin{array}{c}\text { Swiss-prot } \\
\text { /TrEMBL } \\
\text { Accession No. }\end{array}$ & Identified protein & $\begin{array}{l}\text { GST class } \\
\text { identified }\end{array}$ & $\begin{array}{c}\text { Theoretical } \\
\text { M.W./pI }\end{array}$ & $\begin{array}{c}\text { Experimental } \\
\text { M.W./pI }\end{array}$ & $\begin{array}{c}\text { Number of } \\
\text { matched } \\
\text { peptides }\end{array}$ & $\begin{array}{c}\text { Coverage } \\
(\%)\end{array}$ & $\begin{array}{c}\mathrm{Z} \\
\text { score }\end{array}$ \\
\hline 1 & 24654347 & P41043 & GST S1 & Sigma & $27.65 / 4.6$ & $33 / 4.6$ & 11 & 47 & 2.36 \\
\hline 2 & 19922932 & Q9XYZ9 & CG16936 & Epsilon & $25.45 / 5.9$ & $24 / 5.0$ & 6 & 33 & 2.43 \\
\hline 3 & 19922932 & Q9XYZ9 & CG16936 & Epsilon & $25.45 / 5.9$ & $24 / 5.1$ & 10 & 52 & 2.35 \\
\hline 4 & 19922932 & Q9XYZ9 & CG16936 & Epsilon & $25.45 / 5.9$ & $24 / 5.2$ & 6 & 33 & 2.43 \\
\hline 5 & 19922932 & Q9XYZ9 & CG16936 & Epsilon & $25.45 / 5.9$ & $24 / 5.3$ & 8 & 51 & 2.43 \\
\hline 6 & 21355763 & Q9VSL4 & CG6673 & Omega & $28.76 / 6.5$ & $28 / 5.4$ & 10 & 50 & 2.43 \\
\hline 7 & 21355763 & Q9VSL4 & CG6673 & Omega & $28.76 / 6.5$ & $28 / 5.5$ & 15 & 43 & 2.43 \\
\hline
\end{tabular}




\subsubsection{Spectra of spots labelled in Figure 4-21}
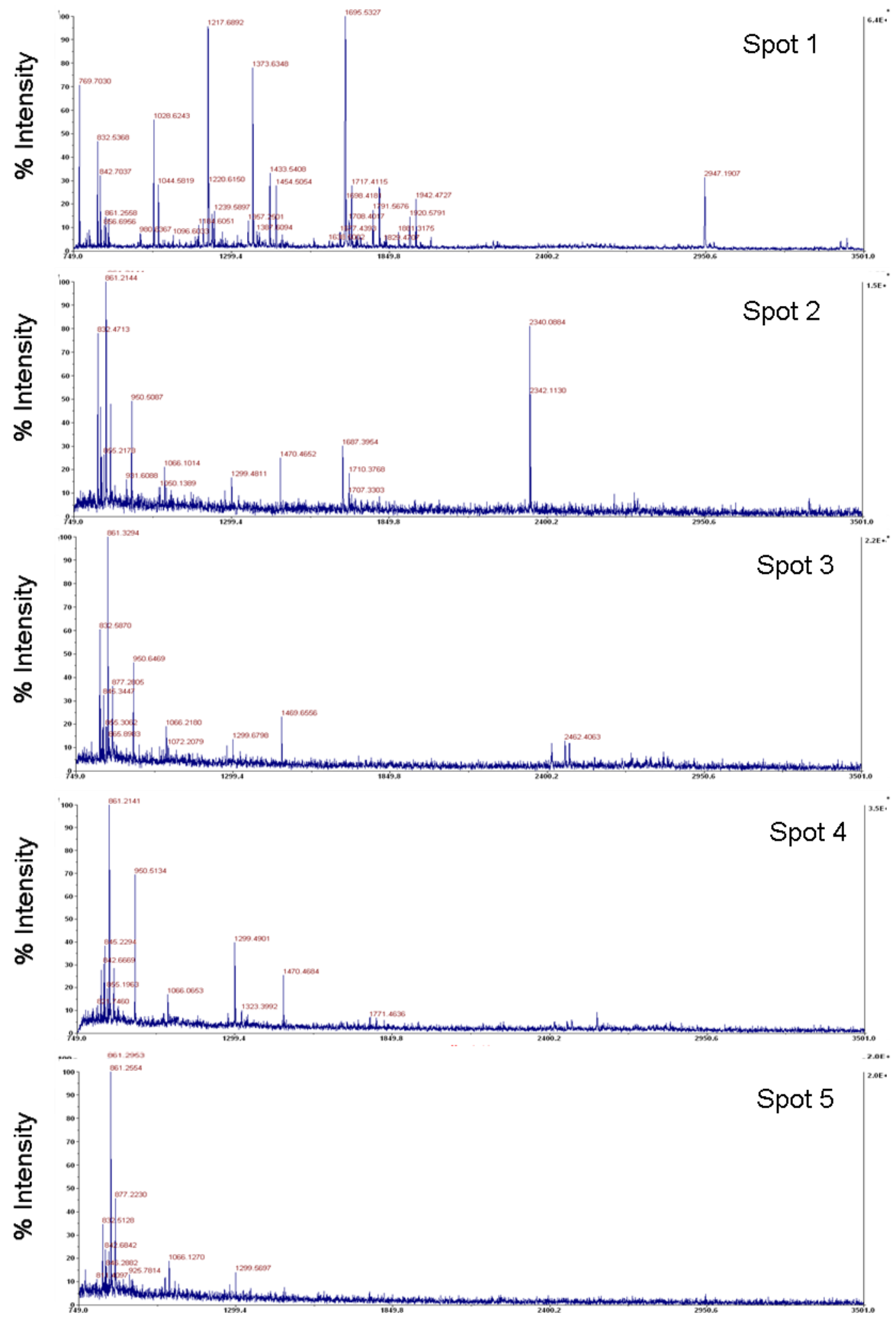

Mass (m/z) 

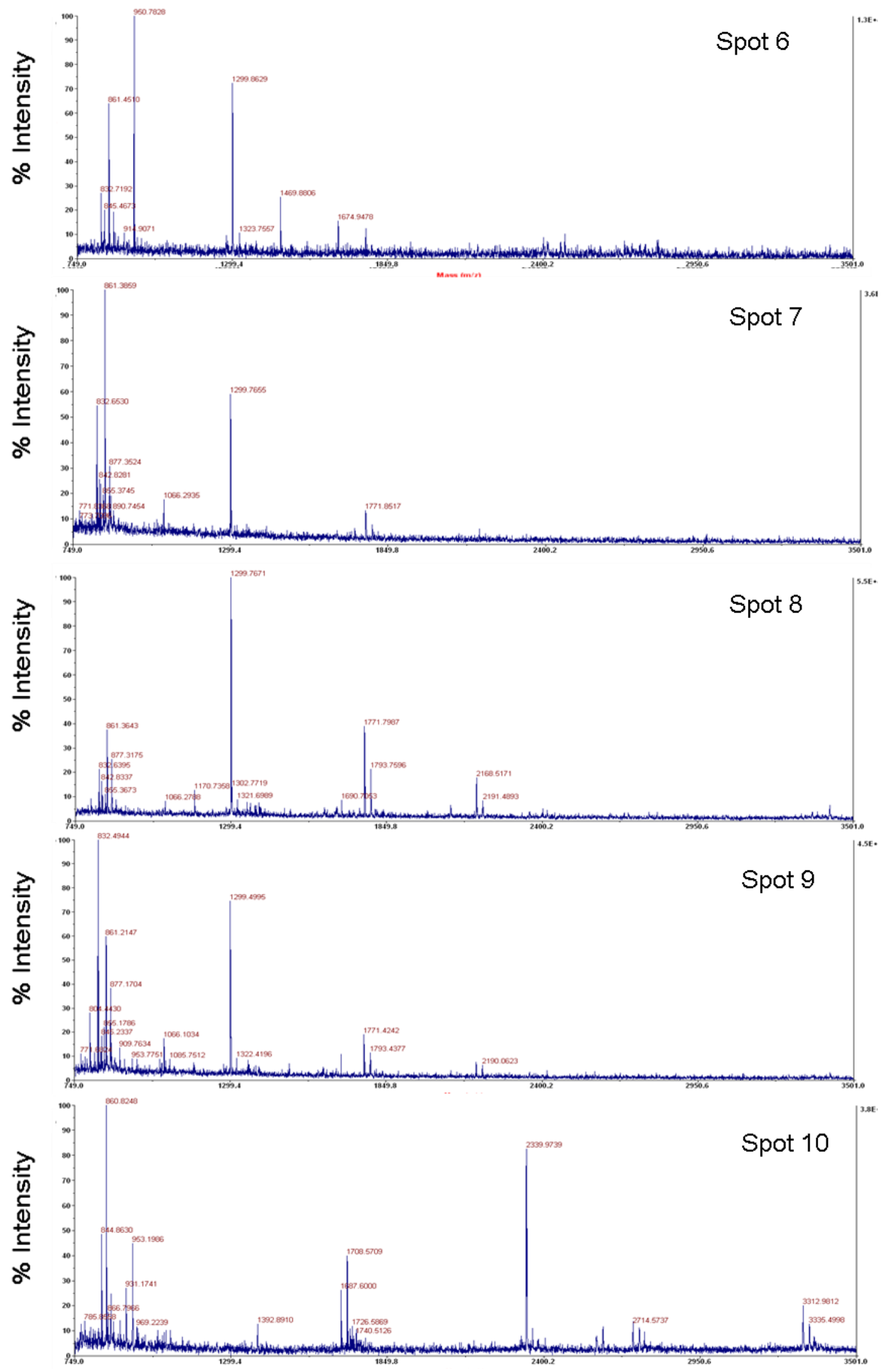

Mass (m/z) 

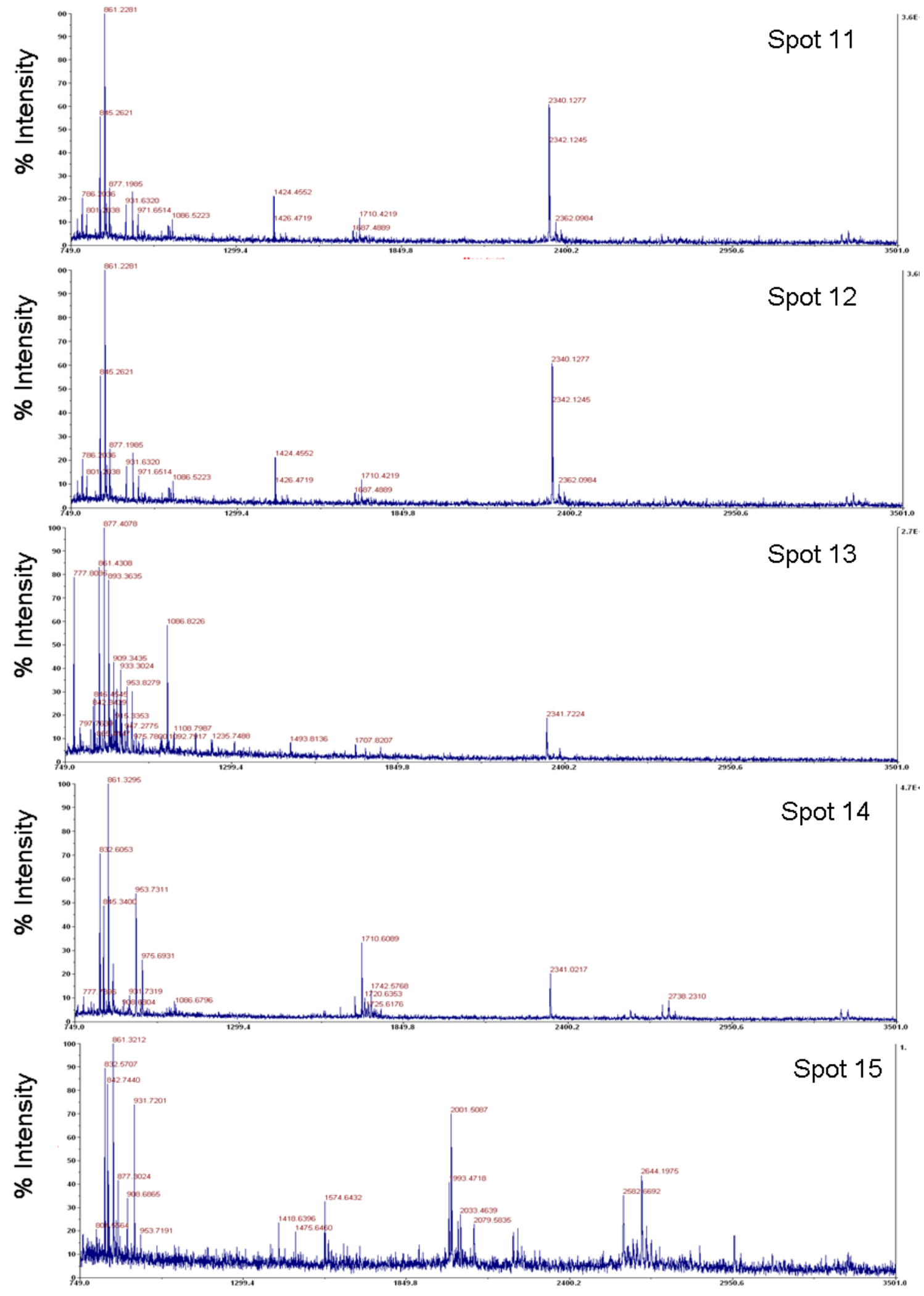

Mass (m/z) 

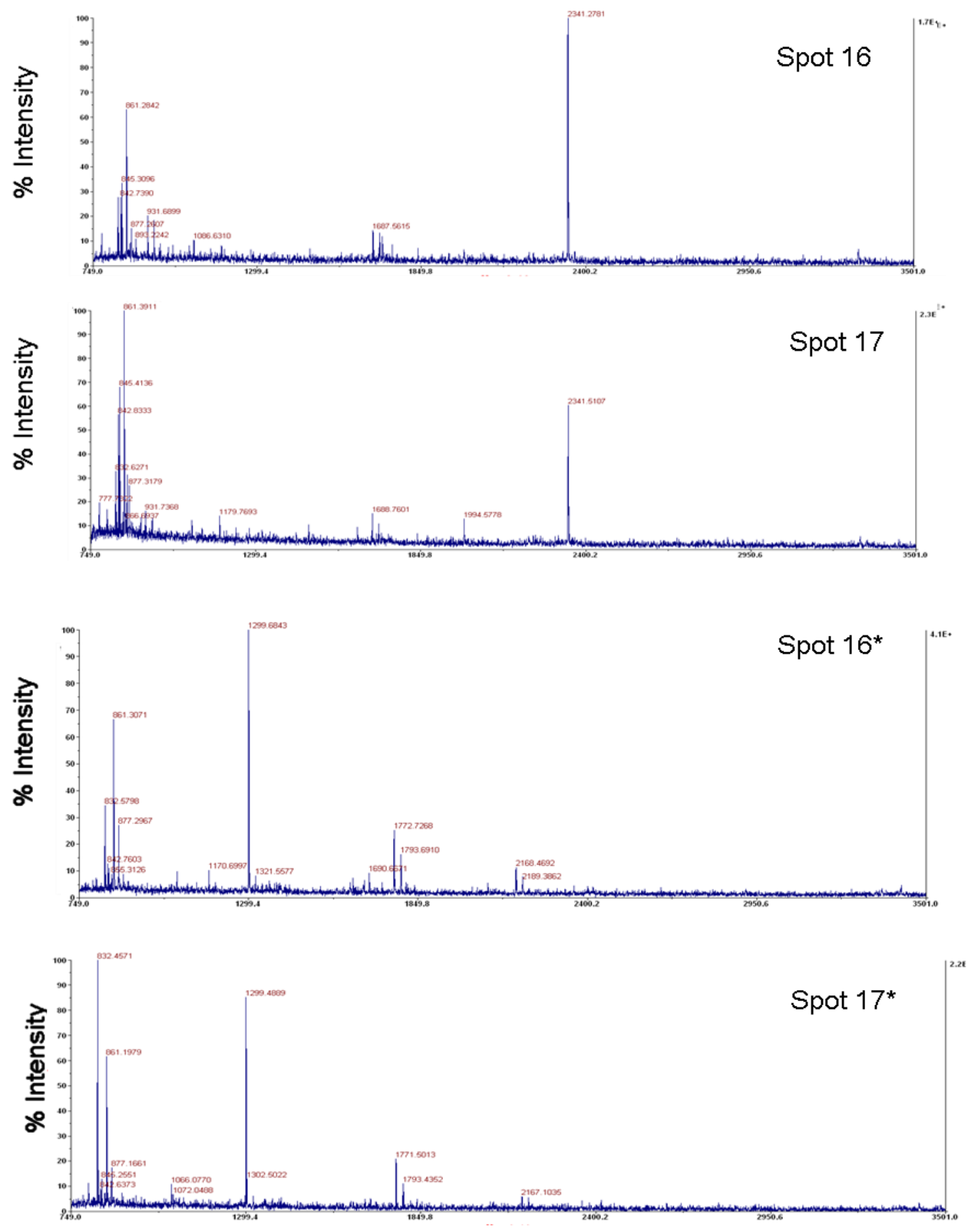

$\operatorname{Mass}(\mathrm{m} / \mathrm{z})$

Figure 10-6: MALDI-TOF spectra of the DNP-GSH affinity-purified L. cuprina spots on 2D gel labelled in Figure 4-21

Spots $16^{*}$ and $17^{*}$ can be found in Figure 6-8. 


\subsubsection{Identification of spots labelled in Figure 4-21 using PROFOUND}

\begin{tabular}{lcccc}
\hline Spot & Rank Probability Est'd $Z$ & $\begin{array}{c}\text { Protein Intormation and Sequence Analyse } \\
\text { No. }\end{array}$ & Tools $(T)$ & pI kDa \\
\hline
\end{tabular}

Measured Avg/ Computed Brror DResidues Missed

Mass (M) Mono Mass D(Da) Start To Cut Peptide sequence

1 gi|1170110|sp|P46437.1|GST_MUSDO RecName:

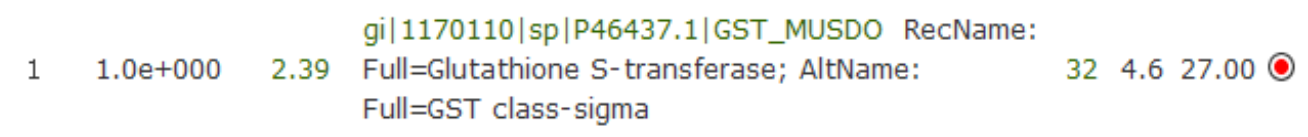

\begin{tabular}{|c|c|c|c|c|c|c|c|}
\hline 768.576 & M & 768.449 & 0.127 & 51 & 57 & 0 & ALAEPLR \\
\hline 1027.642 & M & 1027.523 & 0.119 & 99 & 107 & 0 & VHOSISMAR \\
\hline 1043.612 & M & 1043.518 & 0.094 & 99 & 107 & 0 & $\begin{array}{l}\text { VHQSISMAR } \\
\text { (1) +OQM; }\end{array}$ \\
\hline 1216.804 & M & 1216.645 & 0.159 & 208 & 217 & 0 & DILEQYPALR \\
\hline 1372.896 & M & 1372.746 & 0.151 & 207 & 217 & 1 & RDILEQYPALR \\
\hline 1693.965 & M & 1693.798 & 0.167 & 58 & 71 & 0 & YLFAYGGIEYEDVR \\
\hline 1791.147 & M & 1790.981 & 0.166 & 156 & 170 & 0 & LVTLNNEVIPFYLEK \\
\hline 1919.220 & M & 1919.076 & 0.144 & 155 & 170 & 1 & KLVTLNNEVIPFYLEK \\
\hline 2427.894 & M & 2428.147 & -0.253 & 187 & 206 & 0 & LTWADVYFAGILDYMN \\
\hline
\end{tabular}

(1) $+\mathrm{OeM}_{\text {; }}$

2 $1 \quad 1.0 \mathrm{e}+000 \quad 1.94 \begin{aligned} & \text { gi|3201479|emb|CAA07071.1| glutathione S- } \\ & \text { transferase [Bombyx mori] }\end{aligned} \quad 485.125 .250$

\begin{tabular}{|c|c|c|c|c|c|c|c|}
\hline 952.288 & M & 952.541 & -0.253 & 210 & 217 & 1 & $\begin{array}{l}\text { MLASFLKK } \\
\text { (1) +O@M; }\end{array}$ \\
\hline 1361.738 & M & 1361.679 & 0.058 & 8 & 18 & 0 & LYYLPPSPPCR \\
\hline 2020.025 & M & 2020.109 & -0.085 & 96 & 112 & 1 & AIIDQRLNFDLGILYLR \\
\hline 2138.068 & M & 2138.046 & 0.021 & 8 & 25 & 1 & $\begin{array}{l}\text { LYYLPPSPPCRAVMMTAR } \\
\text { (1) +O@M; }\end{array}$ \\
\hline 2500.973 & M & 2501.146 & -0.172 & 51 & 71 & 0 & MNPQHTIPTMDDNGFILWESR \\
\hline 2622.407 & M & 2622.258 & 0.149 & 194 & 216 & 1 & $\begin{array}{l}\text { MLEPYGYDEIDVTGAKMLASFLK } \\
\text { (2) +0@M; }\end{array}$ \\
\hline 2923.252 & M & 2923.481 & -0.228 & 26 & 50 & 0 & VLELDLHLITTNIMNGEHMTPEYLK \\
\hline 2955.493 & M & 2955.470 & 0.023 & 26 & 50 & 0 & $\begin{array}{l}\text { VLELDLHLITTNIMNGEHMT PEYLK } \\
\text { (2) +0@M; }\end{array}$ \\
\hline
\end{tabular}

3

\begin{tabular}{|c|c|c|c|c|c|c|c|c|c|c|}
\hline \multicolumn{2}{|c|}{$1.0 \mathrm{e}+000$} & $\begin{array}{rr} & g \\
2.43 & \mathrm{t} \\
& \mathrm{g}\end{array}$ & $\begin{array}{l}\text { gi| } 5796758 \\
\text { transferase } \\
\text { gambiae st }\end{array}$ & $\begin{array}{l}\text { ref|X } \\
\text { AGAP } \\
\text { PEST }\end{array}$ & $\begin{array}{l}5626 \\
4164\end{array}$ & 1) & $\begin{array}{l}\text { utathione } \\
\text { opheles }\end{array}$ & \multicolumn{3}{|c|}{$\begin{array}{lll}20 & 5.8 & 25.36\end{array}$} \\
\hline \multicolumn{2}{|c|}{ - } & $\mathrm{g}$ & \multicolumn{5}{|c|}{$\begin{array}{l}\text { gi|3511225|gb|AAC79992.1| glutathione S- } \\
\text { transferase [Anopheles qambiae] }\end{array}$} & 20 & 5.8 & 25.08 \\
\hline 864.413 & M & 864.504 & $4-0.091$ & 96 & 102 & 1 & RAVVHQR & & & \\
\hline 1383.710 & M & 1383.755 & $5-0.044$ & 103 & 113 & 0 & LFFDVAII & & & \\
\hline 1575.588 & M & 1575.684 & $4-0.096$ & 1 & 13 & 0 & MDFYYLPQ & & & \\
\hline 2890.437 & M & 2890.472 & $2-0.036$ & 103 & 125 & 1 & LFFDVAII & EAEYY & YYPQI & IFGK \\
\hline
\end{tabular}




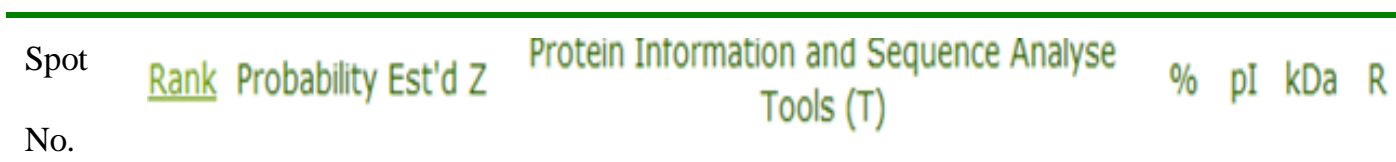

Measured Avg/ Computed Brror Desidues Missed

Mags (M) Mono Mass $\mathbf{D}(\mathrm{Da})$ Start To Cut Peptide sequence

4

\begin{tabular}{|c|c|c|c|}
\hline$+1 \quad 1.0 \mathrm{e}+000$ & 2.43 & $\begin{array}{l}\text { g||57967586|ret|XP_562680.1| glutathıone } \\
\text { transferase (AGAP004164-PD) [Anopheles } \\
\text { gambiae str. PEST] }\end{array}$ & $\begin{array}{lll}32 & 5.825 .360\end{array}$ \\
\hline - & - & $\begin{array}{l}\text { gi|3511225|gb|AAC79992.1| glutathione S- } \\
\text { transferase [Anopheles gambiae] }\end{array}$ & $\begin{array}{lll}33 & 5.8 & 25.08\end{array}$ \\
\hline
\end{tabular}

$\begin{array}{lrrrrrll}1383.762 & \text { M } & 1383.755 & 0.008 & 103 & 113 & 0 & \text { LFFDVAILYQR } \\ 1468.736 & \text { M } & 1468.818 & -0.082 & 205 & 217 & 1 & \text { NLAGAKIFGLYFR } \\ 1575.633 & \text { M } & 1575.684 & -0.051 & 1 & 13 & 0 & \text { MDFYYLPGSAPCR } \\ 1613.900 & \text { M } & 1613.880 & 0.020 & 14 & 29 & 0 & \text { AVQMTAAAVGVELNLK } \\ 2100.025 & \text { M } & 2099.984 & 0.042 & 77 & 94 & 1 & \text { YCAHDPALAERLYPGDPR }\end{array}$

5

$$
\begin{aligned}
& +1 \quad 1.0 \mathrm{e}+000 \quad 2.43 \begin{array}{l}
\text { gi|384151|prf||1905266B glutathione S } \\
\text { transferase:ISOTYPE=GST7 }
\end{array} \quad \begin{array}{ll}
51 \quad 5.925 .410
\end{array}
\end{aligned}
$$

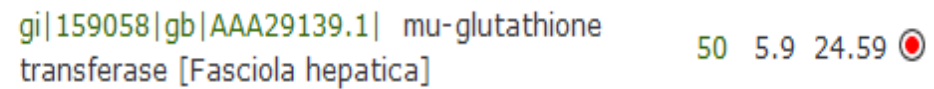

\begin{tabular}{|c|c|c|c|c|c|c|c|}
\hline 1017.500 & $\mathrm{M}$ & 1017.564 & -0.063 & 57 & 65 & 0 & LTQSVAIMR \\
\hline 1240.611 & M & 1240.633 & -0.022 & 107 & 116 & 1 & FEELKGDYLK \\
\hline 1268.356 & M & 1268.622 & -0.266 & 96 & 106 & 0 & IGFGLTCYNPK \\
\hline 1305.544 & M & 1305.689 & -0.145 & 55 & 65 & 1 & CKLTQSVAIMR \\
\hline 1305.544 & M & 1305.641 & -0.097 & 84 & 95 & 0 & ISMIEGAAMDLR \\
\hline 1548.423 & M & 1548.774 & -0.352 & 82 & 95 & 1 & $\begin{array}{l}\text { ARISMIEGA.AMDLR } \\
\text { (1) +o@M; }\end{array}$ \\
\hline 1803.407 & M & 1802.861 & 0.546 & 66 & 81 & 1 & YIADKHGMLGSTPEER \\
\hline 1851.945 & M & 1851.918 & 0.027 & 117 & 132 & 1 & $\begin{array}{l}\text { GLPTTLKMWSDFLGDR } \\
\text { (1) +0@M; }\end{array}$ \\
\hline 2027.371 & M & 2026.970 & 0.401 & 38 & 54 & 0 & FNMGLDLPNLPYYIDDK \\
\hline 2159.130 & M & 2159.020 & 0.110 & 11 & 27 & 0 & LFLEYLGEEYEEHLYGR \\
\hline 2642.131 & M & 2642.271 & -0.140 & 33 & 54 & 1 & $\begin{array}{l}\text { WLGDKFNMGLDLPNLPYYIDDK } \\
\text { (1) +o@M; }\end{array}$ \\
\hline
\end{tabular}




\begin{tabular}{|c|c|c|c|}
\hline Spot & Rank Probability Est'd Z & $\begin{array}{l}\text { Protein Intormation and Sequence Analyse } \\
\text { Tools }(T)\end{array}$ & $\%$ pI kDa R \\
\hline
\end{tabular}

Measured Avg/ Computed Brror DResidues Missed

Mags(M) Mono Mass D(Da) Start To Cut Peptide sequence

6 $\begin{array}{llll}1.0 \mathrm{e}+000 & 1.94 & \begin{array}{l}\text { gi|7387485|gb|AAB33637.2| glutathione S- } \\ \text { transferase [Heligmosomoides polygyrus] }\end{array}\end{array} 56 \quad 6.624 .530$

\begin{tabular}{|c|c|c|c|c|c|c|c|}
\hline 908.614 & M & 908.420 & 0.194 & 185 & 191 & 1 & CDRTVMR \\
\hline 974.491 & M & 974.503 & -0.011 & 140 & 148 & 0 & DSDLVGTIR \\
\hline 2168.392 & M & 2168.075 & 0.317 & 192 & 209 & 0 & QLYCVSQPVLVMDYVQAR \\
\hline 2184.775 & M & 2185.046 & -0.272 & 121 & 139 & 0 & TDVCLPPGSYQVLDLHESR \\
\hline 2339.974 & M & 2340.171 & -0.197 & 192 & 210 & 1 & $\begin{array}{l}\text { QLYYCVSQPVLVMDYVQARR } \\
\text { (1) +OQM; }\end{array}$ \\
\hline 2358.436 & M & 2358.023 & 0.412 & 164 & 184 & 1 & $\begin{array}{l}\text { SSTSMGPMSQPLDCITDTRSR } \\
\text { (2) +0@M; }\end{array}$ \\
\hline 2714.606 & M & 2714.289 & 0.317 & 140 & 163 & 1 & DSDLVGTIRSAGDVCCNILPTVHR \\
\hline 306.781 & M & 2806.296 & 0.485 & 71 & 96 & 0 & TPDFAGATPFESALIDSLADAYTDYR \\
\hline 3265.903 & M & 3265.511 & 0.391 & 71 & 100 & 1 & TPDFAGATPFESALIDSLADAYTDYRAEMK \\
\hline
\end{tabular}

7 $1 \quad 1.0 \mathrm{e}+000 \quad 1.26 \begin{aligned} & \text { gi|12007374|gb|AAG45164.1|AF316636_1 } \\ & \text { glutathione S-transferase E2 [Anopheles gambiae] }\end{aligned} 235.524 .950$ $\begin{array}{rrrrrrrl}836.408 & \text { M } & 836.391 & 0.017 & 82 & 88 & 0 & \text { DDSLYPR } \\ 1176.632 & M & 1176.665 & -0.033 & 183 & 191 & 1 & \text { IYANIDRLK } \\ 1712.030 & \text { M } & 1711.971 & 0.059 & 17 & 32 & 1 & \text { AVELTAKALLLELQQR } \\ 1867.832 & \text { M } & 1867.858 & -0.026 & 192 & 209 & 0 & \text { QLPYYEEANGGGGTDLGK }\end{array}$

8 gi| 19335973 |emb|CAD26834.1| putative $+1 \quad 1.0 \mathrm{e}+000 \quad 2.43$ glutathione S-transferase [Drosophila $28 \quad 5.724 .92 \bigcirc$ melanogaster]

gi|27820029|gb|AAL68213.2 | GM08326p [Drosophila melanogaster] $28 \quad 5.825 .000$ gi|24654992|ref|NP_611330.2 | glutathione S transferase E8 [Drosophila melanogaster]

$\begin{array}{rrrrrrrl}990.676 & M & 990.481 & 0.195 & 215 & 222 & 1 & \text { KFNFTFST } \\ 1084.822 & M & 1084.660 & 0.162 & 205 & 214 & 1 & \text { GARDLVTLLK } \\ 1795.897 & M & 1795.910 & -0.012 & 79 & 93 & 1 & \text { YGQSDTLYPRDLLR } \\ 3404.798 & M & 3404.677 & 0.121 & 49 & 78 & 1 & \text { KNPQHTVPTLEDDGHFIWDSHAISAYLVSK }\end{array}$

9
$19.6 \mathrm{e}-001$
1.47
gi|19922932|ref|NP_611964.1| CG16936 [Drosophila melanogaster]
215.925 .440

$\begin{array}{rrrrrrll}784.575 & \text { M } & 784.405 & 0.170 & 185 & 190 & 0 & \text { MHAWLK } \\ 1172.540 & M & 1172.616 & -0.076 & 182 & 190 & 1 & \begin{array}{l}\text { FPKMHAWLK } \\ (1)+0 \text { GM; }\end{array} \\ 1533.723 & M & 1533.852 & -0.129 & 101 & 113 & 1 & \text { LHLDSGHLFARLR } \\ 2828.526 & M & 2828.615 & -0.089 & 24 & 48 & 1 & \text { AIGLDLELRPINLLKGEHLTPEFLK }\end{array}$




\begin{tabular}{lcccc}
\hline Spot & Rank Probability Est'd $Z$ & Protein Intormation and Sequence Analyse & $\%$ & pI \\
No. & Tools $(T)$ & $R$
\end{tabular}

Measured Avg/ Computed Brror Desidues Missed

Mass (M) Mono Mass $\mathbf{\square}(\mathrm{Da})$ Start To Cut Peptide sequence

10 gi|57967586|ref|XP_562680.1| glutathione $+1 \quad 1.0 \mathrm{e}+000 \quad 2.43$ transferase (AGAP004164-PD) [Anopheles

$33 \quad 5.825 .36 \bigcirc$ gambiae str. PEST]

gi|3511225|gb|AAC79992.1| glutathione Stransferase [Anopheles gambiae]

$33 \quad 5.825 .08 \bigcirc$

$\begin{array}{lrrrrrrc}1915.952 & \text { M } & 1915.989 & -0.037 & 30 & 45 & 1 & \begin{array}{l}\text { LTDLMKGEHMKPEFLK } \\ 2029.039\end{array} \\ \text { M } & 2028.945 & 0.094 & 136 & 152 & 0 & \begin{array}{c}\text { SMEQALEFLNTFLEGER } \\ (1)+0 @ M ;\end{array} \\ 2347.224 & \text { M } & 2347.248 & -0.024 & 14 & 35 & 1 & \begin{array}{c}\text { AVQMTAAAVGVINLKLTDLMK } \\ (2)+0 @ M ;\end{array} \\ 2890.438 & \text { M } & 2890.472 & -0.034 & 103 & 125 & 1 & \text { LFFDVAILYQRFAEYYYPQIFGK }\end{array}$

11 gi|84402 | pir||A26484 glutathione transferase $+1 \quad 1.0 \mathrm{e}+000 \quad 2.43 \quad$ (EC 2.5.1.18) - fluke (Schistosoma japonicum) $35 \quad 6.325 .83$ () (fragment)

gi|121697|sp|P08515.3|GST26_SCHJA RecName: Full=Glutathione S-transferase class-mu $26 \mathrm{kDa}$
isozyme; Short=GST 26; AltName: Full=Sj26 antigen; AltName: Full=SjGST

$\begin{array}{rrrrrrll}751.211 & \text { M } & 751.375 & -0.164 & 115 & 120 & 0 & \text { DFETLK } \\ 1148.613 & \text { M } & 1148.632 & -0.019 & 20 & 28 & 0 & \text { LLLEYLEEK } \\ 1407.923 & \text { M } & 1407.688 & 0.235 & 127 & 137 & 1 & \text { LPEMLKMFEDR } \\ 1423.520 & \text { M } & 1423.683 & -0.163 & 127 & 137 & 1 & \text { LPEMLKMFEDR } \\ & & & & & & & (1)+0 @ M ; \\ 1515.849 & \text { M } & 1515.796 & 0.053 & 91 & 104 & 0 & \text { AEISMLEGAVLDIR } \\ 1682.055 & \text { M } & 1681.721 & 0.334 & 29 & 41 & 1 & \text { YEEHLYERDEGDK } \\ 2627.605 & \text { M } & 2627.291 & 0.314 & 196 & 219 & 1 & \text { SSKYIAWPLQGWQATFGGGDHPPK }\end{array}$

12 gi| 121696 |sp|P28338.1|GSTT1_MUSDO

$1 \quad 1.0 \mathrm{e}+000 \quad 1.75$ RecName: Full=Glutathione S-transferase 1; 367.723 .80 C AltName: Full=GST class- theta

\begin{tabular}{|c|c|c|c|c|c|c|c|}
\hline 764.520 & M & 764.410 & 0.110 & 14 & 20 & 0 & SVLMTAK \\
\hline 1080.713 & M & 1080.588 & 0.125 & 67 & 75 & 0 & $\begin{array}{l}\text { AIMVYLVEK } \\
\text { (1) +oeM; }\end{array}$ \\
\hline 1849.209 & M & 1849.045 & 0.164 & 30 & 45 & 0 & LLNLQAGEHLKPEFLK \\
\hline 2408.290 & M & 2408.211 & 0.078 & 46 & 66 & 0 & INPQHTIPTLVDGDFALWESR \\
\hline 2580.135 & M & 2580.257 & -0.122 & 107 & 128 & 1 & SFADYYYPQIFAKAPADPELFK \\
\hline 3454.604 & M & 3454.794 & -0.190 & 46 & 75 & 1 & INPOHTIPTLVDGDFALWESRAIMVYI \\
\hline
\end{tabular}




\begin{tabular}{|c|c|c|}
\hline Spot & 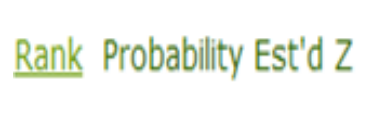 & $\begin{array}{l}\text { Protein Intormation and Sequence Analyse } \\
\qquad \text { Tools }(T)\end{array}$ \\
\hline & $\begin{array}{l}\text { Measured Avg/ Computed } \\
\text { Masg (M) Mono Mass }\end{array}$ & $\begin{array}{l}\text { Brror } \text { QResidues Misged } \\
\text { D(Da) Start To Cut Peptide sequenc }\end{array}$ \\
\hline
\end{tabular}

14

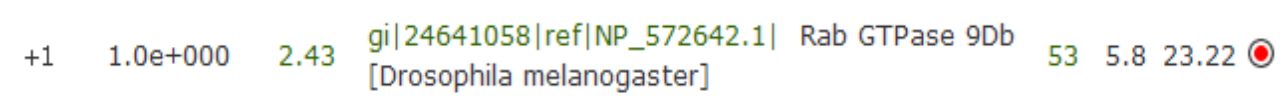

$\begin{array}{rrrrrrll}776.386 & \text { M } & 776.385 & 0.001 & 38 & 44 & 0 & \text { VTVGMDR } \\ 786.868 & \text { M } & 787.401 & -0.532 & 109 & 114 & 1 & \text { RLCPDK } \\ 988.332 & \text { M } & 988.548 & -0.216 & 73 & 80 & 1 & \text { LLKATQCR } \\ 1071.039 & \mathrm{M} & 1070.633 & 0.406 & 10 & 20 & 0 & \text { IIILGDSGVGK } \\ 1296.311 & \mathrm{M} & 1296.601 & -0.290 & 144 & 154 & 0 & \text { QSICFEEVSAR } \\ 1554.394 & \mathrm{M} & 1554.880 & -0.485 & 110 & 123 & 1 & \text { LCPDKVTVLLVGNK } \\ 1762.740 & \mathrm{M} & 1762.932 & -0.192 & 115 & 130 & 1 & \text { VTVLLVGNKSDDPNHR } \\ 1837.784 & \mathrm{M} & 1837.808 & -0.024 & 56 & 70 & 1 & \text { MGRMLQVWDTSDDER } \\ 1844.911 & \mathrm{M} & 1845.020 & -0.109 & 10 & 26 & 1 & \text { IIILGDSGVGKTCLIMR } \\ 2808.727 & \mathrm{M} & 2809.409 & -0.682 & 81 & 105 & 1 & \text { SAHGILLVYDITSSKSFQNIDGWMK }\end{array}$

13

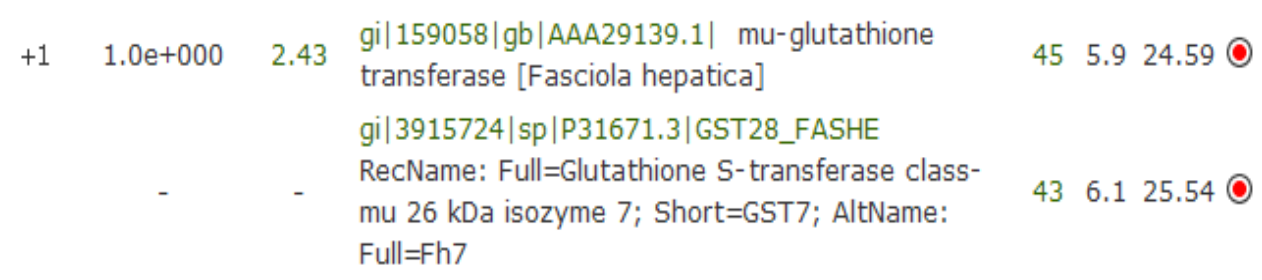

\begin{tabular}{|c|c|c|c|c|c|c|c|}
\hline 796.444 & M & 796.455 & -0.011 & 4 & 10 & 0 & GLQQPVR \\
\hline 1065.590 & M & 1065.640 & -0.050 & 2 & 10 & 1 & IRGLQQPVR \\
\hline 1337.598 & M & 1337.631 & -0.033 & 84 & 95 & 0 & $\begin{array}{l}\text { ISMIEGAAMDLR } \\
\text { (2) +O@M; }\end{array}$ \\
\hline 1707.023 & M & 1706.869 & 0.154 & 156 & 169 & 1 & YLAPQCLDDFPKLK \\
\hline 1852.008 & M & 1851.918 & 0.090 & 117 & 132 & 1 & $\begin{array}{l}\text { GLPTTLKMWSDFLGDR } \\
\text { (1) +o@M; }\end{array}$ \\
\hline 2544.301 & M & 2544.191 & 0.110 & 11 & 30 & 1 & LFLEYLGEBYBEHLYGRNDR \\
\hline 2717.429 & M & 2717.282 & 0.147 & 133 & 155 & 0 & $\begin{array}{l}\text { QYLIGSSVSHVDFMVYEALDCIR } \\
\text { (1) +O@M; }\end{array}$ \\
\hline
\end{tabular}

15 $+1 \quad 1.0 \mathrm{e}+000 \quad 2.43 \quad$ gil $17137218 \mid$ ref $\mid$ NP_477171.1| Rab-protein 14, $\begin{array}{lll}60 & 5.9 & 24.47\end{array}$

\begin{tabular}{|c|c|c|c|c|c|c|c|}
\hline 830.427 & M & 830.438 & -0.011 & 52 & 58 & 0 & IIEVDDK \\
\hline 1014.620 & M & 1014.524 & 0.096 & 75 & 82 & 1 & AVTRSYYR \\
\hline 1493.337 & M & 1492.781 & 0.556 & 83 & 96 & 1 & GAAGALMVYDITRR \\
\hline 1836.108 & M & 1835.844 & 0.264 & 36 & 51 & 0 & FMANCPHTIGVEFGTR \\
\hline 1836.108 & M & 1835.891 & 0.217 & 96 & 110 & 1 & RSTYNHLSSWLTDTR \\
\hline 2077.301 & M & 2077.029 & 0.272 & 181 & 199 & 0 & LDLNASESGVQHRPSQPSR \\
\hline 2408.029 & M & 2408.222 & -0.193 & 14 & 34 & 1 & YIIIGDMGVGKSCLLHQFTEK \\
\hline 2647.868 & M & 2648.271 & -0.403 & 36 & 58 & 1 & FMANCPHTIGVEFGTRIIEVDDK \\
\hline 2741.095 & M & 2741.358 & -0.264 & 1 & 24 & 1 & MTAAPYNYNYIFKYIIIGDMGVGK \\
\hline 2756.684 & M & 2757.353 & -0.670 & 1 & 24 & 1 & $\begin{array}{l}\text { MTAAPYNYNYI FKYI I IGDMGVGK } \\
\text { (1) +o@M; }\end{array}$ \\
\hline 2773.069 & M & 2773.348 & -0.279 & 1 & 24 & 1 & $\begin{array}{l}\text { MTAAPYNYNYI FKYI I I GDMGVGK } \\
\text { (2) +O@M; }\end{array}$ \\
\hline 3479.755 & M & 3479.584 & 0.170 & 141 & 171 & 1 & $\begin{array}{l}\text { EFADENGLMFLEASAMTGQNVEEAFLETARK } \\
\text { (2) +oeM; }\end{array}$ \\
\hline
\end{tabular}




\begin{tabular}{lcccc}
\hline Spot & Rank Probability Est'd Z & Protein Intormation and Sequence Analyse & $\%$ pI kDa R \\
No. & Tools (T) & &
\end{tabular}

$\begin{array}{cllll}\text { Measured Avg/ Computed Brror } & \text { QResidues Missed } \\ \text { Mass (M) Mono Mass } & \mathbf{D}(\mathrm{Da}) & \text { Start } & \text { To Cut Peptide sequence }\end{array}$

16 $+1 \quad 1.0 \mathrm{e}+000 \quad 2.43 \begin{aligned} & \text { gi| } 6517192|\mathrm{dbj}| \text { BAA87878.1| Drab2 [Drosophila } \\ & \text { melanogaster] }\end{aligned} \quad \begin{array}{ll}43 & 5.823 .690\end{array}$

$\begin{array}{rlrrrrll}776.467 & M & 776.410 & 0.057 & 47 & 53 & 0 & \text { MITIDGK } \\ 847.271 & M & 847.414 & -0.143 & 121 & 127 & 1 & \text { SDLDSRR } \\ 908.052 & M & 907.403 & 0.649 & 132 & 139 & 0 & \text { EEGEAFAR } \\ 1474.980 & M & 1474.825 & 0.155 & 78 & 91 & 1 & \text { GAAGALLVYDITRR } \\ 2139.536 & M & 2140.068 & -0.531 & 152 & 170 & 1 & \text { TAANVEEAFINTAKEIYEK } \\ 2340.340 & M & 2340.239 & 0.102 & 9 & 29 & 1 & \text { YIIIGDIGVGKSCLLIQFTDK } \\ 2422.388 & M & 2422.200 & 0.188 & 166 & 186 & 1 & \text { EIYEKIQZGVFDINNEANGIK }\end{array}$

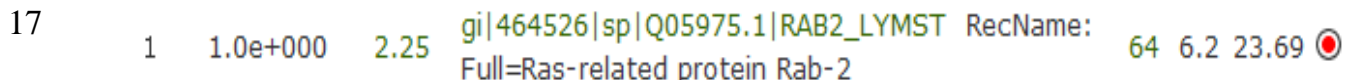

\begin{tabular}{|c|c|c|c|c|c|c|c|}
\hline 776.395 & M & 776.410 & -0.014 & 47 & 53 & 0 & MITIDGK \\
\hline 1021.858 & M & 1021.494 & 0.364 & 1 & 8 & 0 & MSYAYLFK \\
\hline 1134.474 & M & 1134.628 & -0.154 & 9 & 19 & 0 & YIIIGDTGVGK \\
\hline 1474.869 & M & 1474.825 & 0.044 & 78 & 91 & 1 & GAAGALLVYDITRR \\
\hline 1477.490 & M & 1477.741 & -0.251 & 152 & 165 & 0 & TAANVEEAFINTAK \\
\hline 1717.331 & M & 1717.805 & -0.474 & 92 & 105 & 0 & DTFNHLTTWLEDAR \\
\hline 1785.325 & M & 1784.920 & 0.405 & 31 & 46 & 0 & FQPVHDLTIGVEFGAR \\
\hline 1918.629 & M & 1918.989 & -0.360 & 54 & 69 & 1 & QIKLQIWDTAGQESFR \\
\hline 2007.326 & M & 2007.016 & 0.309 & 57 & 73 & 1 & LQIWDTAGQESFRSITR \\
\hline 2340.382 & M & 2340.239 & 0.144 & 9 & 29 & 1 & YIIIGDTGVGKSCLLIQFTDK \\
\hline 2371.808 & M & 2372.156 & -0.349 & 106 & 126 & 1 & $\begin{array}{l}\text { QHSNSNMVIMLIGNKSDLEAR } \\
\text { (1) +O@M; }\end{array}$ \\
\hline 2388.083 & M & 2388.152 & -0.068 & 106 & 126 & 1 & $\begin{array}{l}\text { QHSNSNMVIMLIGNKSDLEAR } \\
\text { (2) +O@M; }\end{array}$ \\
\hline
\end{tabular}

17(2)

\begin{tabular}{|c|c|c|c|c|c|}
\hline$+1 \quad 1.0 \mathrm{e}+000$ & 2.43 & $\begin{array}{l}\text { gi|56967694|gb|AAW31996.1| } \\
\text { [Drosophila melanogaster] }\end{array}$ & CG2885 & 42 & $5.722 .33 \mathrm{C}$ \\
\hline - & - & $\begin{array}{l}\text { gi|24641019|ref|NP_572627.1| } \\
\text { [Drosophila melanogaster] }\end{array}$ & RabX2 & 42 & 5.722 .350 \\
\hline - & - & $\begin{array}{l}\text { gi| } 56967680|\mathrm{gb}| \text { AAW31989.1| } \\
\text { [Drosophila melanogaster] }\end{array}$ & CG2885 & 32 & $5.922 .37 \varnothing$ \\
\hline - & - & $\begin{array}{l}\text { gi| } 56967682 \mid \text { gb } \mid \text { AAW31990.1| } \\
\text { [Drosophila melanogaster] }\end{array}$ & CG2885 & 26 & $5.722 .54 \mathrm{C}$ \\
\hline
\end{tabular}

\begin{tabular}{|c|c|c|c|c|c|c|c|}
\hline 1106.547 & M & 1106.560 & -0.013 & 142 & 152 & 0 & GALGFEEVSAK \\
\hline 1602.935 & M & 1602.861 & 0.074 & 79 & 93 & 0 & SAHGILLVYDITSSK \\
\hline 1803.009 & $\mathrm{M}$ & 1802.974 & 0.036 & 10 & 26 & 1 & VLVLGDSGVGKSCLIMR \\
\hline 2211.257 & M & 2211.139 & 0.118 & 1 & 20 & 1 & MSHFDYLFKVLVLGDSGVGK \\
\hline 2825.205 & M & 2825.405 & -0.200 & 79 & 103 & 1 & $\begin{array}{l}\text { SAHGILLVYDITSSKSFQNIDGWMK } \\
\text { (1) +O@M; }\end{array}$ \\
\hline 3338.738 & $\mathrm{M}$ & 3338.557 & 0.181 & 142 & 171 & 1 & $\begin{array}{l}\text { GALGFEEVSAKSGMNYYIFSSLAMDIYHR } \\
\text { (2) +o@M; }\end{array}$ \\
\hline
\end{tabular}




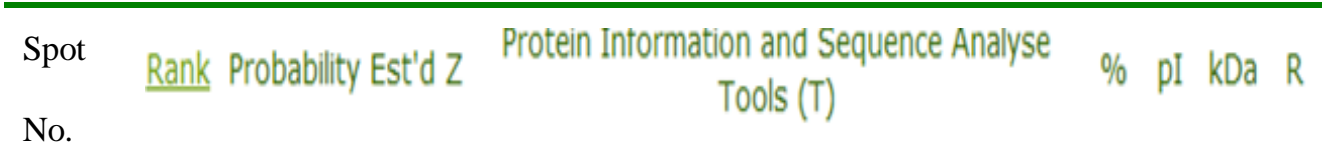

Measured Avg/ Computed Brror Desidues Missed

Mass (M) Mono Mass $\mathbf{D}(\mathrm{Da})$ Start To Cut Peptide sequence

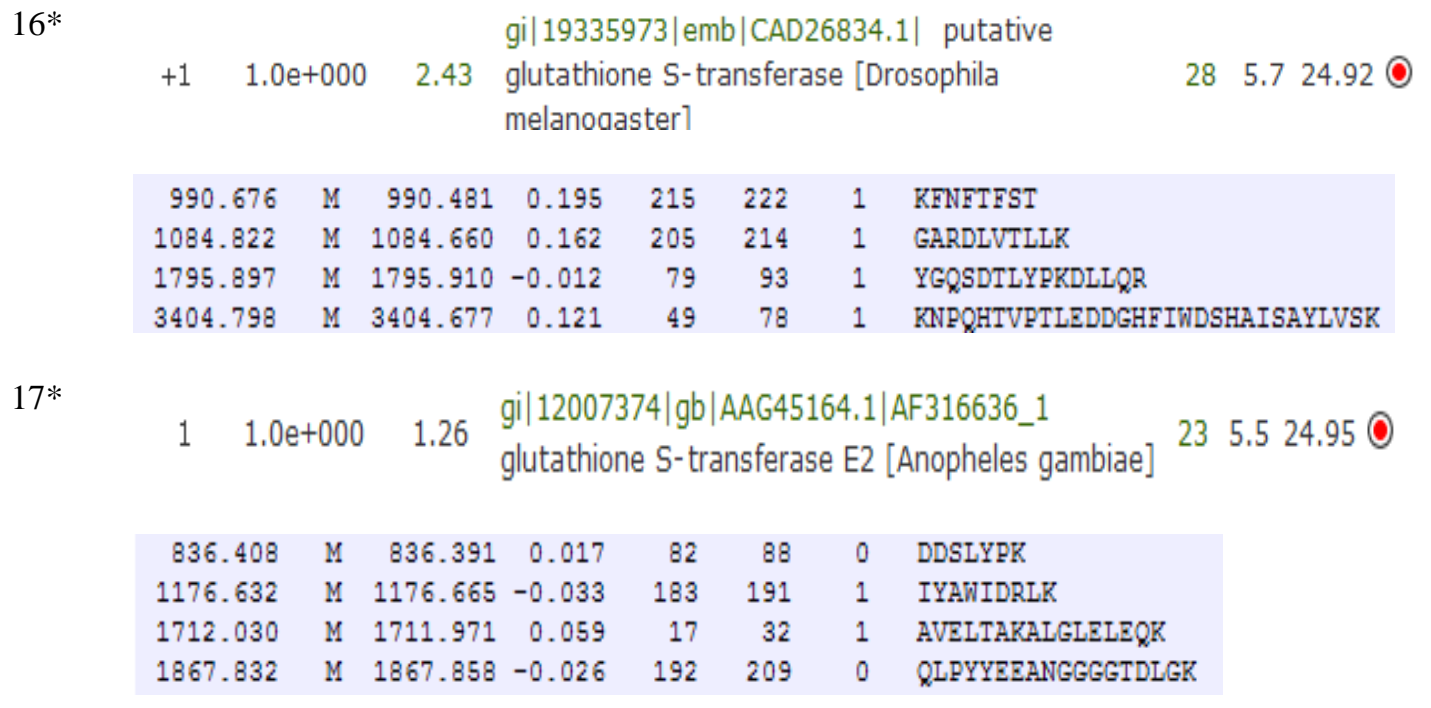

Spots $16^{*}$ and $17^{*}$ can be found in Figure 6-8. 


\subsubsection{Spectra of spots labelled in Figure 5-8 E}
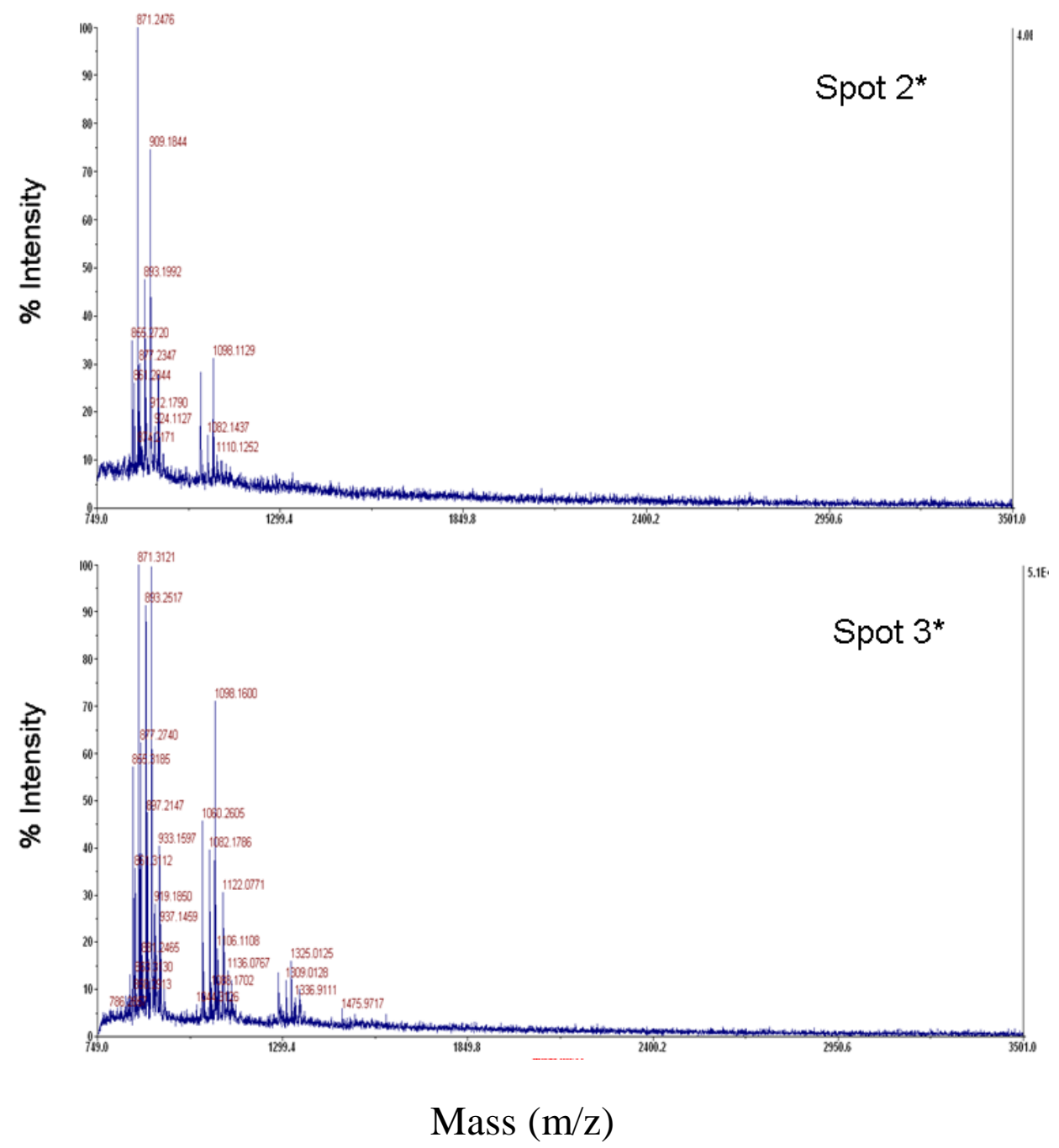

Figure 10-7: MALDI-TOF spectra of the DNP-GSH affinity-purified Lucilia cuprina egg spots on 2D gel labelled in Figure 5-8 E 


\subsubsection{Identification of spots labelled in Figure 5-8 E using PROFOUND}

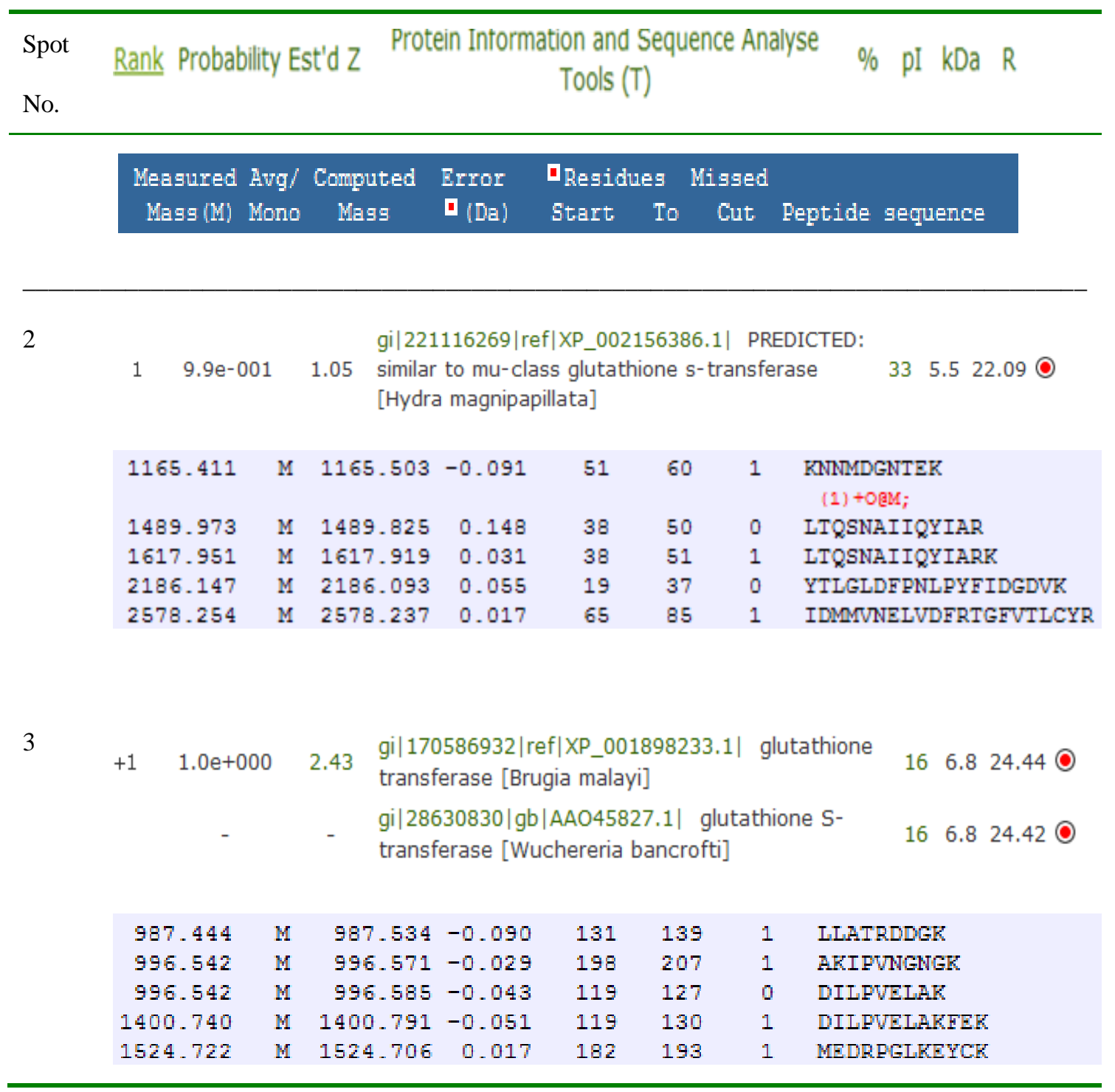

Hydra magnipapillata - a fresh water polyp, commonly known as Hydra

Brugia malayi and Wuchereria bancrofti - nematodes, causative agents of lymphatic filariasis in humans 


\subsection{Consensus sequences for GST classes}

\section{Sigma GST:}

-----MA--A--APPAEGEAPP-----E----A---AP---PAEP

MPSYKLFYFNVKALGEPLRFLLAYGGQEFEDVRITR--

EEWPALKPTMPFGQMPVLEIDGKQVHQSIAIARYLAKQVGLAGATDWEDLQIDIVVDTINDF RLKIAVVSYEPDD-EIKEKKLVTLN

EVIPFYLEKLEEIVKDNDGHLALGKLTWADFYFAGILDYLNYMV

KR-DLLANYPALRGVVDAVLAIPPIKAWIEKRPQTEL

\section{Delta GST:}

M-MDFYYLPGSAPCRSVIMTAKALGVELNKK-

LLNLMAGEHLKPEFLKINPQHTIPTLVDNGFALWESRAIMVYLVEKYGKDDSLYPKDPKKRA LINQRLYFDMGTLYQSFADYYYPQIFAKAPADPENYKKIEAAFEFLNTFLEGQDYVAGDSLT VADIALLATVSTFEVAGFDFSKYPNVARWYENAKKVTPGWDEN-

WAGCLEFKKYFDARKAAAK-

\section{Epsilon GST:}

MGKLVLYGLDASPPVRAVKLTLAALNLPYEYKEVNLLAKEHLSEEFLKKNPQHTVPTLEDD GHFIWDSHAIIAYLVSKYAKSDELYPKDLLKRAVVDQRLHFESGVLFAGGLRNITKPLFFRGQ TEIPKERIDAIIEAYDFLETFLAGNDYLAGDQLTIADFSIVSTVTSLVAFVEIDATKYPKIAAWLKRLEKLPYYEEANGKGARQLVAFLKSKNFTIVDK

\section{Omega GST:}

$-----\mathrm{S}-$

MMSNGKHLAKGSPKPVLPDDGKLRLYSMRFCPYAQRVHLVLDAKNIPYHTIYINLSEKPEW YFEKSPLGKVPALEIPGKEGQPTLYESLIIADYLDEAYPAKERPLYPKDPLQKAQDKILIERFA GAVSAFYYRILFSSDGIPPGAITEFGTGLDIFEKELKERGTPYFGGDKPGMLDYMIWPWCERF DLLKFALGDKYELDKERFPKLLKWRDLMEKDEAVKQSFLSTEDHAKFLQSRKAGENNYDIL AN-AKR-KL------

\section{Zeta GST:}

S----P---MVENQPILYSYWRSSCSWRVRIALNLKEIPYDIKPISLIKSG-G

EQHCNEYREVNPMEQVPALQIDGHTLIESVAIMHYLEETRPQR-

PLLPQDVHKRAKVREICEIICSGIQPLQNLIVLIHVGTEEKKKEWAQHWITRGFRALEKLLSTS AGKYCVGDEITLADCCLVPQVFNGARRFHVDLRPYPIILRIDRELEKEPAFRAAHPSNQPDCPP ELAKK------ 


\section{Theta GST:}

MAMSM[NPT][IL][DKR]YYYDLMSQAPSRALYIFLEATKIPLE[KL][CK]LVNLRKGEHLTPEF LKN[IV]NRF[HQ]KVPCI[DV]DNGFKLAES[IRV]AILRYLAREYGKDDSLYPKDSKKRARVDE YLEWQHGNTR[AL]TCAQYFRYVW[LY]PP[ILM]L[AGT]G[ART]KVD[AEP]EKAKKL[KR][AE ]AMEFLLDFLE[GRT]EWLGRG-[DH]-FIAG[DN]ELT[IV]ADLVAACEIEQP[KR]-

MAGFDPRVGYPNITAWMERV[KR]EAT[NP]PYYD[EL]AHKG[ALV][NY]KFAP[KM][FM]D[A N]LT[GKQ]KKL---

\section{Mu GST:}

MMSKP[IV]LGYWDIRGLAQPIRLLLAYAGVDFEDKRYSCGPAPDFDRSEWLNEK[FH][ST]LG LDFPNLPYYIDGDVKLTQSMAILRYLARKHGLDGKTEAEK[INQ]RI[DS]LLEQQ[AFV][AM]D[ FL]RMAWV[LR][L]CYNPDFEKLKVDYLKNLPDSLKLFS[KN]FLGEH[KP]F[FV]AGD[NS]ITY VDFLLYEYL[AD]QHLV[FL][AV]PG[CV]L[DGK][DQ]FPNLK[AK]FVDRIE[AS]LPRVA[AE]YI KSDKPIKWPFNGPMAK[FW][GN]A[RST]LQKKP

\section{Xi GST:}

MAPIILYHFPGSPPSRSALLAARNLDLDAEVKILNLFAGEHLADEFVAINPDHTVPTLVDDDYI LWESKAIATYLAEQHKPDCTLYPSDPKKRGLINHRLYFDSGTLFAAARAALMPVLRSGATRIP QEKKDAIYEALEKLDGYLDGCDWIAGEECTLADLCALANVASLEEIGVDMEGLANVSAWLE RCKELPGFDENEEGASFFGNAFKSKLEEPF

\section{Iota GST:}

-V-------V-------ES-----P-

MTMKFYAVSDGPPCLAVRMAAKALGIPLNLILVDLGAGEHLTPEYLKMNPQHEIP TLDDNGFFLSESRAILQYLCDKYAKDDSLYPKDPKKRAVVNQRLCFDLGTLYPRFSAYYMPP IFFDYERTPEGLKKLEEALEFLETYLERTGTAFAAGDNLTIADFPLVASVMTLEAINFDDLSKY PNIHKWYANFKQAYPG-DLWEISASGMQEFAEFEKNPPDLTGMEHPIHPIRKVKA- 


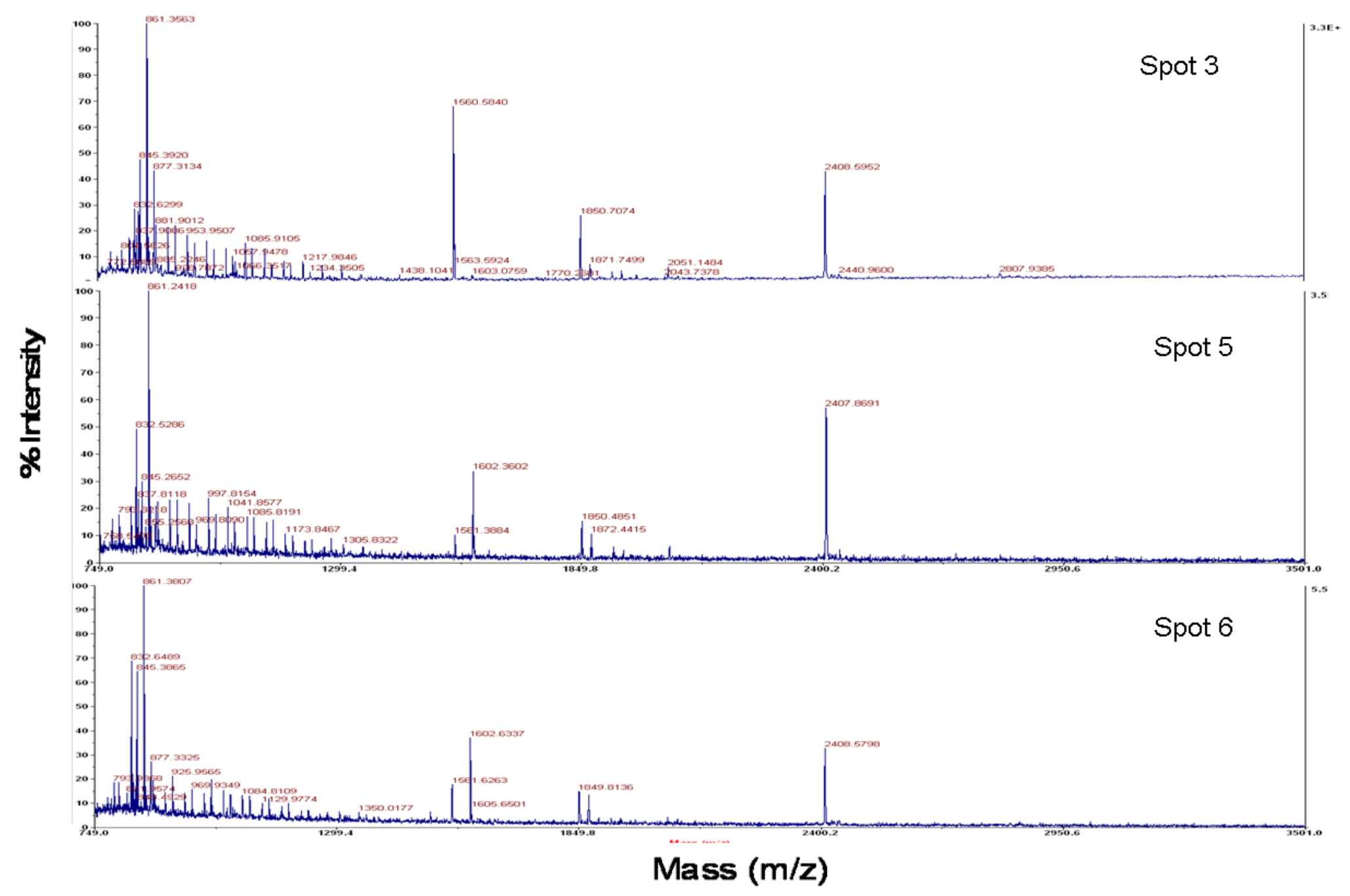

Figure 10-8: Comparison of MALDI-TOF spectra of DmGSTD1 spots from Figure 7-4. 


\section{References}

Abdollahi, M., Ranjbar, A., Shadnia, S., Nikfar, S. and Rezaie, A. (2004) Pesticides and oxidative stress: a review. Medical Science Monitor, 10, RA141-RA147.

Adams, M.D., Celniker, S.E., Holt, R.A., Evans, C.A., Gocayne, J.D., Amanatides, P.G., Scherer, S.E., Li, P.W., Hoskins, R.A., Galle, R.F., George, R.A., Lewis, S.E., Richards, S., Ashburner, M., Henderson, S.N., Sutton, G.G., Wortman, J.R., Yandell, M.D., Zhang, Q., Chen, L.X., Brandon, R.C., Rogers, Y.H.C., Blazej, R.G., Champe, M., Pfeiffer, B.D., Wan, K.H., Doyle, C., Baxter, E.G., Helt, G., Nelson, C.R., Miklos, G.L.G., Abril, J.F., Agbayani, A., An, H.J., Andrews-Pfannkoch, C., Baldwin, D., Ballew, R.M., Basu, A., Baxendale, J., Bayraktaroglu, L., Beasley, E.M., Beeson, K.Y., Benos, P.V., Berman, B.P., Bhandari, D., Bolshakov, S., Borkova, D., Botchan, M.R., Bouck, J., Brokstein, P., Brottier, P., Burtis, K.C., Busam, D.A., Butler, H., Cadieu, E., Center, A., Chandra, I., Cherry, J.M., Cawley, S., Dahlke, C., Davenport, L.B., Davies, A., de Pablos, B., Delcher, A., Deng, Z.M., Mays, A.D., Dew, I., Dietz, S.M., Dodson, K., Doup, L.E., Downes, M., Dugan-Rocha, S., Dunkov, B.C., Dunn, P., Durbin, K.J., Evangelista, C.C., Ferraz, C., Ferriera, S., Fleischmann, W., Fosler, C., Gabrielian, A.E., Garg, N.S., Gelbart, W.M., Glasser, K., Glodek, A., Gong, F.C., Gorrell, J.H., Gu, Z.P., Guan, P., Harris, M., Harris, N.L., Harvey, D., Heiman, T.J., Hernandez, J.R., Houck, J., Hostin, D., Houston, D.A., Howland, T.J., Wei, M.H., Ibegwam, C., Jalali, M., Kalush, F., Karpen, G.H., Ke, Z.X., Kennison, J.A., Ketchum, K.A., Kimmel, B.E., Kodira, C.D., Kraft, C., Kravitz, S., Kulp, D., Lai, Z.W., Lasko, P., Lei, Y.D., Levitsky, A.A., Li, J.Y., Li, Z.Y., Liang, Y., Lin, X.Y., Liu, X.J., Mattei, B., McIntosh, T.C., McLeod, M.P., McPherson, D., Merkulov, G., Milshina, N.V., Mobarry, C., Morris, J., Moshrefi, A., Mount, S.M., Moy, M., Murphy, B., Murphy, L., Muzny, D.M., Nelson, D.L., Nelson, D.R., Nelson, K.A., Nixon, K., Nusskern, D.R., Pacleb, J.M., Palazzolo, M., Pittman, G.S., Pan, S., Pollard, J., Puri, V., Reese, M.G., Reinert, K., Remington, K., Saunders, R.D.C., Scheeler, F., Shen, H., Shue, B.C., Siden-Kiamos, I., Simpson, M., Skupski, M.P., Smith, T., Spier, E., Spradling, A.C., Stapleton, M., Strong, R., Sun, E., Svirskas, R., Tector, C., Turner, R., Venter, E., Wang, A.H.H., Wang, X., Wang, Z.Y., Wassarman, D.A., Weinstock, G.M., Weissenbach, J., Williams, S.M., Woodage, T., Worley, K.C., Wu, D., Yang, S., Yao, Q.A., Ye, J., Yeh, R.F., Zaveri, J.S., Zhan, M., Zhang, G.G., Zhao, Q., Zheng, L.S., Zheng, X.Q.H., Zhong, F.N., Zhong, W.Y., Zhou, X.J., Zhu, S.P., Zhu, X.H., Smith, H.O., Gibbs, R.A., Myers, E.W., Rubin, G.M. and Venter, J.C. (2000) The genome sequence of Drosophila melanogaster. Science, 287, 2185-2195.

Adang, A.E.P., Meyer, D.J., Brussee, J., Vandergen, A., Ketterer, B. and Mulder, G.J. (1989) Interaction of rat glutathione S-transferase -7-7 and 
glutathione S-transferase -8-8 with gamma-glutamyl-modified or glycylmodified glutathione analogs. Biochemical Journal, 264, 759-764.

Adler, V., Yin, Z.M., Fuchs, S.Y., Benezra, M., Rosario, L., Tew, K.D., Pincus, M.R., Sardana, M., Henderson, C.J., Wolf, C.R., Davis, R.J. and Ronai, Z. (1999) Regulation of JNK signaling by GSTp. Embo Journal, 18, 13211334.

Agellon, L.B. and Torchia, E.C. (2000) Intracellular transport of bile acids. Biochimica Et Biophysica Acta-Molecular and Cell Biology of Lipids, 1486, 198-209.

Agianian, B., Tucker, P.A., Schouten, A., Leonard, K., Bullard, B. and Gros, P. (2003) Structure of a Drosophila sigma class glutathione S-transferase reveals a novel active site topography suited for lipid peroxidation products. Journal of Molecular Biology, 326, 151-165.

Alias, Z. (2006) The proteome of insect glutathione S-transferases: its response to toxic challenge. In School of Biological Sciences. Wellington: Victoria University of Wellington, pp. 232.

Alias, Z. and Clark, A.G. (2007) Studies on the glutathione S-transferase proteome of adult Drosophila melanogaster. Responsiveness to chemical challenge. Proteomics, 7, 3618-3628.

Alias, Z. and Clark, A.G. (2010) Adult Drosophila melanogaster glutathione Stransferases: Effects of acute treatment with methyl parathion. Pesticide Biochemistry and Physiology, 98, 94-98.

Allen, R.E., Lo, T.W.C. and Thornalley, P.J. (1993a) A simplified method for the purification of human red-blood-cell glyoxalase. 1. Characteristics, Immunoblotting and Inhibitor studies. Journal of Protein Chemistry, 12, 111119.

Allen, R.E., Theodore, W.C.L. and Thornalley, P.J. (1993b) Purification and characterization of glyoxalase-II from human red-blood-cells. European Journal of Biochemistry, 213, 1261-1267.

Anderson, P.N., Eaton, D.L. and Murphy, S.D. (1992) Comparative metabolism of methyl parathion in intact and subcellular-fractions of isolated rat hepatocytes Fundamental and Applied Toxicology, 18, 221-226.

Annan, R.S. and Carr, S.A. (1996) Phosphopeptide analysis by matrix-assisted laser desorption time-of-flight mass spectrometry. Analytical Chemistry, 68, 3413-3421.

Anuradha, D., Reddy, K.V., Kumar, T.C., Neeraja, S., Reddy, P.R.K. and Reddanna, P. (2000) Purification and characterization of rat testicular glutathione S-transferases: role in the synthesis of eicosanoids. Asian Journal of Andrology, 2, 277-282.

Armstrong, K.F. and Suckling, D.M. (1990) Correlation of azinphosmethyl resistance with detoxication enzyme activity in the light brown apple moth Epiphyas postvittana (Lepidoptera: Tortricidae). Pesticide Biochemistry and Physiology, 36, 281-289. 
Armstrong, R.N. (1991) Glutathione S-transferase - reaction mechanism, structure and function. Chemical Research in Toxicology, 4, 131-140.

Armstrong, R.N. (1994) Glutathione S-transferases: structure and mechanism of an archetypical detoxication enzyme. Adv Enzymol Relat Areas Mol Biol, 69, 144.

Arnold, J.T.A. and Whitten, M.J. (1976) Genetic basis for organophosphorus resistance in Australian sheep blowfly, Lucilia cuprina (wiedemann) (Diptera, calliphoridae). Bulletin of Entomological Research, 66, 561-568.

Arruda, L.K., Vailes, L.D., PlattsMills, T.A.E., Hayden, M.L. and Chapman, M.D. (1997) Induction of IgE antibody responses by glutathione Stransferase from the German cockroach (Blattella germanica). Journal of Biological Chemistry, 272, 20907-20912.

Arthur, J.R. (2000) The glutathione peroxidases. Cellular and Molecular Life Sciences, 57, 1825-1835.

Awasthi, S., Singhal, S.S., Srivastava, S.K., Torman, R.T., Zimniak, P., Bandorowicz-Pikula, J., Singh, S.V., Piper, J.T., Awasthi, Y.C. and Pikula, S. (1998) ATP-dependent human erythrocyte glutathione-conjugate transporter. I. Purification, photoaffinity labeling, and kinetic characteristics of ATPase activity. Biochemistry, 37, 5231-5238.

Awasthi, Y.C., Sharma, R., Sharma, A., Yaclav, S., Singhal, S.S., Chaudhary, P. and Awasthi, S. (2008) Self-regulatory role of 4-hydroxynonenal in signaling for stress-induced programmed cell death. Free Radical Biology and Medicine, 45, 111-118.

Awasthi, Y.C., Yang, Y.S., Tiwari, N.K., Patrick, B., Sharma, A., Li, J. and Awasthi, S. (2004) Regulation of 4-hydroxynonenal-mediated signaling by glutathione S-transferases. Free Radical Biology and Medicine, 37, 607-619.

Ayyadevara, S., Dandapat, A., Singh, S.P., Siegel, E.R., Reis, R.J.S., Zimniak, L. and Zimniak, P. (2007) Life span and stress resistance of Caenorhabditis elegans are differentially affected by glutathione transferases metabolizing 4hydroxynon-2-enal. Mechanisms of Ageing and Development, 128, 196-205.

Baker, W.L., Clark, A.G., Faulds, G. and Nielsen, J.S. (1994) Multiple glutathione S-transferases in Galleria mellonella- their detection with fluorigenic substrates. Insect Biochemistry and Molecular Biology, 24, 301307.

Bates, D.J.P., Lively, M.O., Gorczynski, M.J., King, S.B., Townsend, A.J. and Morrow, C.S. (2009) Noncatalytic Interactions between Glutathione STransferases and Nitroalkene Fatty Acids Modulate Nitroalkene-Mediated Activation of Peroxisomal Proliferator-Activated Receptor gamma. Biochemistry, 48, 4159-4169.

Batterham, P., Hill-Williams, A., Levot, G., Sales, N. and McKenzie, J.A. (2006) The genetic bases of high-level resistance to diflubenzuron and low-level resistance to cyromazine in a field strain of the Australian sheep blowfly, Lucilia cuprina (Wiedemann) (Diptera : Calliphoridae). Australian Journal of Entomology, 45, 87-90. 
Bauche, F., Fouchard, M.H. and Jegou, B. (1994) Antioxidant system in rat testicular cells. Febs Letters, 349, 392-396.

Beall, C., Fyrberg, C., Song, S. and Fyrberg, E. (1992) Isolation of a Drosophila gene encoding glutathione S-transferase. Biochemical Genetics, 30, 515-527.

Beckman, J.K. and Coniglio, J.G. (1979) Comparative study of the lipid composition of isolated rat sertoli and germinal cells. Lipids, 14, 262-267.

Belleville, E., Dufva, M., Aamand, J., Bruun, L., Clausen, L. and Christensen, C.B.V. (2004) Quantitative microarray pesticide analysis. Journal of Immunological Methods, 286, 219-229.

Benke, G.M., Cheever, K.L., Mirer, F.E. and Murphy, S.D. (1974) Comparative toxicity, anticholinesterase action and metabolism of methyl parathion and parathion in sunfish and mice Toxicology and Applied Pharmacology, 28, 97109.

Benke, G.M. and Murphy, S.D. (1975) Influence of age on toxicity and metabolism of methylparathion and parathion in male and female rats. Toxicology and Applied Pharmacology, 31, 254-269.

Berg, J.M., Tymoczko, J.L. and Stryer, L. (2002) Biochemistry 5 edn.

Beuckmann, C.T., Fujimori, K., Urade, Y. and Hayaishi, O. (2000a) Identification of Mu-class glutathione transferases M2-2 and M3-3 as cytosolic prostaglandin E synthases in the human brain. Neurochemical Research, 25, 733-738.

Beuckmann, C.T., Lazarus, M., Gerashchenko, D., Mizoguchi, A., Nomura, S., Mohri, I., Uesugi, A., Kaneko, T., Mizuno, N., Hayaishi, O. and Urade, Y. (2000b) Cellular localization of lipocalin-type prostaglandin D synthase (beta-trace) in the central nervous system of the adult rat. Journal of Comparative Neurology, 428, 62-78.

Blackburn, A.C., Matthaei, K.I., Lim, C., Taylor, M.C., Cappello, J.Y., Hayes, J.D., Anders, M.W. and Board, P.G. (2006) Deficiency of glutathione transferase zeta causes oxidative stress and activation of antioxidant response pathways. Molecular Pharmacology, 69, 650-657.

Board, P.G. (1998) Identification of cDNAs encoding two human Alpha class glutathione transferases (GSTA3 and GSTA4) and the heterologous expression of GSTA4-4. Biochemical Journal, 330, 827-831.

Board, P.G., Baker, R.T., Chelvanayagam, G. and Jermiin, L.S. (1997) Zeta, a novel class of glutathione transferases in a range of species from plants to humans. Biochemical Journal, 328, 929-935.

Board, P.G., Coggan, M., Chelvanayagam, G., Easteal, S., Jermiin, L.S., Schulte, G.K., Danley, D.E., Hoth, L.R., Griffor, M.C., Kamath, A.V., Rosner, M.H., Chrunyk, B.A., Perregaux, D.E., Gabel, C.A., Geoghegan, K.F. and Pandit, J. (2000) Identification, characterization, and crystal structure of the omega class glutathione transferases. Journal of Biological Chemistry, 275, 24798-24806. 
Boatright, K.M. and Salvesen, G.S. (2003) Caspase activation. Biochem Soc Symp, 233-242.

Booth, J., Boyland, E. and Sims, P. (1961) An enzyme from rat liver catalysing conjugations with glutathione. Biochemical Journal, 79, 516-524.

Boyer, T.D. (1989) The glutathione s-transferases- an update. Hepatology, 9, 486496.

Boyland, E. and Chasseaud, L. (1969) Role of glutathione and glutathione Strasnferases in mercapturic acid biosynthesis. Advances in Enzymology and Related Areas of Molecular Biology, 32, 173-219.

Bradford, M.M. (1976) Rapid and sensitive method for quantitation of microgram quantities of protein utilizing principle of protein-dye binding Analytical Biochemistry, 72, 248-254.

Brown, R.S. and Lennon, J.J. (1995) Mass resolution improvement by incorporation of pulsed ion extraction in a matrix-assisted laser-desorption ionization linear time-of-flight mass-spectrometer Analytical Chemistry, 67, 1998-2003.

Burrell, D.H., Merritt, G.C., Watts, J.W. and Walker, K.H. (1982) The role of Pseudomonas aeruginosa in pathogenesis of fleece-rot and the effect of immunization. Australian Veterinary Journal, 58, 34-35.

Cammer, W., Tansey, F., Abramovitz, M., Ishigaki, S. and Listowsky, I. (1989) Differential localization of glutathione S-transferase YP- subunit and YBsubunit in oligodendrocytes and astrocytes of rat brain. Journal of Neurochemistry, 52, 876-883.

Campbell, E., Takahashi, Y., Abramovitz, M., Peretz, M. and Listowsky, I. (1990) A distinct human testis and brain Mu-class glutathione S-transferaseMolecular cloning and characterization of a form present even in individuals lacking hepatic type Mu-isoenzymes. Journal of Biological Chemistry, 265, 9188-9193.

Campbell, P.M., Newcomb, R.D., Russell, R.J. and Oakeshott, J.G. (1998) Two different amino acid substitutions in the ali-esterase, E3, confer alternative types of organophosphorus insecticide resistance in the sheep blowfly, Lucilia cuprina. Insect Biochemistry and Molecular Biology, 28, 139-150.

Cao, P. and Stults, J.T. (2000) Mapping the phosphorylation sites of proteins casing on-line immobilized metal affinity chromatography/capillary electrophoresis/electrospray ionization multiple stage tandem mass spectrometry. Rapid Communications in Mass Spectrometry, 14, 1600-1606.

Carlini, E.J., McPheron, B.A., Felland, C.M. and Hull, L.A. (1995) Biochemical mechanisms of Azinphosmethyl resistance in the tufted apple bud moth Platynota idaeusalis. Pesticide Biochemistry and Physiology, 51, 38-47.

Carr, S.A., Huddleston, M.J. and Annan, R.S. (1996) Selective detection and sequencing of phosphopeptides at the femtomole level by mass spectrometry. Analytical Biochemistry, 239, 180-192. 
Chan, L.M.S., Lowes, S. and Hirst, B.H. (2004) The ABCs of drug transport in intestine and liver: efflux proteins limiting drug absorption and bioavailability. European Journal of Pharmaceutical Sciences, 21, 25-51.

Chang, M., Hong, Y., Burgess, J.R., Tu C-P, D. and Reddy, C.C. (1987) Isoenzyme specificity of rat liver glutathione $\mathrm{S}$-transferases in the formation of $\mathrm{PGF}_{2}$-alpha and $\mathrm{PGE}_{2}$-from $\mathrm{PGH}_{2}$. Archives of Biochemistry and Biophysics, 259, 548-557.

Chase, H.A. (1998) The use of affinity adsorbents in expanded bed adsorption. Journal of Molecular Recognition, 11, 217-221.

Chasseaud, L.F. (1973) Nature and distribution of enzymes catalyzing conjugation of glutathione with foreign compounds. Drug Metabolism Reviews, 2, 185220 .

Chasseaud, L.F. (1979) The role of glutathione and glutathione S-transferases in the metabolism of chemical carcinogens and other electrophilic agents. Adv Cancer Res, 29, 175-274.

Che-Mendoza, A., Penilla, R.P. and Rodriguez, D.A. (2009) Insecticide resistance and glutathione S-transferases in mosquitoes: A review. African Journal of Biotechnology, 8, 1386-1397.

Chekhun, V.F., Zylchuk, V.E., Lukyanova, N.Y., Vorontsova, A.L. and Kudryavets, Y.I. (2009) Expression of drug resistance proteins in triplereceptor-negative tumors as the basis of individualized therapy of the breast cancer patients Experimental Oncology, 31, 123-124.

Chelvanayagam, G., Parker, M.W. and Board, P.G. (2001) Fly fishing for GSTs: a unified nomenclature for mammalian and insect glutathione transferases. Chemico-Biological Interactions, 133, 256-260.

Chemale, G., Morphew, R., Moxon, J.V., Morassuti, A.L., LaCourse, E.J., Barrett, J., Johnston, D.A. and Brophy, P.M. (2006) Proteomic analysis of glutathione transferases from the liver fluke parasite, Fasciola hepatica. Proteomics, 6, 6263-6273.

Chiang, F.M. and Sun, C.N. (1993) Glutathione transferase isozymes of Diamondback moth larvae and their role in the degradation of some organophosphorus insecticides. Pesticide Biochemistry and Physiology, 45, 7-14.

Chiu, V.K., Silletti, J., Dinsell, V., Wiener, H., Loukeris, K., Ou, G., Philips, M.R. and Pillinger, M.H. (2003) Carboxyl methylation of ras regulates membrane targeting and effector engagement. J. Biol.Chem, 279, 7346-7352.

Cho, S.G., Lee, Y.H., Park, H.S., Ryoo, K., Kang, K.W., Park, J., Eom, S.J., Kim, M.J., Chang, T.S., Choi, S.Y., Shim, J., Kim, Y., Dong, M.S., Lee, M.J., Kim, S.G., Ichijo, H. and Choi, E.J. (2001) Glutathione S-transferase $\mathrm{Mu}$ modulates the stress-activated signals by suppressing apoptosis signalregulating kinase 1. Journal of Biological Chemistry, 276, 12749-12755.

Clark, A.G. (1989) The comparative enzymology of the glutathione S-transferases from non-vertebrate organisms. Comparative Biochemistry and Physiology B-Biochemistry \& Molecular Biology, 92, 419-446. 
Clark, A.G. and Dauterman, W.C. (1982) The characterization by affinity chromatography of glutathione S-transferases from different strains of housefly. Pesticide Biochemistry and Physiology, 17, 307-314.

Clark, A.G. and Drake, B. (1984) Purification and properties of glutathione Stransferases from larvae of Wiseana cervinata. Biochemical Journal, 217, 4150 .

Clark, A.G., Letoa, M. and Ting, W.S. (1977) Purification by affinity chromatography of a glutathione S-transferase from larvae of Galleria mellonella. Life Sciences, 20, 141-147.

Clark, A.G., Marshall, S.N. and Qureshi, A.R. (1990) Synthesis and use of an isoform-specific affinity matrix in the purification of glutathione $\mathrm{S}$ transferases from the housefly, Musca domestica (L.). Protein Expression and Purification, 1, 121-126.

Clark, A.G. and Shamaan, N.A. (1984) Evidence that DDT-dehydrochlorinase from the housefly is a glutathione S-transferase. Pesticide Biochemistry and Physiology, 22, 249-261.

Clark, A.G., Shamaan, N.A., Sinclair, M.D. and Dauterman, W.C. (1986) Insecticide metabolism by multiple glutathione $\mathrm{S}$-transferases in 2 strains of the housefly, Musca domestica (L). Pesticide Biochemistry and Physiology, 25, 169-175.

Clark, A.G., Smith, J.N. and Speir, T.W. (1973) Cross specificity in some vertebrate and insect glutathione-transferases with methyl parathion (dimethyl-p-nitrophenyl phosphorothionate), 1-chloro-2,4-dinitrobenzene and S-crotonyl-N-acetylcysteamine as substrates. Biochemical Journal, 135, 385392.

Clayton, J.D., Cripps, R.M., Sparrow, J.C. and Bullard, B. (1998) Interaction of troponin-H and glutathione S-transferase-2 in the indirect flight muscles of Drosophila melanogaster. Journal of Muscle Research and Cell Motility, 19, 117-127.

Cleverley, K.E., Betts, J.C., Blackstock, W.P., Gallo, J.M. and Anderton, B.H. (1998) Identification of novel in vitro PKA phosphorylation sites on the low and middle molecular mass neurofilament subunits by mass spectrometry. Biochemistry, 37, 3917-3930.

Cochrane, B.J., Hargis, M., Debelligny, P.C., Holtsberg, F. and Coronella, J. (1992) Evolution of glutathione S-transferases associated with insecticide resistance in Drosophila. Molecular Mechanisms of Insecticide Resistance, 505, 53-70.

Cohen, S.L., Halaas, J.L., Friedman, J.M., Chait, B.T., Bennett, L., Chang, D., Hecht, R. and Collins, F. (1996) Human leptin characterization. Nature, 382, 589-589.

Crowe, M.L., Serizet, C., Thareau, V., Aubourg, B., Rouze, P., Hilson, P., Beynon, J., Weisbeek, P., van Hummelen, P., Reymond, P., Paz-Ares, J., Nietfeld, W. and Trick, M. (2003) CATMA: a complete Arabidopsis GST database. Nucleic Acids Research, 31, 156-158. 
Cuatrecasas.P and Parikh, I. (1972) Adsorbents for affinity chromatography- Use of N-Hydroxysuccinimide esters of agarose. Biochemistry, 11, 291-299.

Danielson, U.H. and Mannervik, B. (1985) Kinetic independence of the subunits of cytosolic glutathione transferase from the rat Biochemical Journal, 231, 263267.

David, J.P., Strode, C., Vontas, J., Nikou, D., Vaughan, A., Pignatelli, P.M., Louis, C., Hemingway, J. and Ranson, H. (2005) The Anopheles gambiae detoxification chip: A highly specific microarray to study metabolic-based insecticide resistance in malaria vectors. Proceedings of the National Academy of Sciences of the United States of America, 102, 4080-4084.

Davioud-Charvet, E., Delarue, S., Biot, C., Schwobel, B., Boehme, C.C., Mussigbrodt, A., Maes, L., Sergheraert, C., Grellier, P., Schirmer, R.H. and Becker, K. (2001) A prodrug form of a Plasmodium falciparum glutathione reductase inhibitor conjugated with a 4-anilinoquinoline. Journal of Medicinal Chemistry, 44, 4268-4276.

de Rey-Pailhade, J. (1888) Sur un corps d'origine organiques hydrogénant le soufre àfroid. Compt. Rend., 106, 1683-1694.

Dejong, J.L., Morgenstern, R., Depierre, J.W. and Tu, C.P. (1988) Gene expression of rat and human microsomal glutathione S-transferases. FASEB Journal, 2, ABSTRACT 4840.

Del Boccio, G., Di Ilio, C., Miranda, M., Manilla, A., Zarivi, O., Bonfigli, A. and Federici, G. (1987) Glutathione transferase activity during Bufo bufo development. Comparative Biochemistry and Physiology B, 86, 749-754.

Desmots, F., Rissel, M., Pigeon, C., Loyer, P., Loreal, O. and Guillouzo, A. (2002) Differential effects of iron overload on GST isoform expression in mouse liver and kidney and correlation between GSTA4 induction and overproduction of free radicals. Free Radical Biology and Medicine, 32, 93101.

Ding, Y., Ortelli, F., Rossiter, L.C., Hemingway, J. and Ranson, H. (2003) The Anopheles gambiae glutathione transferase supergene family: annotation, phylogeny and expression profiles. BMC Genomics, 4, (13 August 2003).

Ding, Y.C., Hawkes, N., Meredith, J., Eggleston, P., Hemingway, J. and Ranson, H. (2005) Characterization of the promoters of Epsilon glutathione transferases in the mosquito Anopheles gambiae and their response to oxidative stress. Biochemical Journal, 387, 879-888.

Doctor, J.S. and Fristrom, J.W. (1985) Macromolecular changes in imaginal discs during postembryonic development. In In Comprehensive Insect Physiology Biochemistry and Pharmacology. Pergamon, Oxford, pp. 201-238.

Dominey, R.J., Nimmo, I.A., Cronshaw, A.D. and Hayes, J.D. (1991) The major glutathione S-transferase in salmond fish livers is homologous to the mammalian pi-class gst. Comparative Biochemistry and Physiology BBiochemistry \& Molecular Biology, 100, 93-98.

Drapeau, G.R. (1980) Substrate specificity of a proteolytic enzyme isolated from a mutant of Pseudomonas frag. Journal of Biological Chemistry, 255, 839-840. 
Elsby, R., Kitteringham, N.R., Goldring, C.E., Lovatt, C.A., Chamberlain, M., Henderson, C.J., Wolf, C.R. and Park, B.K. (2003) Increased constitutive c-Jun N-terminal kinase signaling in mice lacking glutathione $\mathrm{S}$-transferase Pi. Journal of Biological Chemistry, 278, 22243-22249.

Enayati, A.A., Ranson, H. and Hemingway, J. (2005) Insect glutathione transferases and insecticide resistance. Insect Molecular Biology, 14, 3-8.

Engstrom, Y., Loseva, O. and Theopold, U. (2004) Proteomics of the Drosophila immune response. Trends in Biotechnology, 22, 600-605.

Esterbauer, H., Schaur, R.J. and Zollner, H. (1991) Chemistry and biochemistry of 4-hydroxynonenal, malonaldehyde and related aldehydes. Free Radical Biology and Medicine, 11, 81-128.

Foster, G.G., Whitten, M.J., Konovalov, C., Arnold, J.T.A. and Maffi, G. (1981) Autosomal genetic maps of the australian sheep blowfly, Lucilia cuprina dorsalis R-D (Diptera, calliphoridae), and possible correlations with the linkage maps of Musca domestica and Drosophila melanogaster (MG). Genetical Research, 37, 55-69.

Fournier, D., Bride, J.M., Poirie, M., Berge, J.B. and Plapp, F.W. (1992) Insect glutathione S-transferases: Biochemical characteristics of the major forms from houseflies susceptible and resistant to insecticides. Journal of Biological Chemistry, 267, 1840-1845.

Franciosa, H. and Berge, J.B. (1995) Glutathione S-transferases in housefly (Musca domestica) - Location of GST-1 and GST-2 families. Insect Biochemistry and Molecular Biology, 25, 311-317.

Frova, C. (2006) Glutathione transferases in the genomics era: New insights and perspectives. Biomolecular Engineering, 23, 149-169.

Fujita, S., Kitagawa, H., Ishizawa, H., Suzuki, T. and Kitani, K. (1985) Age associated alterations in hepatic glutathione S-transferase activities. Biochemical Pharmacology, 34, 3891-3894.

Fukami, J.I. and Shishido, T. (1966) Nature of a soluble glutathione -dependent enzyme system active in cleavage of methyl parathion to desmethyl parathion. Journal of Economic Entomology, 59, 1338-1342.

Gagnon, P. (1999) Chromatofocusing: does it really stack up as a process tool? Validated biosystems.

Gillott, C. (1980) Entomology. Gillott, C. Entomology. Xviii+729p. Plenum Press: New York, N.Y., USA; London, England. Illus, XVIII+729P.

Girardini, J., Amirante, A., Zemzoumi, K. and Serra, E. (2002) Characterization of an omega-class glutathione S-transferase from Schistosoma mansoni with glutaredoxin-like dehydroascorbate reductase and thiol transferase activities. European Journal of Biochemistry, 269, 5512-5521.

Gleeson, D.M., Barry, S.C. and Heath, A.C.G. (1994) Insecticide resistance status of Lucilia cuprina in New Zealand using biochemical and toxicological techniques. Veterinary Parasitology, 53, 301-308. 
Gopalakrishnan, B., Aravinda, S., Pawshe, C.H., Totey, S.M., Nagpal, S., Salunke, D.M. and Shaha, C. (1998) Studies on glutathione S-transferases important for sperm function: Evidence of catalytic activity-independent functions. Biochemical Journal, 329, 231-241.

Gopalakrishnan, B. and Shaha, C. (1998) Inhibition of sperm glutathione Stransferase leads to functional impairment due to membrane damage. Febs Letters, 422, 296-300.

Graminski, G.F., Kubo, Y. and Armstrong, R.N. (1989) Spectroscopic and kinetic evidence for the thiolate anion of glutathione at the active site of glutathione S-transferase. Biochemistry, 28, 3562-3568.

Grant, D.F., Dietze, E.C. and Hammock, B.D. (1991) Glutathione S-transferase isozymes in Aedes aegypti- purification, characterization and isozymespecific regulation Insect Biochemistry, 21, 421-433.

Grant, D.F. and Hammock, B.D. (1992) Genetic and molecular evidence for a trans-acting regulatory locus controlling glutathione S-transferase 2 expression in Aedes aegypti. Molecular \& General Genetics, 234, 169-176.

Grant, D.F. and Matsumura, F. (1989) Glutathione S- transferase 1 and 2 in susceptible and insecticide resistant Aedes aegypti. Pesticide Biochemistry and Physiology, 33, 132-143.

Gregus, Z., Varga, F. and Schmelas, A. (1985) Age- development and inducibility of hepatic glutathione S-transferase activities in mice, rats, rabbits and guinea pigs Comparative Biochemistry and Physiology C-Pharmacology Toxicology \& Endocrinology, 80, 85-90.

Grove, G., Zarlengo, R.P., Timmerman, K.P., Li, N.Q., Tam, M.F. and Tu, C.P.D. (1988) Characterization and heterospecific expression of cDNA clones of genes in the maize GSH S-transferase multigene family. Nucleic Acids Research, 16, 425-438.

Guthenberg, C. and Mannervik, B. (1979) Purification of glutathione Stransferases from rat lung by affinity chromatography- evidence for an enzyme form absent in rat liver. Biochemical and Biophysical Research Communications, 86, 1304-1310.

Habig, W.H. and Jakoby, W.B. (1981) Assays for differentiation of glutathione Stransferases. Methods Enzymol, 77, 398-405.

Habig, W.H., Pabst, M.J. and Jakoby, W.B. (1974) Glutathione S-transferasesfirst enzymatic step in mercapturic acid formation. Journal of Biological Chemistry, 249, 7130-7139.

Hart, D.J. (1961) Dieldrin and aldrin resistance in the green blowfly. NZ J. Agric, 102, 368 .

Hayaoka, T. and Dauterman, W.C. (1983) The effect of phenobarbital induction on glutathione conjugation of diazinon in susceptible and resistant houseflies. Pesticide Biochemistry and Physiology, 19, 344-349.

Hayes, J.D. and Clarkson, G.H.D. (1982) Purification and chracterization of 3 forms of glutathione S-transferase -A-a comparative study of the major YaYa 
containing, $\mathrm{YbYb}$ containing and $\mathrm{YcYc}$ containing glutathione S-transferases. Biochemical Journal, 207, 459-470.

Hayes, J.D., Flanagan, J.U. and Jowsey, I.R. (2005) Glutathione transferases. Annual Review of Pharmacology and Toxicology, 45, 51-88.

Hayes, J.D. and Mantle, T.J. (1986a) Anomalous electrophoretic behaviour of the glutathione S-trasnferase Ya and Yk subunits isolated from man and rodentsa potential pitfall for nomenclature. Biochemical Journal, 237, 731-740.

Hayes, J.D. and Mantle, T.J. (1986b) Use of immunoblot techniques to discriminate between the glutathione S-transferase $\mathrm{Yf}, \mathrm{Yk}, \mathrm{Ya}, \mathrm{Yn} / \mathrm{Yb}$ and $\mathrm{Yc}$ subunits and to study their distribution in extrahepatic tissues- evidence for 3 immunochemically distinct groups of transferase in the rat. Biochemical Journal, 233, 779-788.

Hayes, J.D. and Pulford, D.J. (1995) The glutathione S-transferase supergene family: Regulation of GST and the contribution of the isoenzymes to cancer chemoprotection and drug resistance. Critical Reviews in Biochemistry and molecular biology, 30(6), 445-600.

Hayes, J.D. and Wolf, C.R. (1988) Role of glutathione $S$ transferases in drug resistance: Academic press.

Hazelton, G.A. and Lang, C.A. (1983) Glutathione S-transferase EC-2.5.1.18 activities in the yellow fever mosquito Aedes aegypti during growth and aging Biochemical Journal, 210, 281-288.

Hearn, M.T.W., Aguilar, C.T., Mant, C.T. and Hodges, R.S. (1988) HPLC of amino acids, peptides and proteins: Evaluation of the use of hydrophobicity coefficients for the prediction of peptide elution profiles. J. Chromatogr, 438, 197-210.

Heath, A.C.G., Bishop, D.M., Cole, D.J.W. and Dymock, J.J. (1991) Exotic blowflies in New Zealand. New Zealand Journal of Zoology, 18, 85.

Hemachand, T., Gopalakrishnan, B., Salunke, D.M., Totey, S.M. and Shaha, C. (2002) Sperm plasma-membrane-associated glutathione S-transferases as gamete recognition molecules. Journal of Cell Science, 115, 2053-2065.

Hemingway, J., Field, L. and Vontas, J. (2002) An overview of insecticide resistance. Science, 298, 96-97.

Hemingway, J., Hawkes, N.J., McCarroll, L. and Ranson, H. (2004) The molecular basis of insecticide resistance in mosquitoes. Insect Biochemistry and Molecular Biology, 34, 653-665.

Hemingway, J. and Karunaratne, S. (1998) Mosquito carboxylesterases: a review of the molecular biology and biochemistry of a major insecticide resistance mechanism. Medical and Veterinary Entomology, 12, 1-12.

Hemingway, J., Miyamoto, J. and Herath, P.R.J. (1991a) A possible novel link between organophosphorus and DDT insecticide resistance genes in Anopheles - supporting evidence from fenitrothion metabolism studies Pesticide Biochemistry and Physiology, 39, 49-56. 
Henzel, W.J., Billeci, T.M., Stults, J.T., Wong, S.C., Grimley, C. and Watanabe, C. (1993) Identifying proteins from 2-dimensional gels by molecular mass searching of peptide fragments in protein sequence databases. Proceedings of the National Academy of Sciences of the United States of America, 90, 50115015.

Hochstrasser, D.F. (1998) Proteome in perspective. Clinical Chemistry and Laboratory Medicine, 36, 825-836.

Homolya, L., Varadi, A. and Sarkadi, B. (2003) Multidrug resistance-associated proteins: Export pumps for conjugates with glutathione, glucuronate or sulfate (Reprinted from Thiol Metabolism and Redox Regulation of Cellular Functions). Biofactors, 17, 103-114.

Hopkins, F.G. (1929) On glutathione: a reinvestigation. J. Biol.Chem, 84, 269-320.

Houmard, J. and Drapeau, G.R. (1972) Staphylococcal protease - a proteolytic enzyme specific for glutamoyl bonds Proceedings of the National Academy of Sciences of the United States of America, 69, 3506-3509.

Howard, G.A. and Martin, A.J.P. (1950) The separation of the C-12-C-18 fatty acids by reversed phase partition chromatography. Biochemical Journal, 46, 532-538.

Huang, H.S., Hu, N.T., Yao, Y.E., Wu, C.Y., Chiang, S.W. and Sun, C.N. (1998) Molecular cloning and heterologous expression of a glutathione S-transferase involved in insecticide resistance from the diamondback moth, Plutella xylostella. Insect Biochemistry and Molecular Biology, 28, 651-658.

Hughes, P.B. (1981) Spectrum of cross-resistance to insecticides in field samples of the primary sheep blowfly Lucilia cuprina. International Journal for Parasitology, 11, 475-479.

Hughes, P.B. and McKenzie, J.A. (1987) Insecticide resistance in the Australian Sheep blowfly Lucilia cuprina: speculation science and strategies. Combating Resistance to Xenobiotics. Ford MG, Holloman DM, Khambay BPS, Sawicki RM ed. Ellis Horwood, Chichester, 162-177.

Hughes, P.B. and Raftos, D.A. (1985) Genetics of an esterase associated with resistance to organophosphorus insecticides in the sheep blowfly, Lucilia cuprina (Wiedemann) (Diptera, Calliphoridae). Bulletin of Entomological Research, 75, 535-544.

Hunaiti, A.A., Elbetieha, A.M., Obeidat, M.A. and Owais, W.M. (1995) Developmental studies on Drosophila melanogaster glutathione S-transferase and its induction by oxadiazolone. Insect Biochemistry and Molecular Biology, 25, 1115-1119.

Ichijo, H., Nishida, E., Irie, K., tenDijke, P., Saitoh, M., Moriguchi, T., Takagi, M., Matsumoto, K., Miyazono, K. and Gotoh, Y. (1997) Induction of apoptosis by ASK1, a mammalian MAPKKK that activates SAPK/JNK and p38 signaling pathways. Science, 275, 90-94.

Ishigaki, S., Abramovitz, M. and Listowsky, I. (1989) Glutathione S-transferases are major cytosoilc thyroid hormones binding proteins. Arch Biochem Biophys, 273, 265-272. 
Ishikawa, T. (1989) ATP/ $\mathrm{Mg}^{2}+$ dependent cardiac transport system for glutathione S-conjuagtes- a study using rat heart sarcolemma vesicles. Journal of Biological Chemistry, 264, 17343-17348.

Ishikawa, T. (1992) The ATP-dependent glutathione S-conjugate export pump. Trends in Biochemical Sciences, 17, 463-469.

Jaffe, H., Veeranna and Pant, H.C. (1998) Characterization of serine and threonine phosphorylation sites in beta-elimination ethanethiol addition-modified proteins by electrospray tandem mass spectrometry and database searching. Biochemistry, 37, 16211-16224.

Jakobsson, P.J., Mancini, J.A. and FordHutchinson, A.W. (1996) Identification and characterization of a novel human microsomal glutathione S-transferase with leukotriene C-4 synthase activity and significant sequence identity to 5lipoxygenase-activating protein and leukotriene C-4 synthase. Journal of Biological Chemistry, 271, 22203-22210.

Jakoby, W.B. (1978a) The glutathione S-transferases: a group of multifunctional detoxification proteins. Adv Enzymol Relat Areas Mol Biol, 46, 383-414.

Jakoby, W.B. (1978b) Glutathione transferases in detoxification. Hoppe-Seylers Zeitschrift Fur Physiologische Chemie, 359, 1038-1038.

Jakoby, W.B. and Habig, W.H. (1980) Glutathione S transferases: Academic Press Inc.

Jakoby, W.B. and Keen, J.H. (1977) A triple threat in detoxification: the glutathione S-transferases. Trends in Biochemical Sciences, 2, 229-231.

Jang, I.S., Chae, K.R. and Cho, J.S. (2001) Effects of age and strain on small intestinal and hepatic antioxidant defense enzymes in Wistar and Fisher 344 rats. Mechanisms of Ageing and Development, 122, 561-570.

Jedlitschky, G., Leier, I., Buchholz, U., Center, M. and Keppler, D. (1994) ATPdependent transport of glutathione $S$-conjugates by the multidrug resistanceassociated protein. Cancer Research, 54, 4833-4836.

Jekel, P.A., Weijer, W.J. and Beintema, J.J. (1983) Use of endoproteinase Lys-C from Lysobacter enzymogenes in protein sequence analysis Analytical Biochemistry, 134, 347-354.

Jeno, P., Mini, T., Moes, S., Hintermann, E. and Horst, M. (1995) Internal sequences from proteins digested in polyacrylamide gels. Analytical Biochemistry, 224, 75-82.

Johansson, A.S. and Mannervik, B. (2001) Human glutathione transferase A3-3, a highly efficient catalyst of double-bond isomerization in the biosynthetic pathway of steroid hormones. Journal of Biological Chemistry, 276, 3306133065 .

Johansson, K., Ahlen, K., Rinaldi, R., Sahlander, K., Siritantikorn, A. and Morgenstern, R. (2007) Microsomal glutathione transferase 1 in anticancer drug resistance. Carcinogenesis, 28, 465-470.

Johnson, J.A., Finn, K.A. and Siegel, F.L. (1992) Tissue distribution of enzymatic methylation of glutathione S-transferase and its effects on catalytic activity - 
Methylation of glutathione S-transferase 11-11 inhibits conjugating activity towards 1-chloro-2,4-dinitrobenzene. Biochemical Journal, 282, 279-289.

Karas, M. and Hillenkamp, F. (1988) Laser desorption ionisation of proteins with molecular masses exceeding 10000 daltons. Analytical Chemistry, 60, 22992301.

Karunaratne, S., Hemingway, J., Jayawardena, K.G.I., Dassanayaka, V. and Vaughan, A. (1995) Kinetic and molecular differences in the amplified and non-amplified esterases from insecticide-resistant and susceptible Culex quinquefasciatus mosquitoes. Journal of Biological Chemistry, 270, 3112431128.

Kasai, S. and Scott, J.G. (2000) Overexpression of cytochrome P450CYP6D1 is associated with monooxygenase-mediated pyrethroid resistance in house flies from Georgia. Pesticide Biochemistry and Physiology, 68, 34-41.

Keen, J.H. and Jakoby, W.B. (1978) Glutathione transferases EC 2.5.1.18. Catalysis of nucleophilic reactions of glutathione. Journal of Biological Chemistry, 253, 5654-5657.

Keeran, W.S. and Lee, R.F. (1987) The purification and characterization of glutathione S-transferase from the hepatopancreas of the blue-crab, Callinectes sapidus. Archives of Biochemistry and Biophysics, 255, 233-243.

Ketterer, B. (1986) Detoxication reactions of glutathione and glutathione transferases. Xenobiotica, 16, 957-973.

Ketterer, B., Rossmans.P and Whitehea.Jk (1967) Isolation of carcinogen-binding protein from livers of rats given 4-dimethylaminoazobenzene. Biochemical Journal, 103, 316-324.

Khajehali, J., Van Leeuwen, T., Grispou, M., Morou, E., Alout, H., Weill, M., Tirry, L., Vontas, J. and Tsagkarakou, A. (2010) Acetylcholinesterase point mutations in European strains of Tetranychus urticae (Acari: Tetranychidae) resistant to organophosphates. Pest Management Science, 66, 220-228.

Khidekel, N. and Hsieh-Wilson, L.C. (2004) A 'molecular switchboard' - covalent modifications to proteins and their impact on transcription. Organic \& Biomolecular Chemistry, 2, 1-7.

Kim, J., Suh, H., Kim, S., Kim, K., Ahn, C. and Yim, J. (2006) Identification and characteristics of the structural gene for the Drosophila eye colour mutant sepia, encoding PDA synthase, a member of the Omega class glutathione Stransferases. Biochemical Journal, 398, 451-460.

Kim, J.E., Bauer, M.M., Mendoza, K.M., Reed, K.M. and Coulombe, R.A., Jr. (2009) Comparative genomics identifies new \&alpha; class genes within the avian glutathione S-transferase gene cluster. Gene, 452, 45-53.

Kim, K., Kim, J., Suh, H., Kim, S. and Yim*, J. (2007) Functional study of Drosophila melanogaster Omega-class glutathione S-transferase (DmGSTO). Program and Abstracts. 48th Annual Drosophila Research Conference, Philadelphia, PA, 2007, 876C. 
Kinumi, T., Niwa, H. and Matsumoto, H. (2000) Phosphopeptide sequencing by in-source decay spectrum in delayed extraction matrix-assisted laser desorption ionization time-of-flight mass spectrometry. Analytical Biochemistry, 277, 177-186.

Kirsch, R., Fleischner, G., Kamisaka, K. and Arias, I.M. (1975) Structural and functional studies of ligandin, a major renal organic anion binding protein. Journal of Clinical Investigation, 55, 1009-1019.

Kitagawa, E., Takahashi, J., Momose, Y. and Iwahashi, H. (2002) Effects of the pesticide thiuram: Genome-wide screening of indicator genes by yeast DNA microarray. Environmental Science \& Technology, 36, 3908-3915.

Koehler, P.G., Strong, C.A., Patterson, R.S. and Valles, S.M. (1993) Differential susceptibility of german -cockroach (Dictyoptera, Blattellidae) sexes and nymphal age classes to insecticides. Journal of Economic Entomology, 86, 785-792.

Komagata, O., Kasai, S. and Tomita, T. (2010) Overexpression of cytochrome $\mathrm{P} 450$ genes in pyrethroid-resistant Culex quinquefasciatus. Insect Biochemistry and Molecular Biology, 40, 146-152.

Koonin, E.V. and Galperin, M.Y. (2003) Sequence - Evolution - Function: Computational Approaches in Comparative Genomics. In Genome annotation and analysis. Boston.

Kosower, E.M. (1976) Chemical properties of glutathione. Arias, Irwin M. And William B. Jakoby (Ed.). Kroc Foundation Series, Vol. 6. Glutathione: Metabolism and Function. Workshop. Santa Ynez, Calif., U.S.A., June 2-3, 1975. Xiii+382p. Illus. Raven Press: New York, N.Y., U.S.A. Isbn 0-89004062-1, 1-15.

Kostaropoulos, I., Mantzari, A.E. and Papadopoulos, A.I. (1996) Alterations of some glutathione S-transferase characteristics during the development of Tenebrio molitor (Insecta:Coleoptera). Insect Biochemistry and Molecular Biology, 26, 963-969.

Kostaropoulos, I. and Papadopoulos, A.I. (1998) Glutathione S-transferase isoenzymes expressed in the three developmental stages of the insect Tenebrio molitor. Insect Biochemistry and Molecular Biology, 28, 901-909.

Kostaropoulos, I., Papadopoulos, A.I., Metaxakis, A., Boukouvala, E. and Papadopoulou-Mourkidou, E. (2001a) Glutathione S-transferase in the defence against pyrethroids in insects. Insect Biochemistry and Molecular Biology, 31, 313-319.

Kostaropoulos, I., Papadopoulos, A.I., Metaxakis, A., Boukouvala, E. and Papadopoulou-Mourkidou, E. (2001b) The role of glutathione Stransferases in the detoxification of some organophosphorus insecticides in larvae and pupae of the yellow mealworm, Tenebrio molitor (Coleoptera : Tenebrionidae). Pest Management Science, 57, 501-508.

Kotze, A.C. and Rose, H.A. (1987) Glutathione S-transferase in the australian sheep blowfly, Lucilia cuprina (wiedemann). Pesticide Biochemistry and Physiology, 29, 77-86. 
Kotze, A.C. and Sales, N. (1995) Elevated in vitro monooxygenase activity associated with insecticide resistances in field strain larvae of the Australian sheep blowfly (Diptera, Calliphoridae). Journal of Economic Entomology, 88, $782-787$.

Kotze, A.C., Sales, N. and Barchia, I.M. (1997) Diflubenzuron tolerance associated with monooxygenase activity in field strain larvae of the Australian sheep blowfly (Diptera: Calliphoridae). Journal of Economic Entomology, 90, 1520.

Krishna, R. and Wold, F. (1997) Identification of common post translational modifications 2 edn. New York: Oxford University Press.

Kristensen, M. (2005) Glutathione S-transferase and insecticide resistance in laboratory strains and field populations of Musca domestica. Journal of Economic Entomology, 98, 1341-1348.

Krohne-Ehrich, G., Schirmer, R.H. and Untuchtgrau, R. (1977) Glutathione reductase from human erythrocytes - isolation of enzyme and sequence analysis of redox active peptide. European Journal of Biochemistry, 80, 6571.

Kura, T., Takahashi, Y., Takayama, T., Ban, N., Saito, T., Kuga, T. and Niitsu, Y. (1996) Glutathione S-transferase-pi is secreted as a monomer into human plasma by platelets and tumor cells. Biochimica Et Biophysica Acta-Protein Structure and Molecular Enzymology, 1292, 317-323.

Kuzmich, S., Vanderveer, L.A. and Tew, K.D. (1991) Evidence for a glycoconjugate form of glutathione S-transferase pi. International Journal of Peptide and Protein Research, 37, 565-571.

Laborde, E. (2010) Glutathione transferases as mediators of signaling pathways involved in cell proliferation and cell death. Cell Death and Differentiation, 17, 1373-1380.

Laemmli, U.K. (1970) Cleavage of structural proteins during assembly of head of bacteriophage-T4. Nature, 227, 680-685.

Lam, B.K., Penrose, J.F., Freeman, G.J. and Austen, K.F. (1994) Expression cloning of a cDNA for human leukotriene C-4 synthase, an integral membrane-protein conjugating reduced glutathione to leukotriene $\mathrm{A}(4)$. Proceedings of the National Academy of Sciences of the United States of America, 91, 7663-7667.

Larkin, M.A., Blackshields, G., Brown, N.P., Chenna, R., McGettigan, P.A., McWilliam, H., Valentin, F., Wallace, I.M., Wilm, A., Lopez, R., Thompson, J.D., Gibson, T.J. and Higgins, D.G. (2007) Clustal W and clustal X version 2.0. Bioinformatics, 23, 2947-2948.

Laroche, S.D. and Leisinger, T. (1990) Sequence analysis and expression of the bacterial dichloromethane dehalogenase structural gene, a member of the glutathione S-transferase supergene family Journal of Bacteriology, 172, 164-171.

Le Goff, G., Boundy, S., Daborn, P.J., Yen, J.L., Sofer, L., Lind, R., Sabourault, C., Madi-Ravazzi, L. and ffrench-Constant, R.H. (2003) Microarray 
analysis of cytochrome P450 mediated insecticide resistance in Drosophila. Insect Biochemistry and Molecular Biology, 33, 701-708.

Le Goff, G., Hilliou, F., Siegfried, B.D., Boundy, S., Wajnberg, E., Sofer, L., Audant, P., Ffrench-Constant, R.H. and Feyereisen, R. (2006) Xenobiotic response in Drosophila melanogaster: Sex dependence of P450 and GST gene induction. Insect Biochemistry and Molecular Biology, 36, 674-682.

Lee, K.H. (2001) Proteomics: a technology-driven and technology-limited discovery science. Trends in Biotechnology, 19, 217-222.

Leier, I., Jedlitschky, G., Buchholz, U., Cole, S.P.C., Deeley, R.G. and Keppler, D. (1994) The MRP gene encodes an ATPdependent export pump for leukotriene $\mathrm{C} 4$ and structurally related conjugates. Journal of Biological Chemistry, 269, 27807-27810.

Levot, G.W. (1995) Resistance and the control of sheep ectoparasites. International Journal for Parasitology, 25, 1355-1362.

Lewis, J.B. (1969) Detoxification of diazinon by subcellular fractions of diazinon resistant and susceptible houseflies Nature, 224, 917-918.

Lewis, J.B. and Sawicki, R.M. (1971) Characterization of the resistance mechanisms to diazinon, parathion and diazoxon in the organophosphorus resistant SKA strain of houseflies Musca domestica. Pesticide Biochemistry and Physiology, 1, 275-285.

Li, D., Scherfer, C., Korayem, A.M., Zhao, Z., Schmidt, O. and Theopold, U. (2002) Insect hemolymph clotting: evidence for interaction between the coagulation system and the prophenoloxidase activating cascade. Insect Biochemistry and Molecular Biology, 32, 919-928.

Liebman, J.F. and Greenberg, A. (1988) Molecular structure energetics vol. 9. Mechanistic principles of enzyme activity. Liebman, J. F. And A. Greenberg (Ed.). Molecular Structure Energetics, Vol. 9. Mechanistic Principles of Enzyme Activity. Xii+404p. Vch Publishers, Inc.: New York, New York, USA; Vch Verlagsgesellschaft Mbh: Weinheim, West Germany. Illus, XII+404P.

Lindner, H. and Helliger, W. (2001) Age-dependent deamidation of asparagine residues in proteins. Experimental Gerontology, 36, 1551-1563.

Listowsky, I. (2005) Proposed intracellular regulatory functions of glutathione transferases by recognition and binding to S-glutathiolated proteins. Journal of Peptide Research, 65, 42-46.

Listowsky, I., Tew, K.D., Pickett, C.B., Mantle, T.J., Mannervik, B. and Hayes, J.D. (1993) High capacity binding by glutathione S-transferases and glucocorticoid resistance, in structure and function of glutathione transferases. CRC Press, Boca Raton. FL, 199-209.

Liu, L.F. and Tam, M.F. (1991) Nucleotide sequence of a class mu glutathione Stransferase from chicken liver. Biochimica Et Biophysica Acta, 1090, 343344.

Liu, N., Xu, Q., Zhu, F. and Zhang, L. (2006) Pyrethroid resistance in mosquitoes. Insect Science, 13, 159-166. 
Liu, N.N., Liu, H.Q., Zhu, F. and Zhang, L. (2007) Differential expression of genes in pyrethroid resistant and susceptible mosquitoes, Culex quinquefasciatus (S.). Gene, 394, 61-68.

Liu, N.N. and Yue, X. (2001) Genetics of pyrethroid resistance in a strain (ALHF) of house flies (Diptera : Muscidae). Pesticide Biochemistry and Physiology, 70, 151-158.

Lo, H.W. and Ali-Osman, F. (2007) Genetic pollymorphism and function of glutathione S-transferases in tumor drug resistance. Current Opinion in Pharmacology, 7, 367-374.

Low, W.Y., Feil, S.C., Ng, H.L., Gorman, M.A., Morton, C.J., Pyke, J., McConville, M.J., Bieri, M., Mok, Y.F., Robin, C., Gooley, P.R., Parker, M.W. and Batterham, P. (2010) Recognition and Detoxification of the Insecticide DDT by Drosophila melanogaster Glutathione S-Transferase D1. Journal of Molecular Biology, 399, 358-366.

Lumjuan, N., McCarroll, L., Prapanthadara, L.A., Hemingway, J. and Ranson, H. (2005) Elevated activity of an Epsilon class glutathione transferase confers DDT resistance in the dengue vector, Aedes aegypti. Insect Biochemistry and Molecular Biology, 35, 861-871.

Lumjuan, N., Stevenson, B.J., Prapanthadara, L.A., Somboon, P., Brophy, P.M., Loftus, B.J., Severson, D.W. and Ranson, H. (2007) The Aedes aegypti glutathione transferase family. Insect Biochemistry and Molecular Biology, 37, 1026-1035.

Ma, B., Zhang, K.Z., Hendrie, C., Liang, C.Z., Li, M., Doherty-Kirby, A. and Lajoie, G. (2003) PEAKS: powerful software for peptide de novo sequencing by tandem mass spectrometry. Rapid Communications in Mass Spectrometry, 17, 2337-2342.

Maa, W.C.J. and Chuang, M.L. (1983) Esterase of diamond black moth (Plutella xylostella) 1. Enzymatic properties of larval esterases Bulletin of the Institute of Zoology Academia Sinica, 22, 123-131.

Mann, M., Hojrup, P. and Roepstorff, P. (1993) Use of mass spectrometric molecular weight information to identify proteins in sequence databases. Biological Mass Spectrometry, 22, 338-345.

Mann, M., Ong, S.E., Gronborg, M., Steen, H., Jensen, O.N. and Pandey, A. (2002) Analysis of protein phosphorylation using mass spectrometry: deciphering the phosphoproteome. Trends in Biotechnology, 20, 261-268.

Mann, M. and Talbo, G. (1996) Developments in matrix-assisted laser desorption ionization peptide mass spectrometry. Current Opinion in Biotechnology, 7, 11-19.

Mannervik, B. (1985) The isoenzymes of glutathione transferase. Adv Enzymol Relat Areas Mol Biol, 57, 357-417.

Mannervik, B., Alin, P., Guthenberg, C., Jensson, H., Tahir, M.K., Warholm, M. and Jornvall, H. (1985) Identification of 3 classes of cytosolic glutathione transferase common to several mammalian species - Correlation 
between structural data and enzymatic properties Proceedings of the National Academy of Sciences of the United States of America, 82, 7202-7206.

Mannervik, B. and Danielson, U.H. (1988) Glutathione transferases- structure and catalytic activity. Crc Critical Reviews in Biochemistry, 23, 283-337.

Manning, G., Plowman, G.D., Hunter, T. and Sudarsanam, S. (2002) Evolution of protein kinase signaling from yeast to man. Trends in Biochemical Sciences, 27, 514-520.

Margis, R., Dunand, C., Teixeira, F.K. and Margis-Pinheiro, M. (2008) Glutathione peroxidase family - an evolutionary overview. Febs Journal, 275, 3959-3970.

Marten, T.R. and Ruane, R.J. (1980) Drug metabolism studies using HPLC. Chromatographia, 13, 137-140.

Martin, T., Chandre, F., Ochou, O.G., Vaissayre, M. and Fournier, D. (2002) Pyrethroid resistance mechanisms in the cotton bollworm Helicoverpa armigera (Lepidoptera : Noctuidae) from West Africa. Pesticide Biochemistry and Physiology, 74, 17-26.

Martinez, O. and Goud, B. (1998) Rab proteins. Biochimica Et Biophysica ActaMolecular Cell Research, 1404, 101-112.

Martinoia, E., Grill, E., Tommasini, R., Kreuz, K. and Amrhein, N. (1993) ATPdependent glutathione S-conjugate export pump in the vacuolar membrane of plants. Nature, 364, 247-249.

McEwen, G.K., Goode, D.K., Parker, H.J., Woolfe, A., Callaway, H. and Elgar, G. (2009) Early evolution of conserved regulatory sequences associated with development in vertebrates. PLoS Genet, 5, e1000762.

Meijerman, I., Beijnen, J.H. and Schellens, J.H.M. (2008) Combined action and regulation of phase II enzymes and multidrug resistance proteins in multidrug resistance in cancer. Cancer Treatment Reviews, 34, 505-520.

Meyer, D.J., Coles, B., Pemble, S.E., Gilmore, K.S., Fraser, G.M. and Ketterer, B. (1991) Theta, a new class of glutathione transferases purified from rat and man. Biochemical Journal, 274, 409-414.

Meyer, D.J. and Thomas, M. (1995) Characterization of rat spleen prostaglandin-H D isomerase as a sigma class GSH transferase. Biochemical Journal, 311, 739-742.

Michalski, W.P. and Shiell, B.J. (1999) Strategies for analysis of electrophoretically separated proteins and peptides. Analytica Chimica Acta, 383, 27-46.

Misra, S., Crosby, M.A., Mungall, C.J., Matthews, B.B., Campbell, K.S., Hradecky, P., Huang, Y., Kaminker, J.S., Millburn, G.H., Prochnik, S.E., Smith, C.D., Tupy, J.L., Whitfied, E.J., Bayraktaroglu, L., Berman, B.P., Bettencourt, B.R., Celniker, S.E., de Grey, A.D.N.J., Drysdale, R.A., Harris, N.L., Richter, J., Russo, S., Schroeder, A.J., Shu, S.Q., Stapleton, M., Yamada, C., Ashburner, M., Gelbart, W.M., Rubin, G.M. and Lewis, S.E. (2002) Annotation of the Drosophila melanogaster 
euchromatic genome: a systematic review. Genome Biol, 3, RESEARCH0083.

Mittapalli, O., Neal, J.J. and Shukle, R.H. (2007) Tissue and life stage specificity of glutathione S-transferase expression in the Hessian fly, Mayetiola destructor: Implications for resistance to host allelochemicals. Journal of Insect Science (Tucson), 7, Article No.: 20.

Morel, F., Rauch, C., Petit, E., Piton, A., Theret, N., Coles, B. and Guillouzo, A. (2004) Gene and protein characterization of the human glutathione Stransferase Kappa and evidence for a peroxisomal localization*. Journal of Biological Chemistry, 279, 16246-16253.

Morgenstern, R., Guthenberg, C. and Depierre, J.W. (1982) Microsomal glutathione S-transferase- purification, initial characterization and demonstration that it is not identical to the cytosolic glutathione S-transferase -A, S-transferase B and S-transferase C. European Journal of Biochemistry, 128, 243-248.

Morrow, C.S., Peklak-Scott, C., Bishwokarma, B., Kute, T.E., Smitherman, P.K. and Townsend, A.J. (2006) Multidrug resistance protein 1 (MRP1, $\mathrm{ABCC} 1)$ mediates resistance to mitoxantrone via glutathione-dependent drug efflux. Molecular Pharmacology, 69, 1499-1505.

Motoyama, N. and Dauterman, W.C. (1977) Purification and properties of housefly glutathione S-transferase. Insect Biochemistry, 7, 361-369.

Motoyama, N. and Dauterman, W.C. (1980) Glutathione S-transferases: their role in the metabolism of organophosphorus insecticides. Rev. Biochem.Toxicol, 2, 49-69.

Motoyama, N., Kulkarni, A.P., Hodgson, E. and Dauterman, W.C. (1978) Endogenous inhibitors of glutathione S-transferases in houseflies. Pesticide Biochemistry and Physiology, 9, 255-262.

Mouatcho, J.C., Munhenga, G., Hargreaves, K., Brooke, B.D., Coetzee, M. and Koekemoer, L.L. (2009) Pyrethroid resistance in a major African malaria vector Anopheles arabiensis from Mamfene, northern KwaZulu-Natal, South Africa. South African Journal of Science, 105, 127-131.

Nakashita, H., Kozuka, K., Hidaka, T., Hara, O. and Seto, H. (2000) Identification and expression of the gene encoding phosphonopyruvate decarboxylase of Streptomyces hygroscopicus. Biochimica Et Biophysica Acta-Gene Structure and Expression, 1490, 159-162.

Narayanan, S.R. and Crane, L.J. (1990) Affinity chromatography supports -A look at performance requirements. Trends in Biotechnology, 8, 12-16.

Natale, D.A., Shankavaram, U.T., Ggalerin, M.Y., Wolf, Y.I., Aravind, L. and Koonin, E.V. (2000) Towards understanding the first genome sequence of a crenarchaeon by genome annotation using clusters of orthologous groups of proteins (COGs). Genome Biology, 1.

Neubauer, G. and Mann, M. (1999) Mapping of phosphorylation sites of gelisolated proteins by nanoelectrospray tandem mass spectrometry: Potentials and limitations. Analytical Chemistry, 71, 235-242. 
Newcomb, R.D., Campbell, P.M., Ollis, D.L., Cheah, E., Russell, R.J. and Oakeshott, J.G. (1997a) A single amino acid substitution converts a carboxylesterase to an organophosphorus hydrolase and confers insecticide resistance on a blowfly. Proceedings of the National Academy of Sciences of the United States of America, 94, 7464-7468.

Newcomb, R.D., Campbell, P.M., Russell, R.J. and Oakeshott, J.G. (1997b) cDNA cloning, baculovirus-expression and kinetic properties of the esterase, E3, involved in organophosphorus resistance in Lucilia cuprina. Insect Biochemistry and Molecular Biology, 27, 15-25.

Nicholson, D.W., Ali, A., Vaillancourt, J.P., Kargman, S., Zamboni, R.J. and Ford-Hutchinson, A.W. (1993) Purification to homogeneity of human leukotriene C-4 synthase: A unique homodimeric glutathione S-transferase comprised of $18 \mathrm{kDa}$ subunits. FASEB Journal, 7, A217.

Oakley, A.J. (2005) Glutathione transferases: new functions. Current Opinion in Structural Biology, 15, 716-723.

Oda, Y., Nagasu, T. and Chait, B.T. (2001) Enrichment analysis of phosphorylated proteins as a tool for probing the phosphoproteome. Nature Biotechnology, 19, 379-382.

Oppenoorth, F.J. (1984) Biochemistry and genetics of insecticide resistance. In Comprehensive insect physiology biochemistry and pharmacology (Kerkut, G.A. and Gilbert, L.I. eds): Pergamon Press, pp. 731-774.

Oppenoorth, F.J., Vanderpas, L.J.T. and Houx, N.W.H. (1979) Glutathione Stransferase and hydrolytic activity in a tetrachlorvinphos resistant strain of housefly and their influence on resistance Pesticide Biochemistry and Physiology, 11, 176-188.

Orser, C.S., Dutton, J., Lange, C., Jablonski, P., Xun, L.Y. and Hargis, M. (1993) Characterization of a Flavobacterium glutathione S-transferase gene involved in reductive dechlorination Journal of Bacteriology, 175, 26402644.

Ortelli, F., Rossiter, L.C., Vontas, J., Ranson, H. and Hemingway, J. (2003) Heterologous expression of four glutathione transferase genes genetically linked to a major insecticide-resistance locus from the malaria vector Anopheles gambiae. Biochemical Journal, 373, 957-963.

Pandey, A., Podtelejnikov, A.V., Blagoev, B., Bustelo, X.R., Mann, M. and Lodish, H.F. (2000) Analysis of receptor signaling pathways by mass spectrometry: Identification of Vav-2 as a substrate of the epidermal and platelet-derived growth factor receptors. Proceedings of the National Academy of Sciences of the United States of America, 97, 179-184.

Papadopoulos, A.I., Polemitou, I., Laifi, P., Yiangou, A. and Tananaki, C. (2004a) Glutathione S-transferase in the developmental stages of the insect Apis mellifera macedonica. Comparative Biochemistry and Physiology $C$ Toxicology \& Pharmacology, 139, 87-92.

Papadopoulos, A.I., Polemitou, I., Laifi, P., Yiangou, A. and Tananaki, C. (2004b) Glutathione S-transferase in the insect Apis mellifera macedonica - 
Kinetic characteristics and effect of stress on the expression of GST isoenzymes in the adult worker bee. Comparative Biochemistry and Physiology C-Toxicology \& Pharmacology, 139, 93-97.

Pappin, D.J.C., Hojrup, P. and Bleasby, A.J. (1993) Rapid identification of proteins by peptide mass fingerprinting Current Biology, 3, 327-332.

Patterson, S.D. (1995) Matrix-assisted laser desorption ionisation mass spectrometric approaches for the identification of gel separated proteins in the 5-50 pmol range Electrophoresis, 16, 1104-1114.

Patterson, S.D. (2000) Proteomics: the industrialization of protein chemistry. Current Opinion in Biotechnology, 11, 413-418.

Pattinson, N. (1981) Purification by affinity chromatography of glutathione Stransferase A and C from rat liver cytosol Analytical Biochemistry, 115, 424427.

Pearson, W.R. (2005) Phylogenies of glutathione transferase families. Gluthione Transferases and Gamma-Glutamyl Transpeptidases, 401, 186-204.

Pemble, S.E., Wardle, A.F. and Taylor, J.B. (1996) Glutathione S-transferase class Kappa: Characterization by the cloning of rat mitochondrial GST and identification of a human homologue. Biochemical Journal, 319, 749-754.

Pennington, S.R., Wilkins, M.R., Hochstrasser, D.F. and Dunn, M.J. (1997) Proteome analysis: From protein characterization to biological function. Trends in Cell Biology, 7, 168-173.

Petros, W.P., Hopkins, P.J., Spruill, S., Broadwater, G., Vredenburgh, J.J., Colvin, O.M., Peters, W.P., Jones, R.B., Hall, J. and Marks, J.R. (2005) Associations between drug metabolism genotype, chemotherapy pharmacokinetics, and overall survival in patients with breast cancer. Journal of Clinical Oncology, 23, 6117-6125.

Pettersson, P.L. and Mannervik, B. (2000) The isomerization of Delta(5)androstene-3,17-dione catalyzed by human glutathione transferase A1-1. In International Conference on Glutathione Transferases. Uppsala, Sweden, pp. 196-199.

Pettersson, P.L. and Mannervik, B. (2001) The role of glutathione in the isomerization of Delta(5)-androstene-3,17-dione catalyzed by human glutathione transferase A1-1. Journal of Biological Chemistry, 276, 1169811704.

Plant, J.W. (2006) Sheep ectoparasite control and animal welfare. Small Ruminant Research, 62, 109-112.

Posewitz, M.C. and Tempst, P. (1999) Immobilized gallium(III) affinity chromatography of phosphopeptides. Analytical Chemistry, 71, 2883-2892.

Prapanthadara, L., Promtet, N., Koottathep, S., Somboon, P., Suwonkerd, W., McCarroll, L. and Hemingway, J. (2002) Mechanisms of DDT and permethrin resistance in Aedes aegypti from Chiang Mai, Thailand. Dengue Bulletin, 26, 185-189. 
Prapanthadara, L.A., Hemingway, J. and Ketterman, A.J. (1993) Partial purification and characterization of glutathione S-transferases involved in DDT resistance from the mosquito Anopheles gambiae Pesticide Biochemistry and Physiology, 47, 119-133.

Prapanthadara, L.A., Ranson, H., Somboon, P. and Hemingway, J. (1998) Cloning, expression and characterization of an insect class I glutathione Stransferase from Anopheles dirus species B. Insect Biochemistry and Molecular Biology, 28, 321-329.

Prohaska, J.R. (1980) Glutathione -peroxidase activity of glutathione Stransferases. Biochimica Et Biophysica Acta, 611, 87-98.

Prohaska, J.R., Oh, S.H., Hoekstra, W.G. and Ganther, H.E. (1977) Glutathione peroxidase - inhibition by cyanide and release of selenium. Biochemical and Biophysical Research Communications, 74, 64-71.

Radola, B.J., Graesslin, D., Sluyterman, L.A.Æ. and Wijdenes, J. eds (1977) Chromatofocusing: isoelectric focusing on ion exchangers in the absence of an externally applied potential Berlin: In Proc. Int. Symp.

Ranson, H., Claudianos, C., Ortelli, F., Abgrall, C., Hemingway, J., Sharakhova, M.V., Unger, M.F., Collins, F.H. and Feyereisen, R. (2002) Evolution of supergene families associated with insecticide resistance. Science, 298, 179-181.

Ranson, H. and Hemingway, J. (2005) Mosquito glutathione transferases. Glutathione Transferases and Gamma-Glutamyl Transpeptidases, 401, 226238.

Ranson, H., Paton, M.G., Jensen, B., McCarroll, L., Vaughan, A., Hogan, J.R., Hemingway, J. and Collins, F.H. (2004) Genetic mapping of genes conferring permethrin resistance in the malaria vector, Anopheles gambiae. Insect Molecular Biology, 13, 379-386.

Ranson, H., Prapanthadara, L.A. and Hemingway, J. (1997) Cloning and characterization of two glutathione S-transferases from a DDT-resistant strain of Anopheles gambiae. Biochemical Journal, 324, 97-102.

Ranson, H., Rossiter, L., Ortelli, F., Jensen, B., Wang, X.L., Roth, C.W., Collins, F.H. and Hemingway, J. (2001) Identification of a novel class of insect glutathione S-transferases involved in resistance to DDT in the malaria vector Anopheles gambiae. Biochemical Journal, 359, 295-304.

Rao, A. and Shaha, C. (2000) Role of glutathione S-transferases in oxidative stressinduced male germ cell apoptosis. Free Radical Biology and Medicine, 29, 1015-1027.

Rattan, S.I.S. (1996) Synthesis, modifications, and turnover of proteins during aging. Experimental Gerontology, 31, 33-47.

Rebay, I. and Fehon, R.G. (2009a) Preparation of insoluble GST fusion proteins. Cold Spring Harb Protoc, 2009, pdb.prot4997.

Rebay, I. and Fehon, R.G. (2009b) Preparation of soluble GST fusion proteins. Cold Spring Harb Protoc, 2009, pdb.prot4996. 
Reese, M.G., Hartzell, G., Harris, N.L., Ohler, U., Abril, J.F. and Lewis, S.E. (2000) Genome annotation assessment in Drosophila melanogaster. Genome Research, 10, 483-501.

Rice, R.H., Means, G.E. and Brown, W.D. (1977) Stabilization of bovine trypsin by reductive methylation Biochimica Et Biophysica Acta, 492, 316-321.

Ridderstrom, M., Jemth, P., Cameron, A.D. and Mannervik, B. (2000) The active-site residue Tyr-175 in human glyoxalase II contributes to binding of glutathione derivatives. Biochimica Et Biophysica Acta-Protein Structure and Molecular Enzymology, 1481, 344-348.

Robinson, A.B. and Robinson, L.R. (1991) Distribution of glutamine and asparagine residues and their near neighbors in peptides and proteins. Proceedings of the National Academy of Sciences of the United States of America, 88, 8880-8884.

Rodriguez, M.A., Bosch, D., Sauphanor, B. and Avilla, J. (2010) Susceptibility to organophosphate insecticides and activity of detoxifying enzymes in spanish populations of Cydia pomonella (Lepidoptera: Tortricidae). Journal of Economic Entomology, 103, 482-491.

Rogers, I., Hendrie, C. and Li, M. (2004) Protein ID: Comparing de novo based and database search methods. Protein Science, 13, 586.

Rogers, M.E., Jani, M.K. and Vogt, R.G. (1999) An olfactory-specific glutathioneS-transferase in the sphinx moth Manduca sexta. Journal of Experimental Biology, 202, 1625-1637.

Rogers, S.W. and Rechsteiner, M. (1988) Degradation of structurally characterized proteins injected into Hela cells - basic measurements. Journal of Biological Chemistry, 263, 19833-19842.

Rouimi, P., Anglade, P., Benzekri, A., Costet, P., Debrauwer, L., Pineau, T. and Tulliez, J. (2001) Purification and characterization of a glutathione Stransferase Omega in pig: evidence for two distinct organ-specific transcripts. Biochemical Journal, 358, 257-262.

Rufingier, C., Pasteur, N., Lagnel, J., Martin, C. and Navajas, M. (1999) Mechanisms of insecticide resistance in the aphid Nasonovia ribisnigri (Mosley) (Homoptera : Aphididae) from France. Insect Biochemistry and Molecular Biology, 29, 385-391.

Saleh, M.A., Motoyama, N. and Dauterman, W.C. (1978) Reduced glutathione in housefly- concentration during development and variation in strains. Insect Biochemistry, 8, 311-316.

Salzano, M. and Crescenzi, M. (2005) Mass spectrometry for protein identification and the study of post translational modifications. Annali dell'Istituto Superiore di Sanita, 41, 443-450.

Samiec, P.S., Dahm, L.J. and Jones, D.P. (2000) Glutathione S-transferase in mucus of rat small intestine. Toxicological Sciences, 54, 52-59. 
Sau, A., Pellizzari Tregno, F., Valentino, F., Federici, G. and Caccuri, A.M. (2010) Glutathione transferases and development of new principles to overcome drug resistance. Arch Biochem Biophys, 500, 116-122.

Sawicki, R., Singh, S.P., Mondal, A.K., Benes, H. and Zimniak, P. (2003) Cloning, expression and biochemical characterization of one Epsilon-class (GST-3) and ten Delta-class (GST-1) glutathione S-transferases from Drosophila melanogaster, and identification of additional nine members of the Epsilon class. Biochemical Journal, 370, 661-669.

Scheler, C., Lamer, S., Pan, Z.M., Li, X.P., Salnikow, J. and Jungblut, P. (1998) Peptide mass fingerprint sequence coverage from differently stained proteins on two-dimensional electrophoresis patterns by matrix assisted laser desorption/ionization mass spectrometry (MALDI-MS). Electrophoresis, 19, 918-927.

Schmidt-Krey, I., Mitsuoka, K., Hirai, T., Murata, K., Cheng, Y., Fujiyoshi, Y., Morgenstern, R. and Hebert, H. (2000) The three-dimensional map of microsomal glutathione transferase 1 at 6 angstrom resolution. Embo Journal, 19, 6311-6316.

Shanahan, G.J. (1959) Genetics of Dieldrin resistance in Lucilia cuprina Wied. Nature, 183, 1540-1541.

Sharif, M.M., Hejazi, M.J., Mohammadi, A. and Rashidi, M.R. (2007) Resistance status of the Colorado potato beetle, Leptinotarsa decemlineata, to endosulfan in East Azarbaijan and Ardabil provinces of Iran. Journal of Insect Science (Tucson), 7, 7.31.

Sheehan, D. and Casey, J.P. (1993) Evidence for alpha class and mu class glutathione S-transferases in a number of fungal species Comparative Biochemistry and Physiology B-Biochemistry \& Molecular Biology, 104, 713.

Sheehan, D., Meade, G., Foley, V.M. and Dowd, C.A. (2001) Structure, function and evolution of glutathione transferases: implications for classification of non-mammalian members of an ancient enzyme superfamily. Biochemical Journal, 360, 1-16.

Shen, S., Chien, Y. and Chien, C. (2003) Induction of glutathione S-transferases activities in Drosophila melanogaster exposed to phenol. Archives of Insect Biochemistry and Physiology, 53, 80-91.

Simons, P.C. and Vanderjagt, D.L. (1977) Purification of glutathione S transferases from human liver using glutathione affinity chromatography Analytical Biochemistry, 82, 334-341.

Singh, O.P., Raghavendra, K., Nanda, N., Mittal, P.K. and Subbarao, S.K. (2002) Pyrethroid resistance in Anopheles culicifacies in Surat district, Gujarat, west India. Current Science, 82, 547-550.

Singh, S.P., Coronella, J.A., Benes, H., Cochrane, B.J. and Zimniak, P. (2001) Catalytic function of Drosophila melanogaster glutathione S-transferase DmGSTS1-1 (GST-2) in conjugation of lipid peroxidation end products. European Journal of Biochemistry, 268, 2912-2923. 
Singhal, S.S., Ahmad, H., Sharma, R., Gupta, S., Haque, A.K. and Awasthi, Y.C. (1991) Purification and characterization of human muscle glutathione Stransferases: Evidence that glutathione S-transferase zeta corresponds to a locus distinct from GST1, GST2 and GST3. Archives of Biochemistry and Biophysics, 285, 64-73.

Sluyterman, L.A.A. and Wijdenes, J. (1978) Chromatofocusing isoelectricfocusing on ion exchange columns. 2. Experimental verification Journal of Chromatography, 150, 31-44.

Smirle, M.J. and Winston, M.L. (1987) Intercolony variation in pesticide detoxification by the honeybee (Hymenoptera, Apidae). Journal of Economic Entomology, 80, 5-8.

Snyder, L.R., Kirkland, J.J. and Lloyd, R. (1974) Introduction to modern liquid chromatography New York: Wiley-interscience.

Snyder, M.J. and Maddison, D.R. (1997) Molecular phylogeny of glutathione-Stransferases. DNA and Cell Biology, 16, 1373-1384.

Snyder, M.J., Walding, J.K. and Feyereisen, R. (1995) Glutathione S-transferases from larval Manduca sexta midgut- sequence of 2 cDNAs and enzyme induction. Insect Biochemistry and Molecular Biology, 25, 455-465.

Solary, E., Ribrag, V. and Botto, S.D. (2009) Strategies to overcome drug resistance in acute and chronic leukemias. In ABC transporters and multidrug resistance (hcene Boumendjel, J.B., Jacques Robert ed. Hoboken, New Jersey: John Wiley and Sons, Inc.

Sonoda, S., Ashfaq, M. and Tsumuki, H. (2006) Genomic organization and developmental expression of glutathione S-transferase genes of the diamondback moth, Plutella xylostella. Journal of Insect Science (Tucson), 6, 6.35 .

Sonoda, S. and Tsumuki, H. (2005) Studies on glutathione S-transferase gene involved in chlorfluazuron resistance of the diamondback moth, Plutella xylostella L. (Lepidoptera : Yponomeutidae). Pesticide Biochemistry and Physiology, 82, 94-101.

Sorensen, S.B., Sorensen, T.L. and Breddam, K. (1991) Fragmentation of proteins by Staphylococcus aureus strain-V8 protease - Ammonium bicarbonate strongly inhibits the enzyme but does not improve the selectivity for glutamic acid. Febs Letters, 294, 195-197.

Spector, T. (1978) Refinement of coomassie blue method of protein quantitation simple and linear spectrophptometric assay for less than or equal to 0.5 to 50 mu-g of protein Analytical Biochemistry, 86, 142-146.

Srivastava, S.K., Watkins, S.C., Schuetz, E. and Singh, S.V. (2002) Role of glutathione conjugate efflux in cellular protection against benzo[a]pyrene7,8-diol-9,10-epoxide-induced DNA damage. Molecular Carcinogenesis, 33, 156-162.

Steen, H., Jebanathirajah, J.A., Rush, J., Morrice, N. and Kirschner, M.W. (2006) Phosphorylation analysis by mass spectrometry - Myths, facts, and the 
consequences for qualitative and quantitative measurements. Molecular \& Cellular Proteomics, 5, 172-181.

Steinberg, T.H., Agnew, B.J., Gee, K.R., Leung, W.Y., Goodman, T., Schulenberg, B., Hendrickson, J., Beechem, J.M., Haugland, R.P. and Patton, W.F. (2003) Global quantitative phosphoprotein analysis using multiplexed proteomics technology. Proteomics, 3, 1128-1144.

Stenersen, J., Kobro, S., Bjerke, M. and Arend, U. (1987) Glutathione transferases in aquatic and terrestrial animals from 9 phyla Comparative Biochemistry and Physiology C-Pharmacology Toxicology \& Endocrinology, 86, 73-82.

Stensballe, A., Andersen, S. and Jensen, O.N. (2001) Characterization of phosphoproteins from electrophoretic gels by nanoscale $\mathrm{Fe}(\mathrm{III})$ affinity chromatography with off-line mass spectrometry analysis. Proteomics, 1, 207-222.

Sternberg, J. and Kearns, C.W. (1952) The presence of toxins other than DDT in the blood of DDT-poisoned roaches. Science, 175, 448-449

Stewart, A.E., Arfin, S.M. and Bradshaw, R.A. (1995) The sequence of porcine protein $\mathrm{NH}_{2}$-terminal asparagine amidohydrolase. Journal of Biological Chemistry, 270, 25-28.

Sugimoto, M., Takikawa, H., Stolz, A. and Kaplowitz, N. (1987) Subunit heterogeneity of cationic human hepatic glutathione S-transferases Pharmacology, 35, 65-78.

Sun, C.N. and Ku, C.C. (1994) Glutathione transferase isozymes involved in insecticide resistance of diamondback moth larvae. Resistant Pest Management, $6,8$.

Takemori, N. and Yamamoto, M.T. (2009) Proteome mapping of the Drosophila melanogaster male reproductive system. Proteomics, 9, 2484-2493.

Tamaki, H., Kumagai, H. and Tochikura, T. (1990) Glutathione $S$ transferase in yeast: induction of mRNA, cDNA cloning and expression in Escherichia coli. Biochem. Biophys. Res. Commun., 172, 669-675.

Tamaki, H., Kumagai, H. and Tochikura, T. (1991) Nucleotide sequence of the yeast glutathione S-transferase cDNA Biochimica Et Biophysica Acta, 1089, 276-279.

Tang, A.H. and Tu, C.P.D. (1994) Biochemical characterization of Drosophila glutathione S-transferases D1 and D21. Journal of Biological Chemistry, 269, 27876-27884.

Tang, A.H. and Tu, C.P.D. (1995) Pentobarbital induced changes in Drosophila glutathione s-transferase D21 messenger RNA stability. Journal of Biological Chemistry, 270, 13819-13825.

Taniguchi, H. and Pyerin, W. (1989) Glutathione S-transferase is an invitro substrate of ca++ phospholipid dependent protein kinase (Protein kinase c). Biochemical and Biophysical Research Communications, 162, 903-907. 
Tee, L.B.G., Gilmore, K.S., Meyer, D.J., Ketterer, B., Vandenberghe, Y. and Yeoh, G.C.T. (1992) Expression of glutathione S-transferase during rat-liver development. Biochemical Journal, 282, 209-218.

Tew, K.D. (1994) Glutathione-associated enzymes in anticancer drug-resistance. Cancer Research, 54, 4313-4320.

Thompson, J.D., Higgins, D.G. and Gibson, T.J. (1994) ClustalW - Improving the sensitivity of progressive multiple sequence alignment through sequence weighting, position-specific gap penalties and weight matrix choice Nucleic Acids Research, 22, 4673-4680.

Thornalley, P.J. (2003) Glyoxalase I - structure, function and a critical role in the enzymatic defence against glycation. Biochemical Society Transactions, 31, 1343-1348.

Thornton, B., Elthon, T., Cerny, R. and Siegfried, B. (2010) Proteomic analysis of atrazine exposure in Drosophila melanogaster (Diptera: Drosophilidae). Chemosphere, 2, 235-241.

Toba, G. and Aigaki, T. (2000) Disruption of the Microsomal glutathione Stransferase-like gene reduces life span of Drosophila melanogaster. Gene, 253, 179-187.

Tomarev, S.I. and Zinovieva, R.D. (1988) Squid major lens polypeptides are homologous to glutathione S-transferases subunits Nature, 336, 86-88.

Tomarev, S.I., Zinovieva, R.D., Guo, K. and Piatigorsky, J. (1993) Squid glutathione S-transferase - Relationships with other glutathione Stransferases and S-crystallins of cephalopods Journal of Biological Chemistry, 268, 4534-4542.

Toung, Y.P.S., Hsieh, T.S. and Tu, C.P.D. (1990) Drosophila glutathione Stransferase 1-1 shares a region of sequence homology with the maize glutathione S-transferase-III Proceedings of the National Academy of Sciences of the United States of America, 87, 31-35.

Toung, Y.P.S., Hsieh, T.S. and Tu, C.P.D. (1993) The glutathione S-transferase Dgenes: A divergently organized, intronless gene family in Drosophila melanogaster. Journal of Biological Chemistry, 268, 9737-9746.

Trauner, M. and Boyer, J.L. (2003) Bile salt transporters: Molecular characterization, function, and regulation. Physiological Reviews, 83, 633671.

Tu, C.P.D. and Akgul, B. (2005) Drosophila glutathione S-transferases. Gluthione Transferases and Gamma-Glutamyl Transpeptidases, 401, 204-226.

Tyler-Cross, R. and Schirch, V. (1991) Effects of aminoacid sequence, buffers and ionic strength on the rate and mechanism of deamidation of asparagine residues in small peptides. Journal of Biological Chemistry, 266, 2254922556.

Udomsinprasert, R., Bogoyevitch, M.A. and Ketterman, A.J. (2004) Reciprocal regulation of glutathione $S$-transferase spliceforms and the Drosophila c-Jun N-terminal kinase pathway components. Biochemical Journal, 383, 483-490. 
Udomsinprasert, R., Pongjaroenkit, S., Wongsantichon, J., Oakley, A.J., Prapanthadara, L., Wilce, M.C.J. and Ketterman, A.J. (2005) Identification, characterization and structure of a new Delta class glutathione transferase isoenzyme. Biochemical Journal, 388, 763-771.

Urade, Y., Fujimoto, N., Ujihara, M. and Hayaishi, O. (1987) Biochemical and immunological characterization of rat spleen prostaglandin D-synthetase. Journal of Biological Chemistry, 262, 3820-3825.

Vontas, J., David, J.P., Nikou, D., Hemingway, J., Christophides, G.K., Louis, C. and Ranson, H. (2007) Transcriptional analysis of insecticide resistance in Anopheles stephensi using cross-species microarray hybridization. Insect Molecular Biology, 16, 315-324.

Vontas, J.G., Small, G.J. and Hemingway, J. (2001) Glutathione S-transferases as antioxidant defence agents confer pyrethroid resistance in Nilaparvata lugens. Biochemical Journal, 357, 65-72.

Vontas, J.G., Small, G.J., Nikou, D.C., Ranson, H. and Hemingway, J. (2002) Purification, molecular cloning and heterologous expression of a glutathione S-transferase involved in insecticide resistance from the rice brown planthopper, Nilaparvata lugens. Biochemical Journal, 362, 329-337.

Voss, R.M.E. and van Bladeren, P.J. (1990) Glutathione S-transferases in relation to their role in the biotransformation of xenobiotics. Chem. Biol. Interact., 75, 241-265.

Vulule, J.M., Beach, R.F., Atieli, F.K., McAllister, J.C., Brogdon, W.G., Roberts, J.M., Mwangi, R.W. and Hawley, W.A. (1999) Elevated oxidase and esterase levels associated with permethrin tolerance in Anopheles gambiae from Kenyan villages using permethrin-impregnated nets. Medical and Veterinary Entomology, 13, 239-244.

Walsh, K.A. (1970) Trypsinogens and trypsins of various species. Perlmann, Gertrude E. And Lazlo Lorand (Edited by). Methods in Enzymology, Vol. Xix. Proteolytic Enzymes. Xx +1042 p. Illus. Academic Press: New York, N.Y., U.S.A.; London England, 41-63.

Wang, J.Y., McCommas, S. and Syvanen, M. (1991) Molecular-cloning of a glutathione S-transferase overproduced in an insecticide-resistant strain of the housefly (Musca domestica). Molecular \& General Genetics, 227, 260-266.

Wang, L., Amphlett, G., Lambert, J., Blättler, W. and Zhang, W. (2005) Structural characterization of a recombinant monoclonal antibody by electrospray time-of-flight mass spectrometry. Pharm Res, 22, 1338-1349.

Wasinger, V.C., Cordwell, S.J., Cerpapoljak, A., Yan, J.X., Gooley, A.A., Wilkins, M.R., Duncan, M.W., Harris, R., Williams, K.L. and Humpherysmith, I. (1995) Progress with gene product mapping of the mollicutes Mycoplasma genitalium Electrophoresis, 16, 1090-1094.

Weber, K. and Osborn, M. (1969) Reliability of molecular weight determinations by dodecylsulfate polyacrylamide gel electrophoresis Journal of Biological Chemistry, 244, 4406-4412. 
Weech, M., Quash, M. and Walters, E. (2003) Characterization of the mouse olfactory glutathione s-transferases during the acute phase response. Journal of Neuroscience Research, 73, 679-685.

Wei, S.H., Clark, A.G. and Syvanen, M. (2001) Identification and cloning of a key insecticide-metabolizing glutathione S-transferase (MdGST-6A) from a hyper insecticide-resistant strain of the housefly Musca domestica. Insect Biochemistry and Molecular Biology, 31, 1145-1153.

Werle-Schneider, G., Wolfelschneider, A., von Brevern, M.C., Scheel, J., Storck, T., Muller, D., Glockner, R., Bartsch, H. and Bartelmann, M. (2006) Gene expression profiles in rat liver slices exposed to hepatocarcinogenic enzyme inducers, peroxisome proliferators, and 17 alpha-ethinylestradiol. International Journal of Toxicology, 25, 379-395.

Widmann, C., Gibson, S., Jarpe, M.B. and Johnson, G.L. (1999) Mitogenactivated protein kinase: Conservation of a three-kinase module from yeast to human. Physiological Reviews, 79, 143-180.

Wilce, M.C.J., Board, P.G., Feil, S.C. and Parker, M.W. (1995) Crystal structure of a theta class glutathione S transferase. Embo Journal, 14, 2133-2143.

Wilce, M.C.J. and Parker, M.W. (1994) Structure and function of glutathione Stransferases Biochimica Et Biophysica Acta-Protein Structure and Molecular Enzymology, 1205, 1-18.

Wildenburg, G., Liebau, E. and Henkle-Duhrsen, K. (1998) Onchocerca volvulus: Ultrastructural localization of two glutathione S-transferases. Experimental Parasitology, 88, 34-42.

Wilkins, M.R., Pasquali, C., Appel, R.D., Ou, K., Golaz, O., Sanchez, J.C., Yan, J.X., Gooley, A.A., Hughes, G., HumpherySmith, I., Williams, K.L. and Hochstrasser, D.F. (1996) From proteins to proteomes: Large scale protein identification by two-dimensional electrophoresis and amino acid analysis. Bio-Technology, 14, 61-65.

Willoughby, L., Chung, H., Lumb, C., Robin, C., Batterham, P. and Daborn, P.J. (2006) A comparison of Drosophila melanogaster detoxification gene induction responses for six insecticides, caffeine and phenobarbital. Insect Biochemistry and Molecular Biology, 36, 934-942.

Wilm, M., Neubauer, G. and Mann, M. (1996) Parent ion scans of unseparated peptide mixtures. Analytical Chemistry, 68, 527-533.

Wils, P., Escriou, V., Warnery, A., Lacroix, F., Lagneaux, D., Ollivier, M., Crouzet, J., Mayaux, J.F. and Scherman, D. (1997) Efficient purification of plasmid DNA for gene transfer using triple-helix affinity chromatography. Gene Therapy, 4, 323-330.

Wilson, J.A. (1999) Aspects of Insecticide Resistance in New Zealand Strains of the Sheep Blowflies, Lucilia Cuprina and Lucilia Sericata. In School of Biological Sciences. Wellington: Victoria University of Wellington, pp. 467.

Wilson, J.A. and Clark, A.G. (1996) The role of E3 esterase, glutathione Stransferases and other nonoxidative mechanisms in resistance to diazinon and 
other organophosphate insecticides in Lucilia cuprina. Pesticide Biochemistry and Physiology, 54, 85-95.

Wolkoff, A.W., Bhargave, M.M., Chung, C. and Gatmaitan, Z. (1979) Purification of ligands by affinity chromatography on sulfobromophthalein agarose gel Proceedings of the Society for Experimental Biology and Medicine, 160, 150-153.

Wood, E., Casabe, N., Melgar, F. and Zerba, E. (1986) Distribution and properties of glutathione S-transfearse from T. infestans. Comparative Biochemistry and Physiology B-Biochemistry \& Molecular Biology, 84, 607-617.

Wright, H.T. (1991a) Nonenzymatic deamidation of asparaginyl and glutaminyl residues in proteins. Critical Reviews in Biochemistry and Molecular Biology, 26, 1-52.

Wright, H.T. (1991b) Sequence and structure determinants of the nonenzymatic deamidation of asparagine and glutamine residues in proteins. Protein Engineering, 4, 283-294.

Xin, L., Shan, B., Xie, M., Lajoie, G. and Ma, B. (2009) PTMFinder Based on PEAKS De Novo Sequencing Result, ASMS 2009. Session MPL: Proteomics: PTM Determination (Method Development), Poster \# 295

Yamamoto, K., Miake, F. and Aso, Y. (2007) Purification and characterization of a novel sigma-class glutathione S-transferase of the fall webworm, Hyphantria cunea. Journal of Applied Entomology, 131, 466-471.

Yamamoto, K., Nagaoka, S., Banno, Y. and Aso, Y. (2009a) Biochemical properties of an omega-class glutathione S-transferase of the silkmoth, Bombyx mori. Comparative Biochemistry and Physiology C-Toxicology \& Pharmacology, 149, 461-467.

Yamamoto, K., Shigeoka, Y., Aso, Y., Banno, Y., Kimura, M. and Nakashima, T. (2009b) Molecular and biochemical characterization of a Zeta-class glutathione S-transferase of the silkmoth. Pesticide Biochemistry and Physiology, 94, 30-35.

Yamamoto, K., Zhang, P.B., Miake, F., Kashige, N., Aso, Y., Banno, Y. and Fujii, H. (2005) Cloning, expression and characterization of theta-class glutathione S-transferase from the silkworm, Bombyx mori. Comparative Biochemistry and Physiology B-Biochemistry \& Molecular Biology, 141, 340-346.

Yan, C. and Sloan, D.L. (1987) Purification and characterization of nicotinamide deamidase from yeast. Journal of Biological Chemistry, 262, 9082-9087.

Yang, M.L., Zhang, J.Z., Zhu, K.Y., Xuan, T., Liu, X.J., Guo, Y.P. and Ma, E.B. (2009) Mechanisms of organophosphate resistance in a field population of oriental migratory locust, Locusta migratoria manilensis (Meyen). Archives of Insect Biochemistry and Physiology, 71, 3-15. 
Yang, P., Ebbert, J.O., Sun, Z.F. and Weinshilboum, R.M. (2006) Role of the glutathione metabolic pathway in lung cancer treatment and prognosis: A review. Journal of Clinical Oncology, 24, 1761-1769.

Yang, Y.S., Sharma, R., Sharma, A., Awasthi, S. and Awasthi, Y.C. (2003) Lipid peroxidation and cell cycle signaling: 4-hydroxynonenal, a key molecule in stress mediated signaling. Acta Biochimica Polonica, 50, 319-336.

Yu, S.J. (1992) Detection and biochemical characterization of insecticide resistance in Fall armyworm (lepidoptera, Noctuidae) Journal of Economic Entomology, 85, 675-682.

Yu, S.J. (1996) Insect glutathione S-transferases. Zoological Studies, 35, 9-19.

Zdobnov, E.M., von Mering, C., Letunic, I., Torrents, D., Suyama, M., Copley, R.R., Christophides, G.K., Thomasova, D., Holt, R.A., Subramanian, G.M., Mueller, H.M., Dimopoulos, G., Law, J.H., Wells, M.A., Birney, E., Charlab, R., Halpern, A.L., Kokoza, E., Kraft, C.L., Lai, Z.W., Lewis, S., Louis, C., Barillas-Mury, C., Nusskern, D., Rubin, G.M., Salzberg, S.L., Sutton, G.G., Topalis, P., Wides, R., Wincker, P., Yandell, M., Collins, F.H., Ribeiro, J., Gelbart, W.M., Kafatos, F.C. and Bork, P. (2002) Comparative genome and proteome analysis of anopheles gambiae and Drosophila melanogaster. Science, 298, 149-159.

Zhai, B., Villen, J., Beausoleil, S.A., Mintseris, J. and Gygi, S.P. (2008) Phosphoproteome analysis of Drosophila melanogaster embryos. Journal of Proteome Research, 7, 1675-1682.

Zhang, W.Z. and Chait, B.T. (2000) Profound: An expert system for protein identification using mass spectrometric peptide mapping information. Analytical Chemistry, 72, 2482-2489.

Zhou, W., Merrick, B.A., Khaledi, M.G. and Tomer, K.B. (2000) Detection and sequencing of phosphopeptides affinity bound to immobilized metal ion beads by matrix-assisted laser desorption/ionization mass spectrometry. Journal of the American Society for Mass Spectrometry, 11, 273-282.

Zhou, Z.H. and Syvanen, M. (1997) A complex glutathione transferase gene family in the housefly Musca domestica. Molecular \& General Genetics, 256, 187194.

Zhu, Y.C., Snodgrass, G.L. and Chen, M.S. (2007) Comparative study on glutathione S-transferase activity, cDNA, and gene expression between malathion susceptible and resistant strains of the tarnished plant bug, Lygus lineolaris. Pesticide Biochemistry and Physiology, 87, 62-72.

Zimniak, P., Ziller, S.A., Panfil, I., Radominska, A., Wolters, H., Kuipers, F., Sharma, R., Saxena, M., Moslen, M.T., Vore, M., Vonk, R., Awasthi, Y.C. and Lester, R. (1992) Identification of an anion transport ATPase that catalyzes glutathione conjugate dependent ATP hydrolysis in canalicular plasma membranes from normal rats and rats with conjugated hyperbilirubinemia (GY mutant) Archives of Biochemistry and Biophysics, 292, 534-538. 